
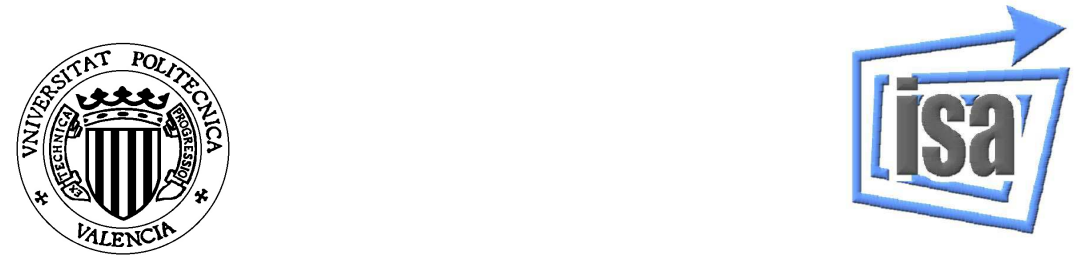

\title{
SENSORES VIRTUALES PARA PROCESOS CON MEDIDAS ESCASAS Y RETARDOS TEMPORALES
}

Autor: Ignacio Peñarrocha Alós

Directores: Pedro Albertos Pérez Roberto Sanchis Llopis 

A mi familia.

A Rebeca. 



\section{Resumen}

En este trabajo se aborda el problema de controlar un proceso cuya salida se muestrea de forma irregular. Para ello se propone utilizar un predictor que estima las salidas del proceso en instantes regulares de tiempo más un controlador convencional que calcula la acción de control a partir de las estimaciones del predictor (técnica conocida como control inferencial).

La predicción consiste en estimar las variables de salida que se desean controlar a partir de las mediciones realizadas con diversos sensores utilizando para ello un modelo matemático del proceso. El filtro de Kalman permite hacer la predicción de forma óptima si las perturbaciones tienen una distribución gaussiana de media cero, pero con el inconveniente de requerir un elevado coste computacional cuando se utilizan diferentes sensores con retardos temporales variantes. En este trabajo se propone una estrategia de predicción alternativa de bajo coste computacional cuyo diseño se basa en el conocimiento de la disponibilidad de mediciones y de los retardos (del proceso, del sistema de medición o del sistema de transmisión de datos) y de la naturaleza de las perturbaciones. Los predictores propuestos minimizan el error de predicción frente al muestreo aleatorio con retardos variantes, perturbaciones, ruido de medida, error de modelado, retardos en la acción de control e incertidumbre en los tiempos de medición. Las diferentes estrategias de diseño que se proponen se clasifican según el tipo de información que se dispone de las perturbaciones y del coste computacional requerido. Se han planteado los diseños para sistemas monovariables, multivariables, lineales y no lineales. Asimismo, también se ha elaborado una forma más eficiente de incluir mediciones escasas con retardo en el filtro de Kalman, con el objetivo de reducir el coste computacional de la predicción.

En este trabajo se demuestra que los sistemas de control inferencial que utilizan los predictores propuestos cumplen con el principio de separación, con lo que el diseño del control con medidas irregulares se reduce al problema del diseño de un predictor estable más el diseño de un controlador para muestreo convencional. 



\section{Resum}

En aquest treball s'aborda el problema de controlar un procés l'eixida del qual es mostreja de forma irregular. Amb eixa finalitat es proposa utilitzar un predictor que estima les eixides del procés en instants regulars de temps més un controlador convencional que calcula l'acció de control a partir de les estimacions del predictor (tècnica coneguda com control inferencial).

La predicció consisteix a estimar les variables d'eixida que es desitgen controlar a partir dels mesuraments realitzats amb diversos sensors utilitzant per a això un model matemàtic del procés. El filtre de Kalman permet fer la predicció de forma òptima si les pertorbacions tenen una distribució gaussiana de mitjana zero, però amb l'inconvenient de requerir un elevat cost computacional quan s'utilitzen diferents sensors amb retards temporals variants. En aquest treball es proposa una estratègia de predicció alternativa de baix cost computacional el disseny del qual es basa en el coneixement de la disponibilitat de mesures i dels retards (del procés, del sistema de mesura o del sistema de transmissió de dades) i de la naturalesa de les pertorbacions. Els predictors proposats minimitzen l'error de predicció enfront del mostreig aleatori amb retards variants, pertorbacions, soroll de mesura, error de modelatge, retards en l'acció de control i incertesa en els temps de mesura. Les diferents estratègies de disseny que es proposen es classifiquen segons el tipus d'informació que es disposa de les pertorbacions i del cost computacional requerit. S'han plantejat els dissenys per a sistemes monovariables, multivariables, lineals i no lineals. Així mateix, també s'ha elaborat una forma més eficient d'incloure mesures escasses amb retard en el filtre de Kalman, amb l'objectiu de reduir el cost computacional de la predicció.

En aquest treball es demostra que els sistemes de control inferencial que utilitzen els predictors proposats compleixen amb el principi de separació, amb què el disseny del control amb mesures irregulars es redueix al problema del disseny d'un predictor estable més el disseny d'un controlador per a mostreig convencional. 



\section{Abstract}

In this work the problem of controlling a process whose output is irregularly measured is addressed. For that purpose a predictor that estimates the outputs of the process at regular instants plus a conventional controller that calculates the control action from the estimations of the predictor is used (technology known as inferential control).

The prediction consists of estimating the output variables that want to be controlled from the measurements taken with several sensors, using for that a mathematical model of the process. The Kalman filter allows to do an optimal prediction if the disturbances have a Gaussian distribution of zero mean, but with the disadvantage of requiring a high computational cost when using several sensors with time-varying delays. In this work an alternative prediction strategy of low computational cost is proposed whose design is based on the knowledge of measurements availability and delays (of the process, measurement system or data transmission line) and the disturbances nature. The proposed predictors minimize the prediction error when dealing with random of time-varying sampling with time-varying delays, disturbances, measurement noise, modelling error, delays on the control action and uncertainty on the knowledge of measurements instants. The different design strategies that are proposed are classified according to the kind of the disturbances information that is known and the required computational cost. Designs for monovariable, multivariable, linear and nonlinear systems have been proposed.

In this work it is demonstrated that the inferential control systems that make use of the proposed predictors fulfil the separation principle, so the design of control systems with irregular measurements is reduced to the problem of designing a stable predictor plus the design of a conventional sampling controller. 



\section{Agradecimientos}

A mis directores de tesis Pedro Albertos y Roberto Sanchis por su apoyo, por la confianza depositada en el trabajo, por sus consejos y por compartir su experiencia durante todo el periodo de formación que he compartido con cada uno de ellos. También agradecer a Romeo Ortega todo el apoyo prestado durante mi estancia en el Supélec.

A mis compañeros en la Universitat Jaume I de Castelló, en la Universitat Politècnica de València y en el Supélec (Francia) con los que he compartido esta etapa de mi formación a la que, sin duda, han contribuido tanto académica como personalmente.

Agradecer sinceramente a mi familia y especialmente a mis padres por haberme dado la educación y un apoyo completo a lo largo de mi carrera. Sin su constante ayuda este trabajo no hubiera sido posible.

Agradecer profundamente a Rebeca su apoyo integral, su paciencia infinita y su transmisión de energía durante el periodo de desarrollo de este trabajo.

A Els Cavallers de la Refesta i la seua Cort d'Honor por apoyarme durante la elaboración de la tesis y comprender mis ausencias.

Finalmente, agradecer a la Generalitat Valenciana por haber financiado durante 48 meses la realización de esta tesis a través del programa de Formación de Personal Investigador (código de la ayuda CTBPRB/2002/245). 



\section{Índice de contenidos}

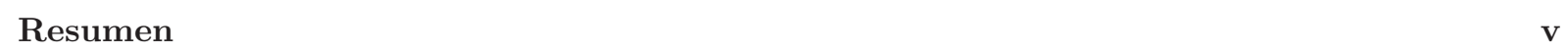

\begin{tabular}{|ll} 
Resum vii & vii
\end{tabular}

\begin{tabular}{|ll}
\hline Abstract ix & ix
\end{tabular}

\begin{tabular}{|l|l|}
\hline Agradecimientos & xi
\end{tabular}

$\begin{array}{ll}\text { Índice de contenidos } & \text { xvi }\end{array}$

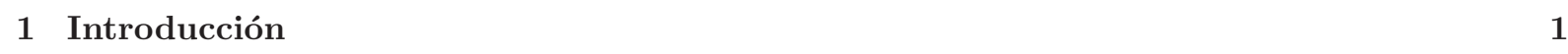

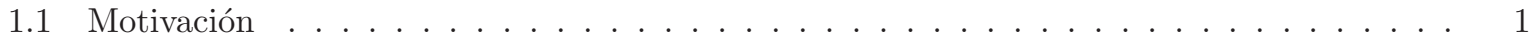

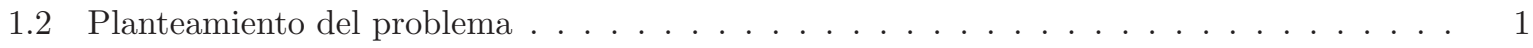

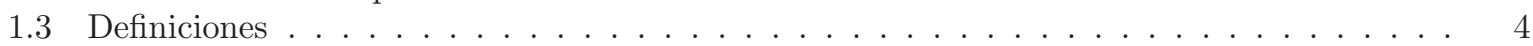

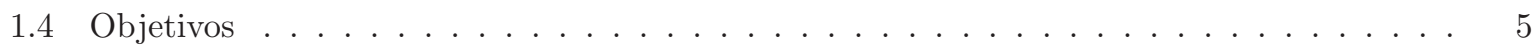

1.5 Estructura de la tesis . . . . . . . . . . . . . . . . . . . . . . . . . 6

2 Estado del arte $\quad 9$

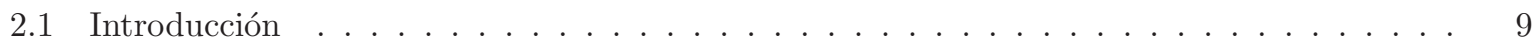

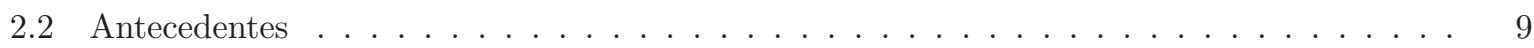

2.3 Resultados preliminares . . . . . . . . . . . . . . . . . . . . . . . . 12

2.3.1 Predicción de salidas mediante interpolación . . . . . . . . . . . . . . . . . . 12

2.3.2 Predicción de salidas basado en modelo entrada-salida . . . . . . . . . . . . . . . . 12

2.3 .3 Predicción de salidas basado en el filtro de Kalman . . . . . . . . . . . . . . . . . . 20

2.4 Conclusiones . . . . . . . . . . . . . . . . . . . . . . . . . . . 21

3 Diseño de predictores en representación entrada salida $\quad 23$

3.1 Descripción del problema . . . . . . . . . . . . . . . . . . . . . . . 23

$3.1 .1 \quad$ Escenario de muestreo . . . . . . . . . . . . . . . . . . . . . . . . . . 23

3.1 .2 Parametrización del escenario de muestreo . . . . . . . . . . . . . . . . . . . . . 25

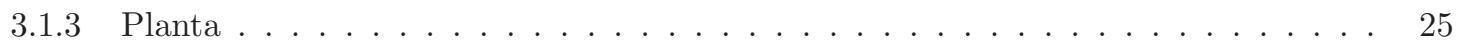

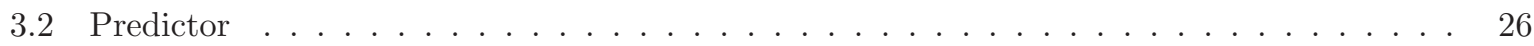

3.2 .1 Implementación del predictor . . . . . . . . . . . . . . . . . . . . . . . 27

3.2 .2 Error de predicción . . . . . . . . . . . . . . . . . . . . . . . . . . 29

3.2 .3 Objetivo del diseño . . . . . . . . . . . . . . . . . . . . . . . . . . . . 31

3.3 Diseño de predictores ante muestreo regular . . . . . . . . . . . . . . . . . . . . . . . . . . 32

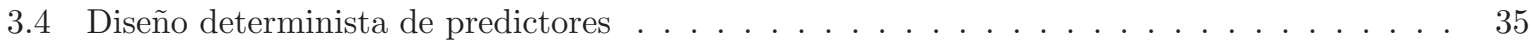

3.4 .1 Diseño de predictores para estabilidad nominal . . . . . . . . . . . . . . . . . . 35

3.4 .2 Diseño de predictores $\mathcal{H}_{\infty} \ldots \ldots \ldots \ldots \ldots \ldots \ldots$

3.4 .3 Diseño de predictores $\mathcal{H}_{2} \ldots \ldots \ldots \ldots \ldots \ldots \ldots \ldots \ldots$

3.4 .4 Diseño de predictores $\mathcal{H}_{2 g} \ldots \ldots \ldots \ldots \ldots \ldots \ldots \ldots$

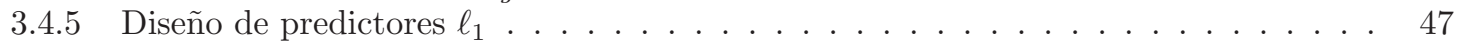

3.5 Diseño estocástico de predictores . . . . . . . . . . . . . . . . . . . . . . . . 48

3.5.1 Diseño de predictores para estabilidad nominal . . . . . . . . . . . . . . . . . . . . 49

3.5.2 Diseño de predictores para atenuar perturbaciones . . . . . . . . . . . . . . 50

3.6 Consideraciones de diseño . . . . . . . . . . . . . . . . . . . . . . . . 54

3.6.1 Robustez frente a perturbaciones de entrada . . . . . . . . . . . . . . 55 
3.6 .2 Robustez al error de modelado . . . . . . . . . . . . . . . . . . . . . . 57

3.6 .3 Retardo en la acción de control . . . . . . . . . . . . . . . . . . . . 59

3.6 .4 Mediciones con etiqueta de tiempo incierta . . . . . . . . . . . . . . . 63

3.6.5 Muestreo asíncrono . . . . . . . . . . . . . . . . . . . . . . 65

3.6 .6 Minimización del error entre mediciones . . . . . . . . . . . . . . . . . . . . 71

3.6.7 Identificación de parámetros en procesos con medidas escasas . . . . . . . . . . . . 72

3.6 .8 Diseño de predictores con objetivos combinados . . . . . . . . . . . . . . . 73

3.7 Simplificaciones . . . . . . . . . . . . . . . . . . . . . . . . 75

3.7 .1 Reducción del número de vectores de ganancia a almacenar . . . . . . . . . . . . 75

3.7 .2 Reducción del coste computacional de las LMI . . . . . . . . . . . . . . . . . . 76

3.7 .3 Interpolación de ganancias . . . . . . . . . . . . . . . . . . . 77

3.7.4 Diseño basado en la minimización de la frecuencia de medición . . . . . . . . . . . 80

3.8 Conclusiones . . . . . . . . . . . . . . . . . . . . . . . . . 80

4 Diseño de predictores en representación interna 83

4.1 Introducción . . . . . . . . . . . . . . . . . . . . . . . . 83

4.2 Descripción del problema $\ldots \ldots \ldots \ldots \ldots$

$4.2 .1 \quad$ Planta . . . . . . . . . . . . . . . . . . . . . . 83

4.2 .2 Escenario de muestreo . . . . . . . . . . . . . . . . . . . . 86

4.2 .3 Parametrización del escenario de muestreo . . . . . . . . . . . . . . . . . 86

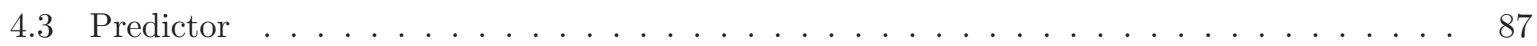

$4.3 .1 \quad$ Implementación del predictor . . . . . . . . . . . . . . . . . 87

4.3 .2 Equivalencia con el predictor entrada salida . . . . . . . . . . . . . . . . . . . 89

4.3 .3 Error de predicción . . . . . . . . . . . . . . . . . . . 90

4.3 .4 Objetivo de diseño . . . . . . . . . . . . . . . . . . . . . . . . 93

4.4 Diseño de predictores ante muestreo regular . . . . . . . . . . . . . . . . . . . . . . . . . . 94

4.5 Diseño determinista de predictores . . . . . . . . . . . . . . . . . . . . . . . . . 95

$4.5 .1 \quad$ Estabilidad nominal . . . . . . . . . . . . . . . . . . . . . . 95

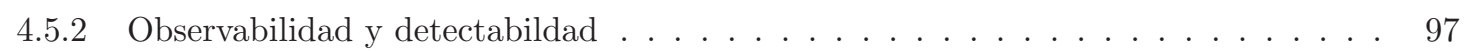

4.5 .3 Atenuación $\mathcal{H}_{\infty} \ldots \ldots \ldots \ldots \ldots$. . . . . . . . . . . . . . . . . . . . 99

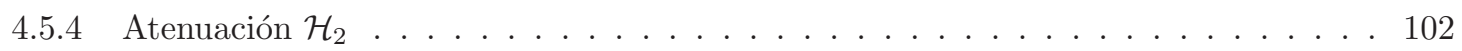

4.5 .5 Atenuación $\mathcal{H}_{2 g} \ldots \ldots \ldots \ldots \ldots \ldots \ldots$

4.5 .6 Atenuación $\ell_{1} \ldots \ldots \ldots \ldots \ldots \ldots \ldots$

4.6 Diseño estocástico de predictores . . . . . . . . . . . . . . . . . . . . . . . 107

4.6 .1 Diseño para estabilidad nominal . . . . . . . . . . . . . . . . . . . 107

4.6 .2 Diseño para atenuar perturbaciones . . . . . . . . . . . . . . . . . . . 108

4.7 Consideraciones de diseño . . . . . . . . . . . . . . . . . . . . . . . . . . . . . . . . . . . 112

4.7 .1 Robustez al error de modelado . . . . . . . . . . . . . . . . . . . . 113

4.7 .2 Retardo en la acción de control . . . . . . . . . . . . . . . . . . . . . 115

4.7 .3 Mediciones con etiqueta de tiempo incierta . . . . . . . . . . . . . . . . . 117

4.7 .4 Muestreo asíncrono . . . . . . . . . . . . . . . . . . . . . . . . . 118

4.7 .5 Minimización del error en bucle abierto . . . . . . . . . . . . . . . . . . 122

4.7 .6 Diseño basado en la minimización de frecuencias de medición . . . . . . . . . . . . 122

4.7 .7 Diseño de predictores con objetivos combinados . . . . . . . . . . . . . . . . . . . 122

4.8 Simplificaciones . . . . . . . . . . . . . . . . . . . . . . . . . . . 123

4.8 .1 Reducción del número de ganancias a almacenar . . . . . . . . . . . . . . . . . . 124

4.8 .2 Simplificación de las LMI . . . . . . . . . . . . . . . . . . . . . . . . . . . . . . . . . . . . . . . . . . . . . . . . . . . . . 124

4.8 .3 Interpolación de ganancias . . . . . . . . . . . . . . . . . . . . . . . . . . . . 124

4.9 Sensores virtuales basados en el filtro de Kalman . . . . . . . . . . . . . . . . . . . . . . . 125

4.9 .1 Sistemas monovariable . . . . . . . . . . . . . . . . . . . 125

4.9 .2 Sistemas multivariables . . . . . . . . . . . . . . . . . . . . . 129

4.9 .3 Predicción óptima . . . . . . . . . . . . . . . . . . . . . . . 132

4.10 Conclusiones . . . . . . . . . . . . . . . . . . . . . . . . . . . 132 
5 Predicción de salidas en sistemas no lineales 135

5.1 Introducción . . . . . . . . . . . . . . . . . . . . . . . . 135

5.2 Descripción del problema . . . . . . . . . . . . . . . . . . . . . 135

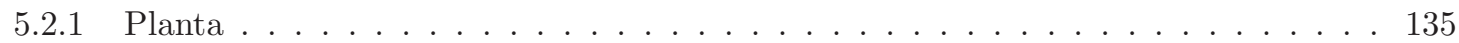

$5.2 .2 \quad$ Escenario de muestreo . . . . . . . . . . . . . . . . . . . . . 136

5.2 .3 Parametrización del escenario de muestreo . . . . . . . . . . . . . . . . . . 137

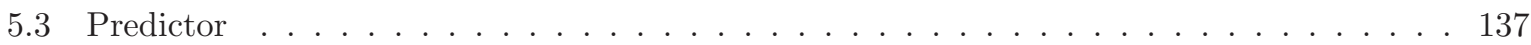

5.3 .1 Error de predicción . . . . . . . . . . . . . . . . . . . 138

5.3 .2 Objetivo del diseño . . . . . . . . . . . . . . . . . . . . . 138

5.4 Diseño de predictores para muestreo convencional . . . . . . . . . . . . . . . . . . . . . . . 139

5.4 .1 Diseño para estabilidad nominal . . . . . . . . . . . . . . . . . . 139

5.4 .2 Diseño basado en la atenuación de perturbaciones . . . . . . . . . . . . . . . 140

5.5 Diseño de predictores para muestreo bifrecuencia . . . . . . . . . . . . . . . . . . . . . . . 142

5.5 .1 Diseño para estabilidad nominal . . . . . . . . . . . . . . . . . . . . 142

5.5 .2 Diseño basado en la atenuación de perturbaciones . . . . . . . . . . . . . . . . 144

5.6 Diseño de predictores para muestreo variante en el tiempo . . . . . . . . . . . . . . . . . . 147

5.6 .1 Diseño para estabilidad nominal . . . . . . . . . . . . . . . . . 147

5.6 .2 Diseño basado en la atenuación de perturbaciones . . . . . . . . . . . . . . . . 148

5.7 Consideraciones de diseño . . . . . . . . . . . . . . . . . . . . . . . . 151

5.7 .1 Minimización del error en bucle abierto . . . . . . . . . . . . . . . . 151

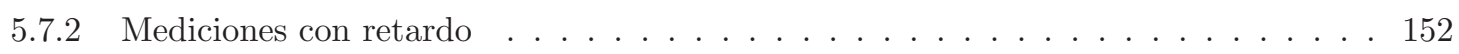

5.7 .3 Simplificaciones . . . . . . . . . . . . . . . . . . . . 152

5.8 Diseño de predictores basados en el filtro de Kalman extendido . . . . . . . . . . . . . . . 153

5.9 Conclusiones . . . . . . . . . . . . . . . . . . . . . . . . . . 153

6 Análisis de sistemas de control inferencial $\quad 155$

6.1 Introducción . . . . . . . . . . . . . . . . . . . . . . . . 155

6.2 Análisis de SCI basado en la representación entrada-salida . . . . . . . . . . . . . . . . . 155

6.2 .1 Planteamiento del problema . . . . . . . . . . . . . . . . . 155

6.2 .2 Dinámica del bucle cerrado . . . . . . . . . . . . . . . . . . . . . . 157

6.2 .3 Principio de separación . . . . . . . . . . . . . . . . . . . . . 160

6.2 .4 Ejemplos . . . . . . . . . . . . . . . . . . . . . . . 162

6.3 Análisis de SCI basado en la representación interna . . . . . . . . . . . . . . . . . . . . 164

6.3 .1 Planteamiento del problema . . . . . . . . . . . . . . . . . 164

6.3 .2 Dinámica del bucle cerrado con muestreo periódico . . . . . . . . . . . . . . . . . 167

6.3 .3 Dinámica del bucle cerrado con muestreo escaso . . . . . . . . . . . . . . . . . . 169

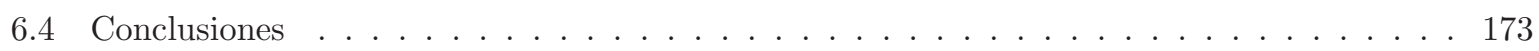

\begin{tabular}{llr}
\hline Aplicaciones & 175
\end{tabular}

7.1 Introducción . . . . . . . . . . . . . . . . . . . . . . . . 175

7.2 Control de posición de un puente grúa . . . . . . . . . . . . . . . . . . . . . . . 175

7.3 Posicionado de un robot móvil mediante detección de azulejos . . . . . . . . . . . . . . . . 181

7.3 .1 Introducción . . . . . . . . . . . . . . . . . . . 181

7.3 .2 Descripción del robot . . . . . . . . . . . . . . . . . . . 181

7.3 .3 Modelo del robot . . . . . . . . . . . . . . . . . . . . . . 182

7.3 .4 Estimación de la posición . . . . . . . . . . . . . . . . . . 185

7.4 Estimación de corriente en un motor de inducción . . . . . . . . . . . . . . . . . 190

7.5 Estimación de sustancias en un biorreactor . . . . . . . . . . . . . . . . . . . . . . 197

8 Conclusiones y trabajo futuro 203

8.1 Conclusiones . . . . . . . . . . . . . . . . . . . . . . . . . . 203

8.1 .1 Diseño de predictores . . . . . . . . . . . . . . . . . . . 203

8.1 .2 Análisis de sistemas de control inferencial . . . . . . . . . . . . . . . . 205

8.2 Trabajo futuro . . . . . . . . . . . . . . . . . . . . . . 205 
\begin{tabular}{|lr} 
A Fundamentos matemáticos & 207
\end{tabular}

A.1 Introducción . . . . . . . . . . . . . . . . . . . . . . . . . . 207

A.2 Normas . . . . . . . . . . . . . . . . . . . . . . . . 207

A.2.1 Definiciones . . . . . . . . . . . . . . . . . . 207

A.3 Análisis de sistemas discretos vía LMI . . . . . . . . . . . . . . . . . . . . . . . . 210

A.3.1 Estabilidad nominal . . . . . . . . . . . . . . . . . . . . . 210

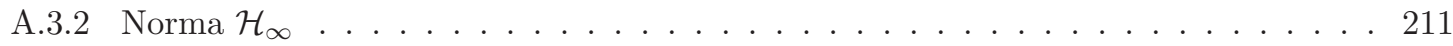

A.3.3 Norma $\mathcal{H}_{2}$. . . . . . . . . . . . . . . . . . . . . . . . . . . . . . . . 212

A.3.4 Norma $\mathcal{H}_{2 g} \ldots \ldots \ldots \ldots \ldots \ldots \ldots \ldots \ldots$

A.3.5 Norma $\ell_{1} \ldots \ldots \ldots \ldots \ldots \ldots \ldots \ldots \ldots \ldots$

A.4 Estabilidad de sistemas variantes en el tiempo de forma paramétrica . . . . . . . . . . . 215

A.5 Observabilidad y detectabilidad . . . . . . . . . . . . . . . . . . . . 216

A.5.1 Sistemas lineales invariantes en el tiempo . . . . . . . . . . . . . . . . . . 217

A.5.2 Sistemas conmutados . . . . . . . . . . . . . . . . . . . . . . 218

A.6 Diseño de predictores para sistemas no lineales . . . . . . . . . . . . . . . . . . . . . 219

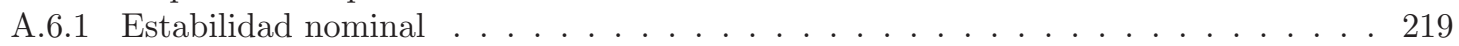

A.6.2 Atenuación de perturbaciones . . . . . . . . . . . . . . . . . . . 221

B Resultados auxiliares en el diseño de predictores 223

B.1 Diseño estocástico de predictores monovariables . . . . . . . . . . . . . . . . . . . . . . . 223

B.1.1 Demostración del teorema $3.5 .2\left(\right.$ diseño $\left.\mathcal{H}_{\infty}\right) \quad \ldots \ldots$. . . . . . . . . . . . . . 223

B.1.2 Demostración del teorema $3.5 .3\left(\right.$ diseño $\left.\mathcal{H}_{2}\right) \ldots \ldots \ldots$. . . . . . . . . . . . . . . 224

B.1.3 Demostración del teorema $3.5 .4\left(\right.$ diseño $\left.\mathcal{H}_{2 g}\right)$. . . . . . . . . . . . . . . . . . . . . 225

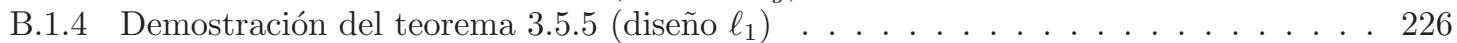

B.2 Diseño determinista de predictores multivariables . . . . . . . . . . . . . . . . . 226

B.2.1 Demostración del teorema $4.5 .2\left(\right.$ diseño $\left.\mathcal{H}_{\infty}\right)$. . . . . . . . . . . . . . . . . . . 226

B.2.2 Demostración del teorema $4.5 .3\left(\right.$ diseño $\left.\mathcal{H}_{2}\right)$. . . . . . . . . . . . . . . . . . . 227

B.2.3 Demostración del teorema $4.5 .4\left(\right.$ diseño $\left.\mathcal{H}_{2 g}\right) \ldots \ldots \ldots$. . . . . . . . . . . . 228

B.2.4 Demostración del teorema $4.5 .5\left(\right.$ diseño $\left.\ell_{1}\right) \ldots \ldots \ldots$. . . . . . . . . . . 229

B.3 Diseño estocástico de predictores multivariables . . . . . . . . . . . . . . . . . . . . . . . 230

B.3.1 Demostración del teorema $4.6 .2\left(\right.$ diseño $\left.\mathcal{H}_{\infty}\right)$. . . . . . . . . . . . . . . . . . . . . 230

B.3.2 Demostración del teorema $4.6 .3\left(\right.$ diseño $\left.\mathcal{H}_{2}\right)$. . . . . . . . . . . . . . . . . . . . . . 231

B.3.3 Demostración del teorema $4.6 .4\left(\right.$ diseño $\left.\mathcal{H}_{2 g}\right)$. . . . . . . . . . . . . . . . . . . . . 232

B.3.4 Demostración del teorema $4.6 .5\left(\right.$ diseño $\left.\ell_{1}\right) \ldots \ldots$. . . . . . . . . . . . . 232

$\begin{array}{lr}\text { C Notación } & 235\end{array}$

\begin{tabular}{lr}
\hline Bibliografía & 240
\end{tabular} 


\section{Introducción}

\subsection{Motivación}

En muchas aplicaciones industriales la señal de control se actualiza a un periodo constante $T$ marcado principalmente por las características dinámicas que se desean en bucle cerrado y por las características del actuador. Sin embargo, puede ocurrir que las salidas no se midan con la misma frecuencia o que las medidas que se tomen correspondan a salidas de un instante pasado. Este muestreo irregular puede estar causado por el dispositivo de medida debido, por ejemplo, al fallo ocasional de un sensor, a la utilización de procedimientos lentos de medición (como procesadores de imágenes o analizadores bioquímicos), o bien por la toma de mediciones basada en eventos (como ocurre con los sensores binarios como un encoder, por ejemplo). El acceso a la información de los sensores a través de una red de comunicaciones es otro causante del muestreo irregular. La red de comunicaciones tiene un ancho de banda finito que tiene que compartir con otros procesos, lo cual implica un acceso limitado a los sensores y un retardo en la transmisión de la información. Cualquiera que sea el causante del muestreo irregular, la consecuencia es que el controlador sólo tendrá acceso a información de la salida desfasada y en instantes de tiempo arbitrarios.

Para poder controlar el proceso a partir del conjunto de muestras irregulares, se propone incluir en el bucle un predictor que estime las salidas en todos los periodos de control y pase esta información al controlador para que éste calcule la acción de control en cada periodo. Si las mediciones del proceso le llegan al sistema de control a través de un canal de comunicación, otra utilidad de calcular la acción de control a partir de estimaciones (en lugar de mediciones) es la de reducir el ancho de banda requerido y contribuir así a la disminución del coste del sistema de control.

El presente trabajo está motivado por diversas problemáticas que no están resueltas en la estimación de salidas con muestreo escaso y retardo. Problemas como el diseño de predictores de bajo coste para muestreo irregular que sean robustos ante perturbaciones, ruidos de medida y errores de modelado. Tampoco se han abordado hasta el momento los problemas de predicción de bajo coste en sistemas no lineales.

\subsection{Planteamiento del problema}

En la figura 1.1 se describe el problema de control de un sistema con una entrada y una salida en el que la información de la salida es escasa. Las señales continuas (marcadas con líneas de trazo lleno en el diagrama) se denotan mediante $u(\tau)$ o $y(\tau)$, siendo $\tau$ el instante de tiempo continuo. Las señales muestreadas de forma irregular (marcadas con líneas punteadas) se denotan mediante $m_{k}$, siendo $k$ el número de muestra.

El sistema de control se supone que está implementado por un computador que puede actualizar la entrada al proceso bien de forma arbitraria o bien de forma periódica (al periodo de control $T$ ). La salida no puede medirse a la frecuencia deseada, sino que sólo se tiene acceso a un conjunto escaso de mediciones que además pueden estar distribuidas de forma irregular en el tiempo. Las muestras $m_{k}$ que se toman de la salida a través del sensor y que llegan al controlador a través de un canal de comunicaciones corresponden a valores medidos de la salida $y(\tau)$ en instantes de tiempo arbitrarios. El tiempo transcurrido entre que se dispone de una muestra $m_{k}$ y la siguiente viene determinado por el tipo de sensor y por la carga del canal de comunicación.

La forma de controlar estos sistemas se puede abordar siguiendo dos estrategias: 


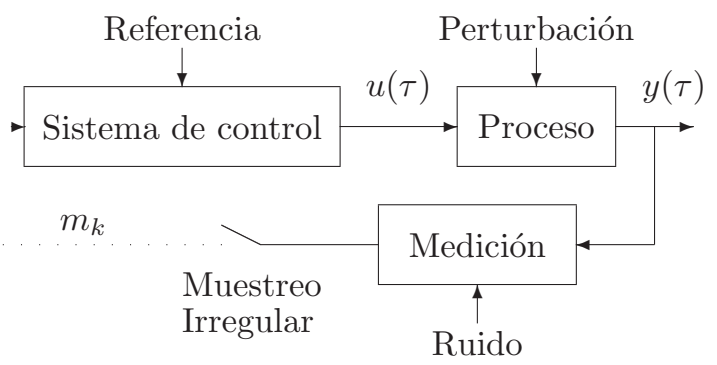

Figura 1.1: Descripción general del problema de control con escasez de información.

Control directo. El controlador actualiza la acción de control $\left(u_{k}\right)$ cada vez que obtiene una nueva medida del proceso $\left(m_{k}\right)$. En la figura 1.2 se muestra un diagrama de bloques que describe el funcionamiento y en la figura 1.3 se muestra la evolución de las señales para un sistema de medición sin retardo, en el que se observa que la acción de control permanece constante entre los instantes de medición debido al retenedor de orden cero $(\mathrm{ZOH})$ presente en el bucle.

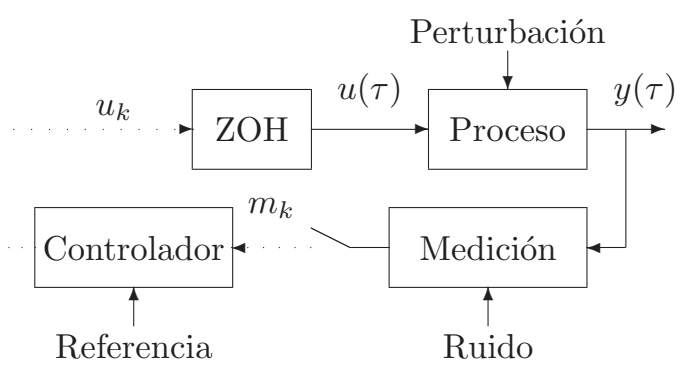

Figura 1.2: Estrategia de control directo.

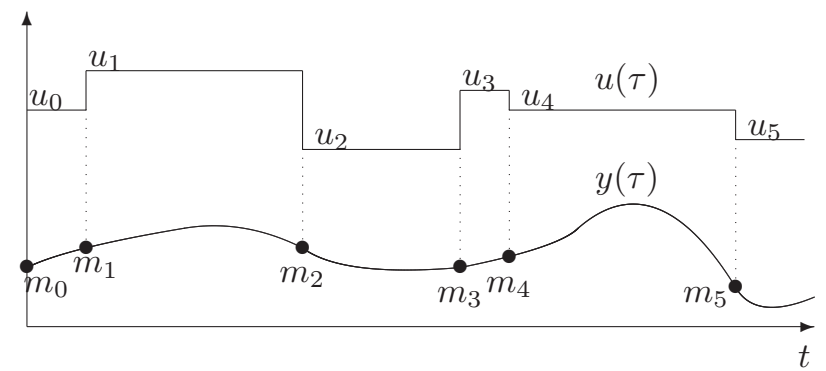

Figura 1.3: Evolución de las señales en el control directo sin retardos.

Control indirecto. El sistema de control recibe como entrada las mediciones disponibles $m_{k}$ pero actualiza la acción de control $u[t]$ a un periodo fijo $T$ a pesar de la irregularidad de las mediciones de la salida. En la figura 1.4 se muestra un diagrama de bloques que describe el funcionamiento. La señal de control actualizada de forma periódica se marca mediante línea de trazos y se denota por $u[t]$, siendo $t \in \mathbb{N}$ el número de actualización, y cumpliéndose que

$$
u(\tau)=u[t], \quad \tau \in[t T, t T+T)
$$

debido a la presencia del retenedor de orden cero.

En la figura 1.5 se muestra la evolución de las señales para un sistema de control indirecto sin retardo en las mediciones.

El ejemplo más simple de control indirecto es el control bifrecuencia, donde las entradas se actualizan a un periodo $T$ mientras que la salida se muestrea de forma síncrona con la entrada pero a un periodo más lento $N T$, siendo $N$ un número entero mayor que uno.

Un caso concreto de control indirecto es el control inferencial, que es aquél en el que el sistema de control se divide en un predictor que proporciona una estimación de las salidas en cada periodo $T(\hat{y}[t])$ y un controlador convencional que opera a periodo fijo $T$ (véanse figuras 1.6 y 1.7). 


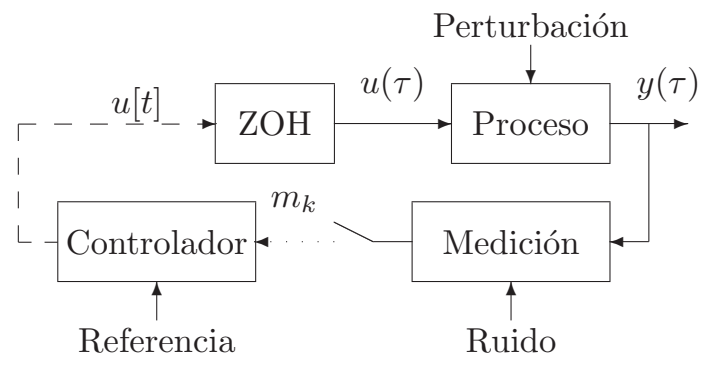

Figura 1.4: Estrategia de control indirecto.

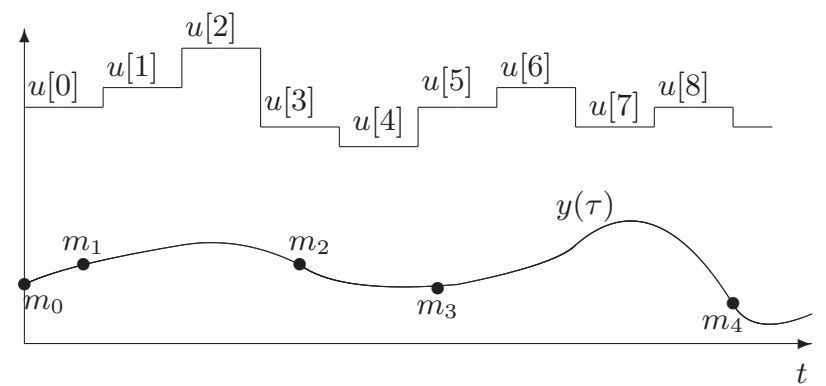

Figura 1.5: Evolución de las señales en el control indirecto sin retardos.

El predictor utiliza las mediciones $m_{k}$ que le llegan en instantes arbitrarios de tiempo y las acciones de control actualizadas en cada periodo $(u[t])$ para proporcionar de forma periódica la estimación de la salida del proceso $\hat{y}[t]$, siendo

$$
\hat{y}[t]=\hat{y}(t T)
$$

la estimación de la salida en el instante $t T$ segundos. El controlador utiliza esta estimación para proporcionar una actualización de la acción de control $u[t]$ cada $T$ segundos. El periodo $T$ se escoge de forma que sea adecuado a la dinámica del proceso a controlar, atendiendo a las restricciones del actuador y del convertidor digital analógico, e independientemente de la frecuencia de muestreo de la variable de salida.

Un ejemplo clásico de control inferencial es el que utiliza el predictor de Smith para controlar un proceso con retardo. En este caso el predictor de Smith utiliza un modelo del proceso y las mediciones para estimar la salida del proceso sin retardo, que luego utiliza el controlador para el cálculo de la acción de control.

El control inferencial también es aplicable a sistemas con varias entradas de control y con varias señales (estados o salidas) medibles, como se muestra en la figura 1.8. En el caso general puede haber diversos sensores que miden diferentes variables a diferentes frecuencias que además varían en el tiempo. Se asume también que algunas salidas pueden medirse con sensores redundantes de diferente precisión y frecuencia de muestreo. Además se asume que el retardo asociado a la adquisición de cada medición puede ser diferente entre sensores y variante en el tiempo. El controlador necesitará los valores de algunas de estas variables ( $u$ otras que no se pueden medir directamente pero que son función de las variables medibles) a una frecuencia fija en instantes de tiempo predefinidos (cuando la acción de control va a calcularse y aplicarse).

En este escenario se define un sensor virtual como el dispositivo inteligente que mide las salidas o los estados disponibles en instantes arbitrarios de tiempo y predice los valores de las señales de salida deseadas en los instantes y a la frecuencia que necesita el sistema de control [58]. Otros usos que se le puede dar a los sensores virtuales son la monitorización de sensores no disponibles, el reemplazamiento de sensores caros por dispositivos de medición baratos, o la disminución de la utilización de los canales de comunicación en sistemas de control distribuido.

En este trabajo se presenta un predictor que toma las mediciones de varios sensores en instantes arbitrarios de tiempo y devuelve una estimación del valor actual de las salidas de interés (las que se quiere controlar) en cada periodo $T$. El diseño del predictor tiene en cuenta la posibilidad de que cada sensor tenga un periodo de muestreo y retardo diferente, además de ser estos valores variantes en el tiempo. También considera el error de modelado, las perturbaciones y los ruidos de medida como factores que afectan al error de predicción.

Además se analiza cómo afecta la presencia del predictor a la estabilidad del lazo de control en bucle cerrado. El resultado obtenido indica que para obtener un lazo cerrado estable con medidas escasas es 


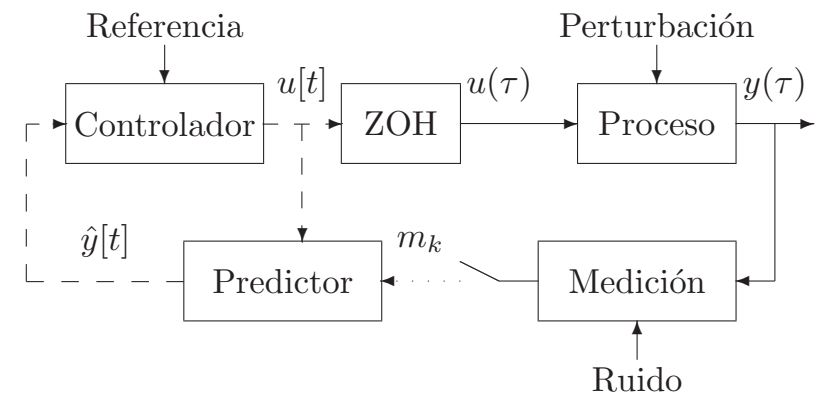

Figura 1.6: Estrategia de control inferencial.

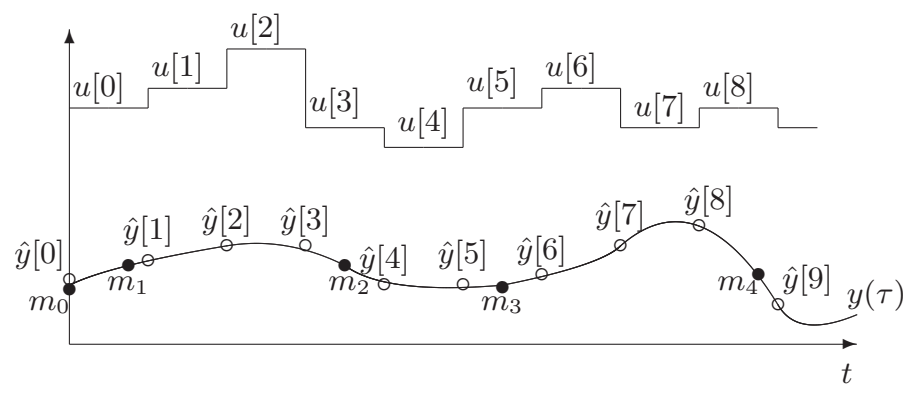

Figura 1.7: Evolución de las señales en el control inferencial sin retardos.

suficiente implementar un control inferencial con un predictor estable (las predicciones convergen al valor de la salida) y un controlador que estabilice la planta con muestreo regular.

\subsection{Definiciones}

Si se dispone de la medida de la salida en todos los instantes en los que se actualiza la entrada $(y[t]=$ $y(t T)$ ), la situación se define como muestreo estándar. Si esto no es así, se pueden distinguir varios casos en función de los instantes en los cuales se obtiene la medida y la frecuencia con la que se obtiene. La clasificación de las situaciones de muestreo irregular es:

Muestreo síncrono con falta de datos. La salida se mide síncronamente con la actualización de la entrada. Algunas de estas medidas, sin embargo, no están disponibles por fallo en los sensores o en la transmisión, o debido a la lentitud de los mismos. Se pueden distinguir dos casos en función de la regularidad de la disponibilidad de datos:

1. Muestreo síncrono regular. Las medidas se obtienen según un patrón de muestreo periódico. El caso más simple es el de muestreo bifrecuencia, en el que la salida se mide cada $N$ periodos de actualización de la entrada. Este caso podría darse debido a la utilización de sensores lentos, como por ejemplo un analizador químico o un sensor de visión artificial.

2. Muestreo síncrono aleatorio. Las medidas se obtienen de forma aleatoria, aunque síncronamente con la entrada. Esta situación puede darse cuando se producen fallos en los sensores o en la transmisión de los datos de forma aleatoria. También puede producirse esta situación cuando los sensores son compartidos por varios procesos, no pudiendo garantizarse la regularidad en la toma de medidas.

Muestreo asíncrono. Las medidas no se obtienen en los instantes en que se actualiza la entrada. No se dispone por tanto de los valores de $y(t T)$, sino de valores intermedios. Se pueden distinguir dos casos de muestreo asíncrono:

1. Muestreo aleatorio en el tiempo. Las medidas simplemente se toman de forma aleatoria en el tiempo.

2. Muestreo por medio de sensores binarios. Las medidas de la salida se obtienen por medio de una batería de sensores binarios cuya posición se conoce con exactitud, y que dan una señal cuando la salida pasa por ese valor. Aunque la naturaleza de este caso es diferente al anterior, 


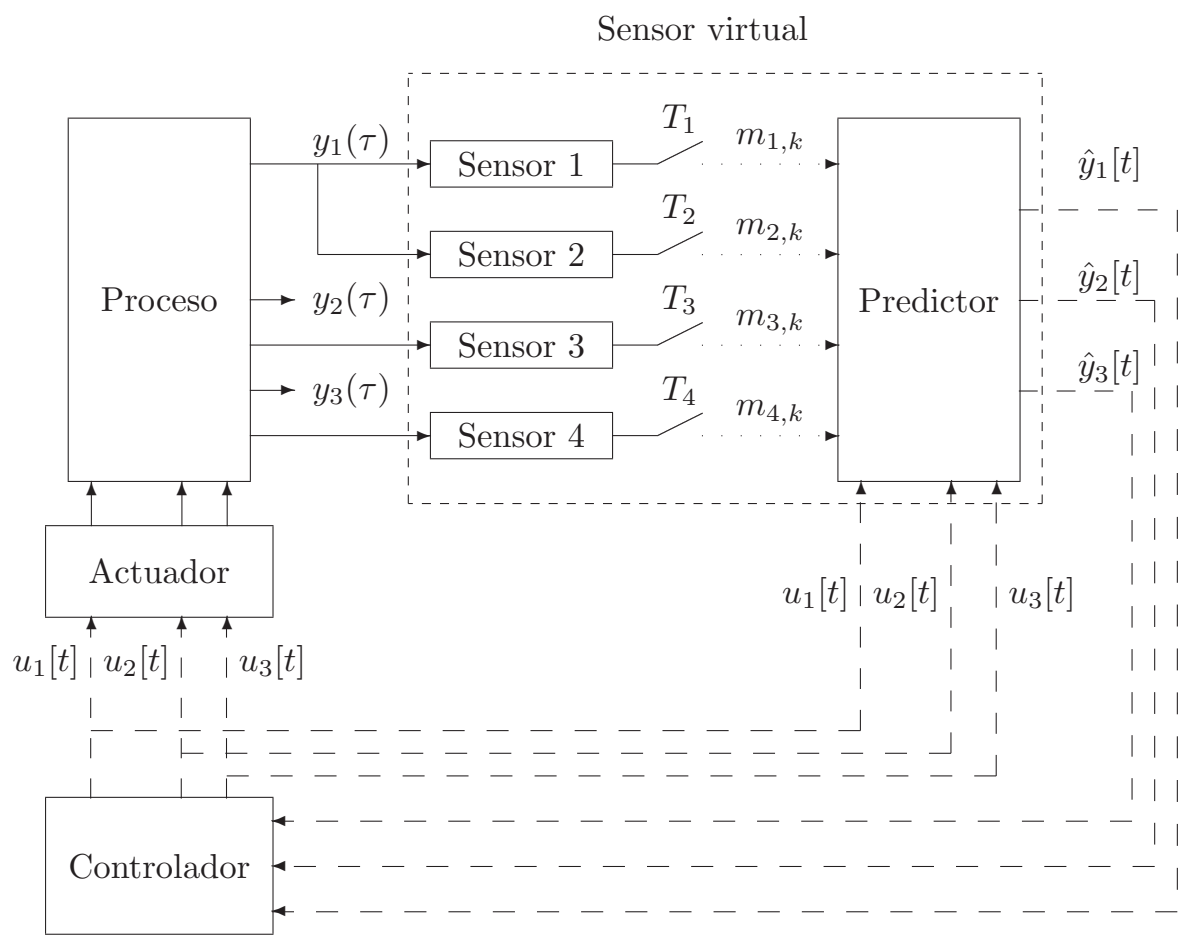

Figura 1.8: Control inferencial de un proceso con ayuda de un sensor virtual.

se puede reducir al mismo, ya que en esencia se desconocen a priori los instantes en los que se obtendrán las medidas. La única diferencia radica en el origen de esa variabilidad en la adquisición de los datos, que en el caso de los sensores binarios depende directamente de la posición y número de esos sensores.

Otra clasificación posible consiste en diferenciar las situaciones de muestreo en función del retardo existente en el sistema de medición o transmisión.

- Medición en un instante arbitrario de tiempo.

- Sin retardo. Es la medición convencional de cualquier sensor analógico.

- Con retardo. El resultado es una medición que corresponde a un instante pasado, como, por ejemplo, la medición que se obtiene mediante la utilización de un sensor bioquímico.

- Medición en instantes fijados por eventos externos.

- Sin retardo. Por ejemplo, la medición de la posición de un motor a través de un encoder.

- Con retardo. Por ejemplo, el análisis de gases de combustión de un motor.

Cabe destacar también la diferencia entre disponer de un solo sensor o de varios sensores que realizan mediciones de diversos tipos, en cuyo caso se pueden tener mediciones de distinta precisión y frecuencia. El tratamiento de este tipo de mediciones se conoce como fusión sensorial.

En función de la frecuencia de pérdida de datos se debe distinguir entre el muestreo escaso o la pérdida de datos escasa. El muestreo escaso es aquél en el que la probabilidad de tener un número determinado de salidas consecutivas es muy pequeña, mientras que la pérdida de datos escasa es el caso en el que la probabilidad de perder un dato es muy baja. El caso de pérdida esporádica de datos es menos problemático y puede incluirse su análisis dentro de los sistemas con medidas escasas, sin perder generalidad en el problema.

\subsection{Objetivos}

El objetivo principal de la tesis es contribuir al desarrollo de métodos de control de procesos con medidas escasas y con retardos temporales, ya sea debido a la presencia de un canal de comunicación, al procesado requerido por el sistema de medición, o a ambos. 
Este objetivo se concreta en el estudio de sistemas de control inferencial, en el que se plantea el control de procesos con medidas escasas y retardos temporales basado en la actualización de la entrada a un periodo $T$ constante adecuado, y la utilización de un predictor para la estimación explícita de las salidas no disponibles en los instantes de actualización de la entrada (al mismo periodo T). Esta secuencia de salidas predichas se puede utilizar para el control y también para la identificación del proceso mediante técnicas convencionales.

El objetivo global de la tesis se puede descomponer en varios objetivos parciales:

1. Análisis del caso general de muestreo aleatorio (síncrono o asíncrono) con múltiples salidas con diferentes retardos variantes en el tiempo.

2. Obtención de predictores de bajo coste alternativos al filtro de Kalman.

3. Análisis del efecto de las perturbaciones, la incertidumbre del modelo, el retardo en la acción de control y la disponibilidad de datos con etiqueta de tiempo incierta sobre el error de predicción. Desarrollo de estrategias de diseño para atenuar estos efectos.

4. Diseño de predictores para plantas no lineales con muestreo aleatorio.

5. Análisis de la dinámica de sistemas de control inferencial. Análisis de estabilidad y atenuación de perturbaciones de sistemas de control que hacen uso de los predictores.

\subsection{Estructura de la tesis}

Los temas abordados en la tesis se estructuran en 8 capítulos:

En el capítulo 1 se plantea la problemática motivadora del presente trabajo. Se introducen los sistemas con medidas escasas introduciendo las definiciones básicas de los sistemas que se van a tratar.

En el capítulo 2 se revisan las técnicas de control de procesos con medidas escasas desarrolladas por otros autores con anterioridad.

En el capítulo 3 se desarrollan predictores de salida para sistemas lineales con muestreo síncrono basados en la representación entrada-salida del proceso que predicen directamente el vector de regresión.

En el capítulo 4 se profundiza en el diseño de predictores basados en la representación interna de sistemas con múltiples entradas y salidas. Se desarrollan predictores de salida robustos que abordan la problemática de la escasez de medidas, la presencia de retardos y la fusión sensorial de forma conjunta. También se analiza la inclusión de retardos en el filtro de Kalman llevando al predictor óptimo.

En el capítulo 5 se aborda la predicción de salidas en plantas no lineales que cumplen con ciertas características.

En el capítulo 6 se analiza la dinámica del bucle cerrado de sistemas de control inferencial que incluyen los predictores propuestos. En este capítulo se obtiene la expresión de la dinámica del bucle cerrado demostrándose el principio de separación entre controlador y predictor que permite un diseño independiente de los dos componentes del sistema de control inferencial. También se incluye un análisis del efecto que tienen las perturbaciones sobre las salidas controladas con la ayuda del predictor.

En el capítulo 7 se describen algunas aplicaciones de control inferencial de plantas reales con los predictores propuestos.

Por último, en el capítulo 8 se hace un resumen de las conclusiones del trabajo y se proponen algunas líneas de investigación futuras. 


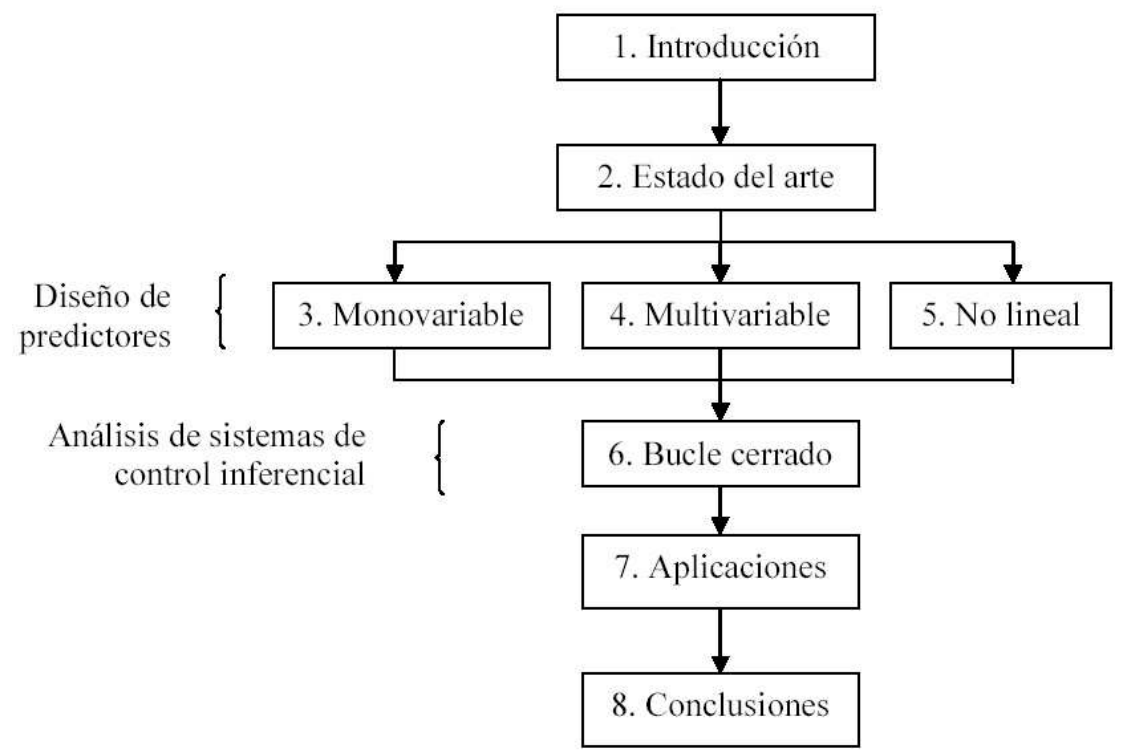

Figura 1.9: Esquema del trabajo de tesis. 



\section{Capítulo 2}

\section{Estado del arte}

\subsection{Introducción}

Este capítulo se estructura en dos bloques. En primer lugar se hace un repaso al estado del arte, comentando los trabajos que se han hecho anteriormente en el campo de predicción y control de procesos con medidas escasas. Finalmente, se exponen cuestiones básicas de la predicción de salidas que sirven como punto de partida para el desarrollo de los capítulos posteriores.

\subsection{Antecedentes}

En la literatura especializada en control se han encontrado referencias que tratan el control de sistemas con muestreo irregular y retardo temporal en varias aplicaciones. Una de las principales aplicaciones que presenta esta problemática es la de los sistemas de control basados en red ${ }^{1}$ (véase por ejemplo [13]).

\section{Control Directo}

Algunos autores han estudiado el control directo de sistemas con periodo de muestreo variante, es decir, actualizando la acción de control cada vez que se tiene una nueva medición de la salida o del estado. En [25, 26] se analiza la estabilidad y robustez de este tipo de sistemas, pero los autores no establecen ninguna técnica de diseño de controladores. En [39] se analiza el mismo problema desde el punto de vista de los sistemas de control basados en red con el objetivo de buscar las condiciones en el retardo y en la pérdida de datos inducidos por la red bajo los cuales la estabilidad del sistema y el rechazo ante perturbaciones se mantienen en un nivel deseado.

En [60] se muestra un sistema continuo muestreado a dos periodos diferentes y se diseñan dos controladores diferentes, uno para cada periodo. Se demuestra que, aunque los diseños llevan a sistemas estables en bucle cerrado para cada uno de los periodos, el sistema resultante (con variaciones entre dos periodos de muestreo) puede ser inestable. Mediante técnicas $\mathrm{LMI}^{2}$ se diseñan controladores de realimentación del estado que sí estabilizan el sistema ante variaciones del periodo de muestreo.

El trabajo [55] trata el diseño mediante LMI de un sistema de control digital para un proceso con periodo de muestreo variante. Utiliza un sistema de control por realimentación del estado observado en el que la estimación del estado y la acción de control se actualizan cuando llega una medición. Para este esquema de control se demuestra la existencia del principio de separación entre observador y controlador en ausencia de retardo en la acción de control. En [17] y [78] se obtienen técnicas de diseño de controladores robustos $\mathcal{H}_{\infty}$ utilizando técnicas LMI, donde se tiene en cuenta el error de modelado y la presencia de perturbaciones en el proceso. En el primer trabajo se diseña un controlador de realimentación de la salida, mientras que en el segundo diseña un controlador de realimentación del estado bajo la suposición de que todo el estado está accesible en cada instante de medición.

\footnotetext{
${ }^{1}$ también conocido como Networked Control Systems (NCS) o Integrated Communication and Control Systems (ICCS) en la literatura inglesa

${ }^{2}$ LMI: Desigualdades lineales matriciales, del inglés Linear matrix inequalities.
} 


\section{Control Multifrecuencia.}

En el caso de control multifrecuencia se han encontrado numerosas publicaciones entre las que se pueden citar $[1,6,7,11,30]$, trabajos en los que se supone que la salida se mide siguiendo un patrón de muestreo periódico y las entradas de control se actualizan siguiendo otro patrón periódico. Más recientemente se han publicado trabajos donde se analiza el control multifrecuencia $\mathcal{H}_{\infty}$ [51], el control óptimo multifrecuencia [67], el control predictivo con muestreo no convencional [62] e incluso su extensión a sistemas no lineales [22]. En trabajos como $[34,53,54,68]$ se aborda el caso de muestreo asíncrono multifrecuencia, en el que las mediciones siguen una pauta periódica, pero el instante de medición no es síncrono con la actualización de la entrada. En [31] se introdujeron las técnicas LMI en el control robusto $\mathcal{H}_{\infty}$ multifrecuencia.

El control multifrecuencia supone las mediciones libres de retardos temporales y que se toman siguiendo alguna pauta, por lo que no puede abordar el control de sistemas con muestreo aleatorio y con retardos temporales. Este tipo de sistemas se pueden controlar introduciendo en el bucle un predictor de salidas que alimenta a un controlador estándar, idea que fue introducida inicialmente por Smith [64] para controlar sistemas con retardo.

\section{Control de Sistemas con Retardo.}

En [52] se ha demostrado que los retardos son la principal causa de inestabilidad y bajas prestaciones de sistemas. En algunos trabajos se ha introducido un predictor de Smith para predecir la salida sin retardo, a pesar de que esta técnica no es aplicable con sistemas inestables. En este sentido se han publicado algunos trabajos para estabilizar sistemas inestables con retardo. En [65] se diseña un controlador para un proceso inestable con retardos mediante un control por modelo interno modificado que separa el problema de la estabilización del bucle de la atenuación de perturbaciones. En [41] se analiza el control robusto basado en predictor de un sistema inestable con retardo con ayuda de un modelo de orden extendido y se considera que se dispone en cada periodo de control de todo el vector de estado. En [44] se demuestra que los controladores diseñados para compensar el retardo se pueden reducir a una estructura de control basada en predictor-observador. Finalmente, en [66, 80] se hace un repaso de las metodologías de control existentes para sistemas basados en red, donde se tiene un retardo aleatorio tanto en la señal medida como en la acción de control, y se exponen diferentes técnicas para tratar estos retardos, entre ellas el control inferencial, mientras que en [28] se propone una técnica que permite obtener la cota del máximo retardo admisible en el sistema de comunicación.

Todos estos trabajos consideran, sin embargo, que hay una salida disponible en cada periodo de control.

\section{Control con Medidas Escasas.}

En [80] se plantea un control con medidas escasas de manera que el controlador supone que la salida del proceso es constante mientras no llegan nuevas mediciones. Se analiza la estabilidad del bucle con esta estructura y se obtiene cuál es la mínima frecuencia de muestreo admisible con esta técnica.

En cuanto a la introducción de un predictor para subsanar la falta de información de la salida en algunos instantes de control, algunos autores ya han utilizado la técnica de control inferencial (véase [20, $33,36])$ utilizando un modelo del proceso para estimar y realimentar las salidas que no se pueden medir. En estos trabajos se alimenta el controlador bien con una estimación de la salida en bucle abierto o bien directamente con la salida medida cuando ésta está disponible. En [77] se plantea este mismo esquema de control, pero esta vez con el objetivo de minimizar la transmisión de medidas del proceso al controlador para liberar la carga del canal de comunicación. En este sentido, los autores de [69, 46] calculan el máximo tiempo posible sin acceder al canal de comunicación que mantiene el sistema en bucle cerrado estable.

En estos trabajos se utiliza un predictor de bucle abierto que no hace uso de las mediciones posibles para mejorar la estimación del proceso y, por tanto, no mejoran las prestaciones ante perturbaciones ni son aplicables en procesos inestables.

El control inferencial que utiliza un predictor que corrige sus estimaciones con las mediciones de salida disponibles (predictor en bucle cerrado) se presentó en un ejemplo en [4] para un sistema con muestreo bifrecuencia. Otro trabajo que también utiliza un predictor en bucle cerrado es [40] donde se estudian las prestaciones de un sistema discreto en el cual el sensor envía la salida de la planta al controlador en cada instante de muestreo. Se asume que los paquetes de datos enviados pueden perderse con cierta probabilidad, y que no hay ningún retardo en la transmisión. Como resultados se establecen las condiciones 
para producirse la estabilidad en valor medio y se calcula la varianza del error de predicción y de la señal de salida como una medida de las prestaciones del sistema de control.

\section{Control con Medidas Escasas y Retardos Temporales.}

En [45] se propone una planta con mediciones del estado completo que se envían a través de un canal de comunicación con retraso y transmisión escasa de datos. El controlador estima el estado (utilizando un modelo) para generar la acción de control (continua) cuando no llegan medidas. La realimentación se produce cuando el estado estimado por el controlador se reemplaza con el estado medido que llega a través del canal de comunicación (predictor de sustitución). En este trabajo se establecen las condiciones que ha de cumplir el bucle para que sea estable con la estructura propuesta.

En [43] se plantea el diseño óptimo de un sistema de control para un proceso controlado por computador mediante canales de comunicación asíncronos. En este trabajo se propone un observador del estado recursivo de mínima varianza análogo al filtro de Kalman para estimar el estado en cada periodo de control con las mediciones de la salida disponibles. La utilización de este estimador eleva el coste computacional del algoritmo, ya que las ecuaciones y las suposiciones sobre las perturbaciones son análogas al filtro de Kalman. En este trabajo no se demuestra la estabilidad del bucle cerrado en su conjunto.

En [81] el autor plantea el control de un proceso donde la salida se realimenta a través de una red que proporciona las medidas con pérdida esporádica de datos y con retardo. Se plantea un control de realimentación del estado observado mediante un observador que utiliza las mediciones disponibles para mejorar la estimación, y un controlador que utiliza estas observaciones para calcular la acción de control. El autor plantea el diseño de un observador estable para un muestreo periódico y calcula cuál es la máxima frecuencia de pérdida de datos que permite que el observador siga siendo estable. En este mismo trabajo, se demuestra la existencia del teorema de separación para sistemas lineales basados en red que realimentan la señal de un observador, así como la estabilidad robusta del bucle cerrado. En la exposición queda patente que el problema de regulación de sistemas basados en red se puede reducir siempre al problema del diseño de un observador estable para un sistema con datos faltantes. Sin embargo, no se establece ninguna metodología de diseño del observador para el tipo de muestreo considerado.

\section{Predictor/observador con medidas escasas.}

En lo que se refiere a la predicción de la salidas, la mayoría de los trabajos encontrados se basan en la aplicación del filtro de Kalman para estimar el estado a partir de las medidas obteniendo después las predicciones de la salida a partir de éste.

En [58] se estudia la aplicación del filtro de Kalman a la predicción de salidas ante cualquier escenario de muestreo (síncrono, asíncrono, periódico y aleatorio), y a la fusión sensorial. En [79] se desarrolla un estimador óptimo para sistemas que reciben mediciones de diferente naturaleza: continuas, multifrecuencia y aleatorias. En [37] se desarrollan las ecuaciones del filtro de Kalman para el caso de control multifrecuencia, donde las mediciones y las actualizaciones de control siguen una pauta. En todos estos trabajos se asume una ruido gaussiano de características conocidas, y se asume que no hay ningún retardo en el sistema de medición.

La introducción de mediciones con retardo en el filtro de Kalman puede realizarse mediante la utilización de un modelo de orden extendido cuando el retardo consiste en un número de periodos reducido (véase por ejemplo [24]). Sin embargo esta implementación es muy poco efectiva para grandes retardos o sistemas de orden elevado debido al incremento del tamaño de las matrices en cálculo del filtro. En [32] se trata la introducción de medidas con retardo de una forma alternativa. El método se basa en extrapolar la medida al instante actual usando las estimaciones anteriores y pasadas del filtro de Kalman y calculando una ganancia óptima para esta medida extrapolada. En [42, 70] se presenta una técnica de reorganización de las mediciones con retardo de manera que se obtienen $d$ filtros de Kalman a calcular en paralelo, siendo $d$ el número máximo de retardos discretos. Ninguno de los autores ha tratado la introducción directa de las mediciones con retardo en las ecuaciones del filtro de Kalman sin necesidad de utilizar un modelo de orden extendido, el cálculo de varios filtros en paralelo, o la extrapolación ${ }^{3}$.

En el diseño de estas técnicas basadas en el filtro de Kalman no se garantizan unas prestaciones óptimas cuando hay incertidumbre en el modelo o cuando las perturbaciones no son ruidos gaussianos con estadísticas conocidas.

Algunos autores, como [29, 21, 23, 35, 47, 48, 71, 73, 74, 75], han estudiado el problema del filtrado robusto de sistemas lineales de datos muestreados utilizando técnicas LMI de forma alternativa al filtro

\footnotetext{
${ }^{3}$ El método de la extrapolación no devuelve la solución óptima del filtro de Kalman
} 
de Kalman. Otros autores como [76] han tratado el problema del filtrado de sistemas no lineales con las mismas técnicas LMI, asumiendo que las no linealidades del sistema cumplen con la condición de Lipschitz. Sin embargo ninguno de estas técnicas de diseño basadas en LMI tiene en cuenta el problema del muestreo escaso con retardos.

Otras técnicas alternativas al filtro de Kalman para la estimación de datos faltantes en sistemas lineales se presentaron y analizaron en [56], donde se estudiaron la predicción mediante técnicas de interpolación y técnicas basadas en el modelo entrada-salida del proceso. En este trabajo se presentan diferentes algoritmos de predicción alternativos al filtro de Kalman aplicables al caso de muestreo irregular sin retardo. Los predictores basados en modelo se diseñaron para alcanzar la estabilidad nominal ante muestreo periódico, y para alcanzar la convergencia a cero del error de predicción en valor medio para el caso de muestreo síncrono aleatorio. En [2] se extendió el uso de este predictor al caso bifrecuencia con retardos temporales en el sistema de medición. En [49] se desarrollaron nuevas técnicas de diseño de predictores basados en modelo entrada-salida mediante la aplicación de técnicas LMI. Este análisis condujo a distintos procedimientos que garantizan la estabilidad del predictor resultante. Sin embargo, las prestaciones frente a perturbaciones no fueron tenidas en cuenta y no había ningún factor de ajuste para cambiar la dinámica del predictor. La presente tesis es la evolución de estos últimos trabajos en los cuales ha participado el autor del presente trabajo.

\subsection{Resultados preliminares}

\subsubsection{Predicción de salidas mediante interpolación}

La predicción de salidas mediante interpolación fue analizada en profundidad en [56]. Si no hay retardos en el sistema de medición, la interpolación es la forma más inmediata de predecir las salidas que no se disponen entre mediciones ya que no es necesario ningún modelo del proceso. Como no hay retardos, se asume que la medición $m_{k}$ tomada en el instante de tiempo continuo $\tau_{k}$ corresponde con la salida en dicho instante, esto es $m_{k}=y\left(\tau_{k}\right)$ (véase figura 2.1).

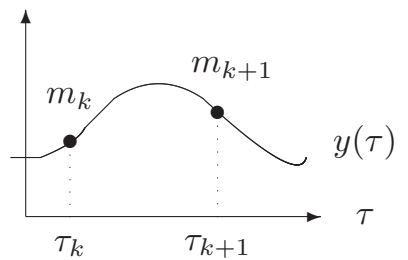

Figura 2.1: Mediciones sin retardo.

La interpolación entre mediciones se puede tomar de diferentes órdenes. Una interpolación de orden cero implica mantener la predicción constante e igual a la última medición disponible, es decir

$$
\hat{y}(\tau)=m_{k}, \quad \tau_{k} \leq \tau<\tau_{k+1},
$$

donde $\tau_{k}$ es el instante de tiempo continuo donde se tiene la $k$-ésima medición. En la interpolación de orden uno se predice una salida en la línea recta que une las dos últimas medidas anteriores al instante de predicción, esto es

$$
\hat{y}(\tau)=m_{k-1}+\frac{m_{k}-m_{k-1}}{\tau_{k}-\tau_{k-1}}\left(\tau-\tau_{k}\right), \quad \tau_{k-1}<\tau_{k} \leq \tau .
$$

En general, esta predicción puede hacerse mediante un polinomio de orden mayor, pero no tiene sentido utilizar un orden muy elevado ya que la salida no sigue una función polinómica.

La utilidad de la interpolación radica en su simplicidad e independencia de un modelo para obtener una predicción de la salida continua. Por este motivo serán útiles cuando se tiene un modelo del proceso impreciso, si el periodo de muestreo es bajo, o si se tienen mediciones asíncronas con la actualización de la acción de control.

\subsubsection{Predicción de salidas basado en modelo entrada-salida}

En trabajos anteriores se han desarrollado algoritmos de predicción basados en una representación interna del sistema, es decir, dan como resultado el estado estimado, pudiendo a partir de él obtener la 
salida estimada. Un ejemplo de ellos es el filtro de Kalman. Cuando no se desea obtener el estado sino las salidas de los instantes de muestreo intermedios, puede resultar interesante la utilización de algoritmos que se basen en la representación entrada-salida, y que, por tanto den lugar directamente a los estimados de las salidas faltantes. Este sería el caso, por ejemplo, si se quiere utilizar un controlador estándar de realimentación de la salida, como un PID, o si se realiza una identificación en línea mediante algoritmos recursivos basados en el vector de regresión de salidas. En principio parece evidente que los predictores basados en la representación entrada-salida pueden ser más simples, y por tanto suponer un coste computacional menor.

Cuando las salidas se miden síncronamente con la entrada, el modelo discreto de entrada-salida es una ecuación en diferencias. Los predictores definidos a partir de esta representación se basan fundamentalmente en la aplicación de la ecuación en diferencias del proceso. La forma básica de incorporar las medidas del sistema en esa ecuación es la sustitución directa del estimado por la medida cuando ésta está disponible. En [56] están desarrolladas diferentes variantes respecto del algoritmo de sustitución básico anterior. Una de las variantes consideradas consiste en sustituir todas las salidas estimadas en el vector de regresión por un valor ponderado de la medida.

A continuación se compara la dinámica obtenida en diferentes predictores de bucle abierto y de bucle cerrado.

Sea un proceso continuo de orden $n$ cuya entrada se actualiza de forma periódica con un computador que dispone de un retenedor de orden cero. Supóngase que la salida se mide de forma síncrona con la actualización de la entrada y sin retardo, de forma que la función de transferencia del proceso puede escribirse como

$$
Y(z)=\frac{B\left(z^{-1}\right)}{A\left(z^{-1}\right)} U(z)=\frac{b_{1} z^{-1}+\cdots+b_{n} z^{n}}{1+a_{1} z^{-1}+\cdots+a_{n} z^{n}} U(z)
$$

que implica la ecuación en diferencias

$$
y[t]=-a_{1} y[t-1]-\cdots-a_{n} y[t-n]+b_{1} u[t-1]+\cdots+b_{n} u[t-n],
$$

donde $y[t]$ es la salida discreta en el instante discreto $t$. Supóngase que algunos de los valores de la secuencia de salida $\{y[0], y[1], y[2], y[3], \ldots\}$ no están disponibles. Definiendo los vectores de regresión de la entrada y la salida como

$$
U[t]=\left[\begin{array}{c}
u[t] \\
u[t-1] \\
\vdots \\
u[t-(n-1)]
\end{array}\right], \quad Y[t]=\left[\begin{array}{c}
y[t] \\
y[t-1] \\
\vdots \\
y[t-(n-1)]
\end{array}\right]
$$

se puede reescribir la ecuación en diferencias (2.2) como

$$
y[t]=-\boldsymbol{\theta}_{a}^{\top} Y[t-1]+\boldsymbol{\theta}_{b}^{\top} U[t-1]
$$

siendo

$$
\begin{aligned}
\boldsymbol{\theta}_{a} & =\left[\begin{array}{lll}
a_{1} & \cdots & a_{n}
\end{array}\right], \\
\boldsymbol{\theta}_{b} & =\left[\begin{array}{lll}
b_{1} & \cdots & b_{n}
\end{array}\right],
\end{aligned}
$$

los vectores de parámetros.

\section{Predictor de bucle abierto}

La forma más simple de estimar la salida del proceso es utilizar el modelo en bucle abierto (predictor en bucle abierto), quedando

$$
\hat{y}[t]=-\boldsymbol{\theta}_{a}^{\top} \hat{Y}[t-1]+\boldsymbol{\theta}_{b}^{\top} U[t-1]
$$

donde

$$
\hat{Y}[t]=\left[\begin{array}{c}
\hat{y}[t] \\
\hat{y}[t-1] \\
\vdots \\
\hat{y}[t-(n-1)]
\end{array}\right]
$$


es la estimación del vector de regresión de salidas compuesto por las estimaciones anteriores de la salida. Este algoritmo no tiene en cuenta las mediciones disponibles de la salida para mejorar la estimación de las salidas y por tanto es muy poco robusto a errores de modelado o a un error inicial en la estimación de las salidas. El siguiente teorema muestra la evolución del error de predicción.

Teorema 2.3.1. La dinámica del error de predicción del predictor en bucle abierto viene dada por

$$
\begin{aligned}
E[t] & =\boldsymbol{A} E[t-1] \\
e[t] & =\boldsymbol{c} E[t]
\end{aligned}
$$

con

$$
\boldsymbol{A}=\left[\begin{array}{cccc}
-a_{1} & \cdots & -a_{n-1} & -a_{n} \\
1 & \cdots & 0 & 0 \\
\vdots & \ddots & & \vdots \\
0 & \cdots & 1 & 0
\end{array}\right], \quad \boldsymbol{c}=\left[\begin{array}{llll}
1 & 0 & \cdots & 0
\end{array}\right]
$$

siendo $E[t]=Y[t]-\hat{Y}[t]$ el vector de regresión del error de predicción y $e[t]=y[t]-\hat{y}[t]$ el error de predicción de la salida actual.

Prueba 2.3.1. Introduciendo la matriz $\boldsymbol{A}$, el proceso puede escribirse como

$$
Y[t]=\boldsymbol{A} Y[t-1]+\boldsymbol{B} U[t],
$$

siendo

$$
\boldsymbol{B}=\left[\begin{array}{ccc}
b_{1} & \cdots & b_{n} \\
0 & \cdots & 0 \\
\vdots & \ddots & \vdots \\
0 & \cdots & 0
\end{array}\right]
$$

mientras que el predictor puede escribirse como

$$
\hat{Y}[t]=\boldsymbol{A} \hat{Y}[t-1]+\boldsymbol{B} U[t] .
$$

Restando (2.6) y (2.7) se tiene que

$$
E[t]=\boldsymbol{A} E[t-1] .
$$

Por otra parte, el error de predicción actual $e[t]$ se obtiene restando el primer elemento del vector $Y[t]$ con el primer elemento del vector $\hat{Y}[t]$ :

$$
e[t]=y[t]-\hat{y}[t]=\boldsymbol{c} Y[t]-\boldsymbol{c} \hat{Y}[t]
$$

Si el sistema es inestable, el algoritmo de predicción en bucle abierto dará como resultado un error de predicción inestable.

\section{Predictor de sustitución}

El siguiente algoritmo (introducido en [4] y analizado en profundidad en [56]) sí que tiene en cuenta las mediciones disponibles y las utiliza para modificar el último valor de la salida estimada sustituyendo su valor en el vector de regresión. Cuando no hay mediciones, la estimación de la salida y el vector de regresión se hace corriendo el modelo en bucle abierto según

$$
\begin{aligned}
\hat{y}[t \mid t-1] & =-\boldsymbol{\theta}_{a}^{\top} \hat{Y}[t-1]+\boldsymbol{\theta}_{b}^{\top} U[t-1] \\
\hat{y}[t-i \mid t] & =\hat{y}[t-i \mid t-1], \quad i=0, \ldots, n-1 \\
\hat{Y}[t] & =\left[\begin{array}{llll}
\hat{y}[t \mid t] & \hat{y}[t-1 \mid t] & \cdots & \hat{y}[t-(n-1) \mid t]
\end{array}\right]^{\top},
\end{aligned}
$$

pero cuando llega una medición $m_{k}$ en el instante $t=t_{k}$, siendo

$$
m_{k}=y\left[t_{k}\right] \equiv y\left(t_{k} T\right)
$$


ésta se utiliza para actualizar el último valor estimado de la salida mediante el algoritmo

$$
\begin{aligned}
& \hat{y}\left[t_{k} \mid t_{k}\right]=m_{k}, \\
& \hat{y}\left[t_{k}-i \mid t_{k}\right]=\hat{y}\left[t_{k}-i \mid t_{k}-1\right], \quad i=1, \ldots, n-1 \\
& \hat{Y}\left[t_{k}\right]=\left[\begin{array}{llll}
\hat{y}\left[t_{k} \mid t_{k}\right] & \hat{y}\left[t_{k}-1 \mid t_{k}\right] & \cdots & \hat{y}\left[t_{k}-(n-1) \mid t_{k}\right.
\end{array}\right]^{\top} .
\end{aligned}
$$

Utilizando las definiciones de las matrices $\boldsymbol{A}, \boldsymbol{B}$ y $\boldsymbol{c}$, este algoritmo puede reescribirse mediante la ecuación

$$
\hat{Y}[t]=\boldsymbol{A} \hat{Y}[t-1]+\boldsymbol{B} U[t-1],
$$

cuando no hay mediciones, más las ecuaciones

$$
\begin{aligned}
\hat{Y}\left[t_{k} \mid t_{k}-1\right] & =\boldsymbol{A} \hat{Y}\left[t_{k}-1\right]+\boldsymbol{B} U\left[t_{k}-1\right], \\
\hat{Y}\left[t_{k}\right] & =\left(\boldsymbol{I}-\boldsymbol{c}^{\top} \boldsymbol{c}\right) \hat{Y}\left[t_{k} \mid t_{k}-1\right]+\boldsymbol{c}^{\top} m_{k},
\end{aligned}
$$

cuando llega una medición $m_{k}$ en el instante $t=t_{k}$. La estimación de la salida en el instante $t$ es el primer elemento de $\hat{Y}[t]$, dado por

$$
y[t]=c \hat{Y}[t]
$$

El siguiente teorema recoge la dinámica del error de predicción de este algoritmo cuando se aplica sobre un proceso con muestreo bifrecuencia como el que se muestra en la figura (2.2).

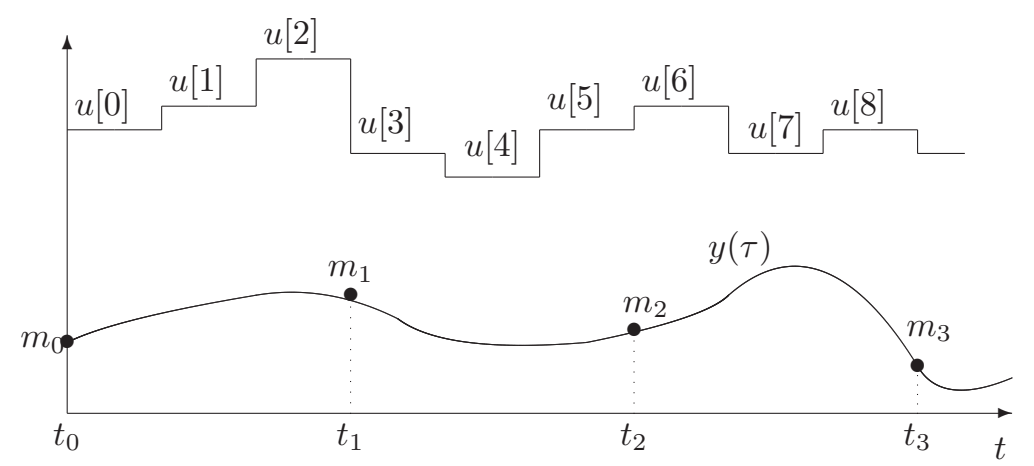

Figura 2.2: Instantes de mediciones y de actualización del control en el muestreo bifrecuencia $(N=3)$.

Teorema 2.3.2. La dinámica del error de predicción del algoritmo (2.9) aplicado al proceso (2.1) cuando hay una medida disponible cada $N$ actualizaciones de la entrada viene dada por las ecuaciones

$$
\begin{aligned}
E_{k} & =\left(\boldsymbol{I}-\boldsymbol{c}^{\top} \boldsymbol{c}\right) \boldsymbol{A}^{N} E_{k-1} \\
e_{k} & =\boldsymbol{c} E_{k}
\end{aligned}
$$

siendo $E_{k}=E\left[t_{k}\right]$ el vector de error de predicción en el instante en que está disponible la $k$-ésima medición de la salida, $y e_{k}=e\left[t_{k}\right]$ el error de predicción en ese mismo instante.

Prueba 2.3.2. En el instante de medición $t=t_{k}$ la predicción de la salida puede expresarse como

$$
\hat{Y}\left[t_{k}\right]=\hat{Y}\left[t_{k} \mid t_{k}-1\right]+c^{\top}\left(\boldsymbol{c} Y\left[t_{k}\right]-\boldsymbol{c} \hat{Y}\left[t_{k} \mid t_{k}-1\right]\right) .
$$

El vector $\hat{Y}\left[t_{k} \mid t_{k}-1\right]$ puede expresarse en función del vector $Y\left[t_{k}-N\right]$ corriendo el modelo, ya que no hay mediciones entre $t_{k}-N$ y $t_{k}-1$, quedando

$$
\hat{Y}\left[t_{k} \mid t_{k}-1\right]=\boldsymbol{A}^{N} \hat{Y}\left[t_{k}-N\right]+\sum_{i=1}^{N} \boldsymbol{A}^{i-1} \boldsymbol{B} U\left[t_{k}-i\right] .
$$

De la misma forma, el vector de regresión de salidas $Y\left[t_{k}\right]$ puede expresarse en función del vector de regresión de salidas en el anterior instante de muestreo $t_{k}-N$ como

$$
Y\left[t_{k}\right]=\boldsymbol{A}^{N} Y\left[t_{k}-N\right]+\sum_{i=1}^{N} \boldsymbol{A}^{i-1} \boldsymbol{B} U\left[t_{k}-i\right] .
$$


Introduciendo las expresiones (2.13) y (2.12) en (2.11) y restando la ecuación resultante de (2.13) se tiene que

$$
Y\left[t_{k}\right]-\hat{Y}\left[t_{k}\right]=A^{N}\left(Y\left[t_{k}-N\right]-\hat{Y}\left[t_{k}-N\right]\right)-c^{\top} \boldsymbol{c} A^{N}\left(Y\left[t_{k}-N\right]-\hat{Y}\left[t_{k}-N\right]\right)
$$

que es exactamente la ecuación (2.10a) debido a que en el instante de toma de medidas $t_{k}-t_{k-1}=N$. El error de predicción de salida instantáneo $e_{k}$ es simplemente el primer valor de $E\left[t_{k}\right]$, y se obtiene por medio del vector $c$

$$
e_{k}=y\left[t_{k}\right]-\hat{y}\left[t_{k}\right]=\boldsymbol{c}\left(Y\left[t_{k}\right]-\hat{Y}\left[t_{k}\right]\right)
$$

El teorema anterior indica que la dinámica del error de predicción viene marcada por la matriz $\left(\boldsymbol{I}-\boldsymbol{c}^{\top} \boldsymbol{c}\right) \boldsymbol{A}^{N}$, por lo que se tiene que ver si para las condiciones de muestreo dadas $(N)$ se obtiene una dinámica estable.

\section{Predictor basado en la actualización completa del vector de regresión}

El siguiente algoritmo (introducido en [4] y analizado en profundidad en [56]) también tiene en cuenta las mediciones disponibles y, además, las utiliza para modificar no sólo el último valor de la salida estimada, sino todo el vector de regresión de salidas. Cuando no hay mediciones, la estimación de la salida y el vector de regresión se hace corriendo el modelo en bucle abierto según

$$
\begin{aligned}
& \hat{y}[t \mid t-1]=-\boldsymbol{\theta}_{a}^{\top} \hat{Y}[t-1]+\boldsymbol{\theta}_{b}^{\top} U[t-1], \\
& \hat{y}[t-i \mid t]=\hat{y}[t-i \mid t-1], \quad i=0, \ldots, n-1 \\
& \hat{Y}[t]=\left[\begin{array}{llll}
\hat{y}[t \mid t-1] & \hat{y}[t-1 \mid t] & \cdots & \hat{y}[t-(n-1) \mid t
\end{array}\right]^{\top},
\end{aligned}
$$

pero cuando llega una medición $m_{k}$ en el instante $t=t_{k}$, siendo

$$
m_{k}=y\left[t_{k}\right] \equiv y\left(t_{k} T\right),
$$

ésta se utiliza para actualizar todo el vector de regresión mediante el algoritmo

$$
\begin{aligned}
& \hat{y}\left[t_{k} \mid t_{k}-1\right]=-\boldsymbol{\theta}_{a}^{\top} \hat{Y}\left[t_{k}-1\right]+\boldsymbol{\theta}_{b}^{\top} U\left[t_{k}-1\right], \\
& \hat{y}\left[t_{k} \mid t_{k}\right]=\hat{y}\left[t_{k} \mid t_{k}-1\right]+l_{1}\left(m_{k}-\hat{y}\left[t_{k} \mid t_{k}-1\right]\right), \\
& \hat{y}\left[t_{k}-1 \mid t_{k}\right]=\hat{y}\left[t_{k}-1 \mid t_{k}-1\right]+l_{2}\left(m_{k}-\hat{y}\left[t_{k} \mid t_{k}-1\right]\right), \\
& \hat{y}\left[t_{k}-(n-1) \mid t_{k}\right]=\hat{y}\left[t_{k}-(n-1) \mid t_{k}-1\right]+l_{n}\left(m_{k}-\hat{y}\left[t_{k} \mid t_{k}-1\right]\right), \\
& \hat{Y}\left[t_{k}\right]=\left[\begin{array}{llll}
\hat{y}\left[t_{k} \mid t_{k}\right] & \hat{y}\left[t_{k}-1 \mid t_{k}\right] & \cdots & \hat{y}\left[t_{k}-(n-1) \mid t_{k}\right.
\end{array}\right]^{\top} .
\end{aligned}
$$

Utilizando las definiciones de las matrices $\boldsymbol{A}, \boldsymbol{B}$ y $\boldsymbol{c}$, este algoritmo puede reescribirse mediante la ecuación

$$
\hat{Y}[t]=\boldsymbol{A} \hat{Y}[t-1]+\boldsymbol{B} U[t-1],
$$

cuando no hay mediciones, más las ecuaciones

$$
\begin{aligned}
\hat{Y}\left[t_{k} \mid t_{k}-1\right] & =\boldsymbol{A} \hat{Y}\left[t_{k}-1\right]+\boldsymbol{B} U\left[t_{k}-1\right], \\
\hat{Y}\left[t_{k}\right] & =\hat{Y}\left[t_{k} \mid t_{k}-1\right]+\boldsymbol{\ell}\left(m_{k}-y\left[t_{k} \mid t_{k}-1\right]\right),
\end{aligned}
$$

cuando llega una medición $m_{k}$ en el instante $t=t_{k}$, siendo

$$
y\left[t_{k} \mid t_{k}-1\right]=c \hat{Y}\left[t_{k} \mid t_{k}-1\right]
$$

la estimación inicial (en bucle abierto) de la salida, y

$$
\ell=\left[\begin{array}{llll}
l_{1} & l_{2} & \cdots & l_{n}
\end{array}\right]^{\top}
$$

el vector de ganancias del predictor. La estimación de la salida en el instante $t$ es el primer elemento de $\hat{Y}[t]$, dado por

$$
y[t]=c \hat{Y}[t] .
$$

En el caso más sencillo $\ell$ se considera un vector constante. El siguiente teorema recoge la dinámica del error de predicción de este algoritmo cuando se aplica sobre un proceso con muestreo bifrecuencia como el que se muestra en la figura (2.2). 
Teorema 2.3.3. La dinámica del error de predicción del algoritmo (2.15) aplicado al proceso (2.1) cuando hay una medida disponible cada $N$ actualizaciones de la entrada viene dada por las ecuaciones

$$
\begin{aligned}
E_{k} & =(\boldsymbol{I}-\ell \boldsymbol{c}) \boldsymbol{A}^{N} E_{k-1} \\
e_{k} & =\boldsymbol{c} E_{k}
\end{aligned}
$$

siendo $E_{k}=E\left[t_{k}\right]$ el vector de error de predicción en el instante en que está disponible la k-ésima medición de la salida, $y e_{k}=e\left[t_{k}\right]$ el error de predicción en ese mismo instante.

Prueba 2.3.3. En el instante de medición $t=t_{k}$ la predicción de la salida puede expresarse como

$$
\hat{Y}\left[t_{k}\right]=\hat{Y}\left[t_{k} \mid t_{k}-1\right]+\boldsymbol{\ell}\left(\boldsymbol{c} Y\left[t_{k}\right]-\boldsymbol{c} \hat{Y}\left[t_{k} \mid t_{k}-1\right]\right) .
$$

El vector $\hat{Y}\left[t_{k} \mid t_{k}-1\right]$ puede expresarse en función del vector $Y\left[t_{k}-N\right]$ corriendo el modelo, ya que no hay mediciones entre $t_{k}-N$ y $t_{k}-1$, quedando

$$
\hat{Y}\left[t_{k} \mid t_{k}-1\right]=\boldsymbol{A}^{N} \hat{Y}\left[t_{k}-N\right]+\sum_{i=1}^{N} \boldsymbol{A}^{i-1} \boldsymbol{B} U\left[t_{k}-i\right] .
$$

De la misma forma, el vector de regresión de salidas $Y\left[t_{k}\right]$ puede expresarse en función del vector de regresión de salidas en el anterior instante de muestreo $t_{k}-N$ como

$$
Y\left[t_{k}\right]=\boldsymbol{A}^{N} Y\left[t_{k}-N\right]+\sum_{i=1}^{N} \boldsymbol{A}^{i-1} \boldsymbol{B} U\left[t_{k}-i\right] .
$$

Introduciendo las expresiones (2.19) y (2.18) en (2.17) y restando la ecuación resultante de (2.19) se tiene que

$$
Y\left[t_{k}\right]-\hat{Y}\left[t_{k}\right]=A^{N}\left(Y\left[t_{k}-N\right]-\hat{Y}\left[t_{k}-N\right]\right)-\ell \boldsymbol{c} A^{N}\left(Y\left[t_{k}-N\right]-\hat{Y}\left[t_{k}-N\right]\right)
$$

que es exactamente la ecuación (2.16a) debido a que en el instante de toma de medidas $t_{k}-t_{k-1}=N$. El error de predicción de salida instantáneo $e_{k}$ es simplemente el primer valor de $E\left[t_{k}\right]$, y se obtiene por medio del vector $c$

$$
e_{k}=y\left[t_{k}\right]-\hat{y}\left[t_{k}\right]=c\left(Y\left[t_{k}\right]-\hat{Y}\left[t_{k}\right]\right)
$$

El teorema anterior indica que la dinámica del error de predicción viene marcada por la matriz $(\boldsymbol{I}-\boldsymbol{\ell} \boldsymbol{c}) \boldsymbol{A}^{N}$, por lo que se pueden utilizar técnicas como la asignación de polos para conseguir que la matriz anterior tenga los polos deseados.

Nótese que las técnicas anteriores son un caso especial de esta última técnica. La técnica de bucle abierto corresponde a una ganancia nula $\boldsymbol{\ell}=\mathbf{0}$, mientras que el predictor de sustitución se corresponde con una ganancia de la forma $\ell=c^{\top}=\left[\begin{array}{llll}1 & 0 & \cdots & 0\end{array}\right]^{\top}$.

En el siguiente ejemplo se comparan las tres técnicas: la de estimación en bucle abierto, la de sustitución y la de actualización del vector de regresión.

Ejemplo 2.3.1. Considérese el sistema continuo invariante en el tiempo definido por la función de transferencia

$$
G(s)=\frac{1}{(s-1)(s+3)}
$$

con un periodo de control de $T=0.1$ segundos. La función de transferencia discreta con retenedor de orden cero es

$$
G(z)=\frac{4.69 z^{-1}+4.39 z^{-2}}{1-1.846 z^{-1}+0.8187 z^{-2}} 10^{-3}
$$

Sin embargo, sólo se tienen mediciones disponibles cada $T_{o}=1.2$ segundos, es decir, $N=12$. Corriendo el modelo en bucle abierto $(\boldsymbol{\ell}=\mathbf{0})$ se tiene un comportamiento inestable del predictor. Esto puede comprobarse calculando los valores propios de la dinámica del error de predicción con predictor en bucle abierto:

$$
\lambda\left((\boldsymbol{I}-\boldsymbol{\ell} \boldsymbol{c}) \boldsymbol{A}^{N}\right)=\lambda\left(\boldsymbol{A}^{N}\right)=\{3.32,0.0273\} .
$$



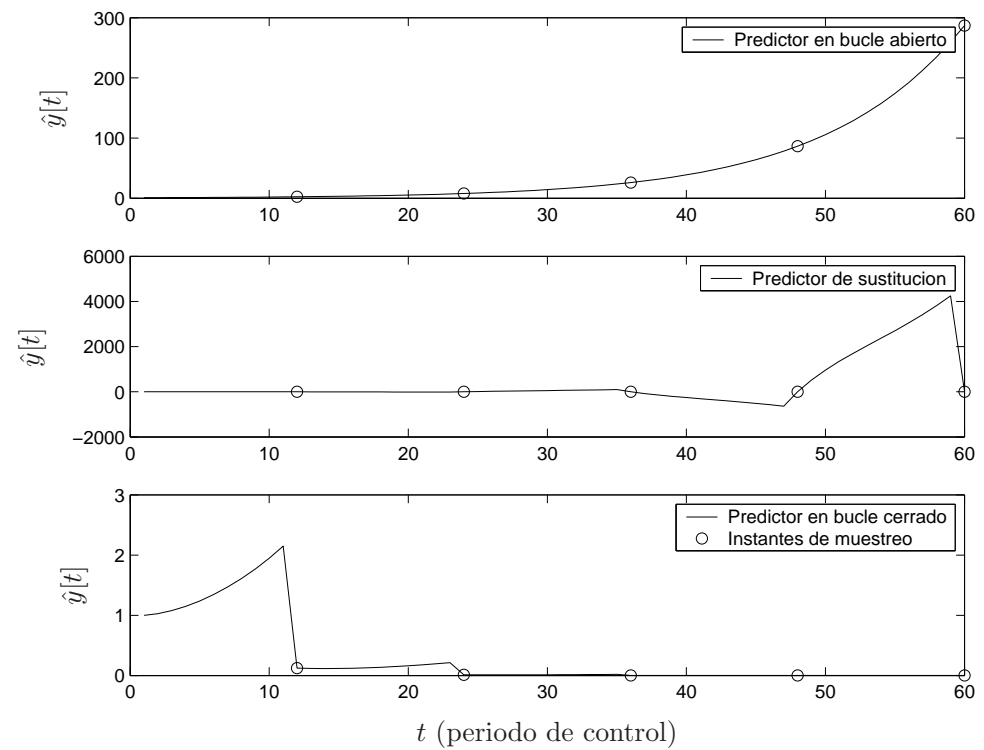

Figura 2.3: Evolución del error de predicción $(N=12)$.

También se obtiene una dinámica inestable si se emplea un predictor de sustitución, ya que los valores propios son

$$
\lambda\left(\left(\boldsymbol{I}-\boldsymbol{c}^{\top} \boldsymbol{c}\right) \boldsymbol{A}^{N}\right)=\{0,-6.67\} .
$$

Sin embargo, si se diseña un predictor para tener unos valores propios entre muestras ubicados en $0.8^{N}$, se obtiene el vector de ganancias

$$
\ell=\left[\begin{array}{l}
0.9479 \\
0.8493
\end{array}\right] \text {. }
$$

En la figura 2.3 se muestra la evolución del error de predicción de salida en cada periodo de control, donde se muestra que el predictor de bucle cerrado disminuye el error cada vez que hay una medición disponible, a diferencia del predictor de bucle abierto o el predictor basado en la sustitución de la medida en el vector de regresión, que llevan a una dinámica inestable del error de predicción

\section{Control de procesos con medidas escasas}

Si se pretende controlar la salida caben varias posibilidades en función de la señal que se realimenta y del predictor que se utiliza. Las diferentes estrategias encontradas en la bibliografía junto con la que se propone en esta tesis (E5) se pueden enumerar como sigue:

E1 Predictor de interpolación manteniendo constante la estimación de la salida mientras no se tiene información. Cálculo de la acción de control con esta estimación constante entre mediciones.

E2 Predictor de bucle abierto. Cálculo de la acción de control con la estimación de bucle abierto.

E3 Predictor de bucle abierto. Cálculo de la acción de control con las medidas si está disponible, y si no, con la predicción en bucle abierto.

E4 Predictor de sustitución. Cálculo de la acción de control con esta estimación. En los instantes de medición se utilizará, por tanto, la medición de la salida para calcular la acción de control.

E5 Predictor de actualización del vector de regresión. Cálculo de la acción de control con la estimación.

En los próximos ejemplos se comparan las diferentes estrategias y se pone de manifiesto la mejoría obtenida con la metodología propuesta en esta tesis, motivo de análisis que se realizan a lo largo del trabajo.

Ejemplo 2.3.2. Considérese el sistema continuo invariante en el tiempo definido por la función de transferencia

$$
G(s)=\frac{5}{(s+1)(s+3)}
$$




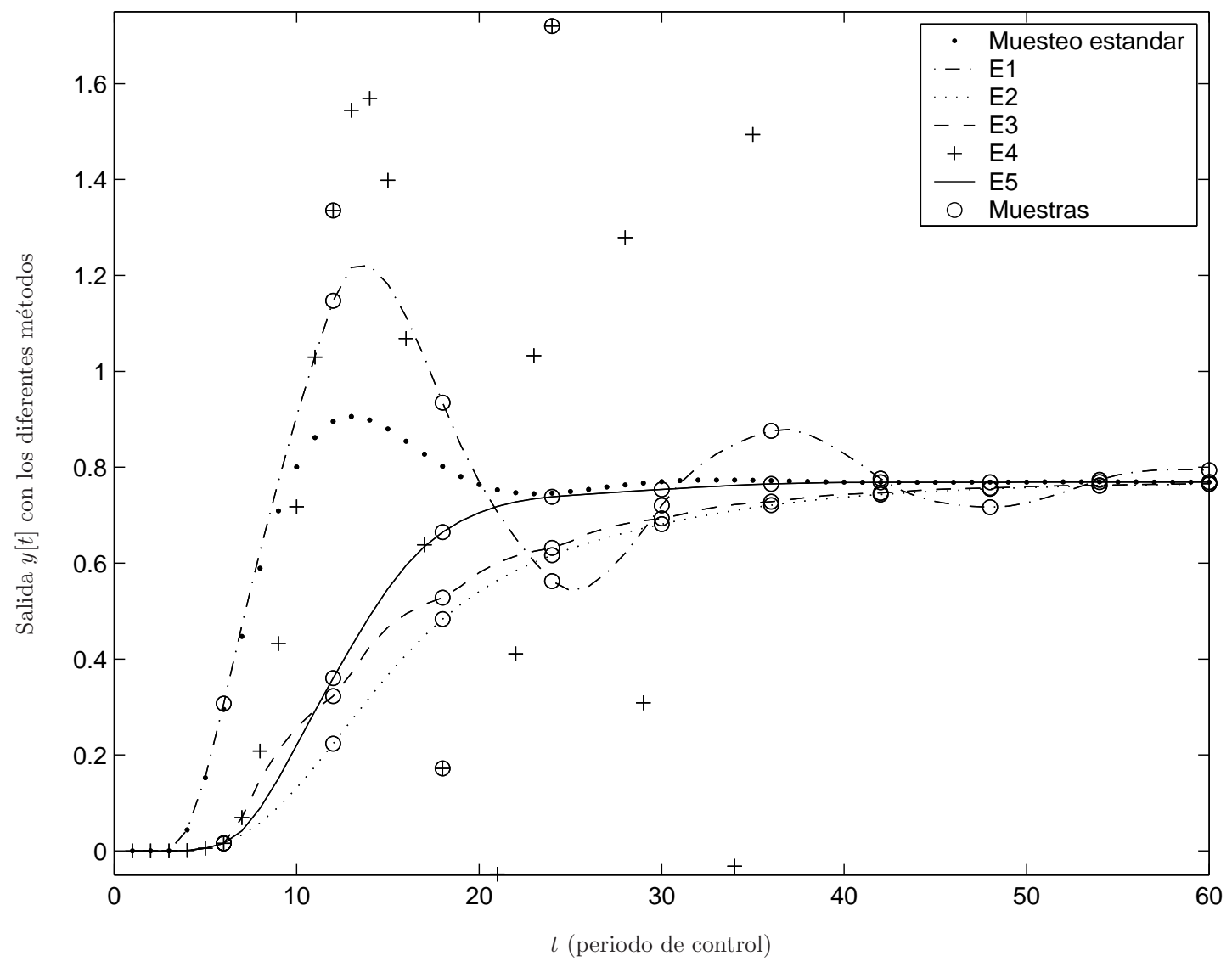

Figura 2.4: Evolución de la salida controlada con $C(z)=2(N=6)$.

con un periodo de control de $T=0.1$ segundos. Supóngase que sólo se tienen mediciones disponibles cada $T_{o}=0.6$ segundos, es decir, $N=6$. Si se utiliza un predictor en bucle abierto $(\boldsymbol{\ell}=\mathbf{0})$ se tiene un comportamiento del predictor marcado por la dinámica del proceso a periodo NT:

$$
\lambda\left((\boldsymbol{I}-\boldsymbol{\ell c}) \boldsymbol{A}^{N}\right)=\lambda\left(\boldsymbol{A}^{N}\right)=\{0.5488,0.1653\}
$$

mientras que si se utiliza un predictor de sustitución la dinámica que se obtiene una dinámica inestable como indican los valores propios

$$
\lambda\left(\left(\boldsymbol{I}-\boldsymbol{c}^{\top} \boldsymbol{c}\right) \boldsymbol{A}^{N}\right)=\{0,-1.5669\}
$$

Sin embargo, si se diseña un predictor para tener unos valores propios entre muestras ubicados en $0.8^{N}$, se obtiene el vector de ganancias

$$
\boldsymbol{\ell}=\left[\begin{array}{l}
0.2425 \\
0.2318
\end{array}\right]
$$

En la figura 2.4 se muestra una simulación en la que se ha controlado la salida del proceso mediante un controlador constante $C(z)=2$. Se muestra la salida controlada con muestreo estándar y también la salida con las diferentes estrategias. Con el predictor de interpolación (E1) se obtiene una respuesta del proceso que es muy oscilatoria, mientras que con el predictor de sustitución (E4) se obtiene una respuesta inestable. Las estrategias que utilizan un predictor de bucle abierto responden (E2 y E3) de forma estable aunque más lento que la respuesta con muestreo estándar. Nótese que la estrategia que realimenta las mediciones cuando éstas están disponibles (E3) trata de corregir la salida con la llegada de cada medición, aunque no consigue mejorar mucho la respuesta respecto de la estrategia que no realimenta las mediciones (E2). Finalmente, con la estrategia que utiliza un predictor en bucle cerrado (E5) se obtiene una salida estable más rápida que con el predictor de bucle abierto. 

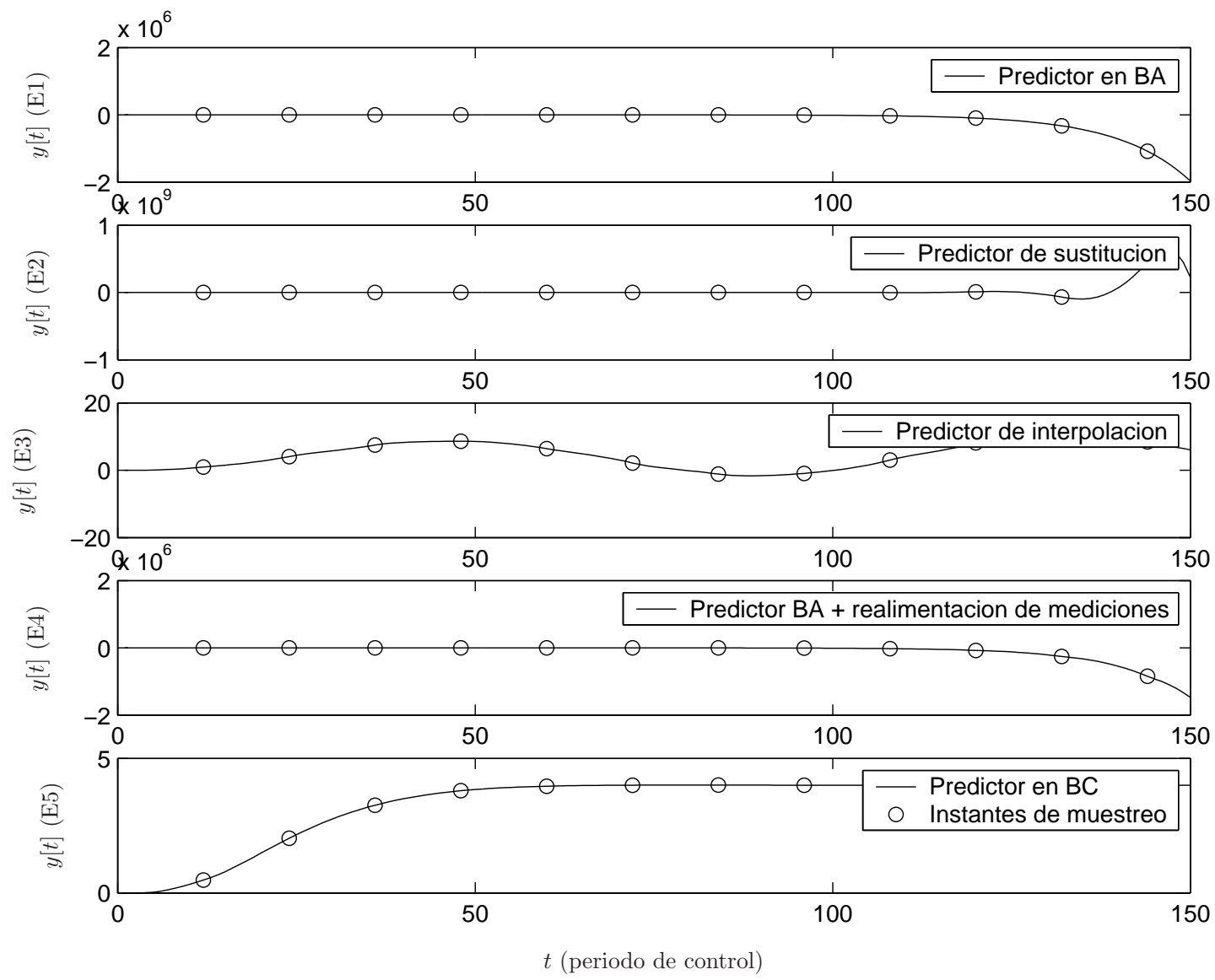

Figura 2.5: Evolución de la salida controlada con $C(z)=4(N=12)$.

Ejemplo 2.3.3. Retómese el proceso inestable del ejemplo 2.3.1 y las condiciones de muestreo allí definidas. Si el muestreo es convencional, un controlador constante $C(z)=4$ es suficiente para controlar el proceso (en ausencia de perturbaciones o errores de modelado), ya que la función de transferencia en bucle cerrado queda

$$
M(z)=\frac{G(z) C(z)}{1+G(z) C(z)}=\frac{0.018715(z+0.9355)}{(z-0.9048)^{2}} .
$$

Si se aplica este controlador con las diferentes estrategias, la respuesta del proceso que se obtiene cuando la referencia es un escalón unitario es la que se muestra en la figura 2.5. Se observa que la única estrategia que permite estabilizar la salida es la que utiliza el predictor en bucle cerrado (E5).

Uno de los objetivos de la presente tesis es demostrar matemáticamente la necesidad de utilizar predictores en bucle cerrado basados en la actualización completa del vector de regresión del modelo, tal y como se ha mostrado en los ejemplos.

\subsubsection{Predicción de salidas basado en el filtro de Kalman}

Supóngase un proceso continuo lineal e invariante en el tiempo descrito por las ecuaciones

$$
\begin{aligned}
\dot{\boldsymbol{x}}(\tau) & =\boldsymbol{A}_{c} \boldsymbol{x}(\tau)+\boldsymbol{B}_{c} \boldsymbol{u}(\tau)+\boldsymbol{v}(\tau) \\
\boldsymbol{y}(\tau) & =\boldsymbol{C}_{y} \boldsymbol{x}(\tau) \\
\boldsymbol{m}(\tau) & =\boldsymbol{C} \boldsymbol{x}(\tau)+\boldsymbol{w}(\tau)
\end{aligned}
$$

Donde $\boldsymbol{x} \in \mathbb{R}^{n}$ es el estado, $\boldsymbol{u} \in \mathbb{R}^{n_{u}}$ es la entrada de control, $\boldsymbol{y} \in \mathbb{R}^{n_{y}}$ es la señal de salida a controlar y $\boldsymbol{m} \in \mathbb{R}^{n_{m}}$ es el vector de señales medidas. Las señales $\boldsymbol{v}(\tau)$ y $\boldsymbol{w}(\tau)$ son las posibles perturbaciones de entrada y ruido de medida, respectivamente. 
Supóngase que las perturbaciones $\boldsymbol{v}(\tau)$ y $\boldsymbol{w}(\tau)$ son ruidos blancos con matrices de varianzas-covarianzas $\boldsymbol{V}$ y $\boldsymbol{W}$ respectivamente. Supóngase que se miden algunos de los elementos del vector $\boldsymbol{m}(\tau)$ en instantes arbitrarios de tiempo $\tau_{k}, k \in \mathbb{N}$ (se mide al menos un elemento de $\boldsymbol{m}(\tau)$ en el instante $\tau_{k}$ ). Defínase la matriz $\boldsymbol{M}_{k}$ de orden $\mu_{k} \times n_{m}$ formada con las filas de una matriz identidad que corresponden a la posición de los elementos de $\boldsymbol{m}(\tau)$ que se miden en el instante $\tau_{k}$, por lo que $\mu_{k}$ es el número de variables medidas en el instante $\tau_{k}$.

El estimador del estado que minimiza la varianza del error (filtro de Kalman) se define mediante las ecuaciones

$$
\begin{aligned}
& \dot{\hat{\boldsymbol{x}}}(\tau)=\boldsymbol{A}_{c} \hat{\boldsymbol{x}}(\tau)+\boldsymbol{B}_{c} \boldsymbol{u}(\tau) \\
& \dot{\hat{\boldsymbol{Q}}}(\tau)=\boldsymbol{A}_{c} \hat{\boldsymbol{Q}}(\tau)+\hat{\boldsymbol{Q}}(\tau) \boldsymbol{A}_{c}^{\top}+\boldsymbol{V}
\end{aligned}
$$

que se corren en el intervalo de tiempo entre mediciones, más las ecuaciones

$$
\begin{aligned}
\boldsymbol{L}\left(\tau_{k}\right) & =\hat{\boldsymbol{Q}}\left(\tau_{k}^{-}\right)\left(\boldsymbol{M}_{k} \boldsymbol{C}\right)^{\boldsymbol{\top}}\left(\boldsymbol{M}_{k}\left(\boldsymbol{C} \hat{\boldsymbol{Q}}\left(\tau_{k}^{-}\right) \boldsymbol{C}_{z}^{\boldsymbol{\top}}+\boldsymbol{W}\right) \boldsymbol{M}_{k}^{\boldsymbol{\top}}\right)^{-1} \\
\hat{\boldsymbol{x}}\left(\tau_{k}^{+}\right) & =\hat{\boldsymbol{x}}\left(\tau_{k}^{-}\right)+\boldsymbol{L}\left(\tau_{k}\right)\left(\boldsymbol{M}_{k} \boldsymbol{m}\left(\tau_{k}\right)-\boldsymbol{M}_{k} \boldsymbol{C} \hat{\boldsymbol{x}}\left(\tau_{k}^{-}\right)\right) \\
\hat{\boldsymbol{Q}}\left(\tau_{k}^{+}\right) & =\left[\boldsymbol{I}-\boldsymbol{L}\left(\tau_{k}\right) \boldsymbol{M}_{k} \boldsymbol{C}\right] \hat{\boldsymbol{Q}}\left(\tau_{k}^{-}\right)
\end{aligned}
$$

que se aplican en los instantes de mediciones. Las ecuaciones diferenciales (2.21a) y (2.21b) pueden integrarse a lo largo de los intervalos intermuestreo. Definiendo el tiempo entre dos muestras consecutivas como $\nu_{k}=\tau_{k}-\tau_{k-1}$, las ecuaciones resultantes son

$$
\begin{aligned}
& \hat{\boldsymbol{x}}\left(\tau_{k}^{-}\right)=e^{\boldsymbol{A}_{c} \nu_{k}} \hat{\boldsymbol{x}}\left(\tau_{k-1}^{+}\right)+\int_{0}^{\nu_{k}} e^{\boldsymbol{A}_{c} \sigma} \boldsymbol{B}_{c} \boldsymbol{u}\left(\tau_{k}-\sigma\right) \mathrm{d} \sigma \\
& \hat{\boldsymbol{Q}}\left(\tau_{k}^{-}\right)=e^{\boldsymbol{A}_{c} \nu_{k}} \hat{\boldsymbol{Q}}\left(\tau_{k-1}^{+}\right)\left(e^{\boldsymbol{A}_{c} \nu_{k}}\right)^{\top}+\overline{\boldsymbol{V}}-e^{\boldsymbol{A}_{c} \nu_{k}} \overline{\boldsymbol{V}}\left(e^{\boldsymbol{A}_{c} \nu_{k}}\right)^{\top}
\end{aligned}
$$

donde $\overline{\boldsymbol{V}}$ es la solución de la ecuación

$$
\boldsymbol{A}_{c} \overline{\boldsymbol{V}}+\overline{\boldsymbol{V}} \boldsymbol{A}_{c}^{\top}=-\boldsymbol{V}
$$

Las ecuaciones anteriores definen el algoritmo del filtro de Kalman para el caso general de instantes de medición arbitrarios. Obsérvese el elevado coste computacional que implica el cálculo de exponenciales de matrices e inversión de matrices cada vez que se tiene una nueva medición. Si esta medición es necesaria para cerrar un lazo de control, el elevado coste computacional puede tener como consecuencia un retardo en el cálculo de la acción de control.

Observación 2.3.1. La matriz de ganancias obtenida con el filtro de Kalman es una matriz variante en el tiempo cuyo tamaño es $n \times \mu_{k}$, es decir, que depende del número de sensores de los que se tiene información en cada muestra. El número de filas de la matriz $\boldsymbol{L}\left(\tau_{k}\right)$ es constante e igual a $n$, y el número de columnas depende del número de sensores disponibles en cada periodo $\left(\mu_{k}\right)$.

A partir de la formulación del filtro de Kalman para sistemas lineales, se puede obtener de forma inmediata las ecuaciones del filtro de Kalman extendido para predecir salidas en sistemas no lineales con medidas escasas.

\subsection{Conclusiones}

En este capítulo se ha realizado una revisión de los trabajos encontrados en la literatura sobre control de procesos y predicción de salidas con retardos y muestreo irregular. Se ha comprobado que ningún autor ha planteado hasta el momento el diseño de predictores robustos con medidas aleatorias y retardos temporales variantes. Tampoco está contemplado en la literatura el control inferencial de procesos multivariables. También se ha visto que las técnicas existentes en la literatura para incorporar las mediciones con retardo en algoritmos de estimación (como el filtro de Kalman), requieren un elevado coste computacional.

Se han reproducido los resultados básicos de predicción con medidas escasas mediante interpolación y predicción basada en modelo entrada-salida, que son los algoritmos de estimación de salidas que tienen un menor coste computacional. Se ha demostrado la necesidad de utilizar predictores de bucle cerrado ante sistemas inestables.

Finalmente se ha expuesto la solución al caso general de predicción mediante el filtro de Kalman utilizando medidas escasas, llevando a un algoritmo de elevado coste computacional. 



\section{Diseño de predictores en representación entrada salida}

Este capítulo se centra en la síntesis de un predictor que estima las salidas de un proceso de una entrada y una salida en cada periodo de control cuando el muestreo es síncrono, aleatorio y con un retardo variante. El predictor utiliza un modelo entrada-salida del proceso y toda la información que dispone de la salida. Se asume una planta lineal afectada por perturbaciones y un sensor afectado de ruido de medida que suministra las mediciones con un retardo variante.

La estructura del capítulo es como sigue. En la segunda sección se plantea el problema de predicción de salidas en un sistema de una entrada y una salida definiendo la planta y el escenario de muestreo. En la tercera sección se introduce el algoritmo de predicción propuesto y se obtiene la dinámica del error de predicción, necesaria para desarrollar las diferentes técnicas de diseño. En la cuarta sección se establece el diseño del predictor para alcanzar la estabilidad nominal ante un escenario de muestreo periódico con retardos constantes. Esta sección sirve de introducción al resto de diseños que consideran muestreo escaso aleatorio con retardos temporales variantes en el tiempo. En la quinta sección se aborda el diseño de predictores robustos frente a perturbaciones y ruido de medida. En esta sección se presenta un catálogo de diferentes formulaciones con las que obtener el diseño más adecuado para diferentes especificaciones en función del tipo de perturbaciones. En la sexta sección se plantea el diseño estocástico de predictores robustos asumiendo conocida la función de distribución de probabilidades del canal de comunicación, es decir, asumiendo conocida la probabilidad de obtener una muestra en un periodo dado. En la séptima sección se analizan las modificaciones que se deben realizar en los diseños anteriores cuando el objetivo del predictor es proporcionar un vector de regresión de salidas que permita identificar el proceso con el menor error posible. En la octava sección se estudian diferentes consideraciones de diseño a tener en cuenta a la hora de seleccionar un tipo de diseño u otro y a la hora de elegir los valores de los parámetros de ajuste. Se estudia además cómo replantear el problema si se tienen perturbaciones de entrada, error de modelado, retardo en la acción de control o mediciones con etiqueta de tiempo incierta. En la octava sección se estudian diferentes simplificaciones que permiten reducir el coste computacional del procedimiento de diseño de los predictores, que se ejecuta fuera de línea.

\subsection{Descripción del problema}

Considérese el sistema de control digital que se muestra en la figura 3.1, donde $G(s)$ es un sistema continuo lineal monovariable cuya entrada se actualiza a periodo $T$ por un controlador digital convencional $C(z)$ con un retenedor de orden cero $(\mathrm{ZOH})$.

\subsubsection{Escenario de muestreo}

Cada periodo de control llega al proceso una nueva entrada $u[t]$, donde $t \in \mathbb{N}$ es el número de actualización de la entrada. La relación entre la señal discreta y la continua es

$$
u(\tau)=u[t] \quad \tau \in[t T, t T+T)
$$

La salida del proceso $y(\tau)$, que está afectada por una perturbación $v(\tau)$, se mide de forma síncrona con la actualización de la entrada pero escasa, con un periodo variante en el tiempo $\left(N_{k} T\right)$. La $k$-ésima salida 


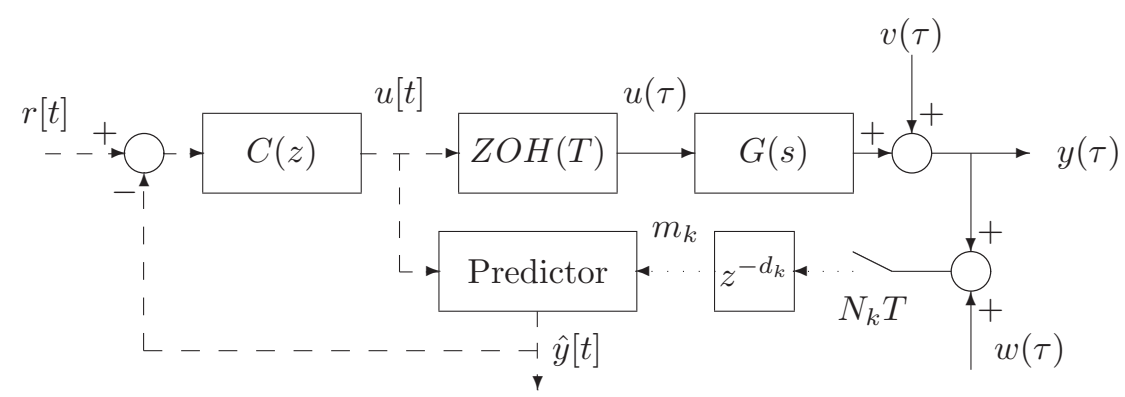

Figura 3.1: Predicción de salidas con mediciones aleatorias con retardo. $z^{-1}$ es el operador retardo

muestreada se denota por $y_{k}$. La medición de la $k$-ésima salida muestreada $\left(m_{k}\right)$ viene afectada por un ruido $w_{k}$ y se asume que está disponible $d_{k} T$ segundos después (debido al retardo temporal presente en el sistema de comunicación o en el procedimiento de medición). Se asume que el retardo variante en el tiempo $\left(d_{k}\right)$ varía en un conjunto finito

$$
d_{k} \in \mathcal{D}=\left\{\delta_{1}, \ldots, \delta_{n_{\mathcal{D}}}\right\}
$$

La $k$-ésima medida está disponible en $t=t_{k}$ y se puede establecer la siguiente relación:

$$
m_{k}=m\left[t_{k}\right]=y\left[t_{k}-d_{k}\right]+w\left[t_{k}-d_{k}\right]=y_{k}+w_{k} .
$$

El número de actualizaciones de la entrada desde $m_{k-1}$ hasta $m_{k}$ se denota con $N_{k}\left(N_{k}=t_{k}-t_{k-1}\right.$, véase figura 3.2) y se asume que varía en un conjunto finito

$$
N_{k} \in \mathcal{N}=\left\{\nu_{1}, \ldots, \nu_{n_{\mathcal{N}}}\right\}
$$

Se puede establecer una relación entre la secuencia de las salidas muestreadas $\left(y_{k}\right)$, la señal discreta de salida a periodo $T(y[t])$, y la señal de salida continua $(y(\tau))$ como

$$
y_{k}=y\left[t_{k}-d_{k}\right]=y\left(\left(t_{k}-d_{k}\right) T\right)
$$

donde

$$
t_{k}=\sum_{i=1}^{k} N_{i}
$$

representa el instante en el cual ocurre la $t_{k}$-ésima actualización de la entrada y se obtiene la $k$-ésima muestra de la salida $\left(m_{k}\right)$.

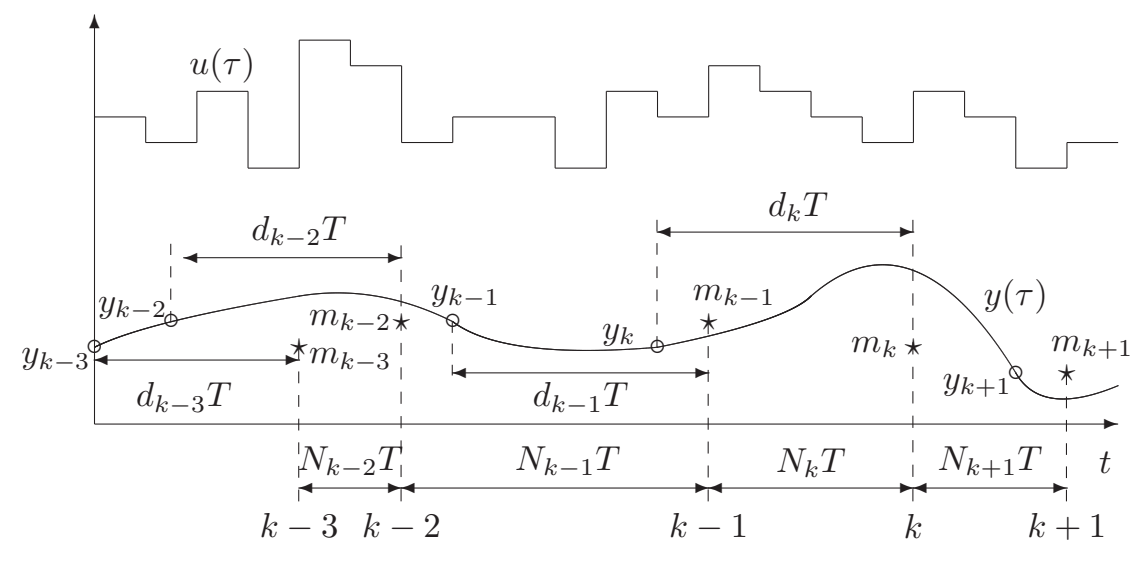

Figura 3.2: Muestreo síncrono aleatorio con retardo variante en el tiempo.

Aunque se ha considerado que existe retardo únicamente entre la salida y la llegada de una medición, realmente en la planta pueden darse otros retardos: el retardo que se produce entre el controlador y el actuador a través del canal de comunicación (si lo hay), y el retardo del proceso, si tiene. En este trabajo se considera que estos posibles retardos son constantes y conocidos, con lo que se puede añadir su valor al retardo de la salida $d_{k}$. De esta forma no se pierde la generalización del problema de predicción de salidas en un proceso libre de retardos. El objetivo del predictor es suministrar al controlador las predicciones de la salida a periodo $T$. 


\subsubsection{Parametrización del escenario de muestreo}

Cada instante de medición viene definido por dos parámetros: el retardo de la medición con respecto a la salida correspondiente $\left(d_{k}\right)$, y el número de veces que se ha actualizado la acción de control entre instantes de medición $\left(N_{k}\right)$. Cada uno de estos parámetros puede tomar valores dentro de unos rangos conocidos (dados por (3.1) y (3.3) ). Sin embargo, según la aplicación, sólo algunas combinaciones $\left(d_{k}, N_{k}\right)$ se darán de forma simultánea. Por este motivo se define un nuevo parámetro $s_{k}$ que indica cuál es la situación del muestreo (combinación $\left(d_{k}, N_{k}\right)$ ) de la $k$-ésima medida. El parámetro $s_{k}$ enumera las combinaciones tomando valores enteros, es decir

$$
s_{k} \in \mathcal{S}=\left\{1,2, \ldots, n_{\mathcal{S}}\right\},
$$

donde $n_{\mathcal{S}}$ es el número de combinaciones posibles $\left(d_{k}, N_{k}\right)$. El número de combinaciones es $n_{\mathcal{S}} \leq n_{\mathcal{N}} \cdot n_{\mathcal{D}}$, siendo $n_{\mathcal{N}}$ el tamaño del conjunto $\mathcal{N}$ y $n_{\mathcal{D}}$ el tamaño del conjunto $\mathcal{D}$.

Con el nuevo parámetro definido se puede establecer una dependencia de los parámetros $N_{k} \mathrm{y} d_{k}$ con respecto de $s_{k}$ de forma que

$$
\begin{aligned}
N_{k} & =N\left(s_{k}\right), \\
d_{k} & =d\left(s_{k}\right) .
\end{aligned}
$$

Como ejemplo supóngase que se tiene un sistema en el que la escasa disponibilidad de medidas se debe al propio retardo del procesado de cada medición, que necesita un tiempo para procesar cada medida antes de tomar la siguiente. Esto es algo usual en las aplicaciones que requieren procesado de imágenes, o un análisis químico de una muestra. También puede darse esta situación cuando la medición se transmite por red, ya que hasta que no lleguen todos los paquetes de datos que transmiten una muestra, no se podrán comenzar a recibir los de la siguiente muestra. En este caso, si se tienen unos conjuntos $\mathcal{N}=\{5,6,7\}$ y $\mathcal{D}=\{5,6,7\}$, la asignación de valores al parámetro de situación de muestreo viene dada por la tabla 3.1 , donde se observa la imposibilidad de que ocurra la situación en la que el retardo $d$ es mayor que el número de periodos intermuestreo $N$, debido a la necesidad de procesar la muestra para tomar una nueva.

\begin{tabular}{|c|ccc|}
\hline & $\nu_{1}=5$ & $\nu_{2}=6$ & $\nu_{3}=7$ \\
\hline$\delta_{1}=5$ & 1 & 2 & 3 \\
$\delta_{2}=6$ & - & 4 & 5 \\
$\delta_{2}=7$ & - & - & 6 \\
\hline
\end{tabular}

Tabla 3.1: Asignación de valores al parámetro escenario de muestreo $s_{k}$ para cada combinación posible de $N_{k}$ y $d_{k}$

\subsubsection{Planta}

A partir del modelo continuo $G(s)$ de la planta se puede obtener la salida del proceso como

$$
y(s)=G(s) u(s)+v(s)
$$

donde $y(s), u(s)$ y $v(s)$ son la transformada de Laplace de la salida, la entrada y la perturbación de salida, respectivamente. Si se obtiene el equivalente discreto con retenedor de orden cero de $G(s)$ a periodo $T$, se obtiene la ecuación en diferencias

$$
y[t]=-\boldsymbol{\theta}_{a}^{\top}(Y[t-1]-V[t-1])+\boldsymbol{\theta}_{b}^{\top} U[t-1]+v[t],
$$

donde

$$
\boldsymbol{\theta}_{a}=\left[\begin{array}{c}
a_{1} \\
\vdots \\
a_{n}
\end{array}\right], \quad \boldsymbol{\theta}_{b}=\left[\begin{array}{c}
b_{1} \\
\vdots \\
b_{n}
\end{array}\right]
$$

son los vectores de parámetros, y

$$
Y[t]=\left[\begin{array}{c}
y[t] \\
\vdots \\
y[t-n+1]
\end{array}\right], \quad U[t]=\left[\begin{array}{c}
u[t] \\
\vdots \\
u[t-n+1]
\end{array}\right], \quad V[t]=\left[\begin{array}{c}
v[t] \\
\vdots \\
v[t-n+1]
\end{array}\right],
$$


son los vectores de salidas, entradas y perturbaciones, respectivamente ( $\operatorname{con} y[t]=y(t T), u[t]=u(t T)$, $v[t]=v(t T))$. Con las ecuaciones (3.2) y (3.6), el modelo de la planta se define con la ecuación 1

$$
\begin{aligned}
Y[t] & =\left[\begin{array}{c}
-\boldsymbol{\theta}_{a}^{\top} \\
\boldsymbol{I}_{(n-1) \times(n)}
\end{array}\right](Y[t-1]-V[t-1])+\left[\begin{array}{c}
\boldsymbol{\theta}_{b}^{\top} \\
\mathbf{0}_{(n-1) \times(n)}
\end{array}\right] U[t-1] \\
& =\boldsymbol{A}(Y[t-1]-V[t-1])+\boldsymbol{B} U[t-1]+V[t],
\end{aligned}
$$

en cada instante de actualización de la entrada, y la ecuación de medición

$$
m_{k}=y\left[t_{k}-d_{k}\right]+w\left[t_{k}-d_{k}\right]
$$

cuando hay disponible un dato de medida $\left(t=t_{k}\right)$. Nótese que si se tuvieran retardos en $G(s)$, la matriz $\boldsymbol{A}$ tendrá tantos polos en el origen como retardos y, por tanto, no será invertible. En los algoritmos que se desarrollan en este trabajo es necesario que $\boldsymbol{A}$ sea invertible, por lo que es imprescindible transferir los retardos al sistema de medición.

\subsection{Predictor}

El controlador digital $C(z)$ necesita la secuencia de salidas a periodo $T$, pero la salida del proceso se mide de forma irregular a una frecuencia menor y con un retardo 2 . Se propone el uso de un predictor de salidas basado en modelo para obtener las estimaciones de las salidas que no se miden a periodo $T$.

El predictor usa el modelo de la planta, todas las entradas anteriores y las mediciones retardadas que se disponen de la salida. Inicialmente, la salida se estima corriendo el modelo en bucle abierto, conduciendo a

$$
\hat{Y}[t \mid t-1]=\boldsymbol{A} \hat{Y}[t-1]+\boldsymbol{B} U[t-1],
$$

donde

$$
\hat{Y}[t \mid t-1]=\left[\begin{array}{l}
\hat{y}[t \mid t-1] \\
\vdots \\
\hat{y}[t-n+1 \mid t-1]
\end{array}\right] .
$$

Cuando una medición está disponible en el instante $t=t_{k}$ (es decir, $m_{k}$ ), el vector de regresión de salidas estimadas se actualiza mediante

$$
\hat{Y}\left[t_{k}\right]=\hat{Y}\left[t_{k} \mid t_{k}\right]=\hat{Y}\left[t_{k} \mid t_{k}-1\right]+\boldsymbol{\ell}_{k}\left(m_{k}-\hat{y}\left[t_{k}-d_{k} \mid t_{k}-1\right]\right),
$$

donde se ha introducido el vector de ganancias del predictor

$$
\boldsymbol{\ell}_{k}=\left[\begin{array}{c}
l_{1} \\
\vdots \\
l_{n}
\end{array}\right]_{k}
$$

Cuando se calcula la actualización del vector de regresión mediante (3.8b), no solo se actualiza el valor de la salida actual, sino de las $n$ últimas salidas estimadas contenidas en $\hat{Y}\left[t_{k} \mid t_{k}-1\right]$. Si no hay medición, la mejor estimación es la de bucle abierto (3.8a), quedando

$$
\hat{Y}[t]=\hat{Y}[t \mid t-1] .
$$

La predicción de la salida es el primer elemento del vector $\hat{Y}[t]$ dado por

$$
\hat{y}[t]=\left[\begin{array}{llll}
1 & 0 & \cdots & 0
\end{array}\right] \hat{Y}[t]=c \hat{Y}[t] .
$$

La estimación de la salida retardada en el instante de medición $\left(\hat{y}\left[t_{k}-d_{k} \mid t_{k}-1\right]\right)$ usada en $(3.8 \mathrm{~b})$, se define como el primer elemento de un vector de estimación de salidas retardado que cumple

$$
\hat{Y}\left[t_{k} \mid t_{k}-1\right]=\boldsymbol{A}^{d_{k}} \hat{Y}\left[t_{k}-d_{k} \mid t_{k}-1\right]+\sum_{i=1}^{d_{k}} \boldsymbol{A}^{i-1} \boldsymbol{B} U\left[t_{k}-i\right]
$$

\footnotetext{
${ }^{1} \boldsymbol{I}_{(n-1) \times(n)}$ indica las $n-1$ primeras filas y $n$ primeras columnas de la matriz identidad, y $\mathbf{0}_{(n-1) \times(n)}$ es una matriz nula de orden $(n-1) \times(n)$

${ }^{2}$ El retardo temporal debido al cómputo de la acción de control se considera despreciable. Si no es el caso, podría considerarse incluido en el retraso global del bucle. Véase §3.6.3 para más detalles en este aspecto.
} 
y, por tanto, puede calcularse como

$$
\hat{y}\left[t_{k}-d_{k} \mid t_{k}-1\right]=\boldsymbol{c}\left(\boldsymbol{A}^{-d_{k}} \hat{Y}\left[t_{k} \mid t_{k}-1\right]+\sum_{i=1}^{d_{k}} \boldsymbol{A}^{i-1-d_{k}} \boldsymbol{B} U\left[t_{k}-i\right]\right) .
$$

\subsubsection{Implementación del predictor}

Con el algoritmo de predicción descrito, cada vez que llega una nueva medición, la estimación de la salida retardada $\hat{y}\left[t_{k}-d_{k} \mid t_{k}-1\right]$ debe calcularse a través de (3.10). Esta es una ecuación relativamente compleja, y, por tanto, se necesita una potencia computacional elevada para evitar un retardo no deseado en la estimación de la salida actual de interés. Con el objetivo de reducir el coste computacional requerido, se propone una implementación alternativa del predictor anterior. La idea es almacenar las estimaciones de la salida anteriores necesarias y actualizarlas cada vez que llega una medida. De esta manera la estimación de la salida con retardo $\hat{y}\left[t_{k}-d_{k} \mid t_{k}-1\right]$ se conoce antes de que la medida $y\left[t_{k}-d_{k}\right]$ esté disponible.

Con este propósito, se utiliza un vector de regresión de orden extendido. La salida se estima inicialmente corriendo el modelo (extendido) en bucle abierto, llevando a

$$
\hat{\mathbb{Y}}[t \mid t-1]=\mathbb{A} \hat{\mathbb{Y}}[t-1]+\mathbb{B} U[t-1],
$$

con $\hat{\mathbb{Y}}[t-1]=\hat{\mathbb{Y}}[t-1 \mid t-1], \mathrm{y}$

$$
\begin{aligned}
& \hat{\mathbb{Y}}[t \mid t-1]=\left[\begin{array}{l}
\hat{Y}[t \mid t-1] \\
\hat{Y}[t-n \mid t-1] \\
\hat{Y}[t-2 n \mid t-1] \\
\vdots
\end{array}\right]_{\eta \times 1}=\left[\begin{array}{l}
\hat{y}[t \mid t-1] \\
\hat{y}[t-2 \mid t-1] \\
\vdots \\
\hat{y}[t-\eta+1 \mid t-1]
\end{array}\right]_{\eta \times 1} \\
& \mathbb{A}_{\eta \times \eta}=\left[\begin{array}{lll|l}
-\boldsymbol{\theta}_{\boldsymbol{a}}^{\top} & 0 & \cdots & 0 \\
\hline \boldsymbol{I} & \vdots \\
& & 0
\end{array}\right] ; \mathbb{B}_{\eta \times n}=\left[\begin{array}{c}
\boldsymbol{\theta}_{\boldsymbol{b}}^{\top} \\
\mathbf{0} \\
\vdots \\
\mathbf{0}
\end{array}\right]
\end{aligned}
$$

donde

$$
\eta=\operatorname{máx}\left(n, \delta_{n_{\mathcal{D}}}+1\right),
$$

$\operatorname{con} \delta_{n_{\mathcal{D}}}$ el máximo retardo posible según (3.1). Cuando hay una nueva medición disponible $\left(t=t_{k}\right)$, el vector de regresión de salidas extendido se actualiza mediante

$$
\hat{\mathbb{Y}}\left[t_{k}\right]=\hat{\mathbb{Y}}\left[t_{k} \mid t_{k}\right]=\hat{\mathbb{Y}}\left[t_{k} \mid t_{k}-1\right]+\mathbb{L}_{k}\left(m_{k}-\hat{y}\left[t_{k}-d_{k} \mid t_{k}-1\right]\right),
$$

donde la estimación de la salida retardada se obtiene como

$$
\hat{y}\left[t_{k}-d_{k} \mid t-1\right]=[\overbrace{0 \cdots 01}^{d_{k}+1} 0 \cdots 0] \cdot \hat{\mathbb{Y}}\left[t_{k} \mid t_{k}-1\right],
$$

y el vector de ganancias extendido se define como

$$
\mathbb{L}_{k(\eta \times 1)}=\left[\begin{array}{c}
\boldsymbol{I} \\
\boldsymbol{A}^{-n} \\
\boldsymbol{A}^{-2 n} \\
\vdots \\
]_{\zeta \times n} \\
0 \\
\vdots
\end{array}\right]_{\eta \times 1}
$$

donde

$$
\zeta=\operatorname{máx}\left\{n, \operatorname{máx}_{s_{k} \in \mathcal{S}}\left(d\left(s_{k}\right)-N\left(s_{k}\right)\right)\right\} \leq \eta .
$$

El vector $\mathbb{L}_{k}$ tiene $\eta-\zeta$ elementos nulos porque las únicas estimaciones retardadas que deben actualizarse son las que se necesitan en actualizaciones posteriores. Procediendo de esta forma se reduce el coste 
computacional por haber un menor número de multiplicaciones y sumas a la hora de actualizar el vector de regresión de salidas extendido $\mathbb{Y}\left[t_{k}\right]$ mediante (3.11b).

La predicción de la salida es el primer elemento del vector $\mathbb{Y}[t]$ dado por

$$
\hat{y}[t]=\left[\begin{array}{llll}
1 & 0 & \cdots & 0
\end{array}\right] \hat{Y}[t] .
$$

Observación 3.2.1 (Actualización de la estimación de salidas con retardo). Supóngase que hay una nueva medición en el instante $t$ con un retardo de $d$. La actualización del vector de regresión de salidas retardadas $(\hat{Y}[t-d \mid t])$ se define como el vector que cumple

$$
\hat{Y}[t \mid t]=\boldsymbol{A}^{d} \hat{Y}[t-d \mid t]+\sum_{j=1}^{d} \boldsymbol{A}^{j-1} \boldsymbol{B} U(t-j)
$$

donde $\hat{Y}[t-d \mid t]$ es una estimación del vector de salidas $Y[t-d]$ con la información conocida hasta el instante $t$. De una forma similar, el vector de salidas no actualizado se define como

$$
\hat{Y}[t \mid t-1]=\boldsymbol{A}^{d} \hat{Y}[t-d \mid t-1]+\sum_{j=1}^{d} \boldsymbol{A}^{j-1} \boldsymbol{B} U(t-j) .
$$

Como $t$ es el instante en que llega una nueva medición, introduciendo la ecuación (3.8b) en (3.13) y restándolo de (3.14) es fácil obtener

$$
\hat{Y}[t-d \mid t]=\hat{Y}[t-d \mid t-1]+\boldsymbol{A}^{-d} \boldsymbol{\ell}_{k}\left(m_{k}-\hat{y}[t-d \mid t-1]\right) .
$$

El vector de regresión $\hat{Y}[t-d \mid t]$ contiene $n$ estimaciones de salidas retardadas (desde $\hat{y}[t-d \mid t]$ hasta $\hat{y}[t-d-n+1 \mid t]])$. La ecuación (3.15) es equivalente (3.8b) para $d=0$, y por lo tanto las estimaciones desde $\hat{y}[t \mid t-1]$ hasta $\hat{y}[t-n+1 \mid t-1]$ se actualizan directamente con esa ecuación. Sin embargo, dependiendo de la disponibilidad de datos y del retardo, también son necesarias estimaciones antiguas. El número de estimaciones con retardo previas que es necesario actualizar depende de los valores del retardo $d$ y del número de periodos de entrada entre mediciones. Si

$$
\rho=\operatorname{máx}_{s_{k} \in \mathcal{S}}\left\{d\left(s_{k}\right)-N\left(s_{k}\right)\right\}
$$

es la máxima diferencia entre retardo y periodos intermuestreo, la estimación más antigua que es necesario actualizar es $\hat{y}(t-\rho \mid t-1)$, porque esta estimación de la salida podría ser necesaria para la proxima actualización con la medición. Entonces, la ecuación (3.15) debe aplicarse desde $d=n$ a $\zeta=\operatorname{máx}(n, \rho)$, lo que lleva a la ecuación (3.12).

Observación 3.2.2. En las aplicaciones más comunes $d\left(s_{k}\right) \leq N\left(s_{k}\right)$, es decir, que no se puede tomar una nueva medición hasta que la anterior se ha procesado y la correspondiente medición está disponible (por ejemplo en procesado de imágenes). En este caso, la ganancia del predictor es simplemente

$$
\mathbb{L}_{k}=\left[\begin{array}{c}
\ell_{k} \\
\mathbf{0} \\
\vdots \\
\mathbf{0}
\end{array}\right] .
$$

La dinámica del predictor (en cualquiera de sus implementaciones, (3.8) o (3.11)) depende del vector de ganancias (3.9) que debe diseñarse para asegurar: la estabilidad del predictor, la robustez a la disponibilidad de datos irregular y una atenuación adecuada de las perturbaciones y el ruido de medida. La ganancia del predictor se asume que es, en general, variante en el tiempo, aunque también se analiza el caso particular de una ganancia constante.

Para diseñar el predictor, es decir, la ganancia del predictor (3.9) con esas propiedades, se debe obtener la ecuación de la dinámica del error de predicción. 


\subsubsection{Error de predicción}

Definiendo el vector de error de predicción como

$$
E[t]=Y[t]-\hat{Y}[t]
$$

se puede obtener la dinámica del error en instantes en los que no hay medición restando las ecuaciones (3.7) y (3.8a), llegándose a

$$
E[t]=\boldsymbol{A} E[t-1]+V[t]-\boldsymbol{A} V[t-1]
$$

Esta ecuación indica que la evolución del error de predicción cuando no hay medición viene marcada por la matriz dinámica del proceso $\boldsymbol{A}$ y por las perturbaciones $v$, con lo que no se puede controlar su evolución. Sólo se puede corregir el error de predicción respecto de su evolución en bucle abierto cuando se tiene información de la salida. El error de predicción interesa mantenerlo en niveles bajos en todos los instantes de control para proporcionar al controlador una estimación de la salida adecuada. El error entre mediciones será tanto menor como menor sea el error conseguido cuando llega una medida de la salida. Es evidente que es necesario obtener la dinámica del error de predicción entre instantes en los que sí que se tiene información de la salida. El siguiente teorema recoge el modelo que sigue el error de predicción cuando no hay incertidumbre en el modelo de la planta.

Lema 3.2.1 (Dinámica del error de predicción entre mediciones). La dinámica del error de predicción del algoritmo (3.8) aplicado al sistema (3.7) cuando no hay error de modelado y las medidas están disponibles cada $N_{k}$ periodos de la entrada (siendo $N_{k}$ variante en el tiempo) con un retardo $d_{k}$, está descrita por el sistema lineal variante en el tiempo

$$
\begin{aligned}
E_{k} & =\left(\boldsymbol{I}-\boldsymbol{\ell}_{k} \boldsymbol{c} \boldsymbol{A}^{-d_{k}}\right) \boldsymbol{A}^{N_{k}}\left(E_{k-1}-V\left[t_{k-1}\right]\right)+V\left[t_{k}\right]-\boldsymbol{\ell}_{k} \boldsymbol{c} V\left[t_{k}-d_{k}\right]-\boldsymbol{\ell}_{k} w_{k}, \\
e_{k} & =\boldsymbol{c} E_{k}
\end{aligned}
$$

que se actualiza en cada instante de medición. El vector de error de estimación se define cuando la medición está disponible $\left(t=t_{k}\right)$ como

$$
E_{k} \equiv E\left[t_{k}\right]=Y\left[t_{k}\right]-\hat{Y}\left[t_{k}\right]
$$

mientras que el error de predicción de salida se define como

$$
e_{k}=e\left[t_{k}\right]=y\left[t_{k}\right]-\hat{y}\left[t_{k}\right]
$$

Prueba 3.2.1. En el instante de medición, $t=t_{k}$, se cumple la expresión (3.2) y el valor medido puede escribirse como

$$
m_{k}=c Y\left[t_{k}-d_{k}\right]+w_{k},
$$

y, por tanto, la predicción de la salida puede expresarse como

$$
\hat{Y}\left[t_{k} \mid t_{k}\right]=\hat{Y}\left[t_{k} \mid t_{k}-1\right]+\ell_{k}\left(\boldsymbol{c}\left(Y\left[t_{k}-d_{k}\right]-\hat{Y}\left[t_{k}-d_{k} \mid t-1\right]\right)+w_{k}\right) .
$$

los vectores $Y\left[t_{k}-d_{k}\right]$ e $\hat{Y}\left[t_{k}-d_{k} \mid t_{k}-1\right]$ pueden expresarse como una función de $Y\left[t_{k}-N_{k}\right]$ e $\hat{Y}\left[t_{k}-N_{k}\right]$ (el instante en el cual se realizó la anterior medición y actualización de la predicción) si se aplican recursivamente las expresiones (3.7) y (3.8a), con lo que se llega a

$$
\begin{aligned}
& Y\left[t_{k}-d_{k}\right]-\hat{Y}\left[t_{k}-d_{k} \mid t_{k}-1\right]= \\
& \quad=\boldsymbol{A}^{N_{k}-d_{k}}\left(Y\left[t_{k}-N_{k}\right]-\hat{Y}\left[t_{k}-N_{k}\right]-V\left[t_{k}-N_{k}\right]\right)+V\left[t_{k}-d_{k}\right] .
\end{aligned}
$$

El vector $\hat{Y}\left[t_{k} \mid t_{k}-1\right]$ también se puede expresar mediante $\hat{Y}\left[t_{k}-N_{k}\right]$ aplicando (3.8a) recursivamente, llegando a

$$
\hat{Y}\left[t_{k} \mid t_{k}-1\right]=\boldsymbol{A}^{N_{k}} \hat{Y}\left[t_{k}-N_{k}\right]+\sum_{i=1}^{N_{k}} \boldsymbol{A}^{i-1} \boldsymbol{B} U\left[t_{k}-i\right] .
$$

La salida $Y\left[t_{k}\right]$ también puede expresarse (mediante (3.7)) como una función de $Y\left[t_{k}-N_{k}\right]$ :

$$
Y\left[t_{k}\right]=\boldsymbol{A}^{N_{k}}\left(Y\left[t_{k}-N_{k}\right]-V\left[t_{k}-N_{k}\right]\right)+\sum_{i=1}^{N_{k}} \boldsymbol{A}^{i-1} \boldsymbol{B} U\left[t_{k}-i\right]+V\left[t_{k}\right] .
$$


Introduciendo las expresiones (3.20) y (3.21) en (3.19) y restando la ecuación resultante de (3.22), se tiene que

$$
\begin{aligned}
Y\left[t_{k}\right]-\hat{Y}\left[t_{k}\right]= & \boldsymbol{A}^{N_{k}}\left(Y\left[t_{k}-N_{k}\right]-\hat{Y}\left[t_{k}-N_{k}\right]-V\left[t_{k}-N_{k}\right]\right)+V\left[t_{k}\right] \\
& -\boldsymbol{\ell}_{k} \boldsymbol{c} \boldsymbol{A}^{N_{k}-d_{k}}\left(Y\left[t_{k}-N_{k}\right]-\hat{Y}\left[t_{k}-N_{k}\right]-V\left[t_{k}-N_{k}\right]\right) \\
& -\boldsymbol{\ell}_{k}\left(\boldsymbol{c} V\left[t_{k}-d_{k}\right]+w_{k}\right),
\end{aligned}
$$

que es exactamente la ecuación (3.17a) debido a que en el instante de toma de medidas $t_{k}-N_{k}=t_{k-1}$.

El error de predicción de salida instantáneo $e_{k}$ es simplemente el primer valor de $E_{k}$, y puede obtenerse mediante el vector $\boldsymbol{c}$

$$
e_{k}=y\left[t_{k}\right]-\hat{y}\left[t_{k}\right]=c\left(Y\left[t_{k}\right]-\hat{Y}\left[t_{k}\right]\right)
$$

Observación 3.2.3. Si se define un nuevo vector agrupando las perturbaciones entre mediciones como

$$
\mathbb{V}_{k}=\left[\begin{array}{c}
V\left[t_{k}\right] \\
V\left[t_{k}-d_{k}\right] \\
V\left[t_{k}-N_{k}\right]
\end{array}\right],
$$

entonces la dinámica del error de predicción puede escribirse en una forma más compacta como

$$
\begin{aligned}
E_{k} & =\mathcal{A}_{k} E_{k-1}+\mathcal{B}_{k}\left[\begin{array}{c}
\mathbb{V}_{k} \\
w_{k}
\end{array}\right], \\
e_{k} & =\boldsymbol{c} E_{k},
\end{aligned}
$$

donde

$$
\begin{aligned}
\mathcal{A}_{k} & =\left(\boldsymbol{I}-\boldsymbol{\ell}_{k} \boldsymbol{c} \boldsymbol{A}^{-d_{k}}\right) \boldsymbol{A}^{N_{k}}, \\
\mathcal{B}_{k} & =\left[\begin{array}{llll}
\boldsymbol{I} & -\boldsymbol{\ell}_{k} \boldsymbol{c} & -\mathcal{A}_{k} & -\boldsymbol{\ell}_{k}
\end{array}\right] .
\end{aligned}
$$

En el diseño de predictores y observadores según el filtro de Kalman, la ganancia variante $\boldsymbol{\ell}_{k}$ se calcula en cada instante de muestreo cuando llega una nueva medición, de forma que se tiene un vector de ganancias que, en general, varía en el tiempo de forma arbitraria. En este trabajo, sin embargo, el diseño de la ganancia $\ell_{k}$ se aborda definiendo un vector diferente para cada posible par $\left(d_{k}, N_{k}\right)$, es decir, para cada posible valor del parámetro de muestreo $s_{k}$. El cálculo de estos vectores se hace fuera de línea una sola vez y da como resultado un conjunto finito de ganancias

$$
\boldsymbol{\ell}_{k}=\boldsymbol{\ell}\left(s_{k}\right) \in \mathcal{L}=\left\{\boldsymbol{\ell}(1), \boldsymbol{\ell}(2), \ldots, \boldsymbol{\ell}\left(n_{\mathcal{S}}\right)\right\}
$$

donde $n_{\mathcal{S}}$ es el número de posible situaciones de muestreo, esto es, de posibles combinaciones $\left(N_{k}, d_{k}\right)$ que pueden darse en el muestreo. El predictor que aquí se propone se implementará con un conjunto de $n_{\mathcal{S}}$ vectores de ganancia constantes. Cada vez que llega una medición de la salida, el predictor utilizará un único vector de ganancias que escogerá en función de las condiciones de muestreo con las que se ha recibido la muestra.

Observación 3.2.4. Definiendo el vector de ganancias del predictor de forma paramétrica según (3.25), la dinámica del error de predicción (3.24) puede reescribirse como

$$
\begin{aligned}
E_{k} & =\mathcal{A}\left(s_{k}\right) E_{k-1}+\mathcal{B}\left(s_{k}\right)\left[\begin{array}{c}
\mathbb{V}_{k} \\
w_{k}
\end{array}\right] \\
e_{k} & =\boldsymbol{c} E_{k},
\end{aligned}
$$

siendo

$$
\begin{aligned}
& \mathcal{A}\left(s_{k}\right)=\left(\boldsymbol{I}-\boldsymbol{\ell}\left(s_{k}\right) \boldsymbol{c} \boldsymbol{A}^{-d\left(s_{k}\right)}\right) \boldsymbol{A}^{N\left(s_{k}\right)}, \\
& \mathcal{B}\left(s_{k}\right)=\left[\begin{array}{llll}
\boldsymbol{I} & -\boldsymbol{\ell}\left(s_{k}\right) \boldsymbol{c} & -\mathcal{A}\left(s_{k}\right) & -\boldsymbol{\ell}\left(s_{k}\right)
\end{array}\right] .
\end{aligned}
$$

Esto indica que la dinámica del error de predicción viene definida por un sistema lineal variante con el tiempo que depende del parámetro variante $s_{k}$. En esta realización interna el vector de $3 n$ perturbaciones $\mathbb{V}$ y el ruido de medida $w$ pueden considerarse como las entradas, los últimos $n$ errores de predicción forman el vector de estados, y el error de predicción es la salida. 


\subsubsection{Objetivo del diseño}

El objetivo del diseño de predictores es encontrar un procedimiento para calcular el vector $\ell_{k}(3.9)$ (variante en el tiempo en función del muestreo $s_{k}$, o constante) tal que el sistema (3.26) logre la estabilidad del predictor ante cualquier escenario de muestreo y el menor error de predicción ante las posibles perturbaciones. La atenuación de las perturbaciones se plantea de forma diferente en función del tipo de señal que las caracteriza, que pueden ser:

- Señales de energía finita. Son perturbaciones cuyo valor cuadrático acumulado (norma $\ell_{2}$ ) es finito en un intervalo de tiempo suficientemente largo. Un ejemplo de este tipo de señal podría ser una perturbación en forma de pulso de una duración determinada. Otro ejemplo puede ser un impulso.

- Señales persistentes. Tienen un valor no necesariamente nulo durante todo el tiempo. Para caracterizar estas señales se puede utilizar bien su valor eficaz (norma RMS), bien su valor de pico (norma $\ell_{\infty}$ ) o bien su varianza, si se asume que es un ruido blanco de media cero.

- Ruido de medida impulsional. Se trata de un ruido que generalmente tiene un valor nulo (medición perfecta) excepto en algunos instantes en los cuales el sistema de medida falla introduciendo de forma puntual un error de valor finito. Se considera que estos fallos puntuales están suficientemente separados en el tiempo, es decir, que el efecto que tiene un ruido impulsional queda atenuado antes de que pueda aparecer otro error de este tipo.

La prestación del predictor en cuanto a la atenuación entre la perturbación y el error de predicción se consigue exigiendo al error de predicción que acote el valor máximo de alguna de sus normas:

- Si se acota la norma $\ell_{\infty}$ del error se le está pidiendo al predictor que no tenga un error de estimación mayor que un umbral dado, aunque ello implique que el error sea persistente.

- Si lo que se acota es la norma $\ell_{2}$ del error se le está pidiendo al predictor que elimine el efecto introducido por las perturbaciones en un tiempo finito, aunque para ello se tenga algún pico elevado.

- Si lo que se le exige al predictor es que mantenga el valor eficaz (norma RMS) por debajo de un valor se está permitiendo que el error de predicción persista a lo largo del tiempo pero con un valor cuadrático medio lo menor posible.

Para poder minimizar la norma del error de predicción es necesario conocer la naturaleza de las perturbaciones y las normas que las caracterizan. Las diferentes situaciones que se pueden presentar se clasifican según el tipo de perturbación que afecta el sistema (véase anexo A.2):

S1 Si las perturbaciones son persistentes con un valor eficaz conocido, se puede plantear un diseño que minimice el valor eficaz del error de predicción minimizando para ello la norma $\mathcal{H}_{\infty}$ de la dinámica del predictor. Si las perturbaciones son ruidos gaussianos de media cero con varianza conocida ${ }^{3}$ es posible plantear el diseño que minimiza el valor eficaz del error de predicción utilizando para ello la norma $\mathcal{H}_{2}$ del predictor. Véase figura 3.3.

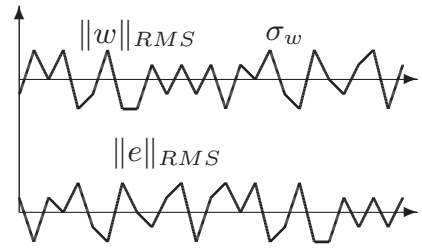

Figura 3.3: Tipos de señales involucradas en las normas $\mathcal{H}_{\infty}$ y $\mathcal{H}_{2}$

S2 Si las perturbaciones son señales persistentes de las cuales se conoce el valor máximo, se puede plantear el diseño que minimiza el valor eficaz del error de predicción por medio de la norma $\mathcal{H}_{\infty}$. Si lo que se quiere es minimizar el valor máximo del error de predicción se puede plantear el diseño por medio de la norma $\ell_{1}$. Véase figura 3.4 .

S3 Si las perturbaciones son señales de energía finita (como un pulso de corta duración) se puede tratar de minimizar el error cuadrático acumulado (la energía) del error de predicción planteando la minimización de la norma $\mathcal{H}_{\infty}$ del predictor, o bien se puede tratar de minimizar el valor máximo del error de predicción usando la norma $\mathcal{H}_{2 g}$ (norma $\mathcal{H}_{2}$ generalizada). Véase figura 3.5. 


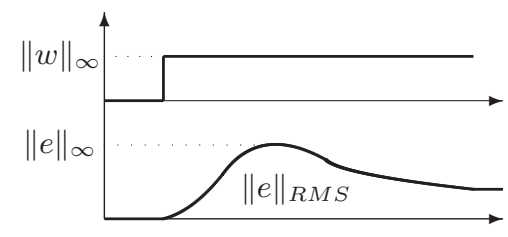

Figura 3.4: Tipos de señales involucradas en las normas $\mathcal{H}_{\infty}$ y $\ell_{1}$

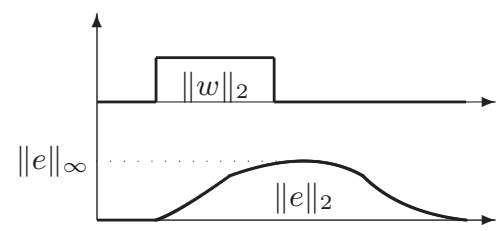

Figura 3.5: Tipos de señales involucradas en las normas $\mathcal{H}_{\infty}$ y $\mathcal{H}_{2 g}$

La tabla 3.2 relaciona las diferentes especificaciones de diseño (error acotado en amplitud, energía o valor eficaz) con los diferentes tipos de perturbación (señal impulsional, de energía finita, de amplitud finita o persistente) y normas del predictor.

\begin{tabular}{|c|cccc|}
\hline & $\left\|w_{k}\right\|_{2}$ & $\left\|w_{k}\right\|_{\infty}$ & $\left\|w_{k}\right\|_{R M S}$ & $\sigma_{w}$ \\
\hline$\left\|e_{k}\right\|_{2}$ & $\mathcal{H}_{\infty}$ & $\infty$ & $\infty$ & $\infty$ \\
$\left\|e_{k}\right\|_{\infty}$ & $\mathcal{H}_{2 g}$ & $\ell_{1}$ & - & - \\
$\left\|e_{k}\right\|_{R M S}$ & 0 & $\mathcal{H}_{\infty}$ & $\mathcal{H}_{\infty}$ & $\mathcal{H}_{2}$ \\
\hline
\end{tabular}

Tabla 3.2: Resumen de especificaciones de diseño

El diseño de predictores se enfoca de la siguiente manera. Dado un escenario de muestreo definido por $s_{k}$, obtener un predictor que consiga las mejores prestaciones en cuanto a atenuación de perturbaciones de salida y ruido de medida.

Además de este enfoque fundamental, también se tratan con posterioridad el efecto de las perturbaciones de entrada, las incertidumbres, las mediciones asíncronas o los retardos de la entrada haciendo equivalente su efecto al de una perturbación.

Finalmente, se tratará un último enfoque que se puede formular de la siguiente forma. Dadas unas exigencias de atenuación de perturbaciones y robustez al error de modelado, encontrar el predictor que minimiza la utilización del canal de comunicación, disminuyendo para ello la frecuencia de muestreo y aumentando el retardo admitido (es decir, aumentando $N_{k} \mathrm{y} d_{k}$ ).

\subsection{Diseño de predictores ante muestreo regular}

Sea una planta libre de perturbaciones y ruidos de medida. Supóngase un muestreo regular y un retardo constante $\left(N_{k}=N\right.$ y $d_{k}=d$ son valores constantes), lo que implica que el parámetro $s_{k}$ no varíe $\left(s_{k}=1\right)$ y que la dinámica del error de predicción del algoritmo (3.8) venga dada por

$$
\begin{aligned}
E_{k+1} & =\mathcal{A} E_{k}, \\
e_{k} & =c E_{k},
\end{aligned}
$$

donde

$$
\mathcal{A}=\left(\boldsymbol{I}-\ell \boldsymbol{c} \boldsymbol{A}^{-d}\right) \boldsymbol{A}^{N}
$$

es una matriz constante. El error de predicción en estas condiciones es estable si y sólo si los valores propios de la matriz $\mathcal{A}$ están dentro del círculo unitario. En este caso se utiliza una ganancia constante $\boldsymbol{\ell}$ debido a la naturaleza invariante en el tiempo de la dinámica del error de predicción. El diseño del predictor para este tipo de muestreo puede abordarse mediante la asignación de polos o utilizando técnicas LMI. El siguiente ejemplo recoge el diseño utilizando la técnica de asignación de polos.

\footnotetext{
${ }^{3}$ En este caso el valor eficaz coincide con la varianza
} 
Ejemplo 3.3.1. Retómese el ejemplo 2.3.1 de la página 17, en el que se tenía un sistema inestable cuya función de transferencia era

$$
G(s)=\frac{1}{(s-1)(s+3)}
$$

con un periodo de control de $T=0.1$ segundos y un periodo de muestreo de $T_{0}=1.2$ segundos $(N=12)$. Supónganse 4 situaciones de muestreo diferentes en las que el retardo es de $d_{1}=2, d_{2}=5, d_{3}=10 y$ $d_{4}=15$ muestras, respectivamente. Si se diseña un predictor para cada situación con el objetivo de tener unos valores propios entre muestras ubicados en $0.8^{N}$, se obtienen los siguientes vectores de ganancias (para cada proceso):

$$
\boldsymbol{\ell}_{1}=\left[\begin{array}{l}
1.1706 \\
1.0546
\end{array}\right], \quad \boldsymbol{\ell}_{2}=\left[\begin{array}{l}
1.5900 \\
1.4368
\end{array}\right], \quad \boldsymbol{\ell}_{3}=\left[\begin{array}{l}
2.6276 \\
2.3771
\end{array}\right], \quad \boldsymbol{\ell}_{4}=\left[\begin{array}{l}
4.3335 \\
3.9211
\end{array}\right]
$$

En la figura 3.6 se muestra la evolución del error de predicción de salida en cada periodo de control, para los 4 escenarios. Se observa que en todos ellos se lleva el error de predicción a cero, con la diferencia de que las prestaciones del predictor entre muestras se degradan conforme aparece un retardo mayor.

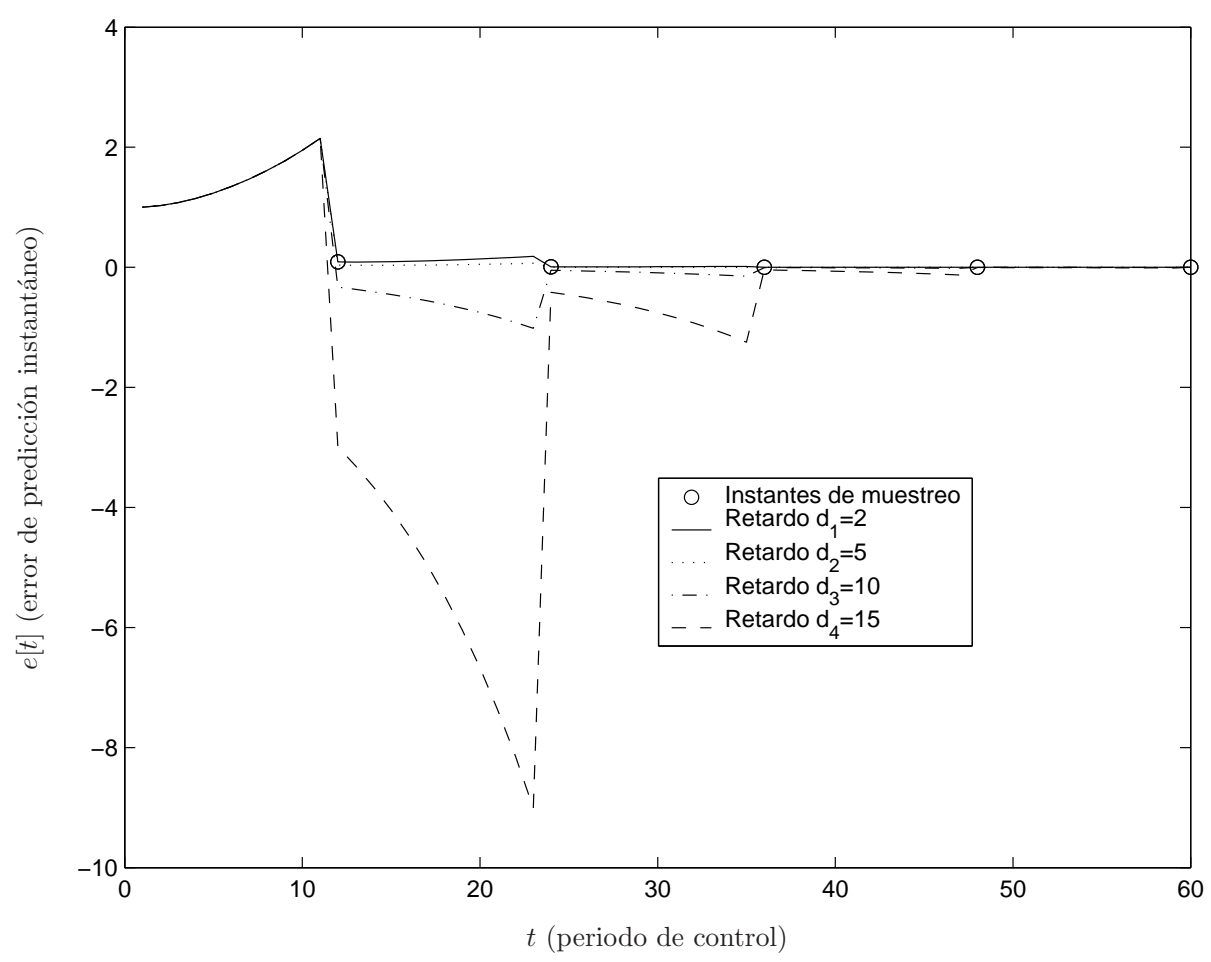

Figura 3.6: Evolución del error de predicción $(N=12)$ para diferentes retardos.

El siguiente teorema recoge el diseño utilizando la técnica de las desigualdades lineales matriciales.

Teorema 3.3.1. Considérese el sistema (3.7) sin perturbaciones ni ruido de medida, y supóngase que hay una medición de la salida disponible cada $N$ periodos de entrada y con un retardo de d periodos, siendo d y $N$ números enteros positivos. Para un $\mu \in(0,1]$ dado, supóngase que existen dos matrices $\boldsymbol{P}=\boldsymbol{P}^{\top} \in \mathbb{R}^{n \times n}$ y $\boldsymbol{X} \in \mathbb{R}^{n \times 1}$ tales que la siguiente desigualdad matricial se cumple

$$
\left[\begin{array}{cc}
\boldsymbol{P} & \left(\boldsymbol{P}-\boldsymbol{X} \boldsymbol{c} \boldsymbol{A}^{-d}\right) \boldsymbol{A}^{N} \\
\left(\left(\boldsymbol{P}-\boldsymbol{X} \boldsymbol{c} \boldsymbol{A}^{-d}\right) \boldsymbol{A}^{N}\right)^{\top} & \mu^{2} \boldsymbol{P}
\end{array}\right] \succ \mathbf{0}
$$

es decir, la LMI es factible. Entonces, si la ganancia del predictor se define como

$$
\ell=P^{-1} \boldsymbol{X}
$$

el error de predicción del algoritmo definido por (3.8) converge a cero de forma asintótica con un ratio de decadencia menor que $\mu$ 
Prueba 3.3.1. Introduciendo la ganancia (3.31) en la LMI (3.30), se puede escribir que

$$
\left[\begin{array}{cc}
\boldsymbol{P} & \boldsymbol{P}\left(\boldsymbol{I}-\boldsymbol{\ell} \boldsymbol{c} \boldsymbol{A}^{-d}\right) \boldsymbol{A}^{N} \\
\left(\boldsymbol{P}\left(\boldsymbol{I}-\boldsymbol{\ell} \boldsymbol{c} \boldsymbol{A}^{-d}\right) \boldsymbol{A}^{N}\right)^{\top} & \mu^{2} \boldsymbol{P}
\end{array}\right]=\left[\begin{array}{cc}
\boldsymbol{P} & \boldsymbol{P} \boldsymbol{A} \\
\mathcal{A}^{\top} \boldsymbol{P} & \mu^{2} \boldsymbol{P}
\end{array}\right] \succ \mathbf{0}
$$

y aplicando los complementos de Schur se obtiene la condición

$$
\mathcal{A}^{\top} \boldsymbol{P} \mathcal{A}-\mu^{2} \boldsymbol{P} \prec \mathbf{0} .
$$

Premultiplicando por $E_{k}^{\top}$ y postmultiplicando por $E_{k}$ se llega a

$$
E_{k+1}^{\top} \boldsymbol{P} E_{k+1} \prec \mu^{2} E_{k}^{\top} \boldsymbol{P} E_{k},
$$

que equivale a la condición

$$
\mathcal{V}_{k+1}<\mu^{2} \mathcal{V}_{k}
$$

si $\mathcal{V}_{k}$ es la función de Lyapunov definida como $\mathcal{V}_{k}=E_{k}^{\top} \boldsymbol{P} E_{k}$. La estabilidad cuadrática queda probada finalmente teniendo en cuenta que (3.30) implica que $\boldsymbol{P} \succ \mathbf{0}$, y, consecuentemente, la función de Lyapunov es definida positiva.

Observación 3.3.1 (Cálculo de la ganancia del predictor). El diseño del predictor queda reducido a la resolución del problema de factibilidad (3.30) con solucionadores LMI estándar, obteniéndose las matrices $\boldsymbol{P}$, y $\boldsymbol{X}$. Uno de los solucionadores LMI más ampliamente utilizados convierte el problema de factibilidad en un problema de minimización convexa de la forma

$$
\text { Minimiza } \quad t \quad \text { sujeto a } \quad \boldsymbol{R}-t \boldsymbol{I} \preceq 0 \text {, }
$$

donde $\boldsymbol{R}$ es una matriz que reúne en su diagonal todas las matrices del conjunto de LMI. Si la LMI es factible, el algoritmo de minimización para cuando encuentra un valor $t<04$.

Las variables matriciales $\boldsymbol{P}$ y $\boldsymbol{X}$ deberían conducir a una tasa de decrecimiento de la función de Lyapunov ligeramente más rápida que la definida por $\mu$. Sin embargo, los algoritmos de minimización tienden a encontrar un valor negativo muy grande para el valor $t$ en muy pocas iteraciones, resultando una solución que conduce a una dinámica del error de predicción mucho más rápida que la predeterminada por $\mu$. El comportamiento de este predictor tan rápido ante la presencia de ruido de medida no es adecuada en general (porque se amplifica el ruido) y por tanto, la estrategia de diseño del predictor debe modificarse para alcanzar una dinámica más realista.

En el siguiente ejemplo se puede observar este efecto de amplificación del ruido observando el valor que toma $l_{1}$ en los casos en los que no hay retardo $\left(l_{1}=1\right.$ para $\left.d=0\right)$, lo que significa que el ruido se transmite directamente a la predicción de la salida que se está midiendo.

Ejemplo 3.3.2. Retómese el sistema de los ejemplos 2.3.1 y 3.3.1. Supónganse 5 situaciones de muestreo diferentes en los que el retardo es de $d_{0}=0, d_{1}=2, d_{2}=5, d_{3}=10 y d_{4}=15$ muestras, respectivamente. Si se diseña un predictor en cada proceso con ayuda del teorema 3.3.1 (tomando $\mu=0.97$ ) se obtienen los siguientes vectores de ganancias (para cada proceso):

$$
\ell_{0}=\left[\begin{array}{c}
1 \\
0.9019
\end{array}\right], \quad \boldsymbol{\ell}_{1}=\left[\begin{array}{l}
1.2312 \\
1.1105
\end{array}\right], \quad \boldsymbol{\ell}_{2}=\left[\begin{array}{l}
1.7202 \\
1.5516
\end{array}\right], \quad \boldsymbol{\ell}_{3}=\left[\begin{array}{l}
3.2271 \\
2.9195
\end{array}\right], \quad \boldsymbol{\ell}_{4}=\left[\begin{array}{l}
4.1458 \\
3.7513
\end{array}\right]
$$

Los valores propios de la dinámica del predictor entre muestras (valores propios de la matriz (I$\left.\boldsymbol{\ell} \boldsymbol{c} \boldsymbol{A}^{-d}\right) \boldsymbol{A}^{N}$ ) con cada diseño son:

$$
\lambda_{0}=\left[\begin{array}{c}
0 \\
0.0059
\end{array}\right], \lambda_{1}=\left[\begin{array}{c}
0 \\
-0.0208
\end{array}\right], \lambda_{2}=\left[\begin{array}{c}
0 \\
-0.1379
\end{array}\right], \lambda_{3}=\left[\begin{array}{c}
0.0240 \\
-0.6190
\end{array}\right], \lambda_{4}=\left[\begin{array}{l}
0.0202 \\
0.2556
\end{array}\right]
$$

Nótese que cuando el retardo es menor, el procedimiento devuelve un predictor que asegura unos valores propios menores y, por tanto, un predictor con una dinámica más rápida. En la figura 3.7 se muestra la evolución del error de predicción de salida en cada periodo de control, para los 5 procesos. Se observa que todos los procesos llevan el error de predicción a cero, y también se puede observar la degradación del error de predicción en bucle abierto en los procesos con mayor retardo.

Supóngase ahora que aparece una perturbación en forma de escalón de tamaño 0.2 en el instante $t=90$. La dinámica del error de predicción en este caso será la indicada en la figura 3.8, donde se 


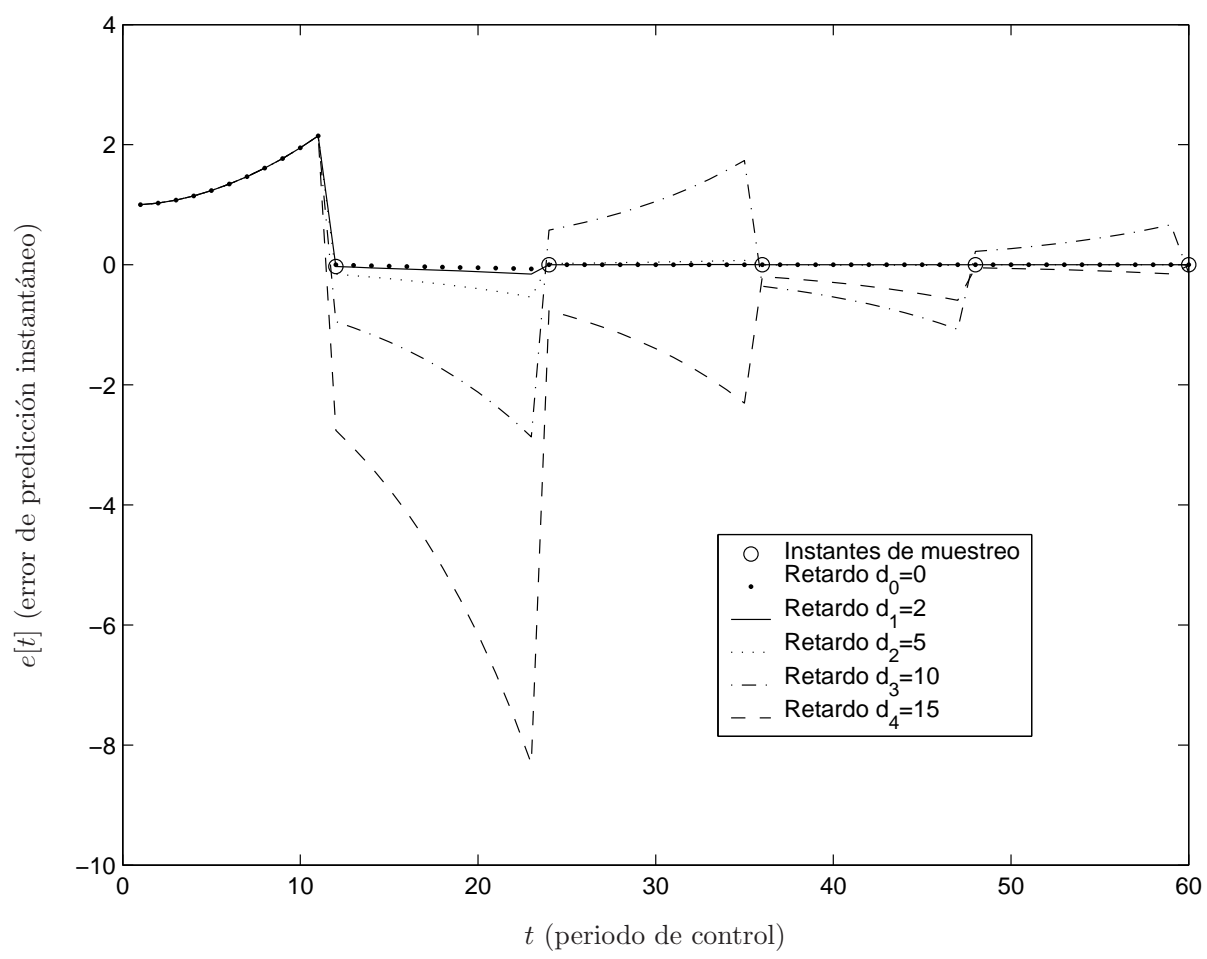

Figura 3.7: Evolución del error de predicción $(N=12)$ para diferentes retardos.

observa que el predictor no es capaz de atenuar dicha perturbación, quedando patente en el elevado error de predicción que se comete entre muestreos.

Supóngase ahora que aparece un ruido en el sensor en forma de escalón de tamaño 0.5 (en ausencia de perturbación) en el instante $t=70$. La dinámica del error de predicción en este caso será la indicada en la figura 3.9, donde se observa que el predictor de nuevo no es capaz de atenuar el ruido de medida, provocando un elevado error de predicción entre muestreos. La incapacidad de los predictores de atenuar la perturbación o el ruido de medida se debe a la dinámica casi de tiempo mínimo (valores de $\lambda$ muy bajos) obtenida con este método.

Si el sistema presenta una perturbación $v(\tau)$, y el dispositivo de medida introduce un ruido $w(\tau)$, se deben tomar en consideración las estrategias de atenuación de perturbaciones introducidas en \$3.2.3 para evitar la solución de tiempo mínimo. En las siguientes secciones se presenta un catálogo de predictores donde cada uno de ellos tiene en cuenta diferentes características en las perturbaciones y su efecto sobre el error de predicción cuando el muestreo es aleatorio con retardo variante en el tiempo.

\subsection{Diseño determinista de predictores}

\subsubsection{Diseño de predictores para estabilidad nominal}

Cuando se asume un muestreo irregular $\left(N_{k}\right.$ y $d_{k}$ varían arbitrariamente con el tiempo), la dinámica del error es variante en el tiempo, y ya no son válidos los argumentos de estabilidad del apartado anterior. En ausencia de perturbaciones, la dinámica del error de predicción se reduce a

$$
\begin{aligned}
E_{k} & =\mathcal{A}\left(s_{k}\right) E_{k-1}, \\
e_{k} & =\boldsymbol{c} E_{k} .
\end{aligned}
$$

La condición de estabilidad cuadrática es suficiente pero no necesaria para que un sistema variante en el tiempo sea estable. Esto significa que un diseño planteado para cumplir con la estabilidad cuadrática llevaría a una solución muy conservadora, reduciendo el espacio de soluciones. Para que el sistema variante

\footnotetext{
${ }^{4} \mathrm{El}$ comando feasp de la $L M I$ Control Toolbox de MATLAB ${ }^{\circledR}$ resuelve este problema
} 


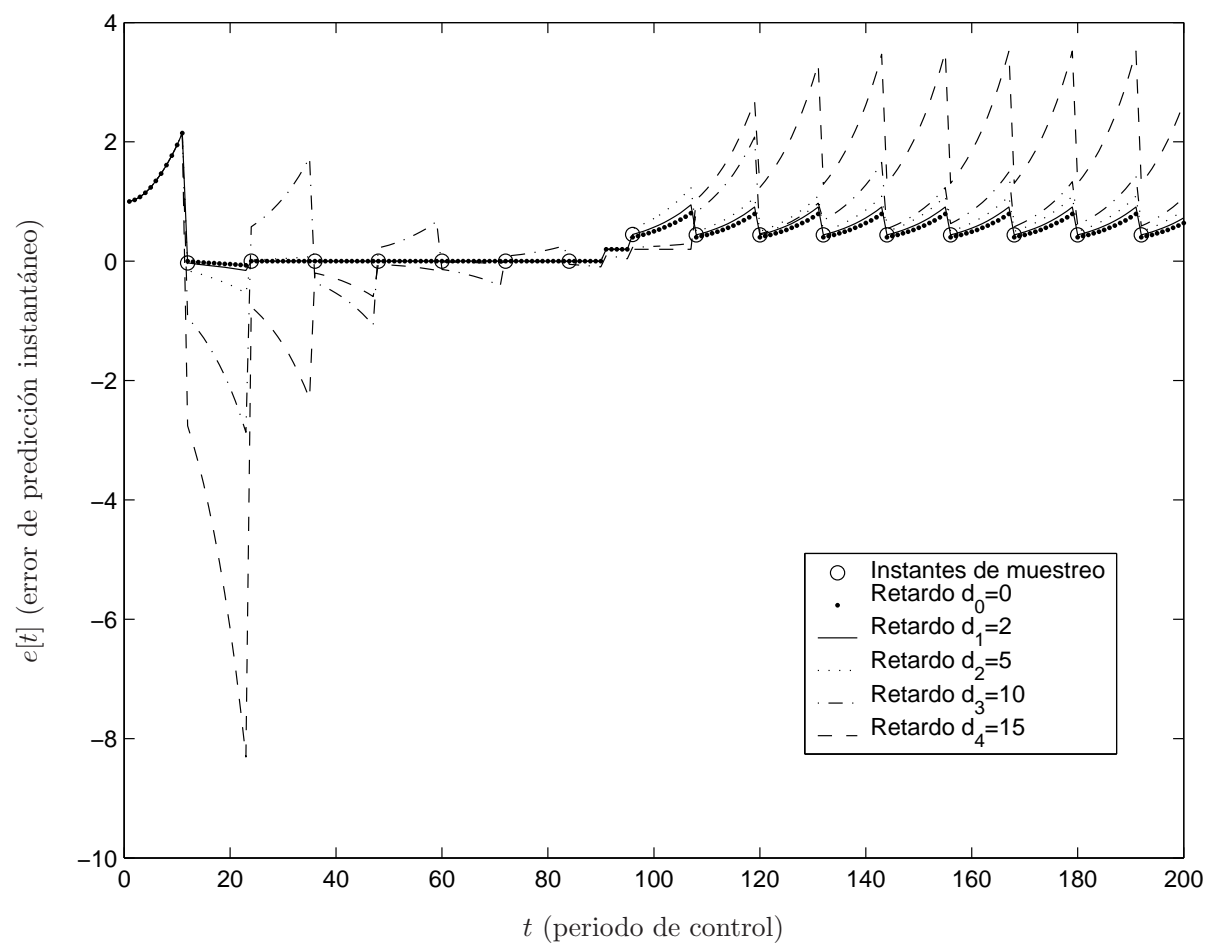

Figura 3.8: Evolución del error de predicción $(N=12)$ para diferentes retardos en presencia de una perturbación en forma de escalón.

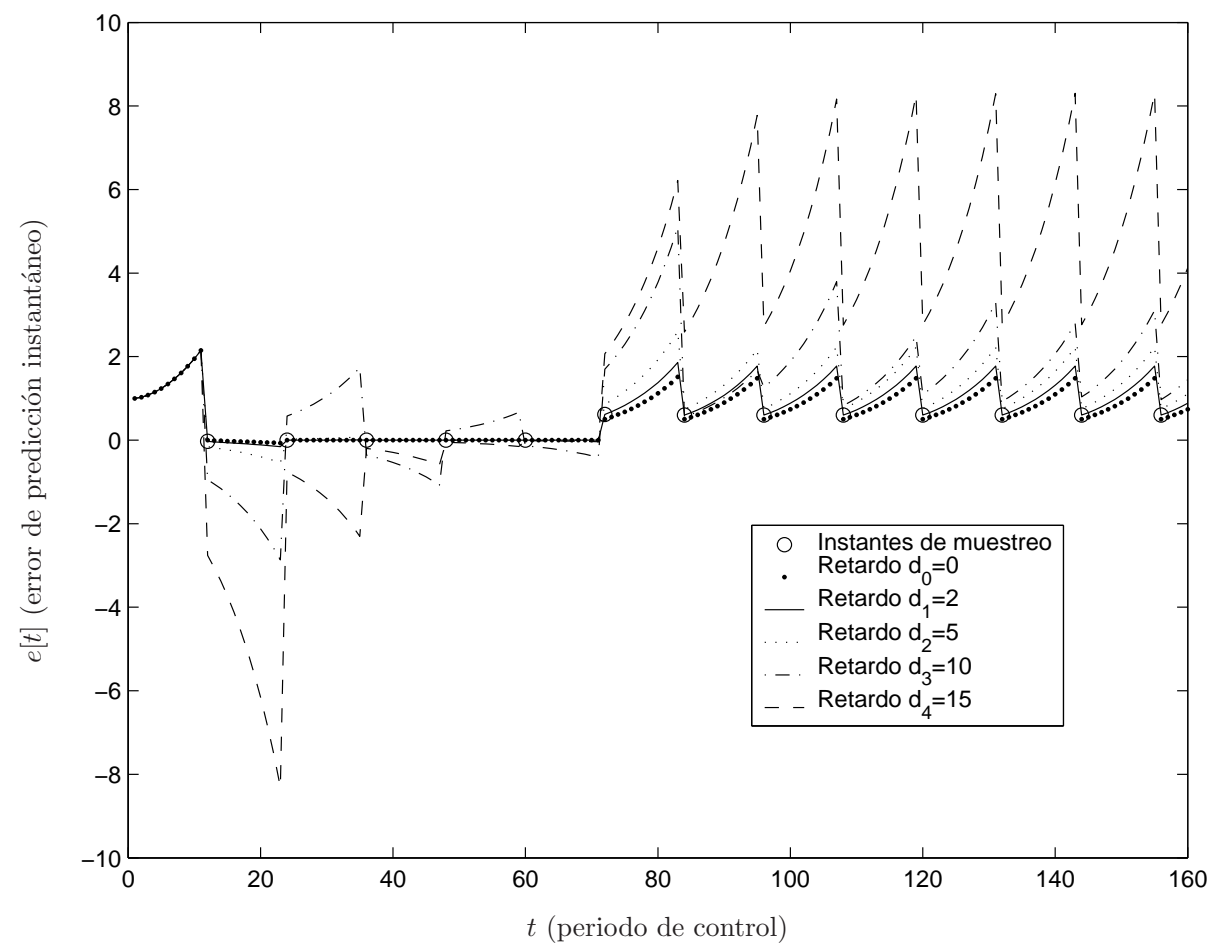

Figura 3.9: Evolución del error de predicción $(N=12)$ para diferentes retardos en presencia de un ruido de medida en forma de escalón.

en el tiempo sea estable es suficiente que se cumpla la condición de estabilidad policuadrática, definida en §A.4 para el caso de un sistema variante en el tiempo dependiente de un parámetro. En esta sección se considera que la secuencia de escenarios de medición puede ser cualquiera.

El siguiente teorema presenta el diseño del predictor que asegura estabilidad policuadrática. 
Teorema 3.4.1. Considérese el sistema (3.7) en ausencia de perturbaciones y ruido de medida $(v=0$ y $w=0)$, y supóngase que hay una medida disponible con un retardo $d_{k}$ cada $N_{k}$ periodos, con $d_{k} y$ $N_{k}$ pertenecientes a los conjuntos (3.1) y (3.3), respectivamente, habiendo $n_{\mathcal{S}}$ escenarios de muestreo $s_{k}$ posibles. Para un $\mu \in(0,1]$ dado, si existen unas matrices $\boldsymbol{P}\left(s_{k}\right)=\boldsymbol{P}\left(s_{k}\right)^{\top} \in \mathbb{R}^{n \times n}, \boldsymbol{Q}\left(s_{k}\right) \in \mathbb{R}^{n \times n}$, $\boldsymbol{X}\left(s_{k}\right) \in \mathbb{R}^{n \times 1}$ tales que la siguiente desigualdad lineal matricial paramétrica (PLMI)

$$
\left[\begin{array}{cc}
\boldsymbol{Q}\left(s_{k}\right)+\boldsymbol{Q}\left(s_{k}\right)^{\top}-\boldsymbol{P}\left(s_{k}\right) & \left(\boldsymbol{Q}\left(s_{k}\right)-\boldsymbol{X}\left(s_{k}\right) \boldsymbol{c} \boldsymbol{A}^{-d\left(s_{k}\right)}\right) \boldsymbol{A}^{N\left(s_{k}\right)} \\
\left(\left(\boldsymbol{Q}\left(s_{k}\right)-\boldsymbol{X}\left(s_{k}\right) \boldsymbol{c} \boldsymbol{A}^{-d\left(s_{k}\right)}\right) \boldsymbol{A}^{N\left(s_{k}\right)}\right)^{\top} & \mu^{2} \boldsymbol{P}\left(s_{k-1}\right)
\end{array}\right] \succ 0,
$$

se cumple para cualquier secuencia de muestreo $\left\{s_{k}\right\}$, entonces, si la ganancia del predictor se define en función del parámetro de muestreo como

$$
\boldsymbol{\ell}\left(s_{k}\right)=\boldsymbol{Q}\left(s_{k}\right)^{-1} \boldsymbol{X}\left(s_{k}\right),
$$

el error de predicción del algoritmo definido por (3.8) converge a cero de forma asintótica con un ratio de decadencia menor que $\mu \in(0,1]$.

Prueba 3.4.1. Si se introduce la ganancia (3.39) en la LMI (3.38), la desigualdad puede escribirse como

$$
\left[\begin{array}{cc}
\boldsymbol{Q}\left(s_{k}\right)+\boldsymbol{Q}\left(s_{k}\right)^{\top}-\boldsymbol{P}\left(s_{k}\right) & \boldsymbol{Q}\left(s_{k}\right) \mathcal{A}\left(s_{k}\right) \\
\mathcal{A}\left(s_{k}\right)^{\top} \boldsymbol{Q}\left(s_{k}\right)^{\top} & \mu^{2} \boldsymbol{P}\left(s_{k-1}\right)
\end{array}\right] \succ 0
$$

La condición $\boldsymbol{Q}\left(s_{k}\right)+\boldsymbol{Q}\left(s_{k}\right)^{\top}-\boldsymbol{P}\left(s_{k}\right) \succ 0$ implica que $\boldsymbol{Q}\left(s_{k}\right)$ es no singular, y además, se cumple la siguiente desigualdad matricial

$$
\left(\boldsymbol{P}\left(s_{k}\right)-\boldsymbol{Q}\left(s_{k}\right)\right) \boldsymbol{P}\left(s_{k}\right)^{-1}\left(\boldsymbol{P}\left(s_{k}\right)-\boldsymbol{Q}\left(s_{k}\right)\right)^{\top} \succeq \mathbf{0},
$$

y por tanto

$$
\boldsymbol{Q}\left(s_{k}\right)+\boldsymbol{Q}\left(s_{k}\right)^{\top}-\boldsymbol{P}\left(s_{k}\right) \preceq \boldsymbol{Q}\left(s_{k}\right) \boldsymbol{P}\left(s_{k}\right)^{-1} \boldsymbol{Q}\left(s_{k}\right)^{\top} .
$$

Aplicando esta condición a (3.40) y tomando complementos de Schur se obtiene la condición

$$
\mathcal{A}\left(s_{k}\right)^{\top} \boldsymbol{P}\left(s_{k}\right) \mathcal{A}\left(s_{k}\right)-\mu^{2} \boldsymbol{P}\left(s_{k-1}\right) \prec 0 .
$$

Si se multiplica esta expresión por la izquierda por $E_{k-1}^{\top}$ y se multiplica por la derecha por $E_{k-1}$ se tiene la desigualdad

$$
E_{k}^{\top} \boldsymbol{P}\left(s_{k}\right) E_{k}<\mu^{2} E_{k-1}^{\top} \boldsymbol{P}\left(s_{k-1}\right) E_{k-1}
$$

Definiendo la función de Lyapunov paramétrica como

$$
\mathcal{V}\left(E_{k}, s_{k}\right)=E_{k}^{\top} \boldsymbol{P}\left(s_{k}\right) E_{k}
$$

la condición (3.42) es equivalente a

$$
\mathcal{V}\left(E_{k}, s_{k}\right)<\mu^{2} \mathcal{V}\left(E_{k-1}, s_{k-1}\right) .
$$

La estabilidad policuadrática queda finalmente probada teniendo en cuenta que la PLMI (3.38) implica claramente que $\boldsymbol{P}\left(s_{k}\right) \succ 0$.

Observación 3.4.1. En general, si los escenarios de muestreo se alternan de forma aleatoria, el número de secuencias $\left\{s_{k-1}, s_{k}\right\}$ diferentes asciende a $n_{\mathcal{S}}^{2}$, y la resolución de la PLMI (3.38) equivale a la resolución del conjunto de LMIs

$$
\left[\begin{array}{cc}
\boldsymbol{Q}(i)+\boldsymbol{Q}(i)^{\top}-\boldsymbol{P}(i) & \left(\boldsymbol{Q}(i)-\boldsymbol{X}(i) \boldsymbol{c} \boldsymbol{A}^{-d(i)}\right) \boldsymbol{A}^{N(i)} \\
\left(\left(\boldsymbol{Q}(i)-\boldsymbol{X}(i) \boldsymbol{c} \boldsymbol{A}^{-d(i)}\right) \boldsymbol{A}^{N(i)}\right)^{\top} & \mu^{2} \boldsymbol{P}(j)
\end{array}\right] \succ 0
$$

$\forall(i, j) \in\left\{1, \ldots, n_{\mathcal{S}}\right\} \times\left\{1, \ldots, n_{\mathcal{S}}\right\}$, siendo $\boldsymbol{P}(i)=\boldsymbol{P}(i)^{\top} \in \mathbb{R}^{n \times n}, \boldsymbol{Q}(i) \in \mathbb{R}^{n \times n}, \boldsymbol{X}(i) \in \mathbb{R}^{n \times 1}$, las variables matriciales del problema LMI. La ganancia del observador vendrá dada por

$$
\boldsymbol{\ell}(i)=\boldsymbol{Q}(i)^{-1} \boldsymbol{X}(i), \quad i=1, \ldots, n_{\mathcal{S}}
$$

Observación 3.4.2. El teorema 3.4 .1 también es válido para muestreo regular con $N_{k}=N$ y $d_{k}=d$ constantes. En ese caso sólo hay un posible valor del parámetro de muestreo $\left(s_{k}=1\right)$, con lo que la LMI (3.40) se reduce a (3.30).

Tal y como muestra la observación 3.3.1, y el ejemplo 3.3.2, la dinámica resultante con este método es muy rápida y, por tanto, si el sistema presenta perturbaciones y ruido de medida, se deben tomar en consideración las estrategias de atenuación de perturbaciones introducidas en §3.2.3. Esto lleva a los diseños que se muestran a continuación. 


\subsubsection{Diseño de predictores $\mathcal{H}_{\infty}$}

El siguiente teorema recoge el diseño de predictores estables que minimizan la cota del error en las situaciones S1, S2 y S3 descritas en la página 31.

Teorema 3.4.2 (Atenuación $\left.\mathcal{H}_{\infty}\right)$. Considérese el predictor (3.8) aplicado al sistema (3.7) y supóngase que hay una medida disponible con un retardo $d_{k}$ cada $N_{k}$ periodos, con $d_{k}$ y $N_{k}$ pertenecientes a los conjuntos (3.1) y (3.3), respectivamente, habiendo $n_{\mathcal{S}}$ escenarios de muestreo $s_{k}$ posibles. Para unas $\gamma_{v}, \gamma_{w}>0$ dadas, supóngase que existen unas matrices $\boldsymbol{P}\left(s_{k}\right)=\boldsymbol{P}\left(s_{k}\right)^{\top} \in \mathbb{R}^{n \times n}, \boldsymbol{Q}\left(s_{k}\right) \in \mathbb{R}^{n \times n}$, $\boldsymbol{X}\left(s_{k}\right) \in \mathbb{R}^{n \times 1}$ tales que

$$
\left[\begin{array}{ccc}
\boldsymbol{Q}\left(s_{k}\right)+\boldsymbol{Q}\left(s_{k}\right)^{\top}-\boldsymbol{P}\left(s_{k}\right) & \boldsymbol{M}_{A}\left(s_{k}\right) & \boldsymbol{M}_{B}\left(s_{k}\right) \\
\boldsymbol{M}_{A}\left(s_{k}\right)^{\top} & \boldsymbol{P}\left(s_{k-1}\right)-\boldsymbol{c}^{\top} \boldsymbol{c} & \mathbf{0} \\
\boldsymbol{M}_{B}\left(s_{k}\right)^{\top} & \mathbf{0} & \boldsymbol{\Gamma}^{2}
\end{array}\right] \succ 0,
$$

para cualquier secuencia de muestreo $\left\{s_{k}\right\}$, siendo

$$
\begin{aligned}
& \boldsymbol{M}_{A}\left(s_{k}\right)=\left(\boldsymbol{Q}\left(s_{k}\right)-\boldsymbol{X}\left(s_{k}\right) \boldsymbol{c} \boldsymbol{A}^{-d\left(s_{k}\right)}\right) \boldsymbol{A}^{N\left(s_{k}\right)}, \\
& \boldsymbol{M}_{B}\left(s_{k}\right)=\left[\begin{array}{llll}
\boldsymbol{Q}\left(s_{k}\right) & -\boldsymbol{X}\left(s_{k}\right) \boldsymbol{c} & -\boldsymbol{M}_{A}\left(s_{k}\right) & -\boldsymbol{X}\left(s_{k}\right)
\end{array}\right] \\
& \boldsymbol{\Gamma}=\left[\begin{array}{cccc}
\frac{\gamma_{v}}{\sqrt{3 n}} & & & \\
& \ddots & & \\
& & \frac{\gamma_{v}}{\sqrt{3 n}} & \\
& & & \gamma_{w}
\end{array}\right]_{(3 n+1)} .
\end{aligned}
$$

Entonces, definiendo la ganancia del predictor en función de la situación de muestreo como $\ell\left(s_{k}\right)=$ $\boldsymbol{Q}\left(s_{k}\right)^{-1} \boldsymbol{X}\left(s_{k}\right)$, el error de predicción del algoritmo definido por (3.8) es estable en ausencia de perturbaciones $y$, bajo condiciones iniciales nulas,

$$
\left\|e_{k}\right\|_{2}^{2} \leq \gamma_{v}^{2}\left\|v_{k}\right\|_{2}^{2}+\gamma_{w}^{2}\left\|w_{k}\right\|_{2}^{2}
$$

Prueba 3.4.2. Como la LMI (3.44) implica obviamente que

$$
\begin{aligned}
& {\left[\begin{array}{cc}
\boldsymbol{Q}\left(s_{k}\right)+\boldsymbol{Q}\left(s_{k}\right)^{\top}-\boldsymbol{P}\left(s_{k}\right) & \left(\boldsymbol{Q}\left(s_{k}\right)-\boldsymbol{X}\left(s_{k}\right) \boldsymbol{c} \boldsymbol{A}^{-d\left(s_{k}\right)}\right) \boldsymbol{A}^{N\left(s_{k}\right)} \\
\left(\left(\boldsymbol{Q}\left(s_{k}\right)-\boldsymbol{X}\left(s_{k}\right) \boldsymbol{c} \boldsymbol{A}^{-d\left(s_{k}\right)}\right) \boldsymbol{A}^{N\left(s_{k}\right)}\right)^{\top} & \boldsymbol{P}\left(s_{k-1}\right)
\end{array}\right]} \\
& -\left[\begin{array}{cc}
\mathbf{0} & \mathbf{0} \\
\mathbf{0} & \boldsymbol{c}^{\boldsymbol{\top}} \boldsymbol{c}
\end{array}\right] \succ 0,
\end{aligned}
$$

y teniendo en cuenta que

$$
\left[\begin{array}{cc}
\mathbf{0} & \mathbf{0} \\
\mathbf{0} & \boldsymbol{c}^{\top} \boldsymbol{c}
\end{array}\right] \succeq 0
$$

entonces se cumple la PLMI (3.38) (con $\mu=1)$ y el predictor converge asintóticamente a cero (teorema 3.4.1).

Aplicando la condición (3.41) en la PLMI (3.44) e introduciendo la ganancia (3.39), se obtiene la expresión

$$
\left[\begin{array}{ccc}
\boldsymbol{Q}\left(s_{k}\right) \boldsymbol{P}\left(s_{k}\right)^{-1} \boldsymbol{Q}\left(s_{k}\right)^{\top} & \boldsymbol{Q}\left(s_{k}\right) \mathcal{A}\left(s_{k}\right) & \boldsymbol{Q}\left(s_{k}\right) \boldsymbol{B}\left(s_{k}\right) \\
\mathcal{A}\left(s_{k}\right)^{\top} \boldsymbol{Q}\left(s_{k}\right)^{\top} & \boldsymbol{P}\left(s_{k-1}\right)-\boldsymbol{c}^{\top} \boldsymbol{c} & \mathbf{0} \\
\mathcal{B}\left(s_{k}\right)^{\top} \boldsymbol{Q}\left(s_{k}\right)^{\top} & \mathbf{0} & \boldsymbol{\Gamma}^{2}
\end{array}\right] \succ 0
$$

donde se ha tenido en cuenta que

$$
\begin{aligned}
& M_{A}\left(s_{k}\right)=\boldsymbol{Q}\left(s_{k}\right) \mathcal{A}\left(s_{k}\right), \\
& \boldsymbol{M}_{B}\left(s_{k}\right)=\boldsymbol{Q}\left(s_{k}\right) \mathcal{B}\left(s_{k}\right) .
\end{aligned}
$$


Aplicando los complementos de Schur sobre la matriz en (3.48) es fácil comprobar que se cumple la LMI

$$
\left[\begin{array}{cc}
\mathcal{A}\left(s_{k}\right)^{\top} \boldsymbol{P}\left(s_{k}\right) \mathcal{A}\left(s_{k}\right)-\boldsymbol{P}\left(s_{k-1}\right)+\boldsymbol{c}^{\top} \boldsymbol{c} & \boldsymbol{\mathcal { A }}\left(s_{k}\right)^{\top} \boldsymbol{P}\left(s_{k}\right) \boldsymbol{B}\left(s_{k}\right) \\
\mathcal{B}\left(s_{k}\right)^{\top} \boldsymbol{P}\left(s_{k}\right) \mathcal{A}\left(s_{k}\right) & \mathcal{B}\left(s_{k}\right)^{\top} \boldsymbol{P}\left(s_{k}\right) \mathcal{B}\left(s_{k}\right)-\boldsymbol{\Gamma}^{2}
\end{array}\right] \prec \mathbf{0} .
$$

Multiplicando esta desigualdad por $\left[E_{k-1}^{\top} \mathbb{V}_{k}^{\top} w_{k}^{\top}\right]$ por la izquierda y por su traspuesta por la derecha se tiene que

$$
E_{k}^{\top} \boldsymbol{P}\left(s_{k}\right) E_{k}-E_{k-1}^{\top} \boldsymbol{P}\left(s_{k-1}\right) E_{k-1}+e_{k-1}^{\top} e_{k-1}-\frac{\gamma_{v}^{2}}{3 n} \mathbb{V}_{k}^{\top} \mathbb{V}_{k}-\gamma_{w}^{2} w_{k}^{\top} w_{k}<0 .
$$

Asumiendo un error de predicción inicial nulo $\left(E_{0}=\mathbf{0}\right)$ y sumando de $k=1$ a $k=K$ se tiene que

$$
E_{K}^{\boldsymbol{\top}} \boldsymbol{P}\left(s_{K}\right) E_{K}+\sum_{k=1}^{K}\left(e_{k-1}^{\top} e_{k-1}-\frac{\gamma_{v}^{2}}{3 n} \mathbb{V}_{k}^{\top} \mathbb{V}_{k}-\gamma_{w}^{2} w_{k}^{\top} w_{k}\right)<0
$$

donde se ha tenido en cuenta la dinámica del error de predicción (3.26). Como $\boldsymbol{P}\left(s_{k}\right)>0$, entonces $E_{K}^{\top} \boldsymbol{P}\left(s_{K}\right) E_{K} \succ \mathbf{0}$, llegándose a

$$
\sum_{k=1}^{K}\left(e_{k-1}^{\top} e_{k-1}-\frac{\gamma_{v}^{2}}{3 n} \mathbb{V}_{k}^{\top} \mathbb{V}_{k}-\gamma_{w}^{2} w_{k}^{\top} w_{k}\right)<0
$$

Cuando $K$ tiende a infinito se obtiene la norma $\ell_{2}$ de las señales cumpliéndose que

$$
\left\|e_{k}\right\|_{2}^{2}<\gamma_{2}^{2}\left\|v_{k}\right\|_{2}^{2}+\gamma_{w}^{2}\left\|w_{k}\right\|_{2}^{2}
$$

donde se ha tenido en cuenta que la norma $\left\|\mathbb{V}_{k}\right\|_{2}$ es $3 n$ veces mayor que $\left\|v_{k}\right\|_{2}$ porque el vector $\mathbb{V}_{k}$ contiene $3 n$ valores de la historia de la señal $v[t]$ según (3.23) (véase [11, capítulo 8]).

Observación 3.4.3. Si se consideran conocidas las normas $\ell_{2}$ de las perturbaciones, se puede minimizar la cota superior de $\left\|e_{k}\right\|_{2}$ minimizando la suma

$$
\gamma_{v}^{2}\left\|v_{k}\right\|_{2}^{2}+\gamma_{w}^{2}\left\|w_{k}\right\|_{2}^{2}
$$

a lo largo de todas las variables $\gamma_{v}, \gamma_{w}, \boldsymbol{P}\left(s_{k}\right), \boldsymbol{Q}\left(s_{k}\right), \boldsymbol{X}\left(s_{k}\right)$ que satisfacen la LMI (3.44).

Observación 3.4.4 (Cálculo de la ganancia del predictor que maximiza la atenuación). La minimización indicada en la observación anterior se puede resolver utilizando solucionadores LMI estándar. Para ello se deben definir $\gamma_{v}^{2}$ y $\gamma_{w}^{2}$ como variables matriciales (de tamaño $1 \times 1$ ) y resolver el problema de minimización de la forma

$$
\text { Minimiza } \quad \boldsymbol{h}^{\top} \boldsymbol{x} \quad \text { sujeto a } \boldsymbol{M}(\boldsymbol{X}) \prec \mathbf{0},
$$

donde $\boldsymbol{h}$ es un vector constante, $\boldsymbol{X}$ denota las variables matriciales, $\boldsymbol{x}$ es un vector con todas las componentes de $\boldsymbol{X}$, y $\boldsymbol{M}(\boldsymbol{X})$ representa las matrices del problema $\mathrm{LMI}^{5}$. Si $\gamma_{v}^{2}$ y $\gamma_{w}^{2}$ son las últimas componentes del vector $\boldsymbol{x}$, entonces el vector $\boldsymbol{h}$ tendrá la forma

$$
\boldsymbol{h}^{\top}=\left[\begin{array}{llllll}
0 & \ldots & 0 & \left\|v_{k}\right\|_{2}^{2} & \left\|w_{k}\right\|_{2}^{2}
\end{array}\right] .
$$

Observación 3.4.5. Para realizar la minimización anterior es necesario conocer el valor de $\left\|v_{k}\right\|_{2}$ (norma $\ell_{2}$ de la perturbación al periodo variante $N_{k} T$ ). Sin embargo, es más frecuente tener una estimación de $\|v[t]\|_{2}$ (norma $\ell_{2}$ de la perturbación a periodo $T$ ). El valor $\left\|v_{k}\right\|_{2}$ se puede relacionar con $\|v[t]\|_{2}$ mediante la relación

$$
\|v[t]\|_{2}^{2}=\sum_{t=0}^{\infty} v[t]^{2} \approx \sum_{k=0}^{\infty} N_{k} v_{k}^{2}
$$

donde se ha aproximado la energía de la señal $v[t]$ acumulada entre muestreos (de $t_{k-1}$ a $t_{k}$ ) como $N_{k}$ veces el valor de $v_{k}^{2}$ :

$$
\sum_{t=t_{k-1}}^{t_{k}} v[t]^{2} \approx N_{k} v_{k}^{2}
$$

\footnotetext{
${ }^{5}$ El comando mincx de la LMI Control Toolbox de MATLAB ${ }^{\circledR}$ resuelve este problema
} 
Si $N_{k}$ es constante (muestreo bifrecuencia) la relación (3.53) queda

$$
\|v[t]\|_{2}^{2} \approx N \sum_{k=0}^{\infty} v_{k}^{2}=N\left\|v_{k}\right\|_{2}^{2}
$$

con lo que se puede calcular un valor aproximado de $\left\|v_{k}\right\|_{2}$ mediante

$$
\left\|v_{k}\right\|_{2}^{2} \approx \frac{\|v[t]\|_{2}^{2}}{N}
$$

Sin embargo, si $N_{k}$ es variante ya no puede aplicarse la relación anterior. En este caso se tiene que obtener una expresión que aproxime el valor de $\left\|v_{k}\right\|_{2}$ a partir del valor conocido de $\|v[t]\|_{2}$. Si se tiene en cuenta la independencia entre las variables $N_{k}$ y $v_{k}$, y se toman esperanzas matemáticas en (3.53), se llega a

$$
\mathcal{E}\left\{\|v[t]\|_{2}^{2}\right\} \approx \sum_{k=0}^{\infty} \mathcal{E}\left\{N_{k}\right\} \mathcal{E}\left\{v_{k}^{2}\right\}
$$

de donde se puede obtener un valor aproximado de $\left\|v_{k}\right\|_{2}$ como

$$
\left\|v_{k}\right\|_{2}^{2} \approx \frac{\|v[t]\|_{2}^{2}}{\bar{N}}
$$

siendo $\bar{N}=\mathcal{E}\left\{N_{k}\right\}$ el valor medio de $N_{k}$.

Observación 3.4.6. Bajo las mismas hipótesis que el teorema 3.4.2, y si se suponen conocidas las normas RMS (o valor eficaz) de las perturbaciones, se puede minimizar la cota superior $\left\|e_{k}\right\|_{R M S}$ minimizando la suma

$$
\gamma_{v}^{2}\left\|v_{k}\right\|_{R M S}^{2}+\gamma_{w}^{2}\left\|w_{k}\right\|_{R M S}^{2}
$$

a lo largo de todas las variables $\gamma_{v}, \gamma_{w}, \boldsymbol{P}\left(s_{k}\right), \boldsymbol{Q}\left(s_{k}\right), \boldsymbol{X}\left(s_{k}\right)$ que satisfacen la LMI (3.44). Para demostrarlo tómese la ecuación (3.51) de la demostración del teorema 3.4.2 y divídase por $K$, llegando a

$$
\left\|e_{k}\right\|_{R M S}^{2}<\gamma_{v}^{2}\left\|v_{k}\right\|_{R M S}^{2}+\gamma_{w}^{2}\left\|w_{k}\right\|_{R M S}^{2} .
$$

La minimización se puede realizar como se ha indicado en la observación 3.4.4, tomando en este caso

$$
\boldsymbol{h}^{\top}=\left[\begin{array}{lllll}
0 & \ldots & 0 & \left\|v_{k}\right\|_{R M S}^{2} & \left\|w_{k}\right\|_{R M S}^{2}
\end{array}\right]
$$

Observación 3.4.7. Para realizar la minimización anterior es necesario conocer el valor de $\left\|v_{k}\right\|_{R M S}$ (valor eficaz de la perturbación al periodo variante $N_{k} T$ ). Sin embargo, es más frecuente tener una estimación de $\|v[t]\|_{R M S}$ (valor eficaz de la perturbación a periodo $T$ ). El valor $\left\|v_{k}\right\|_{R M S}$ se puede relacionar con $\|v[t]\|_{R M S}$ de forma aproximada mediante

$$
\|v[t]\|_{R M S}^{2}=\lim _{T \rightarrow \infty} \frac{1}{T} \sum_{t=0}^{T} v[t]^{2} \approx \lim _{T_{0} \rightarrow \infty} \frac{1}{T_{0}} \sum_{k=0}^{T_{0}} N_{k} v_{k}^{2},
$$

siendo $T_{0}$ el número de muestras necesarias para abarcar el mismo tiempo que abarcan $T$ actualizaciones de la acción de control, y donde se ha considerado de nuevo la aproximación (3.54). Si $N_{k}$ es constante (muestreo bifrecuencia) la relación (3.53) queda

$$
\|v[t]\|_{R M S}^{2} \approx \lim _{T \rightarrow \infty} \frac{N}{T} \sum_{k=0}^{\frac{T}{N}} v_{k}^{2}=\lim _{T_{0} \rightarrow \infty} \frac{1}{T_{0}} \sum_{k=0}^{T_{0}} v_{k}^{2}=\left\|v_{k}\right\|_{R M S}^{2},
$$

ya que $T=N T_{0}$. Si $N_{k}$ es variante, la relación (3.55) se puede aproximar mediante

$$
\|v[t]\|_{R M S}^{2} \approx \lim _{T \rightarrow \infty} \frac{\bar{N}}{T} \sum_{k=0}^{\frac{T}{N}} v_{k}^{2}=\lim _{T_{0} \rightarrow \infty} \frac{1}{T_{0}} \sum_{k=0}^{T_{0}} v_{k}^{2}=\left\|v_{k}\right\|_{R M S}^{2},
$$

donde se ha tomado que el valor esperado del número de muestras necesarias para abarcar $T$ actualizaciones es $T=\bar{N} T_{0}$, siendo $\bar{N}=\mathcal{E}\left\{N_{k}\right\}$ el valor medio de $N_{k}$. 
Por tanto, la mejor aproximación del valor eficaz de la señal $v_{k}$ (a periodo variante) es el valor eficaz de la señal $v[t]$ (a periodo constante):

$$
\left\|v_{k}\right\|_{R M S} \approx\|v[t]\|_{R M S}
$$

Ejemplo 3.4.1. Considérese el sistema

$$
G(s)=\frac{10}{s^{2}+20 s+80}
$$

cuya entrada se actualiza a periodo $T=0.05$ segundos. Supóngase un muestreo variante en el tiempo cuyo número de periodos intermuestreo $N_{k}$, y cuyo retardo variante $d_{k}$ varían dentro de los conjuntos

$$
\mathcal{N}=\{10,11,12,13,14,15\}, \quad \mathcal{D}=\{0,1,2\}
$$

Supóngase también que se tiene una perturbación y un ruido aleatorios con un valor eficaz de

$$
\|v[t]\|_{R M S}=57.7 \cdot 10^{-3}, \quad\|w[t]\|_{R M S}=0.291 .
$$

Se diseña un predictor $\mathcal{H}_{\infty}$ mediante la técnica expuesta anteriormente (teorema 3.4.2) y un predictor diseñado para cumplir con la estabilidad nominal (teorema 3.4.1). En la figura 3.10 se muestra un detalle de la evolución de la salida del proceso y de las salidas estimadas con cada método, obtenido mediante simulación. Se observa que el predictor diseñado para estabilidad nominal tiene un mayor error

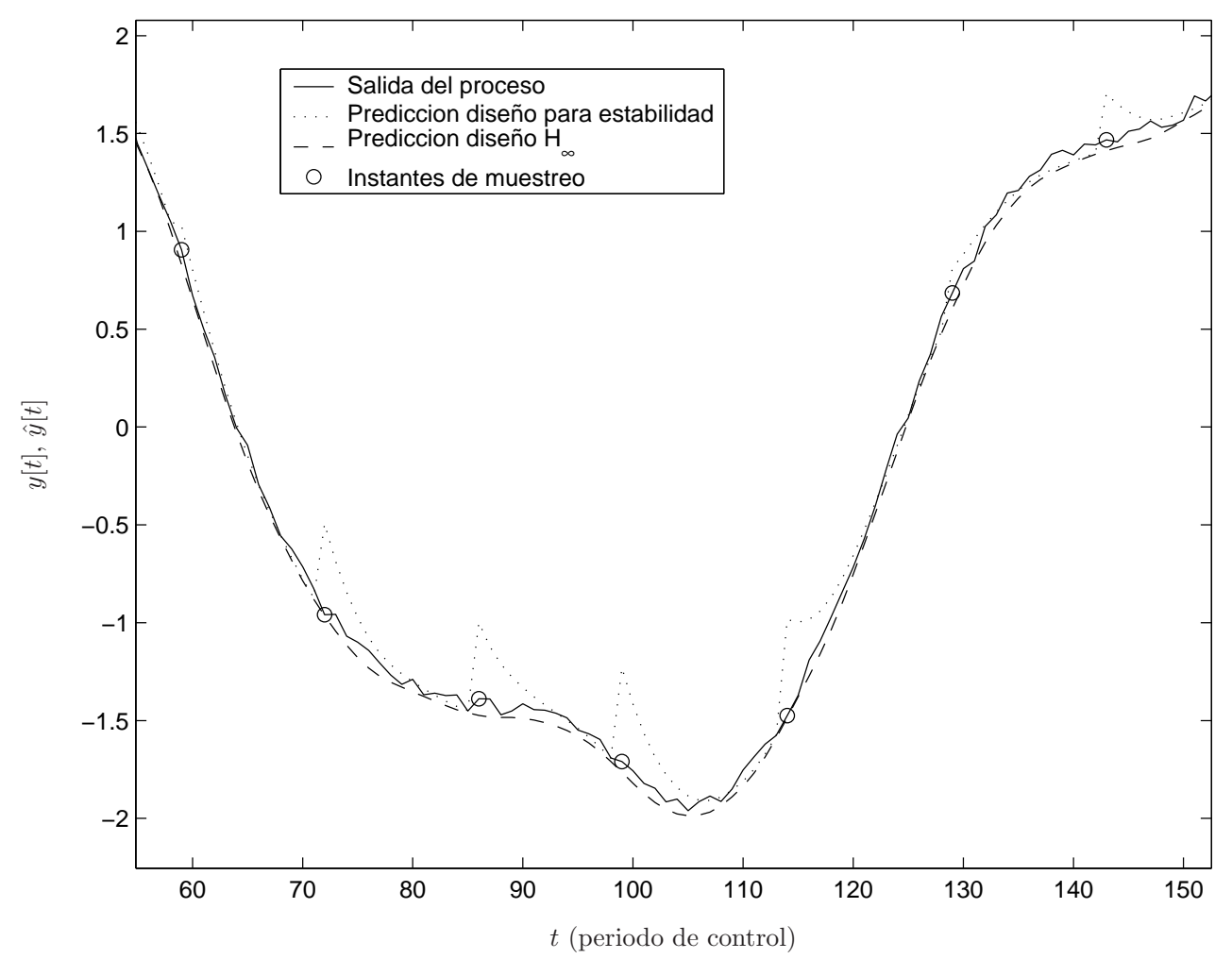

Figura 3.10: Evolución de la salida y de las predicciones con diferentes métodos. $\mathcal{N}=\{10, \ldots, 15\}, \mathcal{D}=\{0,1,2\}$.

de predicción que se acentúa en los instantes de medición debido al ruido de medida. En la simulación realizada se ha obtenido un error de predicción con el predictor diseñado para estabilidad nominal de $\|e[t]\|_{R M S}=94.7 \cdot 10^{-3}$, mientras que con el diseño del predictor para atenuación $\mathcal{H}_{\infty}$ este error se ha reducido a $\|e[t]\|_{R M S}=57.5 \cdot 10^{-3}$.

Nótese que, a pesar de que el diseño $\mathcal{H}_{\infty}$ se realiza para minimizar la cota del error $\left\|e_{k}\right\|$ en los instantes de muestreo, es interesante estudiar el error de predicción en todos los periodos $(\|e[t]\|)$, ya que ayuda a estudiar la bondad de la predicción en los instantes en los que se está prediciendo en bucle abierto. 
Observación 3.4.8. Si se tienen perturbaciones persistentes pero no se conoce su valor eficaz, es necesario proceder de forma diferente para diseñar un predictor que acote el valor eficaz del error de predicción. La idea es que si una señal es persistente, para un intervalo $k=1, \ldots, K$ dado se puede establecer una cota de la energía acumulada como

$$
\sum_{k=1}^{K}\left(\frac{\gamma_{v}^{2}}{3 n} \mathbb{V}_{k}^{\top} \mathbb{V}_{k}+\gamma_{w}^{2} w_{k}^{\top} w_{k}\right) \leq K\left(\gamma_{v}^{2}\left\|v_{k}\right\|_{\infty}^{2}+\gamma_{w}^{2}\left\|w_{k}\right\|_{\infty}^{2}\right)
$$

Nótese que la igualdad se alcanza cuando la perturbación es de tipo escalón. Con esta aproximación, es posible obtener una cota del valor eficaz del error de predicción $\left\|e_{k}\right\|_{R M S}$ si se minimiza la suma

$$
\gamma_{v}^{2}\left\|v_{k}\right\|_{\infty}^{2}+\gamma_{w}^{2}\left\|w_{k}\right\|_{\infty}^{2}
$$

a largo de todas las variables $\gamma_{v}, \gamma_{w}, \boldsymbol{P}\left(s_{k}\right), \boldsymbol{Q}\left(s_{k}\right), \boldsymbol{X}\left(s_{k}\right)$ que satisfacen la LMI (3.44). Para demostrarlo, retómese la demostración del teorema 3.4.2, de manera que (3.51) en combinación con la aproximación (3.56) lleva a

$$
\begin{array}{r}
\frac{1}{K} \sum_{k=1}^{K}\left(e_{k-1}^{\top} e_{k-1}\right)<\gamma_{v}^{2}\left\|v_{k}\right\|_{\infty}^{2}+\gamma_{w}^{2}\left\|w_{k}\right\|_{\infty}^{2} \\
\left\|e_{k}\right\|_{R M S}^{2}<\gamma_{v}^{2}\left\|v_{k}\right\|_{\infty}^{2}+\gamma_{w}^{2}\left\|w_{k}\right\|_{\infty}^{2} .
\end{array}
$$

En el límite cuando $K$ tiende a infinito se tiene que

$$
\left\|e_{k}\right\|_{R M S}^{2}<\gamma_{v}^{2}\left\|v_{k}\right\|_{\infty}^{2}+\gamma_{w}^{2}\left\|w_{k}\right\|_{\infty}^{2}
$$

Nótese que para un caso general en el que las perturbaciones no son del tipo escalón, la aproximación (3.56) lleva a un diseño muy conservador, mientras que si las perturbaciones son de tipo escalón, este diseño coincide con el de la observación 3.4.6. La minimización anterior se puede realizar como se ha indicado en la observación 3.4.4, tomando en este caso

$$
\boldsymbol{h}^{\top}=\left[\begin{array}{llllll}
0 & \ldots & 0 & \left\|v_{k}\right\|_{\infty}^{2} & \left\|w_{k}\right\|_{\infty}^{2}
\end{array}\right]
$$

Observación 3.4.9. Si se conoce el valor de pico (norma $\ell_{\infty}$ ) de la perturbación $v[t]$ (es decir, $\|v[t]\|_{\infty}$ ), se puede asumir este valor como el valor de pico de $\left\|v_{k}\right\|$ ya que, al ser el muestreo variante, no se puede especificar si el valor máximo de $v[t]$ se da en los instantes de muestreo o no. De esta forma, se puede tomar la aproximación

$$
\left\|v_{k}\right\|_{\infty} \approx\|v[t]\|_{\infty}
$$

que permite obtener una solución que minimiza el efecto de la perturbación aún cuando el valor máximo no se produce en los instantes de muestreo. Si la perturbación es en forma de escalón la aproximación anterior se convierte en una igualdad,

$$
\left\|v_{k}\right\|_{\infty}=\|v[t]\|_{\infty}
$$

En el ejemplo anterior se ha tratado un proceso en el que las perturbaciones eran de naturaleza aleatoria y se ha expuesto que la técnica también era válida para el caso en el que las perturbaciones eran constantes. Si embargo es usual encontrarse con procesos que combinan ambas problemáticas, presentando una perturbación constante y un ruido de medida aleatorio de media cero. En estos casos, teniendo en cuenta que una perturbación constante tiene un valor eficaz igual a su norma $\ell_{\infty}$ se puede tratar el problema como si de dos señales aleatorias de valor eficaz conocido se tratara. El próximo ejemplo muestra esta problemática.

Ejemplo 3.4.2. Retómese el sistema del ejemplo 3.4.1 con un muestreo definido por los conjuntos

$$
\mathcal{N}=\{3,4,5,6\}, \quad \mathcal{D}=\{0,1,2\}
$$

Considérese ahora que el sistema está afectado por una perturbación en forma de escalón acotada por

$$
\|v[t]\|_{\infty}=0.4
$$


y por un ruido de medida aleatorio de media cero con un valor eficaz de

$$
\|w[t]\|_{R M S}=0.09
$$

Se diseña un predictor en bucle abierto, que no tiene en cuenta las mediciones, y un predictor $\mathcal{H}_{\infty}$ a partir de las cotas de la perturbación y el ruido de medida. Si se hace una simulación del proceso y las predicciones con las perturbaciones definidas, se obtiene la evolución mostrada en la figura 3.11, donde se observa la mejora conseguida con el predictor $\mathcal{H}_{\infty}$ con respecto del predictor en bucle abierto. En la

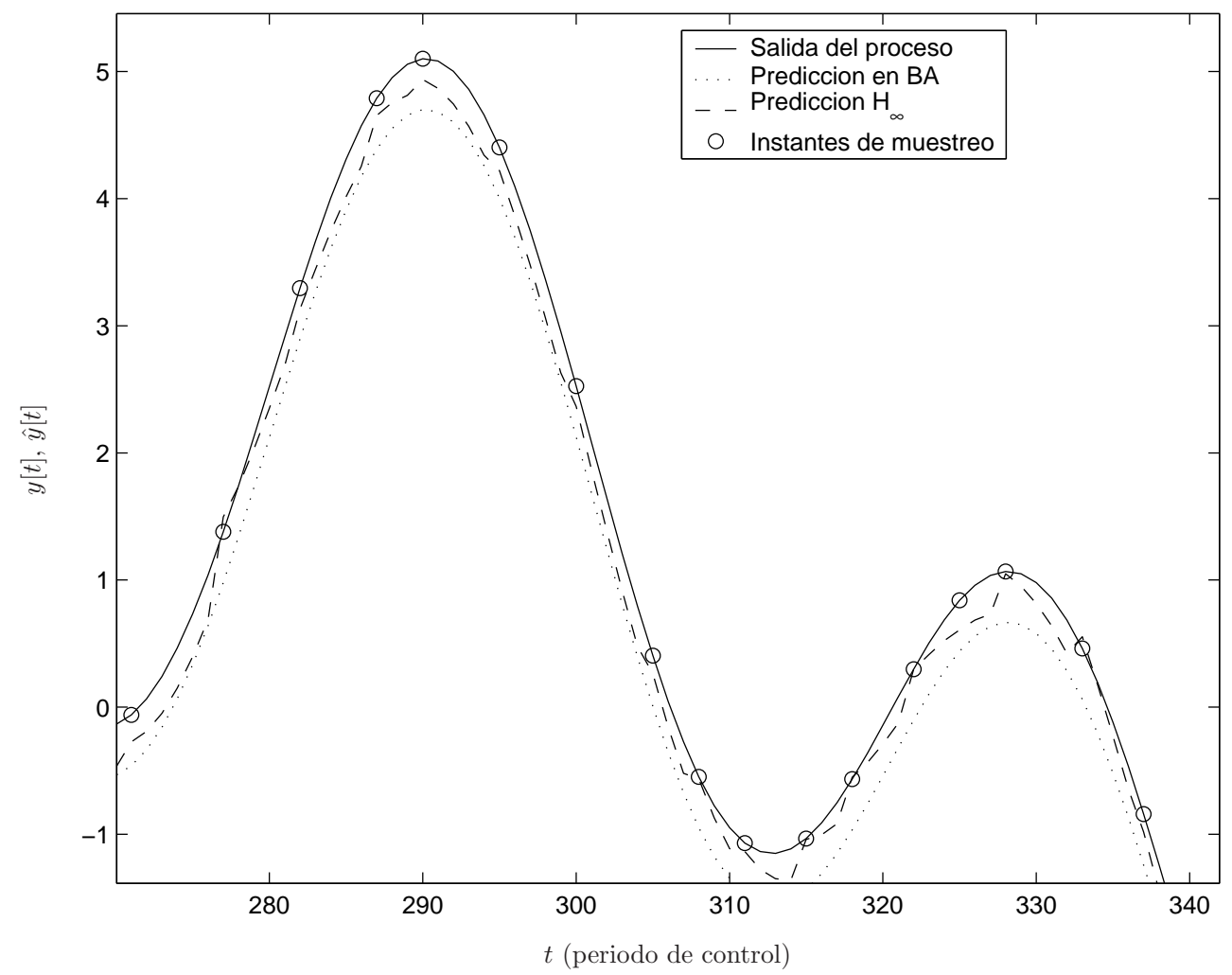

Figura 3.11: Evolución de la salida y de las predicciones con diferentes métodos. $\mathcal{N}=\{3, \ldots, 6\}, \mathcal{D}=\{0,1,2\}$. Perturbación y ruido en forma de escalón

simulación realizada se ha obtenido un error de $\|e[t]\|_{R M S}=399 \cdot 10^{-3}$ para el predictor de bucle abierto, y un error de $\|e[t]\|_{R M S}=217 \cdot 10^{-3}$ para el predictor $\mathcal{H}_{\infty}$.

\subsubsection{Diseño de predictores $\mathcal{H}_{2}$}

Si las perturbaciones son ruidos blancos de media cero se puede plantear el diseño desde el punto de vista de la norma $\mathcal{H}_{2}$ para acotar el valor eficaz del error de predicción (situación S1 descrita en la página 31 ).

Teorema 3.4.3. Considérese el predictor (3.8) aplicado al sistema (3.7) y supóngase que hay una medida disponible con un retardo $d_{k} \in \mathcal{D}$ cada $N_{k} \in \mathcal{N}$ periodos, habiendo $n_{\mathcal{S}}$ escenarios de muestreo $s_{k} \in \mathcal{S}$ posibles. Supóngase que la perturbación y el ruido de medida son ambos ruidos blancos de media cero y varianza $\sigma_{v}$ y $\sigma_{w}$, respectivamente. Para unas $\gamma_{v}, \gamma_{w}>0$ dadas, supóngase que existen unas matrices $\boldsymbol{P}\left(s_{k}\right)=\boldsymbol{P}\left(s_{k}\right)^{\top} \in \mathbb{R}^{n \times n}, \boldsymbol{Q}\left(s_{k}\right) \in \mathbb{R}^{n \times n}, \boldsymbol{X}\left(s_{k}\right) \in \mathbb{R}^{n \times 1}$ tales que

$$
\begin{aligned}
& {\left[\begin{array}{cc}
\boldsymbol{Q}\left(s_{k}\right)+\boldsymbol{Q}\left(s_{k}\right)^{\top}-\boldsymbol{P}\left(s_{k}\right) & \boldsymbol{M}_{A}\left(s_{k}\right) \\
\boldsymbol{M}_{A}\left(s_{k}\right)^{\top} & \boldsymbol{P}\left(s_{k-1}\right)-\boldsymbol{c}^{\top} \boldsymbol{c}
\end{array}\right] \succ \mathbf{0}} \\
& {\left[\begin{array}{cc}
\boldsymbol{Q}\left(s_{k}\right)+\boldsymbol{Q}\left(s_{k}\right)^{\top}-\boldsymbol{P}\left(s_{k}\right) & \boldsymbol{M}_{B}\left(s_{k}\right) \\
\boldsymbol{M}_{B}\left(s_{k}\right)^{\top} & \boldsymbol{\Gamma}^{2}
\end{array}\right] \succ \mathbf{0}}
\end{aligned}
$$

para cualquier secuencia de muestreo $\left\{s_{k}\right\}$, donde $\boldsymbol{M}_{A}\left(s_{k}\right)$ y $\boldsymbol{M}_{B}\left(s_{k}\right)$ vienen dads por (3.45). Entonces, definiendo la ganancia del predictor en función del valor del parámetro de muestreo como $\ell\left(s_{k}\right)=$ 
$\boldsymbol{Q}\left(s_{k}\right)^{-1} \boldsymbol{X}\left(s_{k}\right)$, el error de predicción del algoritmo definido por (3.8) converge asintóticamente a cero en ausencia de ruido de medida y, bajo condiciones iniciales nulas,

$$
\left\|e_{k}\right\|_{R M S}^{2} \leq \gamma_{v}^{2} \sigma_{v}^{2}+\gamma_{w}^{2} \sigma_{w}^{2}
$$

Prueba 3.4.3. La estabilidad del predictor queda probada aplicando los mismos argumentos que en el teorema anterior con la LMI (3.58a).

Tómese ahora la función de Lyapunov $\mathcal{V}_{k}=E_{k}^{\top} \boldsymbol{P}\left(s_{k}\right) E_{k}$. El valor esperado de la función de Lyapunov en un instante $k$ es

$$
\begin{aligned}
\mathcal{E}\left\{\mathcal{V}_{k}\right\} & =\mathcal{E}\left\{E_{k}^{\top} \boldsymbol{P}\left(s_{k}\right) E_{k}\right\} \\
& =\mathcal{E}\left\{\left(\mathcal{A}\left(s_{k}\right) E_{k-1}+\mathcal{B}\left(s_{k}\right)\left[\begin{array}{c}
\mathbb{V}_{k} \\
w_{k}
\end{array}\right]\right)^{\top} \boldsymbol{P}\left(s_{k}\right)\left(\mathcal{A}\left(s_{k}\right) E_{k-1}+\mathcal{B}\left(s_{k}\right)\left[\begin{array}{c}
\mathbb{V}_{k} \\
w_{k}
\end{array}\right]\right)\right\} \\
& =\mathcal{E}\left\{E_{k-1}^{\top} \mathcal{A}\left(s_{k}\right)^{\top} \boldsymbol{P}\left(s_{k}\right) \mathcal{A}\left(s_{k}\right) E_{k-1}\right\}+\mathcal{E}\left\{\left[\begin{array}{c}
\mathbb{V}_{k} \\
w_{k}
\end{array}\right]^{\top} \boldsymbol{B}\left(s_{k}\right)^{\top} \boldsymbol{P}\left(s_{k}\right) \mathcal{B}\left(s_{k}\right)\left[\begin{array}{c}
\mathbb{V}_{k} \\
w_{k}
\end{array}\right]\right\}
\end{aligned}
$$

donde se ha tenido en cuenta que el producto cruzado

$$
\mathcal{E}\left\{E_{k-1}^{\top} \mathcal{A}\left(s_{k}\right)^{\top} \boldsymbol{P}\left(s_{k}\right) \mathcal{B}\left(s_{k}\right)\left[\begin{array}{c}
\mathbb{V}_{k} \\
w_{k}
\end{array}\right]\right\}=\mathcal{E}\left\{E_{k-1}^{\top} \mathcal{A}\left(s_{k}\right)^{\top} \boldsymbol{P}\left(s_{k}\right) \mathcal{B}\left(s_{k}\right)\right\} \mathcal{E}\left\{\left[\begin{array}{c}
\mathbb{V}_{k} \\
w_{k}
\end{array}\right]\right\}=0
$$

es nulo debido a la independencia de $E_{k-1}$ con $\mathbb{V}_{k}$ y $w_{k}$, y que el valor esperado de las perturbaciones es cero.

Utilizando la condición (3.41) en la LMI (3.58a) y aplicando los complementos de Schur se tiene que

$$
\mathcal{A}\left(s_{k}\right)^{\top} \boldsymbol{P}\left(s_{k}\right) \mathcal{A}\left(s_{k}\right)-\boldsymbol{P}\left(s_{k-1}\right)+\boldsymbol{c}^{\top} \boldsymbol{c} .
$$

Premultiplicando y postmultiplicando esta expresión por $E_{k-1}^{\top}$ y $E_{k-1}$ se tiene que

$$
E_{k-1} \mathcal{A}\left(s_{k}\right)^{\top} \boldsymbol{P}\left(s_{k}\right) \mathcal{A}\left(s_{k}\right) E_{k-1}-E_{k-1}^{\top} \boldsymbol{P}\left(s_{k}\right) E_{k-1}+e_{k-1}^{\top} e_{k-1}<0 .
$$

Si se calcula la esperanza matemática de esta desigualdad y se aplica el resultado (3.60) se tiene que

$$
\mathcal{E}\left\{\mathcal{V}_{k}\right\}-\mathcal{E}\left\{\mathcal{V}_{k-1}\right\}+e_{k-1}^{\top} e_{k-1}<\mathcal{E}\left\{\left[\begin{array}{c}
\mathbb{V}_{k} \\
w_{k}
\end{array}\right]^{\top} \mathcal{B}\left(s_{k}\right)^{\top} \boldsymbol{P}\left(s_{k}\right) \mathcal{B}\left(s_{k}\right)\left[\begin{array}{c}
\mathbb{V}_{k} \\
w_{k}
\end{array}\right]\right\}
$$

Utilizando la condición (3.41) en la LMI (3.58b) y aplicando los complementos de Schur se tiene que

$$
\mathcal{B}\left(s_{k}\right)^{\top} \boldsymbol{P}\left(s_{k}\right) \mathcal{B}\left(s_{k}\right)-\boldsymbol{\Gamma}^{2} \prec \mathbf{0} .
$$

Introduciendo este resultado en (3.61) se llega a

$$
\mathcal{E}\left\{\mathcal{V}_{k}\right\}-\mathcal{E}\left\{\mathcal{V}_{k-1}\right\}+e_{k-1}^{\top} e_{k-1}<\mathcal{E}\left\{\left[\begin{array}{c}
\mathbb{V}_{k} \\
w_{k}
\end{array}\right]^{\top} \Gamma^{2}\left[\begin{array}{c}
\mathbb{V}_{k} \\
w_{k}
\end{array}\right]\right\}
$$

y teniendo en cuenta el teorema A.3.3

$$
\mathcal{E}\left\{\left[\begin{array}{c}
\mathbb{V}_{k} \\
w_{k}
\end{array}\right]^{\top} \boldsymbol{\Gamma}^{2}\left[\begin{array}{c}
\mathbb{V}_{k} \\
w_{k}
\end{array}\right]\right\}=\operatorname{tr}\left(\boldsymbol{\Gamma}^{2} \mathcal{E}\left\{\left[\begin{array}{c}
\mathbb{V}_{k} \\
w_{k}
\end{array}\right]^{\top}\left[\begin{array}{c}
\mathbb{V}_{k} \\
w_{k}
\end{array}\right]\right\}\right)=\frac{\gamma_{v}^{2}}{3 n} 3 n \sigma_{v}^{2}+\gamma_{w}^{2} \sigma_{w}^{2}
$$

se tiene que

$$
\mathcal{E}\left\{\mathcal{V}_{k}\right\}-\mathcal{E}\left\{\mathcal{V}_{k-1}\right\}+e_{k-1}^{\top} e_{k-1}<\gamma_{v}^{2} \sigma_{v}^{2}+\gamma_{w}^{2} \sigma_{w}^{2}
$$

Sumando esta expresión de $k=1$ a $N$ y asumiendo condiciones iniciales nulas se obtiene

$$
\mathcal{E}\left\{\mathcal{V}_{N}\right\}+\sum_{k=1}^{N} e_{k-1}^{\top} e_{k-1}<N\left(\gamma_{v}^{2} \sigma_{v}^{2}+\gamma_{w}^{2} \sigma_{w}^{2}\right)
$$

Como $\boldsymbol{P}\left(s_{N}\right) \succ \mathbf{0}$, entonces $\mathcal{E}\left\{\mathcal{V}_{N}\right\}>0$. Tomando el límite cuando $N$ tiende a infinito se llega finalmente a

$$
\lim _{N \rightarrow \infty} \frac{1}{N} \sum_{k=1}^{N} e_{k-1}^{\top} e_{k-1}=\left\|e_{k}\right\|_{R M S}^{2}<\gamma_{v}^{2} \sigma_{v}^{2}+\gamma_{w}^{2} \sigma_{w}^{2} .
$$


Observación 3.4.10. Si se consideran conocidas las varianza de las perturbación y del ruido de medida, se puede minimizar la cota superior de $\left\|e_{k}\right\|_{R M S}$ minimizando la suma

$$
\gamma_{v}^{2} \sigma_{v}^{2}+\gamma_{w}^{2} \sigma_{w}^{2}
$$

a lo largo de todas las variables $\gamma_{v}, \gamma_{w}, \boldsymbol{P}\left(s_{k}\right), \boldsymbol{Q}\left(s_{k}\right), \boldsymbol{X}\left(s_{k}\right)$ que satisfacen las LMI (3.58). La minimización se puede realizar como se ha indicado en la observación 3.4.4, tomando en este caso

$$
\boldsymbol{h}^{\top}=\left[\begin{array}{lllll}
0 & \ldots & 0 & \sigma_{v}^{2} & \sigma_{w}^{2}
\end{array}\right]
$$

Ejemplo 3.4.3. Considérese de nuevo el ejemplo 3.4 .1 con el muestreo definido por

$$
\mathcal{N}=\{3,4,5,6\}, \quad \mathcal{D}=\{0,1,2\}
$$

Si el sistema está afectado por una perturbación y un ruido de medida aleatorios, ambos con media cero y varianza $\sigma_{v}=0.175$ y $\sigma_{w}=0.116$, se puede aplicar la técnica de diseño basada en la atenuación $\mathcal{H}_{2}$. Si se aplica este diseño y se realizan múltiples simulaciones se obtiene un valor eficaz del error de $\|e[t]\|_{R M S}<0.18$, mientras que la cota obtenida con la metodología de la atenuación $\mathcal{H}_{\infty}$ (tomando $\left.\|v[t]\|_{R M S}=\sigma_{v} y\|w[t]\|_{R M S}=\sigma_{w}\right)$ es de $\|e[t]\|_{R M S}<0.20$. Esto indica una reducción de la cota del error con el diseño $\mathcal{H}_{2}$ del $10 \%$ con respecto al diseño $\mathcal{H}_{\infty}$. Esta reducción se ha producido gracias al conocimiento de que las perturbaciones eran de media cero.

Ejemplo 3.4.4. Considérese ahora en el ejemplo anterior que se tiene una perturbación constante de valor $\|v[t]\|_{\infty}=0.4\left(=\|v[t]\|_{R M S}\right)$ y un ruido aleatorio con media cero de valor eficaz $\|w[t]\|_{R M S}=$ $\sigma_{w}=0.115$. Con estos valores se aplica tanto la técnica de atenuación $\mathcal{H}_{2}$ como la técnica $\mathcal{H}_{\infty}$ obteniéndose en el caso de diseño $\mathcal{H}_{2}$ un error definido por $\|e[t]\|_{R M S}=0.27$ y con el diseño $\mathcal{H}_{\infty}$ un error de $\|e[t]\|_{R M S}=0.22$. Con estos resultados se observa que el diseño $\mathcal{H}_{2}$ devuelve peores resultados debido a la consideración errónea de que la perturbación es un ruido blanco como se puede observar en la simulación de la figura 3.12.

\subsubsection{Diseño de predictores $\mathcal{H}_{2 g}$}

El siguiente teorema recoge el diseño de predictores estables que minimizan la cota del máximo valor absoluto del error frente a perturbaciones y ruidos de energía finita (situación S3 de la página 31).

Teorema 3.4.4. Considérese el predictor (3.8) aplicado al sistema (3.7) y supóngase que hay una medida disponible con un retardo $d_{k} \in \mathcal{D}$ cada $N_{k} \in \mathcal{N}$ periodos, habiendo $n_{\mathcal{S}}$ escenarios de muestreo $s_{k} \in \mathcal{S}$ posibles. Para unas $\gamma_{v}, \gamma_{w}>0$ dadas, supóngase que existen unas matrices $\boldsymbol{P}\left(s_{k}\right)=\boldsymbol{P}\left(s_{k}\right)^{\top} \in \mathbb{R}^{n \times n}$, $\boldsymbol{Q}\left(s_{k}\right) \in \mathbb{R}^{n \times n}, \boldsymbol{X}\left(s_{k}\right) \in \mathbb{R}^{n \times 1}$ tales que

$$
\begin{aligned}
& {\left[\begin{array}{ccc}
\boldsymbol{Q}\left(s_{k}\right)+\boldsymbol{Q}\left(s_{k}\right)^{\top}-\boldsymbol{P}\left(s_{k}\right) & \boldsymbol{M}_{A}\left(s_{k}\right) & \boldsymbol{M}_{B}\left(s_{k}\right) \\
\boldsymbol{M}_{A}\left(s_{k}\right)^{\top} & \boldsymbol{P}\left(s_{k-1}\right) & \mathbf{0} \\
\boldsymbol{M}_{B}\left(s_{k}\right)^{\top} & \mathbf{0} & \boldsymbol{\Gamma}^{2}
\end{array}\right]} \\
& \boldsymbol{P}\left(s_{k}\right)-\boldsymbol{c}^{\top} \boldsymbol{c} \succ 0,
\end{aligned}
$$

para cualquier secuencia de muestreo $\left\{s_{k}\right\}$, estando $\boldsymbol{M}_{A}\left(s_{k}\right)$ y $\boldsymbol{M}_{B}\left(s_{k}\right)$ definidas por (3.45). Entonces, definiendo la ganancia del predictor en función del valor del parámetro de muestreo como $\ell\left(s_{k}\right)=$ $\boldsymbol{Q}\left(s_{k}\right)^{-1} \boldsymbol{X}\left(s_{k}\right)$, el error de predicción del algoritmo definido por (3.8) converge asintóticamente a cero en ausencia de ruido de medida y, bajo condiciones iniciales nulas,

$$
\left\|e_{k}\right\|_{\infty}^{2} \leq \gamma_{v}^{2}\left\|v_{k}\right\|_{2}^{2}+\gamma_{w}^{2}\left\|w_{k}\right\|_{2}^{2}
$$

Prueba 3.4.4. La estabilidad asintótica del predictor queda asegurada ya que si la PLMI (3.64a) se cumple también lo hará la PLMI (3.38). Utilizando la condición (3.41) en la LMI (3.64a) y aplicando los complementos de Schur, se tiene que

$$
E_{k}^{\top} \boldsymbol{P}\left(s_{k}\right) E_{k}-E_{k-1}^{\top} \boldsymbol{P}\left(s_{k-1}\right) E_{k-1}<\frac{\gamma_{v}^{2}}{3 n} \mathbb{V}_{k}^{\top} \mathbb{V}_{k}^{\top}+\gamma_{w}^{2} w_{k}^{\top} w_{k}^{\top}
$$




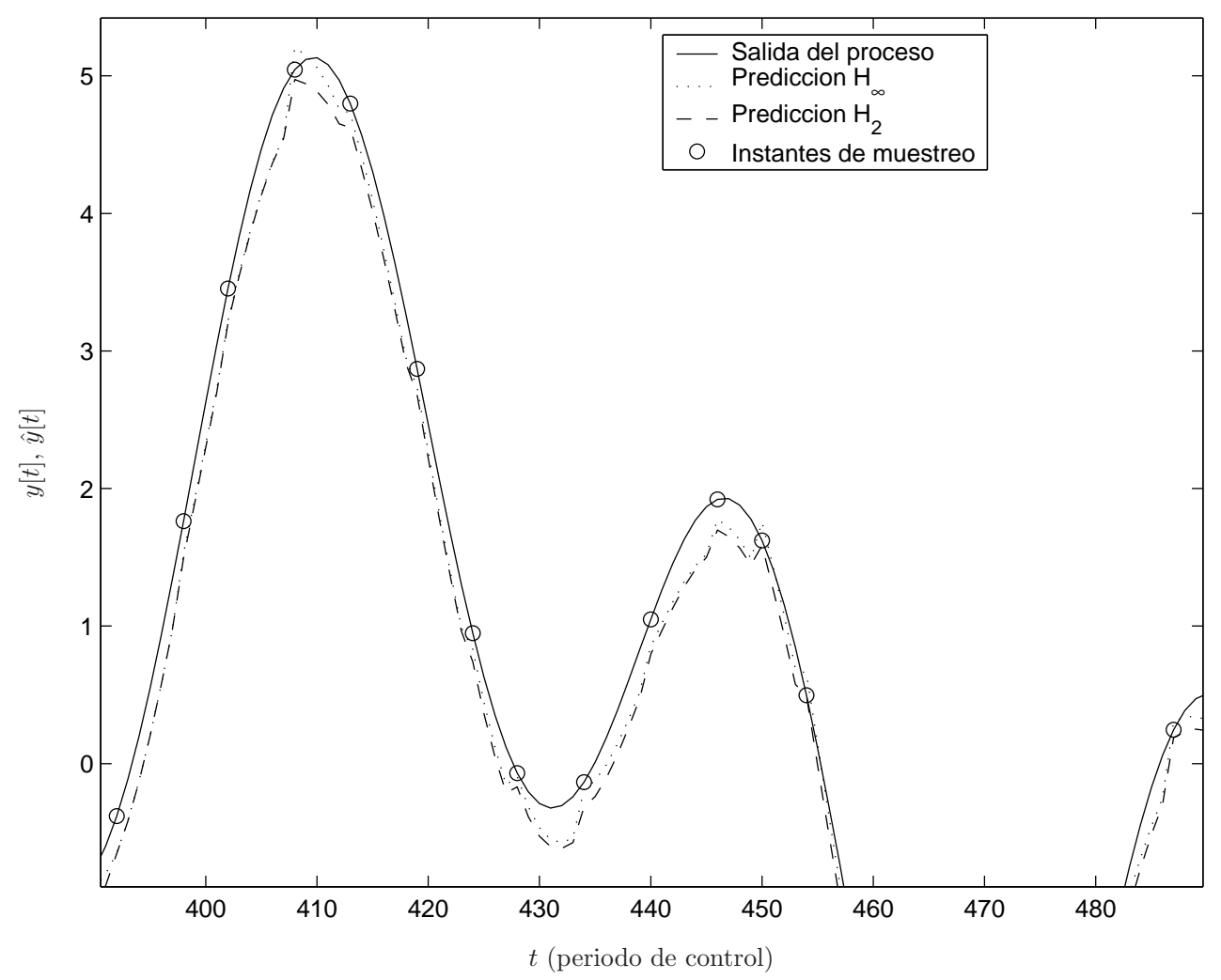

Figura 3.12: Evolución de la salida y de las predicciones con diferentes métodos. $\mathcal{N}=\{3, \ldots, 6\}, \mathcal{D}=\{0,1,2\}$. Perturbación escalón y ruido de medida aleatorio

Considerando condiciones nulas y tomando sumatorio en ambos lados de la desigualdad de 1 a $K$ se tiene que

$$
E_{K}^{\top} \boldsymbol{P}\left(s_{K}\right) E_{K}<\sum_{k=1}^{K}\left(\frac{\gamma_{v}^{2}}{3 n} \mathbb{V}_{k}^{\top} \mathbb{V}_{k}^{\top}+\gamma_{w}^{2} w_{k}^{\top} w_{k}\right) .
$$

Si se multiplica la PLMI (3.64b) por la izquierda por $E_{k}^{\top}$ y se multiplica por la derecha por $E_{k}$ se tiene que

$$
E_{K}^{\top} \boldsymbol{P}\left(s_{K}\right) E_{K}>E_{K}^{\top} \boldsymbol{c}^{\top} \boldsymbol{c} E_{K}=e_{K}^{\top} e_{K}
$$

Con esto, la desigualdad (3.66) lleva a

$$
e_{K}^{\top} e_{K}<\sum_{k=1}^{K}\left(\frac{\gamma_{v}^{2}}{3 n} \mathbb{V}_{k}^{\top} \mathbb{V}_{k}+\gamma_{w}^{2} w_{k}^{\top} w_{k}\right)
$$

Tomando $K$ como el instante en el que el error se hace máximo, y teniendo en cuenta que

$$
\sum_{k=1}^{K}\left(\frac{\gamma_{v}^{2}}{3 n} \mathbb{V}_{k}^{\top} \mathbb{V}_{k}+\gamma_{w}^{2} w_{k}^{\top} w_{k}\right)<\lim _{K \rightarrow \infty} \sum_{k=1}^{K}\left(\frac{\gamma_{v}^{2}}{3 n} \mathbb{V}_{k}^{\top} \mathbb{V}_{k}+\gamma_{w}^{2} w_{k}^{\top} w_{k}\right)
$$

para cualquier $K$, se llega a la cota expresada en (3.65).

Observación 3.4.11. Conocida la norma $\ell_{2}$ de las perturbaciones, se puede diseñar el predictor que minimiza la norma $\left\|e_{k}\right\|_{\infty}$ haciendo una minimización de la suma

$$
\gamma_{v}^{2}\left\|v_{k}\right\|_{2}^{2}+\gamma_{w}^{2}\left\|w_{k}\right\|_{2}^{2}
$$

lo largo de todas las variables $\gamma_{v}, \gamma_{w}, \boldsymbol{P}\left(s_{k}\right)$, las variables $\gamma_{v}, \gamma_{w}, \boldsymbol{P}\left(s_{k}\right), \boldsymbol{Q}\left(s_{k}\right)$, que cumplen la LMI (3.64). La minimización se puede realizar como se ha indicado en la observación 3.4.4, tomando en este caso

$$
\boldsymbol{h}^{\top}=\left[\begin{array}{llllll}
0 & \ldots & 0 & \left\|v_{k}\right\|_{2}^{2} & \left\|w_{k}\right\|_{2}^{2}
\end{array}\right] .
$$




\subsubsection{Diseño de predictores $\ell_{1}$}

Si se conocen únicamente las cotas $\ell_{\infty}$ de la perturbación y el ruido de medida y se desea acotar el error de predicción en amplitud (norma $\ell_{\infty}$ ) hay que plantear el problema de minimización con unas restricciones diferentes a las planteadas en los apartados anteriores. El siguiente teorema muestra el diseño del predictor que minimiza la amplitud del error de predicción frente a perturbaciones de cota de amplitud conocida (situación S2 de la página 31$)^{6}$.

Teorema 3.4.5. Considérese el predictor (3.8) aplicado al sistema (3.7) y supóngase que hay una medida disponible con un retardo $d_{k} \in \mathcal{D}$ cada $N_{k} \in \mathcal{N}$, habiendo $n_{\mathcal{S}}$ escenarios de muestreo $s_{k} \in \mathcal{S}$ posibles. Supóngase que para unos $\gamma_{v}, \gamma_{w}$ dados, existen unas matrices $\boldsymbol{P}\left(s_{k}\right)=\boldsymbol{P}\left(s_{k}\right)^{\top} \in \mathbb{R}^{n \times n}, \boldsymbol{Q}\left(s_{k}\right) \in \mathbb{R}^{n \times n}$, $\boldsymbol{X}\left(s_{k}\right) \in \mathbb{R}^{n \times 1}$ y unos escalares $\lambda, \boldsymbol{\varepsilon}_{v}, \boldsymbol{\varepsilon}_{w} \in \mathbb{R}^{+}$tales que

$$
\begin{aligned}
& \lambda \in] 0,1[ \\
& {\left[\begin{array}{ccc}
\boldsymbol{Q}\left(s_{k}\right)+\boldsymbol{Q}\left(s_{k}\right)^{\top}-\boldsymbol{P}\left(s_{k}\right) & \boldsymbol{M}_{A}\left(s_{k}\right) & \boldsymbol{M}_{B}\left(s_{k}\right) \\
\boldsymbol{M}_{A}\left(s_{k}\right)^{\top} & (1-\lambda) \boldsymbol{P}\left(s_{k-1}\right) & \mathbf{0} \\
\boldsymbol{M}_{B}\left(s_{k}\right)^{\top} & \mathbf{0} & \boldsymbol{\Upsilon}^{2}
\end{array}\right] \succ 0,} \\
& {\left[\begin{array}{cc}
\lambda \boldsymbol{P}\left(s_{k}\right)-\boldsymbol{c}^{\top} \boldsymbol{c} & \mathbf{0} \\
\mathbf{0} & \boldsymbol{\Gamma}^{2}-\boldsymbol{\Upsilon}^{2}
\end{array}\right] \succ 0,}
\end{aligned}
$$

para cualquier secuencia de muestreo $\left\{s_{k}\right\}$, con

$$
\Upsilon=\left[\begin{array}{cccc}
\frac{\varepsilon_{v}^{2}}{3 n} & & & \\
& \ddots & & \\
& & \frac{\varepsilon_{v}^{2}}{3 n} & \\
& & & \varepsilon_{w}^{2}
\end{array}\right]_{(3 n+1)}
$$

y $\boldsymbol{M}_{A}\left(s_{k}\right)$ y $\boldsymbol{M}_{B}\left(s_{k}\right)$ dadas por (3.45). Entonces, definiendo la ganancia del predictor como $\boldsymbol{\ell}\left(s_{k}\right)=$ $\boldsymbol{Q}\left(s_{k}\right)^{-1} \boldsymbol{X}\left(s_{k}\right)$, el error de predicción del algoritmo definido por (3.8) converge asintótica a cero con una tasa de decrecimiento mínima de $1-\lambda$ en ausencia de perturbaciones $y$, bajo condiciones iniciales nulas,

$$
\left\|e_{k}\right\|_{\infty}^{2} \leq \gamma_{v}^{2}\left\|v_{k}\right\|_{\infty}^{2}+\gamma_{w}^{2}\left\|w_{k}\right\|_{\infty}^{2}
$$

Prueba 3.4.5. La prueba de este teorema es similar a la del teorema 3.4.2. La estabilidad asintótica del predictor queda asegurada ya que si las PLMIs (3.68a) y (3.68b) se cumplen también lo hará la PLMI (3.38), con $\mu=1-\lambda$.

Utilizando la condición (3.41) en la LMI (3.68b) y aplicando los complementos de Schur, se tiene que

$$
E_{k}^{\top} \boldsymbol{P}\left(s_{k}\right) E_{k}-E_{k-1} \boldsymbol{P}\left(s_{k-1}\right) E_{k-1}+\lambda E_{k-1} \boldsymbol{P}\left(s_{k-1}\right) E_{k-1}-\frac{\varepsilon_{v}^{2}}{3 n} \mathbb{V}_{k}^{\top} \mathbb{V}_{k}-\varepsilon_{w}^{2} w_{k}^{\top} w_{k}<0
$$

Definiendo las nuevas variables

$$
\mathcal{V}_{k}=E_{k}^{\top} \boldsymbol{P}\left(s_{k}\right) E_{k}
$$

y

$$
\omega_{k}=\frac{\varepsilon_{v}^{2}}{3 n} \mathbb{V}_{k}^{\top} \mathbb{V}_{k}+\varepsilon_{w}^{2} w_{k}^{\top} w_{k},
$$

se puede ver que la expresión (3.70) equivale a una inecuación en diferencias de la forma

$$
\mathcal{V}_{k}-(1-\lambda) \mathcal{V}_{k-1}<\omega_{k},
$$

correspondiente a un sistema de primer orden de entrada $\omega_{k}$ y salida $\mathcal{V}_{k}$. Tomando la entrada de mayor amplitud posible definida como

$$
\left\|\omega_{k}\right\|_{\infty}=\varepsilon_{v}^{2}\left\|v_{k}\right\|_{\infty}^{2}+\varepsilon_{w}^{2}\left\|w_{k}\right\|_{\infty}^{2}
$$

\footnotetext{
${ }^{6}$ La formalización LMI de esta norma sólo se ha encontrado para el caso continuo en [9], y para el caso discreto en [10], aunque esta formulación no permite tratar la LMI con las herramientas estándar. La formulación que aquí se presenta sí que permite tal tratamiento.
} 
se puede obtener el máximo valor $\mathcal{V}_{k}$ como el instante en el que $\mathcal{V}_{k}=\mathcal{V}_{k-1}$ con lo que se llega a

$$
\lambda \mathcal{V}_{k}<\omega_{k} \leq\left\|\omega_{k}\right\|_{\infty}
$$

Deshaciendo los cambios de variable se tiene que

$$
E_{k}^{\top} \boldsymbol{P}\left(s_{k}\right) E_{k}<\frac{1}{\lambda}\left(\varepsilon_{v}^{2}\left\|v_{k}\right\|_{\infty}^{2}+\varepsilon_{w}^{2}\left\|w_{k}\right\|_{\infty}^{2}\right) .
$$

Multiplicando la expresión (3.68c) por $\left[E_{k}^{\top} \mathbb{V}_{k}^{\top} w_{k}^{\top}\right]$ por la izquierda y por su traspuesta por la derecha se tiene que

$$
e_{k}^{\top} e_{k}<\lambda E_{k}^{\top} \boldsymbol{P}\left(s_{k}\right) E_{k}+\frac{\gamma_{v}^{2}-\varepsilon_{v}^{2}}{3 n} \mathbb{V}_{k}^{\top} \mathbb{V}_{k}+\left(\gamma_{w}^{2}-\varepsilon_{w}^{2}\right) w_{k}^{\top} w_{k}
$$

Teniendo en cuenta (3.72) se llega a

$$
e_{k}^{\top} e_{k}<\varepsilon_{v}^{2}\left\|v_{k}\right\|_{\infty}^{2}+\varepsilon_{w}^{2}\left\|w_{k}\right\|_{\infty}^{2}+\frac{\gamma_{v}^{2}-\varepsilon_{v}^{2}}{3 n} \mathbb{V}_{k}^{\top} \mathbb{V}_{k}+\left(\gamma_{w}^{2}-\varepsilon_{w}^{2}\right) w_{k}^{\top} w_{k}
$$

con lo que, teniendo en cuenta que

se llega a

$$
\begin{gathered}
\mathbb{V}_{k}^{\top} \mathbb{V}_{k}<3 n\left\|v_{k}\right\|_{\infty}^{2} \\
w_{k}^{\top} w_{k}<\left\|w_{k}\right\|_{\infty}^{2}
\end{gathered}
$$

$$
e_{k}^{\top} e_{k}<\gamma_{v}^{2}\left\|v_{k}\right\|_{\infty}^{2}+\gamma_{w}^{2}\left\|w_{k}\right\|_{\infty}^{2}
$$

Observación 3.4.12. Conocida la norma $\ell_{\infty}$ de las perturbaciones, se puede minimizar la norma $\left\|e_{k}\right\|_{\infty}$ haciendo una minimización de la suma

$$
\gamma_{v}^{2}\left\|v_{k}\right\|_{\infty}^{2}+\gamma_{w}^{2}\left\|w_{k}\right\|_{\infty}^{2}
$$

a lo largo de todas las variables $\gamma_{v}, \gamma_{w}, \lambda, \boldsymbol{P}\left(s_{k}\right), \boldsymbol{Q}\left(s_{k}\right), \boldsymbol{X}\left(s_{k}\right)$ que satisfacen la LMI (3.68). La minimización se puede realizar como se ha indicado en la observación 3.4.4, tomando en este caso

$$
\boldsymbol{h}^{\top}=\left[\begin{array}{lllll}
0 & \ldots & 0 & \left\|v_{k}\right\|_{\infty}^{2}\left\|w_{k}\right\|_{\infty}^{2}
\end{array}\right]
$$

Observación 3.4.13. Nótese que el conjunto de desigualdades anterior (3.68) no forman un problema PLMI (lineal) porque la variable $\lambda$ entra de forma no lineal en el término $\lambda \boldsymbol{P}\left(s_{k}\right)$ que aparece en (3.68b) y (3.68c). Así pues, para obtener la cota superior ínfima de $\left\|e_{k}\right\|_{\infty}$, se ha de minimizar $\left\|e_{k}\right\|_{\infty}$ también a lo largo de $\lambda \in] 0,1[$, lo que implica resolver otro problema de minimización encadenado con el anterior.

\subsection{Diseño estocástico de predictores}

En el diseño de predictores de la sección anterior se asegura, en ausencia de perturbaciones, un decrecimiento monótono del error de predicción. También asegura, bajo condiciones iniciales del error de predicción nulas, cuál es la cota de la norma del error de predicción. Todo ello se asegura para cualquier secuencia posible que pueda tomar el parámetro de muestreo.

Sin embargo, esta secuencia de muestreo puede que siga cierto modelo probabilístico. En este caso, si se conocen las propiedades estadísticas se pueden derivar diseños menos restrictivos que los de la sección anterior, en los que se asegure el decrecimiento del error de predicción en valor medio o la cota esperada de la norma del error de predicción. En este trabajo se asume que la secuencia de muestreos sigue el modelo de una cadena de Markov.

Definición 3.5.1 (Cadena de Markov). Una cadena de Markov es una colección de variables aleatorias $\left\{s_{k}\right\}(k=0,1, \ldots)$ que tienen la propiedad de que, dado el presente, el futuro es condicionalmente independiente del pasado. Expresado de forma matemática,

$$
\mathcal{P}\left\{s_{k}=j \mid s_{0}=i_{0}, s_{1}=i_{1}, \ldots, s_{k-1}=i_{k-1}\right\}=\mathcal{P}\left\{s_{k}=j \mid s_{k-1}=i_{k-1}\right\} .
$$


Definición 3.5.2 (Probabilidad de transición). Se define la probabilidad de transición

$$
\alpha_{i j}=\mathcal{P}\left\{s_{k}=j \mid s_{k-1}=i\right\},
$$

como la probabilidad de que aparezca la muestra $k$ con un escenario definido por $s_{k}=j$ condicionado a un escenario $s_{k-1}=i$ en la última muestra.

Cuando se trabaja con una red de comunicaciones, esta distribución de probabilidades se puede obtener analizando el funcionamiento del microprocesador que se encarga de las transmisiones o analizando el funcionamiento de la red. A continuación se presentan los teoremas que permiten minimizar el error de predicción esperado.

\subsubsection{Diseño de predictores para estabilidad nominal}

El siguiente teorema muestra el diseño de predictores estables que aseguran la convergencia en valor medio de un proceso cuya distribución de probabilidades de transición es conocida.

Teorema 3.5.1. Considérese el sistema (3.7) en ausencia de perturbaciones y ruido de medida ( $v=0$ and $w=0)$, y supóngase que hay una medida disponible con un retardo $d_{k} \in \mathcal{D}$ cada $N_{k} \in \mathcal{N}$ periodos, habiendo $n_{\mathcal{S}}$ escenarios de muestreo $s_{k} \in \mathcal{S}$ con probabilidad de transición $\alpha_{i j}$ conocida. Para un $\mu \in$ $(0,1]$ dado, supóngase que existen unas matrices $\boldsymbol{P}(i)=\boldsymbol{P}(i)^{\top} \in \mathbb{R}^{n \times n}, \boldsymbol{Q}(i) \in \mathbb{R}^{n \times n}, \boldsymbol{X}(i) \in \mathbb{R}^{n \times 1}$, $\left(i=1, \ldots, n_{\mathcal{S}}\right)$ tales que la siguiente $L M I$

$$
\left[\begin{array}{c:c} 
& \left(\boldsymbol{Q}(1)-\boldsymbol{X}(1) \boldsymbol{c} \boldsymbol{A}^{-d(1)}\right) \boldsymbol{A}^{N(1)} \\
\boldsymbol{\Omega}(i) & \vdots \\
\hdashline \star & \left(\boldsymbol{Q}\left(n_{\mathcal{S}}\right)-\boldsymbol{X}\left(n_{\mathcal{S}}\right) \boldsymbol{c} \boldsymbol{A}^{-d\left(n_{\mathcal{S}}\right)}\right) \boldsymbol{A}^{N\left(n_{\mathcal{S}}\right)} \\
\hdashline \mu^{2} \overline{\boldsymbol{P}}(\bar{i}) &
\end{array}\right] \succ 0, i=1, \ldots, n_{\mathcal{S}}
$$

con

$$
\boldsymbol{\Omega}(i)=\left[\begin{array}{ccc}
\boldsymbol{Q}(1)+\boldsymbol{Q}(1)^{\top}-\alpha_{i 1} \boldsymbol{P}(1) & & \mathbf{0} \\
\mathbf{0} & \ddots & \\
& & \boldsymbol{Q}\left(n_{\mathcal{S}}\right)+\boldsymbol{Q}\left(n_{\mathcal{S}}\right)^{\top}-\alpha_{i n_{\mathcal{S}}} \boldsymbol{P}\left(n_{\mathcal{S}}\right)
\end{array}\right]
$$

se cumple. Entonces, si la ganancia del predictor se define en función del valor del parámetro de muestreo como

$$
\boldsymbol{\ell}(i)=\boldsymbol{Q}(i)^{-1} \boldsymbol{X}(i), \quad i=1, \ldots, n_{\mathcal{S}},
$$

el error de predicción del algoritmo definido por (3.8) converge a cero de forma asintótica con probabilidad uno y con un ratio de decadencia medio menor que $\mu$.

Prueba 3.5.1. Para asegurar la estabilidad asintótica con probabilidad uno se hace uso de una función de Lyapunov cuadrática paramétrica y se analiza el valor esperado de su diferencia entre dos muestras consecutivas. Tomando la función de Lyapunov

$$
\mathcal{V}\left(E_{k}, s_{k}\right)=E_{k}^{\top} \boldsymbol{P}\left(s_{k}\right) E_{k}
$$

el valor esperado de la diferencia es

$$
\begin{aligned}
\mathcal{E}\{ & \left.\Delta \mathcal{V} \mid s_{k-1}=i\right\}=\mathcal{E}\left\{\mathcal{V}\left(E_{k}, s_{k}\right)-\mathcal{V}\left(E_{k-1}, s_{k-1}\right) \mid s_{k-1}=i\right\} \\
& =\mathcal{E}\left\{E_{k}^{\top} \boldsymbol{P}\left(s_{k}\right) E_{k}-E_{k-1}^{\top} \boldsymbol{P}\left(s_{k-1}\right) E_{k-1} \mid s_{k-1}=i\right\} \\
& =\mathcal{E}\left\{E_{k-1}^{\top} \mathcal{A}\left(s_{k}\right)^{\top} \boldsymbol{P}\left(s_{k}\right) \mathcal{A}\left(s_{k}\right) E_{k-1} \mid s_{k-1}=i\right\}-E_{k-1}^{\top} \boldsymbol{P}(i) E_{k-1}
\end{aligned}
$$

Utilizando las probabilidades de transmisión $\left(\alpha_{i j}\right)$ para calcular la esperanza se tiene que

$$
\begin{gathered}
\mathcal{E}\left\{\Delta \mathcal{V} \mid s_{k-1}=i\right\}=\sum_{j=1}^{n_{\mathcal{S}}} \alpha_{i j}\left(E_{k-1}^{\top} \mathcal{A}(j)^{\top} \boldsymbol{P}(j) \mathcal{A}(j) E_{k-1}\right)-E_{k-1}^{\top} \boldsymbol{P}(i) E_{k-1} \\
=E_{k-1}^{\top}\left(\sum_{j=1}^{n_{\mathcal{S}}} \alpha_{i j}\left(\mathcal{A}(j)^{\top} \boldsymbol{P}(j) \mathcal{A}(j)\right)-\boldsymbol{P}(i)\right) E_{k-1}
\end{gathered}
$$


De la última igualdad es obvio que para asegurar la convergencia asintótica en valor medio es necesario que

$$
\sum_{j=1}^{n_{\mathcal{S}}} \alpha_{i j}\left(\boldsymbol{\mathcal { A }}(j)^{\top} \boldsymbol{P}(j) \mathcal{A}(j)\right)-\boldsymbol{P}(i) \prec \mathbf{0} .
$$

Por otra parte, si se introduce la ganancia (3.76) en la LMI (3.74), la desigualdad puede escribirse como

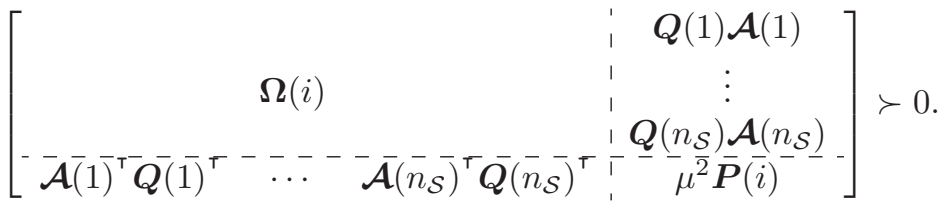

La condición (3.41) se puede aplicar a cada elemento de la matriz $\boldsymbol{\Omega}(i)$ llegándose a

$$
\boldsymbol{Q}(j)+\boldsymbol{Q}(j)^{\top}-\alpha_{i j} \boldsymbol{P}(j) \preceq \alpha_{i j}^{-1} \boldsymbol{Q}(j)^{\top} \boldsymbol{P}(j)^{-1} \boldsymbol{Q}(j),
$$

con lo que

$$
\boldsymbol{\Omega}(i) \preceq\left[\begin{array}{ccc}
\alpha_{i 1}^{-1} \boldsymbol{Q}(1)^{\top} \boldsymbol{P}(1)^{-1} \boldsymbol{Q}(1) & & \mathbf{0} \\
\mathbf{0} & \ddots & \\
& & \alpha_{i n_{\mathcal{S}}}^{-1} \boldsymbol{Q}\left(n_{\mathcal{S}}\right)^{\top} \boldsymbol{P}\left(n_{\mathcal{S}}\right)^{-1} \boldsymbol{Q}\left(n_{\mathcal{S}}\right)
\end{array}\right]
$$

Aplicando esta propiedad a (3.78) y utilizando los complementos de Schur se obtiene la condición

$$
\sum_{j=1}^{n_{\mathcal{S}}} \alpha_{i j}\left(\boldsymbol{\mathcal { A }}(j)^{\top} \boldsymbol{P}(j) \mathcal{A}(j)\right)-\mu^{2} \boldsymbol{P}(i) \prec \mathbf{0} .
$$

Si esta condición se cumple, también se cumple (3.77), ya que $\boldsymbol{P}(i) \succ \mathbf{0}$ y $\mu^{2}<1$, con lo que queda demostrada la convergencia asintótica en valor medio con un ratio menor que $\mu$.

Esta solución a la estabilidad del predictor es menos restrictiva que la obtenida en el teorema 3.4.1. Sin embargo, la convergencia en valor medio puede no impedir la posibilidad de transitorios cortos no deseables para algunos patrones de muestreo (los de menor probabilidad).

\subsubsection{Diseño de predictores para atenuar perturbaciones}

Si el sistema presenta perturbaciones y ruido de medida, se deben tomar en consideración las estrategias de atenuación de perturbaciones introducidas en $\$ 3.2 .3$, llevando a los diseños que se muestran en los apartados siguientes.

\section{Atenuación $\mathcal{H}_{\infty}$}

El siguiente teorema recoge el diseño de predictores estables que minimizan la cota del error en las situaciones S1, S2 y S3 descritas en la página 31.

Teorema 3.5.2 (Atenuación $\mathcal{H}_{\infty}$ en valor medio). Considérese el predictor (3.8) aplicado al sistema (3.7) y supóngase que hay una medida disponible con un retardo $d_{k} \in \mathcal{D}$ cada $N_{k} \in \mathcal{N}$ periodos, habiendo $n_{\mathcal{S}}$ escenarios de muestreo $s_{k} \in \mathcal{S}$ con probabilidad de transición $\alpha_{i j}$ conocida. Para unos $\gamma_{v}, \gamma_{w} \in \mathbb{R}^{+}$dados, supóngase que existen unas matrices $\boldsymbol{P}(i)=\boldsymbol{P}(i)^{\top} \in \mathbb{R}^{n \times n}, \boldsymbol{Q}(i) \in \mathbb{R}^{n \times n}, \boldsymbol{X}(i) \in \mathbb{R}^{n \times 1}\left(i=1, \ldots, n_{\mathcal{S}}\right)$ tales que

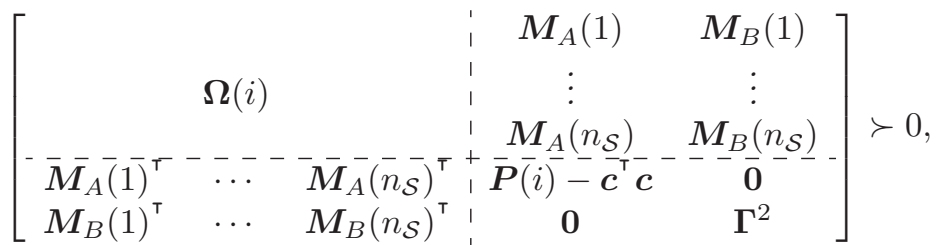

$\left(i=1, \ldots, n_{\mathcal{S}}\right)$. Entonces, definiendo la ganancia del predictor en función de la situación de muestreo $i$ como $\boldsymbol{\ell}(i)=\boldsymbol{Q}(i)^{-1} \boldsymbol{X}(i)$, el error de predicción del algoritmo definido por (3.8) converge asintóticamente a cero en valor medio en ausencia de perturbaciones, $y$, bajo condiciones iniciales nulas,

$$
\mathcal{E}\left\{\left\|e_{k}\right\|_{2}^{2}\right\} \leq \gamma_{v}^{2}\left\|v_{k}\right\|_{2}^{2}+\gamma_{w}^{2}\left\|w_{k}\right\|_{2}^{2}
$$


donde la norma $\mathcal{E}\left\{\left\|e_{k}\right\|_{2}^{2}\right\}$ es la norma de la señal de error esperado en cada muestreo dado el error del muestreo anterior, es decir:

$$
\mathcal{E}\left\{\left\|e_{k}\right\|_{2}^{2}\right\}=\sum_{k=1}^{\infty} \mathcal{E}\left\{e_{k}^{2} \mid k-1\right\}
$$

\section{Prueba 3.5.2. Véase $\S$ B.1.1}

$\mathrm{Al}$ igual que ocurre con el diseño determinista $\mathcal{H}_{\infty}$, este último resultado es fácilmente extendible para obtener los diseños que aseguran

$$
\mathcal{E}\left\{\left\|e_{k}\right\|_{R M S}^{2}\right\} \leq \gamma_{v}^{2}\left\|v_{k}\right\|_{R M S}^{2}+\gamma_{w}^{2}\left\|w_{k}\right\|_{R M S}^{2},
$$

y

$$
\mathcal{E}\left\{\left\|e_{k}\right\|_{R M S}^{2}\right\} \leq \gamma_{v}^{2}\left\|v_{k}\right\|_{\infty}^{2}+\gamma_{w}^{2}\left\|w_{k}\right\|_{\infty}^{2}
$$

donde

$$
\mathcal{E}\left\{\left\|e_{k}\right\|_{R M S}^{2}\right\}=\lim _{K \rightarrow \infty} \frac{1}{K} \sum_{k=1}^{K} \mathcal{E}\left\{e_{k}^{2} \mid k-1\right\} .
$$

Observación 3.5.1. Si se consideran conocidas las normas $\ell_{2}$ de las perturbaciones, se puede minimizar la cota superior de $\mathcal{E}\left\{\left\|e_{k}\right\|_{2}\right\}$ minimizando la suma

$$
\gamma_{v}^{2}\left\|v_{k}\right\|_{2}^{2}+\gamma_{w}^{2}\left\|w_{k}\right\|_{2}^{2}
$$

a lo largo de todas las las variables $\gamma_{v}, \gamma_{w}, \boldsymbol{P}(i), \boldsymbol{Q}(i), \boldsymbol{X}(i)$ que satisfacen la LMI (3.80). La minimización se puede realizar como se ha indicado en la observación 3.4 .4 , tomando en este caso

$$
\boldsymbol{h}^{\top}=\left[\begin{array}{llllll}
0 & \ldots & 0 & \left\|v_{k}\right\|_{2}^{2} & \left\|w_{k}\right\|_{2}^{2}
\end{array}\right] .
$$

Observación 3.5.2. Bajo las mismas hipótesis que el teorema 3.5.2, y si se suponen conocidas las normas RMS de las perturbaciones, se puede minimizar $\mathcal{E}\left\{\left\|e_{k}\right\|_{R M S}\right\}$ minimizando la suma

$$
\gamma_{v}^{2}\left\|v_{k}\right\|_{R M S}^{2}+\gamma_{w}^{2}\left\|w_{k}\right\|_{R M S}^{2}
$$

a lo largo de todas las variables $\gamma_{v}, \gamma_{w}, \boldsymbol{P}(i), \boldsymbol{Q}(i), \boldsymbol{X}(i)$ que satisfacen la LMI (3.80). La minimización se puede realizar como se ha indicado en la observación 3.4 .4 , tomando en este caso

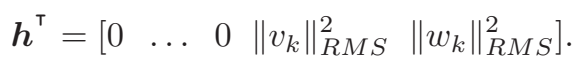

Observación 3.5.3. Utilizando la misma aproximación que en la observación 3.4.8, es posible minimizar la cota superior de $\mathcal{E}\left\{\left\|e_{k}\right\|_{R M S}\right\}$ mediante una minimización de la suma

$$
\gamma_{v}^{2}\left\|v_{k}\right\|_{\infty}^{2}+\gamma_{w}^{2}\left\|w_{k}\right\|_{\infty}^{2}
$$

a lo largo largo de todas las variables $\gamma_{v}, \gamma_{w}, \boldsymbol{P}(i), \boldsymbol{Q}(i), \boldsymbol{X}(i)$ que satisfacen la LMI (3.80). La minimización se puede realizar como se ha indicado en la observación 3.4.4, tomando en este caso

$$
\boldsymbol{h}^{\top}=\left[\begin{array}{lllll}
0 & \ldots & 0 & \left\|v_{k}\right\|_{\infty}^{2}\left\|w_{k}\right\|_{\infty}^{2}
\end{array}\right]
$$

Ejemplo 3.5.1. Retómese el sistema inestable

$$
G(s)=\frac{1}{(s-1)(s+3)}
$$

cuya entrada se actualiza a periodo $T=0.1$ segundos, y cuya salida se muestrea con una frecuencia y un retardo definido por los conjuntos

$$
\mathcal{N}=\{10,11, \ldots, 15\}, \quad \mathcal{D}=\{0,1,2,3\} .
$$




\begin{tabular}{|c|cccccc|}
\hline & $N_{k}=10$ & $N_{k}=11$ & $N_{k}=12$ & $N_{k}=13$ & $N_{k}=14$ & $N_{k}=15$ \\
\hline$d_{k}=0$ & 0.0001 & 0.0004 & 0.0011 & 0.0021 & 0.0035 & 0.0053 \\
$d_{k}=1$ & 0.0076 & 0.0103 & 0.0135 & 0.0172 & 0.0214 & 0.0261 \\
$d_{k}=2$ & 0.0314 & 0.0372 & 0.0436 & 0.0506 & 0.0581 & 0.0663 \\
$d_{k}=3$ & 0.0751 & 0.0845 & 0.0945 & 0.1052 & 0.1165 & 0.1285 \\
\hline
\end{tabular}

Tabla 3.3: Probabilidad de aparición de cada escenario de muestreo

Supóngase que los diferentes muestreos vienen caracterizados por una probabilidad de transición definida por los valores $\alpha_{i}$ (probabilidad de aparición de un escenario de muestro $s_{k}=i$, tras un escenario $s_{k-1}$ cualquiera) dados en la tabla 3.3 Se asume que hay una perturbación constante de valor $\|v[t]\|_{\infty}=0.4$ $y$ un ruido de medida aleatorio de media cero con un valor eficaz $\|w[t]\|_{R M S}=0.1142$. Se diseñan dos predictores $\mathcal{H}_{\infty}$ para este proceso, uno basado en el diseño determinista, y otro basado en el diseño estocástico, ambos mediante una ganancia constante (véase observación 3.7.1 en la página 75). La figura 3.13 muestra una simulación con ambos predictores, donde se observa que el predictor que tiene en cuenta la distribución de probabilidades tiene mejores prestaciones, sobre todo en los muestreos de mayor probabilidad. Sin embargo, el error de predicción obtenido tras una muestra de baja probabilidad (por ejemplo en $t=858$ con $N_{k}=13$ y $d_{k}=1$, y una probabilidad de $\alpha=0.0172$ ), es similar al obtenido con el predictor determinista. El error de predicción también denota esta mejoría, siendo el error obtenido con el predictor determinista de $\|e[t]\|_{R M S}=1.57$ y el error obtenido con el predictor estocástico de $\|e[t]\|_{R M S}=1.22$, con lo que se ha disminuido su valor un 22\%. Para hacer la simulación se ha estabilizado el proceso con muestreo estándar y se ha puesto una referencia variante en el tiempo. La secuencia de acciones de control obtenida en la simulación es la utilizada para las predicciones entre muestreos.

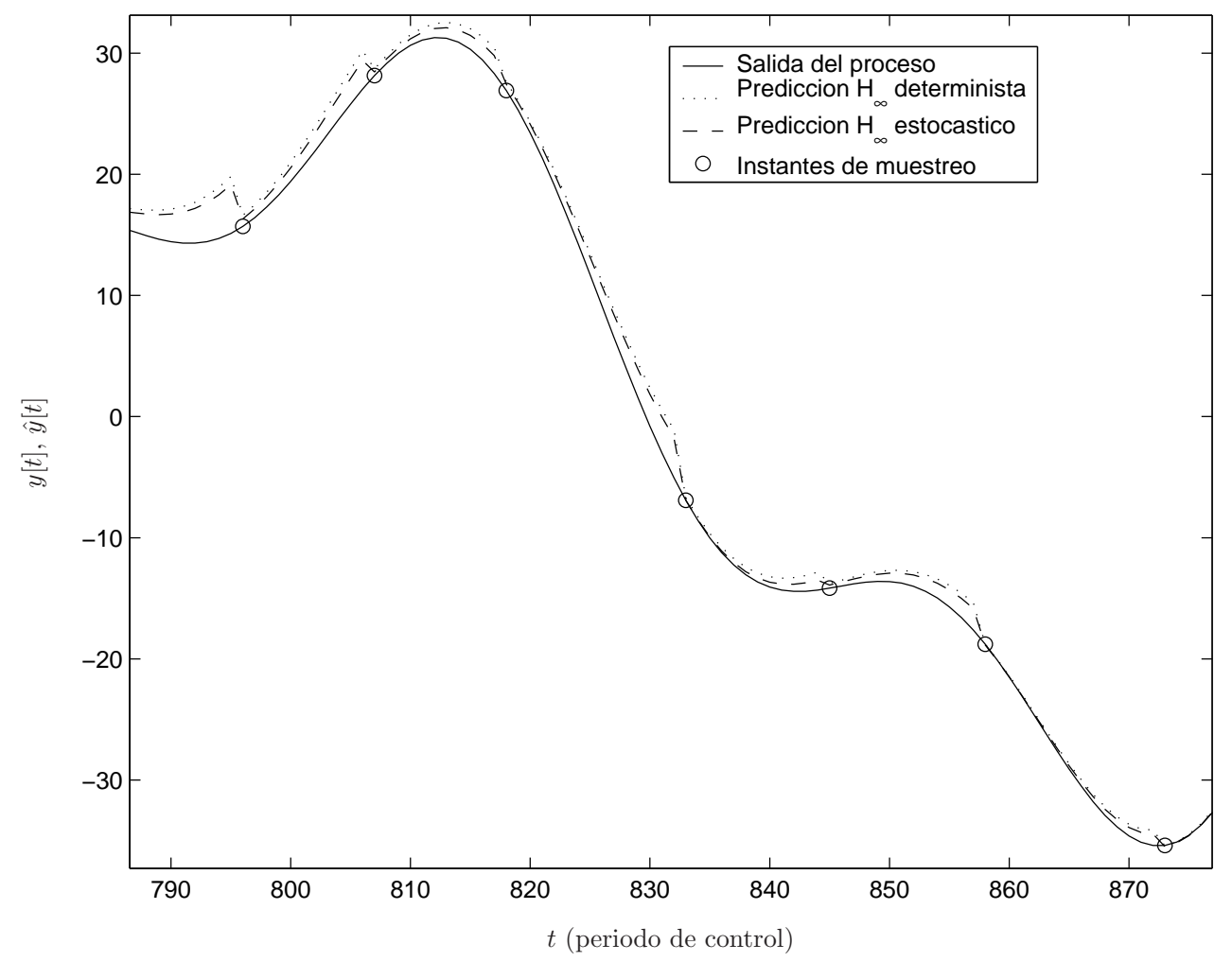

Figura 3.13: Evolución de la salida y de las predicciones con predictores deterministas y estocásticos. $\mathcal{N}=\{10, \ldots, 15\}, \mathcal{D}=\{0,1,2,3\}$. Perturbación constante y ruido blanco de medida

\section{Atenuación $\mathcal{H}_{2}$}

Si las perturbaciones son ruidos blancos de media cero se puede plantear el diseño desde el punto de vista de la norma $\mathcal{H}_{2}$ para acotar el valor eficaz del error de predicción (situación S1 descrita en la página 31 ). 
Teorema 3.5.3. Considérese el predictor (3.8) aplicado al sistema (3.7) y supóngase que hay una medida disponible con un retardo $d_{k} \in \mathcal{D}$ cada $N_{k} \in \mathcal{N}$ periodos, habiendo $n_{\mathcal{S}}$ escenarios de muestreo $s_{k} \in \mathcal{S}$ con probabilidad de transición $\alpha_{i j}$ conocida. Supóngase que la perturbación y el ruido de medida son ambos ruidos blancos de media cero y varianza $\sigma_{v}$ y $\sigma_{w}$, respectivamente. Para unos $\gamma_{v}, \gamma_{w} \in \mathbb{R}^{+}$dados, supóngase que existen unas $\boldsymbol{P}(i)=\boldsymbol{P}(i)^{\top} \in \mathbb{R}^{n \times n}, \boldsymbol{Q}(i) \in \mathbb{R}^{n \times n}, \boldsymbol{X}(i) \in \mathbb{R}^{n \times 1}\left(i=1, \ldots, n_{\mathcal{S}}\right)$ tales que

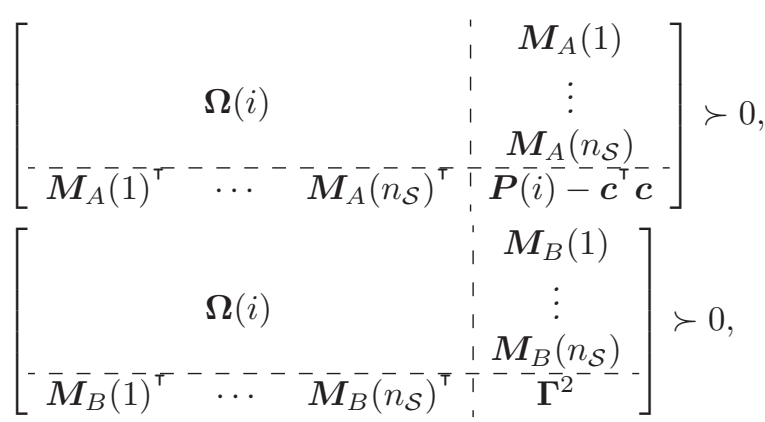

Entonces, definiendo la ganancia del predictor en función del valor del parámetro de muestreo como $\boldsymbol{\ell}(i)=\boldsymbol{Q}(i)^{-1} \boldsymbol{X}(i)$, el error de predicción del algoritmo definido por (3.8) converge asintóticamente a cero en ausencia de ruido de medida y, bajo condiciones iniciales nulas,

$$
\mathcal{E}\left\{\left\|e_{k}\right\|_{R M S}^{2}\right\} \leq \gamma_{v}^{2} \sigma_{v}^{2}+\gamma_{w}^{2} \sigma_{w}^{2} .
$$

Prueba 3.5.3. Véase $\S B .1 .2$

Observación 3.5.4. Si se consideran conocidas las normas $\ell_{2}$ de las perturbaciones, se puede minimizar la cota superior de $\mathcal{E}\left\{\left\|e_{k}\right\|_{\infty}\right\}$ minimizando la suma

$$
\gamma_{v}^{2} \sigma_{v}^{2}+\gamma_{w}^{2} \sigma_{w}^{2}
$$

a lo largo de todas las las variables $\gamma_{v}, \gamma_{w}, \boldsymbol{P}(i), \boldsymbol{Q}(i), \boldsymbol{X}(i)$ que satisfacen la LMI (3.82). La minimización se puede realizar como se ha indicado en la observación 3.4.4, tomando en este caso

$$
\boldsymbol{h}^{\top}=\left[\begin{array}{lllll}
0 & \ldots & 0 & \sigma_{v}^{2} & \sigma_{w}^{2}
\end{array}\right] \text {. }
$$

\section{Atenuación $\mathcal{H}_{2 g}$}

El siguiente teorema recoge el diseño de predictores estables que minimizan la cota de error frente a perturbaciones y ruidos de energía finita (situación S3 de la página 31.

Teorema 3.5.4 (Atenuación $\mathcal{H}_{2 g}$ en valor medio). Considérese el predictor (3.8) aplicado al sistema (3.7) y supóngase que hay una medida disponible con un retardo $d_{k} \in \mathcal{D}$ cada $N_{k} \in \mathcal{N}$, habiendo $n_{\mathcal{S}}$ escenarios de muestreo $s_{k} \in \mathcal{S}$ con probabilidad de transición $\alpha_{i j}$ conocida. Para unos $\gamma_{v}, \gamma_{w} \in \mathbb{R}^{+}$supóngase que

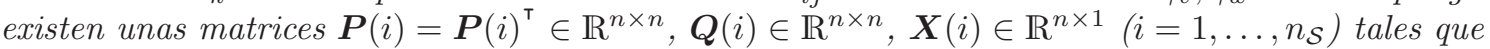

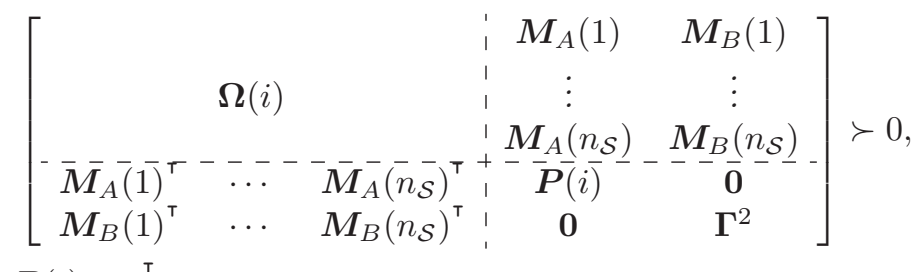

$$
\begin{aligned}
& \boldsymbol{P}(i)-\boldsymbol{c}^{\top} \boldsymbol{c} \succ \mathbf{0}
\end{aligned}
$$

$\left(i=1, \ldots, n_{\mathcal{S}}\right)$. Entonces, definiendo la ganancia del predictor en función de la situación de muestreo $i$ como $\boldsymbol{\ell}(i)=\boldsymbol{Q}(i)^{-1} \boldsymbol{X}(i)$, el error de predicción del algoritmo definido por (3.8) converge a cero en valor medio con probabilidad uno $y$, bajo condiciones iniciales nulas,

$$
\mathcal{E}\left\{\left\|e_{k}\right\|_{\infty}^{2}\right\} \leq \gamma_{v}^{2}\left\|v_{k}\right\|_{2}^{2}+\gamma_{w}^{2}\left\|w_{k}\right\|_{2}^{2}
$$

donde

$$
\mathcal{E}\left\{\left\|e_{k}\right\|_{\infty}^{2}\right\}=\operatorname{máx}_{k} \mathcal{E}\left\{e_{k}^{2} \mid k-1\right\}
$$


Prueba 3.5.4. Véase $\S$ B.1.3

Observación 3.5.5. Si se consideran conocidas las normas $\ell_{2}$ de las perturbaciones, se puede minimizar la cota superior de $\mathcal{E}\left\{\left\|e_{k}\right\|_{\infty}\right\}$ minimizando la suma

$$
\gamma_{v}^{2}\left\|v_{k}\right\|_{2}^{2}+\gamma_{w}^{2}\left\|w_{k}\right\|_{2}^{2}
$$

a lo largo de todas las las variables $\gamma_{v}, \gamma_{w}, \boldsymbol{P}\left(s_{k}\right), \boldsymbol{Q}\left(s_{k}\right)$, que satisfacen la LMI (3.84). La minimización se puede realizar como se ha indicado en la observación 3.4.4, tomando en este caso

$$
\boldsymbol{h}^{\top}=\left[\begin{array}{llllll}
0 & \ldots & 0 & \left\|v_{k}\right\|_{2}^{2} & \left\|w_{k}\right\|_{2}^{2}
\end{array}\right] .
$$

\section{Atenuación $\ell_{1}$}

El siguiente teorema recoge el diseño que permite minimizar el valor esperado del pico del error de predicción frente a perturbaciones con valor de pico conocido (situación S2 de la página 31).

Teorema 3.5.5 (Atenuación $\ell_{1}$ en valor medio). Considérese el predictor (3.8) aplicado al sistema (3.7) y supóngase que hay una medida disponible con un retardo $d_{k} \in \mathcal{D}$ cada $N_{k} \in \mathcal{N}$ periodos, habiendo $n_{\mathcal{S}}$ escenarios de muestreo $s_{k} \in \mathcal{S}$ con probabilidad de transición $\alpha_{i j}$ conocida. Supóngase que existen unas matrices $\boldsymbol{P}(i)=\boldsymbol{P}(i)^{\top} \in \mathbb{R}^{n \times n}, \boldsymbol{Q}(i) \in \mathbb{R}^{n \times n}, \boldsymbol{X}(i) \in \mathbb{R}^{n \times 1}\left(i=1, \ldots, n_{\mathcal{S}}\right)$ tales que

$$
\begin{aligned}
& \lambda \in] 0,1[
\end{aligned}
$$

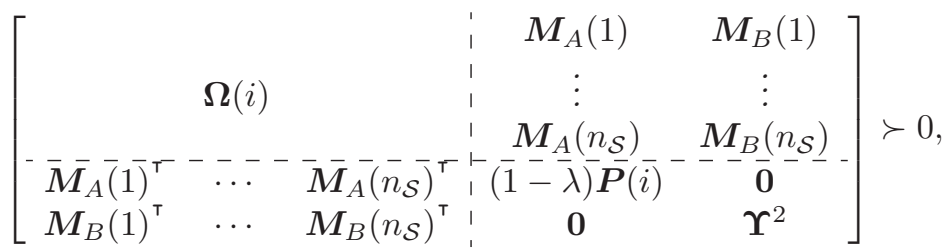

$$
\begin{aligned}
& {\left[\begin{array}{cc}
\lambda \boldsymbol{P}(i)-\boldsymbol{c}^{\boldsymbol{\top}} \boldsymbol{c} & \mathbf{0} \\
\mathbf{0} & \boldsymbol{\Gamma}^{2}-\boldsymbol{\Upsilon}^{2}
\end{array}\right] \succ 0}
\end{aligned}
$$

$\left(i=1, \ldots, n_{\mathcal{S}}\right)$. Entonces, definiendo la ganancia del predictor como $\ell(i)=\boldsymbol{Q}(i)^{-1} \boldsymbol{X}(i)$, el error de predicción del algoritmo definido por (3.8) converge asintóticamente a cero en valor medio y, bajo condiciones iniciales nulas,

$$
\mathcal{E}\left\{\left\|e_{k}\right\|_{\infty}^{2}\right\} \leq \gamma_{v}^{2}\left\|v_{k}\right\|_{\infty}^{2}+\gamma_{w}^{2}\left\|w_{k}\right\|_{\infty}^{2} .
$$

Prueba 3.5.5. Véase $\S B .1 .4$.

Observación 3.5.6. Si se conocen las normas $\ell_{\infty}$ de la perturbación y el ruido de medida, se puede minimizar la cota superior de $\mathcal{E}\left\{\left\|e_{k}\right\|_{\infty}\right\}$ minimizando la suma

$$
\gamma_{v}^{2}\left\|v_{k}\right\|_{\infty}^{2}+\gamma_{w}^{2}\left\|w_{k}\right\|_{\infty}^{2}
$$

a lo largo de todas las las variables $\gamma_{v}, \gamma_{w}, \lambda, \boldsymbol{P}\left(s_{k}\right), \boldsymbol{Q}\left(s_{k}\right)$, que satisfacen la LMI (3.86). La minimización se puede realizar como se ha indicado en la observación 3.4.4, tomando en este caso

$$
\boldsymbol{h}^{\top}=\left[\begin{array}{lllll}
0 & \ldots & 0 & \left\|v_{k}\right\|_{\infty}^{2}\left\|w_{k}\right\|_{\infty}^{2}
\end{array}\right]
$$

Nótese la necesidad de resolver el problema de minimización a lo largo de $\lambda$ encadenado con el anterior (véase observación 3.4.13).

\subsection{Consideraciones de diseño}

En las secciones anteriores se han propuesto diferentes técnicas de diseño cuya idoneidad para cada problema se basa en las características conocidas de las perturbaciones y de la norma que se quiere acotar en el error de predicción. En la tabla 3.4 se muestra una relación entre las diferentes normas de error y perturbación, y el método de diseño a utilizar (indicado mediante el teorema en el cual se expone la 


\begin{tabular}{|c|cccc|}
\hline & $\left\|w_{k}\right\|_{2}$ & $\left\|w_{k}\right\|_{\infty}$ & $\left\|w_{k}\right\|_{R M S}$ & $\sigma_{w}$ \\
\hline$\left\|e_{k}\right\|_{2}$ & $\mathcal{H}_{\infty}(3.4 .2)$ & $\infty$ & $\infty$ & $\infty$ \\
$\left\|e_{k}\right\|_{\infty}$ & $\mathcal{H}_{2 g}(\underline{3.4 .4})$ & $\ell_{1}(3.4 .5)$ & - & - \\
$\left\|e_{k}\right\|_{R M S}$ & 0 & $\mathcal{H}_{\infty}(3.4 .2)$ & $\mathcal{H}_{\infty}(3.4 .2)$ & $\mathcal{H}_{2}(3.4 .3)$ \\
\hline $\mathcal{E}\left\{\left\|e_{k}\right\|_{2}\right\}$ & $\mathcal{H}_{\infty}(3.5 .2)$ & $\infty$ & $\infty$ & $\infty$ \\
$\mathcal{E}\left\{\left\|e_{k}\right\|_{\infty}\right\}$ & $\mathcal{H}_{2 g}(3.5 .4)$ & $\ell_{1}(3.5 .5)$ & - & - \\
$\mathcal{E}\left\{\left\|e_{k}\right\|_{R M S}\right\}$ & 0 & $\mathcal{H}_{\infty}(3.5 .2)$ & $\mathcal{H}_{\infty}(3.5 .2)$ & $\mathcal{H}_{2}(3.5 .3)$ \\
\hline
\end{tabular}

Tabla 3.4: Resumen de especificaciones de diseño

metodología de cálculo). El hecho de diseñar un predictor para acotar el error máximo (teoremas 3.4.4 y 3.4.5) puede tener como contrapartida que el error persista durante un tiempo mayor que si se diseña un predictor para acotar el error cuadrático acumulado (teoremas 3.4.2 y 3.4.3). Dependerá de la aplicación el que sea más conveniente utilizar un tipo de diseño u otro.

El diseño que se ha planteado a lo largo del capítulo se basa en la minimización del error de predicción frente a elementos que pueden tener efecto sobre este error como la perturbación de salida y el ruido de medida. En las aplicaciones reales, sin embargo, aparecen más factores que pueden aumentar el error de predicción. Algunos de estos factores son: el error de modelado, las perturbaciones de entrada, el retardo en el cálculo y transmisión de la acción de control, o el hecho de no conocer con exactitud el instante de tiempo pasado al cual corresponde una medición. Cuando se tiene alguno de estos casos se calculará una perturbación de salida y un ruido de medida equivalentes que tienen el mismo efecto sobre el error de salida. Si hay alguna forma de conocer una norma de esta perturbación y ruido equivalentes, habrá que sumar las normas de las diferentes señales que afectan como una perturbación y las de las que afectan como un ruido de medida para tener la norma total de las señales que afectan al error de predicción. Con estos valores de las normas de la perturbación de salida total y del ruido de medida total, el problema se resuelve aplicando uno de los diseños de las secciones anteriores.

Si las normas que se tienen de la perturbación y del ruido son de diferente naturaleza (norma $\ell_{\infty} \mathrm{y}$ norma $\ell_{2}$, por ejemplo), se tendrá que recurrir a las técnicas de diseño multiobjetivo que se muestran en $§ 3.6 .8$.

\subsubsection{Robustez frente a perturbaciones de entrada}

En los diseños anteriores sólo se ha considerado una planta afectada por una perturbación a la salida y por un ruido de medida. Si el proceso está afectado por una perturbación a la entrada $u_{p}$, la salida vendrá dada por

$$
Y(z)=G(z)\left(U(z)+U_{p}(z)\right)=G(z) U(z)+G(z) U_{p}(z)=G(z) U(z)+V(z),
$$

lo que significa que existe una perturbación de salida $v$ cuyo efecto es equivalente al de la perturbación de entrada. Por lo tanto, el diseño de un predictor robusto a perturbaciones de entrada se puede transformar en el diseño de un predictor robusto a perturbaciones de salida. Para hacer esta transformación es necesario obtener una cota de la norma de la perturbación equivalente $v$ en función de las características del proceso $G(z)$ y de la señal $u_{p}$. La tabla 3.5 permite obtener la norma de la perturbación de salida equivalente en función de las normas del proceso y de la perturbación de entrada. Por ejemplo, si se conoce la norma $\ell_{2}$ de $u_{p}$ es posible tener la cota de la norma $\ell_{2}$ de $v$ mediante $\|v[t]\|_{2} \leq\|G(z)\|_{\infty}\left\|u_{p}[t]\right\|_{2}$. Si la perturbación de entrada es constante de valor $\left\|u_{p}\right\|_{\infty}$, se puede considerar el sistema afectado por una perturbación de salida constante de valor

$$
\|v\|_{\infty}=G(1)\left\|u_{p}\right\|_{\infty}
$$

\begin{tabular}{|c|ccccc|}
\hline & $\left\|u_{p, 0}\right\|$ & $\left\|u_{p}[t]\right\|_{2}$ & $\left\|u_{p}[t]\right\|_{\infty}$ & $\left\|u_{p}[t]\right\|_{R M S}$ & $\sigma_{u}$ \\
\hline$\|v[t]\|_{2}$ & $\|G(z)\|_{2}$ & $\|G(z)\|_{\infty}$ & $\infty$ & $\infty$ & $\infty$ \\
$\|v[t]\|_{\infty}$ & $\|g[t]\|_{\infty}$ & $\|G(z)\|_{2 g}$ & $\|g[t]\|_{1}$ & $\infty$ & $\infty$ \\
$\|v[t]\|_{R M S}$ & 0 & 0 & $\|G(z)\|_{\infty}$ & $\|G(z)\|_{\infty}$ & $\|G(z)\|_{2}$ \\
\hline
\end{tabular}

Tabla 3.5: Obtención de la norma de la perturbación de salida equivalente a la perturbación a la entrada

Nótese que la técnica presentada en este apartado sólo es válida cuando la dinámica del proceso es estable o si éste está controlado en bucle cerrado. Si esto no es así se tiene que recurrir a las técnicas 
de diseño basadas en la representación interna (véase capítulo 4). Sin embargo, se pueden emplear las técnicas de esta sección si se utiliza un valor muy elevado en la norma de la perturbación de la salida para poder obtener un resultado que estabilice la predicción. Esta solución será equivalente al predictor de tiempo mínimo que se obtiene con las técnicas de diseño para estabilidad nominal, con lo que dicha solución no será capaz de minimizar el error de predicción.

Ejemplo 3.6.1. Sea el sistema

$$
G(s)=\frac{10}{s^{2}+20 s+80}
$$

con una actualización de la acción de control a periodo $T=0.05$ segundos y un muestreo variante con retardo definido por los conjuntos

$$
\mathcal{N}=\{1,2,3,4,5\}, \quad \mathcal{D}=\{0,1,2\} .
$$

Supóngase que se tiene un ruido de medida aleatorio con media cero y de valor eficaz $\|w[t]\|_{R M S}=0.98$ y una perturbación a la entrada $u_{p}[t]$ constante de valor $u_{p}[t]=2$. Para diseñar un predictor que atenúe esta perturbación de entrada se obtiene primero el valor eficaz de la perturbación de salida equivalente que tiene el mismo efecto sobre el error de predicción. Ésta se calcula como

$$
\left\|v_{k}\right\|_{R M S} \equiv\|v[t]\|_{R M S} \leq\left. G(z)\right|_{m=1} \cdot\left\|u_{p}[t]\right\|=0.25 .
$$

Con este valor ya se puede aplicar la metodología de diseño $\mathcal{H}_{\infty}$, obteniéndose en este caso un predictor cuyo funcionamiento se muestra en la simulación de la figura 3.14 y se compara con el resultado que se obtiene por aplicación de un predictor en bucle abierto. El error de predicción cometido con el predictor

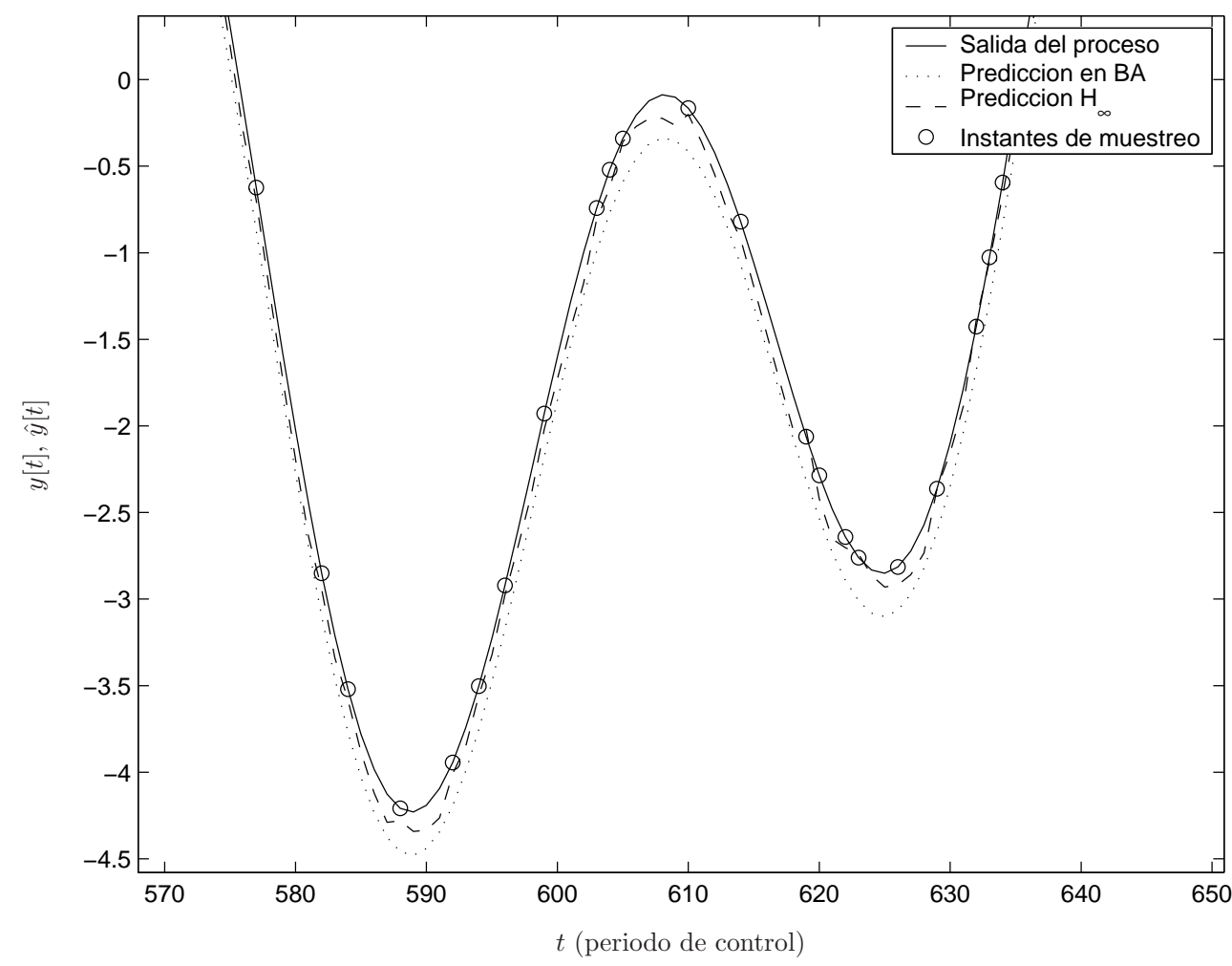

Figura 3.14: Evolución de la salida y de las predicciones con diferentes métodos. $\mathcal{N}=\{1, \ldots, 5\}, \mathcal{D}=\{0,1,2\}$

$\mathcal{H}_{\infty}$ a lo largo de varias simulaciones es de $\|e[t]\|_{R M S}=0.118$, mientras que el obtenido con el predictor simple es de $\|e[t]\|_{R M S}=0.249$.

Ejemplo 3.6.2. Tómese el modelo de un motor de continua en el que la entrada es la tensión en bornes del motor y la salida es el ángulo del eje del motor:

$$
G(s)=\frac{200}{s(s+30)}
$$


Sea un periodo de control de $T=5 \mathrm{~ms}$, y un muestreo escaso y con retardos definido por los conjuntos

$$
\mathcal{N}=\{10,11,12,13,14,15\}, \quad \mathcal{D}=\{0,1,2\} .
$$

Supóngase que hay una perturbación a la entrada en forma de ruido blanco de media cero y desviación típica $\sigma_{v}=\|v[t]\|_{R M S}=2$ y un ruido de medida aleatorio con media cero y una desviación típica de $\sigma_{w}=\|w[t]\|_{R M S}=0.2$. Como el sistema tiene un integrador, la perturbación a la entrada tiene un efecto equivalente al de una perturbación de salida en forma de ruido coloreado (paseo aleatorio). Este tipo de perturbación no tiene una cota finita ni en energía ni en valor eficaz, con lo que no son aplicables las técnicas anteriores. Aún así, es posible obtener una solución por medio de las técnica anteriores tomando un valor muy elevado para la perturbación de salida equivalente. Si se toma una cota de valor $\|v[t]\|_{R M S}=10^{6}$, se puede llevar a cabo el diseño $\mathcal{H}_{\infty}$ que permite utilizar las mediciones escasas para corregir la predicción respecto del bucle abierto. Se comprueba fácilmente que la solución obtenida mediante esta técnica es prácticamente la misma que la obtenida tras realizar un diseño basado únicamente en la estabilidad nominal, obteniéndose un predictor de tiempo mínimo. La figura 3.15 muestra una parte de una simulación de este proceso controlado en bucle cerrado (con una referencia variante en el tiempo) y las diferentes predicciones que se obtienen tanto en bucle abierto como con el diseño $\mathcal{H}_{\infty}$ (predictor de tiempo mínimo). El error de predicción medio con la técnica $\mathcal{H}_{\infty}$ (medido como el valor medio en múltiples simulaciones) tiene un valor eficaz de $\|v[t]\|_{R M S}=0.243$. El error de predicción en bucle abierto se aleja de la salida real conforme avanzan las simulaciones debido a la deriva que introduce integrar el ruido blanco a través del proceso.

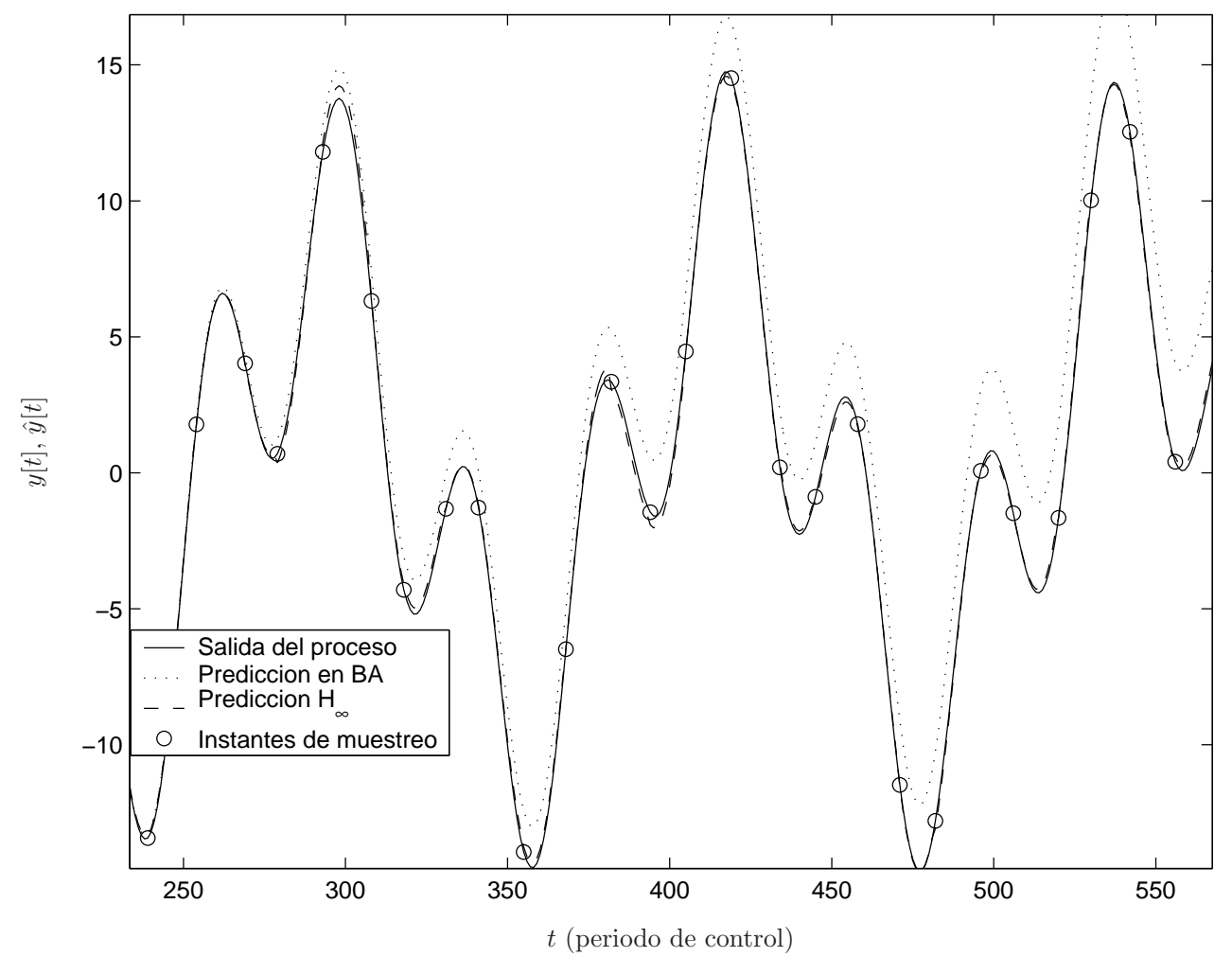

Figura 3.15: Evolución de la salida y de las predicciones con diferentes métodos.

$$
\mathcal{N}=\{10, \ldots, 15\}, \mathcal{D}=\{0,1,2\} .
$$

\subsubsection{Robustez al error de modelado}

En la práctica, además del ruido de medida y las perturbaciones, aparecen otros elementos que provocan error de predicción como es el error de modelado. Si el proceso tiene un error de modelado, de forma que la diferencia entre el modelo teórico y el real es $\Delta G(z)$, la salida del sistema es

$$
Y(z)=(G(z)+\Delta G(z)) U(z)=G(z) U(z)+V(z),
$$


donde se observa que la salida del sistema $\Delta G(z)$ bajo la entrada $u$ es equivalente a una perturbación de salida para el proceso $(v)$. En el capítulo siguiente se demuestra cómo la presencia de incertidumbre en el modelo afecta a la magnitud del error de predicción pero no a la estabilidad de éste (véase §4.7.1). En base a este hecho, se puede afirmar que el diseño robusto de predictores frente a incertidumbre consiste en diseñar un predictor que acote la norma del error de predicción frente a la perturbación de salida que modela la incertidumbre. Por tanto, es necesario obtener una cota de la norma de la perturbación equivalente $v$ en función de las características de la incertidumbre $\Delta G(z)$ y de la señal $u$. Por ejemplo, si se conoce la norma $\mathcal{H}_{\infty}$ de la incertidumbre y la norma $\ell_{2}$ de la acción de control, es posible obtener la norma $\ell_{2}$ de la perturbación de salida equivalente como

$$
\|v[t]\|_{2} \leq\|\Delta G(z)\|_{\infty}\|u[t]\|_{2}
$$

En la tabla 3.6 se establecen todas las relaciones posibles entre normas de la acción de control, de la incertidumbre y de la perturbación de salida equivalente.

\begin{tabular}{|c|cccc|}
\hline & $\|u[t]\|_{2}$ & $\|u[t]\|_{\infty}$ & $\|u[t]\|_{R M S}$ & $\sigma_{u}$ \\
\hline$\|v[t]\|_{2}$ & $\|\Delta G(z)\|_{\infty}$ & $\infty$ & $\infty$ & $\infty$ \\
$\|v[t]\|_{\infty}$ & $\|\Delta G(z)\|_{2 g}$ & $\|\Delta g[t]\|_{1}$ & - & - \\
$\|v[t]\|_{R M S}$ & 0 & $\|\Delta G(z)\|_{\infty}$ & $\|\Delta G(z)\|_{\infty}$ & $\|\Delta G(z)\|_{2}$ \\
\hline
\end{tabular}

Tabla 3.6: Obtención de la norma de la perturbación de salida equivalente para sistemas con incertidumbre

Ejemplo 3.6.3. Considérese el sistema continuo

$$
G(s)=\frac{10(1-0.1 s)}{s^{2}+4 s+2}
$$

donde se asume que la entrada se actualiza a un periodo contante de $T=0.5$ segundos. Supóngase que la salida se mide de forma esporádica y con retardos definiéndose los posibles escenarios de muestreo mediante los conjuntos

$$
\mathcal{N}=\{10,11,12,13,14,15\}, \quad \mathcal{D}=\{0,1,2\} .
$$

Se asume que el sistema está afectado por un ruido aleatorio cuyo valor eficaz es $\left\|w_{k}\right\|_{R M S}=3$. Se asume que se conoce una cota de la incertidumbre del modelo dada por

$$
\|\Delta G(z)\|_{\infty}=0.3
$$

Se asume que, en funcionamiento normal del sistema, la entrada es una señal de valor eficaz $\|u[t]\|_{R M S}=$ 2.15 y, por tanto, se considera para el diseño una perturbación de salida equivalente de valor

$$
\left\|v_{k}\right\|_{R M S} \equiv\|v[t]\|_{R M S}=\|\Delta G(z)\|_{\infty} \cdot\|u[t]\|_{R M S}=0.645 .
$$

Con estos valores, se ha diseñado un predictor $\mathcal{H}_{\infty}$ capaz de atenuar el efecto conjunto del ruido y el error de modelado. Para comprobar el funcionamiento del predictor, también se ha diseñado un predictor basado en la estabilidad nominal y se ha simulado el comportamiento de éstos ante un proceso "real" definido por la función de transferencia

$$
G(s)=\frac{9.9(1-0.12 s)}{s^{2}+4.1 s+2.1}
$$

Los resultados de una simulación se muestran en la figura 3.16. El error eficaz obtenido con el predictor basado en la estabilidad nominal es de $\left\|e_{k}\right\|_{R M S}=1.17$, mientras que el error eficaz con el diseño $\mathcal{H}_{\infty}$ desciende a $\left\|e_{k}\right\|_{R M S}=0.574$.

Ejemplo 3.6.4. Considérese el modelo de un proceso dado por la f.d.t.

$$
G(s)=\frac{1}{s^{3}+2 s^{2}+3 s+4},
$$

en el que la entrada se actualiza a un periodo contante de $T=0.15$ segundos. Supóngase que la salida se mide de forma esporádica y con retardos definiéndose los posibles escenarios de muestreo mediante los conjuntos

$$
\mathcal{N}=\{3,4,5\}, \quad \mathcal{D}=\{0,1,2\}
$$




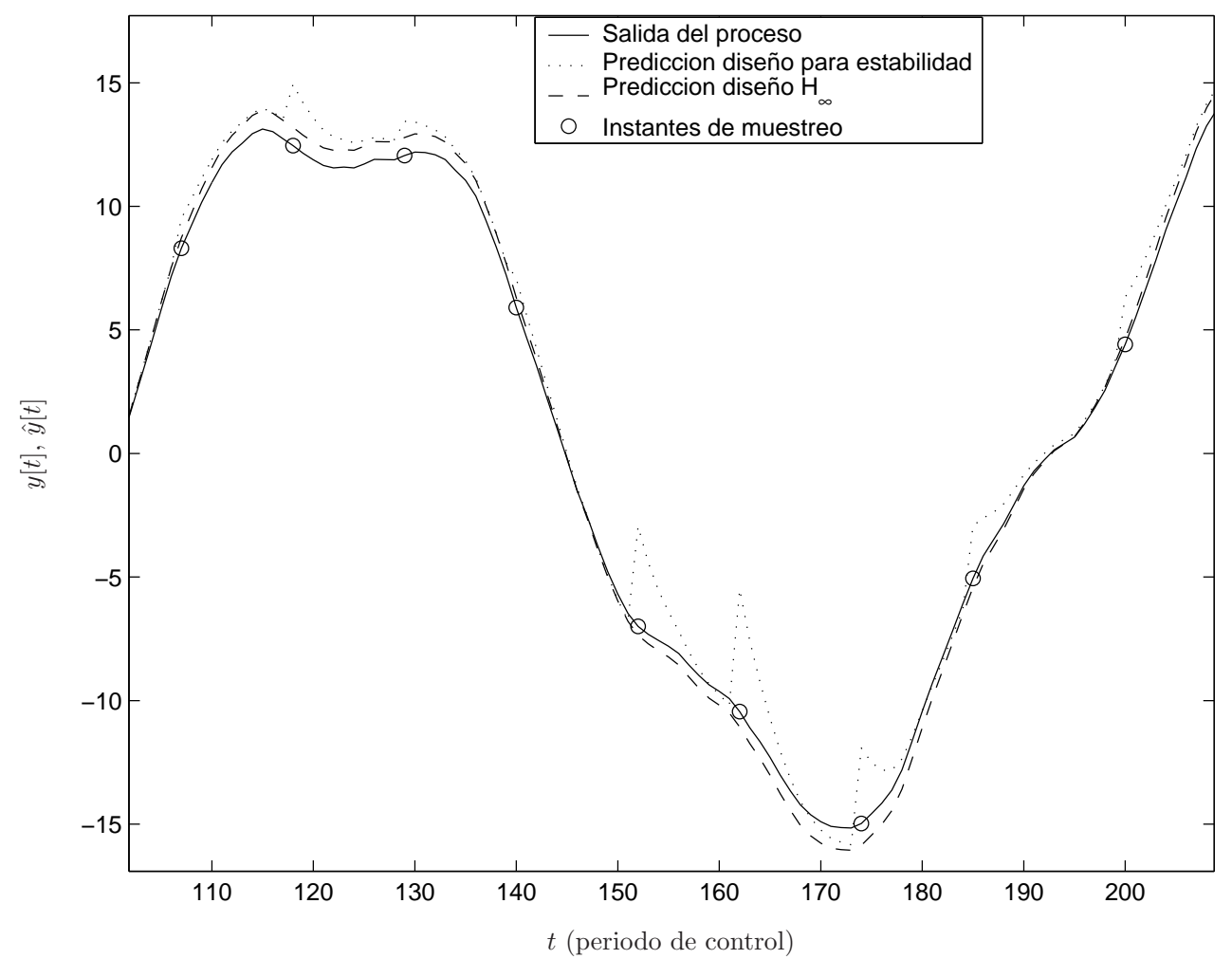

Figura 3.16: Evolución de la salida y de las predicciones con diferentes métodos para un modelo con incertidumbre. $\mathcal{N}=\{10, \ldots, 15\}, \mathcal{D}=\{0,1,2\}$.

Se asume que el sistema está afectado por un ruido aleatorio de media cero y desviación típica $\sigma_{w}=$ $\|w[t]\|_{R M S}=0.01$. Se asume que se conoce una cota de la incertidumbre del modelo dada por

$$
\|\Delta G(z)\|_{\infty}=1
$$

Se asume que, en funcionamiento normal del sistema, la entrada es una señal acotada en el intervalo

$$
-10 \leq u[t] \leq 10
$$

con lo que se considera para el diseño una perturbación de salida equivalente de valor

$$
\left\|v_{k}\right\|_{R M S} \equiv\|v[t]\|_{R M S}=\|\Delta G(z)\|_{\infty} \cdot\|u[t]\|_{\infty}=10 .
$$

Con estos valores, se ha diseñado un predictor $\mathcal{H}_{\infty}$ capaz de atenuar el efecto conjunto del ruido y el error de modelado. Para comprobar el funcionamiento del predictor, también se ha diseñado un predictor basado en la estabilidad nominal y se ha simulado el comportamiento de éstos ante un proceso "real" definido por la función de transferencia

$$
G(s)=\frac{1}{s^{3}+2 s^{2}+2.5 s+4} .
$$

Los resultados de una simulación se muestran en la figura 3.17. El error eficaz obtenido con el predictor de bucle abierto tras múltiples simulaciones es de $\|e[t]\|_{R M S}=0.136$, mientras que con el diseño $\mathcal{H}_{\infty}$ desciende a $\|e[t]\|_{R M S}=0.025$.

\subsubsection{Retardo en la acción de control}

Si el cómputo de la acción de control y su transmisión desde el controlador hasta el actuador introduce un retardo apreciable $d_{u}$ en el bucle de control, éste se tiene que tener en cuenta a la hora de predecir salidas futuras. Si el retardo es múltiplo del periodo de control $T$, se puede trasferir este retardo a la salida y tratarlo como si estuviera en el sistema de medición. De esta manera, el predictor proporcionará 


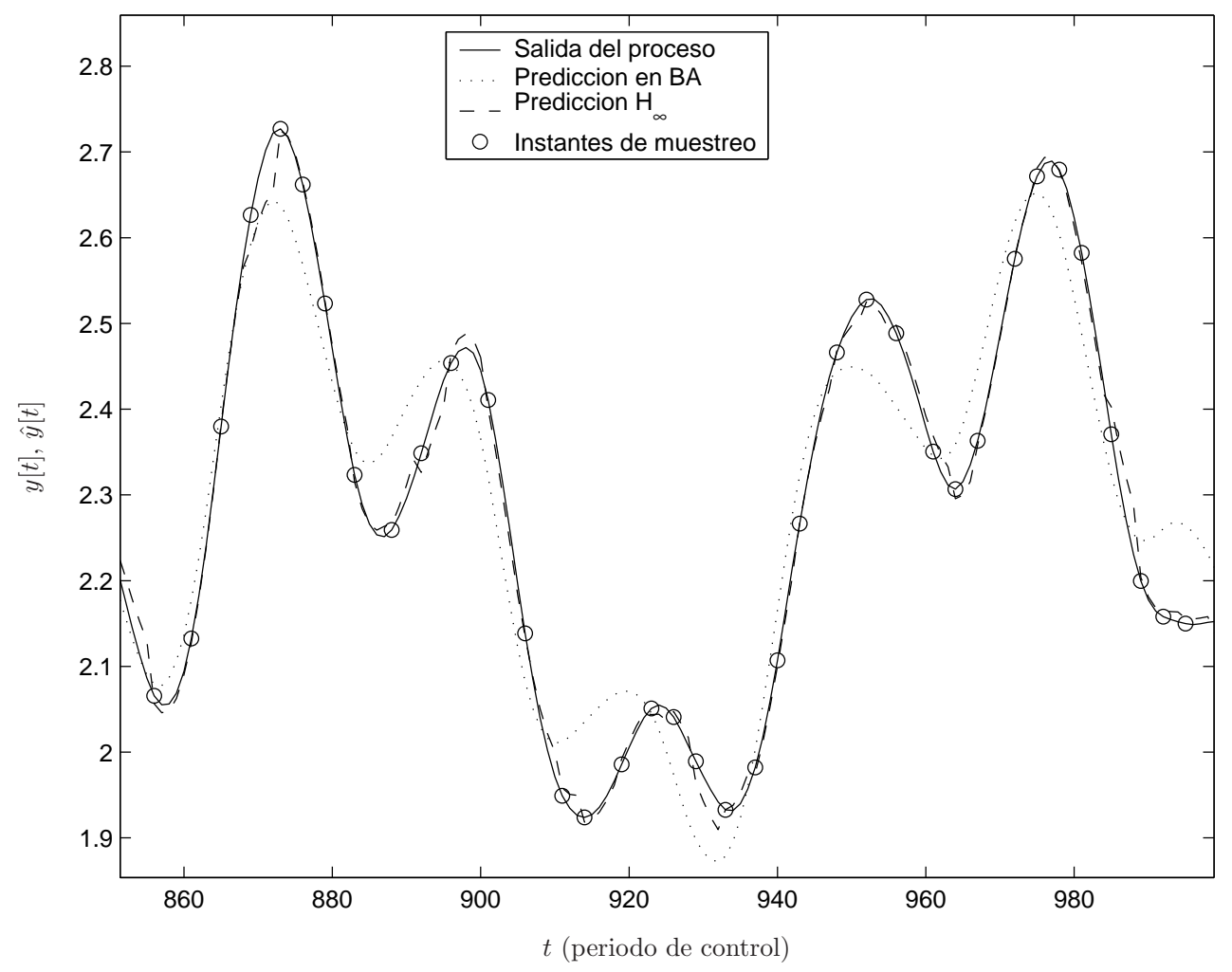

Figura 3.17: Evolución de la salida y de las predicciones con diferentes métodos para un modelo con incertidumbre. $\mathcal{N}=\{3,4,5\}, \mathcal{D}=\{0,1,2\}$.

la salida que se tendría si no hubiera retardos en la entrada del proceso para poder implementar técnicas de control convencionales. Si el retardo $d_{u}$ no es múltiplo de $T$, se divide el retardo en dos partes como

$$
d_{u}[t]=k_{u} T+\sigma[t],
$$

donde $k_{u}$ es un número entero y $\sigma[t] \in[0, T)$. El retardo $k_{u} T$ es la parte del retardo que es múltiplo del periodo de control y que, por tanto, se puede transferir a la salida. Con esta consideración, el problema se reduce al de predicción con retardo en la entrada menor que $T$ para lo que se propone obtener un modelo discreto entrada-salida a partir del modelo continuo que considere el retardo $\sigma[t]$ (véase figura 3.18).

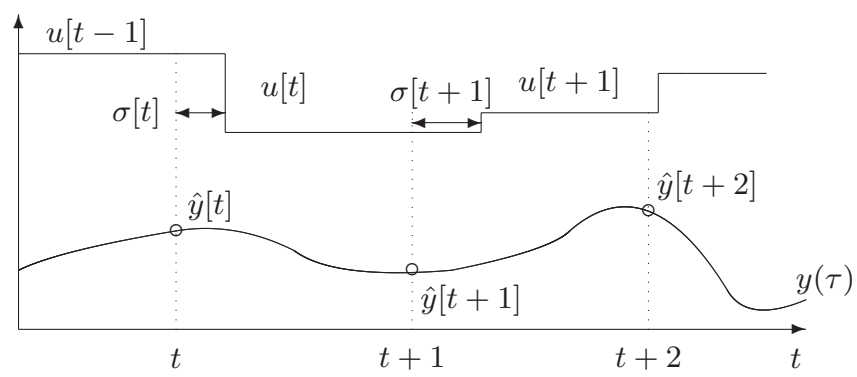

Figura 3.18: Retardo en la acción de control.

\section{Retardo de entrada constante}

Se considera en primer lugar el caso en el que el retardo $\sigma[t]=\sigma_{0}$ es constante. El modelo continuo de la planta es

$$
\begin{aligned}
\dot{\boldsymbol{x}}(\tau) & =\boldsymbol{A}_{c} \boldsymbol{x}(\tau)+\boldsymbol{b}_{c} u(\tau), \\
y(\tau) & =\boldsymbol{c} \boldsymbol{x}(\tau) .
\end{aligned}
$$


Si se aplica un retenedor de orden cero, la acción de control en el periodo $t$ toma los valores

$$
\begin{aligned}
& u(\tau)=u[t-1], \quad \tau \in\left[t T,\left(t+\sigma_{0}\right) T\right), \\
& u(\tau)=u[t], \quad \tau \in\left[\left(t+\sigma_{0}\right) T, T\right) .
\end{aligned}
$$

A partir del modelo continuo, se puede obtener el modelo discreto a periodo $T$ mediante

$$
\begin{aligned}
\boldsymbol{x}[t+1] & =e^{\boldsymbol{A}_{c} T} \boldsymbol{x}[t]+\int_{0}^{T} e^{\boldsymbol{A}_{c} \tau} \boldsymbol{b}_{c} u(t T+\tau) \mathrm{d} \tau, \\
y[t] & =\boldsymbol{c} \boldsymbol{x}[t] .
\end{aligned}
$$

La ecuación de estado se puede escribir como

$$
\boldsymbol{x}[t+1]=\boldsymbol{A} \boldsymbol{x}[t]+\underbrace{\int_{0}^{\sigma_{0}} e^{A_{c} \tau} \mathrm{d} \tau}_{=\boldsymbol{b}_{1}} u[t-1]+\underbrace{\int_{\sigma_{0}}^{T} e^{A_{c} \tau} \boldsymbol{b}_{c} \mathrm{~d} \tau}_{=\boldsymbol{b}_{0}} u[t],
$$

con lo que, tomando transformada en $\mathcal{Z}$ se llega a la función de transferencia discreta

$$
G(z)=\frac{Y(z)}{U(z)}=\boldsymbol{c}(z \boldsymbol{I}-\boldsymbol{A})^{-1}\left(\boldsymbol{b}_{0}+\boldsymbol{b}_{1} z^{-1}\right)
$$

cuyos polinomios del denominador y denominador tendrán la forma

$$
G(z)=\frac{b_{1}^{\prime} z^{-1}+\cdots+b_{n}^{\prime} z^{-n}+b_{n+1}^{\prime} z^{-(n+1)}}{1+a_{1} z^{-1}+\cdots+a_{n} z^{-n}} .
$$

El modelo que se utilizará para realizar la predicción tendrá la forma

$$
Y[t+1]=\boldsymbol{A} Y[t]+\boldsymbol{B}^{\prime} U^{\prime}[t]
$$

con

$$
\boldsymbol{A}=\left[\begin{array}{cccc}
-a_{1} & \cdots & -a_{n-1} & -a_{n} \\
1 & \cdots & 0 & 0 \\
\vdots & \ddots & & \vdots \\
0 & \cdots & 1 & 0
\end{array}\right] ; \quad \boldsymbol{B}^{\prime}=\left[\begin{array}{ccc}
b_{1}^{\prime} & \cdots & b_{n+1}^{\prime} \\
0 & \cdots & 0 \\
\vdots & \ddots & \vdots \\
0 & \cdots & 0
\end{array}\right]
$$

y

$$
Y[t]=\left[\begin{array}{c}
y[t] \\
\vdots \\
y[t-(n-1)]
\end{array}\right] ; \quad U^{\prime}[t]=\left[\begin{array}{c}
u[t] \\
\vdots \\
u[t-n]
\end{array}\right]
$$

Con este modelo se consigue mantener la matriz dinámica $(\boldsymbol{A})$ intacta, con lo que todos los algoritmos de diseño obtenidos siguen siendo válidos ya que ninguno de ellos depende de la matriz $\boldsymbol{B}$ (ahora $\boldsymbol{B}^{\prime}$ ). La implementación del predictor sólo variaría en la forma de definir la matriz $\boldsymbol{B}$ y el vector de entradas $U[t]$ para estimar en bucle abierto.

\section{Retardo de entrada variante}

Si el retardo presenta una componente aleatoria variante en el tiempo, el modelo anterior será incierto porque $b_{0}$ y $b_{1}$ no se conocerán con exactitud. Si el retardo se toma como

$$
\sigma[t]=\sigma_{0}+\Delta \sigma[t]
$$

siendo $\sigma_{0} \geq 0$ el mínimo retardo que se conoce, y $\Delta \sigma[t]>0$ la componente aleatoria de la cual se conoce su cota superior, la ecuación de estado discreta (3.89) vendrá dada por

$$
\boldsymbol{x}[t+1]=\boldsymbol{A} \boldsymbol{x}[t]+\int_{0}^{\sigma[t]} e^{A_{c} \tau} \mathrm{d} \tau u[t-1]+\int_{\sigma[t]}^{T} e^{A_{c} \tau} \boldsymbol{b}_{c} \mathrm{~d} \tau u[t] .
$$


Sumando y restando el término $\int_{\sigma_{0}}^{\sigma[t]} e^{A_{c} \tau} \boldsymbol{b}_{c} \mathrm{~d} \tau u[t]$ se llega a

$$
\boldsymbol{x}[t+1]=\boldsymbol{A} \boldsymbol{x}[t]+\boldsymbol{b}_{0} u[t]+\boldsymbol{b}_{1} u[t-1]+\underbrace{\int_{\sigma_{0}}^{\sigma[t]} e^{A_{c} \tau} \boldsymbol{b}_{c} \mathrm{~d} \tau}_{=\Delta \boldsymbol{b}[t]}(u[t-1]-u[t]),
$$

donde $\Delta \boldsymbol{b}[t]$ es la parte incierta del modelo que varía su valor con $\sigma[t]$. La función de transferencia entrada-salida viene dada por

$$
\frac{Y(z)}{U(z)}=\boldsymbol{c}(z \boldsymbol{I}-\boldsymbol{A})^{-1}\left(\boldsymbol{b}_{0}-\Delta \boldsymbol{b}[t]+\left(\boldsymbol{b}_{1}+\Delta \boldsymbol{b}[t]\right) z^{-1}\right)=G(z)+\Delta G(z, t),
$$

de donde se extrae la parte nominal del modelo $(G(z)$ en (3.90)) y la parte incierta (variante en el tiempo) dada por

que puede escribirse como

$$
\Delta G(z, t)=\boldsymbol{c}(z \boldsymbol{I}-\boldsymbol{A})^{-1}\left(-1+z^{-1}\right) \Delta \boldsymbol{b}[t],
$$

$$
\Delta G(z, \sigma[t])=\boldsymbol{c}(z \boldsymbol{I}-\boldsymbol{A})^{-1}\left(-1+z^{-1}\right) \int_{\sigma_{0}}^{\sigma[t]} e^{A_{c} \tau} \boldsymbol{b}_{c} \mathrm{~d} \tau .
$$

Para calcular el predictor puede utilizarse la norma del peor caso, que viene dada por

$$
\|\Delta G(z)\|_{p}=\operatorname{máx}_{\sigma[t]}\left\|\boldsymbol{c}(z \boldsymbol{I}-\boldsymbol{A})^{-1}\left(-1+z^{-1}\right) \int_{\sigma_{0}}^{\sigma[t]} e^{A_{c} \tau} \boldsymbol{b}_{c} \mathrm{~d} \tau\right\|_{p} .
$$

o bien el valor medio que toma esta norma para todos los posibles valores de $\sigma[t]$ a lo largo del tiempo, es decir

$$
\|\Delta G(z)\|_{p}=\mathcal{E}\left\{\left\|\boldsymbol{c}(z \boldsymbol{I}-\boldsymbol{A})^{-1}\left(-1+z^{-1}\right) \int_{\sigma_{0}}^{\sigma[t]} e^{A_{c} \tau} \boldsymbol{b}_{c} \mathrm{~d} \tau\right\|_{p}\right\} .
$$

Observación 3.6.1. Si se tiene un retardo incierto en la entrada de control será difícil determinar cuál es el retardo mínimo $\sigma_{0}$, con lo que el vector $\boldsymbol{b}_{1}$ será nulo $\left(\boldsymbol{b}_{1}=\mathbf{0}\right)$ y $\boldsymbol{b}_{0}$ será el vector resultado de la discretización estándar a periodo $T$, es decir,

$$
\boldsymbol{b}_{0}=\int_{0}^{T} e^{A_{c} \tau} \boldsymbol{b}_{c} \mathrm{~d} \tau
$$

De la misma forma, el vector incierto $\Delta \boldsymbol{b}[t]$ vendrá dado por

$$
\Delta \boldsymbol{b}[t]=\int_{0}^{\sigma[t]} e^{A_{c} \tau} \boldsymbol{b}_{c} \mathrm{~d} \tau
$$

Ejemplo 3.6.5. Tómese el sistema

$$
G(s)=\frac{10(1-0.1 s)}{s^{2}+4 s+2}
$$

con un periodo de actualización de la acción de control de $T=0.5$ segundos. Supóngase que la acción de control sufre un retardo aleatorio de un valor comprendido entre 0 y 0.5 segundos desde que se calcula hasta que se aplica al proceso. La distribución de este retardo es uniforme. Según lo indicado en esta sección, el efecto de este retardo es equivalente al efecto de una incertidumbre en el modelo del valor indicado en (3.95). La norma de esta incertidumbre equivalente (expresada en (3.96)) para este ejemplo es la que se indica en la figura 3.19 en función del retardo. Se ha indicado tanto la norma $\mathcal{H}_{2}$ como la norma $\mathcal{H}_{\infty}$, aunque otras normas podrían también haberse obtenido. La norma a utilizar en cada caso depende de la información que se tiene sobre la acción de control, para, de esta manera, poder obtener la norma de la perturbación de salida equivalente (véase tabla 3.6). Teniendo en cuenta la distribución uniforme del retardo, se utiliza el valor medio de esta norma a lo largo de $\sigma$ para calcular el predictor. En el caso de la norma $\mathcal{H}_{\infty}$ se utiliza como norma el valor

$$
\|\Delta G(z)\|_{\infty}=0.611
$$

mientras que en el caso de la norma $\mathcal{H}_{2}$ se utiliza el valor

$$
\|\Delta G(z)\|_{2}=0.448 \text {. }
$$



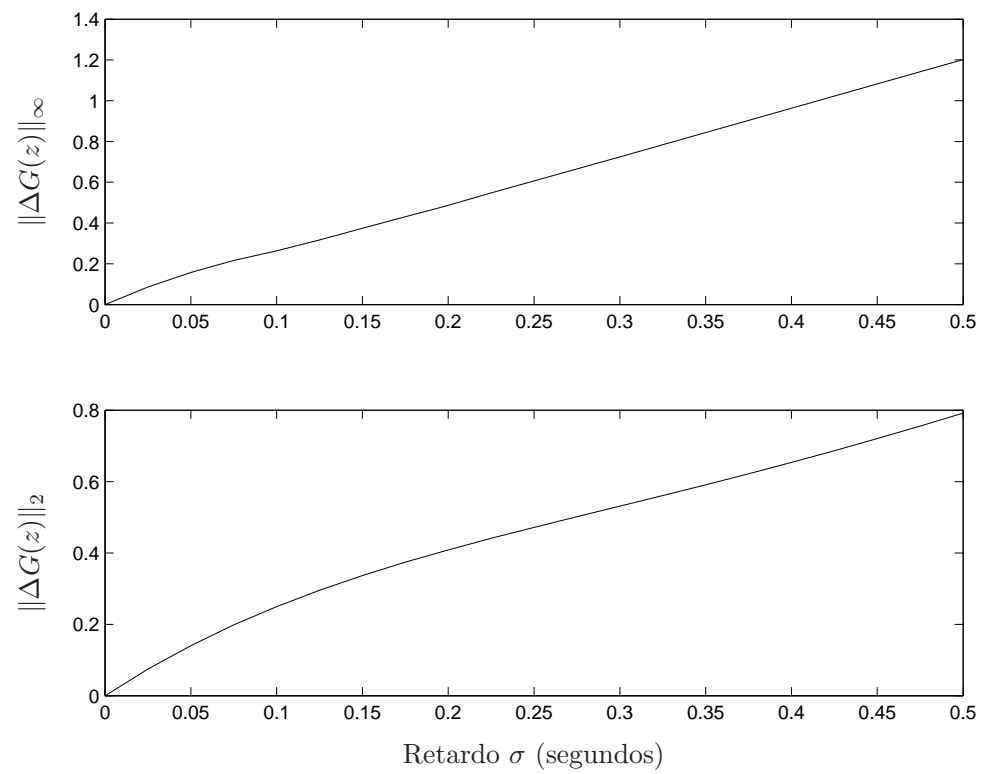

Figura 3.19: Norma de la incertidumbre equivalente a la presencia de retardos en la acción de control

\subsubsection{Mediciones con etiqueta de tiempo incierta}

En el problema de predicción planteado se asume que cada medición $m_{k}$ viene acompañada de una etiqueta de tiempo que indica el instante de tiempo en el cual se dio la salida que se ha medido. Si las mediciones llegan con un retardo $d_{k}$, la etiqueta que acompaña a una medición $m_{k}$ disponible en el instate $t_{k}$ tiene el valor $t_{k}-d_{k}$. El predictor utiliza esta etiqueta para estimar la salida en el instante $t_{k}-d_{k}$ $\left(\hat{y}\left[t_{k}-d_{k} \mid t_{k}-1\right]\right)$ y compararla con la medición $m_{k}$, que se supone de valor $y\left[t_{k}-d_{k}\right]$. Sin embargo, puede ocurrir que la medición corresponda a un instante de tiempo diferente al indicado por la etiqueta. Denotando mediante $i_{k}$ la incertidumbre que acompaña a la etiqueta de tiempo, normalmente asociada al retardo, puede escribirse el valor de la medición $m_{k}$ como (véase figura 3.20)

$$
m_{k}=y\left[t_{k}-d_{k}+i_{k}\right]
$$

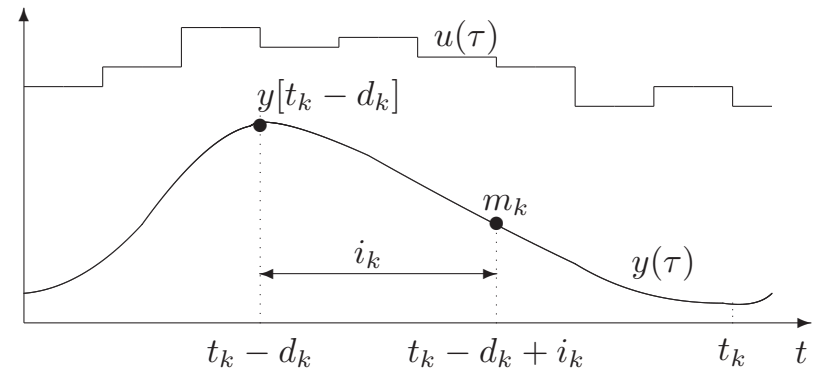

Figura 3.20: Medición disponible $\left(m_{k}\right)$ y salida correspondiente al instante indicado por la etiqueta de tiempo $\left(y\left[t_{k}-d_{k}\right]\right)$.

El objetivo es encontrar una señal $w_{k}$ que tenga en cuenta el error cometido por la incertidumbre $i_{k}$, es decir, se busca el valor de $w_{k}$ tal que

$$
m_{k}=y\left[t_{k}-d_{k}\right]+w_{k}
$$

Esta señal $w_{k}$, que puede considerarse como un ruido de medida, viene dada por

$$
w_{k}=m_{k}-y\left[t_{k}-d_{k}\right]=y\left[t_{k}-d_{k}+i_{k}\right]-y\left[t_{k}-d_{k}\right] .
$$


La aplicación de las estrategias de diseño anteriores que minimizan el error de predicción frente a ruidos de medida y perturbaciones de salida, requieren del conocimiento de una norma del ruido ficticio $w_{k}$. Si se asume conocido el rango de variación de la incertidumbre de la etiqueta $i_{k}$

$$
i_{k} \in \mathcal{I}=\left\{\iota_{1}, \iota_{2}, \ldots, \iota_{r}\right\} \subset \mathbb{N}
$$

se tienen varias formas de obtener una norma característica de $w_{k}$. Una primera aproximación consiste en obtener una secuencia de valores $y[t]$ con una simulación del funcionamiento del proceso para obtener después el valor esperado de la norma de $\left\|y\left[t+i_{k}\right]-y[t]\right\|_{R M S}$ para diferentes valores de $i_{k}$ :

$$
\left\|w_{k}\right\|_{R M S}=\mathcal{E}_{i \in \mathcal{I}}\left\{\|y[t+i]-y[t]\|_{R M S}\right\}
$$

De forma similar se puede obtener el ruido ficticio del peor caso como

$$
\left\|w_{k}\right\|_{\infty}=\operatorname{máx}_{i \in \mathcal{I}}\left\{\|y[t+i]-y[t]\|_{\infty}\right\} .
$$

Otra forma de calcular una cota de esta norma es tomando transformadas en $\mathcal{Z}$ llegando a

$$
\left\|w_{k}\right\|_{p}=\left\|\left(1-z^{-i_{k}}\right) G(z) u(z)\right\|_{p}
$$

Esta expresión permite obtener una cota del valor eficaz esperado del ruido ficticio mediante

$$
\left\|w_{k}\right\|_{R M S}=\mathcal{E}_{i \in \mathcal{I}}\left\{\left\|\left(1-z^{-i}\right) G(z)\right\|_{\infty}\right\}\|u[t]\|_{R M S},
$$

o bien una cota del valor máximo del ruido ficticio mediante

$$
\left\|w_{k}\right\|_{\infty}=\mathcal{E}_{i \in \mathcal{I}}\left\{\left\|\left(1-z^{-i}\right) G(z)\right\|_{1}\right\}\|u[t]\|_{\infty}
$$

donde se han utilizado las definiciones de las normas $\mathcal{H}_{\infty}$ y $\ell_{1}$ de un sistema (véase $\S$ A.2.1).

Observación 3.6.2. Un caso de aplicación de este método es cuando las mediciones no vienen acompañadas de etiqueta de tiempo a pesar de que éstas están afectadas de un retardo que puede ser variante. Este caso se puede obtener a partir del anterior haciendo $d_{k}=0$, y que la incertidumbre $i_{k}$ sea igual al retardo en la medición. Esta situación lleva a un predictor de fácil implementación (no se tienen retardos) pero que puede tener bajas prestaciones según sea el sistema (estable/inestable) y según el número de periodos de retardo.

Ejemplo 3.6.6. Considérese el sistema continuo doble integrador

$$
G(s)=\frac{1}{s^{2}}
$$

cuya acción de control se actualiza cada $T=0.05$ segundos. Sea un muestreo aleatorio en el que llega una medición cada $N_{k}=10$ periodos con un retardo de $d_{k}$ periodos, donde $d_{k}$ toma valores dentro del conjunto

$$
\mathcal{D}=\{0,1,2,3,4,5,6\}
$$

Considérese que hay una perturbación constante a la salida de valor $\|v[t]\|_{\infty}=0.02$. Supóngase también que hay un ruido de medida aleatorio con media cero y desviación típica $\sigma_{w}=\|w[t]\|_{R M S}=0.3$. En este escenario se plantean 3 diseños diferentes:

1. Un primer diseño basado en la atenuación $\mathcal{H}_{\infty}$ y considerando que las mediciones llegan sin retardo.

2. Un segundo diseño en el que se considera que las mediciones llegan con un retardo $d_{k}=0$, de manera que el efecto del retardo real (entre 0 y 6) se considera como un ruido de medida. En este caso, aplicando la expresión (3.100) se considera una cota del ruido de medida de valor $\left\|w_{k}\right\|_{\infty}=50$. Con la norma $\ell_{\infty}$ de la perturbación y del ruido de medida equivalente se realiza un diseño basado en la atenuación $\ell_{1}$.

3. Un tercer diseño en el que se considera que las mediciones llegan con un retardo $d_{k}=3$ (el valor medio de los retardos posibles), de manera que el efecto del retardo real se considera como un ruido de medida que viene acotado por (aplicando (3.100) $)\left\|w_{k}\right\|_{\infty}=31.7$. Con los valores máximos de la perturbación y el ruido de medida equivalente se realiza un diseño basado en la atenuación $\ell_{1}$. 
En la figura 3.21 se comparan los tres predictores donde se observa que el primer predictor tiene muy bajas prestaciones ya que desprecia el efecto del error cometido por el retardo real de las mediciones, que se puede observar por los cambios bruscos que se tienen en el valor de salida predicho cuando se tiene una medición. En este primer caso se tiene un error de predicción caracterizado por los valores $\|e[t]\|_{R M S}=0.98$ y $\|e[t]\|_{\infty}=2.7$. En el segundo caso se mejora el error de predicción por haber introducido en el diseño el ruido equivalente al error cometido por el retardo no considerado. En el segundo caso se tiene un error de predicción caracterizado por los valores $\|e[t]\|_{R M S}=0.43 y\|e[t]\|_{\infty}=1.1$. Con el tercer predictor se vuelve a disminuir el error de predicción por haber tomado un valor de retardo medio, de manera que el ruido equivalente introducido con cada medición (retardada) ha sido menor. Con el último predictor se tiene un error de predicción caracterizado por los valores $\|e[t]\|_{R M S}=0.34$ y $\|e[t]\|_{\infty}=0.77$.

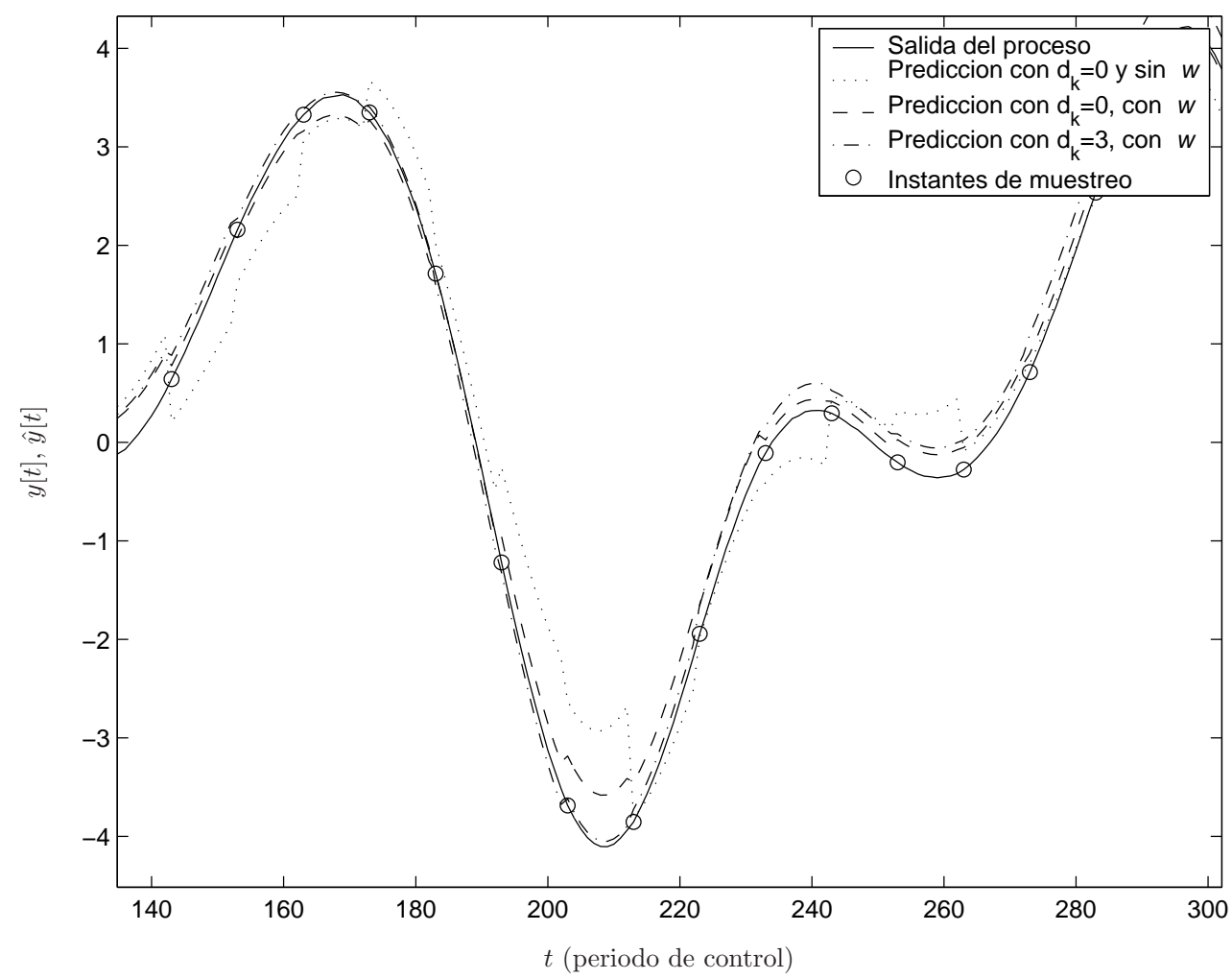

Figura 3.21: Evolución de la salida y de las predicciones con diferentes métodos para un proceso en el que las mediciones no vienen acompañadas de etiqueta de tiempo. $\mathcal{N}=\{10\}, \mathcal{D}=\{0,1, \ldots, 6\}$. Diseño basado en la atenuación $\ell_{1}$

\subsubsection{Muestreo asíncrono}

Cuando se tienen mediciones asíncronas, es necesario utilizar un modelo continuo del proceso, ya que éste sí que tiene en cuenta la dinámica en todos los instantes de tiempo continuo. El filtro de Kalman mostrado en $\S 2.3 .3$ resuelve esta situación para muestreo asíncrono sin retardos con el inconveniente de ser necesario un elevado coste computacional.

Para evitar la utilización de un modelo continuo del proceso y para poder manejar mediciones con retardo, se propone utilizar una interpolación (bien de orden cero, o bien de orden uno, véase §2.3.1) que permita obtener de forma aproximada el valor de la salida en los instantes síncronos con la actualización de la acción de control (cuando $\tau=t T$ ). Supóngase que el instante $\tau_{k}$ en el que se tiene una medición asíncrona corresponde al intervalo de tiempo que se encuentra entre los instantes de control $t \mathrm{y}(t+1)$, es decir

$$
t T<\tau_{k}<(t+1) T
$$


Si se define la fracción del periodo en la que llega la medición como

$$
\alpha_{k}=\frac{\tau_{k}-t T}{T},
$$

el instante continuo $\tau_{k}$ se puede escribir como

$$
\tau_{k}=\left(t+\alpha_{k}\right) T, \quad \alpha_{k} \in(0,1) .
$$

La asincronía del muestreo se puede dar de tres formas:

1. La medición se toma en un instante síncrono con el control pero su valor llega al controlador de forma asíncrona debido al retardo. Este caso no se considera muestreo asíncrono porque el valor medido corresponde a un instante síncrono, y la actualización del predictor con esta medida se realizará en el instante de control inmediatamente posterior.

2. La medición se toma en un instante asíncrono con el control pero su valor llega al controlador de forma síncrona tras el retardo introducido por el canal de comunicación. En este caso es necesario obtener una señal de medición síncrona ficticia a partir del valor asíncrono medido para aplicar la ecuación de actualización del predictor.

3. La medición se toma en un instante asíncrono y le llega al controlador en ese mismo instante u otro posterior que también es asíncrono. Este caso se reduce al segundo si se aplica el razonamiento del caso primero.

Teniendo en cuenta esto último, se considera que no hay retardos entre la salida y la medición, y que la ecuación continua que relaciona la medición de un sensor con la salida continua viene dada por

$$
m(\tau)=y(\tau) .
$$

\section{Interpolación de orden cero}

Estrategia 11. La primera estrategia que se propone consiste en considerar que el instante de medición es el del instante $t+1$ inmediatamente posterior al instante de medición (véase figura 3.22), es decir,

$$
m_{k}=y\left(\left(t+\alpha_{k}\right) T\right) \approx y[t+1],
$$

y utilizar esta aproximación de la salida para correr la ecuación de actualización del predictor en $t+1$.

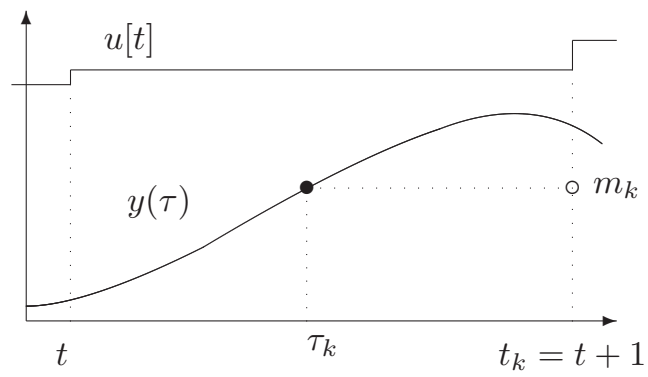

Figura 3.22: Estrategia I1. Interpolación de la medición hasta el siguiente instante de control.

El error entre la salida real $y[t+1]$ y la aproximada por $m_{k}$ que se comete puede considerarse como una señal de ruido que acompaña a la medición y que tiene un valor de

$$
w_{k}=y((t+1) T)-y\left(\left(t+\alpha_{k}\right) T\right) .
$$

Si el periodo de control se considera suficientemente pequeño, la evolución de la salida continua de $y(t T)$ a $y((t+1) T)$ se aproxima a una linea recta, con lo que la salida $y\left(\left(t+\alpha_{k}\right) T\right)$ puede aproximarse por

$$
y\left(\left(t+\alpha_{k}\right) T\right) \approx y[t]+\alpha_{k}(y[t+1]-y[t]) .
$$

Con esta aproximación, el ruido de medida puede expresarse como

$$
w_{k}=\left(1-\alpha_{k}\right)(y[t+1]-y[t]) .
$$


A partir de esta expresión se tienen diferentes formas de obtener una norma característica del ruido de medición. El valor máximo del ruido viene dado por

$$
\left\|w_{k}\right\|_{\infty}=\|\Delta y[t]\|_{\infty}
$$

donde los incrementos de la salida se pueden estimar mediante simulación. También mediante simulación es posible obtener una cota aproximada del valor eficaz mediante la expresión

$$
\left\|w_{k}\right\|_{R M S}=\frac{1}{2}\|\Delta y[t]\|_{R M S}
$$

donde se ha considerado una distribución uniforme de los instantes de medición, es decir de $\alpha_{k}$ y, por tanto, $\mathcal{E}\left(\alpha_{k}\right)=0.5$. Mediante la transformada en $\mathcal{Z}$ también es posible obtener una norma del valor eficaz esperado del ruido ficticio como

$$
\left\|w_{k}\right\|_{R M S}=\frac{1}{2}\left\|\left(1-z^{-1}\right) G(z)\right\|_{\infty}\|u[t]\|_{R M S}
$$

y del valor máximo como

$$
\left\|w_{k}\right\|_{\infty}=\left\|\left(1-z^{-1}\right) G(z)\right\|_{1}\|u[t]\|_{\infty}
$$

Estrategia I2. La segunda estrategia que se propone consiste en considerar que el instante de medición es el del instante de control $t$ inmediatamente anterior al instante de medición (véase figura 3.23), es decir,

$$
m_{k}=y\left(\left(t+\alpha_{k}\right) T\right) \approx y[t]
$$

y utilizar esta aproximación de la salida para correr la ecuación de actualización del predictor en $t$, que, aunque podría haberse ejecutado en el instante $t$, se vuelve a ejecutar para mejorar la estimación. El

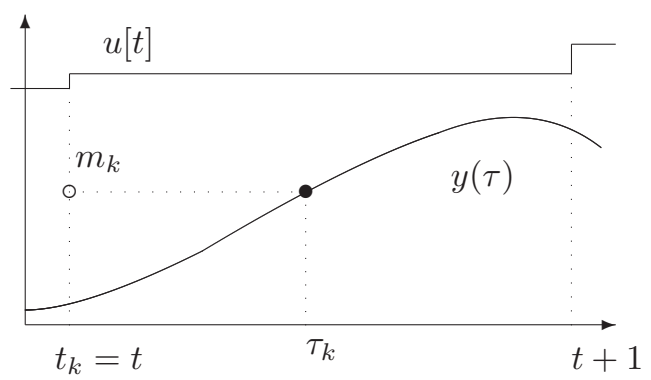

Figura 3.23: Estrategia I2. Interpolación de la medición hacia el instante de control anterior.

error entre la salida real $y[t]$ y la aproximada por $m_{k}$ que se comete puede considerarse como una señal de ruido que acompaña a la medición y que tiene un valor de

$$
w_{k}=y\left(\left(t+\alpha_{k}\right) T\right)-y(t T)
$$

De nuevo, bajo la consideración de transición casi lineal de $y[t]$ a $y[t+1]$, la norma del ruido ficticio se puede acotar mediante alguna de las expresiones (3.105), (3.106) o (3.107).

Si se conoce cuál es el valor más probable de $\alpha_{k}$ se puede elegir entre las dos estrategias I1 e I2 para minimizar el ruido ficticio introducido. Se elegirá la primera estrategia (asumir la medición en $t+1$ ) cuando $\mathcal{P}\left(\alpha_{k}\right)>0.5$ y la segunda estrategia (asumir la medición en $t$ ) cuando $\mathcal{P}\left(\alpha_{k}\right) \leq 0.5$.

Estrategia I3. Si se conoce el instante $\tau_{k}$ en el que llega la medición se pueden utilizar otras estrategias. La más simple consiste en elegir en cada instante entre las dos estrategias anteriores en función de si la medición está más cerca en el tiempo de $t T$ o de $(t+1) T$, es decir en función del valor de $\alpha_{k}$ :

$$
\begin{cases}t_{k}=t, & \text { si } \alpha_{k}<0.5 \\ t_{k}=t+1, & \text { si } \alpha_{k}>0.5\end{cases}
$$


Con este método, el valor esperado del ruido ficticio que se introduce con la medición ficticia es la mitad del indicado por las expresiones (3.105), (3.106), (3.107) y (3.108):

$$
\begin{aligned}
\left\|w_{k}\right\|_{\infty} & =\frac{1}{2}\|\Delta y[t]\|_{\infty}, \\
\left\|w_{k}\right\|_{R M S} & =\frac{1}{4}\|\Delta y[t]\|_{R M S}, \\
\left\|w_{k}\right\|_{R M S} & =\frac{1}{4}\left\|\left(1-z^{-1}\right) G(z)\right\|_{\infty}\|u[t]\|_{R M S} \\
\left\|w_{k}\right\|_{\infty} & =\frac{1}{2}\left\|\left(1-z^{-1}\right) G(z)\right\|_{1}\|u[t]\|_{\infty} .
\end{aligned}
$$

Observación 3.6.3. Como se ha podido comprobar, la utilización de mediciones asíncronas mediante interpolación de orden cero ha resultado introducir un ruido de medida ficticio cuyo valor máximo equivale al que se introduce en el caso de mediciones con etiqueta de tiempo incierta con $i_{k}=1$. Por lo tanto el análisis de los sistemas con mediciones asíncronas con etiqueta de tiempo incierta se puede hacer tomando el peor caso, que consiste simplemente en añadir al conjunto de incertidumbres de etiquetas $(\mathcal{I})$ una unidad. Es decir, que el diseño se tiene aplicar para calcular una ganancia que atenúe los ruidos de medida con norma (3.101) donde

$$
\mathcal{I}=\left\{\iota_{1}, \iota_{1}+1, \iota_{2}, \iota_{2}+1, \ldots, \iota_{r}, \iota_{r}+1\right\} \subset \mathbb{N} .
$$

Si los números $\iota_{j}$ son consecutivos, el conjunto anterior se reducirá a

$$
\mathcal{I}=\left\{\iota_{1}, \iota_{2}, \ldots, \iota_{r}, \iota_{r}+1\right\}
$$

\section{Interpolación de orden uno}

Estrategia 14. La interpolación de orden uno se puede utilizar para obtener una estimación de la medición $m_{k}$ que se tendría en el instante $t+1$ a partir de la salida (o su estimación) en el instante $t$ y de la medición real en $\tau=\tau_{k}$ de la cual se asume conocido $\tau_{k}$ (véase figura 3.24). Para ello se utiliza la ecuación de interpolación

$$
m_{k}=\hat{y}[t]+\frac{T}{\tau_{k}-t T}\left(y\left(\tau_{k}\right)-\hat{y}[t]\right)=\hat{y}[t]+\frac{1}{\alpha_{k}}\left(y\left(\tau_{k}\right)-\hat{y}[t]\right) .
$$

El ruido ficticio que se introduce con la utilización de la señal interpolada es

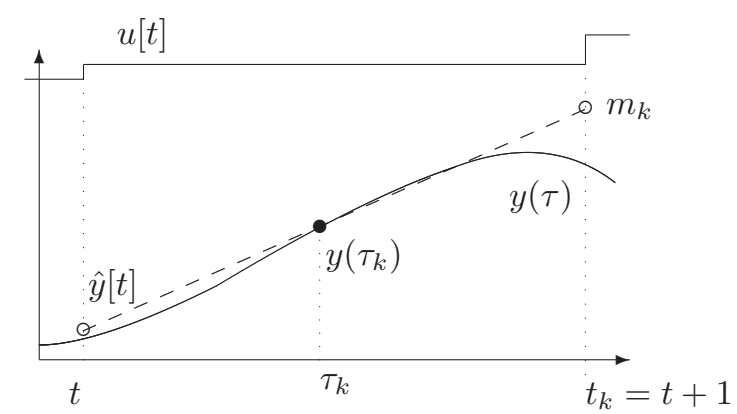

Figura 3.24: Estrategia I4. Extrapolación de la medición hasta el siguiente instante de control.

$$
w_{k}=y[t+1]-m_{k}
$$

Sustituyendo la ecuación de interpolación (3.115) y aplicando la aproximación(3.104) es fácil llegar a la expresión del ruido ficticio

$$
w_{k}=\frac{\alpha_{k}-1}{\alpha_{k}}(y[t]-\hat{y}[t])=\frac{\alpha_{k}-1}{\alpha_{k}} e[t]=\frac{\alpha_{k}-1}{\alpha_{k}} e\left[t_{k}-1\right]
$$

Esto implica que el error cometido con la interpolación aumenta con el error de estimación de partida $y[t]-\hat{y}[t]$ y es mayor cuando aumenta el tiempo entre la toma de medición y la llegada del nuevo control, es decir cuando disminuye $\alpha_{k}$. Nótese que cuando $\alpha_{k}$ es cercana a 0 el error cometido con este método puede llegar a ser muy grande, dependiendo del error de partida $e\left[t_{k}-1\right]$. 
Observación 3.6.4. Con la obtención de este último ruido ficticio se puede concluir que cualquiera de los métodos de interpolación de orden cero puede dar mejores resultados que esta interpolación de orden uno. La razón es que cuando cuando se trabaja con medidas escasas, la estimación de las salidas antes de tener una medición (estimación de bucle abierto) puede tener un error considerable que la interpolación de orden uno amplificaría considerablemente (como se ha visto en (3.116)), sobre todo si $\alpha_{k}$ tiene un valor pequeño, tendiendo el error a infinito cuando $\alpha_{k}$ tiende a cero.

Estrategia I5. La interpolación de orden uno se puede utilizar para obtener una estimación de la medición $m_{k}$ que se tendría en el instante $t+1$ a partir de la salida (o su estimación) en el instante $t$, de la estimación inicial (en bucle abierto) de la salida en $t+1$ y de la medición real en $\tau=\tau_{k}$ de la cual se asume conocido $\tau_{k}$ (véase figura 3.25 )

$$
m_{k}=y\left(\tau_{k}\right)+\left(1-\alpha_{k}\right)(\hat{y}[t+1 \mid t]-\hat{y}[t]) .
$$

Para ello se traza la recta que va desde la estimación $\hat{y}[t]$ hasta $\hat{y}[t+1 \mid t]$ para luego trazar una paralela a

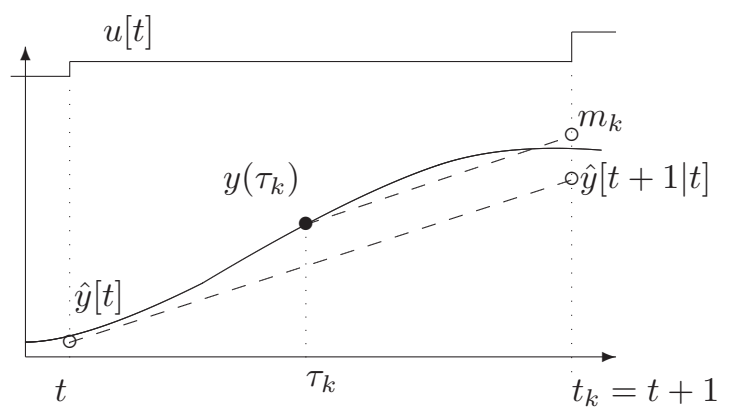

Figura 3.25: Estrategia I5. Extrapolación de la medición hasta el siguiente instante de control.

partir de $y\left(\tau_{k}\right)$ y así obtener el valor de la medición ficticia $m_{k}$ en el instante de control. El ruido ficticio que se introduce con la utilización de la señal interpolada es

$$
w_{k}=y[t+1]-m_{k} .
$$

Utilizando la expresión de interpolación (3.117) y la aproximación (3.104) se llega fácilmente a la expresión aproximada del ruido ficticio

$$
w_{k}=\left(1-\alpha_{k}\right)(y[t+1]-\hat{y}[t+1 \mid t]-y[t]+\hat{y}[t])=\left(1-\alpha_{k}\right)(e[t+1 \mid t]-e[t]) .
$$

Como la evolución en bucle abierto del error de predicción de $t$ a $t+1$ viene dada por

$$
e\left[t_{k} \mid t_{k}-1\right]=e[t+1 \mid t]=\boldsymbol{c} \boldsymbol{A} E[t]=\boldsymbol{c} \boldsymbol{A} E\left[t_{k}-1\right]
$$

siendo $e[t]=c E[t]$, la expresión (3.118) lleva a

$$
w_{k}=\left(1-\alpha_{k}\right) \boldsymbol{c}(\boldsymbol{A}-\boldsymbol{I}) E[t] .
$$

De nuevo, este valor depende del error de estimación de partida aunque, con esta estrategia, el aumento del error debido a $\alpha_{k}$ no es tan grande como con la estrategia I4. Si se puede estimar el error de predicción de partida mediante simulaciones o mediante el conocimiento de las perturbaciones o error de modelado, es posible obtener una cota del ruido ficticio mediante

$$
\left\|w_{k}\right\|_{\infty}=\left\|\boldsymbol{c}(\boldsymbol{A}-\boldsymbol{I}) E\left[t_{k}-1\right]\right\|_{\infty} .
$$

o bien mediante

$$
\left\|w_{k}\right\|_{R M S}=\frac{1}{2}\left\|\boldsymbol{c}(\boldsymbol{A}-\boldsymbol{I}) E\left[t_{k}-1\right]\right\|_{R M S}
$$

Si se considera que la matriz $\boldsymbol{A}$ toma valores cercanos a la unidad (porque el sistema es estable o porque la frecuencia de control es suficientemente rápida), esta última técnica puede dar mejores resultados que la técnica anterior $\mathbf{I 4}$, ya que

$$
1-\alpha_{k}<\frac{1-\alpha_{k}}{\alpha_{k}}, \quad \forall \alpha_{k} \in(0,1)
$$

Esta observación, junto con el hecho de que la estrategia I4 tiene un ruido ficticio que tiende a infinito cuando $\alpha_{k}$ tiende a cero, indica que es preferible la estrategia I5 frente a la $\mathbf{I} 4$ 
Ejemplo 3.6.7. Sea el proceso inestable

$$
G(s)=\frac{1}{(s-1)(s+3)}
$$

con un periodo de control de $T=0.1$ segundos, en el que se conoce que, en funcionamiento normal, la entrada tiene un valor máximo de $\|u[t]\|_{\infty}=26$. Se asume que el proceso está afectado de una perturbación de cota $\left\|v_{k}\right\|_{\infty}=0.5$. Se asume que las muestras llegan al sistema de control cada $N_{k} \in\{10, \ldots, 15\}$ periodos de forma asíncrona con la actualización de la acción de control, pero sin retardo. Se diseñan dos predictores para dicho sistema: uno basado en la estrategia de interpolación I1, y el otro basado en la estrategia I5. El ruido ficticio introducido con la estrategia I1 tiene un valor máximo (obtenido mediante (3.105) ) de valor $\left\|w_{k}\right\|_{\infty}=2.6$, mientras que el ruido ficticio introducido con la interpolación I5 se considera de cota $\left\|w_{k}\right\|_{\infty}=1.0$ (estimación obtenida mediante simulaciones del predictor con medidas síncronas). Con ayuda de estos datos, se calculan las ganancias de dos predictores basados en la atenuación $\ell_{1}$ para cada una de las estrategias (I1, e I5). En la figura 3.26 se muestra un detalle de una simulación del predictor, donde se observa el mejor resultado obtenido con la técnica de interpolación I1. Esto es debido a que el ruido ficticio introducido por la técnica I5 depende del error de estimación inicial que, al tratarse de un sistema inestable, es elevado. En concreto, con la técnica de interpolación I1 se ha obtenido un error de estimación que viene determinado por $\|e[t]\|_{R M S}=0.6366$ y $\|e[t]\|_{\infty}=2.7182$, mientras que con la técnica de interpolación 15 se ha tenido un error de estimación determinado por $\|e[t]\|_{R M S}=0.7773 y\|e[t]\|_{\infty}=2.7271$. Para hacer la simulación se ha estabilizado el proceso con muestreo estándar y se ha puesto una referencia variante en el tiempo. La secuencia de acciones de control obtenida en esa simulación es la utilizada para las predicciones entre muestreos.

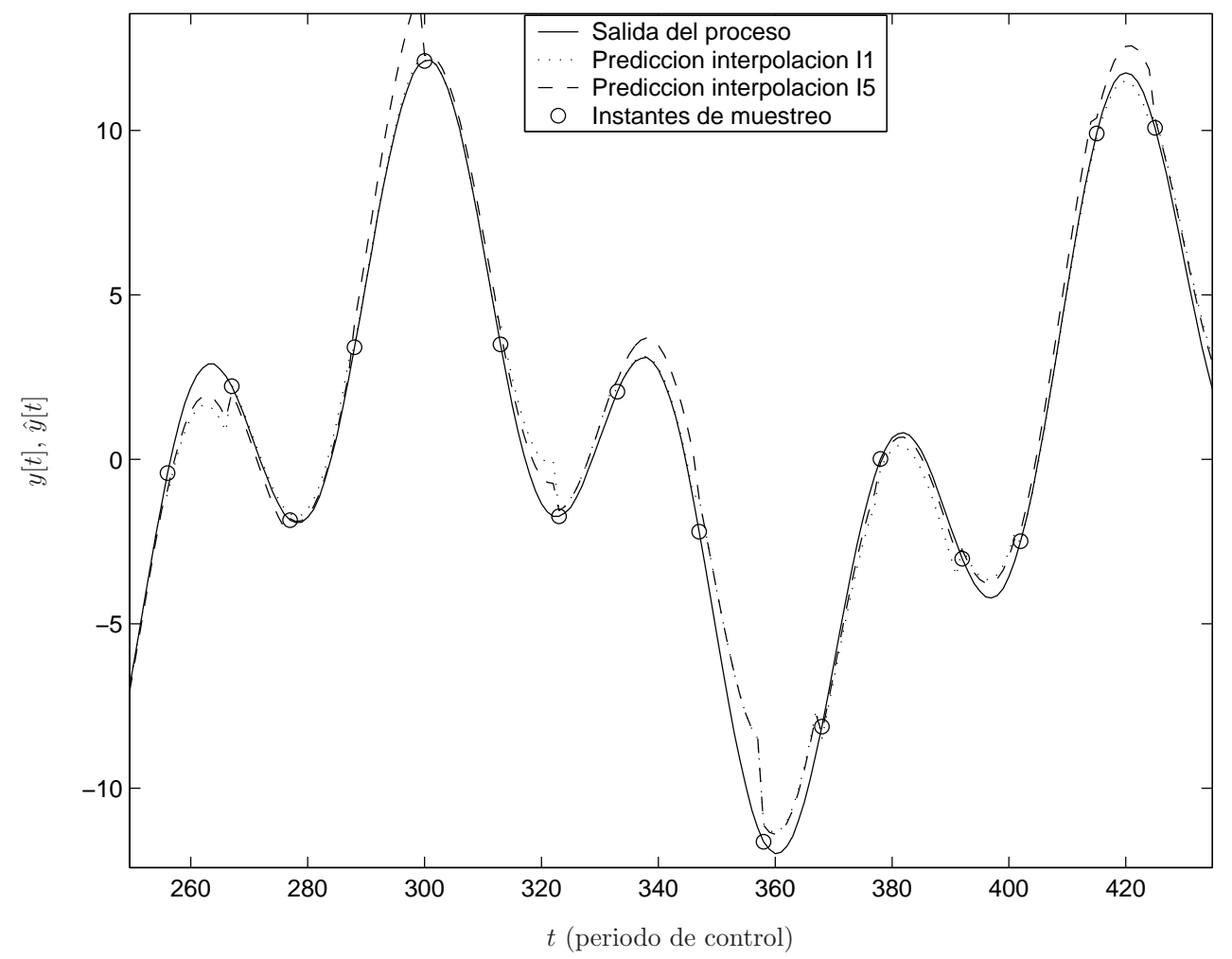

Figura 3.26: Evolución de la salida y de las predicciones con diferentes métodos de interpolación para un proceso con mediciones asíncronas. $\mathcal{N}=$ $\{10, \ldots, 15\}, \mathcal{D}=\{0\}$. Diseño $\ell_{1}$

Considérese ahora el mismo proceso, pero sometido a un muestreo definido por $N_{k} \in\{2,3,4,5\}$, cuyas mediciones se toman de forma asíncrona. Se diseñan de nuevo dos predictores basados en las metodologías de interpolación I1 e I5, y basados en la atenuación $\ell_{1}$. En este caso, el error de predicción obtenido mediante la técnica I1 viene determinado por $\|e[t]\|_{R M S}=0.4896$ y $\|e[t]\|_{\infty}=1.4670$, mientras que el error obtenido con la técnica I5 viene determinado por $\|e[t]\|_{R M S}=0.4345$ y $\|e[t]\|_{\infty}=1.2170$.

Este último resultado indica que según la frecuencia de muestreo puede ser más conveniente utilizar uno u otro método. El método de interpolación I5 será mejor para frecuencias altas de muestreo, ya que 
a bajas frecuencias de muestreo, el error inicial de estimación es elevado y provoca la introducción de un elevado ruido ficticio (véase (3.119)). Para frecuencias bajas de muestreo es más conveniente el método I1, ya que el ruido ficticio que se introduce no depende del error de estimación inicial, que suele ser elevado cuando se trabaja con un proceso inestable.

El ejemplo anterior ha puesto de manifiesto que la mejor manera de decidir la utilización de la estrategia I1 o la I5 es realizando una simulación y estimando el valor del error $\|e[t]\|$ con cada una de las técnicas.

\subsubsection{Minimización del error entre mediciones}

En los diseños de las secciones anteriores se ha tratado de minimizar la norma del error de estimación de la salida en el instante de medición $\left(\left\|e_{k}\right\|\right)$. El error de predicción entre mediciones se puede calcular sabiendo que el modelo se corre en bucle abierto (ecuación (3.16)), con lo que

$$
e\left[t_{k}+r\right]=\boldsymbol{c} E\left[t_{k}+r\right]=\boldsymbol{c} \boldsymbol{A}^{r} E\left[t_{k}\right]+\boldsymbol{c}\left(V\left[t_{k}+r\right]-\boldsymbol{A}^{r} V\left[t_{k}\right]\right)
$$

con $r<N_{k+1}$. La norma del error se puede acotar mediante

$$
\left\|e\left[t_{k}+r\right]\right\|_{p} \leq\left\|\boldsymbol{c} \boldsymbol{A}^{r}\right\|_{i p}\left\|E\left[t_{k}\right]\right\|_{p}+\left\|\boldsymbol{c}\left(\boldsymbol{I}-\boldsymbol{A}^{r}\right)\right\|_{i p}\left\|V\left[t_{k}\right]\right\|_{p}
$$

donde se observa que la norma del error de predicción entre mediciones será tanto menor como menor sea la norma $\left\|E\left[t_{k}\right]\right\|$. Si la escasez de medidas no es muy elevada ( $N_{k}$ pequeño), los algoritmos expuestos hasta el momento en la sección darán un buen funcionamiento. Pero si la escasez de medidas es muy elevada y además el proceso es inestable, es más conveniente acotar la norma del vector de errores $\left\|E_{k}\right\|$ en lugar de $\left\|e_{k}\right\|=\left\|\boldsymbol{c} E_{k}\right\|$ para asegurar que el error entre mediciones es pequeño. Para ello se han de replantear los diseños del capítulo como se ha hecho en $\$ 3.6 .7$, es decir, sustituyendo $\boldsymbol{c}^{\top} \boldsymbol{c}$ por $\boldsymbol{I}$ en las LMI de las secciones $\S 3.4$ y $\$ 3.5$.

Ejemplo 3.6.8. Considérese el sistema

$$
G(s)=\frac{1}{s^{3}+2 s^{2}+3 s+4}
$$

donde la acción de control se actualiza cada $T=0.15$ segundos. Considérese que se toman mediciones de la salida cada $N_{k}$ periodos, siendo

$$
N_{k} \in \mathcal{N}=\{10,11,1,13,14,15\}
$$

Supóngase que las mediciones llegan al predictor sin retardo y que hay una perturbación constante de valor $\|v[t]\|_{\infty}=0.005$ y un ruido de medida aleatorio de media cero con un valor eficaz $\|w[t]\|_{R M S}=0.0293$. Se diseñan dos predictores $\mathcal{H}_{\infty}$, uno de ellos basado en la minimización de la norma de $e_{k}$ (con $\boldsymbol{c}^{\top} \boldsymbol{c}$ en las LMI) y otro basado en la minimización de la norma de $E_{k}$ (con I en las LMI). La figura 3.27 muestra una simulación de ambos predictores, donde se puede observar la mejoría con el predictor basado en la atenuación del error entre mediciones. En efecto, el error cometido con el primer método (minimización de $\left.e_{k}\right)$ viene caracterizado por los valores $\|e[t]\|_{R M S}=0.0392$ y $\|e[t]\|_{\infty}=0.1207$, mientras que con el segundo método (minimización de $E_{k}$ ) se obtiene un error de predicción menor, definido por los valores $\|e[t]\|_{R M S}=0.0236\|e[t]\|_{\infty}=0.0766$, es decir que ha habido una reducción del error del $38 \%$.

Considérese ahora el mismo proceso sin retardos, pero en el que el muestreo se realiza cada $N_{k}=2$ periodos de control. Para este caso se vuelven a diseñar ambos predictores, teniéndose para el predictor basado en la atenuación de $e_{k}$ un error determinado por $\|e[t]\|_{R M S}=0.0335$ y $\|e[t]\|_{\infty}=0.0748$, mientras que con el diseño del predictor basado en la atenuación de $E_{k}$ se obtiene un error determinado por $\|e[t]\|_{R M S}=0.0386$ y $\|e[t]\|_{\infty}=0.1065$, que no mejora el resultado anterior.

El resultado obtenido en el último ejemplo pone de manifiesto que la técnica de minimización del error $E_{k}$ expuesta en esta sección puede mejorar el error de predicción en bucle abierto cuando el muestreo es muy escaso ( $N_{k}$ elevado), pero puede empeorarlo (respecto de la técnica basada en la minimización de $e_{k}$ ) cuando el muestreo es bastante frecuente $\left(N_{k}\right.$ pequeño). En cada ejemplo en concreto se tendrán que estudiar ambas alternativas e implementar aquella que pueda proporcionar mejores resultados. 


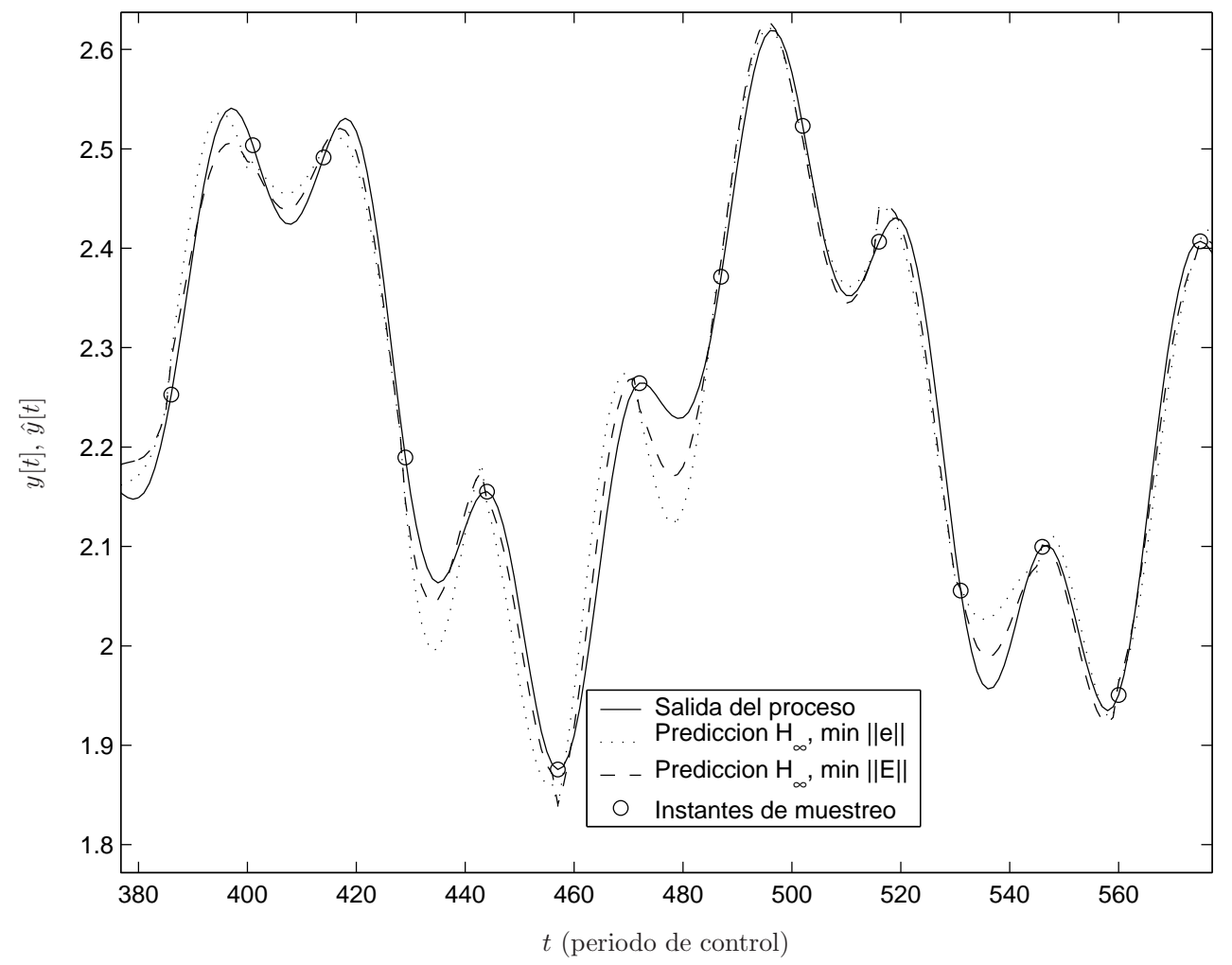

Figura 3.27: Evolución de la salida y de las predicciones con diferentes métodos (minimización de $e_{k}$ o de $\left.E_{k}\right) . \mathcal{N}=\{10,11,12,13,14,15\}, \mathcal{D}=\{0\}$.

\subsubsection{Identificación de parámetros en procesos con medidas escasas}

Una de las ventajas de utilizar el predictor de salidas con modelo entrada salida es el hecho de que se tiene en cada instante el vector de regresión de salidas estimado. Este hecho lo hace idóneo para implementar algoritmos de identificación en línea con medidas escasas (véase [57]). En el trabajo [3] se trata la inicialización de estos algoritmos y queda patente la necesidad de diseñar un predictor con medidas escasas a partir de un modelo preliminar con gran incertidumbre.

Si se utilizan las técnicas expuestas a lo largo del capítulo se consiguen predictores que minimizan la norma del error cometido en la estimación de la salida actual $\left(\left\|e_{k}\right\|\right)$. Sin embargo, cuando este vector de regresión que se actualiza se utiliza para la identificación en línea del proceso, es más razonable minimizar la cota de la norma del vector de error de predicción $\left(\left\|E_{k}\right\|\right)$, es decir del error de predicción en todos los instantes (el error de predicción en bucle abierto y el de los instantes de actualización). Para conseguir esta modificación basta con sustituir la matriz $\boldsymbol{c}^{\top} \boldsymbol{c}$ por la matriz identidad de orden $n(\boldsymbol{I})$. El siguiente teorema muestra, a modo de ejemplo, como queda el diseño del predictor $\mathcal{H}_{\infty}$ que permite minimizar la cota del vector de errores de predicción en el caso determinista tras realizar la sustitución indicada.

Teorema 3.6.1 (Atenuación $\mathcal{H}_{\infty}$ en el vector de regresión). Considérese el predictor (3.8) aplicado al sistema (3.7) y supóngase que hay una medida disponible con un retardo $d_{k} \in \mathcal{D}$ cada $N_{k} \in \mathcal{N}$, habiendo $n_{\mathcal{S}}$ escenarios de muestreo $s_{k}$ posibles. Para unos $\gamma_{v}, \gamma_{w}>0$ dados, supóngase que existen unas matrices $\boldsymbol{P}\left(s_{k}\right)=\boldsymbol{P}\left(s_{k}\right)^{\top} \in \mathbb{R}^{n \times n}, \boldsymbol{Q}\left(s_{k}\right) \in \mathbb{R}^{n \times n}, \boldsymbol{X}\left(s_{k}\right) \in \mathbb{R}^{n \times 1}$ tales que la siguiente PLMI

$$
\left[\begin{array}{ccc}
\boldsymbol{Q}\left(s_{k}\right)+\boldsymbol{Q}\left(s_{k}\right)^{\top}-\boldsymbol{P}\left(s_{k}\right) & \boldsymbol{M}_{A}\left(s_{k}\right) & \boldsymbol{M}_{B}\left(s_{k}\right) \\
\boldsymbol{M}_{A}\left(s_{k}\right)^{\top} & \boldsymbol{P}\left(s_{k-1}\right)-\boldsymbol{I} & \mathbf{0} \\
\boldsymbol{M}_{B}\left(s_{k}\right)^{\top} & \mathbf{0} & \boldsymbol{\Gamma}^{2}
\end{array}\right] \succ \mathbf{0}
$$

se cumple para cualquier secuencia de muestreo $\left\{s_{k}\right\}$. Entonces, definiendo la ganancia del predictor en función de la situación de muestreo según $\boldsymbol{\ell}\left(s_{k}\right)=\boldsymbol{Q}\left(s_{k}\right)^{-1} \boldsymbol{X}\left(s_{k}\right)$, el error de predicción del algoritmo definido por (3.8) en ausencia de perturbaciones converge asintóticamente a cero y, bajo condiciones iniciales nulas,

$$
\left\|E_{k}\right\|_{2}^{2} \leq \gamma_{v}^{2}\left\|v_{k}\right\|_{2}^{2}+\gamma_{w}^{2}\left\|w_{k}\right\|_{2}^{2}
$$


Prueba 3.6.1. La demostración se consigue siguiendo los mismos pasos que en la demostración del teorema 3.4 .2 teniendo en cuenta que la expresión (3.49) será en este caso

$$
E_{k}^{\top} \boldsymbol{P}\left(s_{k}\right) E_{k}-E_{k-1}^{\top} \boldsymbol{P}\left(s_{k-1}\right) E_{k-1}+E_{k-1}^{\top} E_{k-1}-\frac{\gamma_{v}}{3 n} \mathbb{V}_{k}^{\top} \mathbb{V}_{k}-\gamma_{w} w_{k}^{\top} w_{k}<0,
$$

con lo que tomando condiciones iniciales nulas, y que $E_{k}^{\top} \boldsymbol{P}\left(s_{k}\right) E_{k}>0$, se puede sumar la expresión anterior entre $k=1$ y $k=\infty$ para llegar a (3.123).

\subsubsection{Diseño de predictores con objetivos combinados}

Otro problema no contemplado en los diseños anteriores es el caso en el que se desea minimizar el error de predicción frente a perturbaciones y ruidos de diferente naturaleza como, por ejemplo, una perturbación persistente acotada en $\ell_{2}$ y un ruido de medida impulsional. Este caso se resuelve mediante técnicas multiobjetivo o técnicas de objetivos combinados (véase [59]). El diseño de un predictor para un objetivo dado (atenuación estocástica $\mathcal{H}_{\infty}$, por ejemplo) se basa en la resolución de una LMI (la LMI (3.80), por ejemplo). Una forma de diseñar un predictor que cumpla con varios objetivos de forma simultánea (atenuación estocástica $\mathcal{H}_{\infty}$ y $\mathcal{H}_{2 g}$, por ejemplo), consiste en solucionar el conjunto de LMIs que forman ambos objetivos compartiendo las variables matriciales. El siguiente teorema muestra a modo de ejemplo el planteamiento del diseño combinado $\mathcal{H}_{2 g} / \mathcal{H}_{\infty}$ para el caso determinista.

Teorema 3.6.2 (Atenuación $\left.\mathcal{H}_{2 g} / \mathcal{H}_{\infty}\right)$. Considérese el predictor (3.8) aplicado al sistema (3.7) y supóngase que hay una medida disponible con un retardo $d_{k} \in \mathcal{D}$ cada $N_{k} \in \mathcal{N}$ periodos, habiendo $n_{\mathcal{S}}$ escenarios de muestreo $s_{k} \in \mathcal{S}$ posibles. Supóngase que son conocidas las normas $\ell_{2}$ y $\ell_{\infty}$ de las perturbaciones $y$ el ruido de medida. Para unos $\alpha_{\infty}, \alpha_{2}$ dados, defínanse unas matrices $\boldsymbol{P}\left(s_{k}\right)=\boldsymbol{P}\left(s_{k}\right)^{\top} \in \mathbb{R}^{n \times n}$, $\boldsymbol{Q}\left(s_{k}\right) \in \mathbb{R}^{n \times n}, \boldsymbol{X}\left(s_{k}\right) \in \mathbb{R}^{n \times 1}$, que minimizan la función de coste

$$
J=\alpha_{\infty}\left(\gamma_{v, \infty}\left\|v_{k}\right\|_{2}^{2}+\gamma_{w, \infty}\left\|w_{k}\right\|_{2}^{2}\right)+\alpha_{2}\left(\gamma_{v, 2}\left\|v_{k}\right\|_{2}^{2}+\gamma_{w, 2}\left\|w_{k}\right\|_{2}^{2}\right)
$$

a lo largo de todas las variables $P\left(s_{k}\right), \boldsymbol{Q}\left(s_{k}\right), \boldsymbol{X}\left(s_{k}\right), \gamma_{v, \infty}, \gamma_{w, \infty}, \gamma_{v, 2}, \gamma_{w, 2}$ que cumplen

$$
\begin{aligned}
& {\left[\begin{array}{ccc}
\boldsymbol{Q}\left(s_{k}\right)+\boldsymbol{Q}\left(s_{k}\right)^{\top}-\boldsymbol{P}\left(s_{k}\right) & \boldsymbol{M}_{A}\left(s_{k}\right) & \boldsymbol{M}_{B}\left(s_{k}\right) \\
\boldsymbol{M}_{A}\left(s_{k}\right)^{\top} & \boldsymbol{P}\left(s_{k-1}-\boldsymbol{c}^{\top} \boldsymbol{c}\right. & \mathbf{0} \\
\boldsymbol{M}_{B}\left(s_{k}\right)^{\top} & \mathbf{0} & \boldsymbol{\Gamma}_{\infty}^{2}
\end{array}\right] \succ 0,} \\
& {\left[\begin{array}{ccc}
\boldsymbol{Q}\left(s_{k}\right)+\boldsymbol{Q}\left(s_{k}\right)^{\top}-\boldsymbol{P}\left(s_{k}\right) & \boldsymbol{M}_{A}\left(s_{k}\right) & \boldsymbol{M}_{B}\left(s_{k}\right) \\
\boldsymbol{M}_{A}\left(s_{k}\right)^{\top} & \boldsymbol{P}\left(s_{k-1}\right) & \mathbf{0} \\
\boldsymbol{M}_{B}\left(s_{k}\right)^{\top} & \mathbf{0} & \boldsymbol{\Gamma}_{2}^{2}
\end{array}\right] \succ 0,}
\end{aligned}
$$

para cualquier secuencia de muestreo $\left\{s_{k}\right\}$, siendo

$$
\begin{aligned}
& \boldsymbol{\Gamma}_{\infty}=\operatorname{diag}\left\{\frac{\gamma_{v, \infty}}{\sqrt{3 n}}, \cdots, \frac{\gamma_{v, \infty}}{\sqrt{3 n}}, \gamma_{w, \infty}\right\}_{(3 n+1)}, \\
& \boldsymbol{\Gamma}_{\mathbf{2}}=\operatorname{diag}\left\{\frac{\gamma_{v, 2}}{\sqrt{3 n}}, \cdots, \frac{\gamma_{v, 2}}{\sqrt{3 n}}, \gamma_{w, \infty}\right\}_{(3 n+1)} .
\end{aligned}
$$

Entonces, definiendo la ganancia del predictor en función de la situación de muestreo como $\ell\left(s_{k}\right)=$ $\boldsymbol{Q}\left(s_{k}\right)^{-1} \boldsymbol{X}\left(s_{k}\right)$, el error de predicción del algoritmo definido por (3.8) converge asintóticamente a cero en ausencia de perturbación y ruido de medida, y, bajo condiciones iniciales nulas,

$$
\begin{aligned}
\alpha_{\infty}\left\|e_{k}\right\|_{2}^{2}+\alpha_{2}\left\|e_{k}\right\|_{\infty}^{2} \leq & \alpha_{\infty}\left(\gamma_{v, \infty}^{2}\left\|v_{k}\right\|_{2}^{2}+\gamma_{w, \infty}^{2}\left\|w_{k}\right\|_{2}^{2}\right) \\
& +\alpha_{2}\left(\gamma_{v, 2}^{2}\left\|v_{k}\right\|_{2}^{2}+\gamma_{w, 2}^{2}\left\|w_{k}\right\|_{2}^{2}\right) .
\end{aligned}
$$

Observación 3.6.5. El teorema anterior también puede plantearse cuando hay señales persistentes de valor eficaz conocido (diseño $\mathcal{H}_{\infty}$ ) en combinación con señales de energía finita (diseño $\mathcal{H}_{2}$ ), quedando en ese caso la cota del error de predicción como

$$
\begin{aligned}
\alpha_{\infty}\left\|e_{k}\right\|_{R M S}^{2}+\alpha_{2}\left\|e_{k}\right\|_{\infty}^{2} \leq & \alpha_{\infty}\left(\gamma_{v, \infty}^{2}\left\|v_{k}\right\|_{R M S}^{2}+\gamma_{w, \infty}^{2}\left\|w_{k}\right\|_{R M S}^{2}\right) \\
& +\alpha_{2}\left(\gamma_{v, 2}^{2}\left\|v_{k}\right\|_{2}^{2}+\gamma_{w, 2}^{2}\left\|w_{k}\right\|_{2}^{2}\right) .
\end{aligned}
$$


Los coeficientes $\alpha_{\infty}$ y $\alpha_{2}$ indican el peso relativo que se le da a cada tipo de perturbación o ruido. Para obtener un valor que permita ajustarse a cada caso concreto es necesario realizar simulaciones o pruebas con el proceso real.

Ejemplo 3.6.9. Tómese el proceso

$$
G(s)=\frac{200}{s^{2}+30 s}
$$

con un periodo de control de 5 milisegundos y un muestreo definido por los conjuntos

$$
\mathcal{N}=\{10, \ldots, 15\}, \quad \mathcal{D}=\{0,1,2\} .
$$

El sistema está afectado por una perturbación en forma de escalón acotada por $\|v[t]\|_{\infty} \equiv\|v[t]\|_{R M S}=0.4$, y por un ruido de medida aleatorio de media cero con una desviación típica de $\sigma_{w}=\|w[t]\|_{R M S}=0.1$. Supóngase que debido a un comportamiento anómalo del sensor se producen algunas mediciones erróneas en ciertos intervalos de tiempo que no son detectables. Estas mediciones consisten en la adición de dos unidades a la cantidad medida en intervalos de 250 milisegundos (equivalente a 50 periodos de control). Esta secuencia de mediciones erróneas se repite a lo largo del tiempo de forma esporádica y separada en el tiempo un tiempo elevado, con lo que puede considerarse como un ruido de medida de energía finita. Se puede obtener una cota de la norma $\left\|w_{k}\right\|_{2}$ teniendo en cuenta las características de este ruido y el valor medio del número de periodos entre muestreos $(\bar{N}=12.5)$. Con esto se obtiene una estimación de la energía del ruido de medida esporádico de valor $\left\|w_{k}\right\|_{2} \approx 2 \cdot \frac{50}{12.5}=8$.

El predictor tiene que tener en cuenta esta característica para que la aparición de medidas con deriva no afecte a la predicción. Para ello se decide realizar un diseño combinado $\mathcal{H}_{\infty} / \mathcal{H}_{2 g}$ que consiste en realizar una minimización de la forma indicada en el teorema 3.6.2 y que devuelve una cota del error de la forma indicada en (3.131), con $\alpha_{\infty}=0.3$ y $\alpha_{2}=0.7$. Para comprobar su funcionamiento se compara en una simulación el error de predicción obtenido con la técnica $\mathcal{H}_{\infty} / \mathcal{H}_{2 g}$ y el obtenido con la técnica $\mathcal{H}_{\infty}$ diseñado sin tener en cuenta el fallo del sensor. En la figura 3.28 se muestra una parte de la simulación donde se puede observar la atenuación conseguida con el método combinado en los instantes en los que falla el sensor (de 450 a 500, de 550 a 600, de 650 a 700) En la simulación realizada se ha obtenido

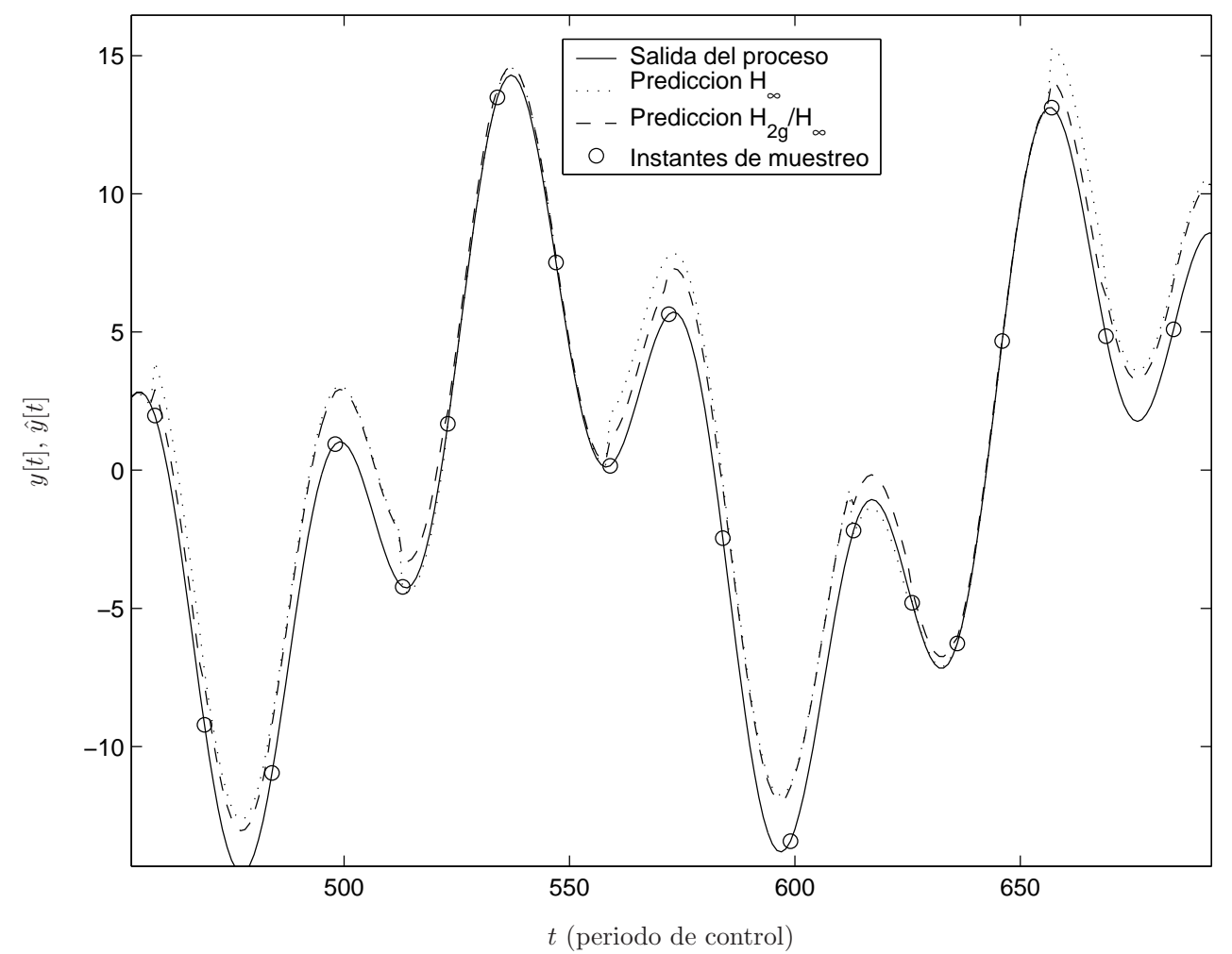

Figura 3.28: Evolución de la salida y de las predicciones con diferentes métodos. $\mathcal{N}=\{10, \ldots, 15\}, \mathcal{D}=\{0,1,2\}$. Perturbación y ruido en forma de escalón 
un error de $\|e[t]\|_{R M S}=1.1$ para el predictor $\mathcal{H}_{\infty}$, y un error de $\|e[t]\|_{R M S}=0.94$ para el predictor $\mathcal{H}_{2 g} / \mathcal{H}_{\infty}$

\subsection{Simplificaciones}

En algunas ocasiones, la solución de los problemas LMI que se han planteado en cada una de las estrategias, puede tener un coste computacional considerable, aún cuando no se trata de un proceso crítico (un proceso estable con perturbaciones de poca magnitud). En otras ocasiones puede ocurrir que la plataforma en la que se implementa el predictor (un microcontrolador de bajo coste, por ejemplo) no disponga de mucha memoria para utilizar por parte del programador del algoritmo de predicción, con lo que no se pueden almacenar todos los vectores de ganancias $\ell\left(s_{k}\right)\left(s_{k}=1, \ldots, n_{\mathcal{S}}\right)$ necesarios. Para estas situaciones se plantean a continuación diferentes estrategias que restringen el espacio de soluciones y llevan a un diseño más conservador a cambio de un menor coste computacional en el cálculo de las ganancias del predictor y una disminución de la memoria requerida para implementar el predictor.

\subsubsection{Reducción del número de vectores de ganancia a almacenar}

En el diseño de predictores basados en un patrón de muestreo variante de forma arbitraria se han asumido diferentes matrices $\boldsymbol{X}\left(s_{k}\right)$ y $\boldsymbol{Q}\left(s_{k}\right)\left(s_{k}=\left\{1, \ldots, n_{\mathcal{S}}\right\}\right)$ para cada uno de los valores del parámetro de muestreo. Esto conduce a conjunto de $n_{\mathcal{S}}$ ganancias diferentes para el predictor que deberían almacenarse para aplicar la ganancia correcta dependiendo de los valores $d_{k}$ y $N_{k}$ que definen el muestreo.

Los teoremas, sin embargo, también son válidos si se imponen algunas restricciones en las matrices $\boldsymbol{Q}\left(s_{k}\right)$ y $\boldsymbol{X}\left(s_{k}\right)$ para reducir el número de ganancias a almacenar. La idea es hacer la ganancia robusta a variaciones de $s_{k}$.

El caso más general puede definirse como sigue. Se divide el conjunto de posibles muestreos $\mathcal{S}$ en $r$ subconjuntos disjuntos, $S_{i}, i=1, \ldots, r$ y se definen $r$ matrices diferentes $\boldsymbol{Q}(i)$ y $\boldsymbol{X}(i), i=1, \ldots, r$, tales que $\boldsymbol{Q}\left(s_{k}\right)=\boldsymbol{Q}(i)$ y $\boldsymbol{X}\left(s_{k}\right)=\boldsymbol{X}(i)$ si $s_{k} \in \mathcal{S}_{i}$. Como resultado, se debe almacenar un conjunto reducido de $r$ vectores de ganancias $\boldsymbol{\ell}(i)=\boldsymbol{Q}(i)^{-1} \boldsymbol{X}(i), i=\ldots, r$. Qué ganancia se utilizará en un instante de muestreo dado dependerá del subconjunto $\mathcal{S}_{i}$ al cual pertenece el par $\left(d_{k}, N_{k}\right)$. El inconveniente de imponer esta restricción en el caso de la búsqueda de un predictor que cumpla con la estabilidad nominal es que el conjunto de LMIs que se ha de resolver es más improbable que sea factible. El inconveniente de esta restricción en el caso de la búsqueda de un predictor que trate de atenuar algún tipo de perturbación es que el conjunto de LMIs devolverá un predictor que hará mayor la cota de la norma del error $e_{k}$.

Un caso particular interesante, que conduce al algoritmo de predicción más simple, se obtiene cuando sólo hay un subconjunto. En este caso se toman dos matrices constantes $\boldsymbol{Q}\left(s_{k}\right)=\boldsymbol{Q}$ y $\boldsymbol{X}\left(s_{k}\right)=\boldsymbol{X}, \forall s_{k}$ en la resolución del conjunto de LMI, y como resultado se obtiene una ganancia constante $\boldsymbol{\ell}=\boldsymbol{Q}^{-1} \boldsymbol{X}$.

Ejemplo 3.7.1. Tómese el sistema inestable

$$
G(s)=\frac{1}{(s-1)(s+3)}
$$

con un periodo de control de $T=0.2$ segundos. Supóngase que se tiene un muestreo síncrono aleatorio con retardo variante definido por los conjuntos

$$
\mathcal{N}=\{1,2,3,4\}, \quad \mathcal{D}=\{0,1,2\}
$$

Supóngase que el proceso está afectado por una perturbación de salida constante y un ruido de medida aleatorio con media cero, de valores

$$
\|v[t]\|_{\infty}=1, \quad\|w[t]\|_{R M S}=1 .
$$

Se quiere diseñar un predictor mediante la técnica $\mathcal{H}_{\infty}$. Para ello se consideran cuatro alternativas posibles

1. Tomar una ganancia constante.

2. Tomar una ganancia diferente para cada posible retardo $d_{k}$ en $\mathcal{D}$.

3. Tomar una ganancia diferente para cada posible $N_{k}$ en $\mathcal{N}$.

4. Tomar una ganancia diferente para cada elemento del conjunto $\mathcal{N} \times \mathcal{D}$. 


\begin{tabular}{|l|c|c|}
\hline Tipo de diseño & Cota de $\left\|e_{k}\right\|_{R M S}$ & Reducción (\%) \\
\hline Ganancia constante & 25.2 & 0 \\
Ganancia diferente para cada $N_{k}$ & 21.9 & 13.3 \\
Ganancia diferente para cada $d_{k}\left(N_{k}, d_{k}\right)$ & 19.6 & 22.2 \\
Ganancia diferente para cada par & 17.1 & 32.4 \\
\hline
\end{tabular}

Tabla 3.7: Cota del error para diferentes tipos de diseño.

Aplicando lo expuesto en la observación anterior, se llegan a diferentes predictores, donde cada uno de ellos consigue una cota en el error de predicción según se indica en la tabla 3.7. Obsérvese la reducción conseguida en cada tipo de diseño con respecto a la utilización de una ganancia constante, que constituye el algoritmo más simple de implementar.

\subsubsection{Reducción del coste computacional de las LMI}

Si lo que se pretende es reducir el coste computacional necesario para calcular (fuera de línea) las ganancias, se pueden aplicar otras simplificaciones que, por contra, llevarán a un problema cuyo espacio de soluciones disminuye.

La primera simplificación consiste en tomar iguales la matriz $\boldsymbol{P}\left(s_{k}\right)$ y $\boldsymbol{Q}\left(s_{k}\right)$.

Otra simplificación consiste en encontrar un predictor que asegure la estabilidad cuadrática (condición suficiente pero no necesaria), haciendo para ello la matriz $\boldsymbol{P}\left(s_{k}\right)=\boldsymbol{P}$ constante. Este caso reduce el espacio de soluciones con el inconveniente de que puede que no se encuentre un predictor estable ante un sistema inestable y/o con muestreo muy escaso, o con el inconveniente de que la atenuación conseguida sea menor que la obtenida con $\boldsymbol{P}\left(s_{k}\right)$ variante.

Si se aplican las dos últimas simplificaciones al mismo tiempo, tomando las matrices $\boldsymbol{P}\left(s_{k}\right)$ y $\boldsymbol{Q}\left(s_{k}\right)$ iguales y de valor constante,

$$
\boldsymbol{Q}\left(s_{k}\right)=\boldsymbol{P}\left(s_{k}\right)=\boldsymbol{P},
$$

la ganancia del predictor vendrá determinada por $\boldsymbol{\ell}\left(s_{k}\right)=\boldsymbol{P}^{-1} \boldsymbol{X}\left(s_{k}\right)$. Nótese que en este caso sólo se cumplirá la condición suficiente de estabilidad cuadrática, con lo que el espacio de soluciones quedará reducido, y el predictor tendrá peores prestaciones.

La mayor simplificación posible consiste en, además de tomar $\boldsymbol{P}\left(s_{k}\right)=\boldsymbol{Q}\left(s_{k}\right)=\boldsymbol{P}$, tomar una matriz $\boldsymbol{X}\left(s_{k}\right)=\boldsymbol{X}$ constante para obtener una matriz de ganancias $\boldsymbol{\ell}_{k}=\boldsymbol{\ell}$ constante, es decir, utilizar todas las simplificaciones expresadas anteriormente. En este caso se tendrá el predictor de peores prestaciones. Aún así, este predictor puede es muy útil como diseño inicial, ya que es el que menor coste computacional tiene tanto en coste de cálculo de la ganancia $\ell$ como en coste de implementación. Como ejemplo, considérese la LMI que devuelve la ganancia $\ell$ en el caso de atenuación $\mathcal{H}_{\infty}$, que queda

$$
\left[\begin{array}{ccc}
\boldsymbol{P} & \boldsymbol{M}_{A}\left(s_{k}\right) & \boldsymbol{M}_{B}\left(s_{k}\right) \\
\boldsymbol{M}_{A}\left(s_{k}\right)^{\top} & \boldsymbol{P}-\boldsymbol{c}^{\top} \boldsymbol{c} & \mathbf{0} \\
\boldsymbol{M}_{B}\left(s_{k}\right)^{\top} & \mathbf{0} & \boldsymbol{\Gamma}^{2}
\end{array}\right] \succ 0,
$$

con

$$
\begin{aligned}
& \boldsymbol{M}_{A}\left(s_{k}\right)=\left(\boldsymbol{P}-\boldsymbol{X} \boldsymbol{c} \boldsymbol{A}^{-d\left(s_{k}\right)}\right) \boldsymbol{A}^{N\left(s_{k}\right)}, \\
& \boldsymbol{M}_{B}\left(s_{k}\right)=\left[\begin{array}{llll}
\boldsymbol{P} & -\boldsymbol{X} \boldsymbol{c} & -\boldsymbol{M}_{A}\left(s_{k}\right) & -\boldsymbol{X}
\end{array}\right] .
\end{aligned}
$$

La ganancia en este caso se calcularía como $\boldsymbol{\ell}=\boldsymbol{P}^{-1} \boldsymbol{X}$.

Ejemplo 3.7.2. Considérese el sistema inestable

$$
G(s)=\frac{1}{(s-1)(s+3)}
$$

cuya entrada se actualiza a periodo $T=0.1$ segundos. Supóngase que se tiene un muestreo síncrono aleatorio con retardo variante definido por los conjuntos

$$
\mathcal{N}=\{1,2,3,4,5\}, \quad \mathcal{D}=\{0,1,2\}
$$


Supóngase que hay una perturbación y un ruido de medida, ambos aleatorios y con un valor eficaz unitario $\left(\left\|v_{k}\right\|_{R M S}=1,\left\|w_{k}\right\|_{R M S}=1\right)$. Se quiere diseñar un predictor mediante la técnica $\mathcal{H}_{\infty}$ y para ello se aplican todas las combinaciones de simplificaciones posibles haciendo bien $\boldsymbol{P}$ constante, bien $\boldsymbol{Q}$ constante o bien $\boldsymbol{X}$ constante. Las prestaciones obtenidas con cada una de las combinaciones de simplificaciones se resumen en la tabla 3.8. Obsérvese la mejora más significativa se produce cuando se utiliza un predictor

\begin{tabular}{cccccccc}
\hline $\boldsymbol{P}$ & $\boldsymbol{P}\left(s_{k}\right)$ & $\boldsymbol{Q}$ & $\boldsymbol{Q}\left(s_{k}\right)$ & $\boldsymbol{X}$ & $\boldsymbol{X}\left(s_{k}\right)$ & Cota de $\left\|e_{k}\right\|_{R M S}$ & Reducción (\%) \\
\hline $\mathrm{x}$ & & $\mathrm{x}$ & & $\mathrm{x}$ & & 30.8 & 0 \\
$\mathrm{x}$ & & $\mathrm{x}$ & & & $\mathrm{x}$ & 19.5 & 36.7 \\
$\mathrm{x}$ & & & $\mathrm{x}$ & & $\mathrm{x}$ & 19.4 & 37.0 \\
& $\mathrm{x}$ & $\mathrm{x}$ & & $\mathrm{x}$ & & 28.1 & 8.8 \\
& $\mathrm{x}$ & $\mathrm{x}$ & & & $\mathrm{x}$ & 19.3 & 37.3 \\
& & $\mathrm{x}$ & & $\mathrm{x}$ & 18.9 & 38.6 \\
\hline
\end{tabular}

Tabla 3.8: Comparación de resultados con diferentes simplificaciones.

de ganancia variante. Cuando, partiendo del predictor más completo, que asegura una cota de $\left\|e_{k}\right\|_{R M S}=$ 18.9 se aplica la simplificación de $\boldsymbol{P}$ y $\boldsymbol{Q}$ constantes, se tiene una cota $\left\|e_{k}\right\|_{R M S}=19.5$, lo que significa una reducción en las prestaciones de sólo un $3.1 \%$ con respecto al predictor completo.

\subsubsection{Interpolación de ganancias}

En la metodología planeada en el capítulo, la obtención de ganancias se basa en la resolución de un problema de optimización por medio de un sistema de inecuaciones lineales matriciales. El coste computacional de estos problemas de optimización vía LMI aumenta con el número de LMIs que conforman el sistema de inecuaciones. El tamaño de este sistema de inecuaciones depende directamente del número de escenarios de muestreo $n_{\mathcal{S}}$. Si se utiliza una matriz $\boldsymbol{P}$ constante, el sistema a resolver está formado por $n_{\mathcal{S}}$ LMIs, pero si se utiliza una matriz $\boldsymbol{P}(i)$ que varía en función del parámetro $s_{k}$, el sistema está formado por $n_{\mathcal{S}}^{2}$ inecuaciones. Esto conlleva un tiempo muy elevado de resolución, que puede llegar a durar varias horas.

Supóngase a partir de ahora que no hay retardos en el proceso y que, por tanto, el número de posibles escenarios de muestreo coincide con el número de periodos intermuestreo. Asúmase que el número de posibles periodos intermuestreo viene definido por el conjunto

$$
\mathcal{N}=\left\{\nu_{1}, \nu_{2} \ldots, \nu_{n_{\mathcal{N}}}\right\}
$$

Para poder reducir el número de LMIs a resolver se toma un subconjunto

$$
\mathcal{N}^{\prime}=\left\{\mu_{1}, \mu_{2} \ldots, \mu_{n_{\mathcal{N}^{\prime}}}\right\} \subset \mathcal{N}, m<n_{\mathcal{S}}
$$

de manera que contenga por lo menos el primer y el último elemento $\left(\mu_{1}=\nu_{1}\right.$ y $\left.\mu_{n_{\mathcal{N}^{\prime}}}=\nu_{n_{\mathcal{N}}}\right)$, y se aplica alguno de los métodos basado en LMIs a lo largo de este nuevo conjunto $\mathcal{N}^{\prime}$. El conjunto de ganancias que se obtendrán como solución viene definido por

$$
\left\{\boldsymbol{\ell}\left(\mu_{i}\right)\right\}, \mu_{i} \in \mathcal{N}^{\prime}
$$

El resto de ganancias asociadas al conjunto $\mathcal{N}$ se obtienen a partir de éstas mediante una interpolación

$$
\begin{aligned}
\ell\left(\nu_{j}\right)= & \ell\left(\mu_{i}\right)+\frac{\nu_{j}-\mu_{i}}{\mu_{i+1}-\mu_{i}}\left(\ell\left(\mu_{i+1}\right)-\ell\left(\mu_{i}\right)\right), \\
& \nu_{j} \in \mathcal{N} \\
& \mu_{i}, \mu_{i+1} \in \mathcal{N}^{\prime} \\
& \mu_{i}<\nu_{j}<\mu_{i+1}
\end{aligned}
$$

El siguiente paso en el diseño mediante esta técnica consiste en comprobar que este nuevo conjunto de ganancias estabiliza el predictor en todo su rango de funcionamiento (es decir, sobre $\mathcal{N}$ ). Este método será adecuado siempre que las variaciones de los elementos del vector $\ell$ sean suaves a lo largo de los elementos de $\mathcal{N}$, tal y como se muestra en los siguientes ejemplos. 
Ejemplo 3.7.3. Sea el proceso continuo

$$
G(s)=\frac{s+1}{s+3}
$$

con un periodo de control de $T=0.1$ segundos. Asúmase un ruido de medida aleatorio de valor eficaz $\|w[t]\|_{R M S}=0.02$ y una perturbación de salida aleatoria de valor eficaz $\|v[t]\|_{R M S}=2.5$. Supóngase un muestreo definido por el conjunto

$$
\mathcal{N}=\{1,2,3,4, \ldots, 46\} .
$$

Con estos datos se puede aplicar un diseño basado en la atenuación $\mathcal{H}_{2}$ mediante las técnicas expuestas en el capítulo. Si se calcula la ganancia $\ell(N)=\left[l_{1}(N), l_{2}(N)\right]^{\top}$ que varía en función del parámetro de muestreo se obtienen los valores que se observan en línea llena en la figura 3.29, mientras que si sólo se calcula la ganancia para un subconjunto

$$
\mathcal{N}^{\prime}=\{1,6,11,16, \ldots, 46\}
$$

se obtienen los valores marcados con una cruz en la figura. El tiempo de cálculo para el subconjunto $\mathcal{N}^{\prime}$ es un quinto del necesario para el subconjunto $\mathcal{N}$. Para mejorar la precisión para muestreos bajos se ha probado con otro subconjunto definido por

$$
\mathcal{N}^{\prime \prime}=\{1,2,3,4,5,6,11,16,21, \ldots, 46\},
$$

con el que se obtienen los valores de ganancia que se muestran con círculos en la figura.

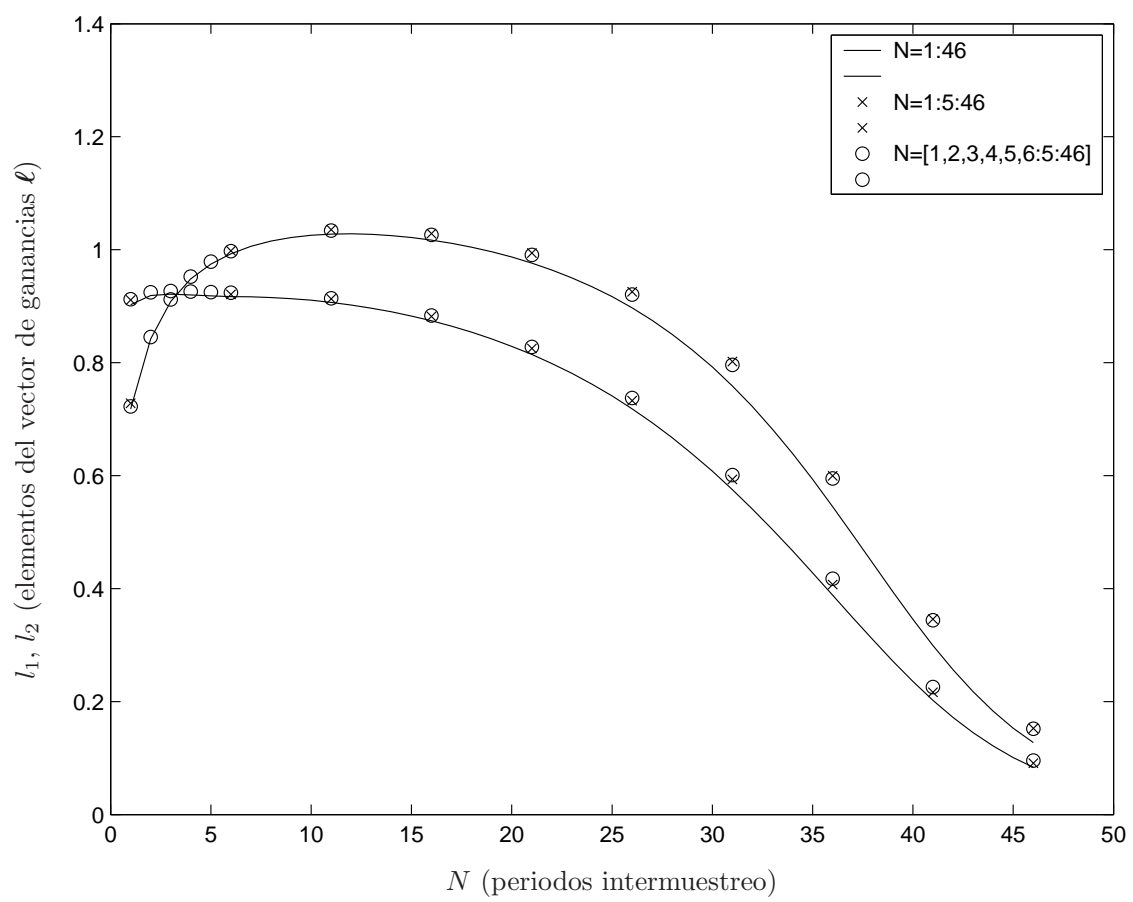

Figura 3.29: Valores de los elementos del vector $\ell(N)$ según los subconjuntos utilizados para realizar los cálculos.

La simulación del predictor con los diferentes métodos $(\ell(N)$ definida para cada $N$, o $\ell(N)$ interpolada a partir de un subconjunto $\{\boldsymbol{\ell}\}$ ) lleva prácticamente a las mismas prestaciones.

Ejemplo 3.7.4. Sea el proceso continuo

$$
G(s)=\frac{1}{s+2 s^{2}+3 s+4}
$$

con un periodo de control de $T=0.15$ segundos. Asúmase un ruido de medida aleatorio de valor eficaz $\|w[t]\|_{R M S}=0.02$ y una perturbación de salida aleatoria de valor eficaz $\|v[t]\|_{R M S}=2.5$. Supóngase un muestreo definido por el conjunto

$$
\mathcal{N}=\{1,2,3,4, \ldots, 46\}
$$


Con estos datos se puede aplicar un diseño basado en la atenuación $\mathcal{H}_{2}$ mediante las técnicas expuestas en el capítulo. Si se calcula la ganancia $\ell(N)=\left[l_{1}(N), l_{2}(N)\right]^{\top}$ que varía en función del parámetro de muestreo se obtienen los valores que se observan en la figura 3.30 en línea llena que, como se observa, no presentan una evolución suave en función de $N$. Si se calcula la ganancia variante para el subconjunto

$$
\mathcal{N}^{\prime}=\{1,6,11,16, \ldots, 46\}
$$

se obtienen las ganancias marcadas con una cruz en la figura. La técnica de interpolación lleva a ganancias intermedias que no se aproximan a las obtenidas con el cálculo mediante el subconjunto $\mathcal{N}$ (por ejemplo, para $N=16$, las ganancias obtenidas con uno y otro método difieren mucho) y, por tanto, es difícil asegurar la estabilidad del predictor.

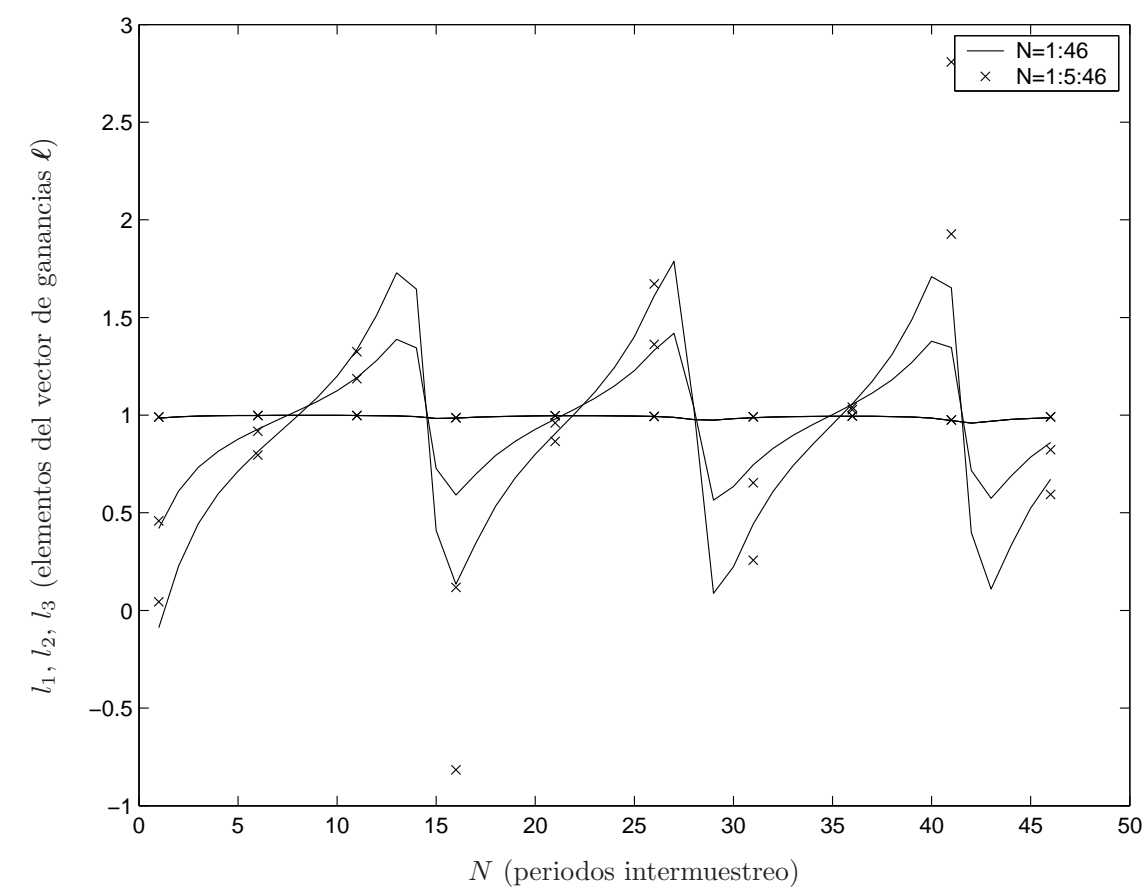

Figura 3.30: Valores de los elementos del vector $\ell(N)$ según los subconjuntos utilizados para realizar los cálculos.

Esta técnica puede ser útil tanto para reducir el coste computacional de obtención de los vectores de ganancia $\ell$, como para reducir los recursos de memoria necesarios para poder implementar el predictor propuesto en microcontroladores de bajo coste.

Un problema diferente que puede darse es que se esté en condiciones de calcular los vectores $\boldsymbol{\ell}$ para un rango de $\mathcal{N}$ amplio, pero, sin embargo, el dispositivo donde se han de almacenar estos valores no tiene memoria suficiente. En este caso, si las variaciones de $\ell$ en función de $N$ son suaves, puede almacenarse sólo un conjunto de vectores $\ell$ correspondientes a un subconjunto $\mathcal{N}^{\prime} \subset \mathcal{N}$, de manera que cada vez que se necesite el vector $\ell(N)$, donde $N$ no pertenece a $\mathcal{N}^{\prime}$, se procederá a la interpolación indicada en (3.135).

\section{Mediciones escasas con retardo}

Si además del muestreo escaso, el proceso o el sistema de medición presenta retardos, de manera que el número de posibles retardos viene definido por el conjunto

$$
\mathcal{D}=\left\{\delta_{1}, \delta_{2}, \ldots, \delta_{n_{\mathcal{D}}}\right\}
$$

se puede partir de un subconjunto de escenarios de muestreo definido por los subconjuntos

$$
\begin{gathered}
\mathcal{N}^{\prime}=\left\{\mu_{1}, \mu_{2} \ldots, \mu_{m}\right\} \subset \mathcal{N}, m<n_{\mathcal{S}} \\
\mathcal{D}^{\prime}=\left\{\gamma_{1}, \gamma_{2} \ldots, \gamma_{n_{\mathcal{D}^{\prime}}}\right\} \subset \mathcal{D}, r<p
\end{gathered}
$$


de manera que contenga por lo menos el primer y el último elemento $\left(\mu_{1}=\nu_{1}, \mu_{n_{\mathcal{N}^{\prime}}}=\nu_{n_{\mathcal{N}}}, \gamma_{1}=\delta_{1} \mathrm{y}\right.$ $\left.\gamma_{r}=\delta_{n_{\mathcal{D}}}\right)$, y se aplica alguno de los métodos de diseño de predictores basado en LMIs a lo largo de este nuevo conjunto $\mathcal{N}^{\prime} \times \mathcal{D}^{\prime}$. El conjunto de ganancias que se obtendrán como solución viene definido por

$$
\left\{\ell\left(\mu_{i}, \gamma_{j}\right)\right\}, \mu_{i} \in \mathcal{N}^{\prime}, \gamma_{j} \in \mathcal{D}^{\prime}
$$

El resto de ganancias asociadas al conjunto $\mathcal{N} \times \mathcal{D}$ se obtienen a partir de éstas mediante una interpolación bidimensional 7 .

\subsubsection{Diseño basado en la minimización de la frecuencia de medición}

Otra de las aplicaciones de los predictores es minimizar la frecuencia de muestro necesaria para controlar un proceso en bucle cerrado. De esta manera se pude minimizar la utilización del canal de comunicación consiguiendo disminuir el ancho de banda necesario y permitiendo compartir el canal con más procesos. Todo esto contribuirá a disminuir los costes del sistema de control. Para obtener este máximo número de periodos hay que tener en cuenta que la norma del error de predicción en bucle abierto viene dada por la ecuación (4.129) y que este error depende de las condiciones iniciales de estimación del estado, del tiempo entre muestras $(N)$ y de la perturbación del estado. También hay que tener en cuenta que el sistema tendrá que ser observable para ese valor de $N$.

Por ejemplo, si se está ante un proceso inestable sin perturbación, puede buscarse el periodo de muestreo más bajo que permita una amplificación de el error de predicción prevista de antemano.

En el siguiente ejemplo se analizan comparativamente las estrategias de simplificación y de diseño (determinista o estocástico) de una forma diferente, mostrando cómo el número de posibles periodos intermuestreo $(\mathcal{N})$ que llevan a un problema LMI factible cambia según la estrategia adoptada, en todos los casos basadas en el diseño para estabilidad nominal.

Ejemplo 3.7.5. Considérese el proceso inestable continuo

$$
G(s)=\frac{10 s-2}{s^{2}-5 s}
$$

con un periodo de actualización de la acción de control de $T=0.02$ segundos. Supóngase que no hay perturbaciones en el proceso y que las mediciones no están afectadas por retardo ni ruidos de medida.

Si se aplica una ganancia constante para asegurar un decrecimiento monótono de la función de Lyapunov con un ratio de decaimiento $\mu=0.6$ (aplicando el teorema 3.4 .1 con $\boldsymbol{Q}\left(s_{k}\right)=\boldsymbol{Q}$ y $\boldsymbol{X}\left(s_{k}\right)=\boldsymbol{X}$ constantes), el rango más ancho de periodos intermuestreo que lleva a una solución factible del problema LMI asociado es $\mathcal{N}_{1}=\{1,2,3\}$, llevando a la ganancia $\ell=\left[\begin{array}{ll}1 & 0.5341\end{array}\right]$. Si el conjunto $\mathcal{N}_{1}$ incluye más periodos intermuestreo, el problema (3.38) no es factible, y no se puede asegurar una convergencia mayor que $\mu=0.6$.

Si la probabilidad asignada a cada escenario de muestreo posible se asume igual, es decir, $\alpha_{i}=1 / \mathrm{m}$ $\left(i=1, \ldots, n_{\mathcal{S}}, n_{\mathcal{S}}=\right.$ length $\left.(\mathcal{N})\right)$, la convergencia en valor medio de un predictor de ganancia constante puede asegurarse para todos los periodos intermuestreo incluidos en el conjunto $\mathcal{N}_{2}=\{1, \ldots, 17\}\left(n_{\mathcal{S}}=17\right.$ aplicando el teorema 3.5.1 con $\boldsymbol{Q}\left(s_{k}\right)=\boldsymbol{Q}$ y $\boldsymbol{X}\left(s_{k}\right)=\boldsymbol{X}$ constantes), lo que muestra que la exigencia de convergencia en valor medio es menos restrictiva que el decrecimiento monótono del error.

Si se utiliza una ganancia variable (aplicando el teorema 3.4.1), el conjunto $\mathcal{N}_{3}$ puede extenderse hasta 30 muestras, por lo menos, tal y como se ha encontrado con las LMI, ya que tanto $\boldsymbol{A}^{N_{k}}$ como $\boldsymbol{\ell}_{k}$ parecen converger para largos periodos intermuestreo, posiblemente hasta un infinito número de muestras.

\subsection{Conclusiones}

En este capítulo se han estudiado distintos métodos de diseño de predictores de la salida aplicables al muestreo síncrono tanto regular como irregular.

En primer lugar se ha definido el escenario de muestreo y las ecuaciones del predictor propuesto, que aborda la problemática de la escasez de medidas y la presencia de retardos de forma conjunta, suponiendo que las mediciones llegan con una etiqueta de tiempo. Este predictor utiliza un modelo del proceso para estimar en bucle abierto la salida y corrige esta estimación cada vez que le llega una medición de la misma. Se ha indicado cómo hacer la implementación que minimiza el coste computacional.

${ }^{7} \mathrm{El}$ comando interp2 de MATLAB ${ }^{\circledR}$ resuelve este problema 
Con la definición del predictor se ha obtenido la dinámica del error de predicción. A diferencia del filtro de Kalman, que utiliza una ganancia que varía en el tiempo de forma arbitraria, se ha propuesto utilizar una ganancia que depende únicamente del escenario de muestreo (retardo $d_{k}$ y número de periodos intermuestreo $N_{k}$ ), consiguiendo así que la implementación del predictor se simplifique considerablemente. Con esta ganancia se ha mostrado que la dinámica del error de predicción es la de un sistema variante en el tiempo dependiente de un parámetro, con lo que se puede analizar la dinámica con técnicas existentes para este tipo de sistemas.

En base al conocimiento de la dinámica del error de predicción se ha desarrollado una metodología basada en técnicas LMI que permite calcular las ganancias que aseguran la estabilidad del predictor a pesar de las variaciones de las condiciones de muestreo, tanto en el caso en el que la disponibilidad de medidas es determinista como en el caso en el que esta disponibilidad es estocástica. También se han aplicado estas técnicas al desarrollo de predictores robustos frente a la presencia de perturbaciones de salida y ruidos de medida. Se ha demostrado que la perturbación a la salida propuesta en el modelo también es válida para modelar perturbaciones de entrada y la incertidumbre en el modelo, con lo que queda cerrado el problema de diseño robusto de forma sencilla. Se ha demostrado también que el retardo aleatorio en la entrada puede modelarse como una incertidumbre en el modelo. Finalmente, se ha demostrado que el ruido de medida propuesto en el modelo también es válido para modelar el error introducido por las mediciones que vienen acompañadas de una etiqueta de tiempo incierta, o por las mediciones asíncronas.

También se ha expuesto cuál es la modificación que se ha de realizar sobre el diseño básico para obtener predictores que minimizan el error del vector de regresión para obtener así predictores más adecuados para la identificación de procesos con medidas escasas.

Finalmente se han expuesto algunas técnicas que permiten reducir el coste computacional de obtención de los vectores de ganancias, así como técnicas que permiten reducir el coste computacional y la memoria requerida para implementar estos predictores. 



\section{Diseño de predictores en representación interna}

\subsection{Introducción}

En el capítulo anterior se han abordado diferentes técnicas de diseño de predictores basado en el modelo entrada-salida de plantas con una entrada y una salida que utilizan un único sensor para medir la salida. Sin embargo, es usual encontrarse con plantas con varias entradas y salidas, incluso con plantas en las que se utilizan diferentes sensores para medir alguna de esas variables (estados o salidas). Además, cada sensor puede tener una dinámica y un retardo diferente (véase figura 1.8, página 5).

Para poder desarrollar predictores en este entorno ya no es válida la representación entrada salida sino que es necesario utilizar una representación interna en la que se incluya la dinámica del proceso (entradas, estados y salidas a controlar) y la de los sensores que relacionan las variables medibles (estados, salidas o combinaciones de ellas) con el valor resultado de la medición.

El objetivo de este capítulo es desarrollar técnicas que permitan estimar el valor de las salidas a un periodo fijo $T$ a partir de las mediciones escasas que se toman de los diferentes sensores y que están afectadas con un retardo variante. En el desarrollo se proponen predictores de bajo coste computacional que tratan de forma conjunta la escasez de medidas, la disponibilidad de sólo algunos sensores cada vez que hay una muestra disponible y el retardo temporal variante asociado a cada sensor. El cálculo de los predictores se realiza mediante técnicas LMI y se diseñan para garantizar la estabilidad frente al muestreo irregular al mismo tiempo que el rechazo a perturbaciones, la robustez frente a errores de modelado o la atenuación del efecto de los retardos en la aplicación de la acción de control. Para obtener los predictores que minimizan el error de predicción es necesario conocer algunas de las características de las perturbaciones, pero si no se conocen, el procedimiento es igualmente válido, ya que hay unos parámetros de ajuste que permiten afinar el predictor.

Otro de los objetivos es estudiar la estimación óptima en plantas con estas características, con lo que se han desarrollado las ecuaciones del filtro de Kalman que incluyen las mediciones escasas con retardo de la misma forma que lo hace el predictor de bajo coste propuesto.

\subsection{Descripción del problema}

La figura 4.1 muestra el problema a tratar, donde se observan diferentes sensores que miden diferentes variables a un periodo diferente (posiblemente variante) y con un retardo diferente. Algunos de estos sensores pueden medir la misma variable (con diferente frecuencia, precisión y retardo) con lo que el caso de fusión sensorial está incluido en el escenario a analizar.

\subsubsection{Planta}

Sea un proceso continuo lineal e invariante en el tiempo descrito por las ecuaciones

$$
\begin{aligned}
\dot{\boldsymbol{x}}(\tau) & =\boldsymbol{A}_{c} \boldsymbol{x}(\tau)+\boldsymbol{B}_{c} \boldsymbol{u}(\tau)+\boldsymbol{B}_{v c} \boldsymbol{v}(\tau), \\
\boldsymbol{y}(\tau) & =\boldsymbol{C}_{y} \boldsymbol{x}(\tau)
\end{aligned}
$$




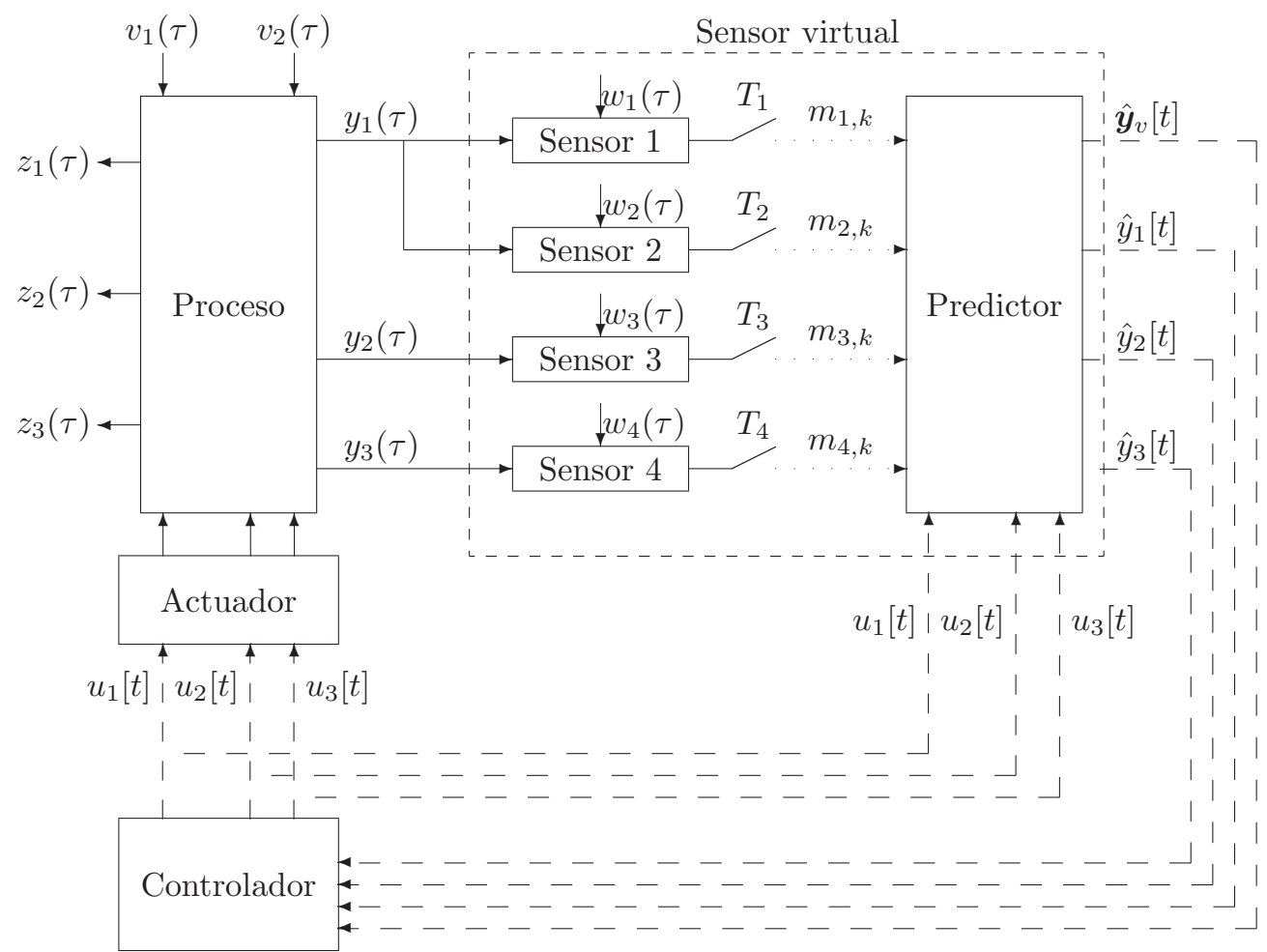

Figura 4.1: Problema general de estimación de salidas de un proceso multivariable con múltiples sensores.

donde $\boldsymbol{x} \in \mathbb{R}^{n}$ es el estado, $\boldsymbol{u} \in \mathbb{R}^{n_{u}}$ es el vector de entradas de control e $\boldsymbol{y} \in \mathbb{R}^{n_{y}}$ es el vector de salidas, que está formado tanto por las salidas medidas $\left(y_{1}, y_{2}, y_{3}\right.$ en la figura) como por el resto de salidas virtuales que necesita el controlador (vector $\boldsymbol{y}_{v}$ en la figura). Para el ejemplo de la figura, el vector $\boldsymbol{y}[t]$ vendría dado por

$$
\boldsymbol{y}[t]=\left[\begin{array}{l}
y_{1}[t] \\
y_{2}[t] \\
y_{3}[4] \\
\boldsymbol{y}_{v}[t]
\end{array}\right] .
$$

La señal $\boldsymbol{v}(\tau) \in \mathbb{R}^{n_{v}}$ es las posible perturbación del estado. Si las señales de control se actualizan a un periodo fijo $T$ a través de un retenedor de orden cero

$$
\boldsymbol{u}(\tau)=\boldsymbol{u}[t] \quad \tau \in[t T, t T+T)
$$

donde $t \in \mathbb{N}$, existe un modelo equivalente discreto que relaciona la secuencia discreta de valores de entrada y la secuencia discreta de los valores de salida en los instantes en los que se actualiza la entrada, que puede expresarse como

$$
\begin{aligned}
\boldsymbol{x}[t+1] & =\boldsymbol{A} \boldsymbol{x}[t]+\boldsymbol{B} \boldsymbol{u}[t]+\boldsymbol{B}_{v} \boldsymbol{v}[t], \\
\boldsymbol{y}[t] & =\boldsymbol{C}_{y} \boldsymbol{x}[t],
\end{aligned}
$$

Las mediciones de los sensores sólo están disponibles en los instantes $t=t_{k}$, y además están afectadas de un retardo variante en el tiempo, con lo que se puede escribir

$$
m_{i, k}=\boldsymbol{c}_{i} \boldsymbol{x}\left[t_{k}-d_{i, k}\right]+w_{i, k}, \quad i=1, \ldots, n_{m}
$$

donde $m_{i, k}$ es la medición disponible del sensor $i$ en el $k$-ésimo muestreo (no todos los sensores están disponibles en cada muestreo), $d_{i, k}$ es el retardo asignado al sensor $i$ en la muestra $k, n_{m}$ es el número de sensores y $w_{i, k}$ es el ruido de medida que tiene el sensor $m_{i}$ en la $k$-ésima muestra.

Si el proceso tiene un retardo entre la entrada $\boldsymbol{u}$ y el estado $\boldsymbol{x}$, se reflejará en que la matriz $\boldsymbol{A}$ tendrá tantos polos en el origen como retardos y, por tanto, no será invertible. En los algoritmos que se desarrollan en este trabajo es necesario que $\boldsymbol{A}$ sea invertible, por lo que es imprescindible transferir los retardos al sistema de medición (añadiendo el valor del retardo en cada $\left.d_{i, k}\right)$ y utilizar un modelo del proceso $(\boldsymbol{A}$, $\boldsymbol{B})$ libre de retardos. 
Observación 4.2.1. La representación anterior también es válida cuando alguno de los sensores tiene una dinámica propia no despreciable. Para demostrarlo, tómense las ecuaciones en diferencias del proceso

$$
\begin{aligned}
\boldsymbol{x}_{p}[t+1] & =\boldsymbol{A}_{p} \boldsymbol{x}_{p}[t]+\boldsymbol{B}_{p} \boldsymbol{u}[t]+\boldsymbol{B}_{v_{p}} \boldsymbol{v}_{p}[t] \\
\boldsymbol{y}[t] & =\boldsymbol{C}_{p} \boldsymbol{x}_{p}[t]
\end{aligned}
$$

y las ecuaciones asociadas a los sensores

$$
\begin{aligned}
\boldsymbol{x}_{s}[t+1] & =\boldsymbol{A}_{s} \boldsymbol{x}_{s}[t]+\boldsymbol{B}_{y} \boldsymbol{y}[t]+\boldsymbol{B}_{v_{s}} \boldsymbol{v}_{s}[t] \\
m_{i, k} & =\boldsymbol{c}_{s, i} \boldsymbol{x}_{s}\left[t_{k}-d_{i, k}\right]+\boldsymbol{d}_{y, i} \boldsymbol{y}\left[t_{k}-d_{i, k}\right]+w_{i, k}, \quad i=1, \ldots, n_{m}
\end{aligned}
$$

Agrupando ambas ecuaciones se puede escribir que

$$
\begin{aligned}
{\left[\begin{array}{l}
\boldsymbol{x}_{p}[t+1] \\
\boldsymbol{x}_{s}[t+1]
\end{array}\right] } & =\underbrace{\left[\begin{array}{cc}
\boldsymbol{A}_{p} & \mathbf{0} \\
\boldsymbol{B}_{y} \boldsymbol{C}_{p} & \boldsymbol{A}_{s}
\end{array}\right]}_{=\boldsymbol{A}}\left[\begin{array}{l}
\left.\boldsymbol{x}_{p}[t]\right] \\
\boldsymbol{x}_{s}[t]
\end{array}\right]+\underbrace{\left[\begin{array}{c}
\boldsymbol{B}_{p} \\
\mathbf{0}
\end{array}\right]}_{=\boldsymbol{B}} \boldsymbol{u}[t]+\underbrace{\left[\begin{array}{cc}
\boldsymbol{B}_{v_{p}} & \mathbf{0} \\
\mathbf{0} & \boldsymbol{B}_{v_{s}}
\end{array}\right]}_{=\boldsymbol{B}_{v}} \underbrace{\left[\begin{array}{l}
\boldsymbol{v}_{p}[t] \\
\boldsymbol{v}_{s}[t]
\end{array}\right]}_{=\boldsymbol{v}[t]} \\
\boldsymbol{y}[t] & =\underbrace{\left[\begin{array}{ll}
\boldsymbol{C}_{p} & \mathbf{0}
\end{array}\right]}_{=\boldsymbol{C}_{y}}\left[\begin{array}{l}
\boldsymbol{x}_{p}[t] \\
\boldsymbol{x}_{s}[t]
\end{array}\right] \\
m_{i, k} & =\underbrace{\left[\begin{array}{ll}
\boldsymbol{d}_{y} \boldsymbol{c}_{p} & \boldsymbol{c}_{s, i}
\end{array}\right]}_{=\boldsymbol{c}_{i}}\left[\begin{array}{l}
\boldsymbol{x}_{p}\left[t_{k}-d_{i, k}\right] \\
\boldsymbol{x}_{s}\left[t_{k}-d_{i, k}\right]
\end{array}\right]+w_{i, k} .
\end{aligned}
$$

Tomando el estado de la planta completa (proceso más sensores) como aquel que agrupa el estado del proceso más el de los sensores

$$
\boldsymbol{x}[t]=\left[\begin{array}{l}
\boldsymbol{x}_{p}[t] \\
\boldsymbol{x}_{s}[t]
\end{array}\right],
$$

se llega a la representación (4.2).

Ejemplo 4.2.1. Sea un proceso cuya dinámica viene definida por las ecuaciones

$$
\begin{aligned}
\boldsymbol{x}_{p}[t+1] & =\left[\begin{array}{ccc}
0.9 & 0.8 & 0.6 \\
0.8 & 0 & 0.7 \\
0 & 0.7 & 0.8
\end{array}\right] \boldsymbol{x}_{p}[t]+\left[\begin{array}{cc}
1 & 2 \\
0 & 1 \\
3 & -2
\end{array}\right] \boldsymbol{u}[t]+\left[\begin{array}{l}
0.1 \\
0.2 \\
0.3
\end{array}\right] \boldsymbol{v}_{p}[t], \\
\boldsymbol{y}[t] & =\left[\begin{array}{ccc}
1 & 0 & 0 \\
0 & 1 & 0
\end{array}\right] \boldsymbol{x}_{p}[t]
\end{aligned}
$$

y sea el sistema de medición de salidas con una dinámica que viene definida por las ecuaciones

$$
\begin{aligned}
\boldsymbol{x}_{s}[t+1] & =\left[\begin{array}{cc}
0.1 & 0 \\
0 & 0.1
\end{array}\right] \boldsymbol{x}_{s}[t]+\left[\begin{array}{ll}
2 & 1 \\
0 & 1
\end{array}\right] \boldsymbol{y}[t] \\
\boldsymbol{m}[t] & =\left[\begin{array}{ll}
1 & 0 \\
0 & 1
\end{array}\right] \boldsymbol{x}_{s}[t]+\boldsymbol{w}[t] .
\end{aligned}
$$

El sistema global que incluye la dinámica del proceso y de los sensores puede escribirse de forma compacta como

$$
\begin{aligned}
\boldsymbol{x}[t+1] & =\left[\begin{array}{ccccc}
0.9 & 0.8 & 0.6 & 0 & 0 \\
0.8 & 0 & 0.7 & 0 & 0 \\
0 & 0.7 & 0.8 & 0 & 0 \\
2 & 0 & 0 & 0.1 & 0 \\
0 & 1 & 0 & 0 & 0.1
\end{array}\right] \boldsymbol{x}[t]+\left[\begin{array}{cc}
1 & 2 \\
0 & 1 \\
3 & -2 \\
0 & 0 \\
0 & 0
\end{array}\right] \boldsymbol{u}[t]+\left[\begin{array}{c}
0.1 \\
0.2 \\
0.3 \\
0 \\
0
\end{array}\right] \boldsymbol{v}[t], \\
\boldsymbol{y}[t] & =\left[\begin{array}{lllll}
1 & 0 & 0 & 0 & 0 \\
0 & 1 & 0 & 0 & 0
\end{array}\right] \boldsymbol{x}[t], \\
\boldsymbol{m}[t] & =\left[\begin{array}{lllll}
0 & 0 & 0 & 1 & 0 \\
0 & 0 & 0 & 0 & 1
\end{array}\right] \boldsymbol{x}[t]+\boldsymbol{w}[t]
\end{aligned}
$$




\subsubsection{Escenario de muestreo}

El sistema de control necesita los valores de $\boldsymbol{y}[t T]$, pero se asume que no están disponibles. En su lugar, se miden los valores de algunas de las variables $m_{i}(\tau)$ en diferentes instantes discretos $t=t_{k}, k \in \mathbb{N}$, definiéndose $N_{k}=t_{k}-t_{k-1}$ como el número de actualizaciones de la entrada desde $t_{k-1}$ hasta $t_{k}$, con lo que

$$
t_{k}=\sum_{i=1}^{k} N_{i}
$$

representa el instante en el cual se da la $t$-ésima actualización del control y se obtiene la $k$-ésima muestra compuesta por los valores de algunos de los sensores (los que están disponibles). Se asume que el número de actualizaciones de la entrada entre mediciones $\left(N_{k}\right)$ varía dentro de un conjunto finito de valores

$$
N_{k} \in \mathcal{N}=\left\{\nu_{1}, \nu_{2} \ldots, \nu_{n_{\mathcal{N}}}\right\}
$$

y que el número de periodos que tarda cada medición en estar disponible $\left(d_{i, k}\right)$ varía dentro de un conjunto finito de valores

$$
d_{i, k} \in \mathcal{D}_{i}=\left\{\delta_{i 1}, \ldots, \delta_{i, p_{i}}\right\}
$$

Si el proceso tiene algún retardo constante en alguna de sus salidas puede asignarse su valor a las señales de los sensores que miden dicha salida. Para ello se debe añadir el valor de este retardo en el valor $d_{i, k}$ correspondiente.

Para denotar qué sensores están disponibles en cada instante de muestreo se define el factor de disponibilidad del sensor $i$ en el instante $t_{k}$ como

$$
\delta_{i}\left[t_{k}\right]= \begin{cases}1, & \text { si } m_{i}\left[t_{k}\right] \text { está disponible, } \\ 0, & \text { si } m_{i}\left[t_{k}\right] \text { no está disponible }\end{cases}
$$

Agrupando los valores del factor de disponibilidad para cada sensor se puede definir la matriz de disponibilidad como

$$
\boldsymbol{\Delta}\left[t_{k}\right]=\left[\begin{array}{ccc}
\delta_{1}\left[t_{k}\right] & & 0 \\
& \ddots & \\
0 & & \delta_{n_{m}}\left[t_{k}\right]
\end{array}\right] .
$$

Dependiendo del patrón de muestreo, pueden haber diferentes valores para la matriz $\left.\boldsymbol{\Delta}\left[t_{k}\right]\right]^{1}$. Se asume que estos valores están dentro de un conjunto finito dado por

$$
\boldsymbol{\Delta}\left[t_{k}\right] \in \Xi=\left\{\boldsymbol{\Delta}_{1}, \ldots, \boldsymbol{\Delta}_{p}\right\}
$$

En el caso general, es posible cualquier combinación de mediciones disponibles, con lo que $p=2^{n_{m}}-1$.

\subsubsection{Parametrización del escenario de muestreo}

Cada instante en el que llega alguna medida viene definido por varios valores: $\boldsymbol{\Delta}_{k}$ que indica de qué sensores ha llegado un dato, $d_{i, k}\left(i=1,2, \ldots, n_{m}\right)$ que indica con qué retardo ha llegado cada medición, y $N_{k}$ que indica el número de veces que se ha actualizado la acción de control desde la última vez que llego algún dato de salida. Aunque cada uno de estos parámetros puede tomar valores dentro de unos rangos conocidos (4.8) y (4.10), puede que sólo algunas de las combinaciones $\left(\boldsymbol{\Delta}_{k}, N_{k}, d_{i, k}\right)$ sean posibles. De forma similar a los sistemas de una entrada y una salida, se define un parámetro de muestreo $s_{k}$ que indica cuál es la situación de muestreo (combinación $\left.\left(\boldsymbol{\Delta}_{k}, N_{k}, d_{i, k}\right)\right)$ con la llegada de la $k$-ésima medida. El parámetro $s_{k}$ enumera las combinaciones tomando valores enteros de 1 a $n_{\mathcal{S}}$,

$$
s_{k} \in \mathcal{S}=\left\{1,2, \ldots, n_{\mathcal{S}}\right\}
$$

donde $n_{\mathcal{S}}$ es el número de combinaciones posibles.

\footnotetext{
${ }^{1} \mathrm{Si}$ en un instante dado $t=t_{k}$ todas las mediciones de salida están disponibles, entonces $\boldsymbol{\Delta}\left[t_{k}\right]=\boldsymbol{I}$.
} 


\subsection{Predictor}

El controlador necesita la secuencia de estados o salidas (dependiendo del tipo de controlador) al periodo $T$, pero sólo se tiene acceso a algunas señales de sensores que miden las salidas de forma irregular y con un retraso. Para estimar los estados y las salidas a periodo $T$ se propone un predictor que tiene una estructura similar al expuesto con la representación externa.

Inicialmente se estima el estado corriendo el modelo en bucle abierto, lo que lleva a

$$
\hat{\boldsymbol{x}}[t \mid t-1]=\boldsymbol{A} \hat{\boldsymbol{x}}[t-1]+\boldsymbol{B} \boldsymbol{u}[t-1] .
$$

Dependiendo de la disponibilidad de una nueva medición en $t=t_{k}$ (es decir, algún $m_{i}[t]$ está disponible), el estado se actualiza mediante

$$
\hat{\boldsymbol{x}}\left[t_{k}\right]=\hat{\boldsymbol{x}}\left[t_{k} \mid t_{k}-1\right]+\sum_{i=1}^{n_{m}} \boldsymbol{\ell}_{i}\left[t_{k}\right]\left(m_{i}\left[t_{k}\right]-\boldsymbol{c}_{i} \hat{\boldsymbol{x}}\left[t_{k}-d_{i, k} \mid t_{k}-1\right]\right) \delta_{i}\left[t_{k}\right] .
$$

donde $\boldsymbol{\ell}_{i}\left[t_{k}\right]$ es el vector de ganancias utilizado para actualizar la estimación del estado con la medición $m_{i}\left[t_{k}\right]$. La predicción de la salida se obtiene mediante la ecuación de salidas

$$
\hat{\boldsymbol{y}}[t]=\boldsymbol{C}_{y} \hat{\boldsymbol{x}}[t]
$$

La ecuación (4.12b) utiliza la estimación retardada del estado $\hat{\boldsymbol{x}}\left[t_{k}-d_{i, k} \mid t_{k}-1\right]$, que se define como la estimación del estado que cumple

$$
\hat{\boldsymbol{x}}\left[t_{k} \mid t_{k}-1\right]=\boldsymbol{A}^{d_{i, k}} \hat{\boldsymbol{x}}\left[t_{k}-d_{i, k} \mid t_{k}-1\right]+\sum_{j=1}^{d_{i, k}} \boldsymbol{A}^{j-1} \boldsymbol{B} \boldsymbol{u}\left[t_{k}-j\right]
$$

y, por tanto, puede calcularse como

$$
\hat{\boldsymbol{x}}\left[t_{k}-d_{i, k} \mid t_{k}-1\right]=\boldsymbol{A}^{-d_{i, k}} \hat{\boldsymbol{x}}\left[t_{k} \mid t_{k}-1\right]+\sum_{j=1}^{d_{i, k}} \boldsymbol{A}^{j-1-d_{i, k}} \boldsymbol{B} \boldsymbol{u}\left[t_{k}-j\right]
$$

\subsubsection{Implementación del predictor}

Con el algoritmo de predicción (4.12), cada vez que llega una nueva medición (en el instante $t=t_{k}$, tal que $\delta_{i}\left[t_{k}\right]=1$ para algún $\left.i\right)$, la estimación del estado con retardo $\hat{\boldsymbol{x}}\left[t_{k}-d_{i, k} \mid t_{k}-1\right]$ debe calcularse con ayuda de la ecuación (4.13). Esta ecuación es relativamente compleja y, por lo tanto, se necesita una potencia de cálculo elevada para evitar un retardo de computación durante la estimación del estado actual. Para reducir la complejidad del cálculo se propone una implementación alternativa de menor coste. La idea es almacenar las estimaciones del estado con retardo y actualizarlas cada vez que llega una medición. De esta forma, la estimación del estado con retardo $\hat{\boldsymbol{x}}\left[t_{k}-d_{i, k} \mid t_{k}-1\right]$ se conoce antes de que la medición $m_{i}\left[t_{k}\right]$ esté disponible.

Para almacenar los estados de instantes anteriores se utiliza un modelo de orden extendido, donde el nuevo vector de estado contiene los estados de los instantes anteriores de interés. La estimación inicial del estado se hace simulando el modelo extendido en bucle abierto,

$$
\hat{\mathbb{X}}[t \mid t-1]=\mathbb{A} \hat{\mathbb{X}}[t-1]+\mathbb{B} \boldsymbol{u}[t-1],
$$

$\operatorname{con} \hat{\mathbb{X}}[t-1]=\hat{\mathbb{X}}[t-1 \mid t-1], y$

$$
\hat{\mathbb{X}}[t \mid t-1]=\left[\begin{array}{l}
\hat{x}[t \mid t-1] \\
\hat{x}[t-1 \mid t-1] \\
\vdots \\
\hat{x}[t-\bar{d} \mid t-1]
\end{array}\right] ; \mathbb{A}_{\eta \times \eta}=\left[\begin{array}{ccc|c}
\boldsymbol{A} & \mathbf{0} & \cdots & \mathbf{0} \\
\hline & \boldsymbol{I} & & \vdots \\
& & & \mathbf{0}
\end{array}\right] ; \mathbb{B}_{\eta \times n}=\left[\begin{array}{c}
B \\
\mathbf{0} \\
\vdots \\
\mathbf{0}
\end{array}\right] .
$$

donde

$$
\bar{d}=\operatorname{máx}_{i=1, \ldots, n_{m}} \delta_{i, p_{i}} ; \quad \delta_{i, p_{i}}=\operatorname{máx} \mathcal{D}_{i}
$$


y $\eta=n(\bar{d}+1)$. Dependiendo de la disponibilidad de una nueva medición en $t=t_{k}$, el vector de estimación del estado se actualiza mediante

$$
\hat{\mathbb{X}}\left[t_{k}\right]=\hat{\mathbb{X}}\left[t_{k} \mid t_{k}-1\right]+\Gamma \cdot \sum_{i=1}^{n_{m}} \boldsymbol{\ell}_{i}\left[t_{k}\right]\left(m_{i}\left[t_{k}\right]-\boldsymbol{c}_{i} \hat{\boldsymbol{x}}\left[t_{k}-d_{i, k} \mid t_{k}-1\right]\right) \delta_{i}\left[t_{k}\right],
$$

donde la estimación del estado con retardo se obtiene como

$$
\hat{\boldsymbol{x}}\left[t_{k}-d_{i, k} \mid t_{k}-1\right]=\left[\begin{array}{llllll}
\overbrace{\mathbf{0}}^{n \cdot d_{i, k}} \mathbf{0} & \boldsymbol{I} & \mathbf{0} & \cdots & \mathbf{0}
\end{array}\right] \cdot \hat{\mathbb{X}}\left[t_{k} \mid t_{k}-1\right],
$$

y $\Gamma$ es la matriz constante definida como

$$
\Gamma=\left[\begin{array}{c}
\boldsymbol{I} \\
\boldsymbol{A}^{-1} \\
\boldsymbol{A}^{-2} \\
\vdots \\
\boldsymbol{A}^{-\zeta} \\
\mathbf{0} \\
\vdots \\
\mathbf{0}
\end{array}\right]_{\eta \times n}
$$

con

$$
\zeta=\operatorname{máx}\left\{0, \operatorname{máx}_{\substack{s_{k} \in \mathcal{S} \\ i=1, \ldots, n_{m}}} d_{i}\left(s_{k}\right)-N\left(s_{k}\right)\right\} .
$$

La matriz $\Gamma$ tiene $\eta-\zeta n$ filas nulas porque las únicas estimaciones retardadas que deben actualizarse son las necesarias para actualizaciones posteriores. Con esto se consigue reducir el número de operaciones (sumas y multiplicaciones) en (4.14b), y, por tanto, reducir el coste computacional requerido en la implementación del algoritmo. La siguiente observación demuestra esta idea.

Observación 4.3.1 (Actualización de la estimación de salidas con retardo). Supóngase que hay al menos una nueva medición en el instante $t_{k}$. La estimación del estado en el instante $t_{k}-d_{i, k}$ condicionado a la medición actual $\left(\hat{\boldsymbol{x}}\left[t_{k}-d_{i, k} \mid t_{k}\right]\right)$ se define como aquella que cumple

$$
\hat{\boldsymbol{x}}\left[t_{k} \mid t_{k}\right]=A^{d_{i, k}} \hat{\boldsymbol{x}}\left[t_{k}-d_{i, k} \mid t_{k}\right]+\sum_{j=1}^{d_{i, k}} \boldsymbol{A}^{j-1} \boldsymbol{B} \boldsymbol{u}\left[t_{k}-j\right] .
$$

De forma similar, se puede obtener la estimación del estado actual sin actualizar $\left(\hat{\boldsymbol{x}}\left[t_{k} \mid t_{k}-1\right]\right)$ en función de la estimación del estado con retardo sin actualizar $\left(\hat{\boldsymbol{x}}\left[t_{k}-d_{i, k} \mid t_{k}-1\right]\right)$ como

$$
\hat{\boldsymbol{x}}\left[t_{k} \mid t_{k}-1\right]=A^{d_{i, k}} \hat{\boldsymbol{x}}\left[t_{k}-d_{i, k} \mid t_{k}-1\right]+\sum_{j=1}^{d_{i, k}} \boldsymbol{A}^{j-1} \boldsymbol{B} \boldsymbol{u}\left[t_{k}-j\right]
$$

Introduciendo (4.12b) en (4.16) y restándolo de (4.17) es fácil obtener

$$
\hat{\boldsymbol{x}}\left[t_{k}-d_{i, k} \mid t_{k}\right]=\hat{\boldsymbol{x}}\left[t_{k}-d_{i, k} \mid t_{k}-1\right]+\sum_{i=1}^{n_{m}} A^{-d_{i, k}} \boldsymbol{\ell}_{i}\left[t_{k}\right]\left(m_{i}\left[t_{k}\right]-\boldsymbol{c}_{i} \hat{\boldsymbol{x}}\left[t_{k}-d_{i, k} \mid t_{k}-1\right]\right) .
$$

El número de estimaciones con retardo previas que es necesario actualizar depende del valor del retardo $d_{i, k}$ y de $N_{k}$. Si

$$
\rho_{i}=\operatorname{máx}_{s_{k} \in \mathcal{S}: \delta_{i, k}=1}\left\{d_{i}\left(s_{k}\right)-N\left(s_{k}\right)\right\}
$$

es la máxima diferencia entre retardo y periodos intermuestreo para un sensor dado, la estimación más antigua que es necesario actualizar es $\hat{\boldsymbol{x}}\left[t_{k}-\rho_{i} \mid t_{k}-1\right]$, porque esta estimación del estado puede ser necesaria para la siguiente actualización. Por lo tanto, la expresión (4.18) se tiene que aplicar de $d=0$ a $\zeta=\operatorname{máx}_{i=1, \ldots, n_{m}}\left\{0, \rho_{i}\right\}$, obteniéndose la ecuación (4.15). 
La dinámica del predictor (en cualquiera de sus implementaciones (4.12) o (4.14)) depende de la matriz de ganancias

$$
\boldsymbol{L}_{k} \equiv \boldsymbol{L}\left[t_{k}\right]=\left[\begin{array}{llll}
\boldsymbol{\ell}_{1}\left[t_{k}\right] & \boldsymbol{\ell}_{2}\left[t_{k}\right] & \cdots & \boldsymbol{\ell}_{n_{m}}\left[t_{k}\right]
\end{array}\right]=\left[\begin{array}{cccc}
l_{11}\left[t_{k}\right] & l_{21}\left[t_{k}\right] & \cdots & l_{n_{m} 1}\left[t_{k}\right] \\
l_{12}\left[t_{k}\right] & l_{22}\left[t_{k}\right] & \cdots & l_{n_{m} 2}\left[t_{k}\right] \\
\vdots & \vdots & & \vdots \\
l_{1 n}\left[t_{k}\right] & l_{2 n}\left[t_{k}\right] & \cdots & l_{n_{m} n}\left[t_{k}\right]
\end{array}\right]
$$

definida en los instantes de medición $\left(t=t_{k}\right)$, que se debe diseñar para asegurar la estabilidad del predictor, la robustez frente a la disponibilidad esporádica de datos, y una atenuación adecuada de las perturbaciones y ruidos de los sensores. La ganancia del predictor se asume en general que es variante en el tiempo, pero el caso particular de una ganancia constante también se analiza.

Con el objetivo de diseñar un predictor, es decir, la matriz de ganancias del predictor (4.19) con esas propiedades, debe obtenerse la ecuación de la dinámica del error de predicción.

\subsubsection{Equivalencia con el predictor entrada salida}

El siguiente teorema muestra la equivalencia que existe en procesos monovariables ausentes de perturbaciones entre el algoritmo (3.8) (basado en la representación entrada salida) y el algoritmo (4.12) (basado en representación la interna).

Teorema 4.3.1. Sea un proceso lineal monovariable definido por las ecuaciones

$$
\begin{aligned}
\boldsymbol{x}[t+1] & =\boldsymbol{A} \boldsymbol{x}[t]+\boldsymbol{b} u[t], \\
\boldsymbol{y}[t] & =\boldsymbol{c} \boldsymbol{x}[t],
\end{aligned}
$$

y supóngase que hay un único sensor para medir la variable de salida (con retardo) cuya ecuación es

$$
m_{k}=y\left[t_{k}-d_{k}\right]=\boldsymbol{c} \boldsymbol{x}\left[t_{k}-d_{k}\right] .
$$

Entonces, los algoritmos (3.8) y (4.12) son equivalentes y la ganancia $\boldsymbol{L}_{k}$ del predictor (4.12) propuesto con la representación en espacio de estados se define en función de la ganancia del predictor basado en el modelo entrada salida $\left(\ell_{k}^{E S}\right)$ del capítulo anterior mediante

$$
\boldsymbol{\ell}_{k}^{E S}=\mathcal{O} \boldsymbol{A}^{1-n} \boldsymbol{L}_{k}
$$

siendo $\boldsymbol{A}$ la matriz de la dinámica de la representación interna, y $\mathcal{O}$ la matriz de observabilidad dada por

$$
\mathcal{O}=\left[\begin{array}{c}
\boldsymbol{c} \boldsymbol{A}^{n-1} \\
\boldsymbol{c} \boldsymbol{A}^{n-2} \\
\vdots \\
\boldsymbol{c} \boldsymbol{A} \\
\boldsymbol{c}
\end{array}\right]
$$

Prueba 4.3.1. La finalidad de la prueba es comparar las ganancias que se utilizan en las ecuaciones de actualización de predicciones tanto en representación interna

$$
\hat{\boldsymbol{x}}\left[t_{k}\right]=\hat{\boldsymbol{x}}\left[t_{k} \mid t_{k}-1\right]-\boldsymbol{L}_{k}\left(m_{k}-\boldsymbol{c} \hat{\boldsymbol{x}}\left[t_{k}-d_{k} \mid t_{k}-1\right]\right),
$$

como en representación externa

$$
\hat{Y}\left[t_{k}\right]=\hat{Y}\left[t_{k} \mid t_{k}-1\right]-\ell_{k}^{E S}\left(m_{k}-\hat{y}\left[t_{k}-d_{k} \mid t_{k}-1\right]\right) .
$$

El vector de regresión $\hat{Y}\left[t_{k} \mid t_{k}-1\right]$ está formado por las estimaciones de la salida desde $t_{k}-(n-1)$ hasta $t_{k}$ con la información disponible hasta $t_{k}-1$. Estos elementos se pueden expresar en función del estado estimado en $t_{k}-(n-1)$ con la información hasta $t_{k}-1$ (es decir, $\hat{\boldsymbol{x}}\left[t_{k}-(n-1) \mid t_{k}-1\right]$ ) con las expresiones

$$
\begin{aligned}
& \hat{y}\left[t_{k} \mid t_{k}-1\right]=\boldsymbol{c} \boldsymbol{A}^{n-1} \hat{\boldsymbol{x}}\left[t_{k}-(n-1) \mid t_{k}-1\right]+\boldsymbol{c}\left(\boldsymbol{b} u\left[t_{k}-1\right]+\cdots+\boldsymbol{A}^{n-2} \boldsymbol{b} u\left[t_{k}-(n-1)\right]\right) \\
& \hat{y}\left[t_{k}-1 \mid t_{k}-1\right]=\boldsymbol{c} \boldsymbol{A}^{n-2} \hat{\boldsymbol{x}}\left[t_{k}-(n-1) \mid t_{k}-1\right]+\boldsymbol{c}\left(\boldsymbol{b} u\left[t_{k}-2\right]+\cdots+\boldsymbol{A}^{n-3} \boldsymbol{b} u\left[t_{k}-(n-1)\right]\right) \\
& \vdots \\
& \hat{y}\left[t_{k}-(n-2) \mid t_{k}-1\right]=\boldsymbol{c}\left(\boldsymbol{A} \hat{\boldsymbol{x}}\left[t_{k}-(n-1) \mid t_{k}-1\right]+\boldsymbol{b} u\left[t_{k}-(n-1)\right]\right) \\
& \hat{y}\left[t_{k}-(n-1) \mid t_{k}-1\right]=\boldsymbol{c} \hat{\boldsymbol{x}}\left[t_{k}-(n-1) \mid t_{k}-1\right]
\end{aligned}
$$


con lo que $\hat{Y}\left[t_{k} \mid t_{k}-1\right]$ se puede escribir como

$$
\hat{Y}\left[t_{k} \mid t_{k}-1\right]=\mathcal{O} \hat{\boldsymbol{x}}\left(t_{k}-(n-1) \mid t_{k}-1\right)+\boldsymbol{H} U\left[t_{k}-1\right]
$$

con

$$
\boldsymbol{H}=\left[\begin{array}{cccc}
\boldsymbol{c} \boldsymbol{b} & \boldsymbol{c A} \boldsymbol{b} & \cdots & \boldsymbol{c} \boldsymbol{A}^{n-2} \boldsymbol{b} \\
\mathbf{0} & \boldsymbol{c b} & & \boldsymbol{c} \boldsymbol{A}^{n-3} \boldsymbol{b} \\
\vdots & & \ddots & \vdots \\
\vdots & & & \boldsymbol{c b} \\
\mathbf{0} & & \cdots & \mathbf{0}
\end{array}\right], \quad U\left[t_{k}-1\right]=\left[\begin{array}{c}
u\left[t_{k}-1\right] \\
u[t-2] \\
\cdots \\
u[t-(n-1)]
\end{array}\right]
$$

Por otra parte, la estimación inicial $\hat{\boldsymbol{x}}\left[t_{k} \mid t_{k}-1\right]$ puede expresarse en función de $\hat{\boldsymbol{x}}\left[t_{k}-(n-1) \mid t_{k}-1\right]$ corriendo el modelo en bucle abierto desde $t_{k}-(n-1)$ hasta $t_{k}$, esto es

$$
\hat{\boldsymbol{x}}\left[t_{k} \mid t_{k}-1\right]=\boldsymbol{A}^{n-1} \hat{\boldsymbol{x}}\left[t_{k}-(n-1) \mid t_{k}-1\right]+\left[\begin{array}{llll}
\boldsymbol{b} & \boldsymbol{A} \boldsymbol{b} & \cdots & \boldsymbol{A}^{n-2} \boldsymbol{b}
\end{array}\right] U\left[t_{k}-1\right] .
$$

Utilizando el resultado (4.25) se puede expresar la relación entre el estado estimado y el vector de regresión estimado como

$$
\hat{\boldsymbol{x}}\left[t_{k} \mid t_{k}-1\right]=\boldsymbol{A}^{n-1} \mathcal{O}^{-1} Y\left[t_{k} \mid t_{k}-1\right]+\left(\left[\begin{array}{llll}
\boldsymbol{b} & \boldsymbol{A} \boldsymbol{b} & \cdots & \boldsymbol{A}^{n-2} \boldsymbol{b}
\end{array}\right]-\boldsymbol{A}^{n-1} \mathcal{O}^{-1} \boldsymbol{H}\right) U\left[t_{k}-1\right] .
$$

Si se procede de igual forma con la estimación corregida del estado $\hat{\boldsymbol{x}}\left[t_{k}\right]$ se llega a

$$
\hat{\boldsymbol{x}}\left[t_{k}\right]=\boldsymbol{A}^{n-1} \mathcal{O}^{-1} Y\left[t_{k}\right]+\left(\left[\begin{array}{llll}
\boldsymbol{b} & \boldsymbol{A} \boldsymbol{b} & \cdots & \boldsymbol{A}^{n-2} \boldsymbol{b}
\end{array}\right]-\boldsymbol{A}^{n-1} \mathcal{O}^{-1} \boldsymbol{H}\right) U\left[t_{k}-1\right] .
$$

Sustituyendo estas dos expresiones en la ecuación (4.23) se tiene que

$$
\boldsymbol{A}^{n-1} \mathcal{O}^{-1} Y\left[t_{k}\right]=\boldsymbol{A}^{n-1} \mathcal{O}^{-1} Y\left[t_{k} \mid t_{k}-1\right]+\boldsymbol{L}_{k}\left(m_{k}-\boldsymbol{c} \hat{\boldsymbol{x}}\left[t_{k}-d_{k} \mid t_{k}-1\right]\right),
$$

y, como $\boldsymbol{c} \hat{\boldsymbol{x}}\left[t_{k}-d_{k} \mid t_{k}-1\right]=\hat{y}\left[t_{k}-d_{k} \mid t_{k}-1\right]$, se llega a

$$
\hat{Y}\left[t_{k}\right]=\hat{Y}\left[t_{k} \mid t_{k}-1\right]+\underbrace{\mathcal{O} \boldsymbol{A}^{1-n} \boldsymbol{L}_{k}}_{=\ell_{k}^{E S}}\left(m_{k}-\hat{y}\left[t_{k}-d_{k} \mid t_{k}-1\right]\right) .
$$

Si las perturbaciones no son nulas, los algoritmos ya no son equivalentes.

\subsubsection{Error de predicción}

Lema 4.3.1 (Dinámica del error de predicción). La dinámica del error de predicción del algoritmo (4.12) aplicado al sistema (4.2) cuando no hay error de modelado y hay al menos una medición disponible cada $N_{k}$ periodos de entrada (con $N_{k}$ variante en el tiempo), se describe mediante el sistema lineal variante en el tiempo

$$
\begin{aligned}
\widetilde{\boldsymbol{x}}_{k}= & \left(\boldsymbol{I}-\sum_{i=1}^{n_{m}} \boldsymbol{\ell}_{i}\left[t_{k}\right] \boldsymbol{c}_{i} \boldsymbol{A}^{-d_{i, k}} \delta_{i}\left[t_{k}\right]\right)\left(\boldsymbol{A}^{N_{k}} \widetilde{\boldsymbol{x}}_{k-1}+\sum_{j=1}^{N_{k}} \boldsymbol{A}^{j-1} \boldsymbol{B}_{v} \boldsymbol{v}\left[t_{k}-j\right]\right) \\
& +\sum_{i=1}^{n_{m}} \boldsymbol{\ell}_{i}\left[t_{k}\right] \boldsymbol{c}_{i} \boldsymbol{A}^{-d_{i, k}} \delta_{i}\left[t_{k}\right] \sum_{j=1}^{d_{i, k}} \boldsymbol{A}^{j-1} \boldsymbol{B}_{v} \boldsymbol{v}\left[t_{k}-j\right]-\sum_{i=1}^{n_{m}} \boldsymbol{\ell}_{i}\left[t_{k}\right] w_{i}\left[t_{k}\right] \delta_{i}\left[t_{k}\right] \\
\boldsymbol{e}_{k}= & \boldsymbol{C}_{y} \widetilde{\boldsymbol{x}}_{k}
\end{aligned}
$$

que se actualiza en cada instante de medición. El vector de error de estimación se define cuando hay una medición disponible (en $t=t_{k}$ ) como

$$
\widetilde{\boldsymbol{x}}_{k} \equiv \widetilde{\boldsymbol{x}}\left[t_{k}\right]=\boldsymbol{x}\left[t_{k}\right]-\hat{\boldsymbol{x}}\left[t_{k}\right],
$$

mientras que el error de predicción de la salida se define como

$$
\boldsymbol{e}_{k} \equiv \boldsymbol{e}\left[t_{k}\right]=\boldsymbol{y}\left[t_{k}\right]-\hat{\boldsymbol{y}}\left[t_{k}\right]
$$


Prueba 4.3.2. En los instantes de medición, $t=t_{k}$, se cumple la expresión (4.2c) y los valores medidos pueden escribirse como

$$
m_{i}\left[t_{k}\right]=\boldsymbol{c}_{i} \boldsymbol{x}\left[t_{k}-d_{i, k}\right]+w_{i}\left[t_{k}\right]
$$

y, por tanto, la predicción de la salida (4.12b) puede expresarse como

$$
\hat{\boldsymbol{x}}\left[t_{k}\right]=\hat{\boldsymbol{x}}\left[t_{k} \mid t_{k}-1\right]+\sum_{i=1}^{n_{m}} \boldsymbol{\ell}_{i}\left[t_{k}\right]\left(\boldsymbol{c}_{i}\left(\boldsymbol{x}\left[t_{k}-d_{i, k}\right]-\hat{\boldsymbol{x}}\left[t_{k}-d_{i, k} \mid t_{k}-1\right]\right)+w_{i}\left[t_{k}\right]\right) \delta_{i}\left[t_{k}\right] .
$$

Los vectores $\boldsymbol{x}\left[t_{k}-d_{i, k}\right]$ y $\hat{\boldsymbol{x}}\left[t_{k}-d_{i, k} \mid t_{k}-1\right]$ pueden expresarse como función de $\boldsymbol{x}\left[t_{k}-N_{k}\right]$ y $\hat{\boldsymbol{x}}\left[t_{k}-N_{k}\right](\mathrm{el}$ instante en el cual se hizo la anterior medición y actualización de la predicción) si las expresiones (4.2a) y (4.12a) se aplican de forma recursiva, conduciendo a

$$
\begin{gathered}
\boldsymbol{x}\left[t_{k}-d_{i, k}\right]-\hat{\boldsymbol{x}}\left[t_{k}-d_{i, k} \mid t_{k}-1\right]=\boldsymbol{A}^{N_{k}-d_{i, k}}\left(\boldsymbol{x}\left[t_{k}-N_{k}\right]-\hat{\boldsymbol{x}}\left[t_{k}-N_{k}\right]\right)+ \\
+\sum_{j=1}^{N_{k}} \boldsymbol{A}^{j-1-d_{i, k}} \boldsymbol{B}_{v} \boldsymbol{v}\left[t_{k}-i\right]-\sum_{j=1}^{d_{i, k}} \boldsymbol{A}^{j-1-d_{i, k}} \boldsymbol{B}_{v} \boldsymbol{v}\left[t_{k}-i\right] .
\end{gathered}
$$

El vector $\hat{\boldsymbol{x}}\left[t_{k} \mid t_{k}-1\right]$ también puede expresarse por medio de $\hat{\boldsymbol{x}}\left[t_{k}-N_{k}\right]$ aplicando (4.12a) de forma recursiva, llegándose a

$$
\hat{\boldsymbol{x}}\left[t_{k} \mid t_{k}-1\right]=\boldsymbol{A}^{N_{k}} \hat{\boldsymbol{x}}\left[t_{k}-N_{k}\right]+\sum_{i=1}^{N_{k}} \boldsymbol{A}^{i-1} \boldsymbol{B} \boldsymbol{u}\left[t_{k}-i\right]
$$

El estado $\boldsymbol{x}\left[t_{k}\right]$ también puede expresarse (por medio de (4.2a)) como una función de $\boldsymbol{x}\left[t_{k}-N_{k}\right]$, es decir,

$$
\boldsymbol{x}\left[t_{k}\right]=\boldsymbol{A}^{N_{k}} \boldsymbol{x}\left[t_{k}-N_{k}\right]+\sum_{i=1}^{N_{k}} \boldsymbol{A}^{i-1} \boldsymbol{B} \boldsymbol{u}\left[t_{k}-i\right]+\sum_{i=1}^{N_{k}} \boldsymbol{A}^{i-1} \boldsymbol{B}_{v} \boldsymbol{v}\left[t_{k}-i\right] .
$$

Introduciendo las expresiones (4.29) y (4.30) en (4.28) y restando la expresión resultante a (4.31), se tiene que

$$
\begin{aligned}
& \underbrace{\boldsymbol{x}\left[t_{k}\right]-\hat{\boldsymbol{x}}\left[t_{k}\right]}_{=\widetilde{\boldsymbol{x}}_{k}}=\boldsymbol{A}^{N_{k}} \underbrace{\left(\boldsymbol{x}\left[t_{k}-N_{k}\right]-\hat{\boldsymbol{x}}\left[t_{k}-N_{k}\right]\right)}_{=\widetilde{\boldsymbol{x}}_{k-1}}+\sum_{j=1}^{N_{k}} \boldsymbol{A}^{j-1} \boldsymbol{B}_{v} \boldsymbol{v}\left[t_{k}-j\right] \\
& \quad-\sum_{i=1}^{n_{m}} \boldsymbol{\ell}_{i}\left[t_{k}\right] \boldsymbol{c}_{i} \boldsymbol{A}^{-d_{i, k}} \boldsymbol{A}^{N_{k}} \underbrace{\left(\boldsymbol{x}\left[t_{k}-N_{k}\right]-\hat{\boldsymbol{x}}\left[t_{k}-N_{k}\right]\right)}_{=\widetilde{\boldsymbol{x}}_{k-1}} \delta_{i}\left[t_{k}\right] \\
& -\sum_{i=1}^{n_{m}} \boldsymbol{\ell}_{i}\left[t_{k}\right] \boldsymbol{c}_{i} \boldsymbol{A}^{-d_{i, k}}\left(\sum_{j=1}^{N_{k}} \boldsymbol{A}^{j-1} \boldsymbol{B}_{v} \boldsymbol{v}\left[t_{k}-j\right]-\sum_{j=1}^{d_{i, k}} \boldsymbol{A}^{j-1} \boldsymbol{B}_{v} \boldsymbol{v}\left[t_{k}-j\right]\right) \delta_{i}\left[t_{k}\right] \\
& \quad-\sum_{i=1}^{n_{m}} \boldsymbol{\ell}_{i}\left[t_{k}\right] w_{i}\left[t_{k}\right] \delta_{i}\left[t_{k}\right]
\end{aligned}
$$

que es exactamente la ecuación (4.26a) porque en el instante de medición $t_{k}-N_{k}=t_{k-1}$.

El error de predicción instantáneo $\boldsymbol{e}_{k}$ se obtiene por medio de la matriz $\boldsymbol{C}_{y}$

$$
\boldsymbol{e}_{k}=\boldsymbol{y}\left[t_{k}\right]-\hat{\boldsymbol{y}}\left[t_{k}\right]=\boldsymbol{C}_{y}\left(\boldsymbol{x}\left[t_{k}\right]-\hat{\boldsymbol{x}}\left[t_{k}\right]\right)=\boldsymbol{C}_{y} \widetilde{\boldsymbol{x}}_{k}
$$

Observación 4.3.2. Defínase un nuevo vector que recoge el valor de las perturbaciones entre mediciones como

$$
\mathbb{V}_{k}=\left[\begin{array}{c}
\boldsymbol{v}\left[t_{k}-1\right] \\
\boldsymbol{v}\left[t_{k}-2\right] \\
\vdots \\
\boldsymbol{v}\left[t_{k}-\beta\right]
\end{array}\right],
$$

siendo

$$
\beta=\operatorname{máx}\left\{\bar{d}, n_{\mathcal{N}}\right\}
$$


el máximo número entero del conjunto definido por todos los posibles retardos $d_{i, k}$ y número de periodos intermuestreo $N_{k}$. Entonces la dinámica del error de predicción puede escribirse de forma compacta como

$$
\begin{aligned}
\widetilde{\boldsymbol{x}}_{k} & =\mathcal{A}_{k} \widetilde{\boldsymbol{x}}_{k-1}+\mathcal{B}_{k}\left[\begin{array}{r}
\mathbb{V}_{k} \\
\boldsymbol{w}_{, k}
\end{array}\right] \\
\boldsymbol{e}_{k} & =\boldsymbol{C}_{y} \widetilde{\boldsymbol{x}}_{k},
\end{aligned}
$$

donde

$$
\begin{aligned}
& \mathcal{A}_{k}=\left(\boldsymbol{I}-\boldsymbol{L}_{k} \boldsymbol{\Delta}_{k} \boldsymbol{C}_{d, k}\right) \boldsymbol{A}^{N_{k}}, \\
& \boldsymbol{C}_{d, k}=\left[\begin{array}{c}
\boldsymbol{c}_{1} \boldsymbol{A}^{-d_{1, k}} \\
\vdots \\
\boldsymbol{c}_{n_{m}} \boldsymbol{A}^{-d_{n_{m}, k}}
\end{array}\right]_{n_{m} \times n}, \\
& \boldsymbol{B}_{k}=\left[\boldsymbol{\Lambda}\left(N_{k}\right)-\boldsymbol{L}_{k} \boldsymbol{\Delta}_{k} \mathcal{C}_{d, k} \quad-\boldsymbol{L}_{k} \boldsymbol{\Delta}_{k}\right]
\end{aligned}
$$

siendo $\boldsymbol{\Delta}_{k} \equiv \boldsymbol{\Delta}\left[t_{k}\right]$ la matriz de disponibilidad, y

$$
\boldsymbol{C}_{d, k}=\left[\begin{array}{c}
\boldsymbol{c}_{1} \boldsymbol{A}^{-d_{1, k}}\left(\boldsymbol{\Lambda}\left(N_{k}\right)-\boldsymbol{\Lambda}\left(d_{1, k}\right)\right) \\
\vdots \\
\boldsymbol{c}_{n_{m}} \boldsymbol{A}^{-d_{n_{m}, k}}\left(\boldsymbol{\Lambda}\left(N_{k}\right)-\boldsymbol{\Lambda}\left(d_{n_{m}, k}\right)\right)
\end{array}\right]_{n_{m} \times \beta n}
$$

con $\boldsymbol{\Lambda}(N)$ la matriz definida como

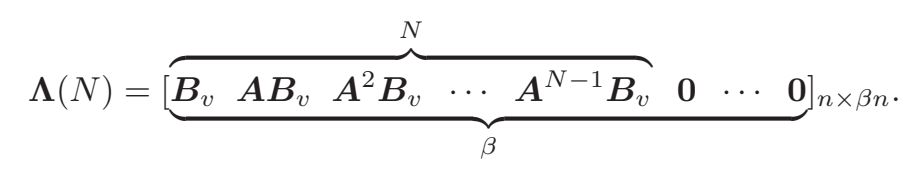

Observación 4.3.3. Otra forma de expresar la dinámica del predictor es mediante la ecuación

$$
\widetilde{\boldsymbol{x}}[t]=\boldsymbol{A} \widetilde{\boldsymbol{x}}[t-1]+\boldsymbol{B}_{v} \boldsymbol{v}[t-1],
$$

en los instantes $t \neq t_{k}$ en los cuales no hay medición, más la ecuación

$$
\widetilde{\boldsymbol{x}}\left[t_{k}\right]=\left(\boldsymbol{I}-\boldsymbol{L}_{k} \boldsymbol{\Delta}_{k} \boldsymbol{C}_{d, k}\right)\left(\boldsymbol{A} \widetilde{\boldsymbol{x}}\left[t_{k}-1\right]+\boldsymbol{\Lambda}\left(N_{k}\right) \mathbb{V}_{k}\right)+\boldsymbol{L}_{k} \boldsymbol{\Delta}_{k} \overline{\boldsymbol{C}}_{d, k} \mathbb{V}_{k}-\boldsymbol{L}_{k} \boldsymbol{\Delta}_{k} \boldsymbol{w}_{k}
$$

con $\overline{\boldsymbol{C}}_{d, k}$ la matriz $n_{m} \times \beta n$ definida como

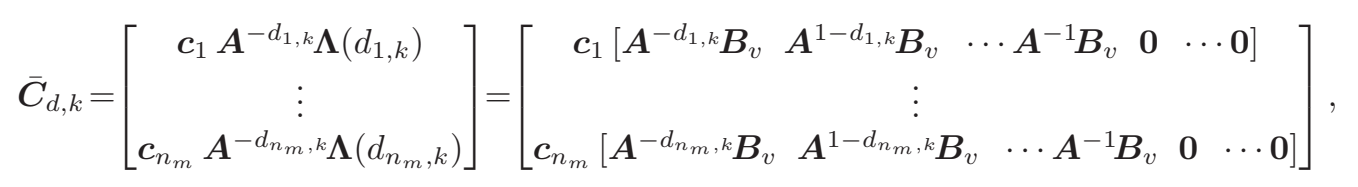

en los instantes de medición $t=t_{k}$. Con esta ecuación se ha separado el efecto que tiene la perturbación $\boldsymbol{v}$ sobre el error de predicción debido al muestreo escaso (término $\left.\left(\boldsymbol{I}-\boldsymbol{L}_{k} \boldsymbol{\Delta}_{k} \boldsymbol{C}_{d, k}\right) \boldsymbol{\Lambda}\left(N_{k}\right) \mathbb{V}_{k}\right)$, del efecto causado por los retardos (término $\boldsymbol{L}_{k} \boldsymbol{\Delta}_{k} \overline{\boldsymbol{C}}_{d, k} \mathbb{V}_{k}$ ). De hecho, si se muestrea en todos los periodos de control $\left(N_{k}=1\right)$, entonces

$$
\left(\boldsymbol{I}-\boldsymbol{L}_{k} \boldsymbol{\Delta}_{k} \boldsymbol{C}_{d, k}\right) \boldsymbol{\Lambda}\left(N_{k}\right) \mathbb{V}_{k}=\left(\boldsymbol{I}-\boldsymbol{L}_{k} \boldsymbol{\Delta}_{k} \boldsymbol{C}_{d, k}\right) \boldsymbol{B}_{v} \boldsymbol{v}\left[t_{k}-1\right],
$$

y si no hay retardos se tiene que

$$
\boldsymbol{L}_{k} \boldsymbol{\Delta}_{k} \overline{\boldsymbol{C}}_{d, k} \mathbb{V}_{k}=\boldsymbol{L}_{k} \boldsymbol{\Delta}_{k} \mathbf{0} \mathbb{V}_{k}=\mathbf{0}
$$

De forma similar a los diseños anteriores para procesos de una entrada y una salida, se aborda el diseño de la ganancia $\boldsymbol{L}_{k}$ definiendo una matriz diferente para cada conjunto $\left(N_{k}, d_{i, k}, \boldsymbol{\Delta}_{k}\right)$, es decir, para 
cada posible valor del parámetro de muestreo $s_{k}$. El cálculo de estas matrices $\boldsymbol{L}_{k}$ se hace fuera de línea una sola vez y da como resultado un conjunto finito de ganancias

$$
\boldsymbol{L}_{k}=\boldsymbol{L}\left(s_{k}\right) \in \mathcal{L}=\left\{\boldsymbol{L}(1), \boldsymbol{L}(2), \ldots, \boldsymbol{L}\left(n_{\mathcal{S}}\right)\right\}
$$

donde $n_{\mathcal{S}}$ es el número de posibles situaciones de muestreo. Cada vez que llega una muestra (con valores de uno o varios sensores), se utiliza una ganancia de $\mathcal{L}$ diferente, dependiendo del valor $s_{k}$, para actualizar la estimación del estado (ecuación (4.18)

Nótese la diferencia de esta implementación con la implementación de un filtro de Kalman (véase \$2.3.3 y §4.9) en el cual la ganancia varía, generalmente, de forma arbitraria a lo largo del tiempo porque la matriz de ganancias $\boldsymbol{L}_{k}$ se calcula en linea con cada nueva muestra. El filtro de Kalman obtiene la estimación óptima (la que minimiza la norma $\ell_{2}$ del error de salida) con cada muestra bajo la suposición de que las perturbaciones son ruidos blancos de media cero con matriz de varianzas-covarianzas conocida. Para ello es necesario correr las ecuaciones de estimación del estado y las de la estimación de la matriz de varianzas-covarianzas, lo que eleva el coste computacional con respecto del predictor propuesto en este trabajo.

Observación 4.3.4. Definiendo la matriz de ganancias del predictor según (4.40), la dinámica del error de predicción (4.32) puede reescribirse de forma paramétrica como

$$
\begin{aligned}
\widetilde{\boldsymbol{x}}_{k} & =\mathcal{A}\left(s_{k}\right) \widetilde{\boldsymbol{x}}_{k-1}+\mathcal{B}\left(s_{k}\right)\left[\begin{array}{c}
\mathrm{V}_{k} \\
\boldsymbol{w}_{k}
\end{array}\right] \\
\boldsymbol{e}_{k} & =\boldsymbol{C}_{y} \widetilde{\boldsymbol{x}}\left(s_{k}\right)
\end{aligned}
$$

con

$$
\mathbb{V}_{k}=\left[\begin{array}{c}
\boldsymbol{v}\left[t_{k}-1\right] \\
\boldsymbol{v}\left[t_{k}-2\right] \\
\vdots \\
\boldsymbol{v}\left[t_{k}-\beta\right]
\end{array}\right], \quad \beta=\operatorname{máx}\left\{d_{1}\left(s_{k}\right), \ldots, d_{n_{m}}\left(s_{k}\right), N\left(s_{k}\right)\right\}
$$

y donde

$$
\begin{aligned}
& \mathcal{A}\left(s_{k}\right)=\left(\boldsymbol{I}-\boldsymbol{L}\left(s_{k}\right) \boldsymbol{\Delta}\left(s_{k}\right) \boldsymbol{C}_{d}\left(s_{k}\right)\right) \boldsymbol{A}^{N\left(s_{k}\right)}, \quad \boldsymbol{C}_{d}\left(s_{k}\right)=\left[\begin{array}{c}
\boldsymbol{c}_{1} \boldsymbol{A}^{-d_{1}\left(s_{k}\right)} \\
\vdots \\
\boldsymbol{c}_{n_{m}} \boldsymbol{A}^{-d_{n_{m}}\left(s_{k}\right)}
\end{array}\right]_{n_{m} \times n}, \\
& \boldsymbol{B}_{k}=\left[\boldsymbol{\Lambda}\left(N\left(s_{k}\right)\right)-\boldsymbol{L}\left(s_{k}\right) \boldsymbol{\Delta}\left(s_{k}\right) \mathcal{C}_{d}\left(s_{k}\right) \quad-\boldsymbol{L}\left(s_{k}\right) \boldsymbol{\Delta}_{k}\right]
\end{aligned}
$$

y

$$
\mathcal{C}_{d}\left(s_{k}\right)=\left[\begin{array}{c}
\boldsymbol{c}_{1} \boldsymbol{A}^{-d_{1}\left(s_{k}\right)}\left(\boldsymbol{\Lambda}\left(N\left(s_{k}\right)\right)-\boldsymbol{\Lambda}\left(d_{1}\left(s_{k}\right)\right)\right) \\
\vdots \\
\boldsymbol{c}_{n_{m}} \boldsymbol{A}^{-d_{n_{m}}\left(s_{k}\right)}\left(\boldsymbol{\Lambda}\left(N\left(s_{k}\right)\right)-\boldsymbol{\Lambda}\left(d_{n_{m}}\left(s_{k}\right)\right)\right)
\end{array}\right]_{n_{m} \times \beta n} .
$$

Con la introducción del parámetro $s_{k}$ se ha llegado a un sistema lineal variante con el tiempo de forma paramétrica. En esta realización interna el vector de perturbaciones y ruido $\mathbb{V}_{k}$ y el ruido $\boldsymbol{w}_{k}$ pueden considerarse como las entradas, el error de estimación como el estado, y el error de predicción como la salida.

\subsubsection{Objetivo de diseño}

En este capítulo se van a estudiar diferentes técnicas de diseño de la matriz $\boldsymbol{L}\left(s_{k}\right)$ para obtener un predictor estable ante errores de modelado y capaz de atenuar perturbaciones. Los objetivos coinciden con aquellos enunciados en \$3.2.3 para el predictor basado en la representación entrada-salida.

El objetivo de diseño de predictores en representación interna es el mismo que se ha presentado en el diseño basado en la representación entrada-salida, que es obtener un procedimiento que permita calcular una matriz de ganancias $\boldsymbol{L}_{k}$ para tener un predictor estable aún con errores de modelado, muestreo escaso y retardo variante, y capaz de atenuar perturbaciones (véase \$3.2.3 en la página 31 para más detalles). 


\subsection{Diseño de predictores ante muestreo regular}

Si se tiene un muestreo periódico de todas las salidas cada $N$ actualizaciones de la entrada $\left(\boldsymbol{\Delta}_{k}=\boldsymbol{I}\right)$ y los retardos en cada sensor tienen un valor constante $d_{i, k}=d,\left(i=1, \ldots, n_{m}\right)$, el error de predicción se reduce a

$$
\begin{aligned}
\widetilde{\boldsymbol{x}}_{k+1} & =\mathcal{A} \widetilde{\boldsymbol{x}}_{k}, \\
\boldsymbol{e}_{k} & =\boldsymbol{C}_{y} \widetilde{\boldsymbol{x}}_{k},
\end{aligned}
$$

con

$$
\mathcal{A}=\left(\boldsymbol{I}-\boldsymbol{L} \boldsymbol{C}_{d}\right) \boldsymbol{A}^{N}, \quad \boldsymbol{C}_{d}=\left[\begin{array}{c}
\boldsymbol{c}_{1} \\
\vdots \\
\boldsymbol{c}_{n_{m}}
\end{array}\right] \boldsymbol{A}^{-d},
$$

donde se ha tomado una matriz de ganancia constante $\boldsymbol{L}$ debido al muestreo constante. Para estabilizar el predictor es suficiente tomar una matriz $\boldsymbol{L}$ tal que todos los valores propios de $\mathcal{A}$ queden dentro del círculo unidad. Para conseguir esto puede abordarse el problema mediante una asignación de polos, o utilizando técnicas LMI. El siguiente teorema recoge la aplicación de esta técnica.

Teorema 4.4.1. Considérese el sistema (4.2) sin perturbaciones ni ruidos de medida, y supóngase que las $n_{m}$ mediciones de los sensores están disponibles de forma simultánea cada $N$ periodos de entrada con un retardo constante de d periodos. Para un $\mu \in(0,1]$ dado, si existen dos matrices $\boldsymbol{P}=\boldsymbol{P}^{\boldsymbol{\top}} \in \mathbb{R}^{n \times n} y$ $\boldsymbol{X}=\in \mathbb{R}^{n \times n_{m}}$ tales que la siguiente desigualdad se cumple

$$
\left[\begin{array}{cc}
\boldsymbol{P} & \left(\boldsymbol{P}-\boldsymbol{X} \boldsymbol{C}_{d}\right) \boldsymbol{A}^{N} \\
\left(\left(\boldsymbol{P}-\boldsymbol{X} \boldsymbol{C}_{d}\right) \boldsymbol{A}^{N}\right)^{\top} & \mu^{2} \boldsymbol{P}
\end{array}\right] \succ \mathbf{0}
$$

es decir, la LMI es factible. Entonces, si la ganancia del predictor se define como

$$
\boldsymbol{L}=\boldsymbol{P}^{-1} \boldsymbol{X},
$$

el error de predicción del algoritmo definido por (4.12) converge a cero de forma asintótica con un ratio de decadencia menor que $\mu \in(0,1]$

Prueba 4.4.1. Introduciendo la ganancia (4.46) en la LMI (4.45), se puede escribir que

$$
\left[\begin{array}{cc}
\boldsymbol{P} & \boldsymbol{P}\left(\boldsymbol{I}-\boldsymbol{L} \boldsymbol{C}_{d}\right) \boldsymbol{A}^{N} \\
\left(\boldsymbol{P}\left(\boldsymbol{I}-\boldsymbol{L} \boldsymbol{C}_{d}\right) \boldsymbol{A}^{N}\right)^{\top} & \mu^{2} \boldsymbol{P}
\end{array}\right]=\left[\begin{array}{cc}
\boldsymbol{P} & \boldsymbol{P} \boldsymbol{A} \\
\mathcal{A}^{\top} \boldsymbol{P} & \mu^{2} \boldsymbol{P}
\end{array}\right] \succ \mathbf{0},
$$

donde, aplicando los complementos de Schur se llega a

$$
\mathcal{A}^{\top} \boldsymbol{P} \mathcal{A}-\boldsymbol{P} \prec \mathbf{0} \text {. }
$$

Premultiplicando por $\widetilde{\boldsymbol{x}}_{k}^{\top}$ y postmultiplicando por $\widetilde{\boldsymbol{x}}_{k}$ se obtiene la condición

$$
\mathcal{V}_{k+1}<\mu^{2} \mathcal{V}_{k}
$$

siendo $\mathcal{V}_{k}=\widetilde{\boldsymbol{x}}_{k}^{\top} \boldsymbol{P} \widetilde{\boldsymbol{x}}_{k}$ la función de Lyapunov. La estabilidad cuadrática queda probada finalmente teniendo en cuenta que la desigualdad (4.45) implica que $\boldsymbol{P} \succ \mathbf{0}$, y, en consecuencia, la función de Lyapunov es definida positiva.

Observación 4.4.1. El problema LMI planteado en el teorema anterior será factible siempre que el par $\left(\boldsymbol{A}^{N}, \boldsymbol{C}_{d} \boldsymbol{A}^{N}\right)$ sea detectable 2 . Para asegurarse de que el sistema es detectable se tratará de trabajar siempre con realizaciones mínimas.

Ejemplo 4.4.1. Sea el proceso de orden 3 con dos entradas, dos salidas y dos sensores definido mediante las ecuaciones

$$
\begin{aligned}
\boldsymbol{x}[t+1] & =\left[\begin{array}{ccc}
0.5 & -0.1 & -0.4 \\
1 & -0.8 & -0.9 \\
-0.8 & 0.4 & 0.9
\end{array}\right] \boldsymbol{x}[t]+\left[\begin{array}{cc}
0.7 & 0.3 \\
0.7 & -0.7 \\
-0.2 & -0.3
\end{array}\right] \boldsymbol{u}[t]+\left[\begin{array}{ll}
1 & 0 \\
0 & 1 \\
1 & 1
\end{array}\right] \boldsymbol{v}[t] \\
\boldsymbol{y}[t] & =\left[\begin{array}{ccc}
-1 & -0.1 & -0.8 \\
0.6 & -1 & -1
\end{array}\right] \boldsymbol{x}[t] \\
\boldsymbol{m}_{k} & =\left[\begin{array}{lll}
1 & 0 & 0.5 \\
0 & 1 & 0.5
\end{array}\right] \boldsymbol{x}\left[t_{k}-d_{k}\right]+\boldsymbol{w}[t] .
\end{aligned}
$$

\footnotetext{
${ }^{2}$ Un sistema es detectable si sus autovalores no observables son estables (véase A.5.1)
} 
Los valores propios de la matriz $\boldsymbol{A}$ son $\lambda=\{1.046,0.113,-0.559\}$ (el proceso es inestable).

Considérese un muestreo periódico en el que se toma una medición de las dos salidas a la vez cada $N=10$ periodos de control, y considérese un retardo constante de $d=3$ periodos en los dos sensores. El par $\left(\boldsymbol{A}^{10}, \boldsymbol{C A}^{7}\right)$ tiene una matriz de observabilidad de rango 3, con lo que el sistema es observable. En estas condiciones es posible aplicar el teorema anterior para llegar a una matriz de ganancias de predicción

$$
\boldsymbol{L}=\left[\begin{array}{cc}
-0.4209 & -0.2937 \\
-0.2449 & -0.9733 \\
-0.9621 & 0.9388
\end{array}\right]
$$

Como el muestreo es regular, la dinámica del predictor vendrá determinada por los valores propios de la matriz $\left(\boldsymbol{I}-\boldsymbol{L} \boldsymbol{C A}^{-3}\right) \boldsymbol{A}^{10}$ que están ubicados en $\{0,0,0\}$, es decir, que se ha obtenido un predictor de tiempo mínimo. En la figura 4.2 se muestra una simulación del proceso controlado en el que no hay perturbaciones ni ruido de medida y se observa como la predicción converge a la salida real con solo una muestra.
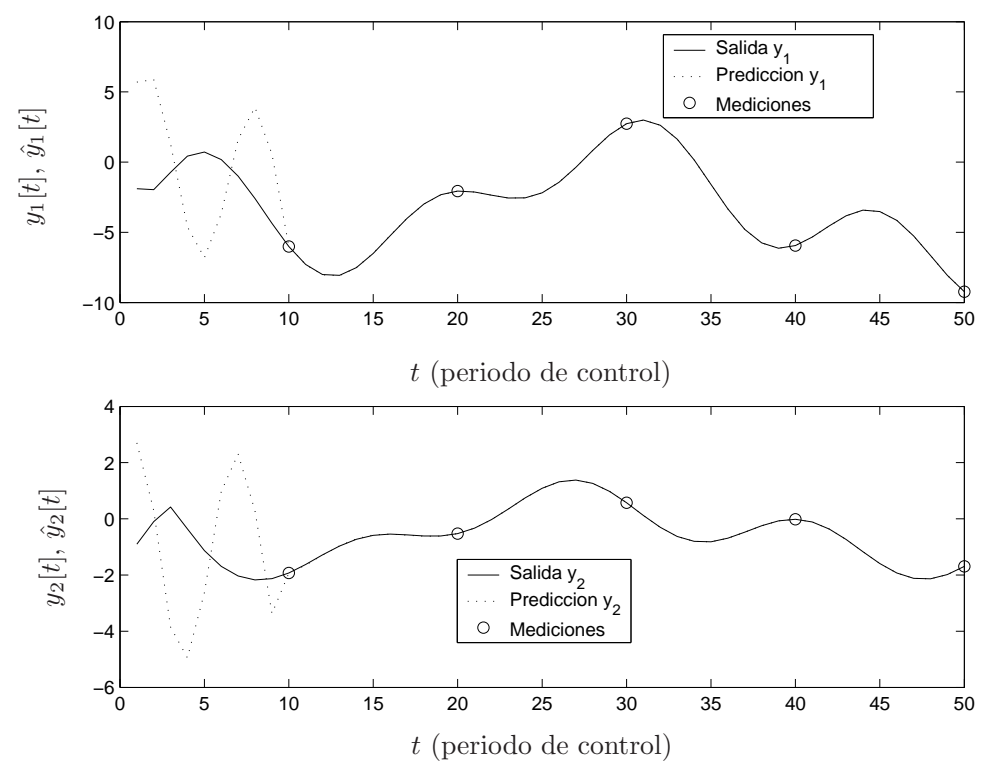

Figura 4.2: Evolución de las salidas y de las predicciones con un predictor de tiempo mínimo. $\mathcal{N}=\{10\}, \mathcal{D}=\{3\}$.

El problema de esta estrategia es que no permite modificar la dinámica, y el procedimiento siempre trata de buscar el predictor con la dinámica más rápida posible (en este ejemplo en concreto se ha llegado al observador de tiempo minimo). Este predictor presenta problemas si se ejecuta en un proceso con perturbaciones o ruido de medida, tal y como muestra la figura 4.3, en la que se ha simulado el funcionamiento del predictor en un proceso con una perturbación escalón de valor $\boldsymbol{v}[t]=[0.50 .5]^{\top}$.

\subsection{Diseño determinista de predictores}

Si el muestreo no es periódico, sino que la información de los sensores llega en instantes de tiempo arbitrarios y no de forma simultánea, o bien si los retardos en los sensores varían en el tiempo, se ha de tener en cuenta que la dinámica del error de predicción es variante en el tiempo y que, en ausencia de perturbaciones, se puede escribir como

$$
\begin{aligned}
\widetilde{\boldsymbol{x}}_{k} & =\mathcal{A}\left(s_{k}\right) \widetilde{\boldsymbol{x}}_{k-1}, \\
\boldsymbol{e}_{k} & =\boldsymbol{C}_{y} \widetilde{\boldsymbol{x}}_{k} .
\end{aligned}
$$

\subsubsection{Estabilidad nominal}

El siguiente teorema trata el diseño del predictor para asegurar la estabilidad ante el cambio irregular de las condiciones de muestreo. 

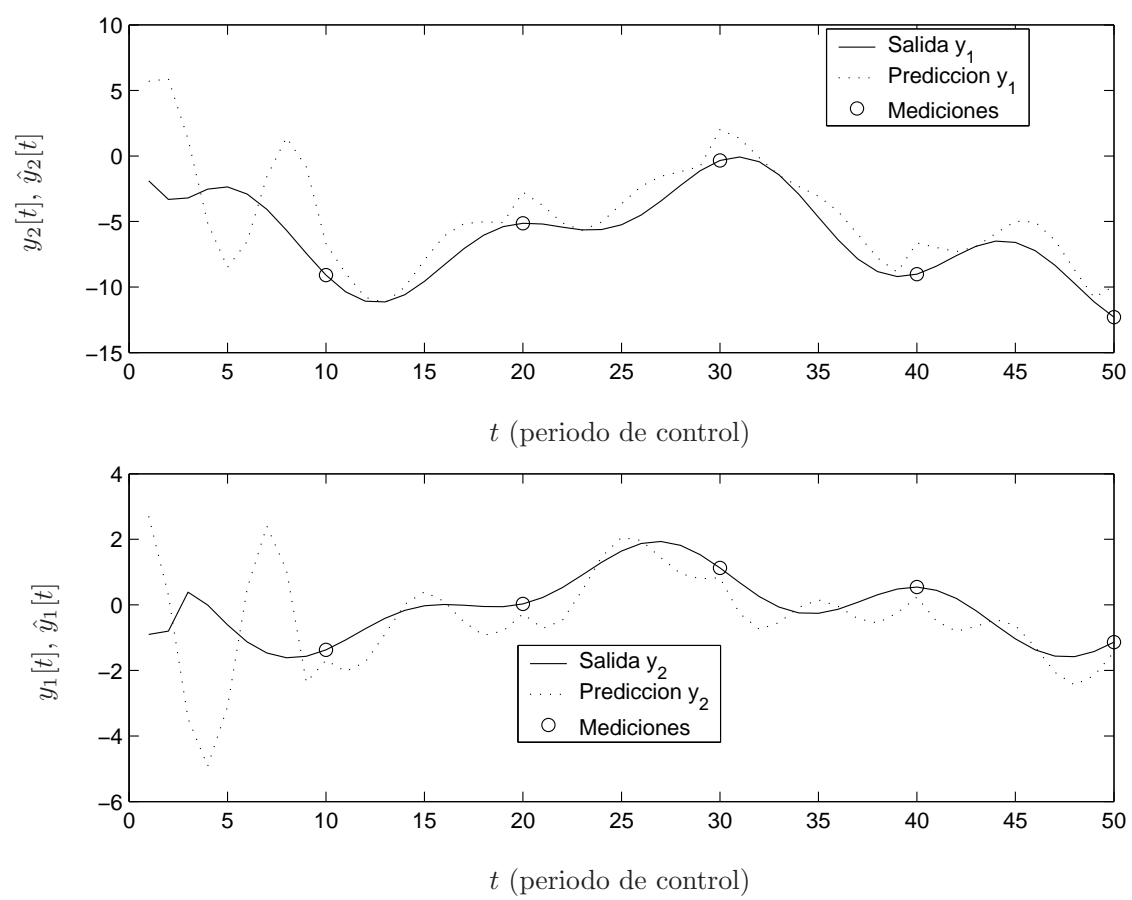

Figura 4.3: Evolución de las salidas y de las predicciones con un predictor de tiempo mínimo cuando el proceso está afectado de una perturbación escalón. $\mathcal{N}=\{10\}, \mathcal{D}=\{3\}$.

Teorema 4.5.1. Considérese el sistema (4.2) en ausencia de perturbaciones y ruido de medida $(\boldsymbol{v}=\mathbf{0}$ y $\boldsymbol{w}=\mathbf{0}$ ), y supóngase que hay por lo menos una medición disponible cada $N_{k} \in \mathcal{N}$ periodos de entrada de manera que los sensores disponibles vienen dados por $\boldsymbol{\Delta}_{k} \in \Xi$ estando cada medición disponible afectada de un retardo $d_{i, k} \in \mathcal{D}_{i}$, de manera que hay $n_{\mathcal{S}}$ escenarios de muestreo $s_{k}$ posibles. Para un $\mu \in(0,1]$ dado, si existen unas matrices $\boldsymbol{P}\left(s_{k}\right)=\boldsymbol{P}\left(s_{k}\right)^{\top} \in \mathbb{R}^{n \times n}, \boldsymbol{Q}\left(s_{k}\right) \in \mathbb{R}^{n \times n}$ and $\boldsymbol{X}\left(s_{k}\right) \in \mathbb{R}^{n \times n_{m}}$, tales que la siguiente desigualdad lineal matricial paramétrica (PLMI)

$$
\left[\begin{array}{cc}
\boldsymbol{Q}\left(s_{k}\right)+\boldsymbol{Q}\left(s_{k}\right)^{\top}-\boldsymbol{P}\left(s_{k}\right) & \left(\boldsymbol{Q}\left(s_{k}\right)-\boldsymbol{X}\left(s_{k}\right) \boldsymbol{\Delta}\left(s_{k}\right) \boldsymbol{C}_{d}\left(s_{k}\right)\right) \boldsymbol{A}^{N\left(s_{k}\right)} \\
\left.\left(\left(\boldsymbol{Q}\left(s_{k}\right)-\boldsymbol{X}\left(s_{k}\right) \boldsymbol{\Delta}\left(s_{k}\right) \boldsymbol{C}_{d}\left(s_{k}\right)\right)\right) \boldsymbol{A}^{N\left(s_{k}\right)}\right)^{\top} & \mu^{2} \boldsymbol{P}\left(s_{k-1}\right)
\end{array}\right] \succ 0,
$$

se cumple para cualquier secuencia de muestreo $\left\{s_{k}\right\}$, entonces, si la matriz de ganancias del predictor se define en función del parámetro de muestreo como

$$
\boldsymbol{L}\left(s_{k}\right)=\boldsymbol{Q}\left(s_{k}\right)^{-1} \boldsymbol{X}\left(s_{k}\right),
$$

el error de predicción del algoritmo definido por (4.12) converge a cero de forma asintótica con un ratio de decadencia menor que $\mu \in(0,1]$.

Prueba 4.5.1. Si se introduce la ganancia (4.52) en la LMI (4.51), la desigualdad puede escribirse como

$$
\left[\begin{array}{cc}
\boldsymbol{Q}\left(s_{k}\right)+\boldsymbol{Q}\left(s_{k}\right)^{\top}-\boldsymbol{P}\left(s_{k}\right) & \boldsymbol{Q}\left(s_{k}\right) \mathcal{A}\left(s_{k}\right) \\
\mathcal{A}\left(s_{k}\right)^{\top} \boldsymbol{Q}\left(s_{k}\right)^{\top} & \mu^{2} \boldsymbol{P}\left(s_{k-1}\right)
\end{array}\right] \succ 0 .
$$

La condición $\boldsymbol{Q}\left(s_{k}\right)+\boldsymbol{Q}\left(s_{k}\right)^{\top}-\boldsymbol{P}\left(s_{k}\right) \succ 0$ implica que $\boldsymbol{Q}\left(s_{k}\right)$ es no singular, y además, se cumple la siguiente desigualdad matricial

$$
\left(\boldsymbol{P}\left(s_{k}\right)-\boldsymbol{Q}\left(s_{k}\right)\right) \boldsymbol{P}\left(s_{k}\right)^{-1}\left(\boldsymbol{P}\left(s_{k}\right)-\boldsymbol{Q}\left(s_{k}\right)\right)^{\boldsymbol{\top}} \succeq \mathbf{0},
$$

y por tanto

$$
\boldsymbol{Q}\left(s_{k}\right)+\boldsymbol{Q}\left(s_{k}\right)^{\top}-\boldsymbol{P}\left(s_{k}\right) \preceq \boldsymbol{Q}\left(s_{k}\right) \boldsymbol{P}\left(s_{k}\right)^{-1} \boldsymbol{Q}\left(s_{k}\right)^{\top} .
$$

Aplicando esta condición a (4.53) y tomando complementos de Schur se obtiene la condición

$$
\boldsymbol{A}\left(s_{k}\right)^{\top} \boldsymbol{P}\left(s_{k}\right) \mathcal{A}\left(s_{k}\right)-\mu^{2} \boldsymbol{P}\left(s_{k-1}\right) \prec 0 .
$$


Si se multiplica esta expresión por la izquierda por $\widetilde{\boldsymbol{x}}_{k-1}^{\top}$ y se multiplica por la derecha por $\widetilde{\boldsymbol{x}}_{k-1}$ se tiene la desigualdad

$$
\widetilde{\boldsymbol{x}}_{k}^{\boldsymbol{\top}} \boldsymbol{P}\left(s_{k}\right) \widetilde{\boldsymbol{x}}_{k}<\mu^{2} \widetilde{\boldsymbol{x}}_{k-1}^{\top} \boldsymbol{P}\left(s_{k-1}\right) \widetilde{\boldsymbol{x}}_{k-1}
$$

Definiendo la función de Lyapunov paramétrica como

$$
\mathcal{V}\left(\widetilde{\boldsymbol{x}}_{k}, s_{k}\right)=\widetilde{\boldsymbol{x}}_{k}^{\top} \boldsymbol{P}\left(s_{k}\right) \widetilde{\boldsymbol{x}}_{k}
$$

la condición (4.55) es equivalente a

$$
\mathcal{V}\left(\widetilde{\boldsymbol{x}}_{k}, s_{k}\right)<\mu^{2} \mathcal{V}\left(\widetilde{\boldsymbol{x}}_{k-1}, s_{k-1}\right)
$$

La estabilidad cuadrática queda finalmente probada teniendo en cuenta que la PLMI (4.51) implica claramente que $\boldsymbol{P}\left(s_{k}\right) \succ 0$.

Observación 4.5.1. En el diseño de predictores con disponibilidad irregular de los sensores se obtiene una matriz de ganancias de la forma

$$
\boldsymbol{L}\left(s_{k}\right)=\left[\begin{array}{llll}
\boldsymbol{\ell}_{1}\left(s_{k}\right) & \boldsymbol{\ell}_{2}\left(s_{k}\right) & \cdots & \boldsymbol{\ell}_{n_{m}}\left(s_{k}\right)
\end{array}\right],
$$

donde cada vector columna $\ell_{i}\left(s_{k}\right)$ es la ganancia que se aplica para actualizar la estimación del estado con la medición del sensor $i$ en un instante cuyo muestreo viene definido por el parámetro $s_{k}$. Si en un instante dado no hay información del sensor $i$ (es decir, $\delta_{i}\left(s_{k}\right)=0$ ), la columna correspondiente $\boldsymbol{\ell}_{i}\left(s_{k}\right)$ es nula. En otras palabras, las columnas no nulas de las matrices $\boldsymbol{L}\left(s_{k}\right)$ y $\boldsymbol{\Delta}\left(s_{k}\right)$ coinciden, siendo el número total de columnas no nulas igual al número de sensores de los cuales se tiene información con la muestra $k\left(m_{k}\right)$. Este resultado es equivalente al obtenido con la predicción basada en filtro de Kalman en $§ 2.3 .3$ y $§ 4.9$ (véase observación 2.3.1).

\subsubsection{Observabilidad y detectabildad}

El teorema anterior llevará a un conjunto de matrices que estabilizan el predictor siempre que el par $\left(\boldsymbol{A}^{N\left(s_{k}\right)}, \boldsymbol{\Delta}\left(s_{k}\right) \boldsymbol{C}_{d}\left(s_{k}\right) \boldsymbol{A}^{N\left(s_{k}\right)}\right)$ sea detectable para cualquier trayectoria $s_{k}$ posible (véase $§ \mathrm{~A} .5 .1$ para la definición general de detectabilidad, y §A.5.2 para las definiciones relativas a sistemas que conmutan con el tiempo). Si el proceso es estable, siempre existirá dicho conjunto de matrices $\left\{\boldsymbol{L}\left(s_{k}\right)\right\}$. Si el sistema es inestable, es necesario realizar previamente un estudio de detectabilidad más detallado para conseguir alcanzar una solución. Para ello se parte de la hipótesis de que existe una cota superior $\bar{N}$ tal que ningún sensor está más de ese número de instantes de muestreo sin obtener una medición.

Considérese por ejemplo el proceso

$$
\begin{aligned}
\boldsymbol{x}[t+1] & =\underbrace{\left[\begin{array}{ccc}
0.7 & 0 & 0.5 \\
0 & 1.1 & 0.8 \\
0 & 0 & 1.5
\end{array}\right]}_{\boldsymbol{A}} \boldsymbol{x}[t]+\left[\begin{array}{ll}
1 & 0 \\
0 & 1 \\
1 & 1
\end{array}\right] \boldsymbol{u}[t] \\
\boldsymbol{m}[t] & =\underbrace{\left[\begin{array}{lll}
1 & 0 & 2 \\
0 & 2 & 0
\end{array}\right]}_{\boldsymbol{C}} \boldsymbol{x}[t]
\end{aligned}
$$

en el que se tiene en cada periodo la medición de algún sensor. Los valores propios del proceso coinciden con los elementos de la diagonal de la matriz $\boldsymbol{A}$. Si todos los sensores están accesibles en cada periodo el sistema es completamente observable. Sin embargo, si sólo se toman medidas del primer sensor, el estado 2 no es observable, puesto que si los estados 1 y 3 están a cero y el estado 2 se inicializa en un valor cualquiera y la acción de control es nula, su evolución no quedaría reflejada en el primer sensor. Además, como el valor propio asociado al estado 2 es inestable $\left(\lambda_{2}=1.1>1\right)$ el sistema no es detectable. Por otra parte, si sólo se toman medidas del segundo sensor, el estado 1 no es observable, pero como su valor propio asociado es estable $\left(\lambda_{1}=0.7<1\right)$, el sistema sí que será detectable.

Supóngase que el escenario de muestreo viene definido por $N=1, d=0$, y por las matrices de disponibilidad

$$
\Xi=\left\{\boldsymbol{\Delta}_{1}=\left[\begin{array}{ll}
1 & 0 \\
0 & 0
\end{array}\right], \boldsymbol{\Delta}_{2}=\left[\begin{array}{ll}
0 & 0 \\
0 & 1
\end{array}\right]\right\},
$$


que indican que nunca se miden los dos sensores de forma simultánea. En este caso el parámetro de muestreo sólo toma dos valores $s_{k}\{1,2\}$ que hacen referencia a la medición de uno u otro sensor y el predictor estará formado por dos matrices

$$
\mathcal{L}=\{\boldsymbol{L}(1), \boldsymbol{L}(2)\}
$$

Si se aplica el método de predicción a este sistema, la solución de las LMI no es factible dado que existe una trayectoria de $s_{k}$ para la cual el sistema no es detectable, que es la trayectoria $s_{k}=\{1,1,1, \ldots\}$, es decir, que sólo se tienen mediciones del sensor 1 a lo largo del tiempo.

Como se parte de la premisa de que todos los sensores van a estar accesibles en algún momento, es necesario eliminar todos los casos de no detectabilidad que se derivan cuando sólo está accesible un subconjunto del total de sensores (en el ejemplo, esto sucede cuando sólo se usa el sensor 1). Para ello se va a intentar que el predictor sea capaz de atenuar con cada medición el error de estimación de, por lo menos, los estados del subespacio detectable. El error de estimación de los estados del subespacio no detectable evolucionará con la dinámica asociada a dichos estados, es decir, que aumentará con la dinámica marcada por su polo inestable asociado.

Para conseguir eliminar este subespacio deben modificarse las LMIs asociadas a trayectorias $s_{k}$ constantes para las cuales el sistema no es detectable. La modificación consiste en eliminar las filas y columnas correspondientes a estados no detectables. Supóngase que se desea obtener el predictor que asegura la estabilidad cuadrática del error de predicción del sistema anterior, para lo cual debe tomarse la matriz $\boldsymbol{P}$ constante (véase la observación 4.8.1 en la página 124). Las LMIs a resolver en este caso son

$$
\begin{aligned}
& {\left[\begin{array}{cc}
\boldsymbol{Q}(1)+\boldsymbol{Q}(1)^{\top}-\boldsymbol{P} & \left(\boldsymbol{Q}(1)-\boldsymbol{X}(1) \boldsymbol{\Delta}_{1} \boldsymbol{C}\right) \boldsymbol{A} \\
\left(\boldsymbol{Q}(1) \boldsymbol{A}-\boldsymbol{X}(1) \boldsymbol{\Delta}_{1} \boldsymbol{C A}\right)^{\top} & \mu^{2} \boldsymbol{P}
\end{array}\right] \succ \mathbf{0}} \\
& {\left[\begin{array}{cc}
\boldsymbol{Q}(2)+\boldsymbol{Q}(2)^{\top}-\boldsymbol{P} & \boldsymbol{Q}(2) \boldsymbol{A}-\boldsymbol{X}(2) \boldsymbol{\Delta}_{2} \boldsymbol{C A} \\
\left(\left(\boldsymbol{Q}(2)-\boldsymbol{X}(2) \boldsymbol{\Delta}_{2} \boldsymbol{C}\right) \boldsymbol{A}\right)^{\top} & \mu^{2} \boldsymbol{P}
\end{array}\right] \succ \mathbf{0} .}
\end{aligned}
$$

Con las LMI planteadas de esta forma el problema resulta no factible (porque está incluido el caso de medición únicamente con el primer sensor). Sin embargo, si se plantean las LMI de forma reducida como

$$
\begin{aligned}
& {\left[\begin{array}{cc}
\boldsymbol{Q}^{\prime}(1)+\boldsymbol{Q}^{\prime}(1)^{\top}-\boldsymbol{P}^{\prime} & \left(\boldsymbol{Q}^{\prime}(1)-\boldsymbol{X}^{\prime}(1) \boldsymbol{\Delta}_{1} \boldsymbol{C}^{\prime}\right) \boldsymbol{A}^{\prime} \\
\left(\left(\boldsymbol{Q}^{\prime}(1)-\boldsymbol{X}^{\prime}(1) \boldsymbol{\Delta}_{1} \boldsymbol{C}^{\prime}\right) \boldsymbol{A}^{\prime}\right)^{\top} & \mu^{2} \boldsymbol{P}^{\prime}
\end{array}\right] \succ \mathbf{0}} \\
& {\left[\begin{array}{cc}
\boldsymbol{Q}(2)+\boldsymbol{Q}(2)^{\top}-\boldsymbol{P} & \boldsymbol{Q}(2) \boldsymbol{A}-\boldsymbol{X}(2) \boldsymbol{\Delta}_{2} \boldsymbol{C} \boldsymbol{A} \\
\left(\boldsymbol{Q}(2) \boldsymbol{A}-\boldsymbol{X}(2) \boldsymbol{\Delta}_{2} \boldsymbol{C} \boldsymbol{A}\right)^{\top} & \mu^{2} \boldsymbol{P}
\end{array}\right] \succ \mathbf{0},}
\end{aligned}
$$

donde las matrices $\boldsymbol{P}^{\prime}, \boldsymbol{Q}^{\prime}(1)$ y $\boldsymbol{A}^{\prime}$ son las matrices resultantes de eliminar a $\boldsymbol{P}, \boldsymbol{Q}(1)$ y $\boldsymbol{A}$ la segunda fila y columna, y $\boldsymbol{X}^{\prime}(1)$ es la matriz resultante de eliminar la segunda fila a la matriz $\boldsymbol{X}(1)$ (la correspondiente al estado no detectable desde la salida 1$)$. La matriz $\boldsymbol{\Delta}_{1} \boldsymbol{C}^{\prime}$ contiene los elementos $(1,1)$ y $(1,3)$ de la matriz $\boldsymbol{C}$. Con las matrices $\boldsymbol{Q}^{\prime}(1)$ y $\boldsymbol{X}^{\prime}(1)$ se pueden calcular los elementos no nulos de la matriz $\boldsymbol{L}(1)$ como

$$
\boldsymbol{L}^{\prime}(1)=\left[\begin{array}{ll}
l_{11} & l_{12} \\
l_{31} & l_{32}
\end{array}\right]=\left(\boldsymbol{Q}^{\prime}(1)\right)^{-1} \boldsymbol{X}^{\prime}(1) .
$$

La matriz $\boldsymbol{L}(1)$ se calcula finalmente añadiendo ceros en la fila correspondiente al estado no detectable:

$$
\boldsymbol{L}(1)=\left[\begin{array}{cc}
l_{11} & l_{12} \\
0 & 0 \\
l_{31} & l_{32}
\end{array}\right]
$$

Para el ejemplo que se está trabajando se obtienen las matrices

$$
\boldsymbol{L}(1)=\left[\begin{array}{cc}
0.1859 & 0 \\
0 & 0 \\
0.4070 & 0
\end{array}\right], \quad \boldsymbol{L}(2)=\left[\begin{array}{ll}
0 & 0.2152 \\
0 & 0.5000 \\
0 & 0.5163
\end{array}\right]
$$

donde se observa que en cada matriz se tiene una columna nula correspondiente al sensor del que no se tiene medición (es el resultado que devuelve la solución de la LMI), y que el elemento $(2,1)$ de la matriz $\boldsymbol{L}(1)$ es nulo siguiendo la metodología explicada. 
Si el sistema es diagonalizable, de forma que se puede obtener una representación en espacio de estados de la forma

$$
\begin{gathered}
\boldsymbol{x}[t]=\left[\begin{array}{cccc}
\lambda_{1} & & & 0 \\
& \lambda_{2} & & \\
& & \ddots & \\
0 & & & \lambda_{n}
\end{array}\right] \boldsymbol{x}[t]+\boldsymbol{B} \boldsymbol{u}[t] \\
\boldsymbol{m}[t]=\left[\begin{array}{cccc}
c_{11} & c_{12} & \cdots & c_{1 n} \\
\vdots & & & \vdots \\
c_{n_{m} 1} & c_{n_{m} 2} & \cdots & c_{n_{m} n}
\end{array}\right] \boldsymbol{x}[t],
\end{gathered}
$$

se tiene que cada estado tiene asignado un único valor propio. La matriz $\boldsymbol{A}^{N_{k}}$ también tiene forma diagonal y los valores propios siguen manteniendo su condición de estabilidad o inestabilidad 3 , Los vectores fila $\boldsymbol{c}_{i, k} \boldsymbol{A}^{N_{k}-d_{i, k}}$ que forman la matriz $\boldsymbol{C}_{d, k}$ mantendrán los elementos nulos de los vectores $\left[c_{i 1}, c_{i 2}, \cdots, c_{i n}\right]^{\top}$ del sistema con muestreo estándar.

De esta manera, si el sistema es diagonalizable, se puede realizar la transformación del estado pertinente para tener una realización en la que se diferencia el subespacio observable y el detectable simplemente teniendo en cuenta cuáles los sensores activos, y sin necesidad de tener en cuenta las condiciones de muestro $N$ y $d$.

A pesar de haber expuesto la necesidad de diferenciar entre espacio detectable y no detectable, cabe notar que para el diseño basado en la estabilidad nominal es suficiente con trabajar con el subespacio observable desde cada sensor. Sin embargo, el hecho de trabajar con el subespacio detectable hace el procedimiento más general y ampliable también al diseño de predictores basados en la atenuación de perturbaciones que se presentan en el siguiente apartado.

Si la planta presenta perturbaciones y los sensores introducen ruido de medida, se deben tomar en consideración las estrategias de diseño basadas en la atenuación de perturbaciones introducidas en $\S 3.2 .3$. En las siguientes secciones se presenta un catálogo de predictores donde cada uno de ellos tiene en cuenta diferentes características en las perturbaciones y su efecto sobre el error de predicción.

En los diseños que se plantean a continuación se asume que el sistema es detectable desde cada sensor de forma individual. Si esto no es así, es necesario aplicar la técnica

\subsubsection{Atenuación $\mathcal{H}_{\infty}$}

El siguiente teorema recoge el diseño de predictores estables que minimizan la cota del error en las situaciones S1, S2 y S3 descritas en la página 31.

Teorema 4.5.2. Considérese el predictor (4.12) aplicado al sistema (4.2) y supóngase que hay al menos una medida disponible cada $N_{k} \in \mathcal{N}$ periodos. Supóngase que la disponibilidad de los sensores en cada muestreo viene definida por la matriz $\boldsymbol{\Delta}_{k} \in \Xi$ y que el retardo asociado a cada uno de los sensores se encuentra en el conjunto $d_{i, k} \in \mathcal{D}_{i}$, de manera que se tienen $n_{\mathcal{S}}$ escenarios de muestreo $s_{k} \in \mathcal{S}$ posibles. Para unos $\gamma_{v_{1}}, \ldots, \gamma_{v_{n_{v}}}, \gamma_{w_{1}}, \ldots, \gamma_{w_{n_{m}}}$ dados, supóngase que existen unas matrices $\boldsymbol{P}\left(s_{k}\right)=\boldsymbol{P}\left(s_{k}\right)^{\top} \in$ $\mathbb{R}^{n \times n}, \boldsymbol{Q}\left(s_{k}\right) \in \mathbb{R}^{n \times n}, \boldsymbol{X}\left(s_{k}\right) \in \mathbb{R}^{n \times n_{m}}$ tales que

$$
\left[\begin{array}{ccc}
\boldsymbol{Q}\left(s_{k}\right)+\boldsymbol{Q}\left(s_{k}\right)^{\top}-\boldsymbol{P}\left(s_{k}\right) & \boldsymbol{M}_{A}\left(s_{k}\right) & \boldsymbol{M}_{B}\left(s_{k}\right) \\
\boldsymbol{M}_{A}\left(s_{k}\right)^{\top} & \boldsymbol{P}\left(s_{k-1}\right)-\boldsymbol{C}_{y}^{\top} \boldsymbol{C}_{y} & \mathbf{0} \\
\boldsymbol{M}_{B}\left(s_{k}\right)^{\top} & \mathbf{0} & \boldsymbol{\Gamma}^{2}
\end{array}\right] \succ 0
$$

con

$$
\begin{aligned}
& \boldsymbol{M}_{A}\left(s_{k}\right)=\left(\boldsymbol{Q}\left(s_{k}\right)-\boldsymbol{X}\left(s_{k}\right) \boldsymbol{\Delta}\left(s_{k}\right) \boldsymbol{C}_{d}\left(s_{k}\right)\right) \boldsymbol{A}^{N\left(s_{k}\right)} \\
& \boldsymbol{M}_{B}\left(s_{k}\right)=\left[\begin{array}{ll}
\boldsymbol{Q}\left(s_{k}\right) \boldsymbol{\Lambda}\left(N\left(s_{k}\right)\right)-\boldsymbol{X}\left(s_{k}\right) \boldsymbol{\Delta}\left(s_{k}\right) \mathcal{C}_{d}\left(s_{k}\right) & -\boldsymbol{X}\left(s_{k}\right) \boldsymbol{\Delta}\left(s_{k}\right)
\end{array}\right]
\end{aligned}
$$

\footnotetext{
${ }^{3}$ Si $\lambda$ es estable, también lo es $\lambda^{N}$. Si $\lambda$ es inestable, también lo es $\lambda^{N}$.

${ }^{4}$ Tampoco añadirán ningún elemento nulo ya que la matriz $\boldsymbol{A}$ nunca tendrá elementos nulos, puesto que significarían retardos y se ha asumido que éstos se transmiten a las salidas.
} 
$y$

$$
\begin{aligned}
& \boldsymbol{\Gamma}=\left[\begin{array}{cc}
\boldsymbol{\Gamma}_{v}^{\prime} & \mathbf{0} \\
\mathbf{0} & \boldsymbol{\Gamma}_{w}
\end{array}\right], \\
& \boldsymbol{\Gamma}_{v}^{\prime}=\frac{1}{\beta}\left[\begin{array}{ccc}
\boldsymbol{\Gamma}_{v} & & \mathbf{0} \\
& \ddots & \\
\mathbf{0} & & \boldsymbol{\Gamma}_{v}
\end{array}\right]_{\beta n \times \beta n}, \\
& \boldsymbol{\Gamma}_{v}=\left[\begin{array}{ccc}
\gamma_{v_{1}} & & 0 \\
& \ddots & \\
0 & & \gamma_{v_{n}}
\end{array}\right], \\
& \boldsymbol{\Gamma}_{w}=\left[\begin{array}{ccc}
\gamma_{w_{1}} & & 0 \\
& \ddots & \\
0 & & \gamma_{w_{n_{m}}}
\end{array}\right] \text {. }
\end{aligned}
$$

La matriz $\boldsymbol{\Lambda}\left(N\left(s_{k}\right)\right)$ viene definida por (4.36). Entonces, definiendo la ganancia del predictor como $\boldsymbol{L}\left(s_{k}\right)=\boldsymbol{Q}\left(s_{k}\right)^{-1} \boldsymbol{X}\left(s_{k}\right)$, el algoritmo de error de predicción definido por (4.12) converge asintóticamente a cero en ausencia de perturbaciones $y$, bajo condiciones iniciales nulas

$$
\left\|\boldsymbol{e}_{k}\right\|_{2}^{2}<\sum_{i=1}^{n_{v}} \gamma_{v_{i}}\left\|v_{i, k}\right\|_{2}^{2}+\sum_{i=1}^{n_{m}} \gamma_{w_{i}}\left\|w_{i, k}\right\|_{2}^{2}=\left\|\boldsymbol{\Gamma}_{v} \boldsymbol{v}_{k}\right\|_{2}^{2}+\left\|\boldsymbol{\Gamma}_{w} \boldsymbol{w}_{k}\right\|_{2}^{2}
$$

Prueba 4.5.2. Véase $\S$ B.2.1

Observación 4.5.2 (Procedimiento de diseño). Si se consideran conocidas las normas $\ell_{2}$ de las perturbaciones, se puede minimizar la cota superior de $\left\|\boldsymbol{e}_{k}\right\|_{2}$ minimizando la suma

$$
\sum_{i=1}^{n_{v}} \gamma_{v_{i}}^{2}\left\|v_{i, k}\right\|_{2}^{2}+\sum_{i=1}^{n_{m}} \gamma_{w_{i}}^{2}\left\|w_{i, k}\right\|_{2}^{2}
$$

a lo largo de todas las variables $\gamma_{v_{i}}, \gamma_{w_{i}}, \boldsymbol{P}\left(s_{k}\right), \boldsymbol{Q}\left(s_{k}\right), \boldsymbol{X}\left(s_{k}\right)$ que satisfacen la LMI (4.64). Para realizar esta minimización se puede proceder de forma similar a como se ha indicado en la observación 3.4.4 para procesos de una entrada y una salida. En primer lugar se definen $\boldsymbol{\Gamma}_{v}^{2}$ y $\boldsymbol{\Gamma}_{w}^{2}$ como variables matriciales. Si $\gamma_{v_{1}}^{2}, \ldots, \gamma_{v_{n_{v}}}^{2}, \gamma_{w_{1}}^{2}, \ldots, \gamma_{w_{n_{m}}}^{2}$ son las últimas componentes del vector $\boldsymbol{x}$ en (3.52), entonces el vector $\boldsymbol{h}^{\top}$ tendrá la forma

$$
\boldsymbol{h}^{\top}=\left[\begin{array}{lllllllll}
0 & \ldots & 0 & \left\|v_{1, k}\right\|_{2}^{2} & \ldots & \left\|v_{n_{v}, k}\right\|_{2}^{2} & \left\|w_{1, k}\right\|_{2}^{2} & \ldots & \left\|w_{n_{m}, k}\right\|_{2}^{2}
\end{array}\right] .
$$

Observación 4.5.3. Para realizar la minimización anterior es necesario conocer el valor $\left\|v_{i, k}\right\|_{2}$ (norma $\ell_{2}$ de la perturbación al periodo variante $N_{k} T$ ). Sin embargo, es más accesible la norma $\left\|v_{i}\right\|_{2}$ (norma $\ell_{2}$ de la perturbación a periodo $T$ ). Para obtener la norma de la señal $\boldsymbol{v}_{k}$ a partir de la norma de la señal $\boldsymbol{v}[t]$ se pueden aplicar las mismas aproximaciones expuestas en la observación (3.4.5) (página 39) del capítulo anterior, llegándose a

$$
\left\|v_{i, k}\right\|_{2}^{2} \approx \frac{\left\|v_{i}[t]\right\|_{2}^{2}}{\bar{N}}
$$

siendo $\bar{N}$ el valor esperado de $N_{k}$.

Observación 4.5.4. Cuando se conoce la norma del vector de perturbación $\boldsymbol{v}$ y no de cada uno de sus componentes se debe plantear el diseño anterior utilizando la misma ganancia $\gamma_{v}>0$ en todo el vector $\boldsymbol{\Gamma}_{v}$,

$$
\boldsymbol{\Gamma}_{v}=\gamma_{v} \boldsymbol{I}
$$

De esta forma, el cálculo del predictor devolverá una cota del error de predicción de la forma

$$
\left\|\boldsymbol{e}_{k}\right\|_{2}^{2}<\gamma_{v}^{2}\left\|\boldsymbol{v}_{k}\right\|_{2}^{2}+\sum_{i=1}^{n_{m}} \gamma_{w_{i}}^{2}\left\|w_{i, k}\right\|_{2}^{2}=\gamma_{v}\left\|\boldsymbol{v}_{k}\right\|_{2}^{2}+\left\|\boldsymbol{\Gamma}_{w} \boldsymbol{w}_{k}\right\|_{2}^{2}
$$

En este caso, se puede minimizar la cota superior de $\left\|\boldsymbol{e}_{k}\right\|_{2}$ minimizando la suma

$$
\gamma_{v}^{2}\left\|v_{k}\right\|_{2}^{2}+\sum_{i=1}^{n_{m}} \gamma_{w_{i}}^{2}\left\|w_{i, k}\right\|_{2}^{2}
$$

a lo largo de todas las variables $\gamma_{v}, \gamma_{w_{i}}, \boldsymbol{P}\left(s_{k}\right), \boldsymbol{Q}\left(s_{k}\right), \boldsymbol{X}\left(s_{k}\right)$ que satisfacen la LMI (4.64). Para realizar la minimización se puede proceder según se ha indicado en la observación 3.4.4, tomando en este caso

$$
\boldsymbol{h}^{\top}=\left[\begin{array}{lllllll}
0 & \ldots & 0 & \left\|\boldsymbol{v}_{k}\right\|_{2}^{2} & \left\|w_{1, k}\right\|_{2}^{2} & \ldots & \left\|w_{n_{m}, k}\right\|_{2}^{2}
\end{array}\right] .
$$


Observación 4.5.5. Bajo las mismas hipótesis que el teorema 4.5.2, y si se suponen conocidos los valores eficaces de las perturbaciones, se puede minimizar la cota superior $\|\boldsymbol{e}\|_{R M S}$ minimizando la suma

$$
\sum_{i=1}^{n_{v}} \gamma_{v_{i}}^{2}\left\|v_{i, k}\right\|_{R M S}^{2}+\sum_{i=1}^{n_{m}} \gamma_{w_{i}}^{2}\left\|w_{i, k}\right\|_{R M S}^{2}
$$

a lo largo de todas las variables $\gamma_{v_{i}}, \gamma_{w_{i}}, \boldsymbol{P}\left(s_{k}\right), \boldsymbol{Q}\left(s_{k}\right), \boldsymbol{X}\left(s_{k}\right)$ que satisfacen la LMI (4.64). Para demostrarlo tómese la ecuación (B.21) y divídase por $K$, llegando a

$$
\left\|\boldsymbol{e}_{k}\right\|_{R M S}^{2}<\left\|\boldsymbol{\Gamma}_{v} \boldsymbol{v}_{k}\right\|_{R M S}^{2}+\left\|\boldsymbol{\Gamma}_{w} \boldsymbol{w}_{k}\right\|_{R M S}^{2} .
$$

El valor eficaz de las perturbaciones $v_{i, k}$ o $w_{i, k}$ se puede calcular de forma aproximada mediante

$$
\left\|v_{i, k}\right\|_{R M S} \approx\left\|v_{i}[t]\right\|_{R M S}
$$

tal y como se detalla en la observación 3.4.7 (página 40).

Para realizar la minimización se puede proceder según se ha indicado en la observación 3.4.4, tomando en este caso

$$
\boldsymbol{h}^{\top}=\left[\begin{array}{lllllllll}
0 & \ldots & 0 & \left\|v_{1, k}\right\|_{R M S}^{2} & \ldots & \left\|v_{n_{v}, k}\right\|_{R M S}^{2} & \left\|w_{1, k}\right\|_{R M S}^{2} & \ldots & \left\|w_{n_{m}, k}\right\|_{R M S}^{2}
\end{array}\right] .
$$

Observación 4.5.6. Si se tienen perturbaciones persistentes pero se desconoce su valor eficaz, es necesario proceder de forma diferente para diseñar un predictor que acote el valor eficaz del error de predicción. La idea es que si una señal es persistente, para un intervalo $k=1, \ldots, K$ dado se puede establecer una cota de la energía acumulada como

$$
\sum_{k=1}^{K}\left(\mathbb{V}_{k}^{\top} \boldsymbol{\Gamma}_{v}^{\prime 2} \mathbb{V}_{k}+\Gamma_{w}^{2} \boldsymbol{w}_{k}^{\top} \boldsymbol{w}_{k}\right) \leq K\left(\boldsymbol{\Gamma}_{v}^{2} \boldsymbol{v}_{k}^{\top} \boldsymbol{v}_{k}+\boldsymbol{\Gamma}_{w}^{2} \boldsymbol{w}_{k}^{\top} \boldsymbol{w}_{k}\right)
$$

Nótese que la igualdad se alcanza cuando las perturbaciones son de tipo escalón. Con esta aproximación, es posible obtener una cota del valor eficaz del error de predicción $\left\|e_{k}\right\|_{R M S}$ si se minimiza la suma

$$
\sum_{i=1}^{n_{v}} \gamma_{v_{i}}^{2}\left\|v_{i, k}\right\|_{\infty}^{2}+\sum_{i=1}^{n_{m}} \gamma_{w_{i}}^{2}\left\|w_{i, k}\right\|_{\infty}^{2}
$$

a largo de todas las variables $\gamma_{v_{i}}, \gamma_{w_{i}}, \boldsymbol{P}\left(s_{k}\right), \boldsymbol{Q}\left(s_{k}\right), \boldsymbol{X}\left(s_{k}\right)$ que satisfacen la LMI (4.64). Para demostrarlo, retómese la demostración del teorema 4.5.2, de manera que (B.21) en combinación con la aproximación (4.69) lleva a

$$
\begin{array}{r}
\frac{1}{K} \sum_{k=1}^{K}\left(\boldsymbol{e}_{k-1}^{\top} \boldsymbol{e}_{k-1}\right)<\left\|\boldsymbol{\Gamma}_{v} \boldsymbol{v}_{k}\right\|_{\infty}^{2}+\left\|\boldsymbol{\Gamma}_{w} \boldsymbol{w}_{k}\right\|_{\infty}^{2}, \\
\left\|\boldsymbol{e}_{k}\right\|_{R M S}<\left\|\boldsymbol{\Gamma}_{v} \boldsymbol{v}_{k}\right\|_{\infty}^{2}+\left\|\boldsymbol{\Gamma}_{w} \boldsymbol{w}_{k}\right\|_{\infty}^{2}
\end{array}
$$

En el límite cuando $K$ tiende a infinito se tiene que

$$
\left\|\boldsymbol{e}_{k}\right\|_{R M S}<\left\|\boldsymbol{\Gamma}_{v} \boldsymbol{v}_{k}\right\|_{\infty}^{2}+\left\|\boldsymbol{\Gamma}_{w} \boldsymbol{w}_{k}\right\|_{\infty}^{2}
$$

Nótese que para un caso general en el que las perturbaciones no son del tipo escalón, la aproximación (4.69) lleva a un diseño muy conservador, mientras que si las perturbaciones son de tipo escalón, este diseño coincide con el de la observación 4.5.5.

Para poder realizar la minimización anterior es necesario aproximar el pico de las perturbaciones muestreadas a periodo $N_{k} T$, que se puede aproximar mediante

$$
\left\|v_{i, k}\right\|_{\infty} \approx\left\|v_{i}[t]\right\|_{\infty}
$$

tal y como se expone en la observación 3.4 .9 (página 42 ).

La minimización anterior se puede realizar como se ha indicado en la observación 3.4.4, tomando en este caso

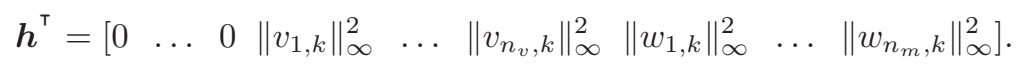


Ejemplo 4.5.1. Tómese el proceso del ejemplo anterior y considérese que se tiene una medida cada 4 o 5 periodos de control. Considérese también que en cada instante de muestreo se tiene información de alguno de los dos sensores, no necesariamente de forma simultánea, y que además el retardo en el primer sensor es fijo de valor 2 periodos, mientras que en el segundo puede ser de 3 o de 4 periodos. Con estas condiciones las posibles condiciones de de muestreo que pueden darse son los que se resumen en la tabla 4.1

\begin{tabular}{l|cccccccccc}
$s_{k}$ & 1 & 2 & 3 & 4 & 5 & 6 & 7 & 8 & 9 & 10 \\
\hline$N_{k}$ & 4 & 5 & 4 & 5 & 4 & 5 & 4 & 5 & 4 & 5 \\
$d_{1, k}$ & 2 & 2 & - & - & 2 & 2 & - & - & 2 & 2 \\
$d_{2, k}$ & - & - & 2 & 2 & 2 & 2 & 3 & 3 & 3 & 3
\end{tabular}

Tabla 4.1: Posibles condiciones de muestreo, "-": sensor no disponible

Considérese también que hay presentes en el proceso un vector de perturbaciones constante cuyo valor máximo es de $\left\|w_{x, i}[t]\right\|_{\infty}=0.2(i=1,2)$ y un vector de ruido de medida aleatorio con media cero y valor eficaz (varianza) $\left\|w_{m, i}[t]\right\|_{R M S}=0.12(i=1,2)$. Con estos datos se ha aplicado el teorema anterior obteniéndose una matriz de ganancias diferente para cada uno de los escenarios detallados en la tabla 4.1 . tal y como se observa en la tabla 4.2, en la que se muestran algunas de las diferentes matrices $\boldsymbol{L}\left(s_{k}\right)$.

\begin{tabular}{|c|c|c|c|c|c|c|}
\hline$s_{k}$ & 1 & 2 & 3 & 4 & \multicolumn{2}{|c|}{5} \\
\hline & $=-0.393 \quad 0$ & {$\left[\begin{array}{ll}-0.477 & 0\end{array}\right.$} & $-0.299^{-}$ & {$\left[\begin{array}{ll}0 & -0.435\end{array}\right.$} & $\lceil-0.180$ & -0.208 \\
\hline$L\left(s_{k}\right)$ & $-1.107 \quad 0$ & $-1.022 \quad 0$ & $\begin{array}{ll}0 & -0.957\end{array}$ & $\begin{array}{ll}0 & -1.019\end{array}$ & -0.263 & -0.824 \\
\hline & 0.8630 & 0.399 & 0.885 & 0.535 & -0.088 & 0.930 \\
\hline
\end{tabular}

Tabla 4.2: Matriz de ganancias del predictor ante diferentes condiciones de muestreo

Aplicando la matriz de ganancias correspondiente en función de las características de las muestras en cada instante se ha hecho una simulación de la predicción obtenida con este método, que se muestra en la figura 4.4. Con este método se ha obtenido un error de predicción caracterizado por las normas $\|\boldsymbol{e}[t]\|_{R M S}=0.3935 y\|\boldsymbol{e}[t]\|_{\infty}=1.0349$ (las normas se ha calculado mediante múltimples simulaciones $y$ sin tener en cuenta el transitorio inicial).

\subsubsection{Atenuación $\mathcal{H}_{2}$}

Si las perturbaciones son ruidos blancos de media cero se puede plantear el diseño desde el punto de vista de la norma $\mathcal{H}_{2}$ para acotar el valor eficaz del error de predicción (situación S1 descrita en la página 31).

Teorema 4.5.3. Considérese el predictor (4.12) aplicado al sistema (4.2) y supóngase que hay al menos una medida disponible cada $N_{k} \in \mathcal{N}$ periodos. Supóngase que la disponibilidad de los sensores en cada muestreo viene definida por la matriz $\boldsymbol{\Delta}_{k} \in \Xi$ y que el retardo asociado a cada uno de los sensores se encuentra en el conjunto $d_{i, k} \in \mathcal{D}_{i}$, de manera que se tienen $n_{\mathcal{S}}$ escenarios de muestreo $s_{k} \in \mathcal{S}$ posibles. Supóngase que las perturbaciones y los ruidos de medida son ruidos blancos de media cero y varianza $\sigma_{v_{i}}(i=1, \ldots, n)$ y $\sigma_{w_{j}}\left(j=1, \ldots, n_{m}\right)$. Para unos $\gamma_{v_{1}}, \ldots, \gamma_{v_{n_{v}}}, \gamma_{w_{1}}, \ldots, \gamma_{w_{n_{m}}}$ dados, supóngase que existen unas matrices $\boldsymbol{P}\left(s_{k}\right)=\boldsymbol{P}\left(s_{k}\right)^{\top} \in \mathbb{R}^{n \times n}, \boldsymbol{Q}\left(s_{k}\right) \in \mathbb{R}^{n \times n}, \boldsymbol{X}\left(s_{k}\right) \in \mathbb{R}^{n \times n_{m}}$ tales que

$$
\begin{aligned}
& {\left[\begin{array}{cc}
\boldsymbol{Q}\left(s_{k}\right)+\boldsymbol{Q}\left(s_{k}\right)^{\top}-\boldsymbol{P}\left(s_{k}\right) & \boldsymbol{M}_{A}\left(s_{k}\right) \\
\boldsymbol{M}_{A}\left(s_{k}\right)^{\top} & \boldsymbol{P}\left(s_{k-1}\right)-\boldsymbol{C}_{y}^{\top} \boldsymbol{C}_{y}
\end{array}\right] \succ 0,} \\
& {\left[\begin{array}{cc}
\boldsymbol{Q}\left(s_{k}\right)+\boldsymbol{Q}\left(s_{k}\right)^{\top}-\boldsymbol{P}\left(s_{k}\right) & \boldsymbol{M}_{B}\left(s_{k}\right) \\
\boldsymbol{M}_{B}\left(s_{k}\right)^{\top} & \boldsymbol{\Gamma}^{2}
\end{array}\right] \succ 0}
\end{aligned}
$$

para cualquier secuencia de muestreo $\left\{s_{k}\right\}$, siendo $\boldsymbol{M}_{A}\left(s_{k}\right)$ y $\boldsymbol{M}_{B}\left(s_{k}\right)$ las matrices definidas en (4.65). Entonces, definiendo la ganancia del predictor como $\boldsymbol{L}\left(s_{k}\right)=\boldsymbol{Q}\left(s_{k}\right)^{-1} \boldsymbol{X}\left(s_{k}\right)$, el error de predicción del algoritmo definido por (4.12) converge a cero en ausencia de perturbaciones y, bajo condiciones iniciales nulas,

$$
\left\|\boldsymbol{e}_{k}\right\|_{2}^{2}<\sum_{i=1}^{n_{v}} \gamma_{v_{i}}^{2} \sigma_{v_{i}}^{2}+\sum_{i=1}^{n_{m}} \gamma_{w_{i}}^{2} \sigma_{w_{i}}^{2}
$$



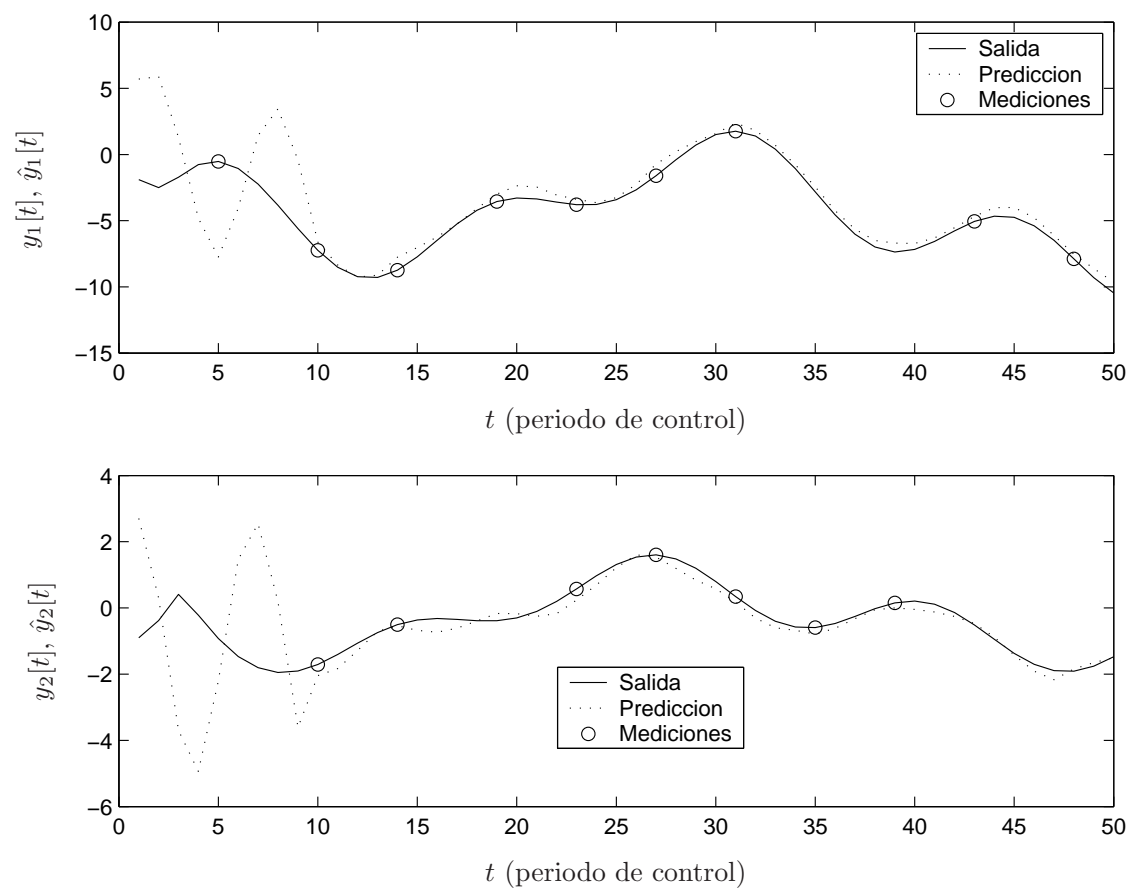

Figura 4.4: Evolución de las salidas y de las predicciones con un predictor $\mathcal{H}_{\infty}$ cuando el proceso está afectado de una perturbación constante y un ruido de medida. $\mathcal{N}=\{4,5\}, \mathcal{D}_{1}=\{2\}, \mathcal{D}_{2}=\{2,3\}, \Xi=$ $\{[1,0 ; 0,0],[0,0 ; 0,1],[1,0 ; 0,1]\}$.

Prueba 4.5.3. Véase $\S B .2 .2$.

Observación 4.5.7. Si se consideran conocidas las varianza de las perturbación y del ruido de medida, se puede minimizar la cota superior de $\left\|\boldsymbol{e}_{k}\right\|_{R M S}$ minimizando la suma

$$
\sum_{i=1}^{n_{v}} \gamma_{v_{i}}^{2} \sigma_{v_{i}}^{2}+\sum_{i=1}^{n_{m}} \gamma_{w_{i}}^{2} \sigma_{w_{i}}^{2}
$$

a lo largo de todas las variables $\gamma_{v_{i}}, \gamma_{w_{i}}, \boldsymbol{P}\left(s_{k}\right), \boldsymbol{Q}\left(s_{k}\right), \boldsymbol{X}\left(s_{k}\right)$ que satisfacen las LMI (4.71). Para realizar la minimización se puede proceder según se ha indicado en la observación 3.4.4, tomando en este caso

$$
\boldsymbol{h}^{\top}=\left[\begin{array}{lllllllll}
0 & \ldots & 0 & \sigma_{v_{1}}^{2} & \ldots & \sigma_{v_{n_{v}}}^{2} & \sigma_{w_{1}}^{2} & \ldots & \sigma_{w_{n_{m}}}^{2}
\end{array}\right] .
$$

Observación 4.5.8. El teorema anterior también es aplicable cuando se conoce la matriz de covarianzas de la perturbación del estado $\boldsymbol{W}$ y del ruido de medida $\boldsymbol{V}$. En ese caso pueden tomarse unas matrices $\boldsymbol{\Gamma}_{v}$ y $\boldsymbol{\Gamma}_{w}$ no necesariamente diagonales y con todos sus elementos positivos para obtener un predictor que acote el error de la siguiente forma

$$
\left\|\boldsymbol{e}_{k}\right\|_{R M S}^{2}<\operatorname{tr}\left(\boldsymbol{\Gamma}_{v}^{2} \boldsymbol{V}\right)+\operatorname{tr}\left(\boldsymbol{\Gamma}_{w}^{2} \boldsymbol{W}\right) .
$$

Para demostrarlo tómese la ecuación (B.23) y aplíquese el teorema A.3.3, llegando a

$$
\begin{aligned}
\mathcal{E}\left\{\mathcal{V}_{k}\right\}-\mathcal{E}\left\{\mathcal{V}_{k-1}\right\}+\boldsymbol{e}_{k-1}^{\top} \boldsymbol{e}_{k-1} & <\mathcal{E}\left\{\left[\begin{array}{c}
\mathbb{V}_{k} \\
\boldsymbol{w}_{k}
\end{array}\right]^{\top} \boldsymbol{\Gamma}^{2}\left[\begin{array}{c}
\mathbb{V}_{k} \\
\boldsymbol{w}_{k}
\end{array}\right]\right\} \\
& =\operatorname{tr}\left(\boldsymbol{\Gamma}^{2} \operatorname{diag}\{\underbrace{\boldsymbol{V}, \ldots, \boldsymbol{V}}_{\beta}, \boldsymbol{W}\}\right)=\operatorname{tr}\left(\boldsymbol{\Gamma}_{v}^{2} \boldsymbol{V}\right)+\operatorname{tr}\left(\boldsymbol{\Gamma}_{w}^{2} \boldsymbol{W}\right),
\end{aligned}
$$

donde

$$
\boldsymbol{\Gamma}^{2}=\operatorname{diag}\left\{\frac{\boldsymbol{\Gamma}_{v}^{2}}{\beta}, \ldots, \frac{\boldsymbol{\Gamma}_{v}^{2}}{\beta}, \boldsymbol{\Gamma}_{w}^{2}\right\}
$$


y $\boldsymbol{\Gamma}_{v} \in \mathbb{R}^{n_{v} \times n_{v}}, \boldsymbol{\Gamma}_{w} \in \mathbb{R}^{n_{m} \times n_{m}}$ son matrices simétricas con elementos positivos. De esta manera, si se realiza una minimización de la suma

$$
\operatorname{tr}\left(\boldsymbol{\Gamma}_{v}^{2} \boldsymbol{V}\right)+\operatorname{tr}\left(\boldsymbol{\Gamma}_{w}^{2} \boldsymbol{W}\right)
$$

a lo largo de las matrices $\boldsymbol{P}\left(s_{k}\right), \boldsymbol{Q}\left(s_{k}\right), \boldsymbol{X}\left(s_{k}\right), \boldsymbol{\Gamma}_{v}$ y $\boldsymbol{\Gamma}_{w}$ que cumplen la LMI (4.71), se minimizará el valor eficaz del error de predicción provocado por la varianza y covarianza de las señales de perturbación y ruido de medida. Nótese la similitud entre este objetivo y el objetivo del filtro de Kalman: minimizar el valor esperado del error en cada muestreo a partir del conocimiento de las varianzas y covarianzas de perturbación y ruido de medida.

Ejemplo 4.5.2. Considérese de nuevo el ejemplo anterior con el muestreo allí definido, pero considérese ahora que el sistema está afectado por una perturbación $\boldsymbol{v}$ y un ruido de medida $\boldsymbol{w}$ ambos de media cero y con matriz de covarianzas dadas por

$$
\boldsymbol{W}=\left[\begin{array}{cc}
0.0033 & -0.0002 \\
-0.0002 & 0.0026
\end{array}\right], \quad \boldsymbol{V}=\left[\begin{array}{cc}
0.1403 & -0.0056 \\
-0.0056 & 0.1198
\end{array}\right] \cdot 10^{-3}
$$

Aplicando la técnica de diseño expuesta en el teorema anterior es posible llegar a un conjunto de matrices $\boldsymbol{L}\left(s_{k}\right)$ que permiten predecir la salida de forma estable y atenuar el efecto de las perturbaciones y ruidos de medida sobre la salida, tal y como se muestra en la simulación de la figura 4.5. Con este método se ha obtenido un error de predicción caracterizado por las normas $\|\boldsymbol{e}[t]\|_{R M S}=0.2661$ y $\|\boldsymbol{e}[t]\|_{\infty}=0.9092$ (la norma infinito se ha calculado sin tener en cuenta el transitorio inicial).
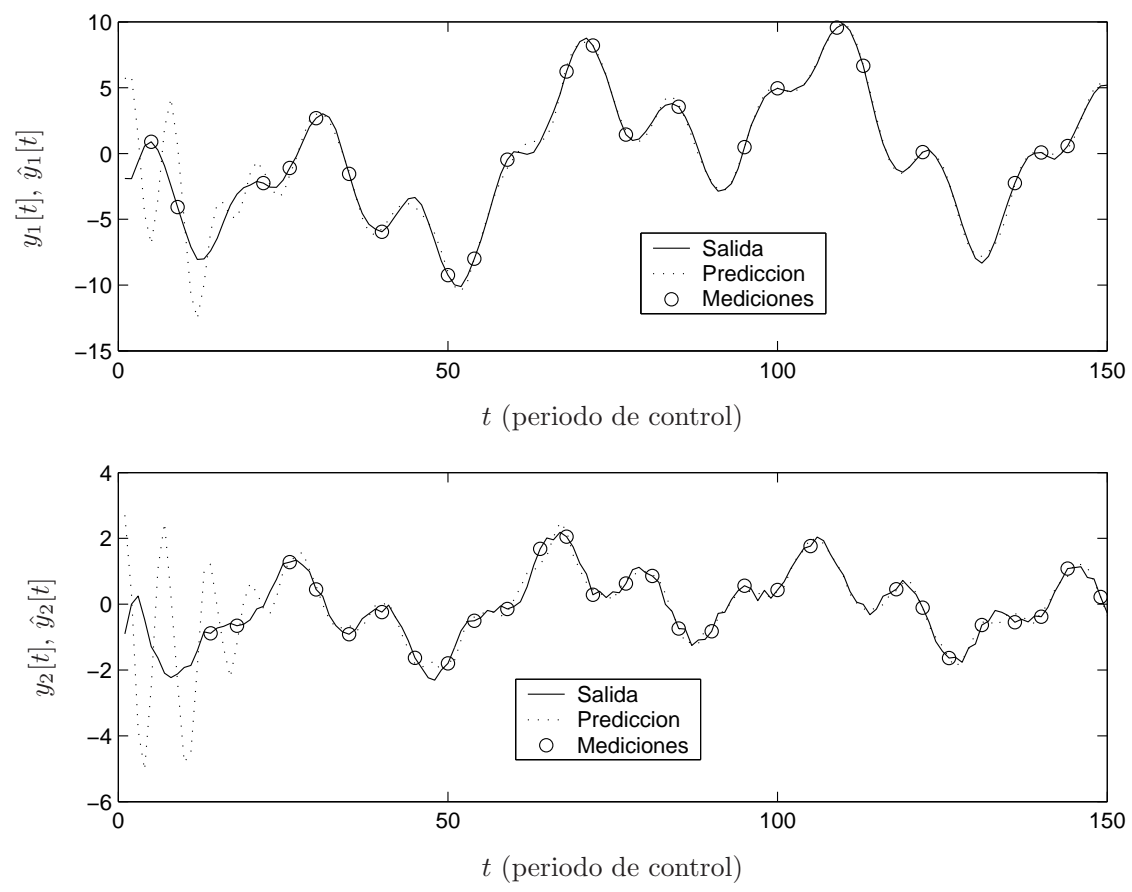

Figura 4.5: Evolución de las salidas y de las predicciones con un predictor $\mathcal{H}_{2}$ cuando el proceso está afectado de perturbaciones y ruidos de medida aleatorios. $\mathcal{N}=\{4,5\}, \mathcal{D}_{1}=\{2\}, \mathcal{D}_{2}=\{2,3\}, \Xi=$ $\{[1,0 ; 0,0],[0,0 ; 0,1],[1,0 ; 0,1]\}$.

Ejemplo 4.5.3. Retómese el proceso del ejemplo anterior y considérense ahora las perturbaciones y ruido propuestas en el ejemplo 4.5 .1 (perturbación constante y ruido de medida aleatorio). Si sobre este proceso se aplican los diseños $\mathcal{H}_{\infty}$ y $\mathcal{H}_{2}$ (tomando $\|\boldsymbol{w}[t]\|_{R M S}=\|\boldsymbol{v}[t]\|_{\infty}$ por tratarse de una perturbación constante), se tiene un resultado similar en régimen permanente. Sin embargo el diseño $\mathcal{H}_{\infty}$ presenta un transitorio más rápido, tal y como se muestra en la simulación de la figura 4.6 

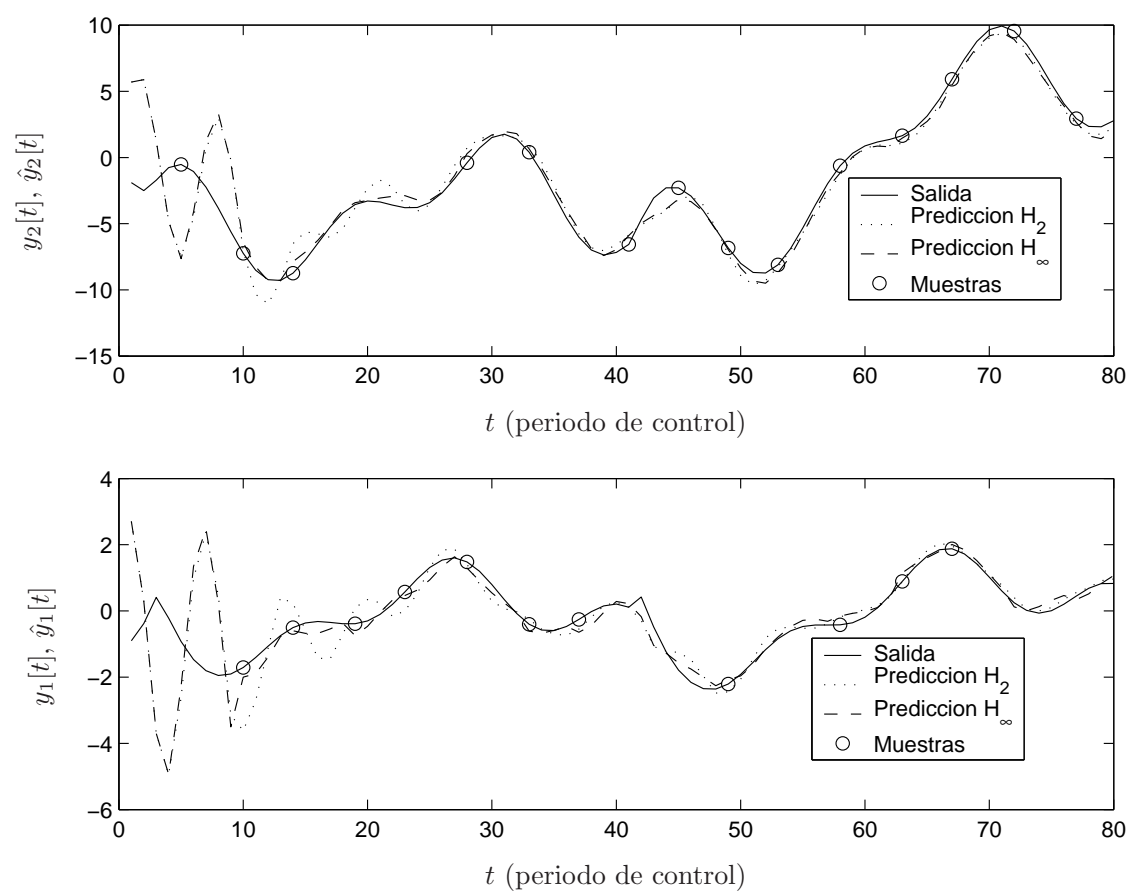

Figura 4.6: Evolución de las salidas y de las predicciones con predictores $\mathcal{H}_{2}$ y $\mathcal{H}_{\infty}$ cuando el proceso está afectado de perturbaciones constantes y ruidos de medida aleatorios. $\mathcal{N}=\{4,5\}, \mathcal{D}_{1}=\{2\}, \mathcal{D}_{2}=\{2,3\}$, $\Xi=\{[1,0 ; 0,0],[0,0 ; 0,1],[1,0 ; 0,1]\}$.

\subsubsection{Atenuación $\mathcal{H}_{2 g}$}

El siguiente teorema recoge el diseño de predictores estables que minimizan la cota de error frente a perturbaciones y ruidos de energía finita (situación S3 de la página 31.

Teorema 4.5.4. Considérese el predictor (4.12) aplicado al sistema (4.2) y supóngase que hay al menos una medida disponible cada $N_{k} \in \mathcal{N}$ periodos. Supóngase que la disponibilidad de los sensores en cada muestreo viene definida por la matriz $\boldsymbol{\Delta}_{k} \in \Xi$ y que el retardo asociado a cada uno de los sensores se encuentra en el conjunto $d_{i, k} \in \mathcal{D}_{i}$, de manera que se tienen $n_{\mathcal{S}}$ escenarios de muestreo $s_{k} \in \mathcal{S}$ posibles. Para unos $\gamma_{v_{1}}, \ldots, \gamma_{v_{n_{v}}}, \gamma_{w_{1}}, \ldots, \gamma_{w_{n_{m}}}$ dados, supóngase que existen unas matrices $\boldsymbol{P}\left(s_{k}\right)=\boldsymbol{P}\left(s_{k}\right)^{\top} \in$ $\mathbb{R}^{n \times n}, \boldsymbol{Q}\left(s_{k}\right) \in \mathbb{R}^{n \times n}, \boldsymbol{X}\left(s_{k}\right) \in \mathbb{R}^{n \times n_{m}}$ tales que

$$
\begin{aligned}
& {\left[\begin{array}{ccc}
\boldsymbol{Q}\left(s_{k}\right)+\boldsymbol{Q}\left(s_{k}\right)^{\top}-\boldsymbol{P}\left(s_{k}\right) & \boldsymbol{M}_{A}\left(s_{k}\right) & \boldsymbol{M}_{B}\left(s_{k}\right) \\
\boldsymbol{M}_{A}\left(s_{k}\right)^{\top} & \mu^{2} \boldsymbol{P}\left(s_{k-1}\right) & \mathbf{0} \\
\boldsymbol{M}_{B}\left(s_{k}\right)^{\top} & \mathbf{0} & \boldsymbol{\Gamma}^{2}
\end{array}\right] \succ 0,} \\
& \boldsymbol{P}\left(s_{k}\right)-\boldsymbol{C}_{y}^{\top} \boldsymbol{C}_{y} \succ 0,
\end{aligned}
$$

para cualquier secuencia de muestreo $\left\{s_{k}\right\}$, siendo $\boldsymbol{M}_{A}\left(s_{k}\right)$ y $\boldsymbol{M}_{B}\left(s_{k}\right)$ las matrices definidas en (4.65). Entonces, definiendo la ganancia del predictor como $\boldsymbol{L}\left(s_{k}\right)=\boldsymbol{Q}\left(s_{k}\right)^{-1} \boldsymbol{X}\left(s_{k}\right)$, el error de predicción del algoritmo definido por (4.12) converge a cero en ausencia de perturbaciones y, bajo condiciones iniciales nulas,

$$
\left\|\boldsymbol{e}_{k}\right\|_{\infty}^{2}<\left\|\boldsymbol{\Gamma}_{v} \boldsymbol{v}_{k}\right\|_{2}^{2}+\left\|\boldsymbol{\Gamma}_{w} \boldsymbol{w}_{k}\right\|_{2}^{2}
$$

Prueba 4.5.4. Véase $\S$ B.2.3.

Observación 4.5.9. Conocida la norma $\ell_{2}$ de las perturbaciones, se puede diseñar el predictor que minimiza la norma $\left\|\boldsymbol{e}_{k}\right\|_{\infty}$ haciendo una minimización de la suma

$$
\sum_{i=1}^{n_{v}} \gamma_{v_{i}}^{2}\left\|v_{i, k}\right\|_{2}^{2}+\sum_{i=1}^{n_{m}} \gamma_{w_{i}}^{2}\left\|w_{i, k}\right\|_{2}^{2}
$$


a lo largo de todas las variables $\gamma_{v_{i}}, \gamma_{w_{i}}, \boldsymbol{P}\left(s_{k}\right), \boldsymbol{Q}\left(s_{k}\right), \boldsymbol{X}\left(s_{k}\right)$, que minimizan la LMI (4.73). Para realizar la minimización se puede proceder según se ha indicado en la observación 3.4.4, tomando en este caso

$$
\boldsymbol{h}^{\top}=\left[\begin{array}{lllllllll}
0 & \ldots & 0 & \left\|v_{1, k}\right\|_{2}^{2} & \ldots & \left\|v_{n_{v}, k}\right\|_{2}^{2} & \left\|w_{1, k}\right\|_{2}^{2} & \ldots & \left\|w_{n_{m}, k}\right\|_{2}^{2}
\end{array}\right] .
$$

\subsubsection{Atenuación $\ell_{1}$}

El siguiente teorema muestra el diseño del predictor que minimiza la amplitud del error de predicción frente a perturbaciones de cota de amplitud conocida (situación S2 de la página 31).

Teorema 4.5.5. Considérese el predictor (4.12) aplicado al sistema (4.2) y supóngase que hay al menos una medida disponible cada $N_{k} \in \mathcal{N}$ periodos. Supóngase que la disponibilidad de los sensores en cada muestreo viene definida por la matriz $\boldsymbol{\Delta}_{k} \in \Xi$ y que el retardo asociado a cada uno de los sensores se encuentra en el conjunto $d_{i, k} \in \mathcal{D}_{i}$, de manera que se tienen $n_{\mathcal{S}}$ escenarios de muestreo $s_{k} \in \mathcal{S}$ posibles. Para unos $\gamma_{v_{1}}, \ldots, \gamma_{v_{n_{v}}}, \gamma_{w_{1}}, \ldots, \gamma_{w_{n_{m}}}$ dados, supóngase que existen unas matrices $\boldsymbol{P}\left(s_{k}\right)=\boldsymbol{P}\left(s_{k}\right)^{\top} \in$ $\mathbb{R}^{n \times n}, \boldsymbol{Q}\left(s_{k}\right) \in \mathbb{R}^{n \times n}, \boldsymbol{X}\left(s_{k}\right) \in \mathbb{R}^{n \times n_{m}}$ y unos escalares $\varepsilon_{v_{1}}, \ldots, \boldsymbol{\varepsilon}_{v_{n_{v}}}, \boldsymbol{\varepsilon}_{w_{1}}, \ldots, \boldsymbol{\varepsilon}_{w_{n_{m}}}$ tales que

$$
\begin{aligned}
& \lambda \in(0,1) \\
& {\left[\begin{array}{ccc}
\boldsymbol{Q}\left(s_{k}\right)+\boldsymbol{Q}\left(s_{k}\right)^{\top}-\boldsymbol{P}\left(s_{k}\right) & \boldsymbol{M}_{A}\left(s_{k}\right) & \boldsymbol{M}_{B}\left(s_{k}\right) \\
\boldsymbol{M}_{A}\left(s_{k}\right)^{\top} & (1-\lambda) \boldsymbol{P}\left(s_{k-1}\right) & \mathbf{0} \\
\boldsymbol{M}_{B}\left(s_{k}\right)^{\top} & \mathbf{0} & \boldsymbol{\Upsilon}^{2}
\end{array}\right] \succ 0,} \\
& {\left[\begin{array}{ccc}
\lambda \boldsymbol{P}\left(s_{k}\right) & \mathbf{0} & \boldsymbol{C}_{y}^{\boldsymbol{\top}} \\
\mathbf{0} & \boldsymbol{\Gamma}^{2}-\boldsymbol{\Upsilon}^{2} & \mathbf{0} \\
\boldsymbol{C}_{y} & \mathbf{0} & \boldsymbol{I}
\end{array}\right] \succ 0,}
\end{aligned}
$$

para cualquier secuencia de muestreo $\left\{s_{k}\right\}$, siendo $\boldsymbol{M}_{A}\left(s_{k}\right)$ y $\boldsymbol{M}_{B}\left(s_{k}\right)$ las matrices definidas en (4.65) y

$$
\begin{aligned}
& \mathbf{\Upsilon}=\left[\begin{array}{cc}
\mathbf{\Upsilon}_{v}^{\prime} & \mathbf{0} \\
\mathbf{0} & \mathbf{\Upsilon}_{w}
\end{array}\right] \\
& \mathbf{\Upsilon}_{v}^{\prime}=\frac{1}{\beta}\left[\begin{array}{ccc}
\boldsymbol{\Upsilon}_{v} & & \mathbf{0} \\
& \ddots & \\
\mathbf{0} & & \boldsymbol{\Upsilon}_{v}
\end{array}\right] \text {, } \\
& \Upsilon_{v}=\left[\begin{array}{ccc}
\varepsilon_{v_{1}} & & 0 \\
& \ddots & \\
0 & & \varepsilon_{v_{n_{v}}}
\end{array}\right] \text {, } \\
& \mathbf{\Upsilon}_{w}=\left[\begin{array}{ccc}
\varepsilon_{w_{1}} & & 0 \\
& \ddots & \\
0 & & \boldsymbol{\varepsilon}_{w_{n_{m}}}
\end{array}\right] \text {. }
\end{aligned}
$$

Entonces, definiendo la ganancia del predictor en función del valor del parámetro de muestreo como $\boldsymbol{L}\left(s_{k}\right)=\boldsymbol{Q}\left(s_{k}\right)^{-1} \boldsymbol{X}\left(s_{k}\right)$, el error de predicción del algoritmo definido por (4.12) converge asintóticamente a cero con una tasa de decrecimiento minima de $\sqrt{1-\lambda}$ en ausencia de perturbaciones y, bajo condiciones iniciales nulas,

$$
\left\|\boldsymbol{e}_{k}\right\|_{\infty}^{2}<\left\|\boldsymbol{\Gamma}_{v} \boldsymbol{v}_{k}\right\|_{\infty}^{2}+\left\|\boldsymbol{\Gamma}_{w} \boldsymbol{w}_{k}\right\|_{\infty}^{2}
$$

Prueba 4.5.5. Véase $\S \mathrm{B} .2 .4$

Observación 4.5.10. Conocida la norma $\ell_{\infty}$ de las perturbaciones, se puede minimizar la norma $\left\|\boldsymbol{e}_{k}\right\|_{\infty}$ haciendo una minimización de la suma

$$
\sum_{i=1}^{n} \gamma_{v_{i}}^{2}\left\|v_{i, k}\right\|_{\infty}^{2}+\sum_{i=1}^{n_{m}} \gamma_{w_{i}}^{2}\left\|w_{i, k}\right\|_{\infty}^{2} .
$$

a lo largo de todas las variables $\gamma_{v_{i}}, \gamma_{w_{i}}, \lambda, \boldsymbol{P}\left(s_{k}\right), \boldsymbol{Q}\left(s_{k}\right), \boldsymbol{X}\left(s_{k}\right)$ que satisfacen la LMI (4.75). Para realizar la minimización se puede proceder según se ha indicado en la observación 3.4.4, tomando en este caso

$$
\boldsymbol{h}^{\top}=\left[\begin{array}{llllllll}
0 & \ldots & 0 & \left\|v_{1, k}\right\|_{\infty}^{2} & \ldots & \left\|v_{n_{v}, k}\right\|_{\infty}^{2}\left\|w_{1, k}\right\|_{\infty}^{2} & \ldots & \left\|w_{n_{m}, k}\right\|_{\infty}^{2}
\end{array}\right]
$$

Observación 4.5.11. Nótese que el conjunto de desigualdades anterior (4.75) no forman un problema PLMI (lineal) porque la variable $\lambda$ entra de forma no lineal en el término $\lambda \boldsymbol{P}\left(s_{k}\right)$ que aparece en (4.75b) y $(4.75 \mathrm{c})$. Así pues, para obtener la cota superior ínfima de $\left\|\boldsymbol{e}_{k}\right\|_{\infty}$, se ha de minimizar $\left\|\boldsymbol{e}_{k}\right\|_{\infty}$ también a lo largo de $\lambda \in] 0,1[$, lo que implica resolver otro problema de minimización encadenado con el anterior. 


\subsection{Diseño estocástico de predictores}

En el diseño de predictores de la sección anterior se asegura, para cualquier secuencia posible que pueda tomar el parámetro de muestreo, tanto la convergencia asintótica del predictor, como la capacidad de éste para atenuar las posibles perturbaciones que afectan a la predicción de la salida.

Sin embargo, es posible que esta secuencia de muestreo siga cierto modelo probabilístico. En este caso, si se considera el proceso de muestreo como una cadena de Markov (véase definición 3.5.1 en la página 48) con probabilidades de transición conocidas (véase definición 3.5.2) se pueden desarrollar diseños menos conservativos que los de la sección anterior, en los que se asegure un decrecimiento del error de predicción en valor medio, a la vez que una atenuación media de las perturbaciones.

\subsubsection{Diseño para estabilidad nominal}

El siguiente teorema muestra el diseño de predictores estables que aseguran la convergencia en valor medio de un proceso cuya distribución de probabilidades de transición es conocida.

Teorema 4.6.1. Considérese el sistema (4.2) en ausencia de perturbaciones y ruido de medida, y supóngase que hay al menos una medición disponible cada $N_{k} \in \mathcal{N}$ periodos de entrada y que cada medición está afectada de un retardo $d_{i, k} \in \mathcal{D}_{i}$. Supóngase que la disponibilidad de sensores en cada muestreo viene dada por $\boldsymbol{\Delta}_{k} \in \Xi$ habiendo en total $n_{\mathcal{S}}$ escenarios de muestreo $s_{k}$ posibles cuya transición viene definida por la probabilidad $\alpha_{i j}$. Para un $\mu \in(0,1]$ dado, supóngase que existen unas matrices $\boldsymbol{P}(i)=\boldsymbol{P}(i)^{\top} \in \mathbb{R}^{n \times n}$, $\boldsymbol{Q}(i) \in \mathbb{R}^{n \times n}, \boldsymbol{X}(i) \in \mathbb{R}^{n \times 1},\left(i=1, \ldots, n_{\mathcal{S}}\right)$ tales que la siguiente $L M I$

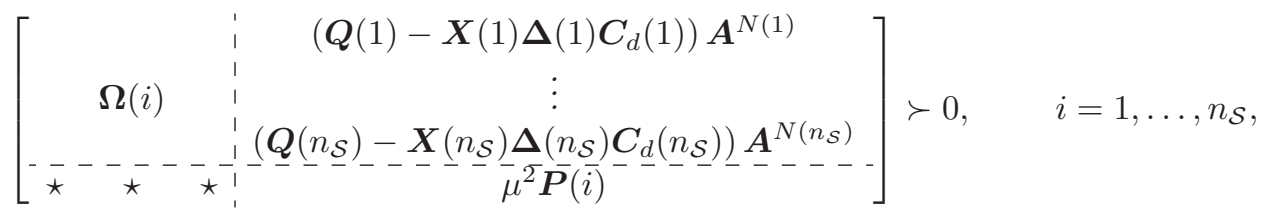

con

$$
\boldsymbol{\Omega}(i)=\left[\begin{array}{ccc}
\boldsymbol{Q}(1)+\boldsymbol{Q}(1)^{\top}-\alpha_{i 1} \boldsymbol{P}(1) & \mathbf{0} \\
\mathbf{0} & \ddots & \boldsymbol{Q}\left(n_{\mathcal{S}}\right)+\boldsymbol{Q}\left(n_{\mathcal{S}}\right)^{\top}-\alpha_{i n_{\mathcal{S}}} \boldsymbol{P}\left(n_{\mathcal{S}}\right)
\end{array}\right]
$$

Entonces, si la ganancia del predictor se define como $\boldsymbol{L}(i)=\boldsymbol{Q}(i)^{-1} \boldsymbol{X}(i)$, el error de predicción del algoritmo definido por (4.12) converge a cero de forma asintótica con probabilidad uno y con un ratio de decadencia menor que $\mu$.

Prueba 4.6.1. Para asegurar la estabilidad asintótica con probabilidad uno se hace uso de una función de Lyapunov cuadrática paramétrica y se analiza el valor esperado de su diferencia entre dos muestras consecutivas. Tomando la función de Lyapunov

$$
\mathcal{V}\left(\widetilde{\boldsymbol{x}}_{k}, s_{k}\right)=\widetilde{\boldsymbol{x}}_{k}^{\top} \boldsymbol{P}\left(s_{k}\right) \widetilde{\boldsymbol{x}}_{k},
$$

el valor esperado de la diferencia es

$$
\begin{aligned}
\mathcal{E} & \left\{\Delta \mathcal{V} \mid s_{k-1}=i\right\}=\mathcal{E}\left\{\mathcal{V}\left(\widetilde{\boldsymbol{x}}_{k}, s_{k}\right)-\mathcal{V}\left(\widetilde{\boldsymbol{x}}_{k-1}, s_{k-1}\right) \mid s_{k-1}=i\right\} \\
& =\mathcal{E}\left\{\widetilde{\boldsymbol{x}}_{k}^{\top} \boldsymbol{P}\left(s_{k}\right) \widetilde{\boldsymbol{x}}_{k}-\widetilde{\boldsymbol{x}}_{k-1}^{\top} \boldsymbol{P}\left(s_{k-1}\right) \widetilde{\boldsymbol{x}}_{k-1} \mid s_{k-1}=i\right\} \\
& =\mathcal{E}\left\{\widetilde{\boldsymbol{x}}_{k-1}^{\top} \boldsymbol{\mathcal { A }}\left(s_{k}\right)^{\top} \boldsymbol{P}\left(s_{k}\right) \mathcal{A}\left(s_{k}\right) \widetilde{\boldsymbol{x}}_{k-1} \mid s_{k-1}=i\right\}-\widetilde{\boldsymbol{x}}_{k-1}^{\top} \boldsymbol{P}(i) \widetilde{\boldsymbol{x}}_{k-1} .
\end{aligned}
$$

Utilizando las probabilidades de transmisión $\left(\alpha_{i j}\right)$ para calcular la esperanza se tiene que

$$
\begin{aligned}
\mathcal{E} & \left\{\Delta \mathcal{V} \mid s_{k-1}=i\right\}=\sum_{j=1}^{n_{\mathcal{S}}} \alpha_{i j}\left(\widetilde{\boldsymbol{x}}_{k-1}^{\top} \mathcal{A}(j)^{\top} \boldsymbol{P}(j) \mathcal{A}(j) \widetilde{\boldsymbol{x}}_{k-1}\right)-\widetilde{\boldsymbol{x}}_{k-1}^{\top} \boldsymbol{P}(i) \widetilde{\boldsymbol{x}}_{k-1} \\
& =\widetilde{\boldsymbol{x}}_{k-1}^{\top}\left(\sum_{j=1}^{n_{\mathcal{S}}} \alpha_{i j}\left(\boldsymbol{\mathcal { A }}(j)^{\top} \boldsymbol{P}(j) \mathcal{A}(j)\right)-\boldsymbol{P}(i)\right) \widetilde{\boldsymbol{x}}_{k-1} .
\end{aligned}
$$


De la última igualdad es obvio que para asegurar la convergencia asintótica en valor medio es necesario que

$$
\sum_{j=1}^{n_{\mathcal{S}}} \alpha_{i j}\left(\boldsymbol{\mathcal { A }}(j)^{\top} \boldsymbol{P}(j) \mathcal{A}(j)\right)-\boldsymbol{P}(i) \prec \mathbf{0} .
$$

Por otra parte, si se introduce la ganancia $\boldsymbol{L}(i)=\boldsymbol{Q}(i)^{-1} \boldsymbol{X}(i)$ en la LMI (4.78), la desigualdad puede escribirse como

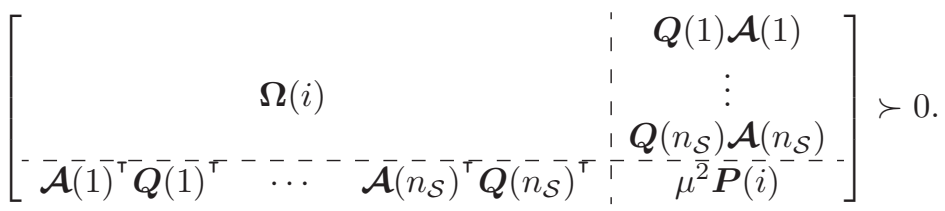

La condición (4.54) se puede aplicar a cada elemento de la matriz $\boldsymbol{\Omega}(i)$ llegándose a

$$
\boldsymbol{Q}(j)+\boldsymbol{Q}(j)^{\top}-\alpha_{i j} \boldsymbol{P}(j) \preceq \alpha_{i j}^{-1} \boldsymbol{Q}(j)^{\top} \boldsymbol{P}(j)^{-1} \boldsymbol{Q}(j),
$$

con lo que

$$
\boldsymbol{\Omega}(i) \preceq\left[\begin{array}{ccc}
\alpha_{i 1}^{-1} \boldsymbol{Q}(1)^{\top} \boldsymbol{P}(1)^{-1} \boldsymbol{Q}(1) & & \mathbf{0} \\
\mathbf{0} & \ddots & \\
& & \alpha_{i n_{\mathcal{S}}}^{-1} \boldsymbol{Q}\left(n_{\mathcal{S}}\right)^{\top} \boldsymbol{P}\left(n_{\mathcal{S}}\right)^{-1} \boldsymbol{Q}\left(n_{\mathcal{S}}\right)
\end{array}\right] .
$$

Aplicando esta propiedad a (4.81) y utilizando los complementos de Schur se obtiene la condición

$$
\sum_{j=1}^{n_{\mathcal{S}}} \alpha_{i j}\left(\boldsymbol{\mathcal { A }}(j)^{\top} \boldsymbol{P}(j) \mathcal{A}(j)\right)-\mu^{2} \boldsymbol{P}(i) \prec \mathbf{0} .
$$

Si esta condición se cumple, también se cumple (4.80), ya que $\boldsymbol{P}(i) \succ \mathbf{0}$ y $\mu^{2}<1$, con lo que queda demostrada la convergencia asintótica en valor medio con un ratio menor que $\mu$.

En el diseño estocástico de predictores mediante técnicas LMI no es necesario extraer el subsistema detectable como se ha expuesto en \$4.5.2 para el diseño determinista de predictores. El hecho de que todos los posibles escenarios de muestreo tengan una probabilidad no nula de aparecer hace que no sean posibles las secuencias de muestreo bajo las cuales el sistema no es detectable.

Si la planta presenta perturbaciones y el sensor introduce ruido de medida, se puede modificar la estrategia de diseño para tratar de atenuar los efectos de estas señales sobre el error de predicción.

\subsubsection{Diseño para atenuar perturbaciones}

Si la planta presenta perturbaciones y los sensores introducen ruido de medida, se deben tomar en consideración las estrategias de diseño basadas en la atenuación de perturbaciones introducidas en $\S 3.2 .3$. En las siguientes secciones se presenta un catálogo de predictores donde cada uno de ellos tiene en cuenta diferentes características en las perturbaciones y su efecto sobre el error de predicción cuando la secuencia de muestreo viene definida por una cadena de Markov.

\section{Atenuación $\mathcal{H}_{\infty}$}

El siguiente teorema recoge el diseño de predictores estables que minimizan la cota del error en las situaciones S1, S2 y S3 descritas en la página 31.

Teorema 4.6.2. Considérese el predictor (4.12) aplicado al sistema (4.2) y supóngase que hay al menos una medida disponible cada $N_{k} \in \mathcal{N}$ periodos. Supóngase que la disponibilidad de los sensores en cada muestreo viene definida por la matriz $\boldsymbol{\Delta}_{k} \in \Xi$ y que el retardo asociado a cada uno de los sensores se encuentra en el conjunto $d_{i, k} \in \mathcal{D}_{i}$, de manera que se tienen $n_{\mathcal{S}}$ escenarios de muestreo $s_{k} \in \mathcal{S}$ posibles posibles cuya transición viene definida por la probabilidad $\alpha_{i j}$. Para unos $\gamma_{v_{1}}, \ldots, \gamma_{v_{n_{v}}}, \gamma_{w_{1}}, \ldots, \gamma_{w_{n_{m}}}$ dados, supóngase que existen unas matrices $\boldsymbol{P}(i)=\boldsymbol{P}(i)^{\top} \in \mathbb{R}^{n \times n}, \boldsymbol{Q}(i) \in \mathbb{R}^{n \times n}, \boldsymbol{X}(i) \in \mathbb{R}^{n \times 1}$ tales que

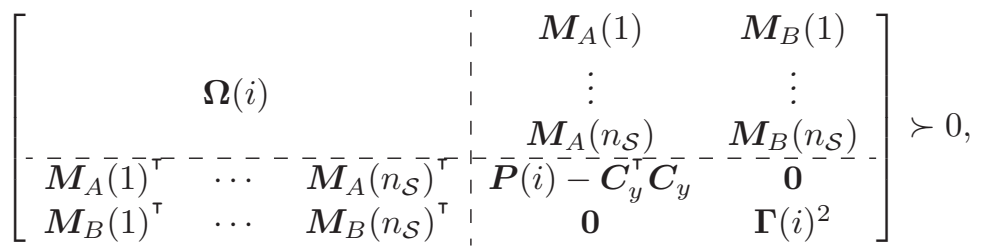


Entonces, definiendo la ganancia del predictor como $\boldsymbol{L}\left(s_{k}\right)=\boldsymbol{Q}\left(s_{k}\right)^{-1} \boldsymbol{X}\left(s_{k}\right)$, el algoritmo de error de predicción definido por (4.12) converge asintóticamente a cero con probabilidad uno en ausencia de perturbaciones y, bajo condiciones iniciales nulas

$$
\mathcal{E}\left\{\left\|\boldsymbol{e}_{k}\right\|_{2}^{2}\right\}<\left\|\boldsymbol{\Gamma}_{v} \boldsymbol{v}_{k}\right\|_{2}^{2}+\left\|\boldsymbol{\Gamma}_{w} \boldsymbol{w}_{k}\right\|_{2}^{2}
$$

donde la norma $\mathcal{E}\left\{\left\|e_{k}\right\|_{2}^{2}\right\}$ es la norma del vector de error esperado en cada muestreo dado el error del muestreo anterior, es decir:

$$
\mathcal{E}\left\{\left\|\boldsymbol{e}_{k}\right\|_{2}^{2}\right\}=\sum_{k=1}^{\infty} \mathcal{E}\left\{\boldsymbol{e}_{k}^{\top} \boldsymbol{e}_{k} \mid k-1\right\}
$$

Prueba 4.6.2. Véase $\S$ B.3.1.

$\mathrm{Al}$ igual que ocurre con el diseño determinista $\mathcal{H}_{\infty}$, este último resultado es fácilmente extendible para obtener los diseños que aseguran

$$
\left\|\boldsymbol{e}_{k}\right\|_{R M S}^{2}<\left\|\boldsymbol{\Gamma}_{v} \boldsymbol{v}_{k}\right\|_{R M S}^{2}+\left\|\boldsymbol{\Gamma}_{w} \boldsymbol{w}_{k}\right\|_{R M S}^{2}
$$

$\mathrm{y}$

$$
\left\|\boldsymbol{e}_{k}\right\|_{R M S}^{2}<\left\|\boldsymbol{\Gamma}_{v} \boldsymbol{v}_{k}\right\|_{\infty}^{2}+\left\|\boldsymbol{\Gamma}_{w} \boldsymbol{w}_{k}\right\|_{\infty}^{2}
$$

donde

$$
\mathcal{E}\left\{\left\|\boldsymbol{e}_{k}\right\|_{R M S}^{2}\right\}=\lim _{K \rightarrow \infty} \frac{1}{K} \sum_{k=1}^{K} \mathcal{E}\left\{\boldsymbol{e}_{k}^{\top} \boldsymbol{e}_{k} \mid k-1\right\} .
$$

Observación 4.6.1. Si se consideran conocidas las normas $\ell_{2}$ de las perturbaciones, se puede minimizar la cota superior de $\mathcal{E}\left\{\left\|e_{k}\right\|_{2}\right\}$ minimizando la suma

$$
\left\|\boldsymbol{\Gamma}_{v} \boldsymbol{v}_{k}\right\|_{2}^{2}+\left\|\boldsymbol{\Gamma}_{w} \boldsymbol{w}_{k}\right\|_{2}^{2}
$$

a lo largo de todas las las variables $\gamma_{v_{i}}, \gamma_{w_{i}}, \boldsymbol{P}(i), \boldsymbol{Q}(i), \boldsymbol{X}(i)$ que satisfacen la LMI (4.83). Para realizar la minimización se puede proceder según se ha indicado en la observación 3.4.4 tomando en este caso

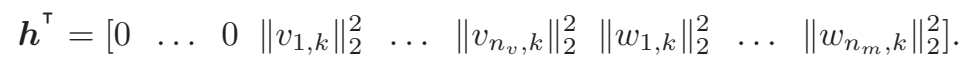

Observación 4.6.2. Bajo las mismas hipótesis que el teorema 4.6.2, y si se suponen conocidas las normas RMS de las perturbaciones, se puede minimizar $\mathcal{E}\left\{\left\|\boldsymbol{e}_{k}\right\|_{R M S}\right\}$ minimizando la suma

$$
\left\|\boldsymbol{\Gamma}_{v} \boldsymbol{v}_{k}\right\|_{R M S}^{2}+\left\|\boldsymbol{\Gamma}_{w} \boldsymbol{w}_{k}\right\|_{R M S}^{2}
$$

a lo largo de todas las variables $\gamma_{v_{i}}, \gamma_{w_{i}}, \boldsymbol{P}(i), \boldsymbol{Q}(i), \boldsymbol{X}(i)$ que satisfacen la LMI (4.83). Para realizar la minimización se puede proceder según se ha indicado en la observación 3.4.4, tomando en este caso

$$
\boldsymbol{h}^{\top}=\left[\begin{array}{lllllllll}
0 & \ldots & 0 & \left\|v_{1, k}\right\|_{R M S}^{2} & \ldots & \left\|v_{n_{v}, k}\right\|_{R M S}^{2} & \left\|w_{1, k}\right\|_{R M S}^{2} & \ldots & \left\|w_{n_{m}, k}\right\|_{R M S}^{2}
\end{array}\right]
$$

Observación 4.6.3. Utilizando la misma aproximación que en la observación 4.5.6, es posible minimizar la cota superior de $\mathcal{E}\left\{\left\|\boldsymbol{e}_{k}\right\|_{R M S}\right\}$ mediante una minimización de la suma

$$
\left\|\boldsymbol{\Gamma}_{v} \boldsymbol{v}_{k}\right\|_{\infty}^{2}+\left\|\boldsymbol{\Gamma}_{w} \boldsymbol{w}_{k}\right\|_{\infty}^{2}
$$

a lo largo largo de todas las variables $\gamma_{v_{i}}, \gamma_{w_{i}}, \boldsymbol{P}(i), \boldsymbol{Q}(i), \boldsymbol{X}(i)$ que satisfacen la LMI (4.83). Para realizar la minimización se puede proceder según se ha indicado en la observación 3.4.4, tomando en este caso

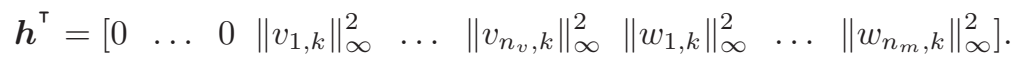




\section{Atenuación $\mathcal{H}_{2}$}

Si las perturbaciones son ruidos blancos de media cero se puede plantear el diseño desde el punto de vista de la norma $\mathcal{H}_{2}$ para acotar el valor eficaz del error de predicción (situación S1 descrita en la página 31).

Teorema 4.6.3. Considérese el predictor (4.12) aplicado al sistema (4.2) y supóngase que hay al menos una medida disponible cada $N_{k} \in \mathcal{N}$ periodos. Supóngase que la disponibilidad de los sensores en cada muestreo viene definida por la matriz $\boldsymbol{\Delta}_{k} \in \Xi$ y que el retardo asociado a cada uno de los sensores se encuentra en el conjunto $d_{i, k} \in \mathcal{D}_{i}$, de manera que se tienen $n_{\mathcal{S}}$ escenarios de muestreo $s_{k} \in \mathcal{S}$ posibles. Supóngase que las perturbaciones y ruidos de medida son señales gaussianas de media cero y varianza $\sigma_{v_{i}}(i=1, \ldots, n)$ y $\sigma_{w_{j}}\left(j=1, \ldots, n_{m}\right)$. Para unos $\gamma_{v_{1}}, \ldots, \gamma_{v_{n_{v}}}, \gamma_{w_{1}}, \ldots, \gamma_{w_{n_{m}}}$ dados, supóngase que existen unas matrices $\boldsymbol{P}(i)=\boldsymbol{P}(i)^{\top} \in \mathbb{R}^{n \times n}, \boldsymbol{Q}(i) \in \mathbb{R}^{n \times n}, \boldsymbol{X}(i) \in \mathbb{R}^{n \times 1}\left(i=1, \ldots, n_{\mathcal{S}}\right)$ tales que

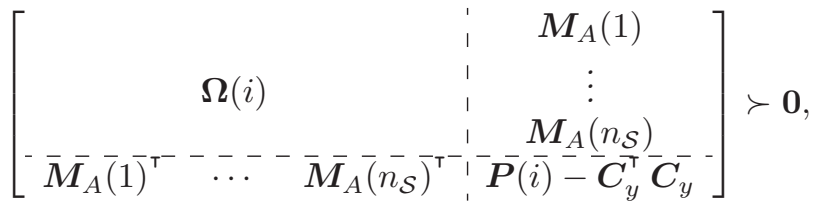

$$
\begin{aligned}
& {\left[\begin{array}{c:c} 
& \boldsymbol{M}_{B}(1) \\
\boldsymbol{\Omega}(i) & \vdots \\
\hdashline \overline{\boldsymbol{M}}_{B}(1)^{\top}-\ldots & \boldsymbol{M}_{B}\left(n_{\mathcal{S}}\right) \\
\left.\hdashline \overline{\boldsymbol{M}}_{\mathcal{S}}\right)^{\top} & \boldsymbol{\Gamma}^{2}-
\end{array}\right] \succ \mathbf{0}, \quad i=1, \ldots, n_{\mathcal{S}}}
\end{aligned}
$$

siendo $\boldsymbol{M}_{A}(i)$ y $\boldsymbol{M}_{B}(i)$ las matrices definidas en (4.65). Entonces, definiendo la ganancia del predictor como $\boldsymbol{L}(i)=\boldsymbol{Q}(i)^{-1} \boldsymbol{X}(i)$, el error de predicción del algoritmo definido por (4.12) converge a cero en valor medio con probabilidad uno en ausencia de perturbaciones y, bajo condiciones iniciales nulas,

$$
\mathcal{E}\left\{\left\|\boldsymbol{e}_{k}\right\|_{R M S}^{2}\right\}<\sum_{i=1}^{n_{v}} \gamma_{v_{i}}^{2} \sigma_{v_{i}}^{2}+\sum_{i=1}^{n_{m}} \gamma_{w_{i}}^{2} \sigma_{w_{i}}^{2}
$$

Prueba 4.6.3. Véase $\S$ B.3.2.

Observación 4.6.4. Si se consideran conocidas las varianza de las perturbación y del ruido de medida, se puede minimizar la cota superior de $\mathcal{E}\left\{\left\|\boldsymbol{e}_{k}\right\|_{R M S}\right\}$ minimizando la suma

$$
\sum_{i=1}^{n_{v}} \gamma_{v_{i}}^{2} \sigma_{v_{i}}^{2}+\sum_{i=1}^{n_{m}} \gamma_{w_{i}}^{2} \sigma_{w_{i}}^{2}
$$

a lo largo de todas las variables $\gamma_{v_{i}}, \gamma_{w_{i}}, \boldsymbol{P}\left(s_{k}\right), \boldsymbol{Q}\left(s_{k}\right), \boldsymbol{X}\left(s_{k}\right)$ que satisfacen las LMI (4.85). Para realizar la minimización se puede proceder según se ha indicado en la observación 3.4.4, tomando en este caso

$$
\boldsymbol{h}^{\top}=\left[\begin{array}{lllllllll}
0 & \ldots & 0 & \sigma_{v_{1}}^{2} & \ldots & \sigma_{v_{n_{v}}}^{2} & \sigma_{w_{1}}^{2} & \ldots & \sigma_{w_{n_{m}}}^{2}
\end{array}\right]
$$

\section{Atenuación $\mathcal{H}_{2 g}$}

El siguiente teorema recoge el diseño de predictores estables que minimizan la cota de error frente a perturbaciones y ruidos de energía finita (situación S3 de la página 31.

Teorema 4.6.4. Considérese el predictor (4.12) aplicado al sistema (4.2) y supóngase que hay al menos una medida disponible cada $N_{k} \in \mathcal{N}$ periodos. Supóngase que la disponibilidad de los sensores en cada muestreo viene definida por la matriz $\boldsymbol{\Delta}_{k} \in \Xi$ y que el retardo asociado a cada uno de los sensores se encuentra en el conjunto $d_{i, k} \in \mathcal{D}_{i}$, de manera que se tienen $n_{\mathcal{S}}$ escenarios de muestreo $s_{k} \in \mathcal{S}$ posibles. Para unos $\gamma_{v_{1}}, \ldots, \gamma_{v_{n_{v}}}, \gamma_{w_{1}}, \ldots, \gamma_{w_{n_{m}}}$ dados, supóngase que existen unas matrices $\boldsymbol{P}(i)=\boldsymbol{P}(i)^{\top} \in$ 
$\mathbb{R}^{n \times n}, \boldsymbol{Q}(i) \in \mathbb{R}^{n \times n}, \boldsymbol{X}(i) \in \mathbb{R}^{n \times 1}\left(i=1, \ldots, n_{\mathcal{S}}\right)$ tales que

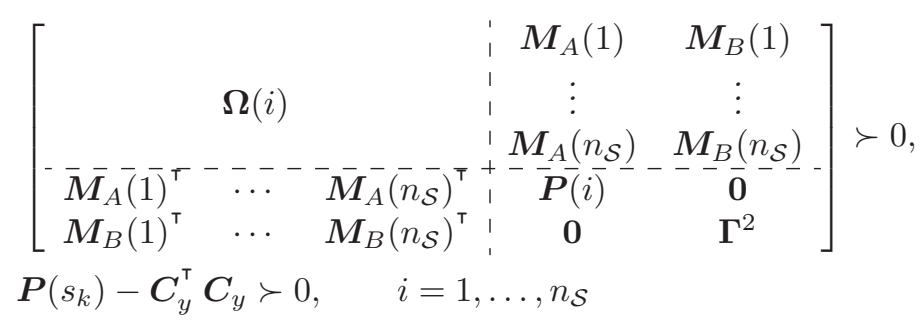

siendo $\boldsymbol{M}_{A}(i)$ y $\boldsymbol{M}_{B}(i)$ las matrices definidas en (4.65). Entonces, definiendo la ganancia del predictor como $\boldsymbol{L}(i)=\boldsymbol{Q}(i)^{-1} \boldsymbol{X}(i)$, el error de predicción del algoritmo definido por (4.12) converge a cero en valor medio con probabilidad uno en ausencia de perturbaciones y, bajo condiciones iniciales nulas,

$$
\mathcal{E}\left\{\left\|\boldsymbol{e}_{k}\right\|_{\infty}^{2}\right\}<\left\|\boldsymbol{\Gamma}_{v} \boldsymbol{v}_{k}\right\|_{2}^{2}+\left\|\boldsymbol{\Gamma}_{w} \boldsymbol{w}_{k}\right\|_{2}^{2}
$$

donde

$$
\mathcal{E}\left\{\left\|\boldsymbol{e}_{k}\right\|_{\infty}^{2}\right\}=\operatorname{máx}_{k} \mathcal{E}\left\{\boldsymbol{e}_{k}^{\top} \boldsymbol{e}_{k} \mid k-1\right\}
$$

Prueba 4.6.4. Véase $\S B .3 .3$

Observación 4.6.5. Conocida la norma $\ell_{2}$ de las perturbaciones, se puede diseñar el predictor que minimiza la norma $\mathcal{E}\left\{\left\|\boldsymbol{e}_{k}\right\|_{\infty}\right\}$ haciendo una minimización de la suma

$$
\sum_{i=1}^{n_{v}} \gamma_{v_{i}}^{2}\left\|v_{i, k}\right\|_{2}^{2}+\sum_{i=1}^{n_{m}} \gamma_{w_{i}}^{2}\left\|w_{i, k}\right\|_{2}^{2}
$$

a lo largo de todas las variables $\gamma_{v_{i}}, \gamma_{w_{i}}, \boldsymbol{P}\left(s_{k}\right), \boldsymbol{Q}\left(s_{k}\right), \boldsymbol{X}\left(s_{k}\right)$, que cumplen la LMI (4.87). Para realizar la minimización se puede proceder según se ha indicado en la observación 3.4.4, tomando en este caso

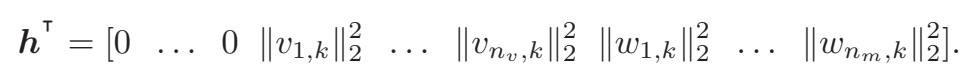

\section{Atenuación $\ell_{1}$}

El siguiente teorema muestra el diseño del predictor que minimiza la amplitud del error de predicción frente a perturbaciones de cota de amplitud conocida (situación S2 de la página 31).

Teorema 4.6.5. Considérese el predictor (4.12) aplicado al sistema (4.2) y supóngase que hay al menos una medida disponible cada $N_{k} \in \mathcal{N}$ periodos. Supóngase que la disponibilidad de los sensores en cada muestreo viene definida por la matriz $\boldsymbol{\Delta}_{k} \in \Xi$ y que el retardo asociado a cada uno de los sensores se encuentra en el conjunto $d_{i, k} \in \mathcal{D}_{i}$, de manera que se tienen $n_{\mathcal{S}}$ escenarios de muestreo $s_{k} \in \mathcal{S}$ posibles. Para unos $\gamma_{v_{1}}, \ldots, \gamma_{v_{n_{v}}}, \gamma_{w_{1}}, \ldots, \gamma_{w_{n_{m}}}$ dados, supóngase que existen unas matrices $\boldsymbol{P}(i)=\boldsymbol{P}(i)^{\top} \in$

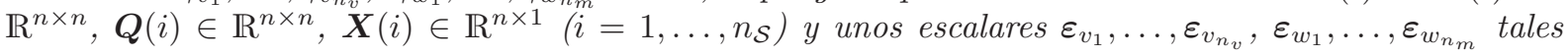
que

$$
\begin{aligned}
& \lambda \in(0,1)
\end{aligned}
$$

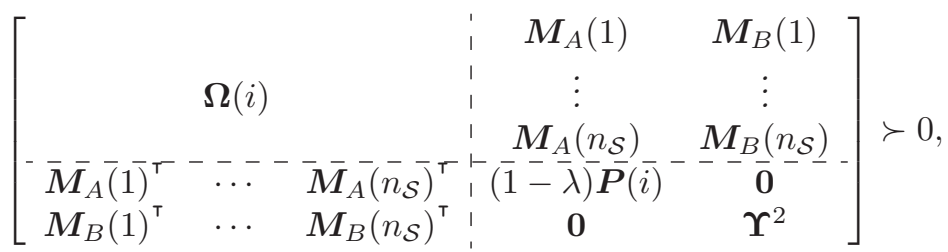

$$
\begin{aligned}
& {\left[\begin{array}{ccc}
\lambda \boldsymbol{P}(i) & \mathbf{0} & \boldsymbol{C}_{y}^{\boldsymbol{\top}} \\
\mathbf{0} & \boldsymbol{\Gamma}^{2}-\boldsymbol{\Upsilon}^{2} & \mathbf{0} \\
\boldsymbol{C}_{y} & \mathbf{0} & \boldsymbol{I}
\end{array}\right] \succ 0, \quad i=1, \ldots, n_{\mathcal{S}}}
\end{aligned}
$$

siendo $\boldsymbol{M}_{A}(i)$ y $\boldsymbol{M}_{B}(i)$ las matrices definidas en (4.65) y $\boldsymbol{\Upsilon}$ definido como en (4.76). Entonces, definiendo la ganancia del predictor en función del valor del parámetro de muestreo como $\boldsymbol{L}(i)=\boldsymbol{Q}(i)^{-1} \boldsymbol{X}(i)$, el 
error de predicción del algoritmo definido por (4.12) convergencia asintóticamente a cero con probabilidad uno en ausencia de perturbaciones y, bajo condiciones iniciales nulas,

$$
\mathcal{E}\left\{\left\|\boldsymbol{e}_{k}\right\|_{\infty}^{2}\right\}<\left\|\boldsymbol{\Gamma}_{v} \boldsymbol{v}_{k}\right\|_{\infty}^{2}+\left\|\boldsymbol{\Gamma}_{w} \boldsymbol{w}_{k}\right\|_{\infty}^{2}
$$

Prueba 4.6.5. Véase $\S$ B.3.4.

Observación 4.6.6. Conocida la norma $\ell_{\infty}$ de las perturbaciones, se puede minimizar la norma $\mathcal{E}\left\{\left\|\boldsymbol{e}_{k}\right\|_{\infty}\right\}$ haciendo una minimización de la suma

$$
\sum_{i=1}^{n} \gamma_{v_{i}}^{2}\left\|v_{i, k}\right\|_{\infty}^{2}+\sum_{i=1}^{n_{m}} \gamma_{w_{i}}^{2}\left\|w_{i, k}\right\|_{\infty}^{2}
$$

a lo largo de todas las variables $\gamma_{v_{i}}, \gamma_{w_{i}}, \lambda, \boldsymbol{P}\left(s_{k}\right), \boldsymbol{Q}\left(s_{k}\right), \boldsymbol{X}\left(s_{k}\right)$ que satisfacen la LMI (4.89). Para realizar la minimización se puede proceder según se ha indicado en la observación 3.4.4, tomando en este caso

$$
\boldsymbol{h}^{\top}=\left[\begin{array}{llllllll}
0 & \ldots & 0 & \left\|v_{1, k}\right\|_{\infty}^{2} & \ldots & \left\|v_{n_{v}, k}\right\|_{\infty}^{2}\left\|w_{1, k}\right\|_{\infty}^{2} & \ldots & \left\|w_{n_{m}, k}\right\|_{\infty}^{2}
\end{array}\right] .
$$

Nótese la necesidad de resolver el problema de minimización a lo largo de $\lambda$ encadenado con el anterior (véase observación 4.5.11).

\subsection{Consideraciones de diseño}

En las secciones anteriores se han propuesto diferentes técnicas de diseño cuya idoneidad para cada problema se basa en las características conocidas de las perturbaciones y de la norma que se quiere acotar en el error de predicción. En la tabla 4.3 se muestra una relación entre las diferentes normas de error y perturbación, y el método de diseño a utilizar (indicado mediante el teorema en el cual se expone la metodología de cálculo). El hecho de diseñar un predictor para acotar el error máximo (teoremas 4.5.4

\begin{tabular}{|c|cccc|}
\hline & $\left\|\boldsymbol{w}_{k}\right\|_{2}$ & $\left\|\boldsymbol{w}_{k}\right\|_{\infty}$ & $\left\|\boldsymbol{w}_{k}\right\|_{R M S}$ & $\sigma_{w}$ \\
\hline$\left\|\boldsymbol{e}_{k}\right\|_{2}$ & $\mathcal{H}_{\infty}(4.5 .2)$ & $\infty$ & - & - \\
$\left\|\boldsymbol{e}_{k}\right\|_{\infty}$ & $\mathcal{H}_{2 g}(4.5 .4)$ & $\ell_{1}(4.5 .5)$ & $\infty$ & $\infty$ \\
$\left\|\boldsymbol{e}_{k}\right\|_{R M S}$ & 0 & $\mathcal{H}_{\infty}(4.5 .2)$ & $\mathcal{H}_{\infty}(4.5 .2)$ & $\mathcal{H}_{2}(4.5 .3)$ \\
\hline $\mathcal{E}\left\{\left\|\boldsymbol{e}_{k}\right\|_{2}\right\}$ & $\mathcal{H}_{\infty}(4.6 .2)$ & $\infty$ & $\infty$ & $\infty$ \\
$\mathcal{E}\left\{\left\|\boldsymbol{e}_{k}\right\|_{\infty}\right\}$ & $\mathcal{H}_{2 g}(4.6 .4)$ & $\ell_{1}(4.6 .5)$ & - & - \\
$\mathcal{E}\left\{\left\|\boldsymbol{e}_{k}\right\|_{R M S}\right\}$ & 0 & $\mathcal{H}_{\infty}(4.6 .2)$ & $\mathcal{H}_{\infty}(4.6 .2)$ & $\mathcal{H}_{2}(4.6 .3)$ \\
\hline
\end{tabular}

Tabla 4.3: Resumen de especificaciones de diseño

y 4.5.5) puede tener como contrapartida que el error persista durante un tiempo mayor que si se diseña un predictor para acotar el error cuadrático acumulado (teoremas 4.5.2 y 4.5.3). Dependerá de la aplicación el que sea más conveniente utilizar un tipo de diseño u otro.

El diseño que se ha planteado a lo largo del capítulo se basa en la minimización del error de predicción frente a elementos que pueden tener efecto sobre este error como las perturbaciones y los ruidos de medida. En las aplicaciones reales, sin embargo, aparecen más factores que pueden aumentar el error de predicción. Algunos de estos factores son: el error de modelado, el retardo en el cálculo y transmisión de la acción de control, el hecho de no conocer con exactitud el instante de tiempo al cual corresponde una medición o la utilización de mediciones asíncronas. En esta sección se demuestra que el efecto de estos factores sobre el error de predicción se puede modelar por medio de determinadas perturbaciones. En este sentido, se presenta un procedimiento para calcular la perturbación y/o ruido equivalente de estos factores con el que poder utilizar el diseño normal de atenuación de perturbaciones y ruido de medida.

Cuando se tiene alguno de estos casos se ha de calcular una perturbación del estado y un ruido de medida equivalentes que tengan el mismo efecto sobre el error de salida. Si hay alguna forma de conocer una norma de esta perturbación y ruido equivalentes, habrá que sumar las normas de las diferentes señales que afectan como una perturbación y las de las que afectan como un ruido de medida para tener la norma total de las señales que afectan al error de predicción. Con estos valores de las normas de la perturbación total y del ruido de medida total, el problema se resuelve aplicando uno de los diseños de las secciones anteriores. 
Si las normas que se tienen de la perturbación y del ruido son de diferente naturaleza (norma $\ell_{\infty}$ y norma $\ell_{2}$, por ejemplo), se tendrá que recurrir a las técnicas de diseño multiobjetivo que se muestran en $\$ 4.7 .7$.

Algunas de las técnicas que se exponen a continuación en esta sección fueron presentadas en \$3.6 para los predictores basados en modelo entrada salida. Las exposiciones de esta sección tratan de adecuar la notación al caso de los predictores multivariables presentados en este capítulo. Para mayor detalle referirse a $\$ 3.6$.

\subsubsection{Robustez al error de modelado}

El diseño del predictor realizado en las secciones anteriores se basaba en un conocimiento exacto del modelo del proceso. Sin embargo, este conocimiento exacto no es posible en general, ya que cualquier método de identificación da como resultado un modelo que solo aproxima al proceso real, siendo además en general de un orden menor que el proceso real. Este error de modelado afectará a las salidas predichas, haciendo que el error de predicción no sea nulo. El siguiente teorema describe la ecuación que permite analizar ese efecto para el caso multivariable en el que todos los sensores tienen el mismo retardo y están disponibles al mismo tiempo. Las ecuaciones del modelo del proceso (4.2) se reducen en este caso a

$$
\begin{aligned}
\boldsymbol{x}[t+1] & =\boldsymbol{A} \boldsymbol{x}[t]+\boldsymbol{B} \boldsymbol{u}[t], \\
\boldsymbol{m}_{k} & =\boldsymbol{C} \boldsymbol{x}\left[t_{k}-d_{k}\right],
\end{aligned}
$$

siendo el vector de salidas virtuales que necesita el controlador

$$
\boldsymbol{y}[t]=\boldsymbol{C}_{y} \boldsymbol{x}[t]
$$

El resultado se puede extender fácilmente al caso general de diferentes retardos en cada sensor.

Teorema 4.7.1. Supóngase que el modelo teórico discreto del sistema a periodo $T$ queda determinado por las matrices $\boldsymbol{A}, \boldsymbol{B}$ y $\boldsymbol{C}$, mientras que el proceso real viene determinado por las matrices $\boldsymbol{A}_{r}, \boldsymbol{B}_{r} y \boldsymbol{C}_{r}$, y supóngase que se aplica el predictor (4.12) cuando no hay perturbaciones ni ruido de medida. Entonces la dinámica del error de predicción cuando se tiene una medición conjunta de todos los sensores cada $N_{k}$ periodos con un retardo de $d_{k}$ periodos en todos los sensores es

$$
\begin{aligned}
\widetilde{\boldsymbol{x}}_{k}= & \left(\boldsymbol{I}-\boldsymbol{L}_{k} \boldsymbol{C} \boldsymbol{A}^{-d_{k}}\right) \boldsymbol{A}^{N_{k}} \widetilde{\boldsymbol{x}}_{k-1}+\left(\boldsymbol{A}_{r}^{N_{k}}-\boldsymbol{A}^{N_{k}}\right) \boldsymbol{x}_{k-1}+\sum_{i=1}^{N_{k}}\left(\boldsymbol{A}_{r}^{i-1} \boldsymbol{B}_{r}-\boldsymbol{A}^{i-1} \boldsymbol{B}\right) \boldsymbol{u}\left[t_{k}-i\right] \\
& +\boldsymbol{L}_{k}\left(\boldsymbol{C} \boldsymbol{A}^{N_{k}-d_{k}}-\boldsymbol{C}_{r} \boldsymbol{A}_{r}^{N_{k}-d_{k}}\right) \boldsymbol{x}_{k-1}+\boldsymbol{L}_{k} \sum_{i=1}^{N_{k}}\left(\boldsymbol{A}^{i-1-d_{k}} \boldsymbol{B}-\boldsymbol{A}_{r}^{i-1-d_{k}} \boldsymbol{B}_{r}\right) \boldsymbol{u}\left[t_{k}-i\right] \\
& -\boldsymbol{L}_{k} \sum_{i=1}^{d_{k}}\left(\boldsymbol{A}^{i-1-d_{k}} \boldsymbol{B}-\boldsymbol{A}_{r}^{i-1-d_{k}} \boldsymbol{B}_{r}\right) \boldsymbol{u}\left[t_{k}-i\right]
\end{aligned}
$$

Prueba 4.7.1. La ecuación de actualización cuando se miden todos los sensores al mismo tiempo y con el mismo retardo es

$$
\hat{\boldsymbol{x}}\left[t_{k}\right]=\hat{\boldsymbol{x}}\left[t_{k} \mid t_{k}-1\right]+\boldsymbol{L}_{k}\left(\boldsymbol{m}_{k}-\boldsymbol{C} \hat{\boldsymbol{x}}\left[t_{k}-d_{k} \mid t_{k}-1\right]\right) .
$$

Como se ha visto en secciones anteriores (véase la demostración del teorema 4.3.1) las estimaciones $\hat{\boldsymbol{x}}\left[t_{k} \mid t_{k}-1\right]$ y $\hat{\boldsymbol{x}}\left[t_{k}-d_{k} \mid t_{k}-1\right]$ pueden escribirse en función de la estimación actualizada con la medición anterior $\left(\hat{\boldsymbol{x}}\left[t_{k}-N_{k}\right]\right)$ mediante

$$
\begin{aligned}
\hat{\boldsymbol{x}}\left[t_{k} \mid t_{k}-1\right] & =\boldsymbol{A}^{N_{k}} \hat{\boldsymbol{x}}\left[t_{k}-N_{k}\right]+\sum_{i=1}^{N_{k}} \boldsymbol{A}^{i-1} \boldsymbol{B} \boldsymbol{u}\left[t_{k}-i\right], \\
\hat{\boldsymbol{x}}\left[t_{k}-d_{k} \mid t_{k}-1\right] & =\boldsymbol{A}^{-d_{k}}\left(\boldsymbol{A}^{N_{k}} \hat{\boldsymbol{x}}\left[t_{k}-N_{k}\right]+\sum_{i=1}^{N_{k}} \boldsymbol{A}^{i-1} \boldsymbol{B} \boldsymbol{u}\left[t_{k}-i\right]-\sum_{i=1}^{d_{k}} \boldsymbol{A}^{i-1} \boldsymbol{B} \boldsymbol{u}\left[t_{k}-i\right]\right),
\end{aligned}
$$


con lo que la ecuación (4.95) queda

$$
\begin{aligned}
\hat{\boldsymbol{x}}\left[t_{k}\right]= & \left(\boldsymbol{I}-\boldsymbol{L}_{k} \boldsymbol{C} \boldsymbol{A}^{-d_{k}}\right)\left(\boldsymbol{A}^{N_{k}} \hat{\boldsymbol{x}}\left[t_{k}-N_{k}\right]+\sum_{i=1}^{N_{k}} \boldsymbol{A}^{i-1} \boldsymbol{B} \boldsymbol{u}\left[t_{k}-i\right]\right) \\
& +\boldsymbol{L}_{k} \boldsymbol{C} \boldsymbol{A}^{-d_{k}} \sum_{i=1}^{d_{k}} \boldsymbol{A}^{i-1} \boldsymbol{B} \boldsymbol{u}\left[t_{k}-i\right]+\boldsymbol{L}_{k} \boldsymbol{m}_{k} .
\end{aligned}
$$

Restando el término

$$
\begin{aligned}
& \left(\boldsymbol{I}-\boldsymbol{L}_{k} \boldsymbol{C A}^{-d_{k}}\right)\left(\boldsymbol{A}^{N_{k}} \boldsymbol{x}\left[t_{k}-N_{k}\right]+\sum_{i=1}^{N_{k}} \boldsymbol{A}^{i-1} \boldsymbol{B} \boldsymbol{u}\left[t_{k}-i\right]\right) \\
& +\boldsymbol{L}_{k} \boldsymbol{C} \boldsymbol{A}^{-d_{k}} \sum_{i=1}^{d_{k}} \boldsymbol{A}^{i-1} \boldsymbol{B} \boldsymbol{u}\left[t_{k}-i\right]
\end{aligned}
$$

en ambos lados de la ecuación (4.96) se tiene que

$$
\begin{aligned}
\widetilde{\boldsymbol{x}}\left[t_{k}\right] & =\left(\boldsymbol{I}-\boldsymbol{L}_{k} \boldsymbol{C} \boldsymbol{A}^{-d_{k}}\right) \widetilde{\boldsymbol{x}}\left[t_{k}-N_{k}\right]+\boldsymbol{x}\left[t_{k}\right]-\boldsymbol{A}^{N_{k}} \boldsymbol{x}\left[t_{k}-N_{k}\right]-\sum_{i=1}^{N_{k}} \boldsymbol{A}^{i-1} \boldsymbol{B} \boldsymbol{u}\left[t_{k}-i\right] \\
& +\boldsymbol{L}_{k} \boldsymbol{C} \boldsymbol{A}^{-d_{k}}\left(\boldsymbol{A}^{N_{k}} \hat{\boldsymbol{x}}\left[t_{k}-N_{k}\right]+\sum_{i=1}^{N_{k}} \boldsymbol{A}^{i-1} \boldsymbol{B} \boldsymbol{u}\left[t_{k}-i\right]-\sum_{i=1}^{d_{k}} \boldsymbol{A}^{i-1} \boldsymbol{B} \boldsymbol{u}\left[t_{k}-i\right]\right)-\boldsymbol{L}_{k} \boldsymbol{m}_{k} .
\end{aligned}
$$

Utilizando las matrices del proceso se pueden escribir los valores de $\boldsymbol{x}\left[t_{k}\right]$ y $\boldsymbol{m}_{k}$ como

$$
\begin{aligned}
\boldsymbol{x}\left[t_{k}\right] & =\boldsymbol{A}_{r}^{N_{k}} \hat{\boldsymbol{x}}\left[t_{k}-N_{k}\right]+\sum_{i=1}^{N_{k}} \boldsymbol{A}_{r}^{i-1} \boldsymbol{B}_{r} \boldsymbol{u}\left[t_{k}-i\right] \\
\boldsymbol{m}_{k} & =\boldsymbol{C}_{r} \boldsymbol{x}\left[t_{k}-d_{k}\right] \\
& =\boldsymbol{C}_{r} \boldsymbol{A}_{r}^{-d_{k}}\left(\boldsymbol{A}_{r}^{N_{k}} \boldsymbol{x}\left[t_{k}-N_{k}\right]+\sum_{i=1}^{N_{k}} \boldsymbol{A}_{r}^{i-1} \boldsymbol{B}_{r} \boldsymbol{u}\left[t_{k}-i\right]-\sum_{i=1}^{d_{k}} \boldsymbol{A}_{r}^{i-1} \boldsymbol{B}_{r} \boldsymbol{u}\left[t_{k}-i\right]\right)
\end{aligned}
$$

con lo que el error de estimación puede escribirse como

$$
\begin{aligned}
\widetilde{\boldsymbol{x}}\left[t_{k}\right]= & \left(\boldsymbol{I}-\boldsymbol{L}_{k} \boldsymbol{C} \boldsymbol{A}^{-d_{k}}\right) \boldsymbol{A}^{N_{k}} \widetilde{\boldsymbol{x}}\left[t_{k}-N_{k}\right]+\left(\boldsymbol{A}_{r}^{N_{k}}-\boldsymbol{A}^{N_{k}}\right) \boldsymbol{x}\left[t_{k}-N_{k}\right] \\
& +\sum_{i=1}^{N_{k}}\left(\boldsymbol{A}_{r}^{i-1} \boldsymbol{B}_{r}-\boldsymbol{A}^{i-1} \boldsymbol{B}\right) \boldsymbol{u}\left[t_{k}-i\right]+\boldsymbol{L}_{k}\left(\boldsymbol{C A}^{N_{k}-d_{k}}-\boldsymbol{C}_{r} \boldsymbol{A}_{r}^{N_{k}-d_{k}}\right) \boldsymbol{x}\left[t_{k}-N_{k}\right] \\
& +\boldsymbol{L}_{k} \sum_{i=1}^{N_{k}}\left(\boldsymbol{A}^{i-1-d_{k}} \boldsymbol{B}-\boldsymbol{A}_{r}^{i-1-d_{k}} \boldsymbol{B}_{r}\right) \boldsymbol{u}\left[t_{k}-i\right] \\
& -\boldsymbol{L}_{k} \sum_{i=1}^{d_{k}}\left(\boldsymbol{A}^{i-1-d_{k}} \boldsymbol{B}-\boldsymbol{A}_{r}^{i-1-d_{k}} \boldsymbol{B}_{r}\right) \boldsymbol{u}\left[t_{k}-i\right] .
\end{aligned}
$$

El teorema anterior muestra que la estabilidad del predictor (relación entre $\widetilde{\boldsymbol{x}}_{k}$ y $\widetilde{\boldsymbol{x}}_{k-1}$ ) sólo depende del modelo teórico $(\boldsymbol{A})$, de la disponibilidad de datos y de los retardos de los sensores, siempre que el proceso sea estable o esté estabilizado con un controlador en lazo cerrado. El error de modelado se manifiesta en el término adicional de la ecuación dinámica, que en general tendrá como consecuencia un error de predicción distinto de cero. El efecto de este error depende tanto de la entrada como del estado y del modelo del proceso. De hecho, puede obtenerse el valor de una perturbación equivalente que tiene el mismo efecto sobre el error de predicción que la presencia del error de modelado de la siguiente forma. Si se toman las matrices reales del proceso como

$$
\begin{aligned}
& \boldsymbol{A}_{r}=\boldsymbol{A}+\Delta \boldsymbol{A}, \\
& \boldsymbol{B}_{r}=\boldsymbol{B}+\Delta \boldsymbol{B}, \\
& \boldsymbol{C}_{r}=\boldsymbol{C}+\Delta \boldsymbol{C},
\end{aligned}
$$


se puede escribir la dinámica real del proceso con el sistema de medición como

$$
\begin{gathered}
\boldsymbol{x}[t+1]=\boldsymbol{A}_{r} \boldsymbol{x}[t]+\boldsymbol{B}_{r} u[t]=\boldsymbol{A} \boldsymbol{x}[t]+\boldsymbol{B} \boldsymbol{u}[t]+\underbrace{\Delta \boldsymbol{A} \boldsymbol{x}[t]+\Delta \boldsymbol{B} \boldsymbol{u}[t]}_{=\boldsymbol{v}[t]}, \\
\boldsymbol{m}_{k}=\boldsymbol{C}_{r} \boldsymbol{x}\left[t_{k}-d_{k}\right]=\boldsymbol{C}_{m} \boldsymbol{x}\left[t_{k}-d_{k}\right]+\underbrace{\Delta \boldsymbol{C} \boldsymbol{x}\left[t_{k}-d_{k}\right]}_{=\boldsymbol{w}_{k}},
\end{gathered}
$$

donde se puede identificar la perturbación del estado (con $\boldsymbol{B}_{v}=\boldsymbol{I}$ ), y el ruido de medición como

$$
\begin{aligned}
\boldsymbol{v}[t] & =\Delta \boldsymbol{A} \boldsymbol{x}[t]+\Delta \boldsymbol{B} \boldsymbol{u}[t], \\
\boldsymbol{w}_{k} & =\Delta \boldsymbol{C} \boldsymbol{x}\left[t_{k}-d_{k}\right] .
\end{aligned}
$$

Para proceder al diseño de predictores robustos a esta incertidumbre es necesario acotar la norma de la perturbación equivalente. De esta forma, si se conoce una cota de las normas de la parte incierta de las matrices del proceso $(\|\Delta \boldsymbol{A}\|,\|\Delta \boldsymbol{B}\|$ y $\|\Delta \boldsymbol{C}\|)$ y una cota del estado y de las entradas del proceso $(\|\boldsymbol{x}[t]\|$ y $\|\boldsymbol{u}[t]\|)$ se puede aplicar cualquiera de las técnicas vistas hasta ahora de atenuación de perturbaciones utilizando como normas conocidas de las perturbaciones los valores

$$
\begin{aligned}
\|\boldsymbol{v}[t]\|_{p} & =\|\Delta \boldsymbol{A}\|_{i p}\|\boldsymbol{x}[t]\|_{p}+\|\Delta \boldsymbol{B}\|_{i p}\|\boldsymbol{u}[t]\|_{p}, \\
\left\|\boldsymbol{w}_{y}[t]\right\|_{p} & =\left\|\Delta \boldsymbol{C}_{y}\right\|_{i p}\|\boldsymbol{x}[t]\|_{p}, \\
\left\|\boldsymbol{w}_{k}\right\|_{p} & =\|\Delta \boldsymbol{C}\|_{i p}\left\|\boldsymbol{x}_{k}\right\|_{p} .
\end{aligned}
$$

En el caso de sensores con diferente retardo y disponibilidad, el valor de la perturbación del estado $v$ es el mismo, pero se el ruido equivalente $\left(w_{i}\right)$ tomará un valor diferente en cada sensor. Si las ecuaciones "reales" de salida de los diferentes sensores son

$$
m_{i, k}=\boldsymbol{c}_{i, r} \boldsymbol{x}\left[t_{k}-d_{i, k}\right]
$$

y la diferencia de cada uno de los vectores $\boldsymbol{c}_{i, r}$ con el modelo $\boldsymbol{c}_{i}$ se denota por $\Delta \boldsymbol{c}_{i}$, el ruido equivalente en cada sensor se puede escribir como

$$
w_{i, k}=\Delta \boldsymbol{c}_{i} \boldsymbol{x}\left[t_{k}-d_{i, k}\right]
$$

Los resultados obtenidos en esta sección ponen de manifiesto que no es necesario que el sistema sea estable, aunque sí debe estar estabilizado por un controlador en el caso de ser inestable, para asegurar que los estados (y las salidas) son señales acotadas. Sin embargo, se encuentran en la bibliografía diseños de observadores (con muestreo regular) que sí requieren la estabilidad de la planta incierta (en bucle abierto) porque plantean un modelo de orden extendido con el estado y el error de estimación del estado (véase, por ejemplo, [76]).

\subsubsection{Retardo en la acción de control}

Si el cómputo de las acciones de control y su transmisión desde el controlador hasta los actuadores introduce un retardo apreciable $d_{u}$ en el bucle de control, éste se tiene que tener en cuenta a la hora de predecir salidas futuras. Si el retardo es múltiplo del periodo de control $T$, se puede trasferir este retardo a la salida y tratarlo como si estuviera en el sistema de medición. De esta manera, el predictor proporcionará la salida que se tendría si no hubiera retardos en la entrada del proceso para poder implementar técnicas de control convencionales. Si el retardo $d_{u}$ no es múltiplo de $T$, se divide el retardo en dos partes como

$$
d_{u}[t]=k_{u} T+\sigma[t],
$$

donde $k_{u}$ es un número entero y $\sigma[t] \in[0, T)$. El retardo $k_{u} T$ es la parte del retardo que es múltiplo del periodo de control y que, por tanto, se puede transferir a la salida. Con esta consideración, el problema se reduce al de predicción con retardo menor que $T$ en la entrada para lo que se propone obtener un modelo discreto entrada-salida a partir del modelo continuo que considere el retardo $\sigma[t]$.

\section{Retardo de entrada constante}

Se considera en primer lugar el caso en el que el retardo $\sigma[t]=\sigma_{0}$ es constante. El modelo continuo de la planta que define la evolución de los estados es

$$
\dot{\boldsymbol{x}}(\tau)=\boldsymbol{A}_{c} \boldsymbol{x}(\tau)+\boldsymbol{B}_{c} \boldsymbol{u}(\tau) .
$$


Si se aplica un retenedor de orden cero, la acción de control en el periodo $t$ toma los valores

$$
\begin{aligned}
& \boldsymbol{u}(\tau)=\boldsymbol{u}[t-1], \quad \tau \in\left[t T,\left(t+\sigma_{0}\right) T\right), \\
& \boldsymbol{u}(\tau)=\boldsymbol{u}[t], \quad \tau \in\left[\left(t+\sigma_{0}\right) T, T\right) .
\end{aligned}
$$

A partir del modelo continuo, se puede obtener el modelo discreto a periodo $T$ mediante

$$
\boldsymbol{x}[t+1]=e^{\boldsymbol{A}_{c} T} \boldsymbol{x}[t]+\int_{0}^{T} e^{\boldsymbol{A}_{c} \tau} \boldsymbol{B}_{c} \boldsymbol{u}(t T+\tau) \mathrm{d} \tau .
$$

La ecuación de estado se puede escribir como

$$
\boldsymbol{x}[t+1]=\boldsymbol{A} \boldsymbol{x}[t]+\underbrace{\int_{0}^{\sigma_{0}} e^{A_{c} \tau} \boldsymbol{B}_{c} \mathrm{~d} \tau}_{=\boldsymbol{B}_{1}} \boldsymbol{u}[t-1]+\underbrace{\int_{\sigma_{0}}^{T} e^{A_{c} \tau} \boldsymbol{B}_{c} \mathrm{~d} \tau}_{=\boldsymbol{B}_{0}} \boldsymbol{u}[t] .
$$

Una vez llegados a este punto se tienen dos opciones:

1. Utilizar un modelo en el que se define una nueva entrada que incluye las $\boldsymbol{u}[t]$ de dos instantes consecutivos,

$$
\boldsymbol{x}[t+1]=\boldsymbol{A} \boldsymbol{x}[t]+\left[\begin{array}{ll}
\boldsymbol{B}_{0} & \boldsymbol{B}_{1}
\end{array}\right]\left[\begin{array}{c}
\boldsymbol{u}[t] \\
\boldsymbol{u}[t-1]
\end{array}\right] .
$$

Con esta primera opción se consigue mantener la matriz dinámica intacta, con lo que todos los algoritmos de diseño obtenidos siguen siendo válidos ya que ninguno de ellos depende de la matriz $\boldsymbol{B}$. La implementación del predictor sólo variaría en la forma de definir la matriz $\boldsymbol{B}$ y el vector de entradas $\boldsymbol{u}[t]$ para estimar en bucle abierto.

2. Utilizar un modelo de orden extendido,

$$
\left[\begin{array}{c}
\boldsymbol{x}[t+1] \\
\xi[t+1]
\end{array}\right]=\underbrace{\left[\begin{array}{ll}
\boldsymbol{A} & 1 \\
\mathbf{0} & 0
\end{array}\right]}_{=\boldsymbol{A}^{\prime}}\left[\begin{array}{c}
\boldsymbol{x}[t] \\
\xi[t]
\end{array}\right]+\left[\begin{array}{l}
\boldsymbol{B}_{0} \\
\boldsymbol{B}_{1}
\end{array}\right] \boldsymbol{u}[t] .
$$

Esta segunda opción tiene el inconveniente de que introduce un polo en el origen de la nueva matriz dinámica $\boldsymbol{A}^{\prime}$, con lo que ya no se pueden aplicar los diseños previstos ya que requieren la invertivilidad de la matriz $\boldsymbol{A}^{\prime}$.

\section{Retardo de entrada variante}

Si el retardo presenta una componente aleatoria variante en el tiempo, el modelo anterior será incierto porque $\boldsymbol{B}_{0}$ y $\boldsymbol{B}_{1}$ no se conocerán con exactitud. Si el retardo se toma como

$$
\sigma[t]=\sigma_{0}+\Delta \sigma[t]
$$

siendo $\sigma_{0} \geq 0$ el mínimo retardo que se conoce, y $\Delta \sigma[t]>0$ la componente aleatoria de la cual se conoce su cota superior, la ecuación de estado discreta (4.101) vendrá dada por

$$
\boldsymbol{x}[t+1]=\boldsymbol{A} \boldsymbol{x}[t]+\int_{0}^{\sigma[t]} e^{A_{c} \tau} \mathrm{d} \tau \boldsymbol{u}[t-1]+\int_{\sigma[t]}^{T} e^{\boldsymbol{A}_{c} \tau} \boldsymbol{B}_{c} \mathrm{~d} \tau \boldsymbol{u}[t] .
$$

Sumando y restando el término $\int_{\sigma_{0}}^{\sigma[t]} e^{\boldsymbol{A}_{c} \tau} \boldsymbol{B}_{c} \mathrm{~d} \tau \boldsymbol{u}[t]$ se llega a

$$
\boldsymbol{x}[t+1]=\boldsymbol{A} \boldsymbol{x}[t]+\boldsymbol{B}_{0} \boldsymbol{u}[t]+\boldsymbol{B}_{1} \boldsymbol{u}[t-1]+\underbrace{\int_{\sigma_{0}}^{\sigma[t]} e^{\boldsymbol{A}_{c} \tau} \boldsymbol{B}_{c} \mathrm{~d} \tau}_{=\Delta \boldsymbol{B}[t]}(\boldsymbol{u}[t-1]-\boldsymbol{u}[t]),
$$

que se puede reescribir como

$$
\boldsymbol{x}[t+1]=\boldsymbol{A} \boldsymbol{x}[t]+(\underbrace{\left[\begin{array}{ll}
\boldsymbol{B}_{0} & \boldsymbol{B}_{1}
\end{array}\right]}_{=\boldsymbol{B}}+\underbrace{\left(\int_{\sigma_{0}}^{\sigma[t]} e^{\boldsymbol{A}_{c} \tau} \boldsymbol{B}_{c} \mathrm{~d} \tau\right)\left[\begin{array}{ll}
\boldsymbol{I} & -\boldsymbol{I}
\end{array}\right]}_{=\Delta \boldsymbol{B}[t]})\left[\begin{array}{c}
\boldsymbol{u}[t] \\
\boldsymbol{u}[t-1]
\end{array}\right]
$$


donde $\Delta \boldsymbol{B}[t]$ es la parte incierta del modelo que varía su valor con $\sigma[t]$. Para calcular el predictor puede utilizarse la norma del peor caso, que viene dada por

$$
\|\Delta \boldsymbol{B}[t]\|_{i p}=\operatorname{máx}_{\sigma[t]}\left\|\left(\int_{\sigma_{0}}^{\sigma[t]} e^{\boldsymbol{A}_{c} \tau} \boldsymbol{B}_{c} \mathrm{~d} \tau\right)\left[\begin{array}{ll}
\boldsymbol{I} & -\boldsymbol{I}
\end{array}\right]\right\|_{i p},
$$

donde ip denota la norma inducida de la matriz, o bien el valor medio que toma esta norma para todos los posibles valores de $\sigma[t]$ asumiendo una distribución uniforme, es decir

$$
\|\Delta \boldsymbol{B}[t]\|_{i p}=\mathcal{E}\left\{\left\|\left(\int_{\sigma_{0}}^{\sigma[t]} e^{\boldsymbol{A}_{c} \tau} \boldsymbol{B}_{c} \mathrm{~d} \tau\right)\left[\begin{array}{ll}
\boldsymbol{I} & -\boldsymbol{I}
\end{array}\right]\right\|_{i p}\right\} .
$$

Para poder utilizar los procedimientos de diseño expuestos en las secciones anteriores a partir de esta incertidumbre asociada al retardo, es necesario utilizar la norma de la perturbación equivalente (con $\left.\boldsymbol{B}_{v}=\boldsymbol{I}\right)$, que se obtiene mediante

$$
\|\boldsymbol{v}[t]\|_{p}=\|\Delta \boldsymbol{B}[t]\|_{i p}\|\boldsymbol{u}[t]\|_{p}
$$

Observación 4.7.1. Normalmente, si se tiene un retardo incierto en la entrada de control será difícil determinar cuál es el retardo mínimo $\sigma_{0}$, con lo que la matriz $\boldsymbol{B}_{1}$ será nula $\left(\boldsymbol{B}_{1}=\mathbf{0}\right)$ y $\boldsymbol{B}_{0}$ será la matriz de la discretización estándar a periodo $T$, es decir,

$$
\boldsymbol{B}_{0}=\int_{0}^{T} e^{A_{c} \tau} \boldsymbol{B}_{c} \mathrm{~d} \tau
$$

De la misma forma, la matriz incierta $\Delta \boldsymbol{B}[t]$ vendrá dada por

$$
\Delta \boldsymbol{B}[t]=\left(\int_{0}^{\sigma[t]} e^{\boldsymbol{A}_{c} \tau} \boldsymbol{B}_{c} \mathrm{~d} \tau\right)\left[\begin{array}{ll}
\boldsymbol{I} & -\boldsymbol{I}
\end{array}\right]
$$

y la norma de la perturbación a utilizar en el diseño podrá ser bien la del peor caso

$$
\|\boldsymbol{v}[t]\|_{p}=\operatorname{máx}_{\sigma[t]}\left\|\left(\int_{0}^{\sigma[t]} e^{\boldsymbol{A}_{c} \tau} \boldsymbol{B}_{c} \mathrm{~d} \tau\right)\left[\begin{array}{ll}
\boldsymbol{I} & -\boldsymbol{I}]
\end{array}\right]\right\|_{i p}\|\boldsymbol{u}[t]\|_{p},
$$

con $\boldsymbol{B}_{v}=\boldsymbol{I}$, o bien la del valor medio

$$
\|\boldsymbol{v}[t]\|_{p}=\mathcal{E}\left\{\left\|\left(\int_{0}^{\sigma[t]} e^{\boldsymbol{A}_{c} \tau} \boldsymbol{B}_{c} \mathrm{~d} \tau\right)\left[\begin{array}{ll}
\boldsymbol{I} & -\boldsymbol{I}]
\end{array}\right]\right\|_{i p}\right\}\|\boldsymbol{u}[t]\|_{p},
$$

asumiendo una distribución uniforme de $\sigma[t]$.

\subsubsection{Mediciones con etiqueta de tiempo incierta}

En el problema de predicción planteado se asume que cada medición $m_{j, k}$ del sensor $j\left(j=1, \ldots, n_{m}\right)$ viene acompañada de una etiqueta de tiempo que indica el instante de tiempo en el cual se dio la salida que se ha medido. Si las mediciones llegan con un retardo $d_{j, k}$, la etiqueta que acompaña a una medición $m_{j, k}$ disponible en el instate $t_{k}$ tiene el valor $t_{k}-d_{j, k}$. El predictor utiliza esta etiqueta para estimar el estado en el instante $t_{k}-d_{j, k}\left(\hat{\boldsymbol{x}}\left[t_{k}-d_{j, k} \mid t_{k}-1\right]\right)$ y compararla con la medición $m_{j, k}$, que se supone de valor $\boldsymbol{c}_{j} \boldsymbol{x}\left[t_{k}-d_{j, k}\right]$. Sin embargo, puede ocurrir que la medición corresponda a un instante de tiempo diferente al indicado por la etiqueta. Denotando mediante $i_{j, k}$ la incertidumbre que acompaña a la etiqueta de tiempo, normalmente asociada al retardo, puede escribirse el valor de la medición $m_{j, k}$ como (véase figura 4.7)

$$
m_{j, k}=\boldsymbol{c}_{j} \boldsymbol{x}\left[t_{k}-d_{j, k}+i_{j, k}\right] .
$$




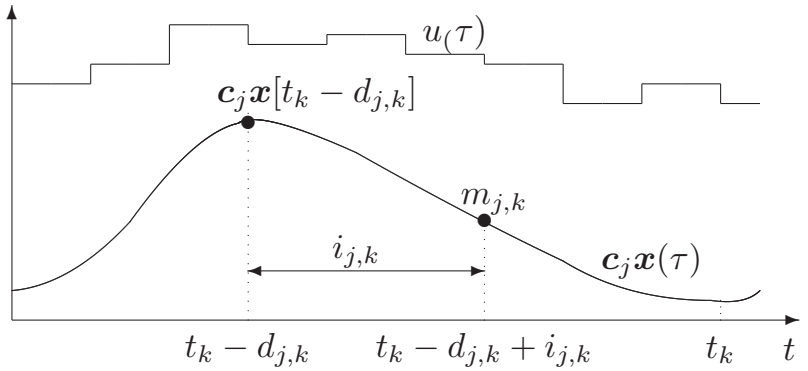

Figura 4.7: Medición disponible $\left(m_{j, k}\right)$ y salida correspondiente al instante indicado por la etiqueta de tiempo $\left(\boldsymbol{c}_{j} \boldsymbol{x}\left[t_{k}-d_{j, k}\right]\right)$.

El objetivo es encontrar una señal $w_{j, k}$ que tenga en cuenta el error cometido por la incertidumbre $i_{j, k}$, es decir, se busca el valor de $w_{j, k}$ tal que

$$
m_{j, k}=\boldsymbol{c}_{j} \boldsymbol{x}\left[t_{k}-d_{j, k}\right]+w_{j, k} .
$$

Esta señal $w_{j, k}$, que puede considerarse como un ruido de medida, viene dada por

$$
w_{j, k}=m_{j, k}-\boldsymbol{c}_{j} \boldsymbol{x}\left[t_{k}-d_{j, k}\right]=\boldsymbol{c}_{j}\left(\boldsymbol{x}\left[t_{k}-d_{j, k}+i_{j, k}\right]-\boldsymbol{x}\left[t_{k}-d_{j, k}\right]\right) .
$$

La aplicación de las estrategias de diseño anteriores que minimizan el error de predicción frente a ruidos de medida y perturbaciones de salida, requieren del conocimiento de una norma del ruido. Si se asume conocido el rango de variación de la incertidumbre de la etiqueta $i_{j, k}$

$$
i_{j, k} \in \mathcal{I}_{j}=\left\{\iota_{j 1}, \iota_{j 2}, \ldots, \iota_{j r}\right\}
$$

se tienen varias formas de obtener una norma característica de $w_{j, k}$. Una primera aproximación consiste en obtener una secuencia de valores $\boldsymbol{x}[t]$ con una simulación para obtener después el valor esperado de la norma de $\left\|\boldsymbol{c}_{j}\left(\boldsymbol{x}\left[t+i_{j, k}\right]-\boldsymbol{x}[t]\right)\right\|_{R M S}$ para diferentes valores de $i_{j, k}$ :

$$
\left\|w_{j, k}\right\|_{R M S}=\mathcal{E}_{i_{j} \in \mathcal{I}_{j}}\left\{\left\|\boldsymbol{c}_{j}\left(\boldsymbol{x}\left[t+i_{j}\right]-\boldsymbol{x}[t]\right)\right\|_{R M S}\right\} .
$$

De forma similar se puede obtener el ruido ficticio del peor caso como

$$
\left\|w_{j, k}\right\|_{\infty}=\operatorname{máx}_{i_{j} \in \mathcal{I}_{j}}\left\{\left\|\boldsymbol{c}_{k}\left(\boldsymbol{x}\left[t+i_{j}\right]-\boldsymbol{x}[t]\right)\right\|_{\infty}\right\} .
$$

Otra forma de calcular una cota de esta norma es tomando transformadas en $\mathcal{Z}$ llegando a

$$
\left\|w_{j, k}\right\|_{p}=\left\|\left(1-z^{-i_{j, k}}\right) G_{j}(z) \boldsymbol{u}(z)\right\|_{p} .
$$

Esta expresión permite obtener una cota del valor eficaz esperado del ruido ficticio mediante

$$
\left\|w_{j, k}\right\|_{R M S}=\mathcal{E}_{i_{j} \in \mathcal{I}_{j}}\left\{\left\|\left(1-z^{-i_{j}}\right) G_{j}(z)\right\|_{\infty}\right\}\|\boldsymbol{u}[t]\|_{R M S},
$$

o bien una cota del valor máximo del ruido ficticio mediante

$$
\left\|w_{j, k}\right\|_{\infty}=\mathcal{E}_{i_{j} \in \mathcal{I}_{j}}\left\{\left\|\left(1-z^{-i_{j}}\right) G_{j}(z)\right\|_{1}\right\}\|\boldsymbol{u}[t]\|_{\infty},
$$

donde se han utilizado las definiciones de las normas $\mathcal{H}_{\infty}$ y $\ell_{1}$ de un sistema (véase $\S$ A.2.1).

\subsubsection{Muestreo asíncrono}

Cuando se tienen mediciones asíncronas, es necesario utilizar un modelo continuo del proceso, ya que éste sí que tiene en cuenta la dinámica en todos los instantes de tiempo. El filtro de Kalman mostrado en §2.3.3 resuelve esta situación para muestreo asíncrono sin retardos. Los inconvenientes de esta estrategia son que no puede aplicarse cuando hay retardos y que tiene un coste computacional muy elevado.

Para evitar la utilización de un modelo continuo del proceso y para poder manejar mediciones con retardo, se propone utilizar una interpolación (bien de orden cero, o bien de orden uno, véase \$2.3.1) que permita obtener de forma aproximada el valor de la salida en los instantes síncronos con la actualización de la acción de control (cuando $\tau=t T$ ). Supóngase que el instante $\tau_{k}$, en el cual se tiene una medición 
asíncrona de algún sensor, corresponde al intervalo de tiempo que se encuentra entre los instantes de control $t$ y $(t+1)$, es decir

$$
t T<\tau_{k}<(t+1) T
$$

Si se define la fracción del periodo en la que llega la medición como

$$
\alpha_{k}=\frac{\tau_{k}-t T}{T}
$$

el instante continuo $\tau_{k}$ se puede escribir como

$$
\tau_{k}=\left(t+\alpha_{k}\right) T, \quad \alpha_{k} \in(0,1) .
$$

Teniendo en cuenta las tres formas de asincronía expuestas en §3.6.5 (página 65), y bajo el mismo razonamiento que allí se expone,se considera que no hay retardos entre el estado y la medición, y que la ecuación continua que relaciona la medición de un sensor $j$ cualquiera con el estado continuo viene dada por

$$
m_{j}(\tau)=\boldsymbol{c}_{j} \boldsymbol{x}(\tau) \quad j=1, \ldots, n_{m} .
$$

El estudio de esta sección se hace sobre una salida individual de forma análoga a como se ha realizado el análisis en los procesos monovariables con mediciones asíncronas. En los apartados siguientes se muestra la adaptación al caso multivariable de las 5 estrategias de interpolación expuestas en \$3.6.5. Con cada una de las estrategias de interpolación se obtiene una forma de estimar el ruido ficticio de medición que acompaña a la medición asíncrona de cada sensor. El procedimiento de cálculo del ruido de medida ficticio debe aplicarse a cada sensor que tome las mediciones de forma asíncrona.

\section{Interpolación de orden cero}

Estrategia I1. La primera estrategia que se propone consiste en considerar que el instante de medición es el del instante $t+1$ inmediatamente posterior al instante de medición, es decir,

$$
m_{j, k}=\boldsymbol{c}_{j} \boldsymbol{x}\left(\left(t+\alpha_{k}\right) T\right) \approx \boldsymbol{c}_{j} \boldsymbol{x}[t+1],
$$

y utilizar esta aproximación de la salida para correr la ecuación de actualización del predictor en $t+1$. El error que se comete entre el valor real de $\boldsymbol{c}_{j} \boldsymbol{x}[t+1]$ y el aproximado por $m_{k}$ puede considerarse como una señal de ruido que acompaña a la medición y que tiene un valor de

$$
w_{j, k}=\boldsymbol{c}_{j}\left(\boldsymbol{x}((t+1) T)-\boldsymbol{x}\left(\left(t+\alpha_{k}\right) T\right)\right) .
$$

Si el periodo de control se considera suficientemente pequeño, la evolución de la salida continua de $\boldsymbol{c}_{j} \boldsymbol{x}(t T)$ a $\boldsymbol{c}_{j} \boldsymbol{x}((t+1) T)$ se aproxima a una linea recta, con lo que la salida $\boldsymbol{c}_{j} \boldsymbol{x}\left(\left(t+\alpha_{k}\right) T\right)$ puede aproximarse por

$$
m_{j}\left(\tau_{k}\right)=m_{j}\left(\left(t+\alpha_{k}\right) T\right)=\boldsymbol{c}_{j} \boldsymbol{x}\left(\left(t+\alpha_{k}\right) T\right) \approx \boldsymbol{c}_{j} \boldsymbol{x}[t]+\alpha_{k} \boldsymbol{c}_{j}(\boldsymbol{x}[t+1]-\boldsymbol{x}[t]) .
$$

Con esta aproximación, el ruido de medida puede expresarse como

$$
w_{j, k}=\left(1-\alpha_{k}\right) \boldsymbol{c}_{j}(\boldsymbol{x}[t+1]-\boldsymbol{x}[t]) .
$$

A partir de esta expresión se tienen diferentes formas de obtener una norma característica del ruido de medición. El valor máximo del ruido viene dado por

$$
\left\|w_{j, k}\right\|_{\infty}=\left\|\boldsymbol{c}_{j} \Delta \boldsymbol{x}[t]\right\|_{\infty},
$$

donde los incrementos del estado se pueden estimar mediante simulación. También mediante simulación es posible obtener una cota aproximada del valor eficaz mediante la expresión

$$
\left\|w_{j, k}\right\|_{R M S}=\frac{1}{2}\left\|\boldsymbol{c}_{j} \Delta \boldsymbol{x}[t]\right\|_{R M S}
$$

donde se ha considerado una distribución uniforme de los instantes de medición, es decir de $\alpha_{k}$ y, por tanto, $\mathcal{E}\left(\alpha_{k}\right)=0.5$. Mediante la transformada en $\mathcal{Z}$ también es posible obtener una norma del valor eficaz esperado del ruido ficticio como

$$
\left\|w_{j, k}\right\|_{R M S}=\frac{1}{2}\left\|\left(1-z^{-1}\right) G_{j}(z)\right\|_{\infty}\|\boldsymbol{u}[t]\|_{R M S},
$$

y del valor máximo como

$$
\left\|w_{j, k}\right\|_{\infty}=\left\|\left(1-z^{-1}\right) G_{j}(z)\right\|_{1}\|\boldsymbol{u}[t]\|_{\infty},
$$

donde $G_{j}(z)$ es la matriz (fila) de transferencia que relaciona las entradas con la medición del sensor $j$ :

$$
G_{j}(z)=\boldsymbol{c}_{j}(z \boldsymbol{I}-\boldsymbol{A})^{-1} \boldsymbol{B} .
$$


Estrategia 12. segunda estrategia que se propone consiste en considerar que el instante de medición es el del instante de control $t$ inmediatamente anterior al instante de medición, es decir,

$$
m_{j, k}=\boldsymbol{c}_{j} \boldsymbol{x}\left(\left(t+\alpha_{k}\right) T\right) \approx \boldsymbol{c}_{j} \boldsymbol{x}[t]
$$

y utilizar esta aproximación de la salida para correr la ecuación de actualización del predictor en $t$, que, aunque podría haberse ejecutado en el instante $t$, se vuelve a ejecutar para mejorar la estimación. El error que se comete entre el valor real de $\boldsymbol{c}_{j} \boldsymbol{x}[t]$ y el aproximado por $m_{j, k}$ puede considerarse como una señal de ruido que acompaña a la medición y que tiene un valor de

$$
w_{j, k}=\boldsymbol{c}_{j} \boldsymbol{x}\left(\left(t+\alpha_{k}\right) T\right)-\boldsymbol{c}_{j} \boldsymbol{x}(t T) .
$$

De nuevo, bajo la consideración de transición casi lineal de $\boldsymbol{x}[t]$ a $\boldsymbol{x}[t+1]$, la norma del ruido ficticio se puede acotar mediante alguna de las expresiones (4.112), (4.113) o (4.114).

Si se conoce cuál es el valor más probable de $\alpha_{k}$ se puede elegir entre las dos estrategias I1 e I2 para minimizar el ruido ficticio introducido. Se elegirá la primera estrategia (asumir la medición en $t+1$ ) cuando $\mathcal{P}\left(\alpha_{k}\right)>0.5$ y la segunda estrategia (asumir la medición en $t$ ) cuando $\mathcal{P}\left(\alpha_{k}\right) \leq 0.5$.

Estrategia I3. Si se conoce el instante $\tau_{k}$ en el que llega la medición se pueden utilizar otras estrategias. La más simple consiste en elegir en cada instante entre las dos estrategias anteriores en función de si la medición está más cerca en el tiempo de $t T$ o de $(t+1) T$, es decir en función del valor de $\alpha_{k}$ :

$$
\begin{cases}t_{k}=t, & \text { si } \alpha_{k}<0.5 \\ t_{k}=t+1, & \text { si } \alpha_{k}>0.5\end{cases}
$$

Con este método, el valor esperado del ruido ficticio que se introduce con la medición ficticia es la mitad del indicado por las expresiones (4.112), (4.113), (4.114) y (4.115), y se puede calcular mediante las expresiones:

$$
\begin{aligned}
\left\|w_{j, k}\right\|_{\infty} & =\frac{1}{2}\left\|\boldsymbol{c}_{j} \Delta \boldsymbol{x}[t]\right\|_{\infty}, \\
\left\|w_{j, k}\right\|_{R M S} & =\frac{1}{4}\left\|\boldsymbol{c}_{j} \Delta \boldsymbol{x}[t]\right\|_{R M S}, \\
\left\|w_{j, k}\right\|_{R M S} & =\frac{1}{4}\left\|\left(1-z^{-1}\right) G_{j}(z)\right\|_{\infty}\|\boldsymbol{u}[t]\|_{R M S} \\
\left\|w_{j, k}\right\|_{\infty} & =\frac{1}{2}\left\|\left(1-z^{-1}\right) G_{j}(z)\right\|_{1}\|\boldsymbol{u}[t]\|_{\infty} .
\end{aligned}
$$

Observación 4.7.2. Como se ha podido comprobar, la utilización de mediciones asíncronas mediante interpolación de orden cero ha resultado introducir un ruido de medida ficticio cuyo valor máximo equivale al que se introduce en el caso de mediciones con etiqueta de tiempo incierta con $i_{j, k}=1$. Por lo tanto el análisis de los sistemas con mediciones asíncronas con etiqueta de tiempo incierta se puede hacer tomando el peor caso, que consiste simplemente en añadir al conjunto de incertidumbres de etiquetas $\left(\mathcal{I}_{j}\right)$ una unidad. Es decir, que el diseño se tiene aplicar para calcular una ganancia que atenúe los ruidos de medida con alguna de las normas indicadas en $\$ 4.7 .3$ donde

$$
\mathcal{I}_{j}=\left\{\iota_{j 1}, \iota_{j 1}+1, \iota_{j 2}, \iota_{j 2}+1, \ldots, \iota_{j r}, \iota_{j r}+1\right\} \subset \mathbb{N} .
$$

Si los números $\iota_{j q}(q=1, \ldots, r)$ son consecutivos, el conjunto anterior se reducirá a

$$
\mathcal{I}_{j}=\left\{\iota_{j 1}, \iota_{j 2}, \ldots, \iota_{j r}, \iota_{j r}+1\right\} .
$$

\section{Interpolación de orden uno}

Estrategia 14. La interpolación de orden uno se puede utilizar para obtener una estimación de la medición $m_{j, k}$ que se tendría en el instante $t+1$ a partir de su estimación en el instante $t$ y de la medición real $m_{j}\left(\tau_{k}\right)$ en $\tau=\tau_{k}$ de la cual se asume conocido $\tau_{k}$. Para ello se utiliza la ecuación de interpolación

$$
m_{j, k}=\boldsymbol{c}_{j} \hat{\boldsymbol{x}}[t]+\frac{T}{\tau_{k}-t T}\left(m_{j}\left(\tau_{k}\right)-\boldsymbol{c}_{j} \hat{\boldsymbol{x}}[t]\right)=\boldsymbol{c}_{j} \hat{\boldsymbol{x}}[t]+\frac{\boldsymbol{c}_{j}}{\alpha_{k}}\left(\boldsymbol{x}\left(\tau_{k}\right)-\hat{\boldsymbol{x}}[t]\right)
$$


El ruido ficticio que se introduce con la utilización de la señal interpolada es

$$
w_{j, k}=\boldsymbol{c}_{j} \boldsymbol{x}[t+1]-m_{j, k}
$$

Sustituyendo la ecuación de interpolación (4.122) y aplicando la aproximación(4.111) es fácil llegar a la expresión del ruido ficticio

$$
w_{j, k}=\frac{\alpha_{k}-1}{\alpha_{k}} \boldsymbol{c}_{j}(\boldsymbol{x}[t]-\hat{\boldsymbol{x}}[t])=\frac{\alpha_{k}-1}{\alpha_{k}} \boldsymbol{c}_{j} \widetilde{\boldsymbol{x}}[t]=\frac{\alpha_{k}-1}{\alpha_{k}} \boldsymbol{c}_{j} \widetilde{\boldsymbol{x}}\left[t_{k}-1\right]
$$

Esto implica que el error cometido con la interpolación aumenta con el error de estimación del estado de partida $\boldsymbol{x}[t]-\hat{\boldsymbol{x}}[t]$, y es mayor cuando aumenta el tiempo entre la toma de medición y la llegada del nuevo control, es decir cuando disminuye $\alpha_{k}$. Nótese que cuando $\alpha_{k}$ es cercana a 0 el error cometido con este método puede llegar a ser muy grande, dependiendo del error de partida $\widetilde{\boldsymbol{x}}\left[t_{k}-1\right]$.

La observación 3.6.4 también es aplicable a este caso de interpolación en representación interna, con lo que es necesario explorar formas alternativas de interpolación de orden uno, como se muestra a continuación.

Estrategia 15. La interpolación de orden uno se puede utilizar para obtener una estimación de la medición $m_{j, k}$ que se tendría en el instante $t+1$ a partir de las estimaciones del estado en $t$ y $t+1, \mathrm{y}$ a partir de la medición real en $\tau=\tau_{k}$ de la cual se asume conocido $\tau_{k}$ :

$$
m_{j, k}=m_{j}\left(\tau_{k}\right)+\left(1-\alpha_{k}\right) \boldsymbol{c}_{j}(\hat{\boldsymbol{x}}[t+1 \mid t]-\hat{\boldsymbol{x}}[t]) .
$$

Para ello se traza la recta que va desde la estimación $\hat{\boldsymbol{x}}[t]$ hasta $\hat{\boldsymbol{x}}[t+1 \mid t]$ para luego trazar una paralela a partir de $m_{j}\left(\tau_{k}\right)$ y así obtener el valor de la medición ficticia $m_{j, k}$ en el instante de control $t+1$. El ruido ficticio que se introduce con la utilización de la señal interpolada es

$$
w_{j, k}=\boldsymbol{c}_{j} \boldsymbol{x}[t+1]-m_{j, k} .
$$

Utilizando la expresión de interpolación (4.124) y la aproximación (4.111) se llega fácilmente a la expresión aproximada del ruido ficticio

$$
w_{j, k}=\left(1-\alpha_{k}\right) \boldsymbol{c}_{j}(\boldsymbol{x}[t+1]-\hat{\boldsymbol{x}}[t+1 \mid t]-\boldsymbol{x}[t]+\hat{\boldsymbol{x}}[t])=\left(1-\alpha_{k}\right) \boldsymbol{c}_{j}(\widetilde{\boldsymbol{x}}[t+1 \mid t]-\widetilde{\boldsymbol{x}}[t]) .
$$

Como la evolución en bucle abierto del error de estimación del estado de $t$ a $t+1$ viene dada por

$$
\widetilde{\boldsymbol{x}}\left[t_{k} \mid t_{k}-1\right]=\widetilde{\boldsymbol{x}}[t+1 \mid t]=\boldsymbol{A} \widetilde{\boldsymbol{x}}[t],
$$

la expresión (4.125) lleva a

$$
w_{j, k}=\left(1-\alpha_{k}\right) \boldsymbol{c}_{j}(\boldsymbol{A}-\boldsymbol{I}) \widetilde{\boldsymbol{x}}[t] .
$$

De nuevo, este valor depende del error de estimación del estado de partida aunque, con esta estrategia, el aumento del error debido a $\alpha_{k}$ no es tan grande como con la estrategia I4. Si se puede estimar el error de predicción de partida mediante simulaciones o mediante el conocimiento de las perturbaciones o error de modelado, es posible obtener una cota del ruido ficticio mediante

$$
\left\|w_{j, k}\right\|_{\infty}=\left\|\boldsymbol{c}_{j}(\boldsymbol{A}-\boldsymbol{I}) \widetilde{\boldsymbol{x}}\left[t_{k}-1\right]\right\|_{\infty}
$$

o bien mediante

$$
\left\|w_{j, k}\right\|_{R M S}=\frac{1}{2}\left\|\boldsymbol{c}_{j}(\boldsymbol{A}-\boldsymbol{I}) \widetilde{\boldsymbol{x}}\left[t_{k}-1\right]\right\|_{R M S} .
$$

Si se considera que la matriz $\boldsymbol{A}$ toma valores cercanos a la unidad (porque el sistema es estable o porque la frecuencia de control es suficientemente rápida), esta última técnica puede dar mejores resultados que la técnica anterior $\mathbf{I} 4$, ya que

$$
1-\alpha_{k}<\frac{1-\alpha_{k}}{\alpha_{k}}, \quad \forall \alpha_{k} \in(0,1)
$$

Esta observación, junto con el hecho de que la estrategia I4 tiene un ruido ficticio que tiende a infinito cuando $\alpha_{k}$ tiende a cero, indica que es preferible la estrategia $\mathbf{I 5}$ frente a la $\mathbf{I 4}$ 


\subsubsection{Minimización del error en bucle abierto}

En los diseños de las secciones anteriores anteriores se ha tratado de minimizar la norma del error de estimación de la salida en el instante de medición $\left(\left\|\boldsymbol{e}_{k}\right\|\right)$. El error de predicción entre mediciones se puede calcular sabiendo que el modelo se corre en bucle abierto, con lo que

$$
\boldsymbol{e}\left[t_{k}+r \mid t\right]=\boldsymbol{C}_{y} \widetilde{\boldsymbol{x}}\left[t_{k}+r\right]=\boldsymbol{C}_{y} \boldsymbol{A}^{r} \widetilde{\boldsymbol{x}}\left[t_{k}\right]+\boldsymbol{C}_{y} \sum_{i=1}^{r} \boldsymbol{A}^{i-1} \boldsymbol{v}\left[t_{k}+r-i\right]
$$

con $r<N_{k+1}$, y siendo $e\left[t_{k}+r \mid t\right]$ el error de predicción en bucle abierto con la información conocida hasta el instante $t$. La norma del error se puede acotar mediante

$$
\left\|\boldsymbol{e}\left[t_{k}+r \mid t\right]\right\|_{p} \leq\left\|\boldsymbol{C}_{y} \boldsymbol{A}^{r}\right\|_{i p}\left\|\widetilde{\boldsymbol{x}}\left[t_{k}\right]\right\|_{p}+\left\|\boldsymbol{C}_{y} \sum_{i=1}^{r} \boldsymbol{A}^{i-1} \boldsymbol{v}\left[t_{k}+r-i\right]\right\|_{p},
$$

donde se observa que la norma del error de predicción entre mediciones será tanto menor como menor sea la norma $\left\|\widetilde{\boldsymbol{x}}\left[t_{k}\right]\right\|$. Si la escasez de medidas no es muy elevada ( $N_{k}$ pequeño), los algoritmos expuestos en el capítulo darán un buen funcionamiento, pero si la escasez de medidas es muy elevada y además el proceso es inestable, es más conveniente acotar la norma del vector de error de estimación del estado para asegurar que el error entre mediciones sea pequeño. Para ello se han de replantear los diseños del capítulo sustituyendo sustituyendo $\boldsymbol{C}_{y}^{\top} \boldsymbol{C}_{y}$ por $\boldsymbol{I}$ en las LMI de las secciones $\$ 4.5$ y $\S 4.6$.

\subsubsection{Diseño basado en la minimización de frecuencias de medición}

Otra de las aplicaciones de los predictores es minimizar la frecuencia de muestro necesaria para controlar un proceso en bucle cerrado. De esta manera se pude minimizar la utilización del canal de comunicación consiguiendo disminuir el ancho de banda necesario y permitiendo compartir el canal con más procesos. Todo esto contribuirá a disminuir los costes del sistema de control. Para obtener este máximo número de periodos hay que tener en cuenta que la norma del error de predicción en bucle abierto viene dado por la ecuación (4.129) y que este error depende de las condiciones iniciales de estimación del estado, del tiempo entre muestras $(N)$ y de la perturbación del estado. También hay que tener en cuenta que el sistema tendrá que ser observable para ese valor de $N$.

Por ejemplo, si se está ante un proceso inestable sin perturbación, puede buscarse el periodo de muestreo más bajo que permita una amplificación de el error de predicción prevista de antemano.

Ejemplo 4.7.1. Sea el proceso inestable descrito en el ejemplo 4.2.1 en el que se supone que se tiene una perturbación del estado constante con un valor acotado de

$$
\left\|v_{1}[t]\right\|_{\infty}<0.1, \quad\left\|v_{2}[t]\right\|_{\infty}<0.1
$$

Suponiendo un error de estimación inicial nulo, el efecto de esta perturbación sobre el error de predicción de las salidas en bucle abierto durante $r$ periodos viene dado por

$$
\left\|\boldsymbol{e}\left[t_{k}+r \mid t\right]\right\|_{2}=\left\|\boldsymbol{C}_{y} \sum_{i=1}^{r} \boldsymbol{A}^{i-1}\left[\begin{array}{l}
0.1 \\
0.1
\end{array}\right]\right\|_{2},
$$

En la figura 4.8 viene representado el valor de la norma anterior para diferentes valores de $r$.

Si se desea que el peor efecto de esta perturbación sobre el error de salida sea de 1 unidad, se tendrá que tomar un periodo de muestreo como mucho de $N=25 T$ segundos, siendo $T$ el periodo de control.

\subsubsection{Diseño de predictores con objetivos combinados}

Otro problema no contemplado en los diseños anteriores es el caso en el que se desea minimizar el error de predicción frente a perturbaciones y ruidos de diferente naturaleza como, por ejemplo, una perturbación persistente acotada en $\ell_{2}$ y un ruido de medida impulsional. Este caso se resuelve mediante técnicas multiobjetivo o técnicas de objetivos combinados (véase [59]). El diseño de un predictor para un objetivo dado (atenuación estocástica $\mathcal{H}_{\infty}$, por ejemplo) se basa en la resolución de una LMI (la LMI (4.83), por ejemplo). Una forma de diseñar un predictor que cumpla con varios objetivos de forma simultánea (atenuación estocástica $\mathcal{H}_{\infty}$ y $\mathcal{H}_{2 g}$, por ejemplo), consiste en solucionar el conjunto de LMIs que forman ambos objetivos compartiendo las variables matriciales. El siguiente teorema muestra a modo de ejemplo el planteamiento del diseño combinado $\mathcal{H}_{2 g} / \mathcal{H}_{\infty}$ para el caso determinista. 


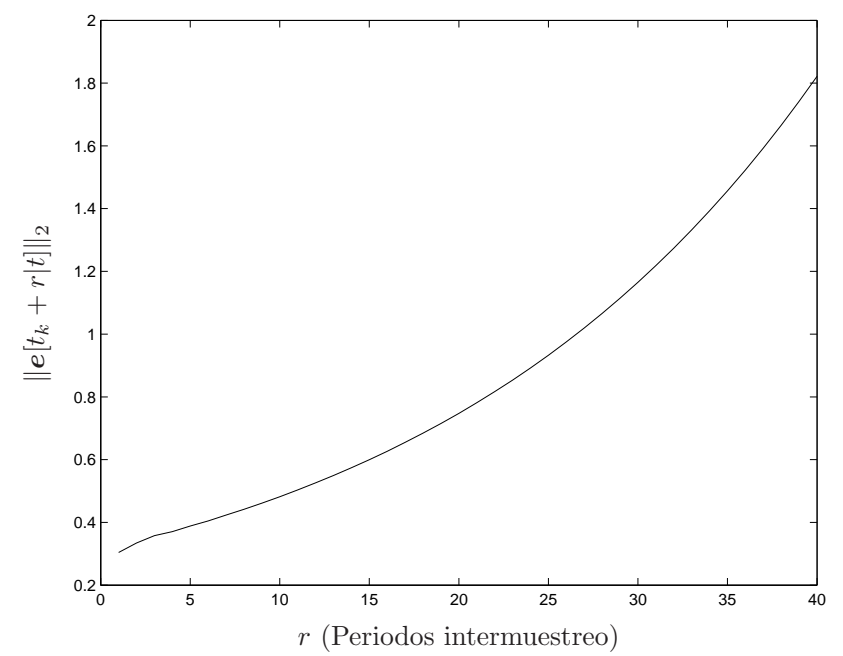

Figura 4.8: Propagación del error en bucle abierto en función del número de periodos intermuestro con una perturbación constante.

Teorema 4.7.2 (Atenuación $\mathcal{H}_{2 g} / \mathcal{H}_{\infty}$ ). Considérese el predictor (4.12) aplicado al sistema (4.2) y supóngase que hay al menos una medida disponible cada $N_{k} \in \mathcal{N}$ periodos, de los sensores indicados por $\boldsymbol{\Delta}_{k} \in \Xi y$ donde el retardo de cada sensor viene indicado por $d_{i, k} \in \mathcal{D}_{i}$, de manera que hay $n_{\mathcal{S}}$ escenarios de muestreo $s_{k} \in \mathcal{S}$ posibles. Supóngase también que son conocidas las normas $\ell_{2}$ y $\ell_{\infty}$ de las perturbaciones y ruido de medida. Para unos $\alpha_{\infty}, \alpha_{2}$ dados, supóngase que existen unas matrices $\boldsymbol{P}\left(s_{k}\right)=\boldsymbol{P}\left(s_{k}\right)^{\top} \in \mathbb{R}^{n \times n}, \boldsymbol{Q}\left(s_{k}\right) \in \mathbb{R}^{n \times n}, \boldsymbol{X}\left(s_{k}\right) \in \mathbb{R}^{n \times n_{m}}$, que minimizan la función de coste

$$
J=\alpha_{\infty}\left(\left\|\boldsymbol{\Gamma}_{v, \infty} \boldsymbol{v}_{k}\right\|_{\infty}^{2}+\left\|\boldsymbol{\Gamma}_{w, \infty} \boldsymbol{w}_{k}\right\|_{\infty}^{2}\right)+\alpha_{2}\left(\left\|\boldsymbol{\Gamma}_{v, 2} \boldsymbol{v}_{k}\right\|_{2}^{2}+\left\|\boldsymbol{\Gamma}_{w, 2} \boldsymbol{w}_{k}\right\|_{\infty}^{2}\right)
$$

a lo largo de todas las variables $\boldsymbol{P}\left(s_{k}\right), \boldsymbol{Q}\left(s_{k}\right), \boldsymbol{X}\left(s_{k}\right), \boldsymbol{\Gamma}_{v, \infty}, \boldsymbol{\Gamma}_{w, \infty}, \boldsymbol{\Gamma}_{v, 2}, \boldsymbol{\Gamma}_{w, 2}$ que cumplen

$$
\begin{aligned}
& {\left[\begin{array}{ccc}
\boldsymbol{Q}\left(s_{k}\right)+\boldsymbol{Q}\left(s_{k}\right)^{\boldsymbol{\top}}-\boldsymbol{P}\left(s_{k}\right) & \boldsymbol{M}_{A}\left(s_{k}\right) & \boldsymbol{M}_{B}\left(s_{k}\right) \\
\boldsymbol{M}_{A}\left(s_{k}\right)^{\top} & \boldsymbol{P}\left(s_{k-1}\right)-\boldsymbol{C}_{y}^{\top} \boldsymbol{C}_{y} & \mathbf{0} \\
\boldsymbol{M}_{B}\left(s_{k}\right)^{\top} & \mathbf{0} & \boldsymbol{\Gamma}_{\infty}
\end{array}\right] \succ 0,} \\
& {\left[\begin{array}{ccc}
\boldsymbol{Q}\left(s_{k}\right)+\boldsymbol{Q}\left(s_{k}\right)^{\top}-\boldsymbol{P}\left(s_{k}\right) & \boldsymbol{M}_{A}\left(s_{k}\right) & \boldsymbol{M}_{B}\left(s_{k}\right) \\
\boldsymbol{M}_{A}\left(s_{k}\right)^{\top} & \boldsymbol{P}\left(s_{k-1}\right) & \mathbf{0} \\
\boldsymbol{M}_{B}\left(s_{k}\right)^{\top} & \mathbf{0} & \boldsymbol{\Gamma}_{2}
\end{array}\right] \succ \mathbf{0}} \\
& \boldsymbol{P}\left(s_{k}\right)-\boldsymbol{C}_{y}^{\top} \boldsymbol{C}_{y} \succ 0,
\end{aligned}
$$

para cualquier secuencia de muestreo $\left\{s_{k}\right\}$, siendo

$$
\begin{aligned}
& \boldsymbol{\Gamma}_{\infty}=\operatorname{diag}\left\{\frac{\boldsymbol{\Gamma}_{v, \infty}}{\sqrt{\beta}}, \cdots, \frac{\boldsymbol{\Gamma}_{v, \infty}}{\sqrt{\beta}}, \boldsymbol{\Gamma}_{w, \infty}\right\}_{\left(n \beta+n_{m}\right)}, \\
& \boldsymbol{\Gamma}_{2}=\operatorname{diag}\left\{\frac{\boldsymbol{\Gamma}_{v, 2}}{\sqrt{\beta}}, \cdots, \frac{\boldsymbol{\Gamma}_{v, 2}}{\sqrt{\beta}}, \boldsymbol{\Gamma}_{w, 2}\right\}_{\left(n \beta+n_{m}\right)} .
\end{aligned}
$$

Entonces, definiendo la ganancia del predictor en función de la situación de muestreo como $\boldsymbol{L}\left(s_{k}\right)=$ $\boldsymbol{Q}\left(s_{k}\right)^{-1} \boldsymbol{X}\left(s_{k}\right)$, el error de predicción del algoritmo definido por (4.12) converge asintóticamente a cero en ausencia de perturbación y ruido de medida, y, bajo condiciones iniciales nulas,

$$
\begin{aligned}
\alpha_{\infty}\left\|\boldsymbol{e}_{k}\right\|_{2}^{2}+\alpha_{2}\left\|\boldsymbol{e}_{k}\right\|_{\infty}^{2} \leq & \alpha_{\infty}\left(\left\|\boldsymbol{\Gamma}_{v, \infty} \boldsymbol{v}_{k}\right\|_{\infty}^{2}+\left\|\boldsymbol{\Gamma}_{w, \infty} \boldsymbol{w}_{k}\right\|_{\infty}^{2}\right) \\
& +\alpha_{2}\left(\left\|\boldsymbol{\Gamma}_{v, 2} \boldsymbol{v}_{k}\right\|_{2}^{2}+\left\|\boldsymbol{\Gamma}_{w, 2} \boldsymbol{w}_{k}\right\|_{\infty}^{2}\right) .
\end{aligned}
$$

\subsection{Simplificaciones}

En algunas ocasiones, la solución de los problemas LMI que se han planteado en cada una de las estrategias, puede tener un coste computacional considerable, aún cuando no se trata de un proceso crítico (un proceso 
estable con perturbaciones de poca magnitud). En otras ocasiones puede ocurrir que la plataforma en la que se implementa el predictor (un microcontrolador de bajo coste, por ejemplo) no disponga de mucha memoria para utilizar por parte del programador del algoritmo de predicción, con lo que no se pueden almacenar todas las matrices de ganancias $\boldsymbol{L}\left(s_{k}\right)\left(s_{k}=1, \ldots, n_{\mathcal{S}}\right)$ necesarias. Para estas situaciones se plantean a continuación diferentes estrategias que restringen el espacio de soluciones y llevan a un diseño más conservador a cambio de un menor coste computacional en el cálculo de las ganancias del predictor y una disminución de la memoria requerida para implementar el predictor.

\subsubsection{Reducción del número de ganancias a almacenar}

En el diseño de predictores basados en un patrón de muestreo variante de forma arbitraria se han asumido diferentes matrices $\boldsymbol{X}\left(s_{k}\right)$ y $\boldsymbol{Q}\left(s_{k}\right)\left(s_{k}=\left\{1, \ldots, n_{\mathcal{S}}\right\}\right)$ para cada uno de los valores del parámetro de muestreo. Esto conduce a conjunto de $n_{\mathcal{S}}$ ganancias diferentes para el predictor que deberían almacenarse para aplicar la ganancia correcta dependiendo de los valores $d_{i, k}, \boldsymbol{\Delta}_{k} \mathrm{y} N_{k}$ que definen el muestreo.

Los teoremas, sin embargo, también son válidos si se imponen algunas restricciones en las matrices $\boldsymbol{Q}\left(s_{k}\right)$ y $\boldsymbol{X}\left(s_{k}\right)$ para reducir el número de ganancias a almacenar. La idea es hacer la ganancia robusta a variaciones de $s_{k}$.

El caso más general puede definirse como sigue. Se divide el conjunto de posibles muestreos $\mathcal{S}$. en $r$ subconjuntos disjuntos, $S_{i}, i=1, \ldots, r$ y se definen $r$ matrices diferentes $\boldsymbol{Q}(i)$ y $\boldsymbol{X}(i), i=1, \ldots, r$, tales que $\boldsymbol{Q}\left(s_{k}\right)=\boldsymbol{Q}(i)$ y $\boldsymbol{X}\left(s_{k}\right)=\boldsymbol{X}(i)$ si $s_{k} \in \mathcal{S}_{i}$. Como resultado, se debe almacenar un conjunto reducido de $r$ vectores de ganancias $\boldsymbol{L}(i)=\boldsymbol{Q}(i)^{-1} \boldsymbol{X}(i), i=, \ldots, r$. Qué ganancia se utilizará en un instante de muestreo dado dependerá del subconjunto $\mathcal{S}_{i}$ al cual pertenece la combinación $\left(d_{j, k}, \boldsymbol{\Delta}_{k}, N_{k}\right)$. El inconveniente de imponer esta restricción es que el conjunto de LMIs que se ha de resolver es más improbable que sea factible

Un caso particular interesante, que conduce al algoritmo de predicción más simple, se obtiene cuando sólo hay un subconjunto. En este caso se toman dos matrices constantes $\boldsymbol{Q}\left(s_{k}\right)=\boldsymbol{Q}$ y $\boldsymbol{X}\left(s_{k}\right)=\boldsymbol{X}, \forall s_{k}$ en la resolución del conjunto de LMI, y como resultado se obtiene una ganancia constante $\boldsymbol{L}=\boldsymbol{Q}^{-1} \boldsymbol{X}$.

\subsubsection{Simplificación de las LMI}

Una simplificación que lleva a un menor coste computacional a costa de disminuir ligeramente el espacio de soluciones consiste en tomar iguales la matriz $\boldsymbol{P}\left(s_{k}\right)$ y $\boldsymbol{Q}\left(s_{k}\right)$.

Otra simplificación consiste en encontrar un predictor que asegure la estabilidad cuadrática (condición suficiente pero no necesaria) haciendo para ello la matriz $\boldsymbol{P}\left(s_{k}\right)=\boldsymbol{P}$ constante. Este caso reduce el espacio de soluciones con el inconveniente de que puede que no se encuentre un predictor estable ante un sistema inestable y/o con muestreo muy escaso.

Si se aplican las dos últimas simplificaciones al mismo tiempo, tomando las matrices $\boldsymbol{P}\left(s_{k}\right)$ y $\boldsymbol{Q}\left(s_{k}\right)$ iguales y de valor constante,

$$
\boldsymbol{Q}\left(s_{k}\right)=\boldsymbol{P}\left(s_{k}\right)=\boldsymbol{P},
$$

la ganancia del predictor vendrá determinada por $\boldsymbol{L}\left(s_{k}\right)=\boldsymbol{P}^{-1} \boldsymbol{X}\left(s_{k}\right)$. Nótese que en este caso sólo se cumplirá la condición suficiente de estabilidad cuadrática, con lo que el espacio de soluciones quedará reducido, y el predictor tendrá peores prestaciones.

Finalmente, la mayor simplificación posible consiste en, además de tomar $\boldsymbol{P}\left(s_{k}\right)=\boldsymbol{Q}\left(s_{k}\right)=\boldsymbol{P}$, tomar una matriz $\boldsymbol{X}\left(s_{k}\right)=\boldsymbol{X}$ constante para obtener una matriz de ganancias $\boldsymbol{L}_{k}=\boldsymbol{L}$ constante. En este caso se tendrá el predictor de peores prestaciones. Aún así, este predictor puede ser muy útil en procesos estables con poca pérdida de datos y poca variación en el retardo.

\subsubsection{Interpolación de ganancias}

En la metodología planeada en el capítulo, la obtención de ganancias se basa en la resolución de un problema de optimización por medio de un sistema de inecuaciones lineales matriciales. El coste computacional de estos problemas de optimización vía LMI aumenta con el número de LMIs que conforman el sistema de inecuaciones. El tamaño de este sistema de inecuaciones depende directamente del número de escenarios de muestreo $n_{\mathcal{S}}$. Si se utiliza una matriz $\boldsymbol{P}$ constante, el sistema a resolver está formado por $n_{\mathcal{S}}$ LMIs, pero si se utiliza una matriz $\boldsymbol{P}(i)$ que varía en función del parámetro $s_{k}$, el sistema está formado por $n_{\mathcal{S}}^{2}$ inecuaciones. Esto conlleva un tiempo muy elevado de resolución, que puede llegar a durar varias horas. 
Supóngase a partir de ahora que los retardos en cada sensor son constantes y que todas las salidas se miden simultáneamente, con lo que el número de posibles escenarios de muestreo coincide con el número de periodos intermuestreo. Asúmase que el número de posibles periodos intermuestreo viene definido por el conjunto

$$
\mathcal{N}=\left\{\nu_{1}, \nu_{2} \ldots, \nu_{n_{\mathcal{N}}}\right\}
$$

Para poder reducir el número de LMIs a resolver se toma un subconjunto

$$
\mathcal{N}^{\prime}=\left\{\mu_{1}, \mu_{2} \ldots, \mu_{n_{\mathcal{N}^{\prime}}}\right\} \subset \mathcal{N}, n_{\mathcal{S}^{\prime}}<n_{\mathcal{S}}
$$

de manera que contenga por lo menos el primer y el último elemento $\left(\mu_{1}=\nu_{1}\right.$ y $\left.\mu_{n_{\mathcal{N}^{\prime}}}=\nu_{n_{\mathcal{N}}}\right)$, y se aplica alguno de los métodos basado en LMIs a lo largo de este nuevo conjunto $\mathcal{N}^{\prime}$. El conjunto de ganancias que se obtendrán como solución viene definido por

$$
\left\{\boldsymbol{L}\left(\mu_{i}\right)\right\}, \mu_{i} \in \mathcal{N}^{\prime}
$$

El resto de ganancias asociadas al conjunto $\mathcal{N}$ se obtienen a partir de éstas mediante una interpolación

$$
\begin{aligned}
\boldsymbol{L}\left(\nu_{j}\right)= & \boldsymbol{L}\left(\mu_{i}\right)+\frac{\nu_{j}-\mu_{i}}{\mu_{i+1}-\mu_{i}}\left(\boldsymbol{L}\left(\mu_{i+1}\right)-\boldsymbol{L}\left(\mu_{i}\right)\right), \\
& \nu_{j} \in \mathcal{N} ; \mu_{i}, \mu_{i+1} \in \mathcal{N}^{\prime} ; \mu_{i}<\nu_{j}<\mu_{i+1} .
\end{aligned}
$$

El siguiente paso en el diseño mediante esta técnica consiste en comprobar que este nuevo conjunto de ganancias estabiliza el predictor en todo su rango de funcionamiento (es decir, sobre $\mathcal{N}$ ). Este método será adecuado siempre que las variaciones de los elementos del vector $\boldsymbol{L}$ sean suaves a lo largo de los elementos de $\mathcal{N}$.

Esta técnica puede ser útil tanto para reducir el coste computacional de obtención de los vectores de ganancia $\boldsymbol{L}$, como para reducir los recursos de memoria necesarios para poder implementar el predictor propuesto en microcontroladores de bajo coste.

Un problema diferente que puede darse es que se esté en condiciones de calcular las matrices $\boldsymbol{L}$ para un rango de $\mathcal{N}$ amplio, pero, sin embargo, el dispositivo donde se han de almacenar estos valores no tiene memoria suficiente. En este caso, si las variaciones de $\boldsymbol{L}$ en función de $N$ son suaves, puede almacenarse sólo un conjunto de vectores $\boldsymbol{L}$ correspondientes a un subconjunto $\mathcal{N}^{\prime} \subset \mathcal{N}$, de manera que cada vez que se necesite el vector $\boldsymbol{L}(N)$, donde $N$ no pertenece a $\mathcal{N}^{\prime}$, se procederá a la interpolación indicada en (4.136).

\subsection{Sensores virtuales basados en el filtro de Kalman}

En esta sección se derivan las ecuaciones del filtro de Kalman para procesos que tienen mediciones síncronas escasas con retardo temporal variante en el tiempo. En primer lugar se desarrollan las ecuaciones para el caso más simple de muestreo regular con retardo constante, para posteriormente obtener el algoritmo general.

\subsubsection{Sistemas monovariable}

\section{Muestreo periódico y retardo constante}

Considérese un proceso discreto lineal descrito por las ecuaciones

$$
\begin{aligned}
\boldsymbol{x}[t+1] & =\boldsymbol{A} \boldsymbol{x}[t]+\boldsymbol{b} u[t]+\boldsymbol{B}_{v} \boldsymbol{v}[t] \\
y[t] & =\boldsymbol{c}_{y} x[t], \\
m[t] & =\boldsymbol{c} x[t-d]+w[t],
\end{aligned}
$$

donde $\boldsymbol{x} \in \mathbb{R}^{n}$ es el vector de estados, $u \in \mathbb{R}$ es la entrada, $y \in \mathbb{R}$ es la salida del proceso a controlar y $m[t]$ es la medición que le llega al controlador cada periodo de control y que viene afectada de un retardo $d$. $\boldsymbol{v}[t]$ es el vector de perturbaciones de entrada y $w[t]$ es el ruido de medida. Se asume que $\boldsymbol{w}$ es un ruido blanco de media cero con matriz de varianzas-covarianzas $\boldsymbol{V}\left(\boldsymbol{V}=\mathcal{E}\left\{\boldsymbol{v}[t] \boldsymbol{v}[t]^{\top}\right\}\right)$, y $w$ es un ruido blanco de media cero con varianza $\boldsymbol{W}\left(\boldsymbol{W}=\mathcal{E}\left\{w[t] w[t]^{\top}\right\}\right)$. 
Teorema 4.9.1 (Filtro de Kalman con retardo constante). Sea el algoritmo de predicción de salidas del proceso (4.137) definido por las ecuaciones

$$
\begin{aligned}
\hat{\boldsymbol{x}}[t \mid t-1] & =\boldsymbol{A} \hat{\boldsymbol{x}}[t-1 \mid t-1]+\boldsymbol{b} u[t-1] \\
\hat{\boldsymbol{x}}[t \mid t] & =\hat{\boldsymbol{x}}[t \mid t-1]+\boldsymbol{\ell}[t](m[t]-\boldsymbol{c} \hat{\boldsymbol{x}}[t-d \mid t-1])
\end{aligned}
$$

donde $\hat{\boldsymbol{x}}[t-d \mid t-1]$ es la mejor estimación del estado en el instante $t-d$ con la información disponible hasta en instante $t-1$, que cumple

$$
\hat{\boldsymbol{x}}[t \mid t-1]=\boldsymbol{A}^{d} \hat{\boldsymbol{x}}[t-d \mid t-1]+\sum_{i=1}^{d} \boldsymbol{A}^{i-1} \boldsymbol{b} u[t-i],
$$

y donde la estimación de la salida viene dada por

$$
\hat{y}[t]=\boldsymbol{c}_{y} \hat{\boldsymbol{x}}[t \mid t] .
$$

El vector de ganancias $\ell[t]$ que minimiza la varianza del error de predicción para el algoritmo anterior viene definido por las ecuaciones

$$
\begin{aligned}
\boldsymbol{P}[t \mid t-1] & =\boldsymbol{A} \boldsymbol{P}[t-1 \mid t-1] \boldsymbol{A}^{\top}+\boldsymbol{B}_{v} \boldsymbol{V} \boldsymbol{B}_{v}^{\top} \\
\boldsymbol{\ell}[t] & =\frac{\boldsymbol{P}[t \mid t-1]\left(\boldsymbol{c} \boldsymbol{A}^{-d}\right)^{\top}}{\boldsymbol{c} \boldsymbol{A}^{-d} \boldsymbol{P}[t \mid t-1]\left(\boldsymbol{c} \boldsymbol{A}^{-d}\right)^{\top}+\boldsymbol{W}+\overline{\boldsymbol{c}} \boldsymbol{V} \overline{\boldsymbol{c}}^{\top}} \\
\boldsymbol{P}[t \mid t] & =\left(\boldsymbol{I}-\boldsymbol{\ell}[t] \boldsymbol{c} \boldsymbol{A}^{-d}\right) \boldsymbol{P}[t \mid t-1]
\end{aligned}
$$

siendo $\overline{\boldsymbol{C}}$ un vector constante que se calcula según

$$
\overline{\boldsymbol{c}}=\boldsymbol{c} \sum_{i=1}^{d} \boldsymbol{A}^{-i} \boldsymbol{B}_{v}
$$

Prueba 4.9.1. El estado del sistema en el instante $t$ se puede relacionar con el del instante $t-d$ corriendo $d$ veces la ecuación (4.137) del sistema, es decir

$$
\boldsymbol{x}[t]=\boldsymbol{A}^{d} \boldsymbol{x}[t-d]+\sum_{i=1}^{d} \boldsymbol{b} u[t-i]+\sum_{i=1}^{d} \boldsymbol{B}_{v} \boldsymbol{v}[t-i]
$$

con lo que la medición $m[t]$ se puede escribir en función del estado actual como

$$
m[t]=\boldsymbol{c} \boldsymbol{A}^{-d}\left(\boldsymbol{x}[t]-\sum_{i=1}^{d} \boldsymbol{A}^{i-1}\left(\boldsymbol{b} u[t-i]+\boldsymbol{B}_{v} \boldsymbol{v}[t-i]\right)\right)+w[t] .
$$

Sustituyendo esta expresión en la ecuación de actualización (4.12b) y restando $x[t]$ en ambos lados de la expresión resultante se llega a

$$
\widetilde{\boldsymbol{x}}[t \mid t]=\left(\boldsymbol{I}-\boldsymbol{\ell}[t] \boldsymbol{c} \boldsymbol{A}^{-d}\right) \widetilde{\boldsymbol{x}}[t \mid t-1]-\boldsymbol{\ell}[t] w[t]+\boldsymbol{\ell}[t] \boldsymbol{c} \boldsymbol{A}^{-d} \sum_{i=1}^{d} \boldsymbol{A}^{i-1} \boldsymbol{B}_{v} \boldsymbol{v}[t-i],
$$

siendo

$$
\begin{aligned}
\widetilde{\boldsymbol{x}}[t \mid t] & =\boldsymbol{x}[t]-\hat{\boldsymbol{x}}[t \mid t], \\
\widetilde{\boldsymbol{x}}[t \mid t-1] & =\boldsymbol{x}[t]-\hat{\boldsymbol{x}}[t \mid t-1] .
\end{aligned}
$$

Definiendo la matriz de varianzas-covarianzas del error de estimación como

$$
\boldsymbol{P}[t \mid t]=\mathcal{E}\left\{\widetilde{\boldsymbol{x}}[t \mid t] \widetilde{\boldsymbol{x}}[t \mid t]^{\top}\right\},
$$


y aplicando la relación (4.140) se tiene que

$$
\begin{aligned}
\boldsymbol{P}[t \mid t]= & \left(\boldsymbol{I}-\boldsymbol{\ell}[t] \boldsymbol{c} \boldsymbol{A}^{-d}\right) \mathcal{E}\left\{\widetilde{\boldsymbol{x}}[t \mid t-1] \widetilde{\boldsymbol{x}}[t \mid t-1]^{\top}\right\}\left(\boldsymbol{I}-\boldsymbol{\ell}[t] \boldsymbol{c} \boldsymbol{A}^{-d}\right)^{\top} \\
& +\boldsymbol{\ell}[t] \mathcal{E}\left\{w[t] w[t]^{\top}\right\} \boldsymbol{\ell}[t]^{\top} \\
& +\sum_{i=1}^{d} \sum_{j=1}^{d}\left(\boldsymbol{\ell}[t] \boldsymbol{c} \boldsymbol{A}^{i-d-1} \mathcal{E}\left\{\boldsymbol{v}[t-i] \boldsymbol{v}[t-j]^{\top}\right\}\left(\boldsymbol{\ell}[t] \boldsymbol{c} \boldsymbol{A}^{j-d-1}\right)^{\top}\right) \\
= & \boldsymbol{P}[t \mid t-1]-\boldsymbol{\ell} \boldsymbol{C} \boldsymbol{A}^{-d} \boldsymbol{P}[t \mid t-1]-\boldsymbol{P}[t \mid t-1]\left(\boldsymbol{\ell} \boldsymbol{C} \boldsymbol{A}^{-d}\right)^{\top} \\
& +\boldsymbol{\ell}[t]\left(\boldsymbol{c} \boldsymbol{A}^{-d} \boldsymbol{P}[t \mid t-1]\left(\boldsymbol{c} \boldsymbol{A}^{-d}\right)^{\top}+\boldsymbol{W}+\overline{\boldsymbol{c}} \boldsymbol{V} \overline{\boldsymbol{c}}^{\boldsymbol{\top}}\right) \boldsymbol{\ell}[t]^{\top}
\end{aligned}
$$

donde se ha considerado que

$$
\mathcal{E}\{\boldsymbol{v}[t-i] \boldsymbol{v}[t-j]\}=\mathbf{0}, \quad i \neq j
$$

Los elementos de la diagonal de $\boldsymbol{P}[t \mid t]$ contienen las varianzas del error de estimación del estado, con lo que la minimización de la varianza puede determinarse minimizando la traza de $\boldsymbol{P}[t \mid t]$. Para obtener la ganancia que minimiza la varianza se deriva la traza de $\boldsymbol{P}[t \mid t)$ con respecto a $\boldsymbol{\ell}[t]$ y se iguala a cero, obteniéndose

$$
-2 \operatorname{tr}\left(\boldsymbol{c} \boldsymbol{A}^{-d} \boldsymbol{P}[t \mid t-1]\right)^{\top}+2 \operatorname{tr}\left(\ell[t]\left(\boldsymbol{c} \boldsymbol{A}^{-d} \boldsymbol{P}[t \mid t-1]\left(\boldsymbol{c} \boldsymbol{A}^{-d}\right)^{\top}+\boldsymbol{W}+\overline{\boldsymbol{c}} \boldsymbol{V} \overline{\boldsymbol{c}}^{\top}\right)\right)=0
$$

de donde se puede despejar $\ell[t]$ para obtener

$$
\ell[t]=\boldsymbol{P}[t \mid t-1]\left(\boldsymbol{C} \boldsymbol{A}^{-d}\right)^{\top}\left(\boldsymbol{C} \boldsymbol{A}^{-d} \boldsymbol{P}[t \mid t-1]\left(\boldsymbol{C} \boldsymbol{A}^{-d}\right)^{\top}+\boldsymbol{W}+\overline{\boldsymbol{C}} \boldsymbol{V} \overline{\boldsymbol{C}}^{\boldsymbol{\top}}\right)^{-1}
$$

La actualización óptima del vector de covarianzas se obtiene sustituyendo este vector $\ell[t]$ en la expresión (4.141). La ecuación que proyecta la matriz de covarianzas al siguiente instante se obtiene a partir de la expresión que relaciona los errores $\widetilde{\boldsymbol{x}}[t \mid t-1]$ y $\widetilde{\boldsymbol{x}}[t-1 \mid t-1]$ :

$$
\begin{aligned}
\widetilde{\boldsymbol{x}}[t \mid t-1] & =\boldsymbol{x}[t]-\hat{\boldsymbol{x}}[t \mid t-1] \\
& =\boldsymbol{A} \boldsymbol{x}[t-1]+\boldsymbol{b} u[t-1]+\boldsymbol{B}_{v} \boldsymbol{v}[t-1]-\boldsymbol{A} \hat{\boldsymbol{x}}[t-1 \mid t-1]-\boldsymbol{b} u[t-1] \\
& =\boldsymbol{A} \widetilde{\boldsymbol{x}}[t-1 \mid t-1]+\boldsymbol{B}_{v} \boldsymbol{v}[t-1] .
\end{aligned}
$$

De esta manera se tiene que

$$
\begin{aligned}
\boldsymbol{P}[t \mid t-1] & =\mathcal{E}\left\{\widetilde{\boldsymbol{x}}[t \mid t-1] \widetilde{\boldsymbol{x}}[t \mid t-1]^{\top}\right\} \\
& =\mathcal{E}\left\{\left(\boldsymbol{A} \widetilde{\boldsymbol{x}}[t-1 \mid t-1]+\boldsymbol{B}_{v} \boldsymbol{v}[t-1]\right)\left(\boldsymbol{A} \widetilde{\boldsymbol{x}}[t-1 \mid t-1]+\boldsymbol{B}_{v} \boldsymbol{v}[t-1]\right)^{\top}\right\} \\
& =\boldsymbol{A} \boldsymbol{P}[t-1 \mid t-1] \boldsymbol{A}^{\top}+\boldsymbol{B}_{v} \boldsymbol{V} \boldsymbol{B}_{v}^{\top} .
\end{aligned}
$$

\section{Muestreo escaso irregular y retardo variante}

Si las medidas son escasas y el retardo asociado a cada medición es variante en el tiempo se utiliza el modelo del proceso

$$
\begin{aligned}
\boldsymbol{x}[t+1] & =\boldsymbol{A} \boldsymbol{x}[t]+\boldsymbol{b} u[t]+\boldsymbol{B}_{v} \boldsymbol{v}[t] \\
y[t] & =\boldsymbol{c}_{y} x[t]
\end{aligned}
$$

más la ecuación de medidas,

$$
m_{k}=\boldsymbol{C} x\left[t_{k}-d_{k}\right]+w\left[t_{k}\right]
$$

que sólo es válida en los instantes $t=t_{k}$ en los que se dispone de un dato $m_{k}$ afectado de un retardo de $d_{k}$ periodos.

Teorema 4.9.2 (Filtro de Kalman para muestreo aleatorio con retardo). Sea el algoritmo de predicción de salidas del proceso (4.142) definido por la ecuación de propagación

$$
\hat{\boldsymbol{x}}[t \mid t-1]=\boldsymbol{A} \boldsymbol{x}[t-1]+\boldsymbol{b} u[t-1]
$$


más la ecuación de actualización

$$
\hat{\boldsymbol{x}}\left[t_{k} \mid t_{k}\right]=\hat{\boldsymbol{x}}\left[t_{k} \mid t_{k}-1\right]+\boldsymbol{\ell}_{k}\left(m_{k}-\boldsymbol{C} \hat{\boldsymbol{x}}\left[t_{k}-d_{k} \mid t_{k}-1\right]\right)
$$

que se utiliza cuando se tiene una medición $m_{k}\left(\right.$ en $\left.t=t_{k}\right)$, siendo $\hat{\boldsymbol{x}}\left[t_{k}-d_{k} \mid t_{k}-1\right]$ la mejor estimación del estado del instante $t_{k}-d_{k}$ con la información disponible hasta en instante $t_{k}-1$, que cumple

$$
\hat{\boldsymbol{x}}\left[t_{k} \mid t_{k}-1\right]=\boldsymbol{A}^{d_{k}} \hat{\boldsymbol{x}}\left[t_{k}-d_{k} \mid t_{k}-1\right]+\sum_{i=1}^{d_{k}} \boldsymbol{A}^{i-1} \boldsymbol{b} u[t-i] .
$$

La predicción de la salida se obtiene mediante

$$
\hat{y}[t]=\boldsymbol{c}_{y} \hat{\boldsymbol{x}}[t] .
$$

El vector de ganancias $\ell[t]$ que minimiza el error de predicción viene dado por la ecuación de propagación

$$
\boldsymbol{P}[t \mid t-1]=\boldsymbol{A P}[t-1 \mid t-1] \boldsymbol{A}+\boldsymbol{B}_{v} \boldsymbol{V} \boldsymbol{B}_{v}^{\top},
$$

más las ecuaciones de actualización

$$
\begin{aligned}
\boldsymbol{\ell}_{k} & =\frac{\boldsymbol{P}\left[t_{k} \mid t_{k}-1\right]\left(\boldsymbol{c} \boldsymbol{A}^{-d_{k}}\right)^{\top}}{\boldsymbol{c} \boldsymbol{A}^{-d_{k}} \boldsymbol{P}\left[t_{k} \mid t_{k}-1\right]\left(\boldsymbol{c} \boldsymbol{A}^{-d_{k}}\right)^{\top}+\boldsymbol{W}+\overline{\boldsymbol{c}}_{d, k} \boldsymbol{V} \overline{\boldsymbol{c}}_{d, k}^{\top}} \\
\boldsymbol{P}\left[t_{k} \mid t_{k}\right] & =\left(\boldsymbol{I}-\boldsymbol{\ell} \boldsymbol{c} \boldsymbol{A}^{-d_{k}}\right) \boldsymbol{P}\left[t_{k} \mid t_{k}-1\right],
\end{aligned}
$$

siendo

$$
\overline{\boldsymbol{c}}_{d, k}=\boldsymbol{c} \sum_{i=1}^{d_{k}} \boldsymbol{A}^{-i} \boldsymbol{B}_{v}
$$

Si no hay ninguna medición, la mejor estimación del estado y de la matriz de varianzas-covarianzas es la de bucle abierto, con lo que

$$
\begin{aligned}
\hat{\boldsymbol{x}}[t \mid t] & =\boldsymbol{x}[t \mid t-1], \\
\boldsymbol{P}[t \mid t] & =\boldsymbol{P}[t \mid t-1] .
\end{aligned}
$$

Prueba 4.9.2. El estado del sistema en el instante $t_{k}$ se puede relacionar con el del instante $t_{k}-d_{k}$ corriendo $d_{k}$ veces la ecuación (4.142) del sistema, es decir

$$
\boldsymbol{x}\left[t_{k}\right]=\boldsymbol{A}^{d_{k}} \boldsymbol{x}\left[t_{k}-d_{k}\right]+\sum_{i=1}^{d_{k}} \boldsymbol{A}^{i-1} \boldsymbol{b} u\left[t_{k}-i\right]+\sum_{i=1}^{d_{k}} \boldsymbol{A}^{i-1} \boldsymbol{B}_{v} \boldsymbol{v}\left[t_{k}-i\right],
$$

con lo que la medición $m_{k}$ se puede escribir en función del estado actual como

$$
m_{k}=\boldsymbol{c} \boldsymbol{A}^{-d_{k}}\left(\boldsymbol{x}\left[t_{k}\right]-\sum_{i=1}^{d_{k}} \boldsymbol{A}^{i-1}\left(\boldsymbol{b} u\left[t_{k}-i\right]+\boldsymbol{B}_{v} \boldsymbol{v}\left[t_{k}-i\right]\right)\right)+w\left[t_{k}\right]
$$

Sustituyendo esta expresión en la ecuación de actualización (4.143b) y restando $x\left[t_{k}\right]$ en ambos lados de la expresión resultante se llega a

$$
\widetilde{\boldsymbol{x}}\left[t_{k} \mid t_{k}\right]=\left(\boldsymbol{I}-\boldsymbol{\ell}_{k} \boldsymbol{c} \boldsymbol{A}^{-d_{k}}\right) \widetilde{\boldsymbol{x}}\left[t_{k} \mid t_{k}-1\right]-\boldsymbol{\ell}_{k} w\left[t_{k}\right]+\boldsymbol{\ell}_{k} \boldsymbol{c} \boldsymbol{A}^{-d_{k}} \sum_{i=1}^{d_{k}} \boldsymbol{A}^{i-1} \boldsymbol{B}_{v} \boldsymbol{v}\left[t_{k}-i\right],
$$

siendo

$$
\begin{aligned}
\widetilde{\boldsymbol{x}}\left[t_{k} \mid t_{k}\right] & =\boldsymbol{x}\left[t_{k}\right]-\hat{\boldsymbol{x}}\left[t_{k} \mid t_{k}\right], \\
\widetilde{\boldsymbol{x}}\left[t_{k} \mid t_{k}-1\right] & =\boldsymbol{x}\left[t_{k}\right]-\hat{\boldsymbol{x}}\left[t_{k} \mid t_{k}-1\right] .
\end{aligned}
$$

Definiendo la matriz de varianzas-covarianzas del error de estimación como

$$
\boldsymbol{P}[t \mid t]=\mathcal{E}\left\{\widetilde{\boldsymbol{x}}[t \mid t] \widetilde{\boldsymbol{x}}[t \mid t]^{\top}\right\}
$$


y aplicando la relación (4.145) se tiene que

$$
\begin{aligned}
\boldsymbol{P}\left[t_{k} \mid t_{k}\right]= & \left(\boldsymbol{I}-\boldsymbol{\ell}_{k} \boldsymbol{c} \boldsymbol{A}^{-d_{k}}\right) \mathcal{E}\left\{\widetilde{\boldsymbol{x}}\left[t_{k} \mid t_{k}-1\right] \widetilde{\boldsymbol{x}}\left[t_{k} \mid t_{k}-1\right]^{\top}\right\}\left(\boldsymbol{I}-\boldsymbol{\ell}_{k} \boldsymbol{c} \boldsymbol{A}^{-d_{k}}\right)^{\top} \\
& +\boldsymbol{\ell}_{k} \mathcal{E}\left\{w\left[t_{k}\right] w\left[t_{k}\right]^{\top}\right\} \boldsymbol{\ell}_{k}^{\top} \\
& +\sum_{i=1}^{d_{k}} \sum_{j=1}^{d_{k}}\left(\boldsymbol{\ell}_{k} \boldsymbol{c} \boldsymbol{A}^{i-d_{k}-1} \mathcal{E}\left\{\boldsymbol{v}\left[t_{k}-i\right] \boldsymbol{v}\left[t_{k}-j\right]^{\top}\right\}\left(\boldsymbol{\ell}_{k} \boldsymbol{c} \boldsymbol{A}^{j-d_{k}-1}\right)^{\boldsymbol{\top}}\right) \\
= & \boldsymbol{P}\left[t_{k} \mid t_{k}-1\right]-\boldsymbol{\ell}_{k} \boldsymbol{c} \boldsymbol{A}^{-d_{k}} \boldsymbol{P}\left[t_{k} \mid t_{k}-1\right]-\boldsymbol{P}\left[t_{k} \mid t_{k}-1\right]\left(\boldsymbol{\ell}_{k} \boldsymbol{c} \boldsymbol{A}^{-d_{k}}\right)^{\top} \\
& +\boldsymbol{\ell}_{k}\left(\boldsymbol{c} \boldsymbol{A}^{-d_{k}} \boldsymbol{P}\left[t_{k} \mid t_{k}-1\right]\left(\boldsymbol{c} \boldsymbol{A}^{-d_{k}}\right)^{\top}+\boldsymbol{W}+\overline{\boldsymbol{c}}_{d, k} \boldsymbol{V} \overline{\boldsymbol{c}}_{d, k}^{\top}\right) \boldsymbol{\ell}_{k}^{\top},
\end{aligned}
$$

donde se ha considerado que

$$
\mathcal{E}\left\{\boldsymbol{v}\left[t_{k}-i\right] \boldsymbol{v}\left[t_{k}-j\right]\right\}=\mathbf{0}, \quad i \neq j .
$$

Los elementos de la diagonal de $\boldsymbol{P}\left[t_{k} \mid t_{k}\right]$ contienen las varianzas del error de estimación del estado, con lo que la minimización de la varianza puede determinarse minimizando la traza de $\boldsymbol{P}\left[t_{k} \mid t_{k}\right]$. Para obtener la ganancia que minimiza la varianza se deriva la traza de $\boldsymbol{P}\left[t_{k} \mid t_{k}\right]$ con respecto a $\boldsymbol{\ell}_{k}$ y se iguala a cero, obteniéndose

$$
-2 \operatorname{tr}\left(\boldsymbol{c} \boldsymbol{A}^{-d_{k}} \boldsymbol{P}\left[t_{k} \mid t_{k}-1\right]\right)^{\top}+2 \operatorname{tr}\left(\boldsymbol{\ell}_{k}\left(\boldsymbol{c} \boldsymbol{A}^{-d_{k}} \boldsymbol{P}\left[t_{k} \mid t_{k}-1\right]\left(\boldsymbol{c} \boldsymbol{A}^{-d_{k}}\right)^{\top}+\boldsymbol{W}+\overline{\boldsymbol{c}}_{d, k} \boldsymbol{V} \overline{\boldsymbol{c}}_{d, k}^{\top}\right)\right)=0,
$$

de donde se puede despejar $\ell_{k}$ para obtener

$$
\boldsymbol{\ell}_{k}=\boldsymbol{P}\left[t_{k} \mid t_{k}-1\right]\left(\boldsymbol{c} \boldsymbol{A}^{-d_{k}}\right)^{\top}\left(\boldsymbol{c} \boldsymbol{A}^{-d_{k}} \boldsymbol{P}\left[t_{k} \mid t_{k}-1\right]\left(\boldsymbol{c} \boldsymbol{A}^{-d_{k}}\right)^{\top}+\boldsymbol{W}+\overline{\boldsymbol{c}}_{d, k} \boldsymbol{V} \overline{\boldsymbol{c}}_{d, k}^{\top}\right)^{-1} .
$$

La actualización óptima del vector de covarianzas se obtiene sustituyendo este vector $\ell_{k}$ en la expresión (4.146). La ecuación que proyecta la matriz de covarianzas al siguiente instante se obtiene a partir de la expresión que relaciona los errores $\widetilde{\boldsymbol{x}}\left[t_{k} \mid t_{k}-1\right]$ y $\widetilde{\boldsymbol{x}}\left[t_{k}-1 \mid t_{k}-1\right]$ :

$$
\begin{aligned}
\widetilde{\boldsymbol{x}}\left[t_{k} \mid t_{k}-1\right] & =\boldsymbol{x}\left[t_{k}\right]-\hat{\boldsymbol{x}}\left[t_{k} \mid t_{k}-1\right] \\
& =\boldsymbol{A} \boldsymbol{x}\left[t_{k}-1\right]+\boldsymbol{b} u[t-1]+\boldsymbol{B}_{v} \boldsymbol{v}\left[t_{k}-1\right]-\boldsymbol{A} \hat{\boldsymbol{x}}\left[t_{k}-1 \mid t_{k}-1\right]-\boldsymbol{b} u[t-1] \\
& =\boldsymbol{A} \widetilde{\boldsymbol{x}}\left[t_{k}-1 \mid t_{k}-1\right]+\boldsymbol{B}_{v} \boldsymbol{v}\left[t_{k}-1\right]
\end{aligned}
$$

De esta manera se tiene que

$$
\begin{aligned}
\boldsymbol{P}\left[t_{k} \mid t_{k}-1\right] & =\mathcal{E}\left\{\widetilde{\boldsymbol{x}}\left[t_{k} \mid t_{k}-1\right] \widetilde{\boldsymbol{x}}\left[t_{k} \mid t_{k}-1\right]^{\top}\right\} \\
& =\mathcal{E}\left\{\left(\boldsymbol{A} \widetilde{\boldsymbol{x}}\left[t_{k}-1 \mid t_{k}-1\right]+\boldsymbol{B}_{v} \boldsymbol{v}[t-1]\right)\left(\boldsymbol{A} \widetilde{\boldsymbol{x}}\left[t_{k}-1 \mid t_{k}-1\right]+\boldsymbol{B}_{v} \boldsymbol{v}\left[t_{k}-1\right]\right)^{\top}\right\} \\
& =\boldsymbol{A} \boldsymbol{P}\left[t_{k}-1 \mid t_{k}-1\right] \boldsymbol{A}^{\top}+\boldsymbol{B}_{v} \boldsymbol{V} \boldsymbol{B}_{v}^{\top} .
\end{aligned}
$$

\subsubsection{Sistemas multivariables}

Si se tiene un proceso multivariable con múltiples entradas, salidas y sensores, las ecuaciones del proceso vienen dadas por

$$
\begin{aligned}
\boldsymbol{x}[t+1] & =\boldsymbol{A} \boldsymbol{x}[t]+\boldsymbol{B} \boldsymbol{u}[t]+\boldsymbol{B}_{v} \boldsymbol{v}[t] \\
\boldsymbol{y}[t] & =\boldsymbol{C}_{y} \boldsymbol{x}[t] \\
m_{i, k} & =\boldsymbol{c}_{i} \boldsymbol{x}\left[t_{k}-d_{i, k}\right]+w_{i, k}, \quad i=1, \ldots, n_{m}
\end{aligned}
$$

donde se asume que en los instantes de medición $t=t_{k}$ se miden $\mu_{k}$ sensores. Defínase la matriz $\boldsymbol{M}_{k}$ de orden $\mu_{k} \times n_{m}$ formada con las filas de una matriz identidad que corresponden a la posición de los elementos de $\boldsymbol{m}\left[t_{k}\right]$ que se miden en el instante $t_{k}$.

Teorema 4.9.3 (Sensor virtual basado en el filtro de Kalman). Sea el algoritmo de predicción de salidas del proceso (4.147a) definido por las ecuación de propagación

$$
\hat{\boldsymbol{x}}[t \mid t-1]=\boldsymbol{A} \boldsymbol{x}[t-1]+\boldsymbol{B} \boldsymbol{u}[t-1],
$$


más la ecuación de actualización

$$
\hat{\boldsymbol{x}}\left[t_{k} \mid t_{k}\right]=\hat{\boldsymbol{x}}\left[t_{k} \mid t_{k}-1\right]+\sum_{i=1}^{n_{m}} \boldsymbol{\ell}_{i, k}\left(m_{i, k}-\boldsymbol{c}_{i} \hat{\boldsymbol{x}}\left[t_{k}-d_{i, k} \mid t_{k}-1\right]\right) \delta_{i, k}
$$

que se utilizan cuando se tiene alguna medición $m_{i, k}$ (en $t=t_{k} \delta_{i, k}=1$ si el sensor $i$ está disponible, sino $\left.\delta_{i, k}=0\right)$ y donde el vector $\hat{\boldsymbol{x}}\left[t_{k}-d_{i, k} \mid t_{k}-1\right]$ es la mejor estimación del estado del instante $t_{k}-d_{i, k}$ con la información disponible hasta en instante $t_{k}-1$, que cumple

$$
\hat{\boldsymbol{x}}\left[t_{k} \mid t_{k}-1\right]=\boldsymbol{A}^{d_{i, k}} \hat{\boldsymbol{x}}\left[t_{k}-d_{i, k} \mid t_{k}-1\right]+\sum_{i=1}^{d_{i, k}} \boldsymbol{A}^{i-1} \boldsymbol{B} \boldsymbol{u}\left[t_{k}-i\right] .
$$

La matriz de ganancias $\boldsymbol{L}_{k}=\left[\begin{array}{llll}\boldsymbol{\ell}_{1, k} & \boldsymbol{\ell}_{2, k} & \cdots & \ell_{n_{m}, k}\end{array}\right]$ que minimiza la varianza del error de predicción del algoritmo anterior se calcula mediante la ecuación de propagación

$$
\boldsymbol{P}[t \mid t-1]=\boldsymbol{A} \boldsymbol{P}[t-1 \mid t-1] \boldsymbol{A}^{\top}+\boldsymbol{B}_{v} \boldsymbol{V} \boldsymbol{B}_{v}^{\top},
$$

más la ecuación de actualización

$$
\begin{aligned}
\boldsymbol{L}_{k} & =\boldsymbol{P}[t \mid t-1]\left(\boldsymbol{M}_{k} \boldsymbol{C}_{d, k}\right)^{\boldsymbol{\top}}\left(\boldsymbol{M}_{k}\left(\boldsymbol{C}_{d, k} \boldsymbol{P}[t \mid t-1] \boldsymbol{C}_{d, k}^{\boldsymbol{\top}}+\boldsymbol{W}+\overline{\boldsymbol{C}}_{d, k} \boldsymbol{V} \overline{\boldsymbol{C}}_{d, k}^{\boldsymbol{\top}}\right) \boldsymbol{M}_{k}^{\top}\right)^{-1} \\
\boldsymbol{P}\left[t_{k} \mid t_{k}\right] & =\left(\boldsymbol{I}-\boldsymbol{L}_{k} \boldsymbol{M}_{k} \boldsymbol{C}_{d, k}\right) \boldsymbol{P}\left[t_{k} \mid t_{k}-1\right]
\end{aligned}
$$

siendo

$$
\begin{aligned}
& \boldsymbol{C}_{d, k}=\left[\begin{array}{c}
\boldsymbol{c}_{1} \boldsymbol{A}^{-d_{1, k}} \\
\vdots \\
\boldsymbol{c}_{n_{m}} \boldsymbol{A}^{-d_{n_{m}, k}}
\end{array}\right]_{n_{m} \times n}, \\
& \overline{\boldsymbol{C}}_{d, k}=\left[\begin{array}{ccc}
\boldsymbol{c}_{1} & \sum_{i=1}^{d_{1, k}} & \boldsymbol{A}^{-i} \boldsymbol{B}_{v} \\
\vdots & \\
\boldsymbol{c}_{n_{m}} & \sum_{i=1}^{d_{n_{m}, k}} & \boldsymbol{A}^{-i} \boldsymbol{B}_{v}
\end{array}\right] .
\end{aligned}
$$

Si no hay ninguna medición, la mejor estimación del estado y de la matriz de varianzas-covarianzas es la de bucle abierto, con lo que

$$
\begin{aligned}
\hat{\boldsymbol{x}}[t \mid t] & =\boldsymbol{x}[t \mid t-1], \\
\boldsymbol{P}[t \mid t] & =\boldsymbol{P}[t \mid t-1],
\end{aligned}
$$

La matriz de ganancias $\boldsymbol{L}_{k}$ tiene un tamaño $n \times m_{k}$ y $\boldsymbol{\ell}_{i, k}$ hace referencia a la $i$-ésima columna de la matriz $\boldsymbol{L}_{k} \boldsymbol{M}_{k}$, es decir la matriz formada por las columnas de $\boldsymbol{L}_{k}$ en las posiciones correspondientes a posiciones de sensores disponibles y por columnas nulas en las posiciones correspondientes a sensores no disponibles (véanse observaciones 2.3.1 y 4.5 .1 en las páginas 21 y 97, respectivamente).

Prueba 4.9.3. El estado del sistema en el instante $t_{k}$ se puede relacionar con el del instante $t_{k}-d_{i, k}$ corriendo $d_{i, k}$ veces la ecuación (4.147a), es decir

$$
\boldsymbol{x}\left[t_{k}\right]=\boldsymbol{A}^{d_{i, k}} \boldsymbol{x}\left[t_{k}-d_{i, k}\right]+\sum_{i=1}^{d_{i, k}} \boldsymbol{A}^{i-1} \boldsymbol{B} \boldsymbol{u}\left[t_{k}-i\right]+\sum_{i=1}^{d_{i, k}} \boldsymbol{A}^{i-1} \boldsymbol{B}_{v} \boldsymbol{v}\left[t_{k}-i\right],
$$

con lo que la medición $m_{i, k}$ se puede escribir en función del estado actual como

$$
m_{i, k}=\boldsymbol{c}_{i} \boldsymbol{A}^{-d_{i, k}}\left(\boldsymbol{x}\left[t_{k}\right]-\sum_{i=1}^{d_{i, k}} \boldsymbol{A}^{i-1}\left(\boldsymbol{B} \boldsymbol{u}\left[t_{k}-i\right]+\boldsymbol{B}_{v} \boldsymbol{v}\left[t_{k}-i\right]\right)\right)+w_{i, k} .
$$

Sustituyendo esta expresión en la ecuación de actualización (4.149) y restando $x[t]$ en ambos lados de la expresión resultante se llega a

$$
\widetilde{\boldsymbol{x}}\left[t_{k} \mid t_{k}\right]=\left(\boldsymbol{I}-\boldsymbol{L}_{k} \boldsymbol{M}_{k} \boldsymbol{C}_{d, k}\right) \widetilde{\boldsymbol{x}}\left[t_{k} \mid t_{k}-1\right]-\boldsymbol{L}_{k} \boldsymbol{M}_{k} \boldsymbol{w}_{k}+\boldsymbol{L}_{k} \boldsymbol{M}_{k} \overline{\boldsymbol{C}}_{d, k} \mathbb{V}_{k},
$$


siendo

$$
\begin{aligned}
\widetilde{\boldsymbol{x}}\left[t_{k} \mid t_{k}\right] & =\boldsymbol{x}\left[t_{k}\right]-\hat{\boldsymbol{x}}\left[t_{k} \mid t_{k}\right], \\
\widetilde{\boldsymbol{x}}\left[t_{k} \mid t_{k}-1\right] & =\boldsymbol{x}\left[t_{k}\right]-\hat{\boldsymbol{x}}\left[t_{k} \mid t_{k}-1\right] .
\end{aligned}
$$

Definiendo la matriz de varianzas-covarianzas del error de estimación como

$$
\boldsymbol{P}[t \mid t]=\mathcal{E}\left\{\widetilde{\boldsymbol{x}}[t \mid t] \widetilde{\boldsymbol{x}}[t \mid t]^{\top}\right\}
$$

y aplicando la relación (4.155) en los instantes de medición $\left(t=t_{k}\right)$ se tiene que

$$
\begin{aligned}
\boldsymbol{P}\left[t_{k} \mid t_{k}\right]= & \left(\boldsymbol{I}-\boldsymbol{L}_{k} \boldsymbol{M}_{k} \boldsymbol{C}_{d, k}\right) \mathcal{E}\left\{\widetilde{\boldsymbol{x}}\left[t_{k} \mid t_{k}-1\right] \widetilde{\boldsymbol{x}}\left[t_{k} \mid t_{k}-1\right]^{\top}\right\}\left(\boldsymbol{I}-\boldsymbol{L}_{k} \boldsymbol{M}_{k} \boldsymbol{C}_{d, k}\right)^{\top} \\
& +\boldsymbol{L}_{k} \boldsymbol{M}_{k} \mathcal{E}\left\{\boldsymbol{w}_{k} \boldsymbol{w}_{k}^{\top}\right\} \boldsymbol{M}_{k}^{\top} \boldsymbol{L}_{k}^{\top} \\
& +\sum_{i=1}^{n_{m}} \sum_{j=1}^{n_{m}} \sum_{\alpha_{k}=1}^{d_{i, k}} \sum_{\beta=1}^{d_{j, k}}\left(\boldsymbol{\ell}_{i, k} \boldsymbol{c}_{i} \boldsymbol{A}^{\alpha_{k}-d_{i, k}-1} \delta_{i, k} \mathcal{E}\left\{\boldsymbol{v}\left[t_{k}-\alpha_{k}\right] \boldsymbol{v}\left[t_{k}-\beta\right]^{\top}\right\}\left(\boldsymbol{\ell}_{j, k} \boldsymbol{c}_{j} \boldsymbol{A}^{\beta-d_{j, k}-1} \delta_{j, k}\right)^{\top}\right) \\
= & \boldsymbol{P}\left[t_{k} \mid t_{k}-1\right]-\boldsymbol{L}_{k} \boldsymbol{M}_{k} \boldsymbol{C}_{d, k} \boldsymbol{P}\left[t_{k} \mid t_{k}-1\right]-\boldsymbol{P}\left[t_{k} \mid t_{k}-1\right]\left(\boldsymbol{L}_{k} \boldsymbol{M}_{k} \boldsymbol{C}_{d, k}\right)^{\top} \\
& +\boldsymbol{L}_{k} \boldsymbol{M}_{k}\left(\boldsymbol{C}_{d, k} \boldsymbol{P}\left[t_{k} \mid t_{k}-1\right]\left(\boldsymbol{C}_{d, k}\right)^{\top}+\boldsymbol{W}+\overline{\boldsymbol{C}}_{d, k} \boldsymbol{V} \overline{\boldsymbol{C}}_{d, k}^{\top}\right) \boldsymbol{M}_{k}^{\top} \boldsymbol{L}_{k}^{\top},
\end{aligned}
$$

donde se ha considerado que

$$
\mathcal{E}\left\{\boldsymbol{v}\left[t_{k}-\alpha_{k}\right] \boldsymbol{v}\left[t_{k}-\beta\right]\right\}=\mathbf{0}, \quad \alpha_{k} \neq \beta .
$$

Los elementos de la diagonal de $\boldsymbol{P}\left[t_{k} \mid t_{k}\right]$ contienen las varianzas del error de estimación del estado (actualizado con la medición), con lo que la minimización de la varianza puede determinarse minimizando la traza de $\boldsymbol{P}\left[t_{k} \mid t_{k}\right]$. Para obtener la ganancia que minimiza la varianza se deriva la traza de $\boldsymbol{P}\left[t_{k} \mid t_{k}\right]$ con respecto a $\boldsymbol{L}_{k}$ y se iguala a cero, obteniéndose

$$
-2 \operatorname{tr}\left(\boldsymbol{M}_{k} \boldsymbol{C}_{d, k} \boldsymbol{P}[t \mid t-1]\right)^{\boldsymbol{\top}}+2 \operatorname{tr}\left(\boldsymbol{L}_{k} \boldsymbol{M}_{k}\left(\boldsymbol{C}_{d, k} \boldsymbol{P}\left[t_{k} \mid t_{k}-1\right] \boldsymbol{C}_{d, k}^{\boldsymbol{\top}}+\boldsymbol{W}+\overline{\boldsymbol{C}}_{d, k} \boldsymbol{V} \overline{\boldsymbol{C}}_{d, k}^{\boldsymbol{\top}}\right) \boldsymbol{M}_{k}\right)=0
$$

de donde se puede despejar $\boldsymbol{L}_{k}$ para obtener

$$
\boldsymbol{L}_{k}=\boldsymbol{P}\left[t_{k} \mid t_{k}-1\right] \boldsymbol{C}_{d, k}^{\boldsymbol{\top}} \boldsymbol{M}_{k}^{\boldsymbol{\top}}\left(\boldsymbol{M}_{k}\left(\boldsymbol{C}_{d, k} \boldsymbol{P}\left[t_{k} \mid t_{k}-1\right] \boldsymbol{C}_{d, k}^{\boldsymbol{\top}}+\boldsymbol{W}+\overline{\boldsymbol{C}}_{d, k} \boldsymbol{V} \overline{\boldsymbol{C}}_{d, k}^{\boldsymbol{\top}}\right) \boldsymbol{M}_{k}\right)^{-1} .
$$

La actualización óptima del vector de covarianzas se obtiene sustituyendo esta matriz $\boldsymbol{L}_{k}$ en la expresión (4.156). La ecuación que proyecta la matriz de covarianzas al siguiente instante (en bucle abierto) se obtiene a partir de la expresión que relaciona los errores $\widetilde{\boldsymbol{x}}[t \mid t-1]$ y $\widetilde{\boldsymbol{x}}[t-1 \mid t-1]$ :

$$
\begin{aligned}
\widetilde{\boldsymbol{x}}[t \mid t-1] & =\boldsymbol{x}[t]-\hat{\boldsymbol{x}}[t \mid t-1] \\
& =\boldsymbol{A} \boldsymbol{x}[t-1]+\boldsymbol{B} \boldsymbol{u}[t-1]+\boldsymbol{B}_{v} \boldsymbol{v}[t-1]-\boldsymbol{A} \hat{\boldsymbol{x}}[t-1 \mid t-1]-\boldsymbol{B} \boldsymbol{u}[t-1] \\
& =\boldsymbol{A} \widetilde{\boldsymbol{x}}[t-1 \mid t-1]+\boldsymbol{B}_{v} \boldsymbol{v}[t-1] .
\end{aligned}
$$

De esta manera se tiene que

$$
\begin{aligned}
\boldsymbol{P}[t \mid t-1] & =\mathcal{E}\left\{\widetilde{\boldsymbol{x}}[t \mid t-1] \widetilde{\boldsymbol{x}}[t \mid t-1]^{\top}\right\} \\
& =\mathcal{E}\left\{(\boldsymbol{A} \widetilde{\boldsymbol{x}}[t-1 \mid t-1]+\boldsymbol{w}[t-1])(\boldsymbol{A} \widetilde{\boldsymbol{x}}[t-1 \mid t-1]+\boldsymbol{w}[t-1])^{\top}\right\} \\
& =\boldsymbol{A P}[t-1 \mid t-1] \boldsymbol{A}^{\boldsymbol{\top}}+\boldsymbol{B}_{v} \boldsymbol{V} \boldsymbol{B}_{v}^{\top} .
\end{aligned}
$$

Observación 4.9.1. Los algoritmos expuestos en esta sección utilizan las estimaciones del estado con retardo en la actualización del estado estimado de la misma forma que lo hace el predictor (4.12) utilizado en este capítulo. Por este motivo, también se puede aplicar la implementación (4.14) para evitar la utilización de la ecuación (4.138c) consiguiendo una reducción del coste computacional. 


\subsubsection{Predicción óptima}

La forma óptima de incorporar mediciones con retardo en el filtro de Kalman consiste en correr el modelo hacia atrás hasta el instante al cual corresponde la medición, actualizar siguiendo las ecuaciones del filtro de Kalman estándar, y luego volver a ejecutar hacia adelante el modelo para obtener la estimación del estado actual. Esta operación debe realizarse para todas las mediciones. A continuación se detalla este procedimiento.

Si no hay mediciones, el modelo se propaga en bucle abierto, dando lugar a

$$
\begin{aligned}
\hat{\boldsymbol{x}}[t \mid t-1] & =\boldsymbol{A} \boldsymbol{x}[t-1]+\boldsymbol{B} \boldsymbol{u}[t-1], \\
\boldsymbol{P}[t \mid t-1] & =\boldsymbol{A} \boldsymbol{P}[t-1 \mid t-1] \boldsymbol{A}^{\top}+\boldsymbol{B}_{v} \boldsymbol{V} \boldsymbol{B}_{v}^{\top},
\end{aligned}
$$

Cuando en $t=t_{k}$ hay una medición

$$
m_{i, k}=\boldsymbol{c}_{i} \boldsymbol{x}\left[t_{k}-d_{i, k}\right]
$$

de la salida $m_{i}$ con retardo $d_{i, k}$, se corre el modelo hacia atrás hasta obtener la estimación del estado para el instante retardado $t_{k}-d_{i, k}$ mediante las ecuaciones

$$
\begin{aligned}
& \hat{\boldsymbol{x}}\left[t_{k}-d_{i, k} \mid t_{k}-1\right]=\boldsymbol{A}^{-d_{i, k}} \hat{\boldsymbol{x}}\left[t_{k} \mid t_{k}-1\right]-\boldsymbol{A}^{-d_{i, k}} \sum_{j=0}^{d_{i, k}-1} \boldsymbol{A}^{j} \boldsymbol{B} \boldsymbol{u}[t-j-1], \\
& \boldsymbol{P}\left[t_{k}-d_{i, k} \mid t_{k}-1\right]=\boldsymbol{A}^{-d_{i, k}} \boldsymbol{P}\left[t_{k} \mid t_{k}-1\right]\left(\boldsymbol{A}^{-d_{i, k}}\right)^{\boldsymbol{\top}}-\sum_{j=1}^{d_{i, k}} \boldsymbol{A}^{-j} \boldsymbol{B}_{v} \boldsymbol{V} \boldsymbol{B}_{v}^{\boldsymbol{\top}}\left(\boldsymbol{A}^{-j}\right)^{\boldsymbol{\top}} .
\end{aligned}
$$

Con este valor se ejecutan las ecuaciones de actualización

$$
\begin{aligned}
\boldsymbol{\ell}_{i, k} & =\boldsymbol{P}[t \mid t-1] \boldsymbol{c}_{i}^{\top}\left(\boldsymbol{c}_{i} \boldsymbol{P}[t \mid t-1] \boldsymbol{c}_{i}^{\top}+\boldsymbol{W}\right)^{-1} \\
\hat{\boldsymbol{x}}\left[t_{k}-d_{i, k} \mid t_{k}\right] & =\boldsymbol{\ell}_{i, k}\left(m_{i, k}-\boldsymbol{c}_{i} \hat{\boldsymbol{x}}\left[t_{k}-d_{i, k} \mid t_{k}-1\right]\right. \\
\boldsymbol{P}\left[t_{k}-d_{i, k} \mid t_{k}\right] & =\left(\boldsymbol{I}-\boldsymbol{\ell}_{i, k} \boldsymbol{c}_{i}\right) \boldsymbol{P}\left[t_{k}-d_{i, k} \mid t_{k}-1\right]
\end{aligned}
$$

y se vuelve a obtener la estimación del estado y de la matriz de covarianzas correspondientes al estado actual $t=t_{k}$ mediante las ecuaciones

$$
\begin{gathered}
\hat{\boldsymbol{x}}\left[t_{k} \mid t_{k}-1\right]=\boldsymbol{A}^{d_{i, k}} \hat{\boldsymbol{x}}\left[t_{k} \mid t_{k}-1\right]+\sum_{j=0}^{d_{i, k}-1} \boldsymbol{A}^{j} \boldsymbol{B} \boldsymbol{u}[t-j-1], \\
\boldsymbol{P}\left[t_{k}-d_{i, k} \mid t_{k}-1\right]=\boldsymbol{A}^{d_{i, k}} \boldsymbol{P}\left[t_{k} \mid t_{k}-1\right]\left(\boldsymbol{A}^{d_{i, k}}\right)^{\top}+\sum_{j=1}^{d_{i, k}} \boldsymbol{A}^{j} \boldsymbol{B}_{v} \boldsymbol{V} \boldsymbol{B}_{v}^{\top}\left(\boldsymbol{A}^{j}\right)^{\top} .
\end{gathered}
$$

Para cada medición $m_{i, k}$ disponible en el instante $t=t_{k}$ se deben ejecutar las ecuaciones (4.159) a (4.165)

El inconveniente de esta última técnica es su elevado coste computacional ya que, dependiendo de la cantidad de sensores disponibles en cada muestreo y de los retardos asociados, puede ser necesario ejecutar un elevado número de inversión de matrices.

\subsection{Conclusiones}

En este capítulo se han estudiado distintos métodos de diseño de predictores de salida aplicables al caso general de multiples entradas, salidas y sensores donde las mediciones de cada sensor llegan al sistema de control en instantes arbitrarios y con retardos aleatorios.

La principal aportación es un procedimiento para diseñar las ganancias de un algoritmo de bajo coste computacional, que garantiza la estabilidad y la atenuación de perturbaciones a pesar de la disponibilidad de datos no periódica, la disponibilidad parcial de los sensores y los retardos variantes que acompañan las mediciones.

El predictor propuesto constituye un sensor virtual que utiliza un modelo interno del sistema, las entradas previas y las mediciones escasas para predecir las salidas en cada periodo de control. El número de periodos de control entre mediciones $\left(N_{k}\right)$, los retardos de cada sensor $\left(d_{i, k}\right)$ y las posibles combinaciones de sensores disponibles $\left(\boldsymbol{\Delta}_{k}\right)$ se asume que son variantes en el tiempo y que pertenecen a conjuntos finitos que se conocen a priori. 
Para analizar la convergencia se ha utilizado una función de Lyapunov del error de estimación del estado en los instantes de medición. El problema de estabilidad de Lyapunov se ha transformado en un problema de factibilidad de un conjunto de desigualdades lineales matriciales. La solución del problema de LMIs (si es factible) se ha utilizado para calcular las matrices de ganancia del sensor virtual.

Para la atenuación de las perturbaciones y los ruidos de medida se ha tenido en cuenta mediante diferentes normas del subsistema predictor (normas $\mathcal{H}_{\infty}, \mathcal{H}_{2}, \mathcal{H}_{2 g}$ y $\ell_{1}$ ), transformando la condición en un problema de factibilidad de un sistema de LMIs. Se han propuesto procedimientos de diseño basados en la información disponible de las perturbaciones. El resultado es un conjunto finito de matrices de ganancia que se aplican dependiendo de las características de la medición (combinación $N_{k}, d_{i, k}$ y $\boldsymbol{\Delta}_{k}$ ). El procedimiento permite definir el tamaño de dicho conjunto (y por tanto, los recursos que necesita la implementación del algoritmo final), desde el caso más simple (ganancia constante), hasta el más complejo (una matriz diferente para cada posible combinación $\left(N_{k}, d_{i, k}, \boldsymbol{\Delta}_{k}\right)$ ). Si no se tiene información sobre las perturbaciones, el procedimiento sigue siendo útil, porque se tienen algunos factores de ajuste que pueden utilizarse para cambiar la dinámica del predictor sin afectar a la estabilidad.

Además de las perturbaciones y el ruido de modelado, también se ha estudiado el efecto de otros factores que aumentan el error de predicción. Se ha demostrado que la incertidumbre en el modelo sólo afecta al valor del error de predicción, pero no a su estabilidad, y se ha demostrado que las perturbaciones consideradas en el diseño, son válidas para minimizar el efecto de este error de modelado, con lo que las técnicas de atenuación de perturbaciones también son útiles para abordar el diseño de predictores robustos. Se ha analizado cómo tratar el retardo en la acción de control y se ha demostrado que los retardos variantes en la acción de control se pueden modelar como una incertidumbre en el modelo. Se ha demostrado también que el ruido de medida también es válido para modelar el error introducido por las mediciones que vienen acompañadas de una etiqueta de tiempo incierta o por las mediciones asíncronas.

Finalmente se ha abordado el problema de predicción de salidas mediante un algoritmo similar al filtro de Kalman, que trata de minimizar la varianza del error de predicción con cada medición. La diferencia respecto del filtro de Kalman estándar reside en la forma de incorporar las mediciones con retardo, y la forma de implementar el algoritmo para evitar la utilización de un modelo de orden extendido. 



\section{Predicción de salidas en sistemas no lineales}

\subsection{Introducción}

En este capítulo se introduce la síntesis de sensores virtuales para plantas no lineales. Se asume una planta formada por múltiples entradas y salidas afectada de perturbaciones, y que se dispone de varios sensores que miden algunas de las señales (estados o salidas) afectadas de un ruido de medida. Se asume que las mediciones son escasas y que no hay retardos en el sistema de transmisión de datos.

La estructura del capítulo es como sigue. En la segunda sección se describe el problema describiendo la planta no lineal y el tipo de muestreo. En la tercera sección se introduce el predictor propuesto y se obtiene la ecuación del error de predicción. En la cuarta sección se plantea el diseño de un estimador de salidas para muestreo convencional, estudiando tanto la estabilidad nominal como la atenuación de perturbaciones. En la quinta sección se extiende el diseño anterior al caso de muestreo bifrecuencia, mientras que en la sexta sección se presentan los resultados para el caso de muestreo irregular. Finalmente, en la séptima sección se exponen las conclusiones del capítulo.

\subsection{Descripción del problema}

\subsubsection{Planta}

Sea un proceso discreto no lineal e invariante en el tiempo que viene descrito por las ecuaciones

$$
\begin{aligned}
\boldsymbol{x}[t+1] & =\boldsymbol{A} \boldsymbol{x}[t]+\boldsymbol{f}(\boldsymbol{x}[t], u[t])+\boldsymbol{B}_{v} \boldsymbol{v}[t] \\
y[t] & =\boldsymbol{g}(\boldsymbol{x}[t])
\end{aligned}
$$

donde $\boldsymbol{x} \in \mathbb{R}^{n}$ es el estado, $\boldsymbol{u} \in \mathbb{R}^{n_{u}}$ es el vector de entradas y $\boldsymbol{y} \in \mathbb{R}^{n_{y}}$ es el vector de salidas que se desean controlar. El vector $\boldsymbol{v}(\tau)$ es la posibles perturbación del estado. $\boldsymbol{f}(\cdot): \mathbb{R}^{n+n_{u}} \rightarrow \mathbb{R}^{n}$ y $\boldsymbol{g}(\cdot): \mathbb{R}^{n} \rightarrow \mathbb{R}^{n_{y}}$ son funciones no lineales conocidas que cumplen con la condición de Lipschitz, es decir,

$$
\begin{aligned}
\left\|\boldsymbol{f}\left(\boldsymbol{x}_{1}, \boldsymbol{u}\right)-\boldsymbol{f}\left(\boldsymbol{x}_{2}, \boldsymbol{u}\right)\right\|_{p} & \leq\left\|\boldsymbol{F} \cdot\left(\boldsymbol{x}_{1}-\boldsymbol{x}_{2}\right)\right\|_{p} \\
\left\|\boldsymbol{g}\left(\boldsymbol{x}_{1}\right)-\boldsymbol{g}\left(\boldsymbol{x}_{2}\right)\right\|_{p} & \leq\left\|\boldsymbol{G} \cdot\left(\boldsymbol{x}_{1}-\boldsymbol{x}_{2}\right)\right\|_{p}
\end{aligned}
$$

para alguna norma vectorial $p$.

La señal de los sensores que se envía al controlador sólo está accesible en los instantes $t=t_{k}$ en los que hay al menos un sensor disponible cuya salida viene dada por

$$
m_{i, k}=\boldsymbol{c}_{i} \boldsymbol{x}_{k}+h_{i}\left(\boldsymbol{x}_{k}\right)+w_{i, k}, \quad i=1, \ldots, n_{m}
$$

siendo $n_{m}$ el número de sensore, $m_{i, k}$ la medición del sensor $i$ en el $k$-ésimo muestreo, y $w_{i, k}$ el ruidos asociado a dicho sensor en dicha muestra. $h_{i}(\cdot): \mathbb{R}^{n} \rightarrow \mathbb{R}$ es una función no lineal conocida que cumple con la condición de Lipschitz, es decir,

$$
\left\|h_{i}\left(\boldsymbol{x}_{1}\right)-h_{i}\left(\boldsymbol{x}_{2}\right)\right\|_{p} \leq\left\|H_{i} \cdot\left(\boldsymbol{x}_{1}-\boldsymbol{x}_{2}\right)\right\|_{p}
$$


para alguna norma vectorial $p$. Nótese que se ha utilizado la notación $\boldsymbol{x}_{k}$ para denotar el estado en el instante de muestreo, es decir $\boldsymbol{x}_{k}=\boldsymbol{x}\left[t_{k}\right]$. A partir de estas señales se pueden definir los vectores

$$
\boldsymbol{m}_{k}=\left[\begin{array}{c}
m_{1, k} \\
\vdots \\
m_{n_{m}, k}
\end{array}\right], \quad \boldsymbol{h}\left(\boldsymbol{x}_{k}\right)=\left[\begin{array}{c}
h_{1}\left(\boldsymbol{x}_{k}\right) \\
\vdots \\
h_{n_{m}}\left(\boldsymbol{x}_{k}\right)
\end{array}\right], \quad \boldsymbol{w}_{k}=\left[\begin{array}{c}
w_{1, k} \\
\vdots \\
w_{n_{m}, k}
\end{array}\right],
$$

y expresar el vector de mediciones como

$$
\boldsymbol{m}_{k}=\boldsymbol{C} \boldsymbol{x}_{k}+\boldsymbol{h}\left(\boldsymbol{x}_{k}\right)+\boldsymbol{w}_{k},
$$

que permite simplificar la notación para el resto de desarrollos.

Observación 5.2.1. La representación anterior también es válida cuando el sensor tiene una dinámica propia no despreciable y está libre de perturbaciones de salida. Para demostrarlo, tómense las ecuaciones en diferencias del proceso

$$
\begin{aligned}
\boldsymbol{x}_{p}[t+1] & =\boldsymbol{A}_{p} \boldsymbol{x}_{p}[t]+\boldsymbol{f}_{p}\left(\boldsymbol{x}_{p}[t], \boldsymbol{u}[t]\right)+\boldsymbol{B}_{v_{p}} \boldsymbol{v}_{p}[t] \\
\boldsymbol{y}[t] & =\boldsymbol{g}_{p}\left(\boldsymbol{x}_{p}[t]\right)
\end{aligned}
$$

y la ecuación asociada al sensor

$$
\begin{aligned}
\boldsymbol{x}_{s}[t+1] & =\boldsymbol{A}_{s} \boldsymbol{x}_{s}[t]+\boldsymbol{f}_{s}\left(\boldsymbol{x}_{s}[t], \boldsymbol{x}_{p}[t], \boldsymbol{y}[t]\right)+\boldsymbol{B}_{v_{s}} \boldsymbol{v}_{s}[t] \\
\boldsymbol{m}_{k} & =\boldsymbol{C}_{s} \boldsymbol{x}_{s, k}+\boldsymbol{h}_{s}\left(\boldsymbol{x}_{s, k}\right)+\boldsymbol{h}_{p}\left(\boldsymbol{x}_{p, k}, \boldsymbol{y}_{k}\right)+\boldsymbol{w}_{k} .
\end{aligned}
$$

Obsérvese que la entrada al sensor es tanto el estado como la salida del proceso para indicar que el sensor puede medir cualquier variable que sea combinación de los estados y la salida. Agrupando ambas ecuaciones se puede escribir que

$$
\begin{aligned}
{\left[\begin{array}{l}
\boldsymbol{x}_{p}[t+1] \\
\boldsymbol{x}_{s}[t+1]
\end{array}\right] } & =\underbrace{\left[\begin{array}{cc}
\boldsymbol{A}_{p} & \mathbf{0} \\
\mathbf{0} & \boldsymbol{A}_{s}
\end{array}\right]}_{=\boldsymbol{A}}\left[\begin{array}{l}
\left.\boldsymbol{x}_{p}[t]\right] \\
\boldsymbol{x}_{s}[t]
\end{array}\right]+\underbrace{\left[\begin{array}{c}
\boldsymbol{f}_{p}\left(\boldsymbol{x}_{p}[t], \boldsymbol{u}[t]\right) \\
\boldsymbol{f}_{s}\left(\boldsymbol{x}_{s}[t], \boldsymbol{x}_{p}[t], \boldsymbol{g}_{p}\left(\boldsymbol{x}_{p}[t]\right)\right)
\end{array}\right]}_{=\boldsymbol{f}(\boldsymbol{x}[t], \boldsymbol{u}[t])}+\underbrace{\left[\begin{array}{l}
\left.\boldsymbol{v}_{p}[t]\right] \\
\boldsymbol{v}_{s}[t]
\end{array}\right]}_{=\boldsymbol{v}} \\
y[t] & =\boldsymbol{g}_{p}\left(\boldsymbol{x}_{p}[t]\right)=\boldsymbol{g}(\boldsymbol{x}[t]) \\
m_{k} & =\underbrace{\left[\begin{array}{ll}
\mathbf{0} & \boldsymbol{C}_{s}
\end{array}\right]}_{\boldsymbol{C}}\left[\begin{array}{l}
\left.\boldsymbol{x}_{p}[t]\right] \\
\boldsymbol{x}_{s}[t]
\end{array}\right]+\underbrace{\boldsymbol{h}_{s}\left(\boldsymbol{x}_{s, k}\right)+\boldsymbol{h}_{p}\left(\boldsymbol{x}_{p, k}, \boldsymbol{g}_{p}\left(\boldsymbol{x}_{p, k}\right)\right)}_{=\boldsymbol{h}\left(\boldsymbol{x}_{k}\right)}+\boldsymbol{w}_{k}=\boldsymbol{C} \boldsymbol{x}_{k}+\boldsymbol{h}\left(\boldsymbol{x}_{k}\right)+\boldsymbol{w}_{k}
\end{aligned}
$$

Tomando $\boldsymbol{x}[t]=\left[\boldsymbol{x}_{p}[t]^{\top} \boldsymbol{x}_{s}[t]^{\top}\right]^{\top}$ se llega a la representación (5.1).

\subsubsection{Escenario de muestreo}

El sistema de control necesita los valores de $y[t T]$, pero se asume que sólo están disponibles las mediciones $\boldsymbol{m}_{k}$ en los instantes discretos $t_{k}, k \in \mathbb{N}$, siendo $N_{k}=t_{k}-t_{k-1}$ el número de actualizaciones de la entrada desde $t_{k-1}$ hasta $t_{k}$. Con esto,

$$
t_{k}=\sum_{i=1}^{k} N_{i}
$$

representa el instante en el cual se da la $t$-ésima actualización del control y se obtiene la $k$-ésima medición. Se asume que el número de actualizaciones de la entrada entre mediciones $\left(N_{k}\right)$ varía dentro de un conjunto finito de valores

$$
N_{k} \in \mathcal{N}=\left\{\nu_{1}, \ldots, \nu_{\mathcal{N}}\right\}
$$

Para denotar qué sensores están disponibles en cada instante de muestreo se define el factor de disponibilidad del sensor $i$ en el instante $t_{k}$ como

$$
\delta_{i}\left[t_{k}\right]= \begin{cases}1, & \text { si } m_{i}\left[t_{k}\right] \text { está disponible, } \\ 0, & \text { si } m_{i}\left[t_{k}\right] \text { no está disponible }\end{cases}
$$

Agrupando los valores del factor de disponibilidad para cada sensor se puede definir la matriz de disponibilidad como

$$
\boldsymbol{\Delta}_{k} \equiv \boldsymbol{\Delta}\left[t_{k}\right]=\left[\begin{array}{ccc}
\delta_{1}\left[t_{k}\right] & & 0 \\
& \ddots & \\
0 & & \delta_{n_{m}}\left[t_{k}\right]
\end{array}\right] .
$$


Dependiendo del patrón de muestreo, pueden haber diferentes valores para la matriz $\boldsymbol{\Delta}\left[t_{k}\right]^{1}$. Se asume que estos valores están dentro de un conjunto finito dado por

$$
\boldsymbol{\Delta}\left[t_{k}\right] \in \Xi=\left\{\boldsymbol{\Delta}_{1}, \ldots, \boldsymbol{\Delta}_{p}\right\} .
$$

En el caso general, es posible cualquier combinación de mediciones disponibles, con lo que $p=2^{n_{m}}-1$.

\subsubsection{Parametrización del escenario de muestreo}

Al igual que con la representación externa e interna se debe definir un parámetro $s_{k}$ que recoja las características temporales que definen a cada una de las muestras escasas de que se dispone (véase sección 3.1.2 en la página 25). Cada instante de medición viene definido por dos parámetros: la matriz de disponibilidad, que indica qué sensores han aportado información, y el número de veces que se ha actualizado la acción de control entre instantes de medición $\left(N_{k}\right)$. Cada uno de estos parámetros puede tomar valores dentro de unos rangos conocidos (dados por $\Xi$ y $\mathcal{N}$ ). Sin embargo, según la aplicación, sólo algunas combinaciones $\left(\boldsymbol{\Delta}_{k}, N_{k}\right)$ se darán de forma simultánea. Por este motivo se define un nuevo parámetro $s_{k}$ que indica cuál es la situación del muestreo (combinación $\left(\boldsymbol{\Delta}_{k}, N_{k}\right)$ ) de la $k$-ésima medida. El parámetro $s_{k}$ enumera las combinaciones tomando valores enteros, es decir

$$
s_{k} \in \mathcal{S}=\left\{1,2, \ldots, n_{\mathcal{S}}\right\},
$$

donde $n_{\mathcal{S}}$ el número de combinaciones posibles $\left(\boldsymbol{\Delta}_{k}, N_{k}\right)$. El número máximo de combinaciones es $m \cdot p$ siendo $m$ el tamaño del conjunto $\mathcal{N}$ y $p$ el tamaño del conjunto $\Xi$.

Con el nuevo parámetro definido se puede establecer una dependencia de los parámetros $N_{k}$ y $\boldsymbol{\Delta}_{k}$ con respecto de $s_{k}$ de forma que

$$
\begin{aligned}
N_{k} & =N\left(s_{k}\right), \\
\boldsymbol{\Delta}_{k} & =\boldsymbol{\Delta}\left(s_{k}\right) .
\end{aligned}
$$

\subsection{Predictor}

Para estimar los estados y las salidas a periodo $T$ se propone un predictor que tiene una estructura similar al expuesto para sistemas lineales. Inicialmente se estima el estado corriendo el modelo en bucle abierto, lo que lleva a

$$
\hat{\boldsymbol{x}}[t \mid t-1]=\boldsymbol{A} \hat{\boldsymbol{x}}[t-1]+\boldsymbol{f}(\hat{\boldsymbol{x}}[t-1], u[t-1]) .
$$

Si no hay ninguna medición en el instante $t$, la mejor estimación es la de bucle abierto

$$
\hat{\boldsymbol{x}}[t]=\hat{\boldsymbol{x}}[t \mid t-1] .
$$

Cuando se tiene una medición en $t=t_{k}$, el estado se actualiza mediante

$$
\hat{\boldsymbol{x}}\left[t_{k}\right]=\hat{\boldsymbol{x}}\left[t_{k} \mid t_{k}-1\right]+\sum_{i=1}^{n_{m}} \boldsymbol{\ell}_{i}\left[t_{k}\right]\left(m_{i}\left[t_{k}\right]-\boldsymbol{c}_{i} \hat{\boldsymbol{x}}\left[t_{k} \mid t_{k}-1\right]-h_{i}\left(\hat{\boldsymbol{x}}\left[t_{k} \mid t_{k}-1\right]\right)\right) \delta_{i}\left[t_{k}\right] .
$$

donde $\boldsymbol{\ell}_{i}\left[t_{k}\right]$ es el vector de ganancias utilizado para actualizar la estimación del estado con la medición $m_{i}\left[t_{k}\right]$. Introduciendo la matriz de disponibilidad (5.11), la ecuación de actualización (5.14b) se reescribe como

$$
\hat{\boldsymbol{x}}\left[t_{k}\right]=\hat{\boldsymbol{x}}\left[t_{k} \mid t_{k}-1\right]+\boldsymbol{L}_{k} \boldsymbol{\Delta}_{k}\left(\boldsymbol{m}_{k}-\boldsymbol{C} \hat{\boldsymbol{x}}\left[t_{k} \mid t_{k}-1\right]-\boldsymbol{h}\left(\hat{\boldsymbol{x}}\left[t_{k} \mid t_{k}-1\right]\right)\right) .
$$

La predicción de la salida se obtiene mediante la ecuación de salidas

$$
\hat{\boldsymbol{y}}[t]=\boldsymbol{g}(\hat{\boldsymbol{x}}[t]) .
$$

La dinámica del sensor virtual no lineal depende de la matriz de ganancias

$$
\boldsymbol{L}_{k} \equiv \boldsymbol{L}\left[t_{k}\right]=\left[\begin{array}{llll}
\boldsymbol{\ell}_{1}\left[t_{k}\right] & \boldsymbol{\ell}_{2}\left[t_{k}\right] & \cdots & \boldsymbol{\ell}_{n_{m}}\left[t_{k}\right]
\end{array}\right]=\left[\begin{array}{cccc}
l_{11}\left[t_{k}\right] & l_{21}\left[t_{k}\right] & \cdots & l_{n_{m} 1}\left[t_{k}\right] \\
l_{12}\left[t_{k}\right] & l_{22}\left[t_{k}\right] & \cdots & l_{n_{m} 2}\left[t_{k}\right] \\
\vdots & \vdots & & \vdots \\
l_{1 n}\left[t_{k}\right] & l_{2 n}\left[t_{k}\right] & \cdots & l_{n_{m} n}\left[t_{k}\right]
\end{array}\right]
$$

\footnotetext{
${ }^{1} \mathrm{Si}$ en un instante dado $t=t_{k}$ todas las mediciones de salida están disponibles, entonces $\boldsymbol{\Delta}\left[t_{k}\right]=\boldsymbol{I}$.
} 
definida en los instantes de medición $\left(t=t_{k}\right)$, que se debe diseñar para asegurar la estabilidad del predictor, la robustez frente a la disponibilidad esporádica de datos, y una atenuación adecuada de las perturbaciones y ruidos de los sensores. La ganancia del predictor se asume en general que es variante en el tiempo, pero el caso particular de una ganancia constante también se analiza.

\subsubsection{Error de predicción}

Con el objetivo de diseñar un predictor debería obtenerse una relación explícita entre el error de predicción en un instante de medición $t_{k}\left(\widetilde{\boldsymbol{x}}_{k}=\widetilde{\boldsymbol{x}}\left[t_{k}\right]\right)$ y el anterior $t_{k-1}\left(\widetilde{\boldsymbol{x}}_{k-1}=\widetilde{\boldsymbol{x}}\left[t_{k-1}\right]=\widetilde{\boldsymbol{x}}\left[t_{k}-N_{k}\right]\right)$. Sin embargo, en el caso de sistemas no lineales, no es posible establecer esta realación. Para demostrarlo, considérese un instante de medición $t_{k}$ en el que se miden todas las salidas (es decir, todo el vector $\boldsymbol{m}_{k}$ está disponible) con lo que se puede escribir

$$
\begin{aligned}
\hat{\boldsymbol{x}}\left[t_{k} \mid t_{k}-1\right] & =\boldsymbol{A} \hat{\boldsymbol{x}}\left[t_{k}-1\right]+\boldsymbol{f}\left(\hat{\boldsymbol{x}}\left[t_{k}-1\right], \boldsymbol{u}\left[t_{k}-1\right]\right) \\
\hat{\boldsymbol{x}}\left[t_{k}\right] & =\hat{\boldsymbol{x}}\left[t_{k} \mid t_{k}-1\right]+\boldsymbol{L}_{k} \boldsymbol{\Delta}_{k}\left(\boldsymbol{m}_{k}-\boldsymbol{C \boldsymbol { x }}\left[t_{k} \mid t_{k}-1\right]-\boldsymbol{h}\left(\hat{\boldsymbol{x}}\left[t_{k} \mid t_{k}-1\right]\right)\right) .
\end{aligned}
$$

Si se introducen las ecuaciones del proceso

$$
\begin{aligned}
\boldsymbol{x}\left[t_{k}\right] & =\boldsymbol{A} \boldsymbol{x}\left[t_{k}-1\right]+\boldsymbol{f}\left(\boldsymbol{x}\left[t_{k}-1\right], \boldsymbol{u}\left[t_{k}-1\right]\right)+\boldsymbol{B}_{v} \boldsymbol{v}\left[t_{k}-1\right] \\
\boldsymbol{m}_{k} & =\boldsymbol{C} \boldsymbol{x}\left[t_{k}\right]+\boldsymbol{h}\left(\boldsymbol{x}\left[t_{k}\right]\right)+\boldsymbol{w}_{k} .
\end{aligned}
$$

se puede expresar el error de estimación como

$$
\begin{aligned}
\widetilde{\boldsymbol{x}}\left[t_{k}\right]= & \boldsymbol{A} \widetilde{\boldsymbol{x}}\left[t_{k}-1\right]+\boldsymbol{f}\left(\boldsymbol{x}\left[t_{k}-1\right], \boldsymbol{u}\left[t_{k}-1\right]\right)-\boldsymbol{f}\left(\hat{\boldsymbol{x}}\left[t_{k}-1\right], \boldsymbol{u}\left[t_{k}-1\right]\right) \\
& -\boldsymbol{L}_{k} \boldsymbol{\Delta}_{k}\left(\boldsymbol{C} \boldsymbol{x}\left[t_{k}\right]+\boldsymbol{h}\left(\boldsymbol{x}\left[t_{k}\right]\right)-\boldsymbol{C} \hat{\boldsymbol{x}}\left[t_{k} \mid t_{k}-1\right]-\boldsymbol{h}\left(\hat{\boldsymbol{x}}\left[t_{k} \mid t_{k}-1\right]\right)\right) \\
& +\boldsymbol{B}_{v} \boldsymbol{v}\left[t_{k}-1\right]-\boldsymbol{L}_{k} \boldsymbol{\Delta}_{k} \boldsymbol{w}_{k}
\end{aligned}
$$

siendo $\widetilde{\boldsymbol{x}}\left[t_{k}\right]=\boldsymbol{x}\left[t_{k}\right]-\hat{\boldsymbol{x}}\left[t_{k}\right]$. Como se observa, no es posible escribir $\widetilde{\boldsymbol{x}}\left[t_{k}\right]$ en función de $\widetilde{\boldsymbol{x}}\left[t_{k}-1\right] \mathrm{y}$, por lo tanto, tampoco en función del anterior instante de medición, es decir, en función de $\widetilde{\boldsymbol{x}}\left[t_{k-1}\right]$. Con el objetivo de simplificar los desarrollos posteriores del capítulo, se introduce la siguiente notación

$$
\begin{aligned}
\widetilde{\boldsymbol{x}}[t \mid t-1] & =\boldsymbol{x}[t]-\hat{\boldsymbol{x}}[t \mid t-1], \\
\widetilde{\boldsymbol{f}}[t] & =\boldsymbol{f}(\boldsymbol{x}[t], \boldsymbol{u}[t])-\boldsymbol{f}(\hat{\boldsymbol{x}}[t], \boldsymbol{u}[t]), \\
\widetilde{\boldsymbol{h}}\left[t_{k} \mid t_{k}-1\right] & =\boldsymbol{h}\left(\boldsymbol{x}\left[t_{k}\right]\right)-\boldsymbol{h}\left(\hat{\boldsymbol{x}}\left[t_{k} \mid t_{k}-1\right]\right) .
\end{aligned}
$$

Nótese que $\widetilde{\boldsymbol{h}}$ sólo se ha definido en los instantes de medición $t_{k}$ porque la función $\boldsymbol{h}$ sólo aparece en la ecuación de actualización del predictor. La dinámica del error de predicción en el instante de medición queda simplificada a

$$
\widetilde{\boldsymbol{x}}\left[t_{k}\right]=\widetilde{\boldsymbol{x}}\left[t_{k} \mid t_{k}-1\right]-\boldsymbol{L}_{k} \boldsymbol{\Delta}_{k}\left(\boldsymbol{C} \widetilde{\boldsymbol{x}}\left[t_{k} \mid t_{k}-1\right]+\widetilde{\boldsymbol{h}}\left[t_{k} \mid t_{k}-1\right]+\boldsymbol{w}_{k}\right),
$$

donde el error de estimación en bucle abierto $(\widetilde{\boldsymbol{x}}[t \mid t-1])$ se puede poner en función de la información del instante anterior como

y las funciones $\widetilde{\boldsymbol{f}}[t]$ y $\widetilde{\boldsymbol{h}}\left[t_{k} \mid t_{k}-1\right]$ cumplen

$$
\widetilde{\boldsymbol{x}}[t \mid t-1]=\boldsymbol{A} \widetilde{\boldsymbol{x}}[t-1]+\widetilde{\boldsymbol{f}}[t-1]+\boldsymbol{B}_{v} \boldsymbol{v}[t-1],
$$

$$
\begin{aligned}
\|\widetilde{\boldsymbol{f}}[t]\| & \leq\|\boldsymbol{F} \widetilde{\boldsymbol{x}}[t]\| \\
\left\|\widetilde{\boldsymbol{h}}\left[t_{k} \mid t_{k}-1\right]\right\| & \leq\left\|\boldsymbol{H} \widetilde{\boldsymbol{x}}\left[t_{k} \mid t_{k}-1\right]\right\|
\end{aligned}
$$

\subsubsection{Objetivo del diseño}

De forma similar a los diseños de los capítulos anteriores, en el caso de procesos no lineales se aborda el diseño de la ganancia $\boldsymbol{L}_{k}$ definiendo una matriz diferente para cada conjunto $\left(\boldsymbol{\Delta}_{k}, N_{k}\right)$, es decir, para cada posible valor del parámetro de muestreo $s_{k}$. El cálculo de estas matrices $\boldsymbol{L}_{k}$ se hace una sola vez y da como resultado un conjunto finito de ganancias en función de los diferentes valores de $s_{k}$ :

$$
\boldsymbol{L}_{k}=\boldsymbol{L}\left(s_{k}\right) \in \mathcal{L}=\left\{\boldsymbol{L}(1), \boldsymbol{L}(2), \ldots, \boldsymbol{L}\left(n_{\mathcal{S}}\right)\right\} .
$$

El predictor que se propone se implementa con un conjunto de $n_{\mathcal{S}}$ matrices constantes. Cada vez que llega un conjunto de mediciones de sensores, el predictor utiliza un único vector de ganancias que se escoge en función de las características de la información recibida (sensores disponibles y $N_{k}$ ).

Para el desarrollo de los procedimientos de cálculo de ganancias es necesario utilizar las herramientas matemáticas cuya obtención se detalla en el anexo A.6. 


\subsection{Diseño de predictores para muestreo convencional}

\subsubsection{Diseño para estabilidad nominal}

Si se dispone de todas las salidas en cada periodo de control (es decir, $N_{k}=1$ y $\boldsymbol{\Delta}_{k}=\boldsymbol{I}$ ) se puede utilizar una matriz de ganancias constante que asegure la estabilidad en todo el rango de funcionamiento.

Teorema 5.4.1. Considérese el sistema (5.1) sin perturbaciones ni ruidos de medida, y supóngase que se tiene información de todos los sensores en cada periodo de control $\left(N_{k}=1\right.$ y $\left.\boldsymbol{\Delta}_{k}=\boldsymbol{I}\right)$. Si existen dos matrices $\boldsymbol{P}=\boldsymbol{P}^{\boldsymbol{\top}} \in \mathbb{R}^{n \times n}$ y $\boldsymbol{X} \in \mathbb{R}^{n \times 1}$ y unos escalares $\boldsymbol{\varepsilon}_{f}, \boldsymbol{\varepsilon}_{h}>0$ tales que la siguiente desigualdad se cumple

$$
\left[\begin{array}{cccc}
\boldsymbol{P} & \boldsymbol{P} \boldsymbol{A}-\boldsymbol{X} \boldsymbol{C} \boldsymbol{A} & \boldsymbol{P}-\boldsymbol{X} \boldsymbol{C} & \boldsymbol{X} \\
\star & \left(\begin{array}{c}
\boldsymbol{P}-\varepsilon_{f} \boldsymbol{F}^{\top} \boldsymbol{F} \\
-\varepsilon_{h} \boldsymbol{A}^{\top} \boldsymbol{H}^{\top} \boldsymbol{H} \boldsymbol{A}
\end{array}\right) & -\varepsilon_{h} \boldsymbol{A}^{\top} \boldsymbol{H}^{\top} \boldsymbol{H} & \mathbf{0} \\
\star & \star & \varepsilon_{f} \boldsymbol{I}-\varepsilon_{h} \boldsymbol{H}^{\top} \boldsymbol{H} & \mathbf{0} \\
\star & \star & \star & \varepsilon_{h} \boldsymbol{I}
\end{array}\right] \succ \mathbf{0} .
$$

Entonces, si la ganancia del predictor se define como

$$
\boldsymbol{L}=\boldsymbol{P}^{-1} \boldsymbol{X},
$$

el error de predicción del algoritmo definido por (5.14) converge a cero de forma asintótica.

Prueba 5.4.1. Tómese la función de Lyapunov

$$
\mathcal{V}[t]=\widetilde{\boldsymbol{x}}[t]^{\top} \boldsymbol{P} \widetilde{\boldsymbol{x}}[t] .
$$

Entonces, el sistema (5.1a) será estable si $\mathcal{V}[t+1]-\mathcal{V}[t]<0$. El incremento de la función de Lyapunov se puede escribir como

$\Delta \mathcal{V}[t]=((\boldsymbol{I}-\boldsymbol{L} \boldsymbol{C}) \widetilde{\boldsymbol{x}}[t \mid t-1]-\boldsymbol{L} \widetilde{\boldsymbol{h}}[t \mid t-1])^{\top} \boldsymbol{P}((\boldsymbol{I}-\boldsymbol{L} \boldsymbol{C}) \widetilde{\boldsymbol{x}}[t \mid t-1]-\boldsymbol{L} \widetilde{\boldsymbol{h}}[t \mid t-1])-\widetilde{\boldsymbol{x}}[t-1]^{\top} \boldsymbol{P} \widetilde{\boldsymbol{x}}[t-1]$

Aplicando el teorema A.6.1 al primer sumando se tiene que

$$
\Delta \mathcal{V}[t] \leq \widetilde{\boldsymbol{x}}[t \mid t-1]^{\top} \boldsymbol{P}_{h} \widetilde{\boldsymbol{x}}[t \mid t-1]-\widetilde{\boldsymbol{x}}[t-1]^{\top} \boldsymbol{P} \widetilde{\boldsymbol{x}}[t-1]
$$

con

$$
\boldsymbol{P}_{h}=(\boldsymbol{I}-\boldsymbol{L} \boldsymbol{C})^{\top}\left(\boldsymbol{P}+\boldsymbol{P} \boldsymbol{L}\left(\varepsilon_{h} \boldsymbol{I}-\boldsymbol{L}^{\top} \boldsymbol{P} \boldsymbol{L}\right)^{-1} \boldsymbol{L}^{\top} \boldsymbol{P}\right)(\boldsymbol{I}-\boldsymbol{L} \boldsymbol{C})+\varepsilon_{h} \boldsymbol{H}^{\top} \boldsymbol{H} .
$$

Introduciendo el valor del error de estimación en bucle abierto (5.21) se tiene que

$$
\Delta \mathcal{V}[t] \leq(\boldsymbol{A} \widetilde{\boldsymbol{x}}[t-1]+\widetilde{\boldsymbol{f}}[t-1])^{\top} \boldsymbol{P}_{h}(\boldsymbol{A} \widetilde{\boldsymbol{x}}[t-1]+\widetilde{\boldsymbol{f}}[t-1])-\widetilde{\boldsymbol{x}}[t-1]^{\top} \boldsymbol{P} \widetilde{\boldsymbol{x}}[t-1]
$$

donde se puede volver a aplicar el teorema A.6.1 al primer sumando, llegándose a

$$
\Delta \mathcal{V}[t] \leq \widetilde{\boldsymbol{x}}[t-1]^{\top} \boldsymbol{P}_{f} \widetilde{\boldsymbol{x}}[t-1]
$$

con

$$
\boldsymbol{P}_{f}=\left(\boldsymbol{A}^{\top}\left(\boldsymbol{P}_{h}+\boldsymbol{P}_{h}\left(\varepsilon_{f} \boldsymbol{I}-P_{h}\right)^{-1} \boldsymbol{P}_{h}\right) \boldsymbol{A}+\varepsilon_{f} \boldsymbol{F}^{\boldsymbol{\top}} \boldsymbol{F}-\boldsymbol{P}\right)
$$

La función de Lyapunov decrecerá si $\boldsymbol{P}_{f} \prec \mathbf{0}$. Aplicando complementos de Schur, esta última condición se puede escribir como

$$
\left[\begin{array}{cc}
\boldsymbol{A}^{\top} \boldsymbol{P}_{h} \boldsymbol{A}-\boldsymbol{P}+\varepsilon_{f} \boldsymbol{F}^{\top} \boldsymbol{F} & \boldsymbol{A}^{\top} \boldsymbol{P}_{h} \\
\boldsymbol{P}_{h} \boldsymbol{A} & \boldsymbol{P}_{h}-\varepsilon_{f} \boldsymbol{I}
\end{array}\right] \prec \mathbf{0}
$$

Introduciendo la matriz $\boldsymbol{P}_{h}$ y aplicando de nuevo los complementos de Schur se tiene la condición

$$
\begin{aligned}
& {\left[\begin{array}{c}
\boldsymbol{A}^{\top}(\boldsymbol{I}-\boldsymbol{L} \boldsymbol{C})^{\top} \\
(\boldsymbol{I}-\boldsymbol{L} \boldsymbol{C})^{\top} \\
\boldsymbol{L}^{\top}
\end{array}\right] \boldsymbol{P}\left[\begin{array}{lll}
(\boldsymbol{I}-\boldsymbol{L} \boldsymbol{C}) \boldsymbol{A} & (\boldsymbol{I}-\boldsymbol{L} \boldsymbol{C}) & \boldsymbol{L}
\end{array}\right]+} \\
& \varepsilon_{h}\left[\begin{array}{c}
\boldsymbol{A}^{\top} \\
\boldsymbol{I} \\
\mathbf{0}
\end{array}\right] \boldsymbol{H}^{\top} \boldsymbol{H}\left[\begin{array}{lll}
\boldsymbol{A} & \boldsymbol{I} & \mathbf{0}
\end{array}\right]+\left[\begin{array}{ccc}
\varepsilon_{f} \boldsymbol{F}^{\top} \boldsymbol{F}-\boldsymbol{P} & \mathbf{0} & \mathbf{0} \\
\mathbf{0} & -\varepsilon_{f} \boldsymbol{I} & \mathbf{0} \\
\mathbf{0} & \mathbf{0} & -\varepsilon_{h}
\end{array}\right] \prec \mathbf{0 .}
\end{aligned}
$$

Aplicando de nuevo los complementos de Schur e introduciendo la matriz $\boldsymbol{X}=\boldsymbol{P} \boldsymbol{L}$ se llega finalmente a (5.25). 


\subsubsection{Diseño basado en la atenuación de perturbaciones}

Teorema 5.4.2. Considérese el predictor (5.14) aplicado al sistema (5.1) y supóngase que todos los sensores proporcionan una medida en cada instante de control $\left(N_{k}=1, \boldsymbol{\Delta}_{k}=\boldsymbol{I}\right)$. Para unas $\gamma_{v_{1}}, \ldots, \gamma_{v_{n}}$, $\gamma_{w_{1}}, \ldots, \gamma_{w_{n_{m}}}$ dadas, supóngase que existen unas matrices $\boldsymbol{P}=\boldsymbol{P}^{\top} \in \mathbb{R}^{n \times n}, \boldsymbol{X} \in \mathbb{R}^{n \times 1}$ y unos escalares $\varepsilon_{f}, \varepsilon_{h}>0$ tales que

$$
\left[\begin{array}{cccccc}
\boldsymbol{P} & \boldsymbol{P} \boldsymbol{A}-\boldsymbol{X} \boldsymbol{C} \boldsymbol{A} & \boldsymbol{P}-\boldsymbol{X} \boldsymbol{C} & \boldsymbol{P} \boldsymbol{B}_{v}-\boldsymbol{X} \boldsymbol{C} \boldsymbol{B}_{v} & \boldsymbol{X} & \boldsymbol{X} \\
& \boldsymbol{P}-\varepsilon_{f} \boldsymbol{F}^{\top} \boldsymbol{F} \\
-\varepsilon_{h} \boldsymbol{A}^{\top} \boldsymbol{H}^{\top} \boldsymbol{H} \boldsymbol{A} & -\varepsilon_{h} \boldsymbol{A}^{\top} \boldsymbol{H}^{\top} \boldsymbol{H} & -\varepsilon_{h} \boldsymbol{A}^{\top} \boldsymbol{H}^{\top} \boldsymbol{H} & \mathbf{0} & \mathbf{0} \\
& +\boldsymbol{G}^{\top} \boldsymbol{G} & & & & \\
\star & \star & \varepsilon_{f} \boldsymbol{I}-\varepsilon_{h} \boldsymbol{H}^{\top} \boldsymbol{H} & -\varepsilon_{h} \boldsymbol{H}^{\top} \boldsymbol{H} & \mathbf{0} & \mathbf{0} \\
\star & \star & \star & \Gamma_{v}^{2}-\varepsilon_{h} \boldsymbol{H}^{\top} \boldsymbol{H} & \mathbf{0} & \mathbf{0} \\
\star & \star & \star & \star & \varepsilon_{h} \boldsymbol{I} & \mathbf{0} \\
\star & \star & \star & \star & \star & \boldsymbol{\Gamma}_{w}^{2}
\end{array}\right] \succ \mathbf{0},
$$

con

$$
\begin{gathered}
\boldsymbol{\Gamma}_{v}=\operatorname{diag}\left\{\gamma_{v_{1}}, \ldots, \gamma_{v_{n}}\right\} \\
\boldsymbol{\Gamma}_{w}=\operatorname{diag}\left\{\gamma_{w_{1}}, \ldots, \gamma_{w_{n_{m}}}\right\} .
\end{gathered}
$$

Entonces, definiendo la ganancia del predictor como $\boldsymbol{L}=\boldsymbol{P}^{-1} \boldsymbol{X}$, el error de predicción converge a cero de forma asintótica en ausencia de perturbaciones $y$, bajo condiciones iniciales nulas,

$$
\|\boldsymbol{e}[t]\|_{2}^{2} \leq\left\|\boldsymbol{\Gamma}_{v} \boldsymbol{v}[t]\right\|_{2}^{2}+\left\|\boldsymbol{\Gamma}_{w} \boldsymbol{w}[t]\right\|_{2}^{2}
$$

Prueba 5.4.2. Considérese el índice

$$
J=\sum_{t=0}^{\infty}\left(\boldsymbol{e}[t]^{\top} \boldsymbol{e}[t]-\boldsymbol{v}[t]^{\top} \boldsymbol{\Gamma}_{v}^{2} \boldsymbol{v}[t]-\boldsymbol{w}[t]^{\top} \boldsymbol{\Gamma}_{w}^{2} \boldsymbol{w}[t]\right) .
$$

Tomando la función de Lyapunov $\mathcal{V}[t]=\widetilde{\boldsymbol{x}}[t] \boldsymbol{P} \widetilde{\boldsymbol{x}}[t]$ y asumiendo condiciones iniciales nulas se tiene que

$$
\begin{aligned}
J & \leq \sum_{t=1}^{\infty}\left(\boldsymbol{e}[t-1]^{\top} \boldsymbol{e}[t-1]-\boldsymbol{v}[t-1]^{\top} \boldsymbol{\Gamma}_{v}^{2} \boldsymbol{v}[t-1]-\boldsymbol{w}[t]^{\top} \boldsymbol{\Gamma}_{w}^{2} \boldsymbol{w}[t]\right)+\left.\mathcal{V}[t]\right|_{t=\infty}-\left.\mathcal{V}[t]\right|_{t=0} \\
& =\sum_{t=0}^{\infty}\left(\boldsymbol{e}[t-1]^{\top} \boldsymbol{e}[t-1]-\boldsymbol{v}[t-1]^{\top} \boldsymbol{\Gamma}_{v}^{2} \boldsymbol{v}[t-1]-\boldsymbol{w}[t]^{\top} \boldsymbol{\Gamma}_{w}^{2} \boldsymbol{w}[t]+\Delta \mathcal{V}[t]\right)
\end{aligned}
$$

Sustituyendo $\Delta \mathcal{V}[t]$ por

$$
\begin{aligned}
\Delta \mathcal{V}[t] & =\widetilde{\boldsymbol{x}}[t]^{\top} \boldsymbol{P} \widetilde{\boldsymbol{x}}[t]-\widetilde{\boldsymbol{x}}[t-1]^{\top} \boldsymbol{P} \widetilde{\boldsymbol{x}}[t-1] \\
& =\underbrace{((\boldsymbol{I}-\boldsymbol{L} \boldsymbol{C}) \widetilde{\boldsymbol{x}}[t \mid t-1]-\boldsymbol{L} \widetilde{\boldsymbol{h}}[t \mid t-1]-\boldsymbol{L} \boldsymbol{w}[t])^{\top} \boldsymbol{P}(\star)-\widetilde{\boldsymbol{x}}[t-1]^{\top} \boldsymbol{P} \widetilde{\boldsymbol{x}}[t-1]}_{\star}
\end{aligned}
$$

y aplicando el teorema A.6.3 para eliminar el término $\boldsymbol{w}[t]^{\top} \boldsymbol{\Gamma}_{w}^{2} \boldsymbol{w}[t]$ se obtiene

$$
\begin{aligned}
J \leq & \sum_{t=1}^{\infty}\left(\boldsymbol{e}[t-1]^{\top} \boldsymbol{e}[t-1]-\boldsymbol{v}[t-1]^{\top} \boldsymbol{\Gamma}_{v}^{2} \boldsymbol{v}[t-1]-\widetilde{\boldsymbol{x}}[t-1]^{\top} \boldsymbol{P} \widetilde{\boldsymbol{x}}[t-1]\right. \\
& +\underbrace{\left.((\boldsymbol{I}-\boldsymbol{L} \boldsymbol{C}) \widetilde{\boldsymbol{x}}[t \mid t-1]-\boldsymbol{L} \widetilde{\boldsymbol{h}}[t \mid t-1])^{\top} \boldsymbol{P}_{w}(\star)\right) .}_{\star}
\end{aligned}
$$

con

$$
\boldsymbol{P}_{w}=\boldsymbol{P}+\boldsymbol{P} \boldsymbol{L}\left(\boldsymbol{\Gamma}_{w}^{2}-\boldsymbol{L}^{\top} \boldsymbol{P} \boldsymbol{L}\right)^{-1} \boldsymbol{L}^{\top} \boldsymbol{P}
$$

Aplicando ahora el teorema A.6.1 para eliminar el término $\widetilde{\boldsymbol{h}}[t \mid t-1]$ se tiene que

$$
J \leq \sum_{t=1}^{\infty}\left(\boldsymbol{e}[t-1]^{\top} \boldsymbol{e}[t-1]-\boldsymbol{v}[t-1]^{\top} \boldsymbol{\Gamma}_{v}^{2} \boldsymbol{v}[t-1]-\widetilde{\boldsymbol{x}}[t-1]^{\top} \boldsymbol{P} \widetilde{\boldsymbol{x}}[t-1]+(\widetilde{\boldsymbol{x}}[t \mid t-1])^{\top} \boldsymbol{P}_{h}(\widetilde{\boldsymbol{x}}[t \mid t-1])\right) .
$$


con

$$
\boldsymbol{P}_{h}=(\boldsymbol{I}-\boldsymbol{L} \boldsymbol{C})^{\top}\left(\boldsymbol{P}_{w}+\boldsymbol{P}_{w} \boldsymbol{L}\left(\varepsilon_{h} \boldsymbol{I}-\boldsymbol{L}^{\top} \boldsymbol{P}_{w} \boldsymbol{L}\right)^{-1} \boldsymbol{L}^{\top} \boldsymbol{P}_{w}\right)(\boldsymbol{I}-\boldsymbol{L} \boldsymbol{C})+\varepsilon_{h} \boldsymbol{H}^{\top} \boldsymbol{H} .
$$

Sustituyendo el error de estimación en bucle abierto por $\widetilde{\boldsymbol{x}}[t \mid t-1]=\boldsymbol{A} \widetilde{\boldsymbol{x}}[t-1]+\widetilde{\boldsymbol{f}}[t-1]+\boldsymbol{B}_{v} \boldsymbol{v}[t-1]$ y aplicando el teorema A.6.3 para eliminar el término $\boldsymbol{v}[t-1]^{\top} \boldsymbol{\Gamma}_{v}^{2} \boldsymbol{v}[t-1]$ se tiene que

$$
J \leq \sum_{t=1}^{\infty}\left(\boldsymbol{e}[t-1]^{\top} \boldsymbol{e}[t-1]+(\boldsymbol{A} \widetilde{\boldsymbol{x}}[t-1]+\widetilde{\boldsymbol{f}}[t-1])^{\top} \boldsymbol{P}_{v}(\boldsymbol{A} \widetilde{\boldsymbol{x}}[t-1]+\widetilde{\boldsymbol{f}}[t-1])-\widetilde{\boldsymbol{x}}[t-1]^{\top} \boldsymbol{P} \widetilde{\boldsymbol{x}}[t-1]\right),
$$

con

$$
\boldsymbol{P}_{v}=\boldsymbol{P}_{h}+\boldsymbol{P}_{h} \boldsymbol{B}_{v}\left(\boldsymbol{\Gamma}_{v}^{2}-\boldsymbol{B}_{v}^{\top} \boldsymbol{P}_{h} \boldsymbol{B}_{v}\right)^{-1} \boldsymbol{B}_{v}^{\top} \boldsymbol{P}_{h}
$$

Aplicando ahora el teorema A.6.1 se tiene que

$$
J \leq \sum_{t=1}^{\infty}\left(\boldsymbol{e}[t-1]^{\top} \boldsymbol{e}[t-1]+\widetilde{\boldsymbol{x}}[t-1]^{\top}\left(\boldsymbol{P}_{f}-\boldsymbol{P}\right) \widetilde{\boldsymbol{x}}[t-1]\right),
$$

con

$$
\boldsymbol{P}_{f}=\boldsymbol{A}^{\boldsymbol{\top}}\left(\boldsymbol{P}_{v}+\boldsymbol{P}_{v}\left(\varepsilon_{f} \boldsymbol{I}-\boldsymbol{P}_{v}\right)^{-1} \boldsymbol{P}_{v}\right) \boldsymbol{A}+\varepsilon_{f} \boldsymbol{F}^{\boldsymbol{\top}} \boldsymbol{F} .
$$

Por otra parte, como $\boldsymbol{e}[t-1]=\boldsymbol{g}(\boldsymbol{x}[t-1])-\boldsymbol{g}(\hat{\boldsymbol{x}}[t-1]) \mathrm{y}$

$$
\boldsymbol{g}(\boldsymbol{x}[t-1])-\boldsymbol{g}(\hat{\boldsymbol{x}}[t-1]) \leq\|\boldsymbol{G} \widetilde{\boldsymbol{x}}[t-1]\|
$$

se puede escribir que

$$
J \leq \sum_{t=1}^{\infty} \widetilde{\boldsymbol{x}}[t-1]^{\top}\left(\boldsymbol{P}_{f}+\boldsymbol{G}^{\top} \boldsymbol{G}-\boldsymbol{P}\right) \widetilde{\boldsymbol{x}}[t-1] .
$$

La condición (5.28) se cumplirá si $J<0$, condición que será cierta siempre que

$$
\boldsymbol{P}_{f}+\boldsymbol{G}^{\top} \boldsymbol{G}-\boldsymbol{P} \prec \mathbf{0} .
$$

Sustituyendo $\boldsymbol{P}_{f}$ en función de $\boldsymbol{P}_{v}$ y aplicando complementos de Schur se tiene que la condición anterior es equivalente a la condición

$$
\left[\begin{array}{cc}
\boldsymbol{A}^{\top} \boldsymbol{P}_{v} \boldsymbol{A}-\boldsymbol{P}+\boldsymbol{G}^{\top} \boldsymbol{G}+\varepsilon_{f} \boldsymbol{F}^{\top} \boldsymbol{F} & \boldsymbol{A}^{\top} \boldsymbol{P}_{v} \\
\boldsymbol{P}_{v} \boldsymbol{A} & \boldsymbol{P}_{v}-\varepsilon_{f} \boldsymbol{I}
\end{array}\right] \prec \mathbf{0 .}
$$

sustituyendo $\boldsymbol{P}_{v}$ en función de $\boldsymbol{P}_{h}$ y aplicando complementos de Schur se llega a

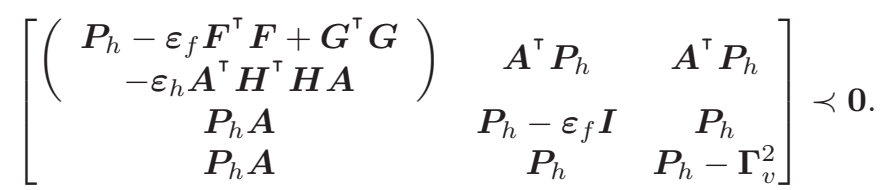

Sustituyendo $\boldsymbol{P}_{h}$ en función de $\boldsymbol{P}_{w}$ y aplicando complementos de Schur se llega a

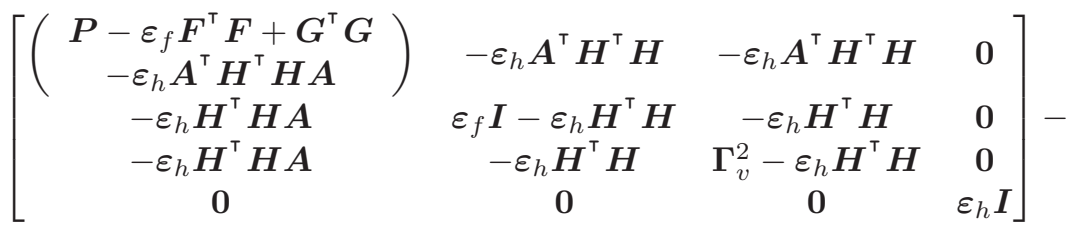

$$
\begin{aligned}
& -\left[\begin{array}{c}
\boldsymbol{A}^{\top}(\boldsymbol{I}-\boldsymbol{L} \boldsymbol{C})^{\top} \\
(\boldsymbol{I}-\boldsymbol{L} \boldsymbol{C})^{\top} \\
\boldsymbol{B}_{v}^{\top}(\boldsymbol{I}-\boldsymbol{L} \boldsymbol{C})^{\top} \\
\boldsymbol{L}^{\top}
\end{array}\right] \boldsymbol{P}_{w}\left[\begin{array}{c}
\boldsymbol{A}^{\top}(\boldsymbol{I}-\boldsymbol{L} \boldsymbol{C})^{\top} \\
(\boldsymbol{I}-\boldsymbol{L} \boldsymbol{C})^{\top} \\
\boldsymbol{B}_{v}^{\top}(\boldsymbol{I}-\boldsymbol{L} \boldsymbol{C})^{\top} \\
\boldsymbol{L}^{\top}
\end{array}\right]^{\top} \succ \mathbf{0} .
\end{aligned}
$$

Sustituyendo finalmente $\boldsymbol{P}_{w}$ en función de $\boldsymbol{P}$, aplicando dos veces consecutivas los complementos de Schur y teniendo en cuenta que $\boldsymbol{P L}=\boldsymbol{X}$ se llega finalmente a (5.27) 
Observación 5.4.1 (Procedimiento de diseño). De forma similar a como se ha expuesto para sistemas lineales en la sección \$4.5.3, si se consideran conocidas las normas $\ell_{2}$ de las perturbaciones, se puede minimizar la cota superior de $\left\|\boldsymbol{e}_{k}\right\|_{2}$ minimizando la suma

$$
\sum_{i=1}^{n_{v}} \gamma_{v_{i}}^{2}\left\|v_{i}[t]\right\|_{2}^{2}+\sum_{i=1}^{n_{m}} \gamma_{w_{i}}^{2}\left\|w_{i}[t]\right\|_{2}^{2}
$$

a lo largo de todas las variables $\gamma_{v_{i}}, \gamma_{w_{i}}, \boldsymbol{P}$ y $\boldsymbol{X}$ que satisfacen la LMI (5.27).

De forma similar, si se consideran conocidas las normas $R M S$ de las perturbaciones, se puede minimizar la cota superior de $\left\|\boldsymbol{e}_{k}\right\|_{R M S}$ minimizando la suma

$$
\sum_{i=1}^{n_{v}} \gamma_{v_{i}}^{2}\left\|v_{i}[t]\right\|_{R M S}^{2}+\sum_{i=1}^{n_{m}} \gamma_{w_{i}}^{2}\left\|w_{i}[t]\right\|_{R M S}^{2}
$$

a lo largo de todas las variables $\gamma_{v_{i}}, \gamma_{w_{i}}, \boldsymbol{P}$ y $\boldsymbol{X}$ que satisfacen la LMI (5.27).

Si sólo se conoce el valor máximo de las perturbaciones, también se puede minimizar la cota superior de $\left\|e_{k}\right\|_{R M S}$ minimizando la suma

$$
\sum_{i=1}^{n_{v}} \gamma_{v_{i}}^{2}\left\|v_{i}[t]\right\|_{\infty}^{2}+\sum_{i=1}^{n_{m}} \gamma_{w_{i}}^{2}\left\|w_{i}[t]\right\|_{\infty}^{2}
$$

a lo largo de todas las variables $\gamma_{v_{i}}, \gamma_{w_{i}}, \boldsymbol{P}$ y $\boldsymbol{X}$ que satisfacen la LMI (5.27).

Para realizar esta minimización se puede proceder de forma similar a como se ha indicado en la observación 3.4.4 para procesos de una entrada y una salida. En primer lugar se definen $\boldsymbol{\Gamma}_{v}^{2} \mathrm{y} \boldsymbol{\Gamma}_{w}^{2}$ como variables matriciales. Si $\gamma_{v_{1}}^{2}, \ldots, \gamma_{v_{n_{v}}}^{2}, \gamma_{w_{1}}^{2}, \ldots, \gamma_{w_{n_{m}}}^{2}$ son las últimas componentes del vector $\boldsymbol{x}$ en (3.52), entonces el vector $\boldsymbol{h}^{\top}$ tendrá la forma

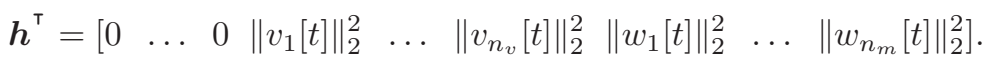

\subsection{Diseño de predictores para muestreo bifrecuencia}

\subsubsection{Diseño para estabilidad nominal}

Teorema 5.5.1. Considérese el sistema (5.1) sin perturbaciones ni ruidos de medida, y supóngase que se tiene información de todos los sensores cada $N$ periodos de control $\left(N_{k}=N\right.$ y $\left.\boldsymbol{\Delta}_{k}=\boldsymbol{I}\right)$. Si existen dos matrices $\boldsymbol{P}=\boldsymbol{P}^{\boldsymbol{\top}} \in \mathbb{R}^{n \times n}$ y $\boldsymbol{X}=\in \mathbb{R}^{n \times 1}$ y unos escalares $\boldsymbol{\varepsilon}_{f}, \boldsymbol{\varepsilon}_{h}>0$ tales que la siguiente desigualdad se cumple

$$
\left[\begin{array}{ccc}
\boldsymbol{P} & \boldsymbol{P} \boldsymbol{\Phi}_{1}(N, N)-\boldsymbol{X} \boldsymbol{C} \boldsymbol{\Phi}_{1}(N, N) & \boldsymbol{X} \\
\star & \operatorname{diag}\{\boldsymbol{P}, \mathbf{0}, \ldots, \mathbf{0}\}+\boldsymbol{M}_{1}(N) & \mathbf{0} \\
\star & \star & \boldsymbol{\varepsilon}_{h} \boldsymbol{I}
\end{array}\right] \succ \mathbf{0}
$$

siendo

$$
\begin{aligned}
& \boldsymbol{\Phi}_{1}(N, i)=\left[\begin{array}{llllllll}
\boldsymbol{A}^{i} & \boldsymbol{A}^{i-1} & \cdots & \boldsymbol{A} & \boldsymbol{I} & \mathbf{0} & \cdots & \mathbf{0}
\end{array}\right]_{(N+1) n \times n}, \\
& \boldsymbol{M}_{1}(N)=\varepsilon_{f} \operatorname{diag}\{\boldsymbol{0}, \underbrace{\boldsymbol{I}, \cdots, \boldsymbol{I}}_{N}\}_{(N+1) n \times(N+1) n}-\boldsymbol{\varepsilon}_{h} \boldsymbol{\Phi}_{1}(N, N)^{\top} \boldsymbol{H}^{\boldsymbol{\top}} \boldsymbol{H} \boldsymbol{\Phi}_{1}(N, N) \\
& -\varepsilon_{f} \sum_{i=0}^{N-1} \boldsymbol{\Phi}_{1}(N, i)^{\top} \boldsymbol{F}^{\top} \boldsymbol{F} \boldsymbol{\Phi}_{1}(N, i)
\end{aligned}
$$

Nótese que

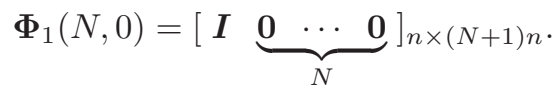

Entonces, si la ganancia del predictor se define como $\boldsymbol{L}=\boldsymbol{P}^{-1} \boldsymbol{X}$, el error de predicción del algoritmo definido por (5.14) converge a cero de forma asintótica. 
Prueba 5.5.1. Tómese la función de Lyapunov

$$
\mathcal{V}_{k}=\widetilde{\boldsymbol{x}}_{k}^{\top} \boldsymbol{P} \widetilde{\boldsymbol{x}}_{k} .
$$

Entonces, el sistema (5.1a) será estable si $\mathcal{V}_{k+1}-\mathcal{V}_{k}<0$. El incremento de la función de Lyapunov se puede escribir como

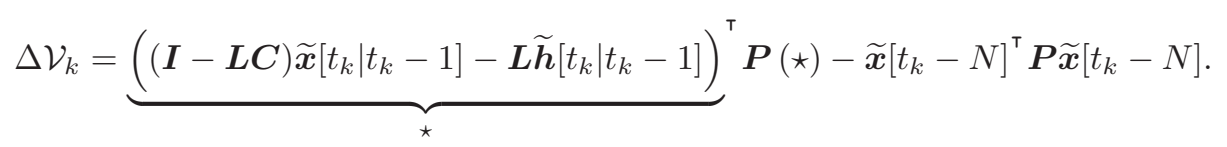

Aplicando el teorema A.6.1 al primer sumando se tiene que

$$
\Delta \mathcal{V}_{k} \leq \widetilde{\boldsymbol{x}}\left[t_{k} \mid t_{k}-1\right]^{\top} \boldsymbol{P}_{h} \widetilde{\boldsymbol{x}}\left[t_{k} \mid t_{k}-1\right]-\widetilde{\boldsymbol{x}}_{k-1}^{\top} \boldsymbol{P} \widetilde{\boldsymbol{x}}_{k-1}
$$

con

$$
\boldsymbol{P}_{h}=(\boldsymbol{I}-\boldsymbol{L} \boldsymbol{C})^{\top}\left(\boldsymbol{P}+\boldsymbol{P L}\left(\varepsilon_{h} \boldsymbol{I}-\boldsymbol{L}^{\top} \boldsymbol{P} \boldsymbol{L}\right)^{-1} \boldsymbol{L}^{\top} \boldsymbol{P}\right)(\boldsymbol{I}-\boldsymbol{L} \boldsymbol{C})+\boldsymbol{\varepsilon}_{h} \boldsymbol{H}^{\top} \boldsymbol{H} .
$$

Introduciendo el valor del error de estimación en bucle abierto (5.21) se tiene que

$$
\Delta \mathcal{V}_{k} \leq\left(\boldsymbol{A} \widetilde{\boldsymbol{x}}\left[t_{k}-1\right]+\widetilde{\boldsymbol{f}}\left[t_{k}-1\right]\right)^{\top} \boldsymbol{P}_{h}\left(\boldsymbol{A} \widetilde{\boldsymbol{x}}\left[t_{k}-1\right]+\widetilde{\boldsymbol{f}}\left[t_{k}-1\right]\right)-\widetilde{\boldsymbol{x}}_{k-1}^{\top} \boldsymbol{P} \widetilde{\boldsymbol{x}}_{k-1}
$$

donde se puede volver a aplicar el teorema A.6.1 al primer sumando, llegándose a

$$
\Delta \mathcal{V}_{k} \leq \widetilde{\boldsymbol{x}}\left[t_{k}-1\right]^{\top} \boldsymbol{P} \widetilde{\boldsymbol{x}}\left[t_{k}-1\right]-\widetilde{\boldsymbol{x}}_{k-1} \boldsymbol{P} \widetilde{\boldsymbol{x}}_{k-1}
$$

con

$$
\boldsymbol{P}_{1}=\boldsymbol{A}^{\top}\left(\boldsymbol{P}_{h}+\boldsymbol{P}_{h}\left(\varepsilon_{f} \boldsymbol{I}-\boldsymbol{P}_{h}\right)^{-1} \boldsymbol{P}_{h}\right) \boldsymbol{A}+\varepsilon_{f} \boldsymbol{F}^{\top} \boldsymbol{F} .
$$

Introduciendo de nuevo el valor del error de estimación en bucle abierto $\left(\widetilde{\boldsymbol{x}}\left[t_{k}-1\right]=\boldsymbol{A} \widetilde{\boldsymbol{x}}\left[t_{k}-2\right]+\widetilde{\boldsymbol{f}}\left[t_{k}-2\right]\right)$ se tiene que

$$
\Delta \mathcal{V}_{k} \leq \widetilde{\boldsymbol{x}}\left[t_{k}-2\right]^{\top} \boldsymbol{P}_{2} \widetilde{\boldsymbol{x}}\left[t_{k}-2\right]-\widetilde{\boldsymbol{x}}_{k-1} \boldsymbol{P} \widetilde{\boldsymbol{x}}_{k-1}
$$

con

$$
\boldsymbol{P}_{2}=\boldsymbol{A}^{\top}\left(\boldsymbol{P}_{1}+\boldsymbol{P}_{1}\left(\varepsilon_{f} \boldsymbol{I}-\boldsymbol{P}_{1}\right)^{-1} \boldsymbol{P}_{1}\right) \boldsymbol{A}+\varepsilon_{f} \boldsymbol{F}^{\top} \boldsymbol{F}
$$

Procediendo recursivamente de esta misma forma hasta llegar a

$$
\Delta \mathcal{V}_{k} \leq \widetilde{\boldsymbol{x}}\left[t_{k}-N\right]^{\top} \boldsymbol{P}_{N} \widetilde{\boldsymbol{x}}\left[t_{k}-N\right]-\widetilde{\boldsymbol{x}}_{k-1} \boldsymbol{P} \widetilde{\boldsymbol{x}}_{k-1}=\widetilde{\boldsymbol{x}}_{k-1}^{\top}\left(\boldsymbol{P}_{N}-\boldsymbol{P}\right) \widetilde{\boldsymbol{x}}_{k-1}
$$

con

$$
\boldsymbol{P}_{N}=\boldsymbol{A}^{\boldsymbol{\top}}\left(\boldsymbol{P}_{N-1}+\boldsymbol{P}_{N-1}\left(\varepsilon_{f} \boldsymbol{I}-\boldsymbol{P}_{N-1}\right)^{-1} \boldsymbol{P}_{N-1}\right) \boldsymbol{A}+\boldsymbol{\varepsilon}_{f} \boldsymbol{F}^{\boldsymbol{\top}} \boldsymbol{F},
$$

se puede establecer que la función de Lyapunov será decreciente si $\boldsymbol{P}_{N}-\boldsymbol{P} \prec \mathbf{0}$. Aplicando complementos de Schur, esta última condición se puede escribir como

$$
\left[\begin{array}{cc}
\boldsymbol{A}^{\top} \boldsymbol{P}_{N-1} \boldsymbol{A}-\boldsymbol{P}+\boldsymbol{\varepsilon}_{f} \boldsymbol{F}^{\boldsymbol{\top}} \boldsymbol{F} & \boldsymbol{A}^{\top} \boldsymbol{P}_{N-1} \\
\boldsymbol{P}_{N-1} \boldsymbol{A} & \boldsymbol{P}_{N-1}-\varepsilon_{f} \boldsymbol{I}
\end{array}\right] \prec \mathbf{0}
$$

Introduciendo la matriz

$$
\boldsymbol{P}_{N-1}=\boldsymbol{A}^{\boldsymbol{\top}}\left(\boldsymbol{P}_{N-2}+\boldsymbol{P}_{N-2}\left(\varepsilon_{f} \boldsymbol{I}-\boldsymbol{P}_{N-2}\right)^{-1} \boldsymbol{P}_{N-2}\right) \boldsymbol{A}+\boldsymbol{\varepsilon}_{f} \boldsymbol{F}^{\top} \boldsymbol{F},
$$

y aplicando de nuevo los complementos de Schur se tiene la condición

$$
\left[\begin{array}{ccc}
\boldsymbol{A}^{\top 2} \boldsymbol{P}_{N-2} \boldsymbol{A}^{2}-\boldsymbol{P}+\boldsymbol{\varepsilon}_{f} \boldsymbol{F}^{\boldsymbol{\top}} \boldsymbol{F}+\boldsymbol{\varepsilon}_{f} \boldsymbol{A}^{\top} \boldsymbol{F}^{\boldsymbol{\top}} \boldsymbol{F} \boldsymbol{A} & \boldsymbol{A}^{\top 2} \boldsymbol{P}_{N-2} \boldsymbol{A}+\boldsymbol{\varepsilon}_{f} \boldsymbol{A}^{\top} \boldsymbol{F}^{\boldsymbol{\top}} \boldsymbol{F} & \boldsymbol{A}^{\top 2} \boldsymbol{P}_{N-2} \\
\boldsymbol{A}^{\top} \boldsymbol{P}_{N-2} \boldsymbol{A}^{2}+\boldsymbol{\varepsilon}_{f} \boldsymbol{F}^{\top} \boldsymbol{F} \boldsymbol{A} & \boldsymbol{A}^{\top} \boldsymbol{P}_{N-2} \boldsymbol{A}-\varepsilon_{f} \boldsymbol{I}+\boldsymbol{\varepsilon}_{f} \boldsymbol{F}^{\top} \boldsymbol{F} & \boldsymbol{A}^{\top} \boldsymbol{P}_{N-2} \\
\boldsymbol{P}_{N-2} \boldsymbol{A}^{2} & \boldsymbol{P}_{N-2} \boldsymbol{A} & \boldsymbol{P}_{N-2}-\boldsymbol{\varepsilon}_{f} \boldsymbol{I}
\end{array}\right] \prec \mathbf{0} .
$$

Realizando esta operación de sustitución y aplicación de complementos de Schur se llega a

$$
\begin{aligned}
\boldsymbol{\Phi}_{1}(N, N)^{\top} \boldsymbol{P}_{h} \boldsymbol{\Phi}_{1}(N, N)-\operatorname{diag}\{\boldsymbol{P}, \mathbf{0}, \ldots, \mathbf{0}\} \\
\quad+\boldsymbol{\varepsilon}_{f} \sum_{i=0}^{N-1} \boldsymbol{\Phi}_{1}(N, i)^{\top} \boldsymbol{F}^{\boldsymbol{\top}} \boldsymbol{F} \boldsymbol{\Phi}_{1}(N, i)-\boldsymbol{\varepsilon}_{f}, \operatorname{diag}\{\mathbf{0}, \boldsymbol{I}, \ldots, \boldsymbol{I}\} \prec \mathbf{0}
\end{aligned}
$$


donde, sustituyendo $\boldsymbol{P}_{h}$ y aplicando de nuevo los complementos de Schur se llega a

$$
\left[\begin{array}{cc}
\boldsymbol{\Phi}_{1}(N, N)^{\top}(\boldsymbol{I}-\boldsymbol{L} \boldsymbol{C})^{\top} \boldsymbol{P}(\boldsymbol{I}-\boldsymbol{L} \boldsymbol{C}) \boldsymbol{\Phi}_{1}(N, N) \\
\left.-\operatorname{diag}^{\top} \boldsymbol{P}, \mathbf{0}, \mathbf{0}, \ldots, \mathbf{0}\right\}-\boldsymbol{M}_{1}(N) & \boldsymbol{\Phi}_{1}(N, N)^{\top}(\boldsymbol{I}-\boldsymbol{L} \boldsymbol{C})^{\top} \boldsymbol{P} \boldsymbol{L} \\
\boldsymbol{L}^{\top} \boldsymbol{P}(\boldsymbol{I}-\boldsymbol{L} \boldsymbol{C}) \boldsymbol{\Phi}_{1}(N, N) & \boldsymbol{L}^{\top} \boldsymbol{P} \boldsymbol{L}-\boldsymbol{\varepsilon}_{h} \boldsymbol{I}
\end{array}\right] \prec \mathbf{0}
$$

Aplicando de nuevo los complementos de Schur e introduciendo la matriz $\boldsymbol{X}=\boldsymbol{P} \boldsymbol{L}$ se llega finalmente a $(5.29)$

\subsubsection{Diseño basado en la atenuación de perturbaciones}

Teorema 5.5.2. Considérese el predictor (5.14) aplicado al sistema (5.1) y supóngase que todos los sensores proporcionan una medida cada $N$ instantes de control $\left(N_{k}=N, \boldsymbol{\Delta}_{k}=\boldsymbol{I}\right)$. Para unas $\gamma_{v_{i}}, \gamma_{w_{j}}>0$ dadas, supóngase que existen unas matrices $\boldsymbol{P}=\boldsymbol{P}^{\top} \in \mathbb{R}^{n \times n}, \boldsymbol{X} \in \mathbb{R}^{n \times 1}$ y unos escalares $\boldsymbol{\varepsilon}_{f}, \boldsymbol{\varepsilon}_{h}>0$ tales que

$$
\left[\begin{array}{cccc}
\boldsymbol{P} & \boldsymbol{P} \boldsymbol{\Phi}_{2}(N, N) & \boldsymbol{X} & \boldsymbol{X} \\
\boldsymbol{\Phi}_{2}(N, N)^{\top} \boldsymbol{P} & \operatorname{diag}\left\{\boldsymbol{P}+\boldsymbol{G}^{\top} \boldsymbol{G}, \mathbf{0}, \ldots, \mathbf{0}\right\}+\boldsymbol{M}_{2}(N) & \mathbf{0} & \mathbf{0} \\
\boldsymbol{X}^{\top} & \mathbf{0} & \varepsilon_{h} \boldsymbol{I} & \mathbf{0} \\
\boldsymbol{X}^{\top} & \mathbf{0} & \mathbf{0} & \boldsymbol{\Gamma}_{w}^{2}
\end{array}\right] \succ \mathbf{0}
$$

siendo

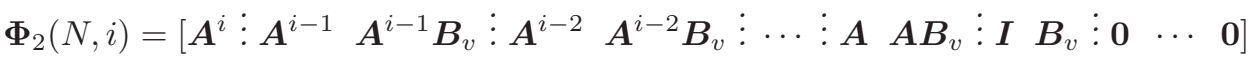

$$
\begin{aligned}
& \boldsymbol{M}_{2}(N)=\boldsymbol{\varepsilon}_{f} \operatorname{diag}\{\mathbf{0}, \boldsymbol{I}, \mathbf{0}, \boldsymbol{I}, \ldots\},-\boldsymbol{\varepsilon}_{f} \sum_{i=0}^{N-1} \boldsymbol{\Phi}_{2}(N, i)^{\boldsymbol{\top}} \boldsymbol{F}^{\boldsymbol{\top}} \boldsymbol{F} \boldsymbol{\Phi}_{2}(N, i) \\
& -\boldsymbol{\varepsilon}_{h} \boldsymbol{\Phi}_{2}(N, N)^{\top} \boldsymbol{H}^{\top} \boldsymbol{H} \boldsymbol{\Phi}_{2}(N, N)+\operatorname{diag}\left\{\mathbf{0}, \mathbf{0}, \boldsymbol{\Gamma}_{v}^{2}, \mathbf{0}, \boldsymbol{\Gamma}_{v}^{2}, \mathbf{0} \ldots\right\} .
\end{aligned}
$$

Entonces, definiendo la ganancia del predictor como $\boldsymbol{L}=\boldsymbol{P}^{-1} \boldsymbol{X}$, el error de predicción converge a cero de forma asintótica y, bajo condiciones iniciales nulas,

$$
\left\|\boldsymbol{e}_{k}\right\|_{2}^{2} \leq\left\|\boldsymbol{\Gamma}_{v} \boldsymbol{v}[t]\right\|_{2}^{2}+\left\|\boldsymbol{\Gamma}_{w} \boldsymbol{w}_{k}\right\|_{2}^{2}
$$

Prueba 5.5.2. Considérese el índice

$$
J=\sum_{k=0}^{\infty}\left(\boldsymbol{e}_{k}^{\top} \boldsymbol{e}_{k}-\sum_{j=1}^{N} \boldsymbol{v}\left[t_{k}-i\right]^{\top} \boldsymbol{\Gamma}_{v}^{2} \boldsymbol{v}\left[t_{k}-i\right]-\boldsymbol{w}_{k}^{\top} \boldsymbol{\Gamma}_{w}^{2} \boldsymbol{w}_{k}\right)
$$

Tomando la función de Lyapunov $\mathcal{V}_{k}=\widetilde{\boldsymbol{x}}_{k} \boldsymbol{P} \widetilde{\boldsymbol{x}}_{k}$ y asumiendo condiciones iniciales nulas se tiene que

$$
\begin{aligned}
J & \leq \sum_{k=1}^{\infty}\left(\boldsymbol{e}_{k-1}^{\top} \boldsymbol{e}_{k-1}-\sum_{j=1}^{N} \boldsymbol{v}\left[t_{k}-j\right]^{\top} \boldsymbol{\Gamma}_{v}^{2} \boldsymbol{v}\left[t_{k}-j\right]-\boldsymbol{w}_{k}^{\top} \boldsymbol{\Gamma}_{w}^{2} \boldsymbol{w}_{k}\right)+\left.\mathcal{V}_{k}\right|_{k=\infty}-\left.\mathcal{V}_{k}\right|_{k=0} \\
& =\sum_{k=1}^{\infty}\left(\boldsymbol{e}_{k-1}^{\top} \boldsymbol{e}_{k-1}-\sum_{j=1}^{N} \boldsymbol{v}\left[t_{k}-j\right]^{\top} \boldsymbol{\Gamma}_{v}^{2} \boldsymbol{v}\left[t_{k}-j\right]-\boldsymbol{w}_{k}^{\top} \boldsymbol{\Gamma}_{w}^{2} \boldsymbol{w}_{k}+\Delta \mathcal{V}_{k}\right) .
\end{aligned}
$$

Sustituyendo $\Delta \mathcal{V}_{k}$ por

$$
\begin{aligned}
\Delta \mathcal{V}_{k} & =\widetilde{\boldsymbol{x}}_{k}^{\top} \boldsymbol{P} \widetilde{\boldsymbol{x}}_{k}-\widetilde{\boldsymbol{x}}_{k-1}^{\top} \boldsymbol{P} \widetilde{\boldsymbol{x}}_{k-1} \\
& =\underbrace{\left((\boldsymbol{I}-\boldsymbol{L} \boldsymbol{C})^{\top} \widetilde{\boldsymbol{x}}\left[t_{k} \mid t_{k}-1\right]-\boldsymbol{L} \widetilde{\boldsymbol{h}}\left[t_{k} \mid t_{k}-1\right]-\boldsymbol{L} \boldsymbol{w}_{k}\right)^{\top} \boldsymbol{P}(\star)-\widetilde{\boldsymbol{x}}_{k-1}^{\top} \boldsymbol{P} \widetilde{\boldsymbol{x}}_{k-1}}_{\star}
\end{aligned}
$$


donde $\widetilde{\boldsymbol{x}}_{k} \equiv \widetilde{\boldsymbol{x}}\left[t_{k}\right]$ y $\widetilde{\boldsymbol{x}}_{k-1} \equiv \widetilde{\boldsymbol{x}}\left[t_{k}-N\right]$, se puede aplicar el teorema A.6.3 para eliminar el término $\boldsymbol{w}_{k}^{\top} \boldsymbol{\Gamma}_{w}^{2} \boldsymbol{w}_{k}$, quedando

$$
\begin{aligned}
J \leq & \sum_{k=1}^{\infty}\left(\boldsymbol{e}_{k-1}^{\top} \boldsymbol{e}_{k-1}-\sum_{j=1}^{N} \boldsymbol{v}\left[t_{k}-j\right]^{\top} \boldsymbol{\Gamma}_{v}^{2} \boldsymbol{v}\left[t_{k}-j\right]-\widetilde{\boldsymbol{x}}_{k-1}^{\boldsymbol{\top}} \boldsymbol{P} \widetilde{\boldsymbol{x}}_{k-1}\right. \\
& +\underbrace{\left.\left((\boldsymbol{I}-\boldsymbol{L} \boldsymbol{C}) \widetilde{\boldsymbol{x}}\left[t_{k} \mid t_{k}-1\right]-\boldsymbol{L} \widetilde{\boldsymbol{h}}\left[t_{k} \mid t_{k}-1\right]\right)^{\top} \boldsymbol{P}_{w}(\star)\right)}_{\star} .
\end{aligned}
$$

con

$$
\boldsymbol{P}_{w}=\boldsymbol{P}+\boldsymbol{P} \boldsymbol{L}\left(\boldsymbol{\Gamma}_{w}^{2}-\boldsymbol{L}^{\top} \boldsymbol{P} \boldsymbol{L}\right)^{-1} \boldsymbol{L}^{\top} \boldsymbol{P}
$$

Aplicando ahora el teorema A.6.1 para eliminar el término $\widetilde{\boldsymbol{h}}\left[t_{k} \mid t_{k}-1\right]$ se tiene que

$$
J \leq \sum_{t=1}^{\infty}\left(\boldsymbol{e}_{k-1}^{\boldsymbol{\top}} \boldsymbol{e}_{k-1}-\sum_{j=1}^{N} \boldsymbol{v}\left[t_{k}-j\right]^{\top} \boldsymbol{\Gamma}_{v}^{2} \boldsymbol{v}\left[t_{k}-j\right]-\widetilde{\boldsymbol{x}}_{k-1}^{\boldsymbol{\top}} \boldsymbol{P} \widetilde{\boldsymbol{x}}_{k-1}+\widetilde{\boldsymbol{x}}\left[t_{k} \mid t_{k}-1\right]^{\top} \boldsymbol{P}_{h} \widetilde{\boldsymbol{x}}\left[t_{k} \mid t_{k}-1\right]\right) .
$$

con

$$
\boldsymbol{P}_{h}=(\boldsymbol{I}-\boldsymbol{L} \boldsymbol{C})^{\top}\left(\boldsymbol{P}_{w}+\boldsymbol{P}_{w} \boldsymbol{L}\left(\varepsilon_{h} \boldsymbol{I}-\boldsymbol{L}^{\top} \boldsymbol{P}_{w} \boldsymbol{L}\right)^{-1} \boldsymbol{L}^{\top} \boldsymbol{P}_{w}\right)(\boldsymbol{I}-\boldsymbol{L} \boldsymbol{C})+\boldsymbol{\varepsilon}_{h} \boldsymbol{H}^{\top} \boldsymbol{H}
$$

Sustituyendo el error de estimación en bucle abierto por $\widetilde{\boldsymbol{x}}\left[t_{k} \mid t_{k}-1\right]=\boldsymbol{A} \widetilde{\boldsymbol{x}}\left[t_{k}-1\right]+\widetilde{\boldsymbol{f}}\left[t_{k}-1\right]+\boldsymbol{v}\left[t_{k}-1\right]$ y aplicando el teorema A.6.3 para eliminar el término $\boldsymbol{v}\left[t_{k}-1\right]^{\top} \boldsymbol{\Gamma}_{v}^{2} \boldsymbol{v}\left[t_{k}-1\right]$ se tiene que

$$
\begin{aligned}
J \leq \sum_{t=1}^{\infty} & \left(\boldsymbol{e}_{k-1}^{\top} \boldsymbol{e}_{k-1}-\sum_{j=2}^{N} \boldsymbol{v}\left[t_{k}-j\right]^{\top} \boldsymbol{\Gamma}_{v}^{2} \boldsymbol{v}\left[t_{k}-j\right]\right. \\
& \left.+\left(\boldsymbol{A} \widetilde{\boldsymbol{x}}\left[t_{k}-1\right]+\widetilde{\boldsymbol{f}}\left[t_{k}-1\right]\right)^{\top} \boldsymbol{P}_{x_{1}}\left(\boldsymbol{A} \widetilde{\boldsymbol{x}}\left[t_{k}-1\right]+\widetilde{\boldsymbol{f}}\left[t_{k}-1\right]\right)-\widetilde{\boldsymbol{x}}_{k-1}^{\top} \boldsymbol{P} \widetilde{\boldsymbol{x}}_{k-1}\right),
\end{aligned}
$$

con

$$
\boldsymbol{P}_{x_{1}}=\boldsymbol{P}_{h}+\boldsymbol{P}_{h} \boldsymbol{B}_{v}\left(\boldsymbol{\Gamma}_{v}^{2}-\boldsymbol{B}_{v}^{\top} \boldsymbol{P}_{h} \boldsymbol{B}_{v}\right)^{-1} \boldsymbol{B}_{v}^{\top} \boldsymbol{P}_{h}
$$

Aplicando ahora el teorema A.6.1 se tiene que

$$
J \leq \sum_{k=1}^{\infty}\left(\boldsymbol{e}_{k-1}^{\top} \boldsymbol{e}_{k-1}-\sum_{j=2}^{N} \boldsymbol{v}\left[t_{k}-j\right]^{\top} \boldsymbol{\Gamma}_{v}^{2} \boldsymbol{v}\left[t_{k}-j\right]+\widetilde{\boldsymbol{x}}\left[t_{k}-1\right]^{\top} \boldsymbol{P}_{f_{1}} \widetilde{\boldsymbol{x}}\left[t_{k}-1\right]-\widetilde{\boldsymbol{x}}_{k-1}^{\top} \boldsymbol{P} \widetilde{\boldsymbol{x}}_{k-1}\right),
$$

con

$$
\boldsymbol{P}_{f_{1}}=\boldsymbol{A}^{\boldsymbol{\top}}\left(\boldsymbol{P}_{x_{1}}+\boldsymbol{P}_{x_{1}}\left(\varepsilon_{f}-\boldsymbol{P}_{x_{1}}\right)^{-1} \boldsymbol{P}_{x_{1}}\right) \boldsymbol{A}+\boldsymbol{\varepsilon}_{f} \boldsymbol{F}^{\boldsymbol{\top}} \boldsymbol{F} .
$$

Como en $t_{k}-1$ no hay medición, el error de estimación de este instante se relaciona con el instante anterior mediante $\widetilde{\boldsymbol{x}}\left[t_{k}-1\right]=\boldsymbol{A} \widetilde{\boldsymbol{x}}\left[t_{k}-2\right]+\widetilde{\boldsymbol{f}}\left[t_{k}-2\right]+\boldsymbol{v}\left[t_{k}-2\right]$. Sustituyendo este valor en (5.37) y aplicando el teorema A.6.3 para eliminar el término $\boldsymbol{v}\left[t_{k}-2\right]^{\top} \boldsymbol{\Gamma}_{v}^{2} \boldsymbol{v}\left[t_{k}-2\right]$ se tiene que

$$
\begin{aligned}
J \leq \sum_{t=1}^{\infty} & \left(\boldsymbol{e}_{k-1}^{\top} \boldsymbol{e}_{k-1}-\sum_{j=3}^{N} \boldsymbol{v}\left[t_{k}-j\right]^{\top} \boldsymbol{\Gamma}_{v}^{2} \boldsymbol{v}\left[t_{k}-j\right]\right. \\
& \left.+\left(\boldsymbol{A} \widetilde{\boldsymbol{x}}\left[t_{k}-2\right]+\widetilde{\boldsymbol{f}}\left[t_{k}-2\right]\right)^{\top} \boldsymbol{P}_{x_{2}}\left(\boldsymbol{A} \widetilde{\boldsymbol{x}}\left[t_{k}-2\right]+\widetilde{\boldsymbol{f}}\left[t_{k}-2\right]\right)-\widetilde{\boldsymbol{x}}_{k-1}^{\top} \boldsymbol{P} \widetilde{\boldsymbol{x}}_{k-1}\right),
\end{aligned}
$$

con

$$
\boldsymbol{P}_{x_{2}}=\boldsymbol{P}_{f_{1}}+\boldsymbol{P}_{f_{1}} \boldsymbol{B}_{v}\left(\boldsymbol{\Gamma}_{v}^{2}-\boldsymbol{B}_{v}^{\top} \boldsymbol{P}_{f_{1}} \boldsymbol{B}_{v}\right)^{-1} \boldsymbol{B}_{v}^{\top} \boldsymbol{P}_{f_{1}} .
$$

Aplicando ahora el teorema A.6.1 se tiene que

$$
J \leq \sum_{k=1}^{\infty}\left(\boldsymbol{e}_{k-1}^{\top} \boldsymbol{e}_{k-1}-\sum_{j=3}^{N} \boldsymbol{v}\left[t_{k}-j\right]^{\top} \boldsymbol{\Gamma}_{v}^{2} \boldsymbol{v}\left[t_{k}-j\right]+\widetilde{\boldsymbol{x}}\left[t_{k}-2\right]^{\top} \boldsymbol{P}_{f_{2}} \widetilde{\boldsymbol{x}}\left[t_{k}-2\right]-\widetilde{\boldsymbol{x}}_{k-1}^{\top} \boldsymbol{P} \widetilde{\boldsymbol{x}}_{k-1}\right),
$$


con

$$
\boldsymbol{P}_{f_{2}}=\boldsymbol{A}^{\boldsymbol{\top}}\left(\boldsymbol{P}_{x_{2}}+\boldsymbol{P}_{x_{2}}\left(\varepsilon_{f}-\boldsymbol{P}_{x_{2}}\right)^{-1} \boldsymbol{P}_{x_{2}}\right) \boldsymbol{A}+\varepsilon_{f} \boldsymbol{F}^{\boldsymbol{\top}} \boldsymbol{F} .
$$

Aplicando este procedimiento de forma recursiva se llega finalmente a

$$
J \leq \sum_{k=1}^{\infty}\left(\boldsymbol{e}_{k-1}^{\top} \boldsymbol{e}_{k-1}+\widetilde{\boldsymbol{x}}\left[t_{k}-N\right]^{\top} \boldsymbol{P}_{f_{N}} \widetilde{\boldsymbol{x}}\left[t_{k}-N\right]-\widetilde{\boldsymbol{x}}_{k-1}^{\top} \boldsymbol{P} \widetilde{\boldsymbol{x}}_{k-1}\right),
$$

con

$$
\boldsymbol{P}_{f_{N}}=\boldsymbol{A}^{\boldsymbol{\top}}\left(\boldsymbol{P}_{x_{N}}+\boldsymbol{P}_{x_{N}}\left(\varepsilon_{f}-\boldsymbol{P}_{x_{N}}\right)^{-1} \boldsymbol{P}_{x_{N}}\right) \boldsymbol{A}+\varepsilon_{f} \boldsymbol{F}^{\boldsymbol{\top}} \boldsymbol{F} .
$$

$\mathrm{y}$

$$
\boldsymbol{P}_{x_{N}}=\boldsymbol{P}_{f_{N-1}}+\boldsymbol{P}_{f_{N-1}} \boldsymbol{B}_{v}\left(\boldsymbol{\Gamma}_{v}^{2}-\boldsymbol{B}_{v}^{\top} \boldsymbol{P}_{f_{N-1}} \boldsymbol{B}_{v}\right)^{-1} \boldsymbol{B}_{v}^{\top} \boldsymbol{P}_{f_{N-1}} .
$$

Por otra parte, como $\boldsymbol{e}_{k-1}=\boldsymbol{g}\left(\boldsymbol{x}_{k-1}\right)-\boldsymbol{g}\left(\hat{\boldsymbol{x}}_{k-1}\right)$ y

$$
\boldsymbol{g}\left(\boldsymbol{x}_{k-1}\right)-\boldsymbol{g}\left(\hat{\boldsymbol{x}}_{k-1}\right) \leq\left\|\boldsymbol{G} \widetilde{\boldsymbol{x}}_{k-1}\right\|,
$$

se puede escribir que

$$
J \leq \sum_{t=1}^{\infty} \widetilde{\boldsymbol{x}}_{k-1}^{\top}\left(\boldsymbol{P}_{f_{N}}+\boldsymbol{G}^{\top} \boldsymbol{G}-\boldsymbol{P}\right) \widetilde{\boldsymbol{x}}_{k-1} .
$$

La condición (5.36) se cumplirá si $J<0$, condición que será cierta siempre que

$$
\boldsymbol{P}_{f_{N}}+\boldsymbol{G}^{\top} \boldsymbol{G}-\boldsymbol{P} \prec \mathbf{0} .
$$

Sustituyendo $\boldsymbol{P}_{f_{N}}$ en función de $\boldsymbol{P}_{x_{N}}$ y aplicando complementos de Schur se tiene que la condición anterior es equivalente a la condición

$$
\left[\begin{array}{cc}
\boldsymbol{A}^{\top} \boldsymbol{P}_{x_{N}} \boldsymbol{A}-\boldsymbol{P}+\boldsymbol{G}^{\top} \boldsymbol{G}+\varepsilon_{f} \boldsymbol{F}^{\boldsymbol{\top}} \boldsymbol{F} & \boldsymbol{A}^{\top} \boldsymbol{P}_{x_{N}} \\
\boldsymbol{P}_{x_{N}} \boldsymbol{A} & \boldsymbol{P}_{x_{N}}-\varepsilon_{f} \boldsymbol{I}
\end{array}\right] \prec \mathbf{0} .
$$

sustituyendo $\boldsymbol{P}_{x_{N}}$ en función de $\boldsymbol{P}_{f_{N-1}}$ y aplicando complementos de Schur se llega a

$$
\left[\begin{array}{ccc}
\boldsymbol{A}^{\boldsymbol{\top}} \boldsymbol{P}_{f_{N-1}} \boldsymbol{A}-\boldsymbol{P}+\boldsymbol{G}^{\boldsymbol{\top}} \boldsymbol{G}+\boldsymbol{\varepsilon}_{f} \boldsymbol{F}^{\boldsymbol{\top}} \boldsymbol{F} & \boldsymbol{A}^{\boldsymbol{\top}} \boldsymbol{P}_{f_{N-1}} & \boldsymbol{A}^{\boldsymbol{\top}} \boldsymbol{P}_{f_{N-1}} \\
\boldsymbol{P}_{f_{N-1}} \boldsymbol{A} & \boldsymbol{P}_{f_{N-1}}-\varepsilon_{f} \boldsymbol{I} & \boldsymbol{P}_{f_{N-1}} \\
\boldsymbol{P}_{f_{N-1}} \boldsymbol{A} & \boldsymbol{P}_{f_{N-1}} & \boldsymbol{P}_{f_{N-1}}-\boldsymbol{\Gamma}_{v}^{2}
\end{array}\right] \prec \mathbf{0 .}
$$

A partir de este momento, la prueba consiste en:

- sustituir $\boldsymbol{P}_{f_{i}}$ en función de $\boldsymbol{P}_{x_{i}}$,

- aplicar los complementos de Schur,

- sustituir $\boldsymbol{P}_{x_{i}}$ en función de $\boldsymbol{P}_{f_{i-1}}$,

- aplicar los complements de Schur,

y proceder de esta manera hasta tener una desigualdad en la que aparece $\boldsymbol{P}_{x_{1}}$. Esta matriz se sustituye finalmente en función de $\boldsymbol{P}_{h}$ y se aplican los complementos de Schur llegándose a

$$
\begin{aligned}
\boldsymbol{\Phi}_{2}(N, N)^{\top} \boldsymbol{P}_{h} \boldsymbol{\Phi}_{2}(N, N)-\operatorname{diag}\left\{\boldsymbol{P}+\boldsymbol{G}^{\boldsymbol{\top}} \boldsymbol{G}, \mathbf{0}, \ldots, \mathbf{0}\right\}-\boldsymbol{\varepsilon}_{f} \operatorname{diag}\{\mathbf{0}, \boldsymbol{I}, \mathbf{0}, \boldsymbol{I}, \ldots\} \\
+\boldsymbol{\varepsilon}_{f} \sum_{i=0}^{N-1} \boldsymbol{\Phi}_{2}(N, i)^{\top} \boldsymbol{F}^{\boldsymbol{\top}} \boldsymbol{F} \boldsymbol{\Phi}_{2}(N, i)-\operatorname{diag}\left\{\mathbf{0}, \mathbf{0}, \boldsymbol{\Gamma}_{v}^{2}, \mathbf{0}, \boldsymbol{\Gamma}_{v}^{2}, \mathbf{0}, \ldots\right\} \prec \mathbf{0} .
\end{aligned}
$$

Sustituyendo $\boldsymbol{P}_{h}$ en función de $\boldsymbol{P}_{w}$ y aplicando complementos de Schur se llega a

$$
\left[\begin{array}{cc}
\boldsymbol{\Phi}_{2}(N, N)^{\top}(\boldsymbol{I}-\boldsymbol{L} \boldsymbol{C})^{\top} \boldsymbol{P}_{w}(\boldsymbol{I}-\boldsymbol{L} \boldsymbol{C}) \boldsymbol{\Phi}_{2}(N, N) \\
-\operatorname{diag}\left\{\boldsymbol{P}+\boldsymbol{G}^{\top} \boldsymbol{G}, \mathbf{0}, \mathbf{0}, \ldots, \mathbf{0}\right\}-\boldsymbol{M}_{2}(N) & \boldsymbol{\Phi}_{2}(N, N)^{\top}(\boldsymbol{I}-\boldsymbol{L} \boldsymbol{C})^{\top} \boldsymbol{P}_{w} \boldsymbol{L} \\
\boldsymbol{L}^{\top} \boldsymbol{P}_{w}(\boldsymbol{I}-\boldsymbol{L} \boldsymbol{C}) \boldsymbol{\Phi}_{2}(N, N) & \boldsymbol{L}^{\top} \boldsymbol{P}_{w} \boldsymbol{L}-\boldsymbol{\varepsilon}_{h} \boldsymbol{I}
\end{array}\right] \prec \mathbf{0} .
$$

Sustituyendo $\boldsymbol{P}_{w}$ en función de $\boldsymbol{P}$, aplicando dos veces consecutivas los complementos de Schur y teniendo en cuenta que $\boldsymbol{P} \boldsymbol{L}=\boldsymbol{X}$ se llega finalmente a (5.34).

En este caso también es aplicable el procedimiento de diseño expuesto en la observación 5.4.1, teniendo en cuenta que en este caso las normas que se deben utilizar no son las normas $\left\|v_{i}[t]\right\|$ o $\left\|w_{i}[t]\right\|$ sino las normas $\left\|v_{i, k}\right\|$ y $\left\|w_{i, k}\right\|$, cuya relación con las anteriores debe obtenerse mediante las relaciones expuestas en las observaciones 4.5 .3 y 4.5 .6 (páginas 100 y 101 , respectivamente). 


\subsection{Diseño de predictores para muestreo variante en el tiempo}

\subsubsection{Diseño para estabilidad nominal}

A partir del resultado que permite diseñar el predictor para muestreo bifrecuencia, es fácil obtener el predictor para muestreo irregular aplicando el concepto de estabilidad policuadrática (véase anexo $§$ A.4). El siguiente teorema recoge el diseño de predictores para este tipo de muestreo.

Teorema 5.6.1. Considérese el sistema (5.1) sin perturbaciones ni ruidos de medida, y supóngase que cada $N_{k} \in \mathcal{N}$ periodos de control se tiene información de algunos de los sensores según lo indicado en la matriz de disponibilidad $\boldsymbol{\Delta}_{k} \in \Xi$, de manera que hay $n_{\mathcal{S}}$ escenarios de muestreo $s_{k} \in \mathcal{S}$ diferentes. Supóngase que existen unas matrices $\boldsymbol{P}\left(s_{k}\right)=\boldsymbol{P}\left(s_{k}\right)^{\top} \in \mathbb{R}^{n \times n}, \boldsymbol{Q}\left(s_{k}\right) \in \mathbb{R}^{n \times 1}, \boldsymbol{X}\left(s_{k}\right) \in \mathbb{R}^{n \times 1}$ y unos escalares $\varepsilon_{f}, \varepsilon_{h}>0$ tales que la siguiente desigualdad se cumple para cualquier secuencia de muestreo $\left\{s_{k}\right\}$

$$
\left[\begin{array}{ccc}
\boldsymbol{Q}\left(s_{k}\right)+\boldsymbol{Q}\left(s_{k}\right)^{\top}-\boldsymbol{P}\left(s_{k}\right) & \left(\boldsymbol{Q}\left(s_{k}\right)-\boldsymbol{X}\left(s_{k}\right) \boldsymbol{\Delta}\left(s_{k}\right) \boldsymbol{C}\right) \boldsymbol{\Phi}_{1}\left(N\left(s_{k}\right), N\left(s_{k}\right)\right) & \boldsymbol{X}\left(s_{k}\right) \boldsymbol{\Delta}\left(s_{k}\right) \\
\star & \operatorname{diag}\left\{\boldsymbol{P}\left(s_{k-1}\right), 0, \ldots \mathbf{0}\right\}+\boldsymbol{M}_{1}\left(N\left(s_{k}\right)\right) & \mathbf{0} \\
\star & \star & \boldsymbol{\varepsilon}_{h} \boldsymbol{I}
\end{array}\right] \succ \mathbf{0} .
$$

Entonces, si la ganancia del predictor se define como $\boldsymbol{L}\left(s_{k}\right)=\boldsymbol{Q}\left(s_{k}\right)^{-1} \boldsymbol{X}\left(s_{k}\right)$, el error de predicción del algoritmo definido por (5.14) converge a cero de forma asintótica.

Prueba 5.6.1. Tómese la función de Lyapunov paramétrica

$$
\mathcal{V}_{k}=\mathcal{V}\left(\widetilde{\boldsymbol{x}}_{k}, s_{k}\right)=\widetilde{\boldsymbol{x}}_{k}^{\top} \boldsymbol{P}\left(s_{k}\right) \widetilde{\boldsymbol{x}}_{k} .
$$

Si $\mathcal{V}_{k+1}-\mathcal{V}_{k}<0$, el sistema (5.1a) será estable. El incremento de la función de Lyapunov se puede escribir como

$$
\begin{aligned}
\Delta \mathcal{V}_{k}= & \underbrace{\left(\left(\boldsymbol{I}-\boldsymbol{L}\left(s_{k}\right) \boldsymbol{\Delta}\left(s_{k}\right) \boldsymbol{C}\right) \widetilde{\boldsymbol{x}}\left[t_{k} \mid t_{k}-1\right]-\boldsymbol{L}\left(s_{k}\right) \boldsymbol{\Delta}\left(s_{k}\right) \widetilde{\boldsymbol{h}}\left[t_{k} \mid t_{k}-1\right]\right)^{\top}}_{\star} \boldsymbol{P}(\star) \\
& -\widetilde{\boldsymbol{x}}\left[t_{k}-N\left(s_{k}\right)\right]^{\top} \boldsymbol{P}\left(s_{k-1}\right) \widetilde{\boldsymbol{x}}\left[t_{k}-N\left(s_{k}\right)\right] .
\end{aligned}
$$

Aplicando el teorema A.6.1 al primer sumando se tiene que

$$
\Delta \mathcal{V}_{k} \leq \widetilde{\boldsymbol{x}}\left[t_{k} \mid t_{k}-1\right]^{\top} \boldsymbol{P}_{h}\left(s_{k}\right) \widetilde{\boldsymbol{x}}\left[t_{k} \mid t_{k}-1\right]-\widetilde{\boldsymbol{x}}_{k-1}^{\boldsymbol{T}} \boldsymbol{P}\left(s_{k}\right) \widetilde{\boldsymbol{x}}_{k-1}
$$

con

$$
\begin{aligned}
\boldsymbol{P}_{h}\left(s_{k}\right)= & \left(\boldsymbol{I}-\boldsymbol{L}\left(s_{k}\right) \boldsymbol{\Delta}\left(s_{k}\right) \boldsymbol{C}\right)^{\boldsymbol{\top}} \boldsymbol{P}\left(s_{k}\right)\left(\boldsymbol{I}-\boldsymbol{L}\left(s_{k}\right) \boldsymbol{\Delta}\left(s_{k}\right) \boldsymbol{C}\right)+\boldsymbol{\varepsilon}_{h} \boldsymbol{H}^{\boldsymbol{\top}} \boldsymbol{H}+ \\
& +\underbrace{\left(\left(\boldsymbol{I}-\boldsymbol{L}\left(s_{k}\right) \boldsymbol{\Delta}\left(s_{k}\right) \boldsymbol{C}\right) \boldsymbol{P}\left(s_{k}\right) \boldsymbol{L}\left(s_{k}\right) \boldsymbol{\Delta}\left(s_{k}\right)\right)}_{\star}\left(\boldsymbol{\varepsilon}_{h} \boldsymbol{I}-\boldsymbol{\Delta}\left(s_{k}\right)^{\boldsymbol{\top}} \boldsymbol{L}\left(s_{k}\right)^{\boldsymbol{\top}} \boldsymbol{P}\left(s_{k}\right) \boldsymbol{L}\left(s_{k}\right) \boldsymbol{\Delta}\left(s_{k}\right)\right)^{-1}(\star) .
\end{aligned}
$$

Introduciendo el valor del error de estimación en bucle abierto (5.21) se tiene que

$$
\Delta \mathcal{V}_{k} \leq\left(\boldsymbol{A} \widetilde{\boldsymbol{x}}\left[t_{k}-1\right]+\widetilde{\boldsymbol{f}}\left[t_{k}-1\right]\right)^{\top} \boldsymbol{P}_{h}\left(s_{k}\right)\left(\boldsymbol{A} \widetilde{\boldsymbol{x}}\left[t_{k}-1\right]+\widetilde{\boldsymbol{f}}\left[t_{k}-1\right]\right)-\widetilde{\boldsymbol{x}}_{k-1}^{\top} \boldsymbol{P}\left(s_{k-1}\right) \widetilde{\boldsymbol{x}}_{k-1}
$$

donde se puede volver a aplicar el teorema A.6.1 al primer sumando, llegándose a

$$
\Delta \mathcal{V}_{k} \leq \widetilde{\boldsymbol{x}}\left[t_{k}-1\right]^{\top} \boldsymbol{P}_{1}\left(s_{k}\right) \widetilde{\boldsymbol{x}}\left[t_{k}-1\right]-\widetilde{\boldsymbol{x}}_{k-1} \boldsymbol{P}\left(s_{k-1}\right) \widetilde{\boldsymbol{x}}_{k-1}
$$

con

$$
\boldsymbol{P}_{1}\left(s_{k}\right)=\boldsymbol{A}^{\boldsymbol{\top}}\left(\boldsymbol{P}_{h}\left(s_{k}\right)+\boldsymbol{P}_{h}\left(s_{k}\right)\left(\varepsilon_{f} \boldsymbol{I}-\boldsymbol{P}_{h}\left(s_{k}\right)\right)^{-1} \boldsymbol{P}_{h}\left(s_{k}\right)\right) \boldsymbol{A}+\boldsymbol{\varepsilon}_{f} \boldsymbol{F}^{\boldsymbol{\top}} \boldsymbol{F} .
$$

Introduciendo de nuevo el valor del error de estimación en bucle abierto $\left(\widetilde{\boldsymbol{x}}\left[t_{k}-1\right]=\boldsymbol{A} \widetilde{\boldsymbol{x}}\left[t_{k}-2\right]+\widetilde{\boldsymbol{f}}\left[t_{k}-2\right]\right)$ se tiene que

$$
\Delta \mathcal{V}_{k} \leq \widetilde{\boldsymbol{x}}\left[t_{k}-2\right]^{\top} \boldsymbol{P}_{2}\left(s_{k}\right) \widetilde{\boldsymbol{x}}\left[t_{k}-2\right]-\widetilde{\boldsymbol{x}}_{k-1} \boldsymbol{P}\left(s_{k-1}\right) \widetilde{\boldsymbol{x}}_{k-1}
$$

con

$$
\boldsymbol{P}_{2}\left(s_{k}\right)=\boldsymbol{A}^{\boldsymbol{\top}}\left(\boldsymbol{P}_{1}\left(s_{k}\right)+\boldsymbol{P}_{1}\left(s_{k}\right)\left(\varepsilon_{f} \boldsymbol{I}-\boldsymbol{P}_{1}\left(s_{k}\right)\right)^{-1} \boldsymbol{P}_{1}\left(s_{k}\right)\right) \boldsymbol{A}+\boldsymbol{\varepsilon}_{f} \boldsymbol{F}^{\boldsymbol{\top}} \boldsymbol{F}
$$


Procediendo recursivamente de esta misma forma hasta llegar a

$$
\begin{aligned}
\Delta \mathcal{V}_{k} & \leq \widetilde{\boldsymbol{x}}\left[t_{k}-N\left(s_{k}\right)\right]^{\top} \boldsymbol{P}_{N\left(s_{k}\right)}\left(s_{k}\right) \widetilde{\boldsymbol{x}}\left[t_{k}-N\left(s_{k}\right)\right]-\widetilde{\boldsymbol{x}}_{k-1} \boldsymbol{P}\left(s_{k-1}\right) \widetilde{\boldsymbol{x}}_{k-1} \\
& \leq \widetilde{\boldsymbol{x}}_{k-1}^{\top}\left(\boldsymbol{P}_{N\left(s_{k}\right)}\left(s_{k}\right)-\boldsymbol{P}\left(s_{k-1}\right)\right) \widetilde{\boldsymbol{x}}_{k-1}
\end{aligned}
$$

con

$$
\boldsymbol{P}_{N\left(s_{k}\right)}\left(s_{k}\right)=\boldsymbol{A}^{\boldsymbol{\top}}\left(\boldsymbol{P}_{N\left(s_{k}\right)-1}\left(s_{k}\right)+\boldsymbol{P}_{N\left(s_{k}\right)-1}\left(s_{k}\right)\left(\varepsilon_{f} \boldsymbol{I}-\boldsymbol{P}_{N\left(s_{k}\right)-1}\left(s_{k}\right)\right)^{-1} \boldsymbol{P}_{N\left(s_{k}\right)-1}\left(s_{k}\right)\right) \boldsymbol{A}+\boldsymbol{\varepsilon}_{f} \boldsymbol{F}^{\boldsymbol{\top}} \boldsymbol{F},
$$

se puede establecer que la función de Lyapunov será decreciente si $\boldsymbol{P}_{N\left(s_{k}\right)}\left(s_{k}\right)-\boldsymbol{P}\left(s_{k-1}\right) \prec \mathbf{0}$. Aplicando complementos de Schur, esta última condición se puede escribir como

$$
\left[\begin{array}{cc}
\boldsymbol{A}^{\boldsymbol{\top}} \boldsymbol{P}_{N\left(s_{k}\right)-1}\left(s_{k}\right) \boldsymbol{A}-\boldsymbol{P}\left(s_{k-1}\right)+\boldsymbol{\varepsilon}_{f} \boldsymbol{F}^{\boldsymbol{\top}} \boldsymbol{F} & \boldsymbol{A}^{\boldsymbol{\top}} \boldsymbol{P}_{N\left(s_{k}\right)-1}\left(s_{k}\right) \\
\boldsymbol{P}_{N\left(s_{k}\right)-1}\left(s_{k}\right) \boldsymbol{A} & \boldsymbol{P}_{N\left(s_{k}\right)-1}\left(s_{k}\right)-\boldsymbol{\varepsilon}_{f} \boldsymbol{I}
\end{array}\right] \prec \mathbf{0}
$$

El procedimiento a seguir ahora es el mismo que en la demostración del teorema 5.4.1: sustituir $\boldsymbol{P}_{N\left(s_{k}\right)-1}\left(s_{k}\right)$ en función de $\boldsymbol{P}_{N\left(s_{k}\right)-2}\left(s_{k}\right)$, aplicar complementos de Schur, y realizar esta operación de forma recursiva hasta llegar a sustituir $\boldsymbol{P}_{1}\left(s_{k}\right)$ en función de $\boldsymbol{P}_{h}\left(s_{k}\right)$. Con esto se llega a

$$
\begin{aligned}
& \boldsymbol{\Phi}_{1}\left(N\left(s_{k}\right), N\left(s_{k}\right)\right)^{\boldsymbol{\top}} \boldsymbol{P}_{h}\left(s_{k}\right) \boldsymbol{\Phi}_{1}\left(N\left(s_{k}\right), N\left(s_{k}\right)\right)-\operatorname{diag}\left\{\boldsymbol{P}\left(s_{k}\right), \mathbf{0}, \mathbf{0}, \ldots, \mathbf{0}\right\} \\
& \quad+\boldsymbol{\varepsilon}_{f} \sum_{i=0}^{N\left(s_{k}\right)-1} \boldsymbol{\Phi}_{1}\left(N\left(s_{k}\right), i\right)^{\boldsymbol{\top}} \boldsymbol{F}^{\boldsymbol{\top}} \boldsymbol{F} \boldsymbol{\Phi}_{1}\left(N\left(s_{k}\right), i\right)-\boldsymbol{\varepsilon}_{f} \operatorname{diag}\{\mathbf{0}, \boldsymbol{I}, \boldsymbol{I}, \ldots, \boldsymbol{I}\} \prec \mathbf{0}
\end{aligned}
$$

donde, sustituyendo $\boldsymbol{P}_{h}\left(s_{k}\right)$ y aplicando de nuevo los complementos de Schur se llega a

$$
\begin{aligned}
& {\left[\left(\begin{array}{c}
(\star)^{\top} \boldsymbol{P}\left(s_{k}\right) \underbrace{\star} \underbrace{\left(\boldsymbol{I}-\boldsymbol{L}\left(s_{k}\right) \boldsymbol{\Delta}\left(s_{k}\right) \boldsymbol{C}\right) \boldsymbol{\Phi}_{1}\left(N\left(s_{k}\right), N\left(s_{k}\right)\right)} \\
-\operatorname{diag}\left\{\boldsymbol{P}\left(s_{k}\right), \mathbf{0}, \mathbf{0}, \ldots, \mathbf{0}\right\}-\boldsymbol{M}_{1}\left(N\left(s_{k}\right)\right)
\end{array}\right)\right.} \\
& \boldsymbol{\Delta}\left(s_{k}\right)^{\top} \boldsymbol{L}\left(s_{k}\right)^{\top} \boldsymbol{P}\left(s_{k}\right)\left(\boldsymbol{I}-\boldsymbol{L}\left(s_{k}\right) \boldsymbol{\Delta}\left(s_{k}\right) \boldsymbol{C}\right) \boldsymbol{\Phi}_{1}\left(N\left(s_{k}\right), N\left(s_{k}\right)\right) \\
& \left.\begin{array}{c}
\boldsymbol{\Phi}_{1}\left(N\left(s_{k}\right), N\left(s_{k}\right)\right)^{\top}\left(\boldsymbol{I}-\boldsymbol{L}\left(s_{k}\right) \boldsymbol{\Delta}\left(s_{k}\right) \boldsymbol{C}\right)^{\top} \boldsymbol{P}\left(s_{k}\right) \boldsymbol{L}\left(s_{k}\right) \boldsymbol{\Delta}\left(s_{k}\right) \\
\boldsymbol{\Delta}\left(s_{k}\right)^{\top} \boldsymbol{L}\left(s_{k}\right)^{\top} \boldsymbol{P}\left(s_{k}\right) \boldsymbol{L}\left(s_{k}\right) \boldsymbol{\Delta}\left(s_{k}\right)-\boldsymbol{\varepsilon}_{h} \boldsymbol{I}
\end{array}\right] \prec \mathbf{0} .
\end{aligned}
$$

Para terminar la prueba se debe retomar la LMI (5.39) y aplicar la condición

$$
\boldsymbol{Q}\left(s_{k}\right)+\boldsymbol{Q}\left(s_{k}\right)^{\top}-\boldsymbol{P}\left(s_{k}\right) \preceq \boldsymbol{Q}\left(s_{k}\right) \boldsymbol{P}\left(s_{k}\right)^{-1} \boldsymbol{Q}\left(s_{k}\right)^{\top}
$$

para llegar a

$$
\left[\begin{array}{ccc}
\boldsymbol{Q}\left(s_{k}\right) \boldsymbol{P}\left(s_{k}\right)^{-1} \boldsymbol{Q}\left(s_{k}\right)^{\top} & \boldsymbol{Q}\left(s_{k}\right)\left(\boldsymbol{I}-\boldsymbol{L}\left(s_{k}\right) \boldsymbol{\Delta}\left(s_{k}\right) \boldsymbol{C}\right) \boldsymbol{\Phi}_{1}\left(N\left(s_{k}\right), N\left(s_{k}\right)\right) & \boldsymbol{X}\left(s_{k}\right) \boldsymbol{\Delta}\left(s_{k}\right) \\
\star & \boldsymbol{M}_{1}\left(N\left(s_{k}\right)\right) & \mathbf{0} \\
\star & \star & \boldsymbol{\varepsilon}_{h} \boldsymbol{I}
\end{array}\right] \succ \mathbf{0} .
$$

Sustituyendo $\boldsymbol{X}\left(s_{k}\right)$ por $\boldsymbol{Q}\left(s_{k}\right) \boldsymbol{L}\left(s_{k}\right)$ en esta matriz y aplicando los complementos de Schur se llega a (5.39), con lo que queda probado el teorema.

\subsubsection{Diseño basado en la atenuación de perturbaciones}

Teorema 5.6.2. Considérese el predictor (5.14) aplicado al sistema (5.1) y que cada $N_{k} \in \mathcal{N}$ periodos de control se tiene información de algunos de los sensores según lo indicado en la matriz de disponibilidad $\boldsymbol{\Delta}_{k} \in \Xi$, de manera que hay $n_{\mathcal{S}}$ escenarios de muestreo $s_{k} \in \mathcal{S}$ diferentes. Para unas $\boldsymbol{\Gamma}_{v}, \boldsymbol{\Gamma}_{w}>0$ dadas, supóngase que existen uunas matrices $\boldsymbol{P}\left(s_{k}\right)=\boldsymbol{P}\left(s_{k}\right)^{\top} \in \mathbb{R}^{n \times n}, \boldsymbol{Q}\left(s_{k}\right) \in \mathbb{R}^{n \times 1}, \boldsymbol{X}\left(s_{k}\right) \in \mathbb{R}^{n \times 1}$ y unos escalares $\varepsilon_{f}, \varepsilon_{h}>0$ tales que la siguiente desigualdad se cumple para cualquier secuencia de muestreo $\left\{s_{k}\right\}$

$$
\left[\begin{array}{cccc}
\boldsymbol{Q}\left(s_{k}\right)+\boldsymbol{Q}\left(s_{k}\right)^{\top}-\boldsymbol{P}\left(s_{k}\right) & \boldsymbol{Q}\left(s_{k}\right) \boldsymbol{\Phi}_{2}\left(N\left(s_{k}\right), N\left(s_{k}\right)\right) & \boldsymbol{X}\left(s_{k}\right) \boldsymbol{\Delta}\left(s_{k}\right) & \boldsymbol{X}\left(s_{k}\right) \boldsymbol{\Delta}\left(s_{k}\right) \\
\boldsymbol{\Phi}_{2}\left(N\left(s_{k}\right), N\left(s_{k}\right)\right)^{\top} \boldsymbol{Q}\left(s_{k}\right) & \left(\begin{array}{c}
\operatorname{diag}\left\{\boldsymbol{P}\left(s_{k-1}\right), \mathbf{0}, \ldots, \mathbf{0}\right\} \\
+\boldsymbol{M}_{2}\left(N\left(s_{k}\right)\right)
\end{array}\right. & \mathbf{0} & \mathbf{0} \\
\boldsymbol{\Delta}\left(s_{k}\right)^{\top} \boldsymbol{X}\left(s_{k}\right)^{\top} & \mathbf{0} & \boldsymbol{\varepsilon}_{h} \boldsymbol{I} & \mathbf{0} \\
\boldsymbol{\Delta}\left(s_{k}\right)^{\top} \boldsymbol{X}\left(s_{k}\right)^{\top} & \mathbf{0} & \mathbf{0} & \boldsymbol{\Gamma}_{w}^{2}
\end{array}\right] \succ \mathbf{0}
$$


Entonces, definiendo la ganancia del predictor como $\boldsymbol{L}\left(s_{k}\right)=\boldsymbol{Q}\left(s_{k}\right)^{-1} \boldsymbol{X}\left(s_{k}\right)$, el error de predicción converge a cero asintóticamente y, bajo condiciones iniciales nulas,

$$
\left\|\boldsymbol{e}_{k}\right\|_{2}^{2} \leq\left\|\boldsymbol{\Gamma}_{v} \boldsymbol{w}_{x}[t]\right\|_{2}^{2}+\left\|\boldsymbol{\Gamma}_{w} \boldsymbol{w}_{k}\right\|_{2}^{2}
$$

La prueba de este teorema es una generalización de la prueba del teorema 5.5 .2 (caso bifrecuencia), tomando la variable $N$ variante en función del parámetro de muestreo $s_{k}\left(N_{k} \equiv N\left(s_{k}\right)\right)$.

Prueba 5.6.2. Considérese el índice

$$
J=\sum_{k=0}^{\infty}\left(\boldsymbol{e}_{k}^{\top} \boldsymbol{e}_{k}-\sum_{j=1}^{N_{k}} \boldsymbol{v}\left[t_{k}-i\right]^{\top} \boldsymbol{\Gamma}_{v}^{2} \boldsymbol{v}\left[t_{k}-i\right]-\boldsymbol{w}_{k}^{\top} \boldsymbol{\Gamma}_{w}^{2} \boldsymbol{w}_{k}\right)
$$

Tomando la función de Lyapunov $\mathcal{V}_{k}=\mathcal{V}\left(\widetilde{\boldsymbol{x}}_{k}, s_{k}\right)=\widetilde{\boldsymbol{x}}_{k} \boldsymbol{P}\left(s_{k}\right) \widetilde{\boldsymbol{x}}_{k}$ y asumiendo condiciones iniciales nulas se tiene que

$$
\begin{aligned}
J & \leq \sum_{k=1}^{\infty}\left(\boldsymbol{e}_{k-1}^{\top} \boldsymbol{e}_{k-1}-\sum_{j=1}^{N_{k}} \boldsymbol{v}\left[t_{k}-j\right]^{\top} \boldsymbol{\Gamma}_{v}^{2} \boldsymbol{v}\left[t_{k}-j\right]-\boldsymbol{w}_{k}^{\top} \boldsymbol{\Gamma}_{w}^{2} \boldsymbol{w}_{k}\right)+\left.\mathcal{V}_{k}\right|_{k=\infty}-\left.\mathcal{V}_{k}\right|_{k=0} \\
& =\sum_{k=1}^{\infty}\left(\boldsymbol{e}_{k-1}^{\top} \boldsymbol{e}_{k-1}-\sum_{j=1}^{N_{k}} \boldsymbol{v}\left[t_{k}-j\right]^{\top} \boldsymbol{\Gamma}_{v}^{2} \boldsymbol{v}\left[t_{k}-j\right]-\boldsymbol{w}_{k}^{\top} \boldsymbol{\Gamma}_{w}^{2} \boldsymbol{w}_{k}+\Delta \mathcal{V}_{k}\right)
\end{aligned}
$$

Sustituyendo $\Delta \mathcal{V}_{k}$ por

$$
\begin{aligned}
\Delta \mathcal{V}_{k} & =\widetilde{\boldsymbol{x}}_{k}^{\top} \boldsymbol{P}\left(s_{k}\right) \widetilde{\boldsymbol{x}}_{k}-\widetilde{\boldsymbol{x}}_{k-1}^{\top} \boldsymbol{P}\left(s_{k-1}\right) \widetilde{\boldsymbol{x}}_{k-1} \\
& =\underbrace{\left(\left(\boldsymbol{I}-\boldsymbol{L}\left(s_{k}\right) \boldsymbol{\Delta}\left(s_{k}\right) \boldsymbol{C}\right) \widetilde{\boldsymbol{x}}\left[t_{k} \mid t_{k}-1\right]-\boldsymbol{L}\left(s_{k}\right) \widetilde{\boldsymbol{h}}\left[t_{k} \mid t_{k}-1\right]-\boldsymbol{L}\left(s_{k}\right) \boldsymbol{w}_{k}\right)^{\top} \boldsymbol{P}\left(s_{k}\right)(\star)-\widetilde{\boldsymbol{x}}_{k-1}^{\top} \boldsymbol{P}\left(s_{k-1}\right) \widetilde{\boldsymbol{x}}_{k-1}}_{\star}
\end{aligned}
$$

donde $\widetilde{\boldsymbol{x}}_{k} \equiv \widetilde{\boldsymbol{x}}\left[t_{k}\right]$ y $\widetilde{\boldsymbol{x}}_{k-1} \equiv \widetilde{\boldsymbol{x}}\left[t_{k}-N_{k}\right]$, se puede aplicar el teorema A.6.3 para eliminar el término $\boldsymbol{w}_{k}^{\top} \boldsymbol{\Gamma}_{w}^{2} \boldsymbol{w}_{k}$, quedando

$$
\begin{aligned}
J \leq & \sum_{k=1}^{\infty}\left(\boldsymbol{e}_{k-1}^{\top} \boldsymbol{e}_{k-1}-\sum_{j=1}^{N_{k}} \boldsymbol{v}\left[t_{k}-j\right]^{\top} \boldsymbol{\Gamma}_{v}^{2} \boldsymbol{v}\left[t_{k}-j\right]-\widetilde{\boldsymbol{x}}_{k-1}^{\top} \boldsymbol{P}\left(s_{k-1}\right) \widetilde{\boldsymbol{x}}_{k-1}\right. \\
& +\underbrace{\left.\left(\left(\boldsymbol{I}-\boldsymbol{L}\left(s_{k}\right) \boldsymbol{\Delta}\left(s_{k}\right) \boldsymbol{C}\right) \widetilde{\boldsymbol{x}}\left[t_{k} \mid t_{k}-1\right]-\boldsymbol{L}\left(s_{k}\right) \widetilde{\boldsymbol{h}}\left[t_{k} \mid t_{k}-1\right]\right)^{\top} \boldsymbol{P}_{w}\left(s_{k}\right)(\star)\right) .}_{\star} .
\end{aligned}
$$

con

$$
\boldsymbol{P}_{w}\left(s_{k}\right)=\boldsymbol{P}\left(s_{k}\right)+\boldsymbol{P}\left(s_{k}\right) \boldsymbol{L}\left(s_{k}\right)\left(\boldsymbol{\Gamma}_{w}^{2}-\boldsymbol{L}\left(s_{k}\right)^{\top} \boldsymbol{P}\left(s_{k}\right) \boldsymbol{L}\left(s_{k}\right)\right)^{-1} \boldsymbol{L}\left(s_{k}\right)^{\top} \boldsymbol{P}\left(s_{k}\right) .
$$

Aplicando ahora el teorema A.6.1 para eliminar el término $\widetilde{\boldsymbol{h}}\left[t_{k} \mid t_{k}-1\right]$ se tiene que

$J \leq \sum_{t=1}^{\infty}\left(\boldsymbol{e}_{k-1}^{\top} \boldsymbol{e}_{k-1}-\sum_{j=1}^{N_{k}} \boldsymbol{v}\left[t_{k}-j\right]^{\top} \boldsymbol{\Gamma}_{v}^{2} \boldsymbol{v}\left[t_{k}-j\right]-\widetilde{\boldsymbol{x}}_{k-1}^{\top} \boldsymbol{P}\left(s_{k-1}\right) \widetilde{\boldsymbol{x}}_{k-1}+\widetilde{\boldsymbol{x}}\left[t_{k} \mid t_{k}-1\right]^{\top} \boldsymbol{P}_{h}\left(s_{k}\right) \widetilde{\boldsymbol{x}}\left[t_{k} \mid t_{k}-1\right]\right)$

con

$$
\begin{aligned}
\boldsymbol{P}_{h}\left(s_{k}\right)= & \left(\boldsymbol{I}-\boldsymbol{L}\left(s_{k}\right) \boldsymbol{\Delta}\left(s_{k}\right) \boldsymbol{C}\right)^{\top} \boldsymbol{P}_{w}\left(s_{k}\right)\left(\boldsymbol{I}-\boldsymbol{L}\left(s_{k}\right) \boldsymbol{\Delta}\left(s_{k}\right) \boldsymbol{C}\right)+\boldsymbol{\varepsilon}_{h} \boldsymbol{H}^{\boldsymbol{\top}} \boldsymbol{H} \\
& +\underbrace{\left(\boldsymbol{I}-\boldsymbol{L}\left(s_{k}\right) \boldsymbol{\Delta}\left(s_{k}\right) \boldsymbol{C}\right)^{\top} \boldsymbol{P}_{w}\left(s_{k}\right) \boldsymbol{L}\left(s_{k}\right)}_{\star}\left(\varepsilon_{h} \boldsymbol{I}-\boldsymbol{L}\left(s_{k}\right)^{\top} \boldsymbol{P}_{w}\left(s_{k}\right) \boldsymbol{L}\left(s_{k}\right)\right)^{-1}(\star)^{\top} .
\end{aligned}
$$


Sustituyendo el error de estimación en bucle abierto por $\widetilde{\boldsymbol{x}}\left[t_{k} \mid t_{k}-1\right]=\boldsymbol{A} \widetilde{\boldsymbol{x}}\left[t_{k}-1\right]+\widetilde{\boldsymbol{f}}\left[t_{k}-1\right]+\boldsymbol{v}\left[t_{k}-1\right]$ y aplicando el teorema A.6.3 para eliminar el término $\boldsymbol{v}\left[t_{k}-1\right]^{\top} \boldsymbol{\Gamma}_{v}^{2} \boldsymbol{v}\left[t_{k}-1\right]$ se tiene que

$$
\begin{aligned}
J \leq \sum_{t=1}^{\infty} & \left(\boldsymbol{e}_{k-1}^{\top} \boldsymbol{e}_{k-1}-\sum_{j=2}^{N_{k}} \boldsymbol{v}\left[t_{k}-j\right]^{\top} \boldsymbol{\Gamma}_{v}^{2} \boldsymbol{v}\left[t_{k}-j\right]\right. \\
& \left.+\left(\boldsymbol{A} \widetilde{\boldsymbol{x}}\left[t_{k}-1\right]+\widetilde{\boldsymbol{f}}\left[t_{k}-1\right]\right)^{\top} \boldsymbol{P}_{x_{1}}\left(s_{k}\right)\left(\boldsymbol{A} \widetilde{\boldsymbol{x}}\left[t_{k}-1\right]+\widetilde{\boldsymbol{f}}\left[t_{k}-1\right]\right)-\widetilde{\boldsymbol{x}}_{k-1}^{\top} \boldsymbol{P}\left(s_{k-1}\right) \widetilde{\boldsymbol{x}}_{k-1}\right),
\end{aligned}
$$

con

$$
\boldsymbol{P}_{x_{1}}\left(s_{k}\right)=\boldsymbol{P}_{h}\left(s_{k}\right)+\boldsymbol{P}_{h}\left(s_{k}\right) \boldsymbol{B}_{v}\left(\boldsymbol{\Gamma}_{v}^{2}-\boldsymbol{B}_{v}^{\boldsymbol{\top}} \boldsymbol{P}_{h}\left(s_{k}\right) \boldsymbol{B}_{v}\right)^{-1} \boldsymbol{B}_{v}^{\top} \boldsymbol{P}_{h}\left(s_{k}\right) .
$$

Aplicando ahora el teorema A.6.1 se tiene que

$$
J \leq \sum_{k=1}^{\infty}\left(\boldsymbol{e}_{k-1}^{\top} \boldsymbol{e}_{k-1}-\sum_{j=2}^{N_{k}} \boldsymbol{v}\left[t_{k}-j\right]^{\top} \boldsymbol{\Gamma}_{v}^{2} \boldsymbol{v}\left[t_{k}-j\right]+\widetilde{\boldsymbol{x}}\left[t_{k}-1\right]^{\top} \boldsymbol{P}_{f_{1}}\left(s_{k}\right) \widetilde{\boldsymbol{x}}\left[t_{k}-1\right]-\widetilde{\boldsymbol{x}}_{k-1}^{\top} \boldsymbol{P}\left(s_{k-1}\right) \widetilde{\boldsymbol{x}}_{k-1}\right),
$$

con

$$
\boldsymbol{P}_{f_{1}}\left(s_{k}\right)=\boldsymbol{A}^{\boldsymbol{\top}}\left(\boldsymbol{P}_{x_{1}}\left(s_{k}\right)+\boldsymbol{P}_{x_{1}}\left(s_{k}\right)\left(\boldsymbol{\varepsilon}_{f}-\boldsymbol{P}_{x_{1}}\left(s_{k}\right)\right)^{-1} \boldsymbol{P}_{x_{1}}\left(s_{k}\right)\right) \boldsymbol{A}+\boldsymbol{\varepsilon}_{f} \boldsymbol{F}^{\boldsymbol{\top}} \boldsymbol{F} .
$$

Como en $t_{k}-1$ no hay medición, el error de estimación de este instante se relaciona con el instante anterior mediante $\widetilde{\boldsymbol{x}}\left[t_{k}-1\right]=\boldsymbol{A} \widetilde{\boldsymbol{x}}\left[t_{k}-2\right]+\widetilde{\boldsymbol{f}}\left[t_{k}-2\right]+\boldsymbol{v}\left[t_{k}-2\right]$. Sustituyendo este valor en (5.46) y aplicando el teorema A.6.3 para eliminar el término $\boldsymbol{v}\left[t_{k}-2\right]^{\top} \boldsymbol{\Gamma}_{v}^{2} \boldsymbol{v}\left[t_{k}-2\right]$ se tiene que

$$
\begin{aligned}
J \leq \sum_{t=1}^{\infty} & \left(\boldsymbol{e}_{k-1}^{\top} \boldsymbol{e}_{k-1}-\sum_{j=3}^{N_{k}} \boldsymbol{v}\left[t_{k}-j\right]^{\top} \boldsymbol{\Gamma}_{v}^{2} \boldsymbol{v}\left[t_{k}-j\right]\right. \\
& \left.+\left(\boldsymbol{A} \widetilde{\boldsymbol{x}}\left[t_{k}-2\right]+\widetilde{\boldsymbol{f}}\left[t_{k}-2\right]\right)^{\top} \boldsymbol{P}_{x_{2}}\left(s_{k}\right)\left(\boldsymbol{A} \widetilde{\boldsymbol{x}}\left[t_{k}-2\right]+\widetilde{\boldsymbol{f}}\left[t_{k}-2\right]\right)-\widetilde{\boldsymbol{x}}_{k-1}^{\top} \boldsymbol{P}\left(s_{k-1}\right) \widetilde{\boldsymbol{x}}_{k-1}\right),
\end{aligned}
$$

con

$$
\boldsymbol{P}_{x_{2}}\left(s_{k}\right)=\boldsymbol{P}_{f_{1}}\left(s_{k}\right)+\boldsymbol{P}_{f_{1}}\left(s_{k}\right) \boldsymbol{B}_{v}\left(\boldsymbol{\Gamma}_{v}^{2}-\boldsymbol{B}_{v}^{\top} \boldsymbol{P}_{f_{1}}\left(s_{k}\right) \boldsymbol{B}_{v}\right)^{-1} \boldsymbol{B}_{v}^{\top} \boldsymbol{P}_{f_{1}}\left(s_{k}\right) .
$$

Aplicando ahora el teorema A.6.1 se tiene que

$$
J \leq \sum_{k=1}^{\infty}\left(\boldsymbol{e}_{k-1}^{\top} \boldsymbol{e}_{k-1}-\sum_{j=3}^{N_{k}} \boldsymbol{v}\left[t_{k}-j\right]^{\top} \boldsymbol{\Gamma}_{v}^{2} \boldsymbol{v}\left[t_{k}-j\right]+\widetilde{\boldsymbol{x}}\left[t_{k}-2\right]^{\top} \boldsymbol{P}_{f_{2}}\left(s_{k}\right) \widetilde{\boldsymbol{x}}\left[t_{k}-2\right]-\widetilde{\boldsymbol{x}}_{k-1}^{\top} \boldsymbol{P}\left(s_{k-1}\right) \widetilde{\boldsymbol{x}}_{k-1}\right),
$$

con

$$
\boldsymbol{P}_{f_{2}}\left(s_{k}\right)=\boldsymbol{A}^{\boldsymbol{\top}}\left(\boldsymbol{P}_{x_{2}}\left(s_{k}\right)+\boldsymbol{P}_{x_{2}}\left(s_{k}\right)\left(\varepsilon_{f}-\boldsymbol{P}_{x_{2}}\left(s_{k}\right)\right)^{-1} \boldsymbol{P}_{x_{2}}\left(s_{k}\right)\right) \boldsymbol{A}+\boldsymbol{\varepsilon}_{f} \boldsymbol{F}^{\boldsymbol{\top}} \boldsymbol{F} .
$$

Aplicando este procedimiento de forma recursiva se llega finalmente a

$$
J \leq \sum_{k=1}^{\infty}\left(\boldsymbol{e}_{k-1}^{\top} \boldsymbol{e}_{k-1}+\widetilde{\boldsymbol{x}}\left[t_{k}-N_{k}\right]^{\top} \boldsymbol{P}_{f_{N_{k}}}\left(s_{k}\right) \widetilde{\boldsymbol{x}}\left[t_{k}-N_{k}\right]-\widetilde{\boldsymbol{x}}_{k-1}^{\top} \boldsymbol{P}\left(s_{k-1}\right) \widetilde{\boldsymbol{x}}_{k-1}\right),
$$

con

$$
\boldsymbol{P}_{f_{N_{k}}}=\boldsymbol{A}^{\boldsymbol{\top}}\left(\boldsymbol{P}_{x_{N_{k}}}+\boldsymbol{P}_{x_{N_{k}}}\left(\varepsilon_{f}-\boldsymbol{P}_{x_{N_{k}}}\right)^{-1} \boldsymbol{P}_{x_{N_{k}}}\right) \boldsymbol{A}+\boldsymbol{\varepsilon}_{f} \boldsymbol{F}^{\boldsymbol{\top}} \boldsymbol{F} .
$$

y

$$
\boldsymbol{P}_{x_{N_{k}}}\left(s_{k}\right)=\boldsymbol{P}_{f_{N_{k}-1}}\left(s_{k}\right)+\boldsymbol{P}_{f_{N_{k}-1}}\left(s_{k}\right) \boldsymbol{B}_{v}\left(\boldsymbol{\Gamma}_{v}^{2}-\boldsymbol{B}_{v}^{\top} \boldsymbol{P}_{f_{N_{k}-1}}\left(s_{k}\right) \boldsymbol{B}_{v}\right)^{-1} \boldsymbol{B}_{v}^{\top} \boldsymbol{P}_{f_{N_{k}-1}}\left(s_{k}\right) .
$$

Por otra parte, como $\boldsymbol{e}_{k-1}=\boldsymbol{g}\left(\boldsymbol{x}_{k-1}\right)-\boldsymbol{g}\left(\hat{\boldsymbol{x}}_{k-1}\right) \mathrm{y}$

$$
\boldsymbol{g}\left(\boldsymbol{x}_{k-1}\right)-\boldsymbol{g}\left(\hat{\boldsymbol{x}}_{k-1}\right) \leq\left\|\boldsymbol{G} \widetilde{\boldsymbol{x}}_{k-1}\right\|,
$$

se puede escribir que

$$
J \leq \sum_{t=1}^{\infty} \widetilde{\boldsymbol{x}}_{k-1}^{\top}\left(\boldsymbol{P}_{f_{N_{k}}}\left(s_{k}\right)+\boldsymbol{G}^{\top} \boldsymbol{G}-\boldsymbol{P}\left(s_{k-1}\right)\right) \widetilde{\boldsymbol{x}}_{k-1} .
$$


La condición (5.45) se cumplirá si $J<0$, condición que será cierta siempre que

$$
\boldsymbol{P}_{f_{N_{k}}}\left(s_{k}\right)+\boldsymbol{G}^{\top} \boldsymbol{G}-\boldsymbol{P}\left(s_{k-1}\right) \prec \mathbf{0} .
$$

Sustituyendo $\boldsymbol{P}_{f_{N_{k}}}\left(s_{k}\right)$ en función de $\boldsymbol{P}_{x_{N_{k}}}\left(s_{k}\right)$ y aplicando complementos de Schur se tiene que la condición anterior es equivalente a la condición

$$
\left[\begin{array}{cc}
\boldsymbol{A}^{\top} \boldsymbol{P}_{x_{N_{k}}}\left(s_{k}\right) \boldsymbol{A}-\boldsymbol{P}+\boldsymbol{G}^{\top} \boldsymbol{G}+\boldsymbol{\varepsilon}_{f} \boldsymbol{F}^{\boldsymbol{\top}} \boldsymbol{F} & \boldsymbol{A}^{\top} \boldsymbol{P}_{x_{N_{k}}}\left(s_{k}\right) \\
\boldsymbol{P}_{x_{N_{k}}}\left(s_{k}\right) \boldsymbol{A} & \boldsymbol{P}_{x_{N_{k}}}\left(s_{k}\right)-\boldsymbol{\varepsilon}_{f} \boldsymbol{I}
\end{array}\right] \prec \mathbf{0} .
$$

sustituyendo $\boldsymbol{P}_{x_{N_{k}}}\left(s_{k}\right)$ en función de $\boldsymbol{P}_{f_{N_{k}-1}}\left(s_{k}\right)$ y aplicando complementos de Schur se llega a

$$
\left[\begin{array}{ccc}
\boldsymbol{A}^{\boldsymbol{\top}} \boldsymbol{P}_{f_{N_{k}-1}}\left(s_{k}\right) \boldsymbol{A}-\boldsymbol{P}\left(s_{k-1}\right)+\boldsymbol{G}^{\boldsymbol{\top}} \boldsymbol{G}+\boldsymbol{\varepsilon}_{f} \boldsymbol{F}^{\boldsymbol{\top}} \boldsymbol{F} & \boldsymbol{A}^{\boldsymbol{\top}} \boldsymbol{P}_{f_{N_{k}-1}}\left(s_{k}\right) & \boldsymbol{A}^{\boldsymbol{\top}} \boldsymbol{P}_{f_{N_{k}-1}}\left(s_{k}\right) \\
\boldsymbol{P}_{f_{N_{k}-1}}\left(s_{k}\right) \boldsymbol{A} & \boldsymbol{P}_{f_{N_{k}-1}}\left(s_{k}\right)-\boldsymbol{\varepsilon}_{f} \boldsymbol{I} & \boldsymbol{P}_{f_{N_{k}-1}}\left(s_{k}\right) \\
\boldsymbol{P}_{f_{N_{k}-1}}\left(s_{k}\right) \boldsymbol{A} & \boldsymbol{P}_{f_{N_{k}-1}}\left(s_{k}\right) & \boldsymbol{P}_{f_{N_{k}-1}}\left(s_{k}\right)-\boldsymbol{\Gamma}_{v}^{2}
\end{array}\right] \prec \mathbf{0} .
$$

A partir de este momento, la prueba consiste en:

- sustituir $\boldsymbol{P}_{f_{i}}\left(s_{k}\right)$ en función de $\boldsymbol{P}_{x_{i}}\left(s_{k}\right)$,

- aplicar los complementos de Schur,

- sustituir $\boldsymbol{P}_{x_{i}}\left(s_{k}\right)$ en función de $\boldsymbol{P}_{f_{i-1}}\left(s_{k}\right)$,

- aplicar los complements de Schur,

y proceder de esta manera hasta tener una desigualdad en la que aparece $\boldsymbol{P}_{x_{1}}\left(s_{k}\right)$. Esta matriz se sustituye finalmente en función de $\boldsymbol{P}_{h}\left(s_{k}\right)$ y se aplican los complementos de Schur llegándose a

$$
\begin{gathered}
\boldsymbol{\Phi}_{2}\left(N_{k}, N_{k}\right)^{\top} \boldsymbol{P}_{h}\left(s_{k}\right) \boldsymbol{\Phi}_{2}\left(N_{k}, N_{k}\right)-\operatorname{diag}\left\{\boldsymbol{P}\left(s_{k-1}\right)+\boldsymbol{G}^{\boldsymbol{\top}} \boldsymbol{G}, \mathbf{0}, \ldots, \mathbf{0}\right\}-\boldsymbol{\varepsilon}_{f} \operatorname{diag}\{\mathbf{0}, \boldsymbol{I}, \mathbf{0}, \boldsymbol{I}, \ldots\} \\
+\boldsymbol{\varepsilon}_{f} \sum_{i=0}^{N_{k}-1} \boldsymbol{\Phi}_{2}\left(N_{k}, i\right)^{\boldsymbol{\top}} \boldsymbol{F}^{\boldsymbol{\top}} \boldsymbol{F} \boldsymbol{\Phi}_{2}\left(N_{k}, i\right)-\operatorname{diag}\left\{\mathbf{0}, \mathbf{0}, \boldsymbol{\Gamma}_{v}^{2}, \mathbf{0}, \boldsymbol{\Gamma}_{v}^{2}, \mathbf{0}, \ldots\right\} \prec \mathbf{0} .
\end{gathered}
$$

Nótese que se ha utilizado la notación $\boldsymbol{\Phi}_{2}\left(N_{k}, \cdot\right)$, en lugar de $\boldsymbol{\Phi}_{2}\left(N\left(s_{k}\right), \cdot\right)$ para simplificar la expresión. Sustituyendo $\boldsymbol{P}_{h}\left(s_{k}\right)$ en función de $\boldsymbol{P}_{w}\left(s_{k}\right)$ y aplicando complementos de Schur se llega a

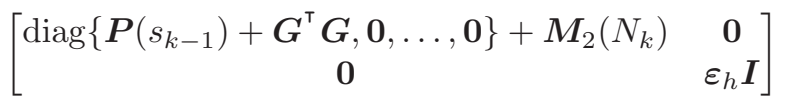

$$
\begin{aligned}
& -\left[\begin{array}{c}
\boldsymbol{\Phi}_{2}\left(N_{k}, N_{k}\right)^{\top}\left(\boldsymbol{I}-\boldsymbol{L}\left(s_{k}\right) \boldsymbol{\Delta}\left(s_{k}\right) \boldsymbol{C}\right)^{\top} \\
\boldsymbol{L}\left(s_{k}\right)^{\top}
\end{array}\right] \boldsymbol{P}_{w}\left(s_{k}\right)\left[\left(\boldsymbol{I}-\boldsymbol{L}\left(s_{k}\right) \boldsymbol{\Delta}\left(s_{k}\right) \boldsymbol{C}\right) \boldsymbol{\Phi}_{2}\left(N_{k}, N_{k}\right) \quad \boldsymbol{L}\left(s_{k}\right)\right] \succ \mathbf{0 .}
\end{aligned}
$$

Sustituyendo $\boldsymbol{P}_{w}\left(s_{k}\right)$ en función de $\boldsymbol{P}\left(s_{k}\right)$, aplicando dos veces consecutivas los complementos de Schur y teniendo en cuenta que $\boldsymbol{P} \boldsymbol{L}\left(s_{k}\right)=\boldsymbol{X}$ se llega finalmente a (5.44).

En este caso también es aplicable el procedimiento de diseño expuesto en la observación 5.4 .1 , teniendo en cuenta que en este caso las normas que se deben utilizar no son las normas $\left\|v_{i}[t]\right\|$ o $\left\|w_{i}[t]\right\|$ sino las normas $\left\|v_{i, k}\right\| \mathrm{y}\left\|w_{i, k}\right\|$, cuya relación con las anteriores debe obtenerse mediante las relaciones expuestas en las observaciones 4.5.3 y 4.5.6 (páginas 100 y 101, respectivamente).

\subsection{Consideraciones de diseño}

\subsubsection{Minimización del error en bucle abierto}

En los diseños de las secciones anteriores se ha tratado de minimizar la norma del error de estimación de la salida en el instante de medición $\left(\left\|\boldsymbol{e}_{k}\right\|\right)$. La norma del error de predicción entre mediciones se puede acotar teniendo en cuenta que el modelo se corre en bucle abierto, con lo que

$$
\begin{aligned}
\left\|\boldsymbol{e}\left[t_{k}+r\right]\right\| & =\| \boldsymbol{g}\left(\boldsymbol{x}\left[t_{k}+r\right]-\boldsymbol{g}\left(\hat{\boldsymbol{x}}\left[t_{k}+r\right]\right)\|\leq\| \boldsymbol{G} \widetilde{\boldsymbol{x}}\left[t_{k}+r\right] \|\right. \\
& =\left\|\boldsymbol{G}\left(\boldsymbol{A} \widetilde{\boldsymbol{x}}\left[t_{k}+r-1\right]+\widetilde{\boldsymbol{f}}\left[t_{k}+r-1\right]+\boldsymbol{v}\left[t_{k}+r-1\right]\right)\right\| \\
& \leq\|G\|(\|\boldsymbol{A}\|+\|\boldsymbol{F}\|)\left\|\widetilde{\boldsymbol{x}}\left[t_{k}+r-1\right]\right\|+\|\boldsymbol{G}\|\left\|\boldsymbol{v}\left[t_{k}+r-1\right]\right\|,
\end{aligned}
$$


con $r<N_{k+1}$. Sustituyendo de forma recursiva $\widetilde{\boldsymbol{x}}\left[t_{k}+r-1\right]$ hasta llegar a $\widetilde{\boldsymbol{x}}\left[t_{k}\right]$ se puede acotar la norma del error mediante

$$
\left\|\boldsymbol{e}\left[t_{k}+r\right]\right\| \leq\|G\|(\|\boldsymbol{A}\|+\|\boldsymbol{F}\|)^{r}\left\|\widetilde{\boldsymbol{x}}\left[t_{k}\right]\right\|+\sum_{i=1}^{r}\|\boldsymbol{G}\|(\|\boldsymbol{A}\|+\|\boldsymbol{F}\|)^{i-1}\left\|\boldsymbol{v}\left[t_{k}+r-i\right]\right\|,
$$

donde se observa que la norma del error de predicción entre mediciones será tanto menor como menor sea la norma $\left\|\widetilde{\boldsymbol{x}}\left[t_{k}\right]\right\|$. Si la escasez de medidas no es muy elevada ( $N_{k}$ pequeño), los algoritmos expuestos darán un buen funcionamiento, pero si la escasez de medidas es muy elevada y además el proceso cumple que

$$
\|\boldsymbol{A}\|+\|\boldsymbol{F}\| \geq 1
$$

lo que debería acotar el predictor es la norma del vector de error de estimación del estado. De esta forma se conseguirá un error entre mediciones (predicción en bucle abierto) menor. Para ello se han de replantear los diseños del capítulo sustituyendo $\boldsymbol{G}^{\top} \boldsymbol{G}$ por $\boldsymbol{I}$ en las LMI (véase la analogía de este planteamiento con el realizado en $\S 4.7 .5$

\subsubsection{Mediciones con retardo}

Si las mediciones disponibles llegan con un retardo $d_{i, k}$, pero éste es menor que el tiempo intermuestreo $\left(d_{i, k}<N_{k}, i=1, \ldots, n_{y}\right)$, es igualmente aplicable el algoritmo de predicción propuesto. El algoritmo de predicción quedaría en este caso de la siguiente forma. La estimación en bucle abierto se realizaría de forma normal

$$
\hat{\boldsymbol{x}}[t \mid t-1]=\boldsymbol{A} \hat{\boldsymbol{x}}[t-1]+\boldsymbol{f}(\hat{\boldsymbol{x}}[t-1], u[t-1]),
$$

y se almacenaría en un nuevo vector esta estimación junto a las obtenidas en instantes anteriores

$$
\hat{\mathbb{X}}[t \mid t-1]=\left[\begin{array}{c}
\hat{\boldsymbol{x}}[t \mid t-1] \\
\hat{\boldsymbol{x}}[t-1 \mid t-1] \\
\vdots \\
\hat{\boldsymbol{x}}[t-\alpha \mid t-1]
\end{array}\right]
$$

siendo $\alpha$ el máximo retardo posible que se puede dar en los sensores. Cuando en un instante $t_{k}$ hay una o más mediciones disponibles con retardos $d_{i, k}$, se actualiza el vector de estado almacenado en $\hat{\mathbb{X}}[t \mid t-1]$ correspondiente a ese instante mediante la ecuación de actualización

$$
\hat{\boldsymbol{x}}\left[t-d_{i, k} \mid t\right]=\hat{\boldsymbol{x}}\left[t-d_{i, k} \mid t-1\right]+\boldsymbol{\ell}_{i, k}\left(m_{i, k}-\boldsymbol{c}_{i} \hat{\boldsymbol{x}}\left[t-d_{i, k} \mid t-1\right]-g_{i}\left(\hat{\boldsymbol{x}}\left[t-d_{i, k} \mid t-1\right], \boldsymbol{u}\left[t-d_{i, k}\right]\right)\right)
$$

y se actualizan también todas las entradas del vector $\hat{\mathbb{X}}[t \mid t-1]$ corriendo el modelo en bucle abierto desde el instante $t-d_{i, k}$ hasta el siguiente instante del cual se tiene una medición $t-d_{j, k}\left(\operatorname{con} d_{j, k}<d_{i, k}\right)$ :

$\hat{\boldsymbol{x}}\left[t-d_{i, k}+l \mid t\right]=\boldsymbol{A} \hat{\boldsymbol{x}}\left[t-d_{i, k}+l-1 \mid t\right]+\boldsymbol{f}\left(\hat{\boldsymbol{x}}\left[t-d_{i, k}+l-1 \mid t\right], \boldsymbol{u}\left[t-d_{i, k}+l-1\right]\right), ; l=1,2, \ldots, d_{i, k}-d_{j, k}$.

Aplicando la ecuación (5.49) para los instantes en los que se dispone de una medición y (5.50) para los periodos intermuestreo, se obtiene finalmente la actualización de $\hat{\boldsymbol{x}}[t \mid t]$ para el instante $t=t_{k}$, y también la actualización del vector $\hat{\mathbb{X}}[t \mid t]$. Nótese la diferencia entre esta forma de incorporar mediciones con retardo, y la forma en que se incluían en 4.3.1, donde la corrección del estado a partir de la medición se hacía sobre la estimación del estado actual (utilizando para ello la expresión (4.13)), y no sobre el estado retardado que hay almacenado. La razón de no poder aplicar el método utilizado en sistemas lineales es que en el caso no lineal no existe una expresión equivalente a (4.13) que permita obtener la estimación de un instante pasado a partir de la estimación actual.

\subsubsection{Simplificaciones}

En el diseño de los predictores no lineales ante muestreo no convencional pueden aplicarse las técnicas de reducción de coste computacional expuestas en \$4.8.1 (página 124) teniendo en cuenta que no hay retardos. De esta manera pueden diseñarse, por ejemplo, predictores que aseguren sólo la estabilidad cuadrática, sin más que tomar $\boldsymbol{P}\left(s_{k}\right)=\boldsymbol{P}$ constante, o puede conseguirse un diseño con ganancia constante haciendo para ello $\boldsymbol{X}\left(s_{k}\right)=\boldsymbol{X}$ y $\boldsymbol{Q}\left(s_{k}\right)=\boldsymbol{Q}$ constantes. 


\subsection{Diseño de predictores basados en el filtro de Kalman extendido}

Considérese un proceso discreto no lineal descrito por las ecuaciones

$$
\begin{aligned}
\boldsymbol{x}[t+1] & =\boldsymbol{f}(\boldsymbol{x}[t], \boldsymbol{u}[t], \boldsymbol{v}[t]) \\
\boldsymbol{y}_{k} & =\boldsymbol{g}(\boldsymbol{x}[t]), \\
\boldsymbol{m}_{k} & =\boldsymbol{h}\left(\boldsymbol{x}\left[t_{k}\right]\right)+\boldsymbol{w}_{k},
\end{aligned}
$$

donde $\boldsymbol{x} \in \mathbb{R}^{n}$ es el vector de estados, $\boldsymbol{u} \in \mathbb{R}$ es la entrada, $\boldsymbol{y} \in \mathbb{R}$ es la salida del proceso a controlar y $\boldsymbol{m}_{k}=\left[m_{1, k} \cdots m_{n_{m}, k}\right]$ es el vector de mediciones de los sensores que le llega al controlador en cada periodo de control. $\boldsymbol{v}[t]$ es el vector de perturbaciones de entrada y $\boldsymbol{w}[t]=\left[w_{1, k} \cdots w_{n_{m}, k}\right]$ es el vector de ruidos de medida que afecta a cada sensor. Se asume que $\boldsymbol{v}$ es un ruido blanco de media cero con matriz de varianzas-covarianzas $\boldsymbol{W}\left(\boldsymbol{W}=\mathcal{E}\left\{\boldsymbol{v}[t] \boldsymbol{v}[t]^{\top}\right\}\right)$, y $\boldsymbol{w}$ es un ruido blanco de media cero con varianza $\boldsymbol{V}$ $\left(\boldsymbol{V}=\mathcal{E}\left\{\boldsymbol{w}[t] \boldsymbol{w}[t]^{\top}\right\}\right)$. Se asume que en los instantes de medición $t=t_{k}$ se miden $m_{k}$ sensores. Defínase la matriz $\boldsymbol{M}_{k}$ de orden $m_{k} \times n_{m}$ formada con las filas de una matriz identidad que corresponden a la posición de los elementos de $\boldsymbol{m}\left[t_{k}\right]$ que se miden en el instante $t_{k}$.

El algoritmo que minimiza la varianza del error de predicción viene definido por las ecuaciones

$$
\begin{aligned}
\hat{\boldsymbol{x}}[t \mid t-1] & =\boldsymbol{f}(\hat{\boldsymbol{x}}[t-1], \boldsymbol{u}[t-1], \mathbf{0}) \\
\boldsymbol{P}[t \mid t-1] & =\boldsymbol{A}[t] \boldsymbol{P}[t-1 \mid t-1] \boldsymbol{A}[t]^{\top}+\boldsymbol{B}_{v}[t] \boldsymbol{V} \boldsymbol{B}_{v}[t]^{\top},
\end{aligned}
$$

más las ecuaciones

$$
\begin{aligned}
\boldsymbol{L}_{k} & =\boldsymbol{P}[t \mid t-1]\left(\boldsymbol{M}_{k} \boldsymbol{C}_{k}\right)^{\boldsymbol{\top}}\left(\boldsymbol{M}_{k}\left(\boldsymbol{C}_{k} \boldsymbol{P}[t \mid t-1] \boldsymbol{C}_{k}^{\top}+\boldsymbol{W}\right) \boldsymbol{M}_{k}^{\boldsymbol{\top}}\right)^{-1} \\
\hat{\boldsymbol{x}}\left[t_{k} \mid t_{k}\right] & =\hat{\boldsymbol{x}}\left[t_{k} \mid t_{k}-1\right]+\sum_{i=1}^{n_{m}} \boldsymbol{\ell}_{i, k}\left(m_{i, k}-\boldsymbol{c}_{i} \hat{\boldsymbol{x}}\left[t_{k}-d_{i, k} \mid t_{k}-1\right]\right) \delta_{i, k} \\
\boldsymbol{P}\left[t_{k} \mid t_{k}\right] & =\left(\boldsymbol{I}-\boldsymbol{L}_{k} \boldsymbol{M}_{k} \boldsymbol{C}_{k}\right) \boldsymbol{P}\left[t_{k} \mid t_{k}-1\right]
\end{aligned}
$$

que se utilizan cuando se tiene alguna medición $m_{i, k}$ (en $t=t_{k}, \delta_{i, k}=1$ si el sensor $i$ está disponible, si no $\left.\delta_{i, k}=0\right)$, siendo

$$
\begin{aligned}
\boldsymbol{A}[t] & =\left.\frac{\partial \boldsymbol{f}}{\partial \boldsymbol{x}[t]}\right|_{(\hat{\boldsymbol{x}}[t], \boldsymbol{u}[t], \mathbf{0})}, \\
\boldsymbol{B}_{v}[t] & =\left.\frac{\partial \boldsymbol{f}}{\partial \boldsymbol{v}[t]}\right|_{(\hat{\boldsymbol{x}}[t], \boldsymbol{u}[t], \mathbf{0})}, \\
\boldsymbol{C}_{k} & =\left.\frac{\partial \boldsymbol{h}}{\partial \boldsymbol{x}\left[t_{k}\right]}\right|_{\left(\hat{\boldsymbol{x}}\left[t_{k} \mid t_{k}-1\right]\right)},
\end{aligned}
$$

matrices jacobianas.

Si no hay ninguna medición, la mejor estimación del estado y de la matriz de varianzas-covarianzas es la de bucle abierto, con lo que

$$
\begin{aligned}
\hat{\boldsymbol{x}}[t \mid t] & =\boldsymbol{x}[t \mid t-1], \\
\boldsymbol{P}[t \mid t] & =\boldsymbol{P}[t \mid t-1],
\end{aligned}
$$

La matriz de ganancias $\boldsymbol{L}_{k}$ tiene un tamaño $n \times m_{k}$ y $\boldsymbol{\ell}_{i, k}$ hace referencia a la $i$-ésima columna de la matriz $\boldsymbol{L}_{k} \boldsymbol{M}_{k}$, es decir la matriz formada por las columnas de $\boldsymbol{L}_{k}$ en las posiciones correspondientes a posiciones de sensores disponibles y por columnas nulas en las posiciones correspondientes a sensores no disponibles (véanse observaciones 2.3.1 y 4.5.1 en las páginas 21 y 97, respectivamente).

\subsection{Conclusiones}

En este capítulo se han establecido procedimientos de diseño de sensores virtuales para sistemas no lineales con mediciones escasas. El predictor propuesto utiliza el modelo no lineal del proceso y todas las mediciones disponibles para estimar la salida en todos los instantes de tiempo. 
Se han establecido procedimientos para obtener predictores estables y predictores capaces de atenuar las perturbaciones y ruidos de medida por medio de la norma $\mathcal{H}_{\infty}$. Para ello se asume que las funciones no lineales que definen el proceso cumplen con la condición de Lipschitz.

Para mostrar el correcto funcionamiento de los predictores de bajo coste propuestos se han realizado diversos ejemplos y se ha comparado su funcionamiento con el filtro de Kalman extendido. 


\section{Análisis de sistemas de control inferencial}

\subsection{Introducción}

En este capítulo se analiza la dinámica de lazo cerrado de un sistema de control inferencial (SCI) con muestreo irregular y retardos temporales. La estrategia de control se basa en la utilización de un controlador convencional a periodo $T$ combinado con un predictor de salidas de los analizados en los capítulos 3 y 4. El resultado principal de este capítulo es la existencia del principio de separación entre la dinámica del sensor virtual y la del bucle cerrado. De esta forma, el diseño del sistema de control inferencial queda simplificado, ya que el controlador y el predictor pueden diseñarse de forma separada.

Otro resultado interesante es el efecto que tienen las perturbaciones sobre las salidas controladas con ayuda de un predictor. Los resultados demuestran que la minimización del efecto de las perturbaciones sobre el error de predicción minimiza, a su vez, el efecto de las perturbaciones sobre el error de seguimiento en el sistema de control.

Para el desarrollo del capítulo se analizan en primer lugar los SCI basados en predictores con representación entrada salida (los desarrollados en el capítulo 3). Este análisis se restringe a sistemas no perturbados y sin retardos en el sistema de medición. Los resultados obtenidos se utilizan como base para el análisis en el caso general de sistemas con múltiples entradas, salidas y sensores que utilizan un predictor basado en la representación interna (de los desarrollados en el capítulo 4). Este caso incluye también el caso monovariable como caso especial y los resultados son extrapolables al caso de sistemas de control inferencial que incluyen un predictor basado en la representación entrada-salida, gracias a la relación existente entre ambos predictores (véase $\$ 4.3 .2$, página 89 ).

Algunos ejemplos muestran como un predictor adecuado mejora la atenuación de perturbaciones del lazo cerrado con respecto del sistema de control inferencial con predictor de bucle abierto presentado por otros autores como [36], con lo que queda solucionado el problema de inestabilidad que aparece cuando la dinámica del proceso es inestable.

\subsection{Análisis de sistemas de control inferencial basado en la repre- sentación entrada-salida}

\subsubsection{Planteamiento del problema}

\section{Esquema de control}

Considérese el sistema de control digital mostrado en la figura 6.1, donde $G(s)$ es un sistema lineal continuo monovariable cuya entrada se actualiza a periodo constante $T$ por medio de un ordenador con un retenedor de orden cero. Cada periodo de control llega una nueva medición $u[t]$, donde $t$ es el número de actualización de la entrada. La relación entre la señal continua y la discreta es

$$
u(\tau)=u[t], \quad t T \leq \tau<(t+1) T .
$$

La salida del proceso $y(\tau)$ se mide de forma síncrona con la actualización de la entrada pero a un periodo diferente no necesariamente constante. Los instantes en los cuales la salida $y[t]$ están disponibles vienen 
dados por la secuencia $\left\{t_{0}, t_{1}, \ldots, t_{k}, \ldots\right\}$, donde el instante $t_{k}$ indica el instante de tiempo en el cual está disponible la $k$-ésima medición $\left(m_{k}=y\left[t_{k}\right]\right)$. El número de actualizaciones de la entrada entre mediciones es $N_{k}=t_{k}-t_{k-1} \geq 1$.

En el diagrama de control, $C(z)$ representa el controlador digital convencional que opera a periodo $T$ e $\hat{y}[t]$ es la salida estimada que se envía al controlador para calcular la acción de control.

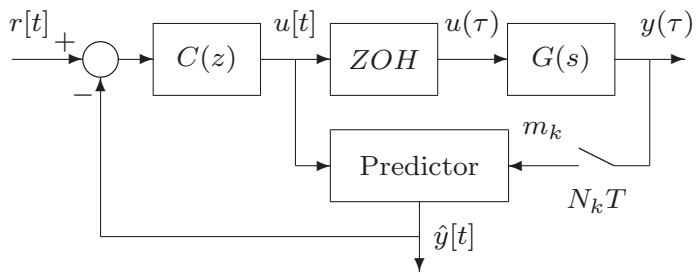

Figura 6.1: Esquema de control (sistemas monovariables).

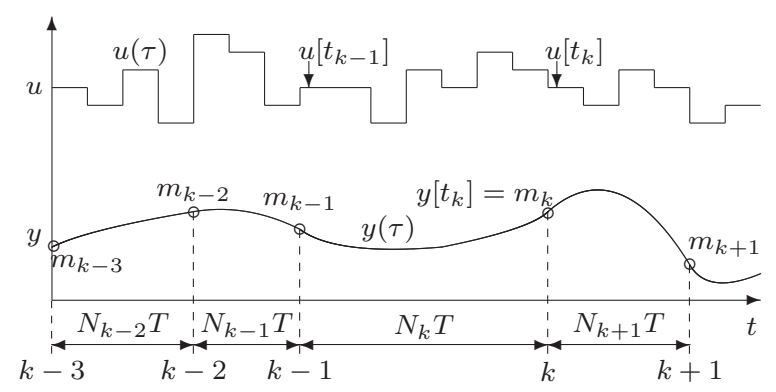

Figura 6.2: Muestreo síncrono irregular sin retardos.

\section{Planta}

El equivalente discreto de $G(s)$ con retenedor de orden cero a periodo $T$ se define mediante la ecuación en diferencias

$$
y[t]=-\boldsymbol{\theta}_{a}^{\top} Y[t-1]+\boldsymbol{\theta}_{b}^{\top} U[t-1],
$$

donde $\boldsymbol{\theta}_{a}=\left[\begin{array}{lll}a_{1} & \cdots & a_{n}\end{array}\right]^{\top}$ y $\boldsymbol{\theta}_{b}=\left[\begin{array}{lll}b_{1} & \cdots & b_{n}\end{array}\right]^{\top}$ son los vectores de parámetros, $Y[t-1]=[y[t-1] \cdots y[t-n]]^{\top}$ es el vector de salidas y $U[t-1]=[u[t-1] \cdots u[t-n]]^{\top}$ es el vector de entradas (cuyos elementos son los definidos es (6.1) ). La ecuación (6.2) puede reescribirse como ${ }^{1}$

$$
\begin{aligned}
Y[t] & =\left[\begin{array}{c}
-\boldsymbol{\theta}_{a}^{\boldsymbol{\top}} \\
\boldsymbol{I}_{(n-1) \times(n)}
\end{array}\right] Y[t-1]+\left[\begin{array}{c}
\boldsymbol{\theta}_{b}^{\boldsymbol{\top}} \\
\mathbf{0}_{(n-1) \times(n)}
\end{array}\right] U[t-1] \\
& =\boldsymbol{A} Y[t-1]+\boldsymbol{B} U[t-1] .
\end{aligned}
$$

\section{Estimación de salidas}

El controlador digital $C(z)$ necesita la secuencia de salidas a periodo $T$, pero la salida del proceso se mide de forma irregular a una frecuencia menor $y$, por tanto, se tienen que estimar las salidas entre muestreos. Para este propósito, se ha añadido un predictor de salidas basado en modelo. El estimador utiliza los parámetros del modelo del proceso, y todas las entradas anteriores, mediciones y estimaciones anteriores de la salida para obtener una estimación de las salidas no conocidas. Cuando no hay ninguna medición no disponible, la salida se estima corriendo el modelo en bucle abierto, llevando a

$$
\hat{Y}[t \mid t-1]=\boldsymbol{A} \hat{Y}[t-1]+\boldsymbol{B} U[t-1],
$$

donde $\hat{Y}[t \mid t-1]=[\hat{y}[t \mid t-1] \cdots \hat{y}[t-n+1 \mid t-1]]^{\top}$ es la estimación inicial del vector de regresión de salidas, y $\hat{Y}[t]=\hat{Y}[t \mid t]=[\hat{y}[t] y[t-1 \mid t] \cdots \hat{y}[t-n+1 \mid t]]^{\top}$ representa la estimación actualizada. Dependiendo de si se dispone de una nueva medición, el vector de regresión de salidas se actualiza mediante

$$
\hat{Y}[t]=\hat{Y}[t \mid t]=\hat{Y}[t \mid t-1]+\boldsymbol{\ell}[t](y[t]-\hat{y}[t \mid t-1]) \delta[t]
$$

\footnotetext{
${ }^{1} \boldsymbol{I}_{(n-1) \times(n)}$ son las primeras $n-1$ filas de una matriz identidad de orden $n, \mathrm{y} \mathbf{0}_{(n-1) \times(n)}$ es una matriz nula de orden $(n-1) \times(n)$.
} 
donde $\delta[t]$ es el factor de disponibilidad $(\delta[t]=1$ si la medición está disponible y $\delta[t]=0$ si no lo está). El vector de ganancias $\ell[t]=\left[l_{1}[t] \cdots l_{n}[t]\right]^{\top}$ debe diseñarse para asegurar la estabilidad de la dinámica del error de estimación de salidas. La estimación de la salida es el primer valor de $\hat{Y}[t]$ dado por $\hat{y}[t]=c \hat{Y}[t]$,

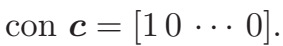

Aplicando el teorema 3.2 .1 con $d_{k}=0, v[t]=0$ y $w_{k}=0$ es fácil demostrar que la dinámica del error de predicción (3.8) aplicado al sistema (3.7) cuando no hay error de modelado viene definido por el sistema lineal variante en el tiempo (que se actualiza cada instante de medición)

$$
E_{k}=\left(\boldsymbol{I}-\boldsymbol{\ell}_{k} \boldsymbol{c}\right) \boldsymbol{A}^{N_{k}} E_{k-1}=\mathcal{A}_{k} E_{k-1}
$$

donde $N_{k}$ puede cambiar de forma arbitraria en el tiempo. El vector de error de estimación se define cuando hay una medición disponible $\left(t=t_{k}\right)$ como $E_{k}=Y\left[t_{k}\right]-\hat{Y}\left[t_{k}\right]$.

Definiendo la función de Lyapunov como $\mathcal{V}_{k}=E_{k}^{\top} \boldsymbol{P}_{k} E_{k}$, la existencia de una matriz $\boldsymbol{P}_{k} \succ \mathbf{0}$ tal que $\mathcal{V}_{k}<\mathcal{V}_{k-1}, \forall k$ es una condición necesaria para asegurar la convergencia del error de estimación. Utilizando la ecuación de la dinámica del error (6.5), esta condición es equivalente a

$$
\mathcal{A}_{k}^{\top} \boldsymbol{P}_{k} \mathcal{A}_{k}-\boldsymbol{P}_{k-1} \prec \mathbf{0}
$$

Esta condición estará asegurada siempre que el predictor se diseñe con cualquiera de las técnicas vistas en el capítulo 3 , prestando atención a que es necesario tomar $\boldsymbol{Q}\left(s_{k}\right)$ y $\boldsymbol{X}\left(s_{k}\right)$ constantes para obtener un vector de ganancias constante (véase observación 3.7.1).

\section{Controlador digital}

El controlador convencional monofrecuencia $C(z)$ se considera un sistema discreto lineal e invariante en el tiempo de orden $n$, que opera a periodo $T$. Se asume que la señal de referencia es nula, porque no es relevante para obtener la dinámica del bucle cerrado. La ecuación en diferencias del controlador puede expresarse como

$$
u[t]=-\boldsymbol{\theta}_{p}^{\top} U[t-1]+\boldsymbol{\theta}_{q}^{\top} \tilde{Y}[t-1]+q_{0} \hat{y}[t],
$$

donde $\boldsymbol{\theta}_{p}=\left[p_{1} \cdots p_{n}\right]^{\top}, \boldsymbol{\theta}_{q}=\left[q_{1} \cdots q_{n}\right]^{\top}$ y $q_{0}$ son los parámetros del controlador 2 , y

$$
\tilde{Y}[t-1]=[\hat{y}[t-1] \cdots \hat{y}[t-n]]^{\top}=[\hat{y}[t-1 \mid t-1] \cdots \hat{y}[t-n \mid t-n]]^{\top}
$$

es la historia de las estimaciones de la salida en cada periodo de control (nótese la diferencia con $\hat{Y}[t]$ ). La ecuación (6.7) puede escribirse de forma matricial como

$$
\begin{aligned}
U[t]= & {\left[\begin{array}{c}
-\boldsymbol{\theta}_{p}^{\top} \\
\boldsymbol{I}_{(n-1) \times(n)}
\end{array}\right] U[t-1]+\left[\begin{array}{c}
\boldsymbol{\theta}_{q}^{\top} \\
\mathbf{0}_{(n-1) \times(n)}
\end{array}\right] \tilde{Y}[t-1] } \\
& +q_{0} \boldsymbol{c}^{\top} \boldsymbol{c} \hat{Y}[t] \\
= & \boldsymbol{P} U[t-1]+\boldsymbol{Q} \tilde{Y}[t-1]+\boldsymbol{Q}_{0} \hat{Y}[t],
\end{aligned}
$$

donde $\boldsymbol{Q}_{0}$ es una matriz nula de orden $n \times n$ con el elemento $(1,1)$ igual a $q_{0}$.

\subsubsection{Dinámica del bucle cerrado}

En esta sección se obtiene la matriz variante en el tiempo que define la dinámica del bucle cerrado. Para ello, se establece una relación entre el vector de entradas, salidas y errores de estimación, primero al periodo de control $T$ y luego al periodo global variante en el tiempo $N_{k} T$.

\section{Dinámica del bucle cerrado con muestreo estándar}

Considérese un sistema en bucle cerrado de datos muestreados que funciona a periodo $T$ con la salida medida cada vez que se actualiza la acción de control. En este escenario no es necesario ningún predictor y el controlador sólo recibe salidas medidas $(\hat{y}[t]=y[t])$, con lo que $E_{k}$ es un vector nulo. La ecuación del controlador (6.8) puede combinarse con la ecuación (6.3) llegándose a

$$
U[t]=\left(\boldsymbol{P}+\boldsymbol{Q}_{0} \boldsymbol{B}\right) U[t-1]+\left(\boldsymbol{Q}+\boldsymbol{Q}_{0} \boldsymbol{A}\right) Y[t-1] .
$$

\footnotetext{
${ }^{2} \mathrm{Si}$ los polinomios del proceso y del controlador tienen diferente orden, $n$ será el mayor de los dos, y los vectores de parámetros se completarán con ceros.
} 
Combinando las ecuaciones del proceso (6.3) y del controlador (6.9), la dinámica del lazo puede expresarse como

$$
\left[\begin{array}{c}
Y[t+1] \\
U[t+1]
\end{array}\right]=\left[\begin{array}{cc}
\boldsymbol{A} & \boldsymbol{B} \\
\boldsymbol{Q}+\boldsymbol{Q}_{0} \boldsymbol{A} & \boldsymbol{P}+\boldsymbol{Q}_{0} \boldsymbol{B}
\end{array}\right]\left[\begin{array}{c}
Y[t] \\
U[t]
\end{array}\right] \equiv \boldsymbol{M}_{B C}\left[\begin{array}{c}
Y[t] \\
U[t]
\end{array}\right]
$$

donde los autovalores de la matriz $\boldsymbol{M}_{B C}$ definen el comportamiento en lazo cerrado. Si están dentro del círculo unitario, la estabilidad está asegurada.

\section{Reconstrucción de la señal de realimentación}

Para expresar la dinámica del bucle cerrado del sistema con estimador de salidas perdidas, las cuatro ecuaciones (6.3), (6.4) y (6.8) deben agruparse. Para este propósito, el vector de señales de realimentación $\tilde{Y}[t]$ debe expresarse como una función del vector de salidas $Y[t]$ y del vector error de estimación $E[t]$. Como $\hat{Y}[t]$ contiene las estimaciones actualizadas $\hat{y}[t-i \mid t]$, éstas deben relacionarse con las realimentadas $\hat{y}[t-i]=\hat{y}[t-i \mid t-i]$. El segundo elemento en $\hat{Y}[t]$ es

$$
\hat{y}[t-1 \mid t]=\hat{y}[t-1]+l_{2}[t](y[t]-\hat{y}[t \mid t-1]) \delta[t],
$$

siendo

que lleva a

$$
\hat{y}[t \mid t-1]=\frac{1}{1-l_{1}[t] \delta[t]}\left(\hat{y}[t]-l_{1}[t] y[t] \delta[t]\right)
$$

$$
\hat{y}[t-1 \mid t]=\hat{y}[t-1]+\frac{l_{2}[t] \delta[t]}{1-l_{1}[t]}(y[t]-\hat{y}[t]) .
$$

Cuando se expresa el tercer elemento de $\hat{Y}[t]$ como

$$
\hat{y}[t-2 \mid t]=\hat{y}[t-2 \mid t-1]+l_{3}[t](y[t]-\hat{y}[t \mid t-1]) \delta[t]
$$

los elementos $\hat{y}[t \mid t-1]$ y $\hat{y}[t-2 \mid t-1]$ deben obtenerse en función de $\hat{y}[t], \hat{y}[t-1]$ y $\hat{y}[t-2]$ llevando a

$$
\hat{y}[t-2 \mid t]=\hat{y}[t-2]+\frac{l_{3}[t](y[t]-\hat{y}[t]) \delta[t]+l_{2}[t](y[t-1]-\hat{y}[t-1]) \delta[t-1]}{1-l_{1}[t]} .
$$

Para una entrada genérica en $\hat{Y}[t]$ la expresión anterior toma la forma

$$
\hat{y}[t-i \mid t]=\hat{y}[t-i]+\sum_{j=0}^{i-1} \frac{l_{i-j+1}[t]}{1-l_{1}[t]}(y[t-j]-\hat{y}[t-j]) \delta[t-j]
$$

$i=1, \ldots, n-1$. Este conjunto de igualdades pueden unirse en la expresión

$$
\hat{Y}[t]=\boldsymbol{R}[t] \tilde{Y}[t]+(\boldsymbol{I}-\boldsymbol{R}[t]) Y[t]
$$

con

$$
\boldsymbol{R}[t]=\left[\begin{array}{ccccc}
1 & 0 & \cdots & \cdots & 0 \\
\frac{-l_{2}[t] \delta[t]}{1-l_{1}[t]} & 1 & \ddots & & \vdots \\
\frac{-l_{3}[t] \delta[t]}{1-l_{1}[t]} & \frac{-l_{2}[t-1] \delta[t-1]}{1-l_{1}[t-1]} & \ddots & \ddots & \vdots \\
\vdots & \vdots & \ddots & 1 & 0 \\
\frac{-l_{n}[t] \delta[t]}{1-l_{1}[t]} & \frac{-l_{n-1}[t-1] \delta[t-1]}{1-l_{1}[t-1]} & \cdots & \frac{-l_{2}[t-n+2] \delta[t-n+2]}{1-l_{1}[t-n+2]} & 1
\end{array}\right] .
$$

La relación entre el regresor de salidas realimentadas y el error de estimación puede establecerse como

$$
\tilde{Y}[t]=Y[t]-\boldsymbol{W}[t] E[t]
$$

donde $\boldsymbol{W}[t]$ es la inversa de $\boldsymbol{R}[t]$ que se define como

$$
\boldsymbol{W}[t]=\boldsymbol{R}[t]^{-1}=2 \boldsymbol{I}-\boldsymbol{R}[t],
$$

y toma la forma

$$
\boldsymbol{W}[t]=\left[\begin{array}{ccccc}
1 & 0 & \ldots & \ldots & 0 \\
\frac{l_{2}[t] \delta[t]}{1-l_{1}[t]} & 1 & \ddots & & \vdots \\
\frac{l_{3}[t] \delta[t]}{1-l_{1}[t]} & \frac{l_{2}[t-1] \delta[t-1]}{1-l_{1}[t-1]} & \ddots & \ddots & \vdots \\
\vdots & \vdots & \ddots & 1 & 0 \\
\frac{l_{n}[t] \delta[t]}{1-l_{1}[t]} & \frac{l_{n-1}[t-1] \delta[t-1]}{1-l_{1}[t-1]} & \ldots & \frac{l_{2}[t-n+2] \delta[t-n+2]}{1-l_{1}[t-n+2]} & 1
\end{array}\right]
$$




\section{Dinámica del bucle cerrado con muestreo irregular}

Teorema 6.2.1. Considérese el esquema de control mostrado en la figura 6.1 done se asume que el proceso es un sistema lineal monovariable de tiempo continuo definido por la ecuación (6.3). Se asume que el controlador es un sistema lineal invariante en el tiempo que opera a periodo $T$ y viene definido por la ecuación (6.8), y el estimador viene definido por las ecuaciones (6.4). Supóngase también un muestreo irregular síncrono como el mostrado en la figura 6.2. Entonces, la dinámica del bucle cerrado del sistema resultante actualizado en cada instante de medición viene definido por

$$
\boldsymbol{\xi}_{k}=\boldsymbol{\Gamma}_{k}\left(\prod_{j=1}^{N_{k}} \boldsymbol{M}\left[t_{k}-j\right]\right) \boldsymbol{\xi}_{k-1} \equiv \mathcal{M}_{k} \boldsymbol{\xi}_{k-1}
$$

con el vector de estado global $\boldsymbol{\xi}[t]=\left[E[t]^{\top} Y[t]^{\top} U[t]^{\top}\right]^{\top}$ y donde la matriz $\boldsymbol{M}[t]$ viene definida por

$$
M[t]=\left[\begin{array}{ccc}
\boldsymbol{A} & \mathbf{0} & \mathbf{0} \\
\mathbf{0} & \boldsymbol{A} & \boldsymbol{B} \\
-\left(\boldsymbol{Q W}[t]+\boldsymbol{Q}_{0} \boldsymbol{A}\right) & \boldsymbol{Q}+\boldsymbol{Q}_{0} \boldsymbol{A} & \boldsymbol{P}+\boldsymbol{Q}_{0} \boldsymbol{B}
\end{array}\right]
$$

y la matriz $\mathbf{\Gamma}$ está definida mediante

$$
\boldsymbol{\Gamma}_{k}=\left[\begin{array}{ccc}
\boldsymbol{I}-\boldsymbol{\ell}_{k} \boldsymbol{c} & \mathbf{0} & \mathbf{0} \\
\mathbf{0} & \boldsymbol{I} & \mathbf{0} \\
l_{1} \boldsymbol{Q}_{0} & \mathbf{0} & \boldsymbol{I}
\end{array}\right]
$$

Prueba 6.2.1. Para un instante arbitrario $t \neq t_{k}$ sin medición, el predictor (6.4) se aplica con $\delta[t]=0$ y la ecuación del controlador (6.8) se expresa como

$$
U[t]=\boldsymbol{P} U[t-1]+\boldsymbol{Q} \tilde{Y}[t-1]+\boldsymbol{Q}_{0}(\boldsymbol{A} \hat{Y}[t-1]+\boldsymbol{B} U[t-1])
$$

Aplicando la expresión (6.12) al vector $\tilde{Y}[t-1]$

$$
\begin{aligned}
U[t]= & \left(\boldsymbol{P}+\boldsymbol{Q}_{0} \boldsymbol{B}\right) U[t-1]+\left(\boldsymbol{Q}+\boldsymbol{Q}_{0} \boldsymbol{A}\right) Y[t-1] \\
& -\left(\boldsymbol{Q} \boldsymbol{W}[t-1]+\boldsymbol{Q}_{0} \boldsymbol{A}\right) E[t-1] .
\end{aligned}
$$

y agrupando las ecuaciones (6.3), (6.4) y (6.19) se obtiene la siguiente expresión cuando no hay medición disponible, es decir, cuando $t=t_{k-1}+1, \ldots, t_{k}-1$

$$
\boldsymbol{\xi}[t]=\boldsymbol{M}[t-1] \boldsymbol{\xi}[t-1]
$$

Cuando se tiene una nueva medición en el instante $t=t_{k}$, la expresión (6.8) conduce a

$$
\begin{gathered}
U\left[t_{k}\right]=\left(\boldsymbol{P}+\boldsymbol{Q}_{0} \boldsymbol{B}\right) U\left[t_{k}-1\right]+\left(\boldsymbol{Q}+\boldsymbol{Q}_{0} \boldsymbol{A}\right) Y\left[t_{k}-1\right] \\
-\left(\boldsymbol{Q} \boldsymbol{W}\left[t_{k}-1\right]+\boldsymbol{Q}_{0} \boldsymbol{A}\right) E\left[t_{k}-1\right]+\boldsymbol{Q}_{0} \boldsymbol{\ell}_{k} \boldsymbol{c} \boldsymbol{A} E\left[t_{k}-1\right],
\end{gathered}
$$

donde $\boldsymbol{Q}_{0} \boldsymbol{\ell} \boldsymbol{c}=l_{1} \boldsymbol{Q}_{0}$ debido a la forma especial de las matrices $\boldsymbol{c}$ y $\boldsymbol{Q}_{0}$. Agrupando las ecuaciones (6.3), (6.4) y (6.21) se puede escribir la siguiente expresión

$$
\boldsymbol{\xi}\left[t_{k}\right]=\boldsymbol{\Gamma}_{k} \boldsymbol{M}\left[t_{k}-1\right] \boldsymbol{\xi}\left[t_{k}-1\right]
$$

Aplicando la ecuación (6.20) de forma recursiva para $t=t_{k-1}+1, \ldots, t_{k}-1$ y la ecuación (6.22) para $t=t_{k}$, se obtiene la expresión deseada (6.15)

$$
\boldsymbol{\xi}\left[t_{k}\right]=\boldsymbol{\Gamma}_{k} \boldsymbol{M}\left[t_{k}-1\right] \boldsymbol{M}\left[t_{k}-2\right] \cdots \boldsymbol{M}\left[t_{k-1}\right] \boldsymbol{\xi}\left[t_{k-1}\right] .
$$




\subsubsection{Principio de separación}

En esta sección, se demuestra que el esquema de la figura 6.1 cumple con el principio de separación, en el sentido que la estabilidad del estimador (6.4), y del sistema en lazo cerrado estándar (6.10) son condiciones necesarias y suficientes para asegurar la estabilidad del sistema completo (6.15).

Observación 6.2.1 (Triangualarización del bucle cerrado). Escribiendo (6.16) como

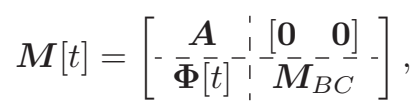

con

$$
\boldsymbol{\Phi}[t]=\left[\begin{array}{c}
\mathbf{0} \\
-\left(\boldsymbol{Q} \boldsymbol{W}[t]+\boldsymbol{Q}_{0} \boldsymbol{A}\right)
\end{array}\right]
$$

y $\boldsymbol{M}_{B C}$ la matriz de la dinámica del lazo cerrado con muestreo convencional (6.10), la ecuación de la dinámica en lazo cerrado (6.15) se define entonces mediante la matriz

$$
\mathcal{M}_{k}=\boldsymbol{\Gamma}_{k} \boldsymbol{M}\left[t_{k}-1\right] \cdots \boldsymbol{M}\left[t_{k-1}\right]=\left[\begin{array}{c:c}
\mathcal{A}_{k} & {\left[\begin{array}{ll}
\mathbf{0} & \mathbf{0}
\end{array}\right]} \\
\hdashline\left[\begin{array}{c}
\mathbf{0} \\
l_{1} \boldsymbol{Q}_{0}
\end{array}\right] \boldsymbol{A}^{N_{k}+\boldsymbol{\Delta}_{k}} & \boldsymbol{M}_{B C} N_{k}
\end{array}\right],
$$

donde $\mathcal{A}_{k}=\left(\boldsymbol{I}-\boldsymbol{\ell}_{k} \boldsymbol{c}\right) \boldsymbol{A}^{N_{k}}$ es la matriz (6.5) que define la dinámica del error de predicción, y

$$
\boldsymbol{\Delta}_{k}=\sum_{j=0}^{N_{k}} \boldsymbol{M}_{B C}{ }^{N_{k}-j} \boldsymbol{\Phi}[t+j] \boldsymbol{A}^{j}
$$

Teorema 6.2.2. Considérese un sistema lineal variante en el tiempo definido por la ecuación

$$
\boldsymbol{x}_{k}=\mathcal{M}_{k} \boldsymbol{x}_{k-1}
$$

donde la matriz $\mathcal{M}_{k}$ es una matriz particionada de la forma

$$
\mathcal{M}_{k}=\left[\begin{array}{cc}
\boldsymbol{A}_{k} & \mathbf{0} \\
\boldsymbol{B}_{k} & \boldsymbol{C}_{k}
\end{array}\right]
$$

donde las matrices $\boldsymbol{A}_{k}$ y $\boldsymbol{C}_{k}$ representan la dinámica de dos subsistemas que son parte del sistema global representado por $\boldsymbol{\mathcal { M }}_{k}$, siendo $\boldsymbol{A}_{k}$ una matriz invertible. Si existen unas matrices $\boldsymbol{Z}=\boldsymbol{Z}^{\top} \succ \mathbf{0}$ y $\boldsymbol{P}_{3, k}=$ $\boldsymbol{P}_{3, k}^{\top} \succ \mathbf{0}$ tales que

$$
\begin{aligned}
\boldsymbol{A}_{k}^{\top} \boldsymbol{Z}_{k} \boldsymbol{A}_{k}-\boldsymbol{Z}_{k-1} & \prec \mathbf{0}, \\
\boldsymbol{C}_{k}^{\boldsymbol{\top}} \boldsymbol{P}_{3, k} \boldsymbol{C}_{k}-\boldsymbol{P}_{3, k-1} & \prec \mathbf{0},
\end{aligned}
$$

es decir, los subsistemas $\boldsymbol{A}_{k}$ y $\boldsymbol{C}_{k}$ son policuadráticamente estables, entonces existe una matriz $\boldsymbol{P}_{k} \succ 0$ tal que

$$
\mathcal{M}_{k}^{\top} \boldsymbol{P}_{k} \mathcal{M}_{k}-\boldsymbol{P}_{k-1} \prec \mathbf{0},
$$

y, por tanto, el sistema $\boldsymbol{M}_{k}$ es policuadráticamente estable.

Reciprocamente, si existe una matriz $\boldsymbol{P}_{k} \succ \mathbf{0}$ tal que se cumple (6.27), entonces existen dos matrices $\boldsymbol{Z}_{k} \succ \mathbf{0}$ y $\boldsymbol{P}_{3, k} \succ \mathbf{0}$ tales que (6.25) y (6.26) son ciertas, y, por tanto, los subsistemas $\boldsymbol{A}_{k}$ y $\boldsymbol{C}_{k}$ son policuadráticamente estables.

Prueba 6.2.2. (Demostración de que si $\boldsymbol{M}_{k}$ es estable, entonces $\boldsymbol{A}_{k}$ y $\boldsymbol{C}_{k}$ son estables.) Si se divide la matriz $\boldsymbol{P}_{k}(6.27)$ en bloques de la forma

$$
\boldsymbol{P}=\left[\begin{array}{ll}
\boldsymbol{P}_{1} & \boldsymbol{P}_{2}^{\top} \\
\boldsymbol{P}_{2} & \boldsymbol{P}_{3}
\end{array}\right]
$$

entonces la existencia de una matriz $\boldsymbol{P}_{k}$ tal que se cumple (6.27) implica directamente (6.26) debido a la estructura triangular de la matriz (6.24). Si (6.27) tiene una solución $\boldsymbol{P}_{k} \succ \mathbf{0}$, entonces también se cumple la ecuación

$$
\mathcal{M}_{k} Q_{k-1} \mathcal{M}_{k}^{\top}-Q_{k} \prec \mathbf{0}
$$


con $\boldsymbol{Q}_{k}=\boldsymbol{P}_{k}^{-1}$. Si $\boldsymbol{Q}_{k}$ se divide en bloques

$$
\boldsymbol{Q}=\left[\begin{array}{ll}
\boldsymbol{Q}_{1, k} & \boldsymbol{Q}_{2, k}^{\top} \\
\boldsymbol{Q}_{2, k} & \boldsymbol{Q}_{3, k}
\end{array}\right]
$$

se comprueba que la desigualdad (6.28) implica

$$
\boldsymbol{A}_{k} \boldsymbol{Q}_{1, k-1} \boldsymbol{A}_{k}^{\top}-\boldsymbol{Q}_{1, k} \prec \mathbf{0} .
$$

Realizando el cambio de variable $\boldsymbol{Z}_{k}=\boldsymbol{Q}_{1, k}^{-1}$, y teniendo en cuenta la invertibilidad de $\boldsymbol{A}_{k}$, la desigualdad anterior implica (6.25).

(Demostración de que si $\boldsymbol{A}_{k}$ y $\boldsymbol{C}_{k}$ son estables, entonces $\boldsymbol{\mathcal { M }}_{k}$ es estable.) Supóngase que hay dos matrices $\boldsymbol{Z}_{k} \succ \mathbf{0}$ y $\boldsymbol{P}_{3, k} \succ \mathbf{0}$ tales que (6.25) y (6.26) se cumplen. Se va a demostrar que existe un $\gamma>0$ tal que la matriz

$$
\boldsymbol{P}_{k}=\left[\begin{array}{cc}
\gamma \boldsymbol{Z}_{k} & \mathbf{0} \\
\mathbf{0} & \boldsymbol{P}_{3_{k}}
\end{array}\right]
$$

satisface (6.27), con lo que la estabilidad del sistema (6.24) quedará probada. La ecuación (6.27) puede escribirse como

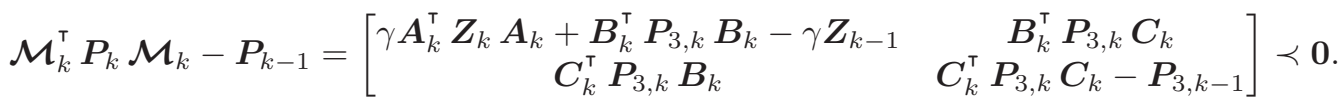

Utilizando los complementos de Schur es fácil probar que la condición anterior se cumple, (y, por tanto, el sistema (6.24) es estable) si $\gamma$ satisface

$$
\gamma\left(\boldsymbol{A}_{k}^{\top} \boldsymbol{M} \boldsymbol{A}_{k}-\boldsymbol{M}\right)+\boldsymbol{B}_{k}^{\top} \boldsymbol{P}_{3} \boldsymbol{B}_{k}-\boldsymbol{B}_{k}^{\top} \boldsymbol{P}_{3} \boldsymbol{C}_{k}\left(\boldsymbol{C}_{k}^{\top} \boldsymbol{P}_{3} \boldsymbol{C}_{k}-\boldsymbol{P}_{3}\right)^{-1} \boldsymbol{C}_{k}^{\top} \boldsymbol{P}_{3} \boldsymbol{B}_{k} \prec 0
$$

Como se satisface (6.25) y $\gamma>0$, el primer sumando es definido negativo. El término $\boldsymbol{B}_{k}^{\top} \boldsymbol{P}_{3, k} \boldsymbol{B}_{k}$ es definido positivo porque $\boldsymbol{P}_{3, k} \succ \mathbf{0}$. El último sumando es definido positivo porque la inversa de una matriz definida positiva también es definida positiva, y porque está pre y postmultiplicada por una matriz $\left(\boldsymbol{B}_{k}^{\boldsymbol{\top}} \boldsymbol{P}_{3, k} \boldsymbol{C}_{k}\right)$ y su traspuesta $\left(\boldsymbol{C}_{k}^{\boldsymbol{\top}} \boldsymbol{P}_{3, k} \boldsymbol{B}_{k}\right)$. Con esto, la condición (6.29) se cumple para cualquier $\gamma>0$ tal que

$$
\gamma \cdot \mu>\nu
$$

donde

$$
\mu=\lambda_{\min }\left(\boldsymbol{Z}_{k-1}-\boldsymbol{A}_{k}^{\top} \boldsymbol{Z}_{k} \boldsymbol{A}_{k}\right)
$$

y

$$
\nu=\lambda_{\text {máx }}\left(\boldsymbol{B}_{k}^{\boldsymbol{\top}} \boldsymbol{P}_{3, k} \boldsymbol{B}_{k}-\boldsymbol{B}_{k}^{\boldsymbol{\top}} \boldsymbol{P}_{3, k} \boldsymbol{C}_{k}\left(\boldsymbol{C}_{k}^{\boldsymbol{\top}} \boldsymbol{P}_{3, k} \boldsymbol{C}_{k}-\boldsymbol{P}_{3, k-1}\right)^{-1} \boldsymbol{C}_{k}^{\boldsymbol{\top}} \boldsymbol{P}_{3, k} \boldsymbol{B}_{k}\right)
$$

Como (6.25) y (6.26) se cumplen, dicho $\gamma$ siempre existe.

Teorema 6.2.3. Considérese la misma hipótesis descrita en el teorema 6.2.1. Supóngase que se cumplen las siguientes condiciones

(i) existe una matriz $\boldsymbol{P}_{k} \succ \mathbf{0}$ tal que

$$
\mathcal{A}_{k}^{\top} \boldsymbol{P}_{k} \mathcal{A}_{k}-\boldsymbol{P}_{k-1} \prec \mathbf{0}
$$

es decir, el error de predicción (cuya dinámica viene definida por la matriz $\mathcal{A}_{k}(6.5)$ ) converge a cero, $y$

(ii) existe una matriz $\boldsymbol{Q}[t] \succ \mathbf{0}$ tal que

$$
\boldsymbol{M}_{B C}^{\top} \boldsymbol{Q}[t] \boldsymbol{M}_{B C}-\boldsymbol{Q}[t-1] \prec \mathbf{0},
$$

es decir, el sistema en lazo cerrado con muestreo convencional (definido por la matriz $\boldsymbol{M}_{B C}(6.10)$ ) es estable. 
Entonces, existe una matriz $\boldsymbol{X}_{k} \succ \mathbf{0}$ tal que

$$
\mathcal{M}_{k}^{\top} \boldsymbol{X}_{k} \mathcal{M}_{k}-\boldsymbol{X}_{k-1} \prec \mathbf{0}
$$

es decir, el sistema en lazo cerrado descrito en (6.15) es estable.

Recíprocamente, si existe una matriz $\boldsymbol{X}_{k} \succ \mathbf{0}$ tal que se satisface la condición (6.32), entonces el subsistema del estimador (6.5), y el subsistema del lazo cerrado convencional (6.10) satisfacen las condiciones (i) y (ii) y, por tanto, ambos son estables.

Prueba 6.2.3. Como la dinámica del bucle cerrado viene definida por la matriz triangular en bloques (6.23), se puede aplicar directamente el teorema 6.2.2 a esta matriz, demostrando fácilmente el principio de separación para el sistema (6.15) que implica la existencia de una matriz $\boldsymbol{P}_{k} \succ \mathbf{0}$ tal que

$$
\mathcal{A}_{k}^{\top} \boldsymbol{P}_{k} \mathcal{A}_{k}-\boldsymbol{P}_{k-1} \prec \mathbf{0}
$$

y una matriz $\boldsymbol{Q}_{k} \succ \mathbf{0}$ tal que

$$
\left(\boldsymbol{M}_{B C}{ }^{N_{k}}\right)^{\top} \boldsymbol{Q}_{k} \boldsymbol{M}_{B C}{ }^{N_{k}}-\boldsymbol{Q}_{k-1} \prec \mathbf{0} .
$$

Falta comprobar que esta última desigualdad es equivalente a la desigualdad (6.31). Tómese el instante $t=t_{k}$ en el que ocurre la $k$-ésima medición. Si es sistema es estable se cumple que

$$
\boldsymbol{M}_{B C}^{\top} \boldsymbol{Q}\left[t_{k}\right] \boldsymbol{M}_{B C} \prec \boldsymbol{Q}\left[t_{k}-1\right] .
$$

Premultiplicando por $\boldsymbol{M}_{B C}^{\top}$ y postmultiplicando por su traspuesta la expresión anterior se tiene que

$$
\left(\boldsymbol{M}_{B C}^{\top}\right)^{2} \boldsymbol{Q}\left[t_{k}\right] \boldsymbol{M}_{B C}^{2} \prec \boldsymbol{M}_{B C}^{\top} \boldsymbol{Q}\left[t_{k}-1\right] \boldsymbol{M}_{B C} .
$$

si se aplica la condición de estabilidad (6.31), la expresión anterior implica que

$$
\left(\boldsymbol{M}_{B C}^{\top}\right)^{2} \boldsymbol{Q}\left[t_{k}\right] \boldsymbol{M}_{B C}^{2}-\boldsymbol{Q}\left[t_{k}-2\right] \prec \mathbf{0},
$$

con lo que aplicando esta propiedad $N_{k}$ veces se llega a

$$
\left(\boldsymbol{M}_{B C}^{\top}\right)^{N_{k}} \boldsymbol{Q}\left[t_{k}\right] \boldsymbol{M}_{B C}^{N_{k}}-\boldsymbol{Q}\left[t_{k}-N_{k}\right] \prec \mathbf{0},
$$

y aplicando la equivalencia en la notación $\boldsymbol{Q}\left[t_{k}\right] \equiv \boldsymbol{Q}_{k}$ se llega a

$$
\left(M_{B C}{ }^{N_{k}}\right)^{\top} \boldsymbol{Q}_{k} \boldsymbol{M}_{B C}{ }^{N_{k}}-\boldsymbol{Q}_{k-1} \prec \mathbf{0} .
$$

Este último resultado simplifica el procedimiento para comprobar la estabilidad cuadrática del sistema completo, porque es suficiente probar la estabilidad del estimador y la del lazo de control convencional. El diseño del sistema completo también queda simplificado, porque el estimador y el controlador pueden diseñarse de forma separada.

El esquema de control estudiado en esta sección (con el estimador propuesto basado en el modelo entrada-salida) no necesita que la planta sea estable en bucle abierto para tener un sistema en lazo cerrado estable, como se ilustra en los ejemplos.

\subsubsection{Ejemplos}

Para ilustrar los resultados anteriores, se analiza el comportamiento en dos ejemplos diferentes.

Ejemplo 6.2.1 (Control de un sistema estable). Considérese un sistema estable que puede aproximarse por la función de transferencia de segundo orden

$$
G(s)=\frac{10}{s^{2}+2 s+8}
$$




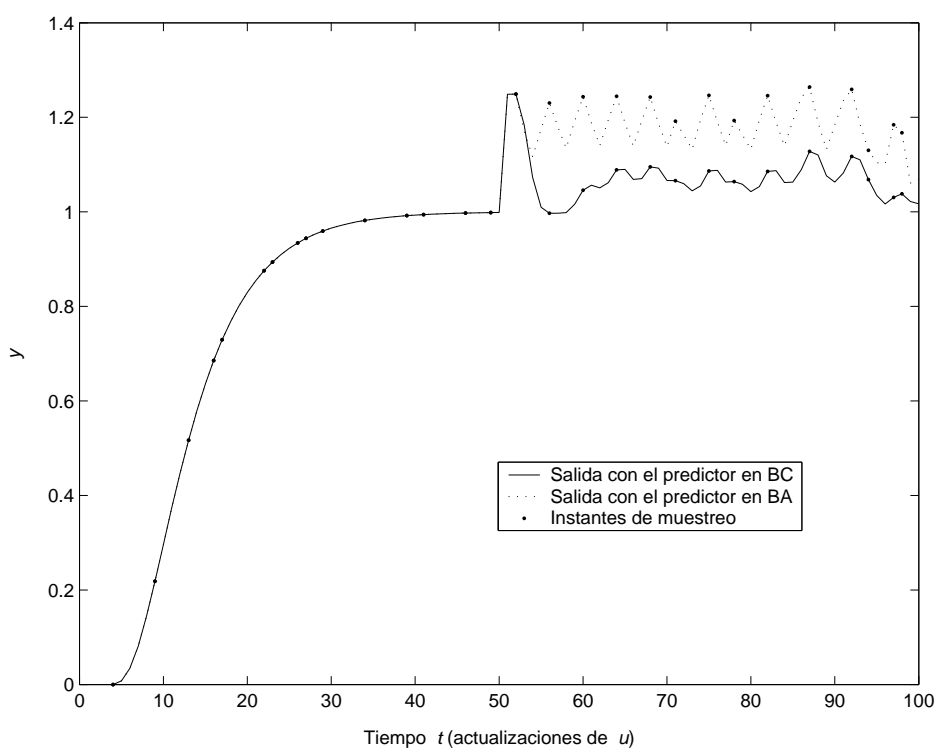

Figura 6.3: Respuesta ante escalón y rechazo de perturbación de salida.

Se asume que la entrada se actualiza a un periodo de $T=0.06$ segundos. La función de transferencia discreta equivalente con $\mathrm{ZOH}$ es

$$
G(z)=\frac{0.0173 z^{-1}+0.0166 z^{-2}}{1-1.86 z^{-1}+0.8869 z^{-2}}
$$

Diseñando un controlador digital $C(z)$ por asignación de polos para unos polos en bucle cerrado $m=$ $\{0.85,0.7,0.4,0.3,0.2\}$, se tiene

$$
C(z)=\frac{18.32-31.79 z^{-1}+13.92 z^{-2}}{\left(1-z^{-1}\right)\left(1+0.0937 z^{-1}+0.0161 z^{-2}\right)} .
$$

Supóngase que las salidas se miden cada $\nu$ periodos de entrada, con $\nu \in \mathcal{N}=\{1, \ldots, 5\}$ y que se calcula un estimador estable definido por el vector de ganancias constante con ayuda de uno de los métodos expuestos en el capítulo 3:

$$
\ell=\left[\begin{array}{l}
0.8314 \\
0.7921
\end{array}\right]
$$

Aplicando el teorema 6.2.3 se concluye que el sistema de control en lazo cerrado también es estable: el estimador y el lazo de control convencional son estables y, por tanto, se verifica la ecuación (6.32). La respuesta ante escalón y el rechazo a una perturbación del sistema de control se muestra en la figura 6.3 y se compara con el control inferencial de bucle abierto (estimador en bucle abierto, con $\boldsymbol{\ell}=\mathbf{0}$ ).

Ejemplo 6.2.2 (Control de un sistema inestable). Considérese ahora un sistema inestable definido por la función de transferencia

$$
G(s)=\frac{10}{s^{2}-s},
$$

en el que la entrada se actualiza a un periodo constante $T=0.03$ segundos. El equivalente discreto con $\mathrm{ZOH}$ es

$$
G(z)=\frac{0.0045 z^{-1}+0.0046 z^{-2}}{1-2.0305 z^{-1}+1.0305 z^{-2}}
$$

Supóngase que se mide la salida de forma aleatoria cada 2, 3, 4, 5 o 6 periodos de entrada. Se diseña un controlador digital mediante asignación de polos asignando los polos en lazo cerrado en $m=\{0.9,0.7,0.5\}$, llegándose a

$$
C(z)=\frac{27.05-25.407 z^{-1}}{1-0.1925 z^{-1}}
$$




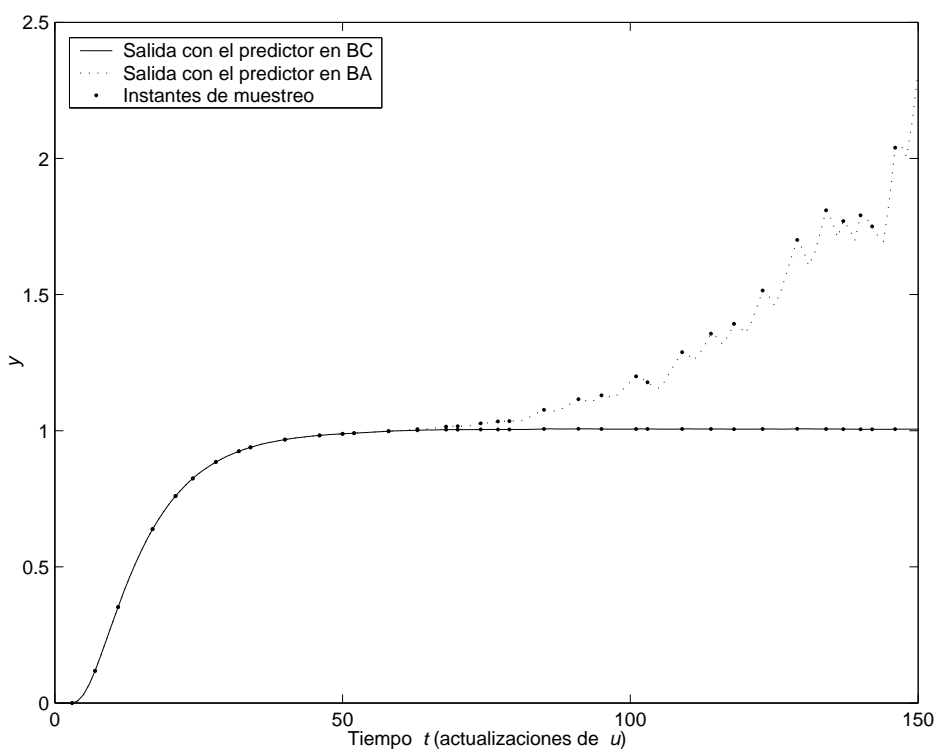

Figura 6.4: Respuesta ante escalón y rechazo de la perturbación a la entrada.

Para el estimador se calcula una ganancia

$$
\ell=\left[\begin{array}{c}
0.9489 \\
0.787
\end{array}\right]
$$

con ayuda de las técnicas expuestas en el capítulo 3, con lo que el predictor cumple con la condición necesaria de estabilidad policuadrática. La estabilidad del bucle cerrado se concluye aplicando el teorema 6.2.3. En este caso, si se utiliza un estimador en bucle abierto $(\boldsymbol{\ell}=\mathbf{0})$ el predictor no cumple con la condición de estabilidad policuadrática y, por tanto, la estrategia de control en bucle cerrado con este estimador en bucle abierto también es inestable.

La respuesta del sistema de control en lazo cerrado a un cambio de la referencia (en $t=0)$ y en la perturbación de la entrada (en $t=50)$ se muestra en la figura 6.4. El comportamiento (rechazo a la perturbación y seguimiento de la referencia) de la estrategia con predictor en lazo cerrado (-) es correcta. Sin embargo, la estrategia de estimador en bucle abierto (- -) lleva a una respuesta inestable debido a la dinámica inestable del proceso incluida en el lazo cerrado (la dinámica del predictor en bucle abierto coincide con la del proceso).

\subsection{Análisis de sistemas de control inferencial basado en la repre- sentación interna}

En esta sección se extienden los resultados obtenidos para sistemas multivariables con retardos en los diferentes sensores. En este caso sí que se tienen en consideración las perturbaciones y ruidos de medición para analizar la dinámica -comprobándose la existencia del principio de separación- y el efecto de las perturbaciones en el bucle cerrado.

\subsubsection{Planteamiento del problema}

\section{Esquema de control}

Considérese el sistema de control digital mostrado en la figura 6.5, donde el proceso es un sistema multivariable continuo cuyas entradas se actualizan a periodo $T$ por medio de un retenedor de orden cero. El vector de entradas $\boldsymbol{u}[t]$ se actualiza cada periodo de control, donde $t$ es el número de actualización de la entrada. La relación entre la señal discreta y la continua es

$$
\boldsymbol{u}(\tau)=\boldsymbol{u}[t] \quad \tau \in[t T, t T+T)
$$


Las mediciones del proceso $\left(m_{i}, i=1, \ldots, n_{m}\right)$ se toman de forma síncrona con la actualización de la entrada pero a un periodo no necesariamente constante, ni tampoco de forma simultánea. Las mediciones que llegan al sistema de control están afectadas de un retraso que puede ser variante en el tiempo. Los

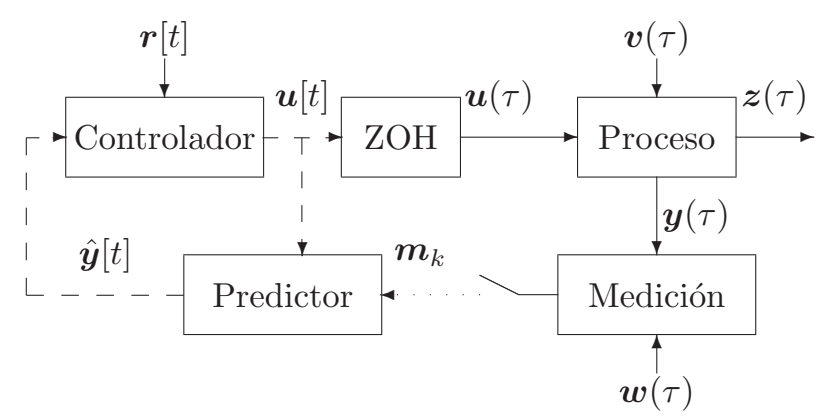

Figura 6.5: Sistema de control inferencial multivariable con el predictor propuesto.

instantes en los que llegan las mediciones están dados por la secuencia $\left\{t_{0}, t_{1}, \ldots, t_{k}, \ldots\right\}$, donde el instante $t_{k}$ indica el instante de tiempo en el cual está disponible el $k$-ésimo conjunto de mediciones de sensores. El número de actualizaciones de la entrada (periodos de control) entre mediciones disponibles es

$$
N_{k}=t_{k}-t_{k-1} \leq 1
$$

El controlador representado en el diagrama de control es un controlador digital que opera a periodo $T$ e $\hat{\boldsymbol{y}}[t]$ el vector de salidas virtuales estimado que se envía al controlador para calcular la acción de control.

\section{Planta}

El equivalente discreto $(\mathrm{ZOH})$ de la planta a periodo $T$, se define mediante las ecuaciones

$$
\begin{aligned}
\boldsymbol{x}[t+1] & =\boldsymbol{A} \boldsymbol{x}[t]+\boldsymbol{B} \boldsymbol{u}[t]+\boldsymbol{v}[t] \\
\boldsymbol{z}[t] & =\boldsymbol{C}_{z} \boldsymbol{x}[t]
\end{aligned}
$$

donde $\boldsymbol{A}, \boldsymbol{B}$ y $\boldsymbol{C}_{y}$ son las matrices del proceso, $\boldsymbol{x}$ es el vector de estados, $\boldsymbol{u}$ es el vector de entradas, $\boldsymbol{y}$ es el vector de salidas y $\boldsymbol{v}$ es la perturbación del estado. Las mediciones de los sensores sólo están disponibles en los instantes $t=t_{k}$, y vienen afectadas de un retardo variante en el tiempo quedando definidas por las ecuaciones

$$
m_{i, k}=\boldsymbol{c}_{i} \boldsymbol{x}\left[t_{k}-d_{i, k}\right]+w_{i, k}, \quad i=1, \ldots, n_{m},
$$

donde $m_{i, k}$ es la medición del sensor $i$ en el instante de medición $k$, que viene afectada de un retardo $d_{i, k}$ y un ruido $w_{i, k}$. Los sensores activos en cada instante vienen definidos por la matriz de disponibilidad

$$
\boldsymbol{\Delta}_{k}=\left[\begin{array}{lll}
\delta_{1}\left[t_{k}\right] & & \\
& \ddots & \\
& & \delta_{n_{m}}\left[t_{k}\right]
\end{array}\right]
$$

\section{Predictor}

El controlador digital necesita la secuencia de salidas a periodo $T$, pero sólo se miden algunas señales del proceso de forma irregular, con un periodo menor y afectadas de un retardo. Por tanto, la salida debe ser estimada. Para ello se incluye en el lazo cerrado el predictor de salidas introducido en $\$ 4.3$ y que viene definido por la ecuación de bucle abierto

$$
\hat{\boldsymbol{x}}[t \mid t-1]=\boldsymbol{A} \hat{\boldsymbol{x}}[t-1]+\boldsymbol{B} \boldsymbol{u}[t-1],
$$

más la ecuación de actualización (cuando hay algún $m_{i}$ disponible)

$$
\hat{\boldsymbol{x}}\left[t_{k}\right]=\hat{\boldsymbol{x}}\left[t_{k} \mid t_{k}-1\right]+\sum_{i=1}^{n_{m}} \boldsymbol{\ell}_{i}\left[t_{k}\right]\left(m_{i}\left[t_{k}\right]-\boldsymbol{c}_{i} \hat{\boldsymbol{x}}\left[t_{k}-d_{i, k} \mid t_{k}-1\right]\right) \delta_{i}\left[t_{k}\right]
$$


Si no se tienen mediciones, el estado se actualiza mediante

$$
\hat{\boldsymbol{x}}[t]=\hat{\boldsymbol{x}}[t \mid t-1]
$$

La salida que se envía al controlador digital viene finalmente dada por

$$
\hat{\boldsymbol{y}}[t]=\boldsymbol{C}_{y} \hat{\boldsymbol{x}}[t]
$$

La dinámica del error de predicción entre instantes de medición viene dada por

$$
\begin{aligned}
\widetilde{\boldsymbol{x}}_{k} & =\mathcal{A}_{k} \widetilde{\boldsymbol{x}}_{k-1}+\mathcal{B}_{k}\left[\begin{array}{c}
\mathrm{V}_{x, k} \\
\boldsymbol{w}_{k}
\end{array}\right] \\
\boldsymbol{e}_{k} & =\boldsymbol{C}_{y} \widetilde{\boldsymbol{x}}_{k},
\end{aligned}
$$

donde

$$
\begin{aligned}
& \boldsymbol{A}_{k}=\left(\boldsymbol{I}-\boldsymbol{L}_{k} \boldsymbol{\Delta}_{k} \boldsymbol{C}_{d, k}\right) \boldsymbol{A}^{N_{k}}, \quad \boldsymbol{C}_{d, k}=\left[\begin{array}{c}
\boldsymbol{c}_{1} \boldsymbol{A}^{-d_{1, k}} \\
\vdots \\
\boldsymbol{c}_{n_{m}} \boldsymbol{A}^{-d_{n_{m}, k}}
\end{array}\right]_{n_{m} \times n}, \\
& \boldsymbol{B}_{k}=\left[\boldsymbol{\Lambda}\left(\alpha_{k}, N_{k}\right)-\boldsymbol{L}_{k} \boldsymbol{\Delta}_{k} \mathcal{C}_{d, k} \quad-\boldsymbol{L}_{k} \boldsymbol{\Delta}_{k}\right]
\end{aligned}
$$

siendo $\boldsymbol{\Delta}_{k} \equiv \boldsymbol{\Delta}\left[t_{k}\right]$ la matriz de disponibilidad, y

$$
\mathcal{C}_{d, k}=\left[\begin{array}{c}
\boldsymbol{c}_{1} \boldsymbol{A}^{-d_{1, k}}\left(\boldsymbol{\Lambda}\left(\alpha_{k}, N_{k}\right)-\boldsymbol{\Lambda}\left(\alpha_{k}, d_{1, k}\right)\right) \\
\vdots \\
\boldsymbol{c}_{n_{m}} \boldsymbol{A}^{-d_{n_{m}}}\left(\boldsymbol{\Lambda}\left(\alpha_{k}, N_{k}\right)-\boldsymbol{\Lambda}\left(\alpha_{k}, d_{n_{m}, k}\right)\right)
\end{array}\right]_{n_{m} \times \alpha_{k} n},
$$

$\operatorname{con} \boldsymbol{\Lambda}(\alpha, N)$ la matriz definida como

$$
\boldsymbol{\Lambda}(\alpha, N)=[\underbrace{\boldsymbol{I} \boldsymbol{A} \boldsymbol{A}^{2} \cdots \boldsymbol{A}^{N-1} \mathbf{0} \cdots \mathbf{0}}_{\alpha}]_{n \times \alpha n} .
$$

Observación 6.3.1. La dinámica del error de predicción entre instantes de control (a periodo $T$ ) cuando no se tiene ninguna medición de la salida, viene dada por

$$
\widetilde{\boldsymbol{x}}[t]=\boldsymbol{A} \widetilde{\boldsymbol{x}}[t-1]+\boldsymbol{v}[t-1],
$$

mientras que cuando se tiene alguna medición de la salida viene dada por

$$
\widetilde{\boldsymbol{x}}\left[t_{k}\right]=\left(\boldsymbol{I}-\boldsymbol{L}_{k} \boldsymbol{\Delta}_{k} \boldsymbol{C}_{d, k}\right)\left(\boldsymbol{A} \widetilde{\boldsymbol{x}}\left[t_{k}-1\right]+\boldsymbol{v}\left[t_{k}-1\right]\right)+\boldsymbol{L}_{k} \boldsymbol{\Delta}_{k} \overline{\boldsymbol{C}}_{d, k} \mathbb{V}_{x, k}-\boldsymbol{L}_{k} \boldsymbol{\Delta}_{k} \boldsymbol{w}_{m, k}
$$

con

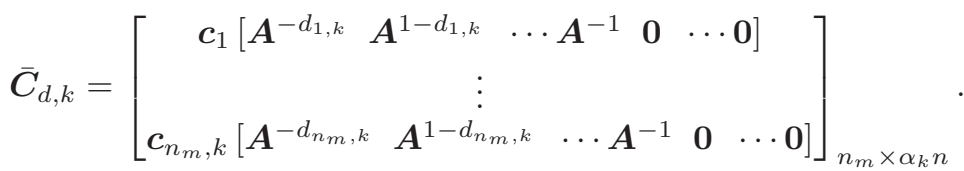

Esta expresión se puede obtener haciendo $N_{k}=1$ en (6.35), o restando la ecuación de estado del proceso y la ecuación de actualización (6.34b) del predictor

\section{Controlador digital}

El controlador digital opera a periodo $T$ y utiliza la estimación de la salida para calcular la acción de control. Se asume que la referencia es nula porque no es relevante para obtener la dinámica del lazo cerrado. El controlador viene definido por las ecuaciones

$$
\begin{aligned}
\boldsymbol{\eta}[t+1] & =\boldsymbol{A}_{c} \boldsymbol{\eta}[t]+\boldsymbol{B}_{c} \hat{\boldsymbol{y}}[t] \\
\boldsymbol{u}[t] & =\boldsymbol{C}_{c} \boldsymbol{\eta}[t]+\boldsymbol{D}_{c} \hat{\boldsymbol{y}}[t] .
\end{aligned}
$$




\subsubsection{Dinámica del bucle cerrado con muestreo periódico}

\section{Sistemas sin retardos}

Considérese un sistema de control muestreado estándar corriendo a periodo $T$ en el que todas las salidas se miden $\left(\boldsymbol{\Delta}_{k}=\boldsymbol{I}\right)$ sin retardo $\left(d_{i, k}=0\right)$ cada vez que se actualizan las acciones de control $\left(N_{k}=1\right)$ y, por tanto, no se usa ningún predictor. En este caso, el controlador sólo recibe mediciones de la salida afectadas de ruido de medida $(\hat{\boldsymbol{y}}[t]=\boldsymbol{y}[t]+\boldsymbol{w}[t])$. La ecuación del proceso (6.33) puede combinarse con la del controlador (6.37) (con $\boldsymbol{y}[t]$ ) para obtenerse la ecuación que determina la dinámica en bucle cerrado:

$$
\begin{aligned}
{\left[\begin{array}{c}
\boldsymbol{x}[t+1] \\
\boldsymbol{\eta}[t+1]
\end{array}\right] } & =\underbrace{\left[\begin{array}{cc}
\boldsymbol{A}+\boldsymbol{B D}_{c} \boldsymbol{C}_{y} & \boldsymbol{B} \boldsymbol{C}_{c} \\
\boldsymbol{B}_{c} \boldsymbol{C}_{y} & \boldsymbol{A}_{c}
\end{array}\right]}_{=\boldsymbol{M}_{B C}}\left[\begin{array}{c}
\boldsymbol{x}[t] \\
\boldsymbol{\eta}[t]
\end{array}\right]+\left[\begin{array}{cc}
\boldsymbol{I} & \boldsymbol{B} \boldsymbol{D}_{c} \\
\mathbf{0} & \boldsymbol{B}_{c}
\end{array}\right]\left[\begin{array}{c}
\boldsymbol{v}[t] \\
\boldsymbol{w}[t]
\end{array}\right], \\
\boldsymbol{z}[t] & =\left[\begin{array}{ll}
\boldsymbol{C}_{z} & \mathbf{0}
\end{array}\right]\left[\begin{array}{l}
\boldsymbol{x}[t] \\
\boldsymbol{\eta}[t]
\end{array}\right] .
\end{aligned}
$$

El comportamiento dinámico viene determinado por los autovalores de la matriz $\boldsymbol{M}_{B C}$. Si están dentro del círculo unitario, la estabilidad está asegurada.

Efecto de las perturbaciones Como la ecuación de la dinámica en bucle cerrado presentada anteriormente es una ecuación lineal e invariante, se pueden aplicar herramientas estándar de análisis.

Para analizar el efecto de las perturbaciones, aplíquese la transformada en $\mathcal{Z}$ en (6.38a) para obtener

$$
\boldsymbol{z}(z)=\left[\begin{array}{ll}
\boldsymbol{C}_{z} & \mathbf{0}
\end{array}\right]\left(z \boldsymbol{I}-\boldsymbol{M}_{B C}\right)^{-1}\left(\left[\begin{array}{l}
\boldsymbol{I} \\
\mathbf{0}
\end{array}\right] \boldsymbol{v}(z)+\mathbf{\Phi} \boldsymbol{w}(z)\right)
$$

con

$$
\boldsymbol{\Phi}=\left[\begin{array}{c}
\boldsymbol{B} \boldsymbol{D}_{c} \\
\boldsymbol{B}_{c}
\end{array}\right]
$$

Esta expresión será retomada posteriormente para comparar el efecto de las perturbaciones y el ruido de medida, cuando el muestreo es estándar y cuando éste es irregular y con retardos.

\section{Sistemas con retardos}

Predictor Considérese ahora la situación de muestreo en la que se tienen las mediciones de los sensores $\left(\boldsymbol{\Delta}_{k}=\boldsymbol{I}\right)$ en todos los periodos de control $\left(N_{k}=1\right)$ afectadas por un retardo constante $\left(d_{i, k}=d\right)$. Para obtener una estimación de las salidas que necesita el controlador se utiliza un predictor de los definidos por el algoritmo (6.34). En este caso la dinámica del error de predicción es invariante en el tiempo y viene determinada por las ecuaciones

$$
\begin{aligned}
\widetilde{\boldsymbol{x}}[t+1] & =\underbrace{\left(\boldsymbol{I}-\boldsymbol{L} \boldsymbol{C}_{d}\right) \boldsymbol{A}}_{=\mathcal{A}} \tilde{\boldsymbol{x}}[t]+\left(\boldsymbol{I}-\boldsymbol{L} \boldsymbol{C}_{d}\right) \boldsymbol{v}[t]+\boldsymbol{L} \overline{\boldsymbol{C}}_{d} \mathbb{V}[t]-\boldsymbol{L} \boldsymbol{w}[t+1], \\
\boldsymbol{e}[t] & =\boldsymbol{C}_{y} \widetilde{\boldsymbol{x}}[t]
\end{aligned}
$$

done las matrices $\boldsymbol{C}_{d, k}$ y $\overline{\boldsymbol{C}}_{d, k}$ son constantes y vienen dadas por

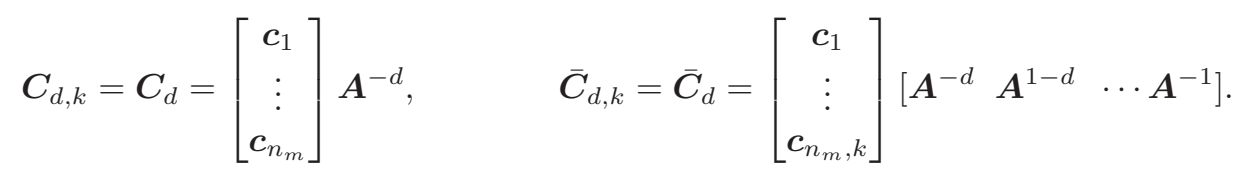

Como las matrices que definen la dinámica son constantes, es posible aplicar la transformada en $\mathcal{Z}$ para obtener la matriz de transferencia discreta que relaciona el error de predicción con las perturbaciones, llegándose a

$$
\boldsymbol{e}(z)=\boldsymbol{C}_{y}(z \boldsymbol{I}-\mathcal{A})^{-1}\left(\left(\boldsymbol{I}-\boldsymbol{L} \boldsymbol{C}_{d}\right) \boldsymbol{v}(z)+\boldsymbol{L} \overline{\boldsymbol{C}}_{d} \mathbb{V}(z)-z \boldsymbol{L} \boldsymbol{w}(z)\right)
$$


Dinámica del bucle cerrado Combinando las ecuaciones del controlador (6.37), el proceso (6.33) y el predictor (6.40) se tiene la dinámica en bucle cerrado

$$
\begin{gathered}
{\left[\begin{array}{c}
\widetilde{\boldsymbol{x}}[t+1] \\
\boldsymbol{x}[t+1] \\
\boldsymbol{\eta}[t+1]
\end{array}\right]=\left[\begin{array}{ccc}
\left(\boldsymbol{I}-\boldsymbol{L} \boldsymbol{C}_{d}\right) \boldsymbol{A} & \mathbf{0} & \mathbf{0} \\
-\boldsymbol{B} \boldsymbol{D}_{c} \boldsymbol{C}_{y} & \boldsymbol{A}+\boldsymbol{B}_{c} \boldsymbol{C}_{y} & \boldsymbol{B} \boldsymbol{C}_{c} \\
-\boldsymbol{B}_{c} \boldsymbol{C}_{y} & \boldsymbol{B}_{c} \boldsymbol{C}_{y} & \boldsymbol{A}_{c}
\end{array}\right]\left[\begin{array}{l}
\widetilde{\boldsymbol{x}}[t]] \\
\boldsymbol{x}[t] \\
\boldsymbol{\eta}[t]
\end{array}\right]} \\
+\left[\begin{array}{cc}
\left(\boldsymbol{I}-\boldsymbol{L} \boldsymbol{C}_{d}\right) & -\boldsymbol{L} \\
\boldsymbol{I} & \mathbf{0} \\
\mathbf{0} & \mathbf{0}
\end{array}\right]\left[\begin{array}{c}
\boldsymbol{v}[t] \\
\boldsymbol{w}[t+1]
\end{array}\right]+\left[\begin{array}{c}
\boldsymbol{L} \overline{\boldsymbol{C}}_{d} \\
\mathbf{0} \\
\mathbf{0}
\end{array}\right] \mathbb{V}[t], \\
\boldsymbol{z}[t]=\left[\begin{array}{lll}
\mathbf{0} & \boldsymbol{C}_{z} & \mathbf{0}
\end{array}\right]\left[\begin{array}{c}
\widetilde{\boldsymbol{x}}[t] \\
\boldsymbol{x}[t] \\
\boldsymbol{\eta}[t]
\end{array}\right] .
\end{gathered}
$$

donde se ha tenido en cuenta que la predicción de la salida $\hat{\boldsymbol{y}}[t]$ necesaria en el controlador se relaciona con el error de estimación del estado mediante

$$
\hat{\boldsymbol{y}}[t]=\boldsymbol{C}_{y} \hat{\boldsymbol{x}}[t]=\boldsymbol{C}_{y}(\boldsymbol{x}[t]+\widetilde{\boldsymbol{x}}[t])
$$

Llamando $\boldsymbol{\xi}[t]$ al vector de estado ampliado

$$
\boldsymbol{\xi}[t]=\left[\begin{array}{l}
\widetilde{\boldsymbol{x}}[t+1] \\
\boldsymbol{x}[t+1] \\
\boldsymbol{\eta}[t+1]
\end{array}\right]
$$

se puede escribir la ecuación anterior como

$$
\begin{aligned}
\boldsymbol{\xi}[t+1] & =\underbrace{\left[\begin{array}{c:c}
\mathcal{A} & \mathbf{0} \\
\hdashline-\overline{\boldsymbol{\Phi}} \overline{\boldsymbol{C}}_{y} & \overline{\boldsymbol{M}_{B C}}
\end{array}\right]}_{=\mathcal{M}} \boldsymbol{\xi}[t]+\left[\begin{array}{cc}
\left(\boldsymbol{I}-\boldsymbol{L} \boldsymbol{C}_{d}\right) & -\boldsymbol{L} \\
\boldsymbol{I} & \mathbf{0} \\
\mathbf{0} & \mathbf{0}
\end{array}\right]\left[\begin{array}{c}
\boldsymbol{v}[t] \\
\boldsymbol{w}[t+1]
\end{array}\right]+\left[\begin{array}{c}
\boldsymbol{L} \overline{\boldsymbol{C}}_{d} \\
\mathbf{0} \\
\mathbf{0}
\end{array}\right] \mathrm{V}[t], \\
\boldsymbol{z}[t] & =\left[\begin{array}{lll}
\mathbf{0} & \boldsymbol{C}_{z} & \mathbf{0}
\end{array}\right] \boldsymbol{\xi}[t] .
\end{aligned}
$$

donde $\mathcal{A}=\left(\boldsymbol{I}-\boldsymbol{L} \boldsymbol{C}_{d}\right) \boldsymbol{A}$ es la dinámica del predictor y $\boldsymbol{M}_{B C}$ es la dinámica del lazo de control con muestreo convencional y sin retardos.

Principio de separación. La dinámica del bucle cerrado con el predictor propuesto ha resultado tener una estructura triangular, con lo que los valores propios del bucle cerrado son los del predictor más los del controlador convencional, es decir

$$
\lambda(\mathcal{M})=\lambda(\mathcal{A}) \cup \lambda\left(\boldsymbol{M}_{B C}\right)
$$

Por tanto, para asegurar la estabilidad del lazo cerrado (valores propios de $\mathcal{M}$ dentro del círculo unitario) se ha de diseñar un predictor estable (con los valores propios de $\mathcal{A}$ en el circulo unitario) y un controlador que estabilice el bucle estándar (con los valores propios de $\boldsymbol{M}_{B C}$ en el circulo unitario). La estabilización del bucle estándar consiste en diseñar un controlador que estabilice un hipotético lazo en el que no hay retardos y en el que se miden todas las salidas de forma periódica, como en el ítem anterior.

Efecto de las perturbaciones. Teniendo en cuenta que los coeficientes de las matrices en (6.44) son constantes y que la ecuación es lineal, se puede aplicar la transformada en $\mathcal{Z}$ para obtener la matriz de transferencia que relaciona la salida con las perturbaciones, llegándose a

$$
\boldsymbol{z}(z)=\left[\begin{array}{lll}
\mathbf{0} & \boldsymbol{C}_{z} & \mathbf{0}
\end{array}\right](z \boldsymbol{I}-\boldsymbol{\mathcal { M }})^{-1}\left(\left[\begin{array}{cc}
\left(\boldsymbol{I}-\boldsymbol{L} \boldsymbol{C}_{d}\right) & -\boldsymbol{L} \\
\boldsymbol{I} & \mathbf{0} \\
\mathbf{0} & \mathbf{0}
\end{array}\right]\left[\begin{array}{c}
\boldsymbol{v}[t] \\
\boldsymbol{w}[t+1]
\end{array}\right]+\left[\begin{array}{c}
\boldsymbol{L} \overline{\boldsymbol{C}}_{d} \\
\mathbf{0} \\
\mathbf{0}
\end{array}\right] \mathbb{V}[t]\right)
$$

Debido a la estructura triangular de $\mathcal{M}$, la matriz $(z \boldsymbol{I}-\mathcal{M})^{-1}$ viene dada por

$$
(z \boldsymbol{I}-\mathcal{M})^{-1}=\left[\begin{array}{cc}
(z \boldsymbol{I}-\mathcal{A})^{-1} & \mathbf{0} \\
-\left(z \boldsymbol{I}-\boldsymbol{M}_{B C}\right)^{-1} \boldsymbol{\Phi} \boldsymbol{C}_{y}(z \boldsymbol{I}-\mathcal{A})^{-1} & \left(z \boldsymbol{I}-\boldsymbol{M}_{B C}\right)^{-1}
\end{array}\right] .
$$


Introduciendo esta matriz en (6.45) se llega a

$$
\begin{aligned}
\boldsymbol{z}(z)= & {\left[\begin{array}{ll}
\boldsymbol{C}_{z} & \mathbf{0}
\end{array}\right]\left[-\left(z \boldsymbol{I}-\boldsymbol{M}_{B C}\right)^{-1} \boldsymbol{\Phi} \boldsymbol{C}_{y}(z \boldsymbol{I}-\boldsymbol{\mathcal { A }})^{-1} \quad\left(z \boldsymbol{I}-\boldsymbol{M}_{B C}\right)^{-1}\right] . } \\
& \cdot\left(\left[\begin{array}{cc}
\left(\boldsymbol{I}-\boldsymbol{L} \boldsymbol{C}_{d}\right) & -\boldsymbol{L} \\
\boldsymbol{I} & \mathbf{0} \\
\mathbf{0} & \mathbf{0}
\end{array}\right]\left[\begin{array}{c}
\boldsymbol{v}[t] \\
\boldsymbol{w}[t+1]
\end{array}\right]+\left[\begin{array}{c}
\boldsymbol{L} \overline{\boldsymbol{C}}_{d} \\
\mathbf{0} \\
\mathbf{0}
\end{array}\right] \mathbb{V}[t]\right) \\
= & {\left[\begin{array}{cc}
\boldsymbol{C}_{z} & \mathbf{0}
\end{array}\right]\left(z \boldsymbol{I}-\boldsymbol{M}_{B C}\right)^{-1} . } \\
& \cdot\left(-\boldsymbol{\Phi} \boldsymbol{C}_{y}(z \boldsymbol{I}-\mathcal{A})^{-1}\left(\left(\boldsymbol{I}-\boldsymbol{L} \boldsymbol{C}_{d}\right) \boldsymbol{v}(z)+\boldsymbol{L} \overline{\boldsymbol{C}}_{d} \mathbb{V}(z)-z \boldsymbol{L} \boldsymbol{w}(z)\right)+\left[\begin{array}{l}
\boldsymbol{I} \\
\mathbf{0}
\end{array}\right] \boldsymbol{v}(z)\right) .
\end{aligned}
$$

Teniendo en cuenta la matriz de transferencia entre perturbaciones y error de predicción (6.41), la matriz de transferencia anterior se puede expresar como

$$
\boldsymbol{z}(z)=\left[\begin{array}{ll}
\boldsymbol{C}_{z} & \mathbf{0}
\end{array}\right]\left(z \boldsymbol{I}-\boldsymbol{M}_{B C}\right)^{-1}\left(\left[\begin{array}{l}
\boldsymbol{I} \\
\mathbf{0}
\end{array}\right] \boldsymbol{v}(z)-\boldsymbol{\Phi} \boldsymbol{e}(z)\right) .
$$

Comparando esta matriz de transferencia con (6.39) se observa que la matriz de transferencia que relaciona el error $\boldsymbol{e}(z)$ con la salida $\boldsymbol{z}(z)$ en el sistema de control inferencial, es la misma que la que relaciona el ruido $\boldsymbol{w}(z)$ con la salida de un sistema de control con muestreo convencional, pero de signo contrario. De esta manera, el diseño del controlador se puede mejorar siguiendo los siguientes pasos:

1. Diseñar el predictor para que minimice el error de predicción frente a las perturbaciones y ruidos del sistema de medición.

2. Calcular la cota de la norma del error de predicción $(\|e[t]\|)$ según la estrategia de diseño que haya sido utilizada $\left(\mathcal{H}_{\infty}, \mathcal{H}_{2}\right.$, etc.).

3. Diseñar un controlador para muestreo convencional que trate de minimizar el efecto de la perturbación y del ruido de medida, tomando como cota de la norma de éste $(\|\boldsymbol{w}[t]\|)$, la cota de la norma del error de predicción. $\|\boldsymbol{e}[t]\|$. Para realizar este diseño se pueden utilizar técnicas como la de Loop Shaping, LQG, $\mathcal{H}_{2}, \mathcal{H}_{\infty}$ (véase [63] capítulo 9), que incluso pueden resolverse de forma óptima mediante técnicas LMI (véase [59])

\subsubsection{Dinámica del bucle cerrado con muestreo escaso}

En esta sección se amplían los resultados de la sección anterior al caso de sistemas en los que las medidas de los sensores están disponibles de forma escasa y no simultánea, además de estar afectadas de retardos variantes en el tiempo. En primer lugar se deduce la expresión de la dinámica del bucle cerrado global.

Teorema 6.3.1. Considérese el sistema de control mostrado en la figura 6.5 donde se asume que el proceso es lineal y viene definido por las ecuaciones (6.33). Se asume que el controlador es un sistema lineal operando a periodo $T$ y definido por las ecuaciones (6.37), mientras que el predictor viene dado por las ecuaciones (6.34). Supóngase una perturbación y ruido de medida nulos y un escenario de muestreo síncrono irregular con retardos variantes en los sensores y disponibilidad parcial de éstos. Entonces, la dinámica del bucle cerrado resultante actualizada en cada instante de medición viene definida por

$$
\boldsymbol{\xi}_{k}=\boldsymbol{\Gamma}_{k} \boldsymbol{M}^{N_{k}} \boldsymbol{\xi}_{k-1}=\mathcal{M}_{k} \boldsymbol{\xi}_{k-1}
$$

con el vector de estado ampliado

$$
\boldsymbol{\xi}_{k}=\left[\begin{array}{c}
\widetilde{\boldsymbol{x}}_{k} \\
\boldsymbol{x}_{k} \\
\boldsymbol{\eta}_{k}
\end{array}\right]
$$

y donde la matriz $\boldsymbol{M}$ viene definida por

$$
\boldsymbol{M}=\left[\begin{array}{ccc}
\boldsymbol{A} & \mathbf{0} & \mathbf{0} \\
-\boldsymbol{B} \boldsymbol{D}_{c} \boldsymbol{C}_{y} & \boldsymbol{A}+\boldsymbol{B} \boldsymbol{D}_{c} \boldsymbol{C}_{y} & \boldsymbol{B} \boldsymbol{C}_{c} \\
-\boldsymbol{B}_{c} \boldsymbol{C}_{y} & \boldsymbol{B}_{c} \boldsymbol{C}_{y} & \boldsymbol{A}_{c}
\end{array}\right],
$$

y la matriz $\boldsymbol{\Gamma}_{k}$ por

$$
\boldsymbol{\Gamma}_{k}=\left[\begin{array}{ccc}
\left(\boldsymbol{I}-\boldsymbol{L}_{k} \boldsymbol{\Delta}_{k} \boldsymbol{C}_{d, k}\right) & \mathbf{0} & \mathbf{0} \\
\mathbf{0} & \boldsymbol{I} & \mathbf{0} \\
\mathbf{0} & \mathbf{0} & \boldsymbol{I}
\end{array}\right]
$$


Prueba 6.3.1. Para un instante de tiempo arbitrario $t \neq t_{k}$ sin medición la dinámica del controlador (6.37) puede expresarse como

$$
\begin{aligned}
\boldsymbol{\eta}[t] & =\boldsymbol{A}_{c} \boldsymbol{\eta}[t-1]+\boldsymbol{B}_{c} \boldsymbol{C}_{y} \hat{\boldsymbol{x}}[t-1] \\
\boldsymbol{u}[t] & =\boldsymbol{C}_{c} \boldsymbol{\eta}[t]+\boldsymbol{D}_{c} \boldsymbol{C}_{y} \hat{\boldsymbol{x}}[t]
\end{aligned}
$$

con

$$
\hat{\boldsymbol{y}}[t]=\boldsymbol{C}_{y} \hat{\boldsymbol{x}}[t]=\boldsymbol{C}_{y}(\boldsymbol{x}[t]-\widetilde{\boldsymbol{x}}[t]) .
$$

Teniendo en cuenta la dinámica del error de predicción en bucle abierto (6.36a), y la dinámica propia del proceso (6.33a), puede expresarse que

$$
\left[\begin{array}{c}
\widetilde{\boldsymbol{x}}[t] \\
\boldsymbol{x}[t] \\
\boldsymbol{\eta}[t]
\end{array}\right]=\left[\begin{array}{ccc}
\boldsymbol{A} & \mathbf{0} & \mathbf{0} \\
-\boldsymbol{B} \boldsymbol{D}_{c} \boldsymbol{C}_{y} & \boldsymbol{A}+\boldsymbol{B}_{c} \boldsymbol{C}_{y} & \boldsymbol{B} \boldsymbol{C}_{c} \\
-\boldsymbol{B}_{c} \boldsymbol{C}_{y} & \boldsymbol{B}_{c} \boldsymbol{C}_{y} & \boldsymbol{A}_{c}
\end{array}\right]\left[\begin{array}{c}
\widetilde{\boldsymbol{x}}[t-1] \\
\boldsymbol{x}[t-1] \\
\boldsymbol{\eta}[t-1]
\end{array}\right]=\boldsymbol{M} \boldsymbol{\xi}[t-1], \quad t_{k}<t<t_{k-1}
$$

Cuando se tiene una medición en un instante $t=t_{k}$, la dinámica del sistema (6.33a) y del controlador (6.37) tienen que unirse a la dinámica (6.36b), quedando

$$
\left[\begin{array}{l}
\widetilde{\boldsymbol{x}}\left[t_{k}\right] \\
\boldsymbol{x}\left[t_{k}\right] \\
\boldsymbol{\eta}\left[t_{k}\right]
\end{array}\right]=\left[\begin{array}{ccc}
\left(\boldsymbol{I}-\boldsymbol{L}_{k} \boldsymbol{\Delta}_{k} \boldsymbol{C}_{d, k}\right) \boldsymbol{A} & \mathbf{0} & \mathbf{0} \\
-\boldsymbol{B} \boldsymbol{D}_{c} \boldsymbol{C}_{y} & \boldsymbol{A}+\boldsymbol{B D}_{c} \boldsymbol{C}_{y} & \boldsymbol{B} \boldsymbol{C}_{c} \\
-\boldsymbol{B}_{c} \boldsymbol{C}_{y} & \boldsymbol{B}_{c} \boldsymbol{C}_{y} & \boldsymbol{A}_{c}
\end{array}\right]\left[\begin{array}{l}
\widetilde{\boldsymbol{x}}\left[t_{k}-1\right] \\
\boldsymbol{x}\left[t_{k}-1\right] \\
\boldsymbol{\xi}\left[t_{k}-1\right]
\end{array}\right] .
$$

Esta última expresión puede expresarse como

$$
\boldsymbol{\xi}\left[t_{k}\right]=\boldsymbol{\Gamma}_{k} \boldsymbol{M} \boldsymbol{\xi}\left[t_{k}-1\right]
$$

Aplicando la ecuación (6.53) de forma recursiva desde $t=t_{k}-1$ hasta $t=t_{k-1}$ para definir $\boldsymbol{\xi}\left[t_{k}-1\right]$ en función de $\boldsymbol{\xi}\left[t_{k-1}\right]$ se tiene que

$$
\boldsymbol{\xi}_{k} \equiv \boldsymbol{\xi}\left[t_{k}\right]=\boldsymbol{\Gamma}_{k} \underbrace{\boldsymbol{M \cdots M}}_{N_{k}} \boldsymbol{\xi}\left[t_{k-1}\right]=\boldsymbol{\Gamma}_{k} \boldsymbol{M}^{N_{k}} \boldsymbol{\xi}_{k-1} .
$$

\section{Principio de separación}

En esta sección se demuestra que el esquema de la figura 6.5 cumple con el principio de separación, en el sentido de que la estabilidad del predictor (6.34), y del lazo cerrado estándar (6.38a) son condiciones necesarias y suficientes para asegurar la estabilidad del sistema completo (6.49)

Observación 6.3.2 (Triangularización del bucle cerrado). Escribiendo (6.49) como

$$
M=\left[\begin{array}{c:c}
\boldsymbol{A} & \mathbf{0} \\
\hdashline-\overline{\boldsymbol{\Phi}} \bar{C}_{y} & \bar{M}_{B C}
\end{array}\right]
$$

con

$$
\boldsymbol{\Phi}=\left[\begin{array}{c}
\boldsymbol{B} \boldsymbol{D}_{c} \\
\boldsymbol{B}_{c}
\end{array}\right]
$$

y $\boldsymbol{M}_{B C}$ la dinámica del lazo cerrado con muestreo convencional (6.38a), la ecuación de la dinámica en lazo cerrado (6.48) se puede escribir como

$$
\mathcal{M}_{k}=\boldsymbol{\Gamma}_{k} \boldsymbol{M} \cdots \boldsymbol{M}=\left[\begin{array}{c:c}
\left(\boldsymbol{I}-\boldsymbol{L}_{k} \boldsymbol{\Delta}_{k} \boldsymbol{C}_{d, k}\right) \boldsymbol{A}^{N_{k}} & \mathbf{0} \\
\hdashline-\boldsymbol{\Theta}_{k} & \boldsymbol{M}_{B C}^{N_{k}^{-}}
\end{array}\right],
$$

con

$$
\boldsymbol{\Theta}_{k}=\sum_{j=0}^{N_{k}} \boldsymbol{M}_{B C}^{N_{k}-j} \boldsymbol{\Phi} \boldsymbol{A}^{j} .
$$

Teorema 6.3.2. Considérese la misma hipótesis descrita en el teorema 6.3.1. Supóngase que se cumplen las siguientes condiciones 
(i) existe una matriz $\boldsymbol{P}_{k} \succ \mathbf{0}$ tal que

$$
\mathcal{A}_{k}^{\top} \boldsymbol{P}_{k} \mathcal{A}_{k}-\boldsymbol{P}_{k-1} \prec \mathbf{0},
$$

es decir, el error de predicción del algoritmo (6.34) (cuya dinámica viene definida por la matriz $\mathcal{A}_{k}(6.35)$ ) converge a cero, $y$

(ii) existe una matriz $\boldsymbol{Q}[t] \succ \mathbf{0}$ tal que

$$
\boldsymbol{M}_{B C}^{\top} \boldsymbol{Q}[t] \boldsymbol{M}_{B C}-\boldsymbol{Q}[t-1] \prec \mathbf{0},
$$

es decir, el sistema en lazo cerrado con muestreo convencional (definido por la matriz $\boldsymbol{M}_{B C}$ (6.38a)) es estable.

Entonces, existe una matriz $\boldsymbol{X}_{k} \succ \mathbf{0}$ tal que

$$
\mathcal{M}_{k}^{\top} \boldsymbol{X}_{k} \mathcal{M}_{k}-\boldsymbol{X}_{k-1} \prec \mathbf{0}
$$

es decir, el sistema en lazo cerrado descrito en (6.49) es estable.

Recíprocamente, si existe una matriz $\boldsymbol{X}_{k} \succ \mathbf{0}$ tal que se satisface la condición (6.60), entonces el predictor (6.34), y el subsistema del lazo cerrado convencional (6.38a) satisfacen las condiciones (i) y (ii) $y$, por tanto, ambos son estables.

Prueba 6.3.2. Como la dinámica del bucle cerrado viene definida por la matriz triangular en bloques (6.57), se puede aplicar directamente el teorema 6.2.2 a esta matriz, demostrando fácilmente el principio de separación para el sistema (6.49) que implica la existencia de una matriz $\boldsymbol{P}_{k} \succ \mathbf{0}$ tal que

$$
\mathcal{A}_{k}^{\top} \boldsymbol{P}_{k} \mathcal{A}_{k}-\boldsymbol{P}_{k-1} \prec \mathbf{0}
$$

y una matriz $\boldsymbol{Q}_{k} \succ \mathbf{0}$ tal que

$$
\left(M_{B C}{ }^{N_{k}}\right)^{\top} \boldsymbol{Q}_{k} \boldsymbol{M}_{B C}{ }^{N_{k}}-\boldsymbol{Q}_{k-1} \prec \mathbf{0} .
$$

Esta última desigualdad es equivalente a la desigualdad (6.59), como ya quedó demostrado en la prueba del teorema 6.2.3 (página 161).

Observación 6.3.3 (Estabilidad de sistemas de control inferencial no lineales). Sea una planta no lineal de la forma

$$
\begin{aligned}
\boldsymbol{x}[t+1] & =\boldsymbol{A} \boldsymbol{x}[t]+\boldsymbol{f}(\boldsymbol{x}[t], \boldsymbol{u}[t]), \\
\boldsymbol{y}[t] & =\boldsymbol{g}(\boldsymbol{x}[t]),
\end{aligned}
$$

y sea $\mathcal{C}$ un controlador diseñado para estabilizar la planta con muestreo convencional. Supóngase que se tiene un muestreo escaso, con lo que se puede aplicar alguno de los diseños expuestos en el capítulo 5 para obtener un predictor $\mathcal{P}$ estable que estime las salidas que se desean controlar. Entonces, si el proceso acepta una linealización de la forma

$$
\begin{aligned}
\boldsymbol{x}[t+1] & =\boldsymbol{A}^{\prime} \boldsymbol{x}[t]+\boldsymbol{B}^{\prime} \boldsymbol{u}[t], \\
\boldsymbol{y}[t] & =\boldsymbol{C}_{y}^{\prime} \boldsymbol{x}[t],
\end{aligned}
$$

alrededor de algún $\boldsymbol{x}_{0}$, se puede asegurar (por aplicación del teorema anterior) que el bucle cerrado que incluye el predictor será estable alrededor del punto $\boldsymbol{x}_{0}$.

\section{Análisis del efecto de las perturbaciones}

El efecto de las perturbaciones sobre la salida del sistema de control inferencial no se puede analizar al hiperperiodo variante $N_{k} T$ mediante la aplicación de la transformada en $\mathcal{Z}$, ya que las matrices son variantes en el tiempo.

Sin embargo, se puede estudiar el efecto del error de predicción en cada instante de control. En los instantes en los que no hay medición, la dinámica del bucle cerrado se define mediante la expresión

$$
\left[\begin{array}{l}
\widetilde{\boldsymbol{x}}[t] \\
\boldsymbol{x}[t] \\
\boldsymbol{\eta}[t]
\end{array}\right]=\left[\begin{array}{cc}
\boldsymbol{A} & \mathbf{0} \\
-\boldsymbol{\Phi} \boldsymbol{C}_{y} & \boldsymbol{M}_{B C}
\end{array}\right]\left[\begin{array}{l}
\widetilde{\boldsymbol{x}}[t-1] \\
\boldsymbol{x}[t-1] \\
\boldsymbol{\eta}[t-1]
\end{array}\right]+\left[\begin{array}{l}
\boldsymbol{I} \\
\boldsymbol{I} \\
\mathbf{0}
\end{array}\right] \boldsymbol{v}[t-1]
$$


que, teniendo en cuenta que $\boldsymbol{e}[t]=\boldsymbol{C}_{y} \widetilde{\boldsymbol{x}}[t]$ se puede reescribir como el sistema de ecuaciones en diferencias

$$
\left\{\begin{array}{l}
\widetilde{\boldsymbol{x}}[t]=\boldsymbol{A} \widetilde{\boldsymbol{x}}[t-1]+\boldsymbol{v}[t-1] \\
{\left[\begin{array}{l}
\boldsymbol{x}[t] \\
\boldsymbol{\eta}[t]
\end{array}\right]=\boldsymbol{M}_{B C}\left[\begin{array}{l}
\boldsymbol{x}[t-1] \\
\boldsymbol{\eta}[t-1]
\end{array}\right]+\left[\begin{array}{l}
\boldsymbol{I} \\
\mathbf{0}
\end{array}\right] \boldsymbol{v}[t-1]-\boldsymbol{\Phi} \boldsymbol{C}_{y} \boldsymbol{e}[t-1] .}
\end{array}\right.
$$

Cuando hay una medición en $t=t_{k}$, la dinámica del bucle cerrado se define como

$$
\begin{aligned}
{\left[\begin{array}{l}
\widetilde{\boldsymbol{x}}\left[t_{k}\right] \\
\boldsymbol{x}\left[t_{k}\right] \\
\boldsymbol{\eta}\left[t_{k}\right]
\end{array}\right]=} & {\left[\begin{array}{ccc}
\left(\boldsymbol{I}-\boldsymbol{L}_{k} \boldsymbol{\Delta}_{k} \boldsymbol{C}_{d, k}\right) \boldsymbol{A} & \mathbf{0} & \mathbf{0} \\
-\boldsymbol{B D}_{c} \boldsymbol{C}_{y} & \boldsymbol{A}+\boldsymbol{B}_{c} \boldsymbol{C}_{y} & \boldsymbol{B} \boldsymbol{C}_{c} \\
-\boldsymbol{B}_{c} \boldsymbol{C}_{y} & \boldsymbol{B}_{c} \boldsymbol{C}_{y} & \boldsymbol{A}_{c}
\end{array}\right]\left[\begin{array}{c}
\widetilde{\boldsymbol{x}}\left[t_{k}-1\right] \\
\boldsymbol{x}\left[t_{k}-1\right] \\
\boldsymbol{\xi}\left[t_{k}-1\right]
\end{array}\right] } \\
& +\left[\begin{array}{cc}
\left(\boldsymbol{I}-\boldsymbol{L}_{k} \boldsymbol{\Delta}_{k} \boldsymbol{C}_{d, k}\right) & -\boldsymbol{L}_{k} \\
\boldsymbol{I} & \mathbf{0} \\
\mathbf{0} & \mathbf{0}
\end{array}\right]\left[\begin{array}{c}
\boldsymbol{v}\left[t_{k}-1\right] \\
\boldsymbol{w}\left[t_{k}\right]
\end{array}\right]+\left[\begin{array}{c}
\boldsymbol{L}_{k} \boldsymbol{\Delta}_{k} \overline{\boldsymbol{C}}_{d, k} \\
\mathbf{0} \\
\mathbf{0}
\end{array}\right] \mathbb{V}\left[t_{k}-1\right]
\end{aligned}
$$

que se puede reescribir como el sistema de ecuaciones en diferencias

$$
\left\{\begin{aligned}
& \widetilde{\boldsymbol{x}}\left[t_{k}\right]=\left(\boldsymbol{I}-\boldsymbol{L}_{k} \boldsymbol{\Delta}_{k} \boldsymbol{C}_{d, k}\right) \boldsymbol{A} \widetilde{\boldsymbol{x}}\left[t_{k}-1\right]+\left(\boldsymbol{I}-\boldsymbol{L}_{k} \boldsymbol{\Delta}_{k} \boldsymbol{C}_{d, k}\right) \boldsymbol{v}\left[t_{k}-1\right] \\
&+\boldsymbol{L}_{k} \boldsymbol{\Delta}_{k} \overline{\boldsymbol{C}}_{d, k} \mathbb{V}\left[t_{k}-1\right]-\boldsymbol{L}_{k} \boldsymbol{w}\left[t_{k}\right] \\
& {\left[\begin{array}{l}
\left.\boldsymbol{x}\left[t_{k}\right]\right] \\
\boldsymbol{\eta}\left[t_{k}\right]
\end{array}\right]=\boldsymbol{M}_{B C}\left[\begin{array}{l}
\boldsymbol{x}\left[t_{k}-1\right] \\
\boldsymbol{\eta}\left[t_{k}-1\right]
\end{array}\right]+\left[\begin{array}{l}
\boldsymbol{I} \\
\mathbf{0}
\end{array}\right] \boldsymbol{v}\left[t_{k}-1\right]-\boldsymbol{\Phi} \boldsymbol{C}_{y} \boldsymbol{e}\left[t_{k}-1\right] . }
\end{aligned}\right.
$$

Si se comparan las ecuaciones (6.61) y (6.64) con (6.38a), se observa que el efecto que tiene el error de predicción sobre el subsistema de control a muestreo convencional en el SCI es el mismo que tiene el ruido en un sistema de control con muestreo convencional. De esta manera, el diseño del controlador se puede mejorar (igual que se ha propuesto en muestreo periódico) siguiendo los siguientes pasos:

1. Diseñar un predictor que minimice el error de predicción frente a las perturbaciones y ruidos de medida en el sistema global con muestreo irregular y retardos variantes en el sistema de medición.

2. Calcular la cota del error de predicción a periodo $T(\|\boldsymbol{e}[t]\|)$ con ayuda del resultado obtenido en el proceso de diseño mediante alguna de las técnicas $\mathcal{H}_{\infty}, \mathcal{H}_{2}, \mathcal{H}_{2 g}$, etc. Para ello pueden ser útiles las relaciones entre normas de señales a distinto periodo, los resultados de las secciones \$3.6.6 (página 71) y §4.7.5 (página 122), o incluso la realización de una simulación del funcionamiento del predictor.

3. Diseñar un controlador para muestreo convencional que trate de minimizar el efecto de la perturbación y del ruido de medida, tomando como cota de la norma de éste $(\|\boldsymbol{w}[t]\|)$, la cota del error de predicción a periodo $T(\|\boldsymbol{e}[t]\|)$. Para ello se pueden utilizar cualquiera de las técnicas expuestas en [63] (capítulo 9) o en [59].

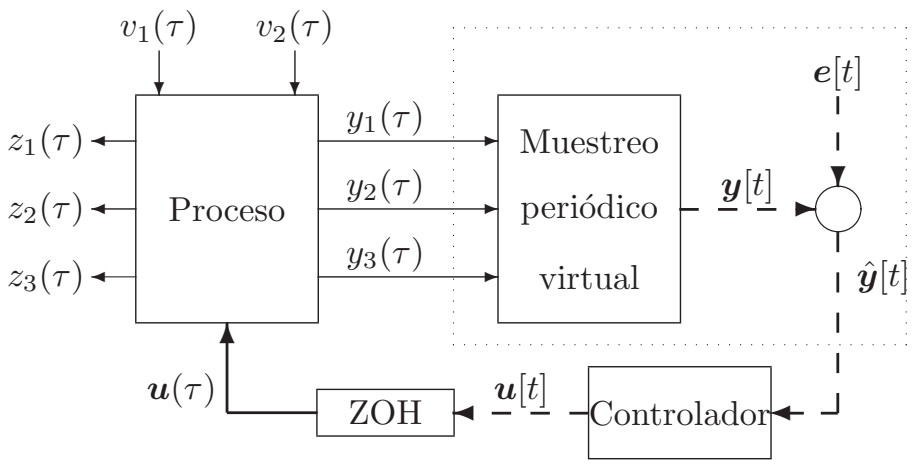

Figura 6.6: Esquema de control con el sensor virtual. 


\subsection{Conclusiones}

En este capítulo se ha tratado el análisis de la dinámica en bucle cerrado de un sistema de control que utiliza las mediciones escasas con retardo proporcionadas por un conjunto de sensores que, en general, no están disponibles de forma simultánea. Se ha analizado un sistema de control que consiste en un controlador convencional monofrecuencia en combinación con un sensor virtual basado en modelo que estima las salidas que se desean controlar a periodo rápido. El estimador propuesto tiene en cuenta las mediciones previas, a diferencia de otros esquemas que realizan la estimación en bucle abierto (véase [36]).

Se ha derivado la ecuación de la dinámica del bucle cerrado global del sistema controlador-estimador, relacionado con los instantes de medición (hiperperiodo variante). El resultado principal es la demostración del principio de separación entre el predictor y el controlador (ambos multivariables), en el sentido que la estabilidad del estimador y del lazo de control con muestreo convencional implican la estabilidad del sistema global en lazo cerrado. Por tanto, el sensor virtual y el controlador pueden diseñarse separadamente, tanto en el caso monovariable como en multivariable.

El esquema propuesto puede llevar a sistemas de control estables en lazo cerrado aún cuando el proceso en bucle abierto es inestable, en diferencia con esquemas de control que utilizan la estimación en bucle abierto y en los que la inestabilidad del proceso en bucle abierto implica la inestabilidad del lazo cerrado.

También se ha estudiado cuál es el efecto que tiene el error de predicción sobre la salida del sistema de control inferencial, y se ha visto que es equivalente al efecto que tiene el ruido de medida en un sistema de control con muestreo convencional. De esta manera se puede mejorar el diseño del controlador (que se diseña para muestreo convencional) tomando el error de predicción del predictor como un ruido de medición.

Finalmente, algunos ejemplos ilustran los principales resultados y muestran la mejora de las prestaciones en bucle cerrado (respecto de los esquemas con estimador en bucle abierto) cuando se diseña un estimador adecuado en lazo cerrado (mediante las técnicas descritas en los capítulos 3 y 4 ). 



\section{Aplicaciones}

\subsection{Introducción}

En este capítulo se presentan algunas aplicaciones de estimación de variables y control de procesos con medidas escasas.

\subsection{Control de posición de un puente grúa}

Considérese el puente grúa mostrado en la figura. La entrada al sistema es la fuerza $u$ y se asume que se actualiza a un periodo constante $T=0.2$ s. Las señales medidas son $\theta$ y $x$, y se miden mediante unos sensores que están conectados a una red de comunicaciones compartida con más procesos y sensores. Sólo se tiene acceso a estas señales cada 2 o 4 segundos $(N=\{10,20\})$, pudiéndose medir sólo una señal (la posición $x$ o el ángulo $\theta$ ). El funcionamiento de la red de comunicaciones es tal que la transmisión de las señales medidas viene acompañada de una etiquea de tiempo, y dicha transmisión tarda 0.4 o 0.8 segundos $\left(d_{i}=\{2,4\}\right)$ en alcanzar el controlador. En este ejemplo se van a comparar diferentes estrategias para controlar el proceso en bucle cerrado haciendo uso de técnicas de predicción alternativas: predicción en bucle abierto, predicción de sustitución, predicción $\mathcal{H}_{\infty}$ con ganancia variable, predicción $\mathcal{H}_{\infty}$ con ganancia constante y, finalmente, predicción mediante el filtro de Kalman.

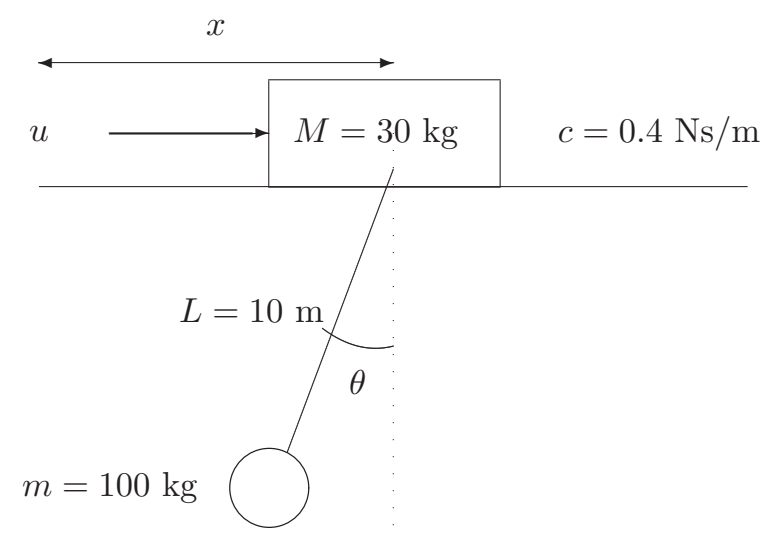

Figura 7.1: Puente grúa.

Las ecuaciones continuas que definen el sistema son:

$$
\begin{aligned}
& \dot{\boldsymbol{x}}(\tau)=\left[\begin{array}{l}
\dot{x}(\tau) \\
\ddot{x}(\tau) \\
\dot{\theta}(\tau) \\
\ddot{\theta}(\tau)
\end{array}\right]=\left[\begin{array}{cccc}
0 & 1 & 0 & 0 \\
0 & \frac{-c}{M} & \frac{-m g}{M} & 0 \\
0 & 0 & 0 & 1 \\
0 & \frac{-c}{M L} & \frac{-(M+m) g}{M L} & 0
\end{array}\right]\left[\begin{array}{c}
x(\tau) \\
\dot{x}(\tau) \\
\theta(\tau) \\
\dot{\theta}(\tau)
\end{array}\right]+\left[\begin{array}{c}
0 \\
\frac{1}{M} \\
0 \\
\frac{1}{M L}
\end{array}\right](u(\tau)+v(\tau)) \\
& \boldsymbol{y}(\tau)=\left[\begin{array}{llll}
1 & 0 & 0 & 0 \\
0 & 0 & 1 & 0
\end{array}\right] \boldsymbol{x}(\tau)
\end{aligned}
$$


donde $v(\tau)$ simboliza una perturbación de entrada. Para aplicar el predictor propuesto es necesario en primer lugar obtener un modelo discreto equivalente con retenedor de orden cero, quedando las matrices

$$
\boldsymbol{A}=\left[\begin{array}{cccc}
=1.0000 & 0.1997 & -0.6442 & -0.0432 \\
0 & 0.9974 & -6.3477 & -0.6442 \\
0 & -0.0000 & 0.9162 & 0.1944 \\
0 & -0.0003 & -0.8255 & 0.9162
\end{array}\right], \quad \boldsymbol{B}=\boldsymbol{B}_{v}=\left[\begin{array}{c}
0.0007 \\
0.0065 \\
0.0001 \\
0.0006
\end{array}\right]
$$

La ecuación de mediciones dependerá del sensor activo en cada instante, pudiendo ser

$$
m_{1}[t]=\left[\begin{array}{llll}
1 & 0 & 0 & 0
\end{array}\right] \boldsymbol{x}[t-d], \quad d=\{1,2\}
$$

cuando se mide la posición $x$, o

$$
m_{2}[t]=\left[\begin{array}{llll}
0 & 0 & 1 & 0
\end{array}\right] \boldsymbol{x}[t-d], \quad d=\{1,2\}
$$

cuando se mide el ángulo $\theta$. Se asume que la entrada puede sufrir una perturbación constante de valor máximo $\|v\|_{\infty}=0.2$, y que el ruido de medida asociado a los sensores ubicados para medir la posición $x$ y la posición $\theta$ puede modelarse como un ruido blanco de media cero y desviación típica $\sigma_{x}=0.01 \mathrm{~m}$ y $\sigma_{\theta}=0.001 \mathrm{rad}$. Con estos valores es posible diseñar un predictor $\mathcal{H}_{\infty}$ siguiendo las indicaciones mostradas en el capítulo 4 (sección \$4.5.3). La predicción en bucle abierto se realizará mediante la ecuación

$$
\begin{aligned}
& \hat{\boldsymbol{x}}[t \mid t-1]=\boldsymbol{A} \hat{\boldsymbol{x}}[t-1]+\boldsymbol{B} u[t-1] \\
& \hat{\boldsymbol{y}}[t \mid t-1]=\left[\begin{array}{llll}
1 & 0 & 0 & 0 \\
0 & 0 & 1 & 0
\end{array}\right] \hat{\boldsymbol{x}}[t \mid t-1]
\end{aligned}
$$

mientras no se tengan mediciones. Como en cada muestro sólo se tienen mediciones de uno de los sensores, las estimaciones anteriores se corregirán mediante la ecuación

$$
\begin{aligned}
\hat{\boldsymbol{x}}\left[t_{k}\right] & =\hat{\boldsymbol{x}}\left[t_{k} \mid t_{k}-1\right]+\boldsymbol{\ell}_{i}\left(s_{k}\right)\left(m_{i}\left[t_{k}-d_{i}\left(s_{k}\right)\right]-\hat{y}_{i}\left[t_{k}-d_{i}\left(s_{k}\right)\right]\right), \quad i=1,2 \\
\hat{\boldsymbol{y}}\left[t_{k}\right] & =\left[\begin{array}{llll}
1 & 0 & 0 & 0 \\
0 & 0 & 1 & 0
\end{array}\right] \hat{\boldsymbol{x}}\left[t_{k}\right]
\end{aligned}
$$

donde $i$ indica el sensor del cual se ha obtenido medición, $m_{i}$ es la medición de dicho sensor, $d_{i}\left(s_{k}\right)$ su retardo y $s_{k}$ hace referencia al tipo de muestreo que se ha dado y que se detalla en la tabla 7.1 , $\boldsymbol{\ell}_{i}\left(s_{k}\right)$

\begin{tabular}{ccccccccc}
\hline$s_{k}$ & 1 & 2 & 3 & 4 & 5 & 6 & 7 & 8 \\
\hline$N_{k}$ & 10 & 20 & 10 & 20 & 10 & 20 & 10 & 20 \\
$d_{1, k}$ & 2 & 2 & 4 & 4 & - & - & - & - \\
$d_{2, k}$ & - & - & - & - & 2 & 2 & 4 & 4 \\
$\Delta\left(s_{k}\right)$ & {$\left[\begin{array}{ll}1 & 0 \\
0 & 0\end{array}\right]$} & {$\left[\begin{array}{ll}1 & 0 \\
0 & 0\end{array}\right]$} & {$\left[\begin{array}{ll}1 & 0 \\
0 & 0\end{array}\right]$} & {$\left[\begin{array}{ll}1 & 0 \\
0 & 0\end{array}\right]$} & {$\left[\begin{array}{ll}0 & 0 \\
0 & 1\end{array}\right]$} & {$\left[\begin{array}{cc}0 & 0 \\
0 & 1\end{array}\right]$} & {$\left[\begin{array}{cc}0 & 0 \\
0 & 1\end{array}\right]$} & {$\left[\begin{array}{ll}0 & 0 \\
0 & 1\end{array}\right]$} \\
\hline
\end{tabular}

Tabla 7.1: Escenarios de muestreo en el puente grúa. "_" indica que no hay medición de esa variable.

es la columna $i$-ésima de la matriz de ganancias $\boldsymbol{L}\left(s_{k}\right)=\left[\boldsymbol{\ell}_{1}\left(s_{k}\right) \boldsymbol{\ell}_{2}\left(s_{k}\right)\right]$ cuyo valor se detalla en función del tipo de muestreo en la tabla 7.2. En dicha tabla se observa que la corrección que se realiza cuando se tienen mediciones del ángulo $\left(s_{k}=\{5,6,7,8\}\right)$ afecta al tercer y cuarto estado, debido a que el sistema no es completamente detectable desde dicho sensor, y sólo es detectable el subespacio formado por el tercer y cuarto estado. Sin embargo el sistema sí que es completamente detectable desde la medición de la posición $x$, y por eso la primera columna completa es de valores no nulos para los muestreos $s_{k}=1,2,3,4$. Si se diseña un predictor $\mathcal{H}_{\infty}$ mediante una ganancia constante se obtiene la matriz

$$
\boldsymbol{L}\left(s_{k}\right)=\boldsymbol{L}=\left[\begin{array}{cc}
1.1122 & -2.3155 \\
0.1735 & -9.0656 \\
-0.0001 & 0.2000 \\
-0.0005 & -1.1135
\end{array}\right], \quad s_{k}=1,2, \ldots, 8
$$

que se aplicará en todos los instantes en los que haya medición, independientemente de las características de la muestra obtenida. 


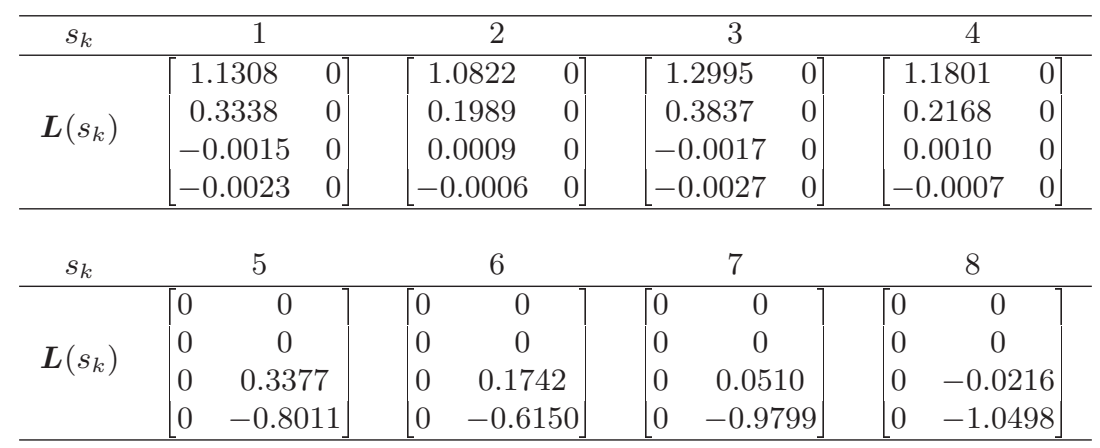

Tabla 7.2: Diferentes valores de la matriz $\boldsymbol{L}\left(s_{k}\right)$ en función del escenario de muestreo.

Las observaciones del estado proporcionadas por el predictor se utilizarán para controlar el proceso en bucle cerrado. De esta forma se diseña un controlador de realimentación del estado a periodo $T$ mediante asignación de polos para conseguir una dinámica en el bucle cerrado con muestreo convencional marcada por los polos $\{0.9,0.8,0.7,0.6\}$, obteniéndose la ecuación:

$$
u[t]=\boldsymbol{K}\left(\boldsymbol{x}_{\text {ref }}[t]-\hat{\boldsymbol{x}}[t]\right)=[46.5892179 .7428560 .3323-631.2319]\left(\boldsymbol{x}_{\text {ref }}[t]-\hat{\boldsymbol{x}}[t]\right) .
$$

Si se tuviera un muestreo convencional (mediciones sin retardo de ambos sensores cada $T$ segundos) la respuesta del sistema controlado sería la que se muestra en la figura 7.2 , donde se ha incluido una perturbación de $v(\tau)=0.2$ a partir del instante $\tau=200$ segundos $(t=1000)$.

Si se controla el sistema mediante un predictor de bucle abierto $\sqrt{1}$, se obtiene una respuesta que se hace inestable a partir del instante en el que aparece la perturbación (figura 7.3). Si se utiliza un predictor de sustitución 2 , se obtiene la respuesta de la figura 7.4, donde se observa que el sistema de control no es capaz de seguir la referencia a partir del instante en el que aparece la perturbación. Sin embargo, si se controla con el predictor $\mathcal{H}_{\infty}$ propuesto con ganancia variante se obtiene la respuesta de la figura 7.5, en la que se aprecia que el sistema si que es capaz de llevar el proceso a la referencia. En este caso, el error cometido entre la estimación de la salida $x$ y su valor real tiene un valor eficaz de $\|e\|_{R M S}=26.3 \mathrm{~mm}$, y un valor máximo de $\|e\|_{\infty}=207 \mathrm{~mm}$, medidos a partir del instante en el que aparece la perturbación. Si se utiliza el predictor $\mathcal{H}_{\infty}$ de ganancia constante propuesto se obtiene la respuesta de la figura 7.6 que es igualmente estable, pero con un ligero aumento del error de predicción, que en este caso viene definido por los valores $\|e\|_{R M S}=36.7 \mathrm{~mm}, \mathrm{y}\|e\|_{\infty}=322 \mathrm{~mm}$, medidos a partir del instante en el que aparece la perturbación. Si la predicción se hace mediante el filtro de Kalman expuesto en la sección §4.9 se obtiene la respuesta indicada en la figura 7.7, donde se advierte que el controlador atenúa en menor medida la perturbación introducida debido a que la estimación que proporciona el filtro de Kalman parte de la suposición de que las perturbaciones son de media nula. Con el filtro de Kalman el error de predicción de la variable $x$ viene definido por los valores $\|e\|_{R M S}=46.9 \mathrm{~mm}, \mathrm{y}\|e\|_{\infty}=330 \mathrm{~mm}$, medidos a partir del instante en el que aparece la perturbación y ligeramente superiores a los obtenidos con cualquiera de las dos técnicas $\mathcal{H}_{\infty}$. A la vista de los resultados, la implementación que presenta mejor relación de coste computacional e implementación frente a grado de atenuación de perturbaciones es el predictor $\mathcal{H}_{\infty}$ de ganancia constante.

\footnotetext{
${ }^{1}$ que no tiene en cuenta las mediciones

${ }^{2}$ cada vez que se tiene alguna medida se introduce su valor en la posición correspondiente del vector de estados observado, es decir, que se implementa mediante una ganancia constante de valor $\boldsymbol{L}=\left[\begin{array}{ll}1 & 0 \\ 0 & 0 \\ 0 & 1 \\ 0 & 0\end{array}\right]$
} 

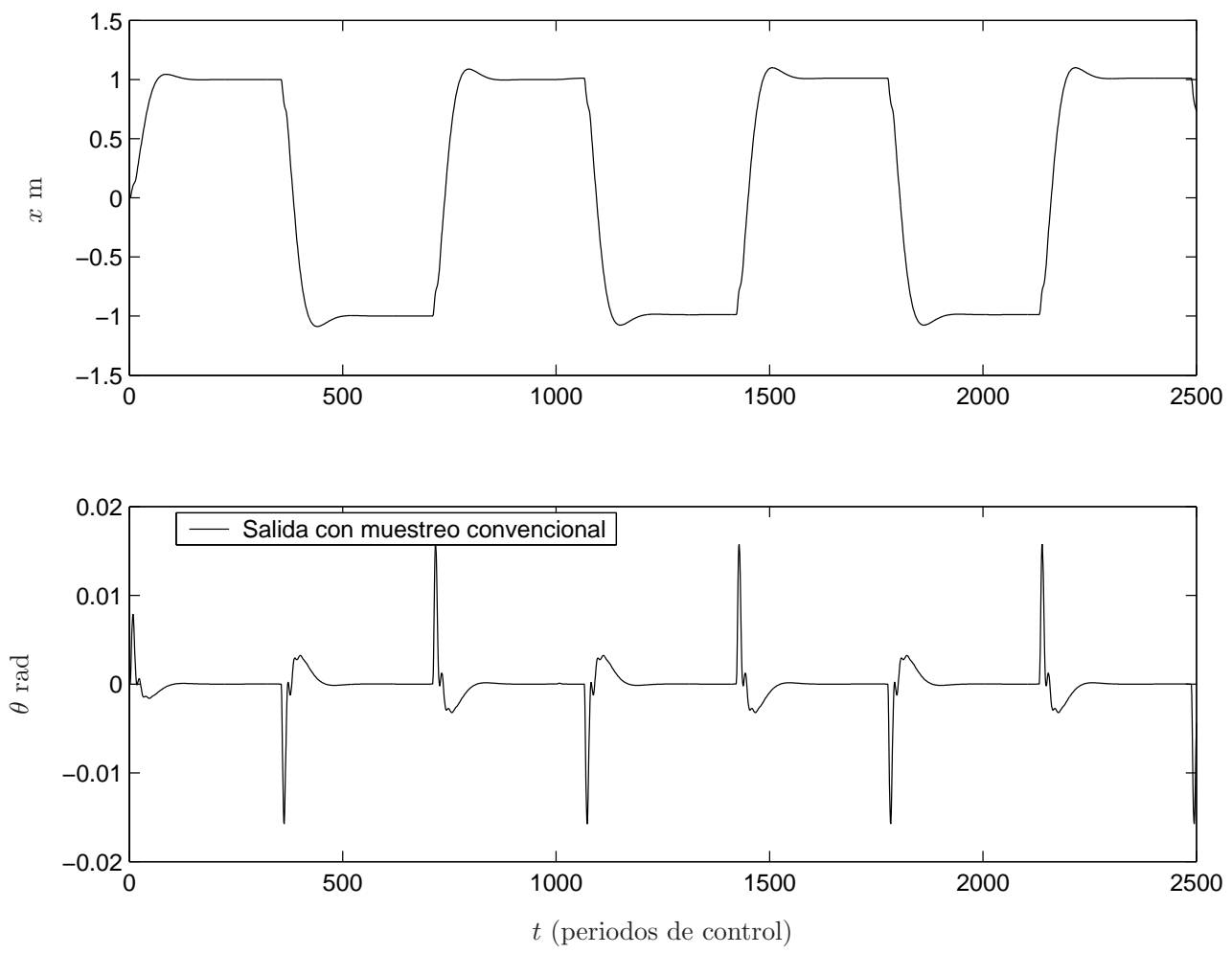

Figura 7.2: Control del puente grúa con un predictor de bucle abierto
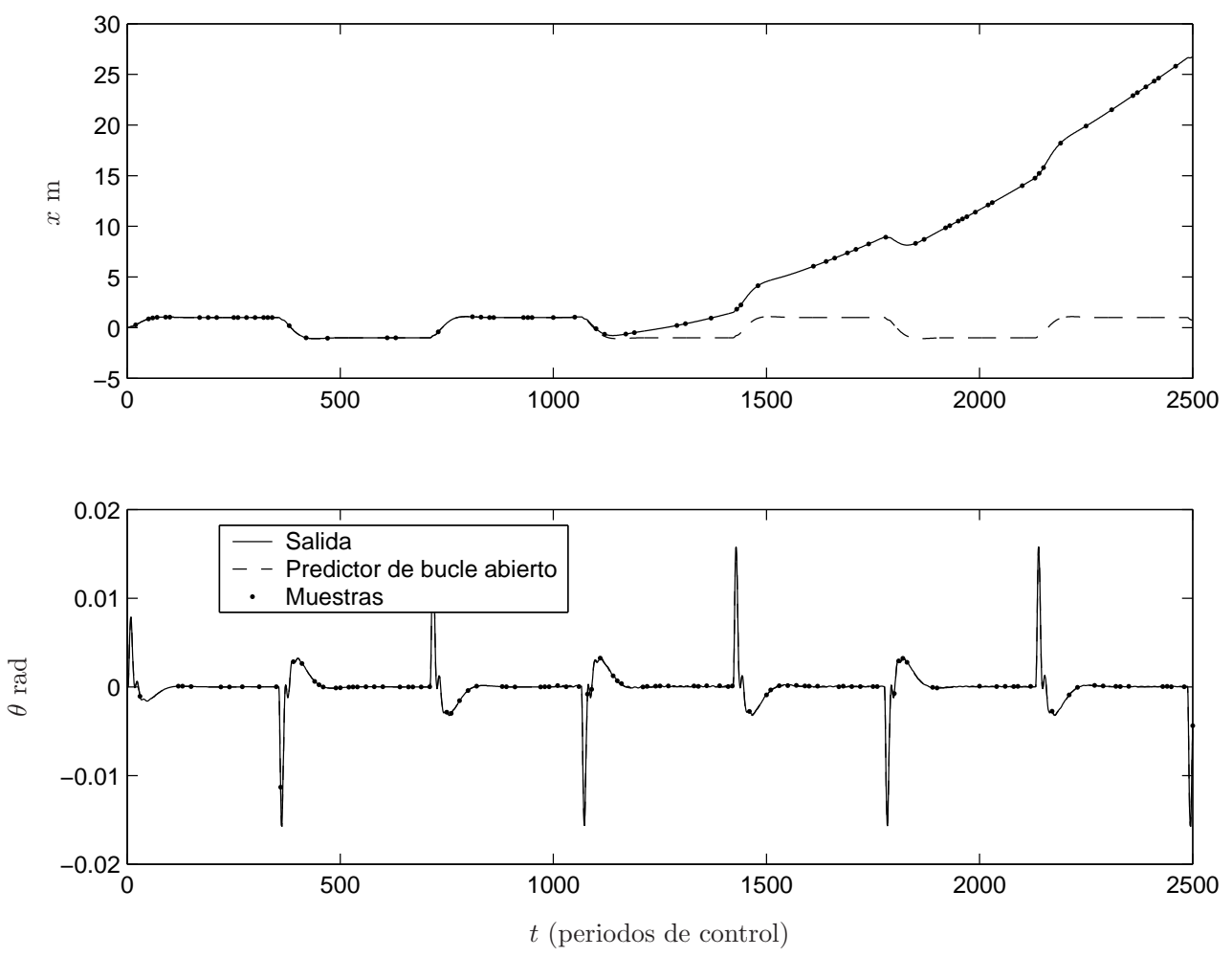

Figura 7.3: Control del puente grúa con un predictor de bucle abierto 

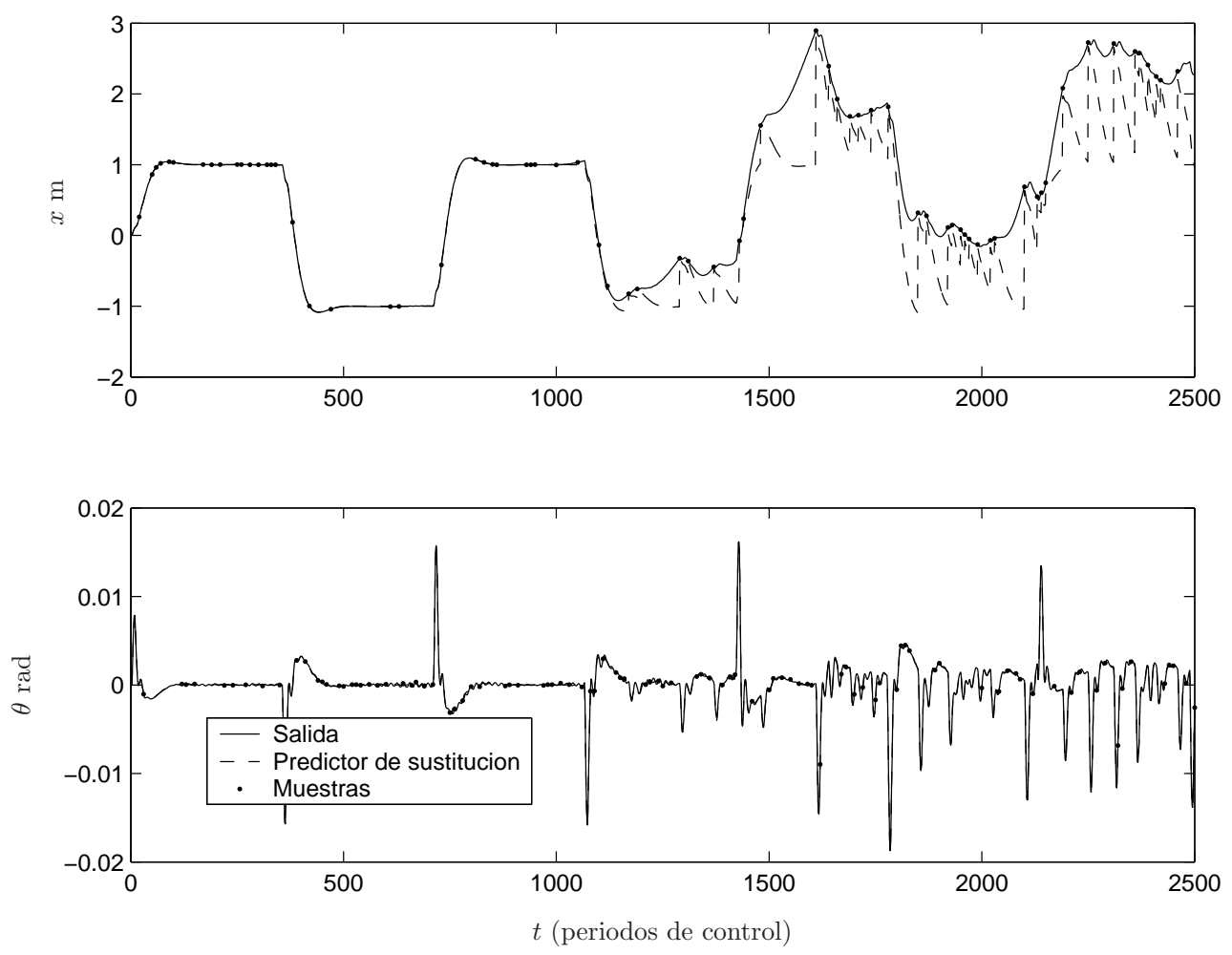

Figura 7.4: Control del puente grúa con un predictor de sustitución
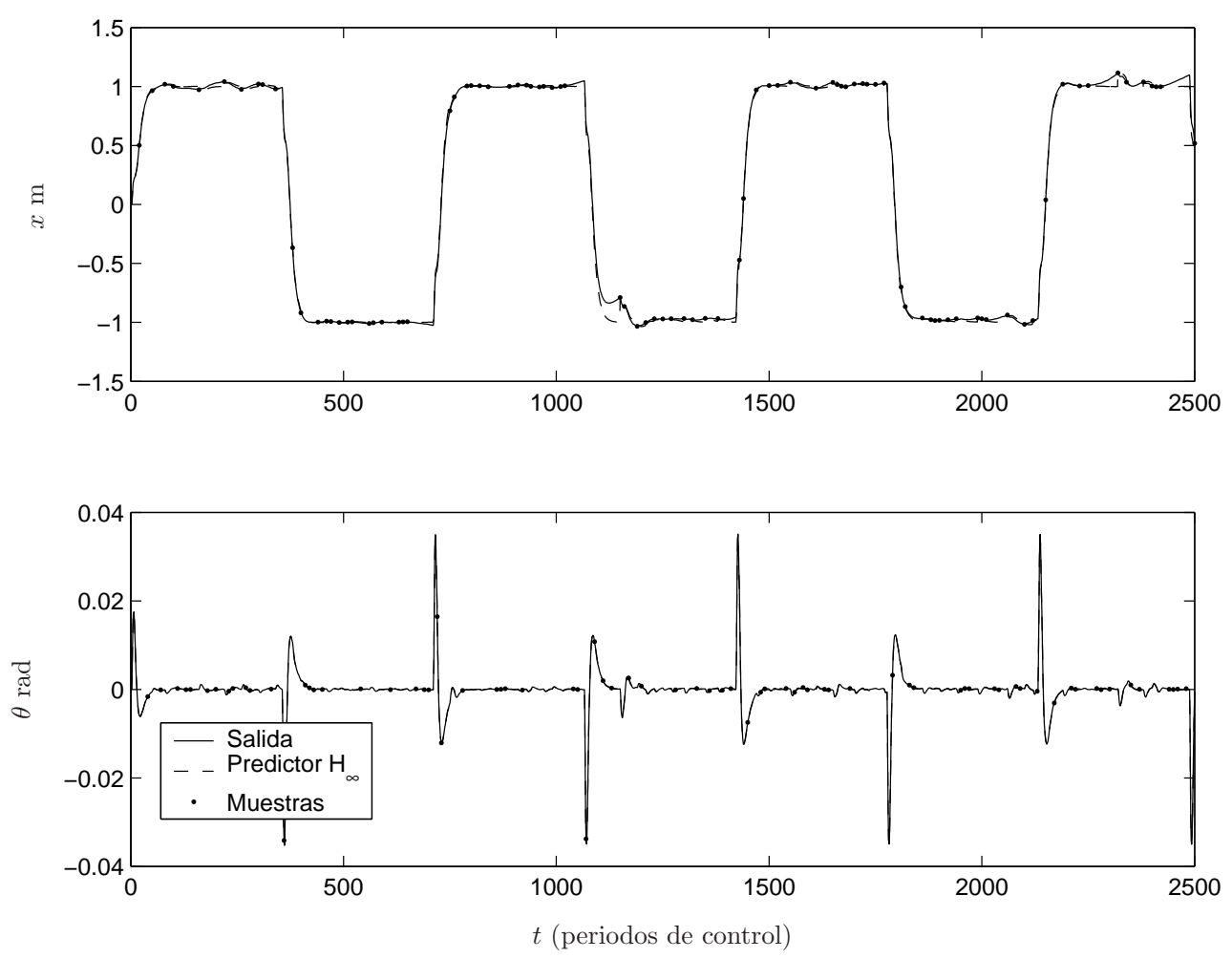

Figura 7.5: Control del puente grúa con un predictor $\mathcal{H}_{\infty}$ de ganancia variable 

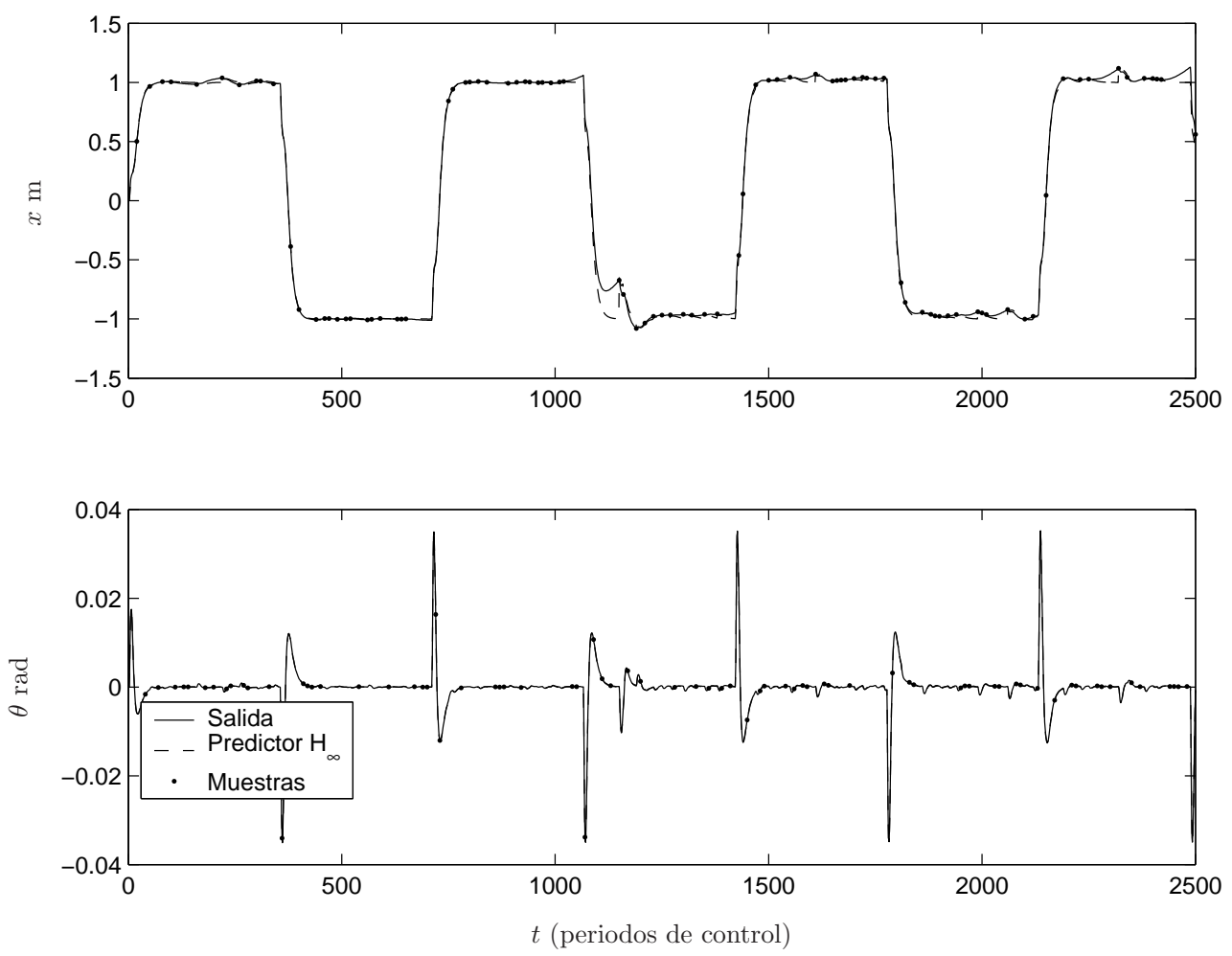

Figura 7.6: Control del puente grúa con un predictor $\mathcal{H}_{\infty}$ de ganancia constante
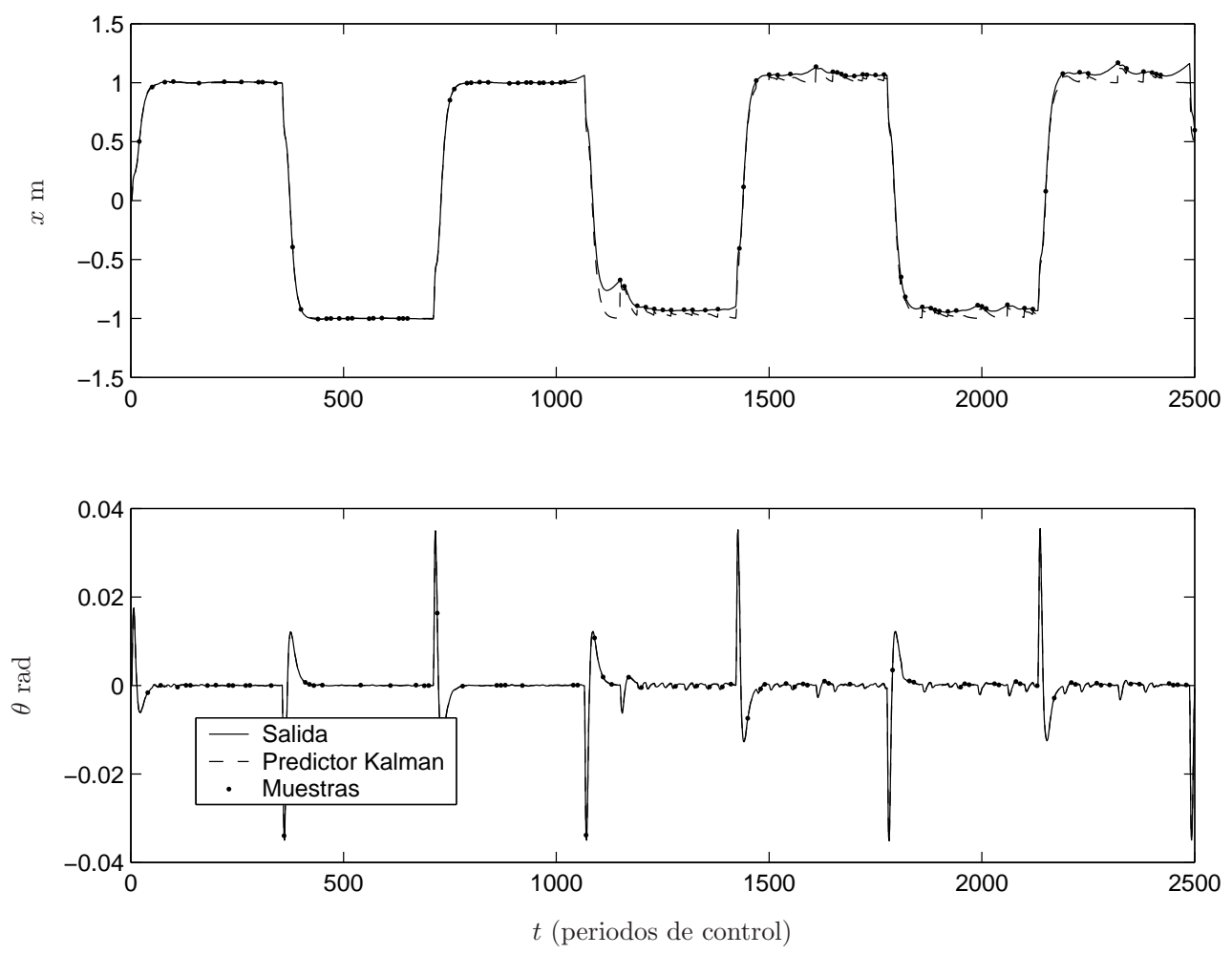

Figura 7.7: Control del puente grúa con un filtro de Kalman 


\subsection{Posicionado de un robot móvil mediante detección de azulejos}

\subsubsection{Introducción}

En este ejemplo se estudia la mejora de la estimación de la posición de un robot móvil a partir de la detección del paso del robot sobre las juntas de azulejos. Para fusionar esta información con los datos proporcionados por los codificadores incrementales de las ruedas, se ha utilizado un modelo odométrico y diferentes predictores de los propuestos en este trabajo de tesis.

Existen diversos métodos para estimar la posición absoluta de los robots móviles, que se han tratado extensamente en la literatura (véase [19]). Uno de los métodos más extendidos se basa en la utilización de codificadores incrementales en las ruedas, que permiten medir el avance de cada rueda. La utilización de un modelo matemático que relaciona dicho avance con la posición y orientación del robot permite estimar estas variables. El problema de esta estrategia es que el error cometido en la estimación aumenta inexorablemente con el tiempo, por lo que se requiere de alguna estrategia adicional para garantizar un error pequeño en la posición estimada.

En este ejemplo se propone utilizar como estrategia adicional a los codificadores de las ruedas, la detección del paso del robot sobre las juntas de los azulejos del suelo. Esta detección se realiza mediante un sensor óptico de reflexión de muy bajo coste. Si se conoce cuál es la línea detectada, se tiene información precisa de una de las coordenadas de la posición, por lo que se puede corregir la estimación, manteniendo el error en valores pequeños. Una de las dificultades de esta idea consiste en determinar cuál es la línea detectada. Para ello se han dispuesto tres sensores alineados, de manera que el robot puede diferenciar con qué tipo de linea (horizontal o vertical) se cruza cada vez, si se tiene una buena estimación del ángulo del vehículo. El vehículo lleva incorporado un microcontrolador de bajo coste para realizar el posicionamiento.

\subsubsection{Descripción del robot}

La figura 7.8 muestra el robot móvil, que se mueve sobre un suelo formado por azulejos rectangulares en los que se distingue la junta de separación entre ellos por ser de distinto color. De esta forma el suelo forma una rejilla cuya detección se utiliza para la mejora de la estimación de la posición del móvil. El robot móvil tiene un pivote delantero y dos ruedas motoras independientes situadas sobre un mismo eje. Al lado de cada rueda motora hay una segunda rueda con un codificador incremental de 500 pulsos/vuelta. El robot también incorpora tres detectores ópticos de reflexión en la parte inferior para detectar el paso entre dos azulejos. Si el ángulo que lleva el vehículo es conocido, o se tiene una buena aproximación de él, cada vez que se activan de forma consecutiva un sensor lateral y el central, se está en condiciones de diferenciar qué tipo de línea (horizontal o vertical) es la que se ha atravesado.
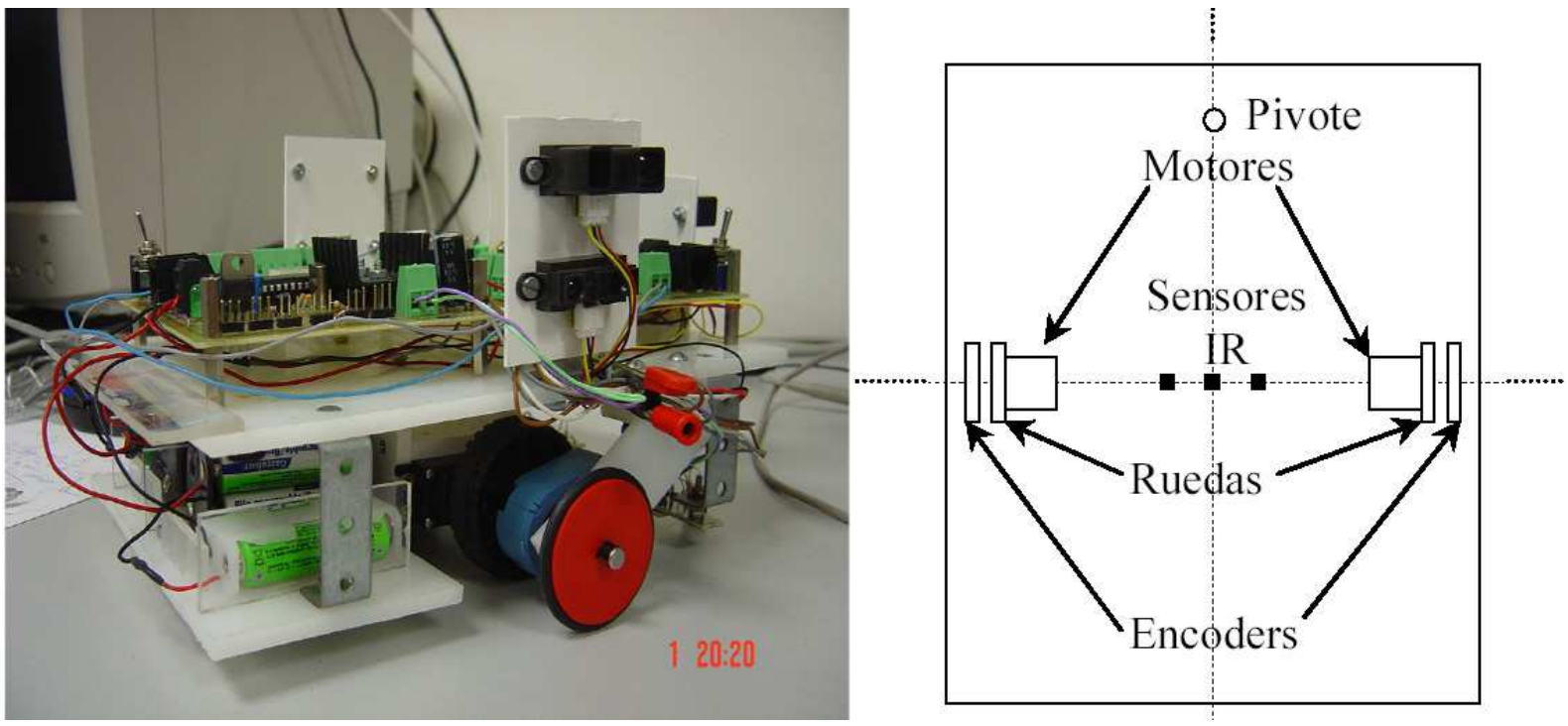

Figura 7.8: Robot móvil y sistema de odometría 


\subsubsection{Modelo del robot}

La figura 7.9 muestra la posición del eje de las ruedas sensoras del robot en dos instantes de tiempo consecutivos $t$ y $t+1$. En el instante $t$ la posición del robot viene definida por el vector de estado

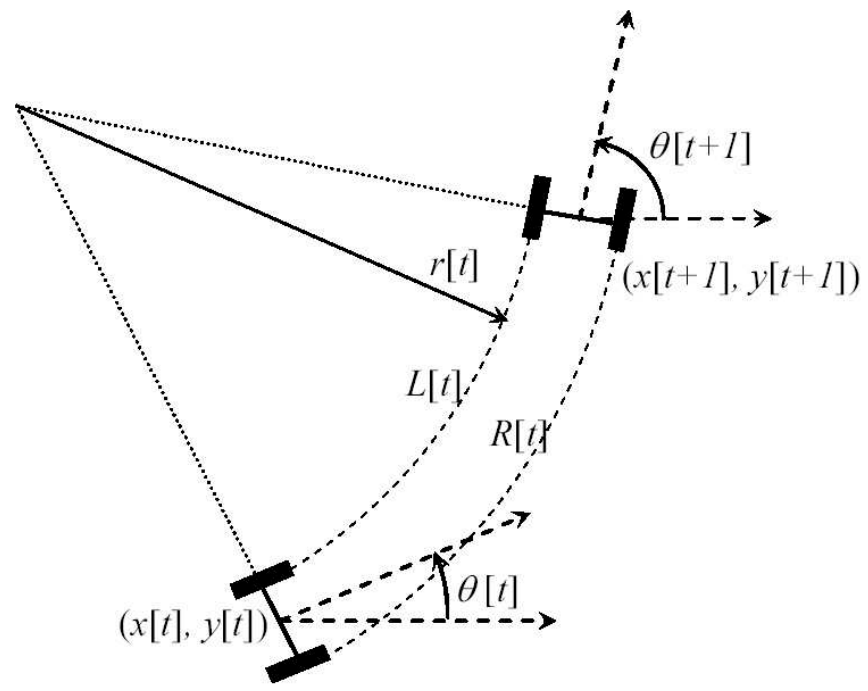

Figura 7.9: Estado inicial y final del móvil tras seguir una trayectoria de arco

$$
\boldsymbol{x}[t]=\left[\begin{array}{l}
x[t] \\
y[t] \\
\theta[t]
\end{array}\right] .
$$

Entre el instante $t$ y el instante $t+1$, el arco recorrido por cada una de las ruedas es $L[t]$ y $R[t]$. Si se asume una velocidad constante de las ruedas entre los instantes $t$ y $t+1$, el radio de curvatura $r[t]$ será constante y se puede obtener la posición en el instante $t+1$ como (véase [12])

$$
\begin{aligned}
{\left[\begin{array}{l}
x[t+1] \\
y[t+1] \\
\theta[t+1]
\end{array}\right] } & =\left[\begin{array}{l}
x[t] \\
y[t] \\
\theta[t]
\end{array}\right]+\left[\begin{array}{c}
r[t]\left[\sin \theta[t]-\sin \left(\theta[t]+\frac{R[t]-L[t]}{B}\right)\right] \\
r[t]\left[\cos \left(\theta[t]+\frac{R[t]-L[t]}{B}\right)-\cos \theta[t]\right] \\
\frac{R[t]-L[t]}{B}
\end{array}\right], \\
r[t] & =\frac{B}{2}\left(\frac{L[t]+R[t]}{L[t]-R[t]}\right),
\end{aligned}
$$

siendo $r[t]$ el radio de curvatura instantáneo y $B$ la distancia entre ruedas. El vector de entradas del sistema es

$$
\boldsymbol{u}[t]=\left[\begin{array}{c}
R[t] \\
L[t]
\end{array}\right] .
$$

En [12] se propone un modelo estocástico del proceso suponiendo que para un desplazamiento pequeño se puede considerar un ruido blanco de media cero asociado a $L[t]$ y $R[t]$, y sin correlación con los desplazamientos anteriores y posteriores. La varianza asociada a $L[t]$ y $R[t]$ se modela como

$$
\begin{aligned}
& \sigma_{L}^{2}=k_{L}^{2}\left|d_{L}\right| \\
& \sigma_{R}^{2}=k_{R}^{2}\left|d_{R}\right|
\end{aligned}
$$

donde $d_{L}$ y $d_{R}$ son las distancias recorridas por cada rueda $(|L[t]|$ y $|R[t]|)$ y $k_{L}$ y $k_{R}$ son constantes que dependen de la precisión del montaje.

En este ejemplo se van a tomar como parámetros característicos del modelo los indicados en la tabla 7.3, y como entrada la secuencia indicada en la figura 7.10.

Para poder aplicar el predictor desarrollado en este trabajo de tesis es necesario obtener un modelo del sistema de la forma

$$
\boldsymbol{x}[t+1]=\boldsymbol{A} \boldsymbol{x}[t]+\boldsymbol{f}(\boldsymbol{x}[t], \boldsymbol{u}[t])+\boldsymbol{B}_{v} \boldsymbol{v}[t]
$$




\begin{tabular}{lll} 
Parámetro & Valor & Definición \\
\hline$B$ & $19 \mathrm{~cm}$ & Distancia entre ejes \\
$k_{L}$ & $0.01 \sqrt{\mathrm{m}}$ & Constante de montaje \\
$k_{R}$ & $0.01 \sqrt{\mathrm{m}}$ & Constante de montaje \\
$L_{\text {máx }}$ & $1.9 \mathrm{~cm}$ & Máximo avance de la rueda izquierda en un periodo \\
$R_{\text {máx }}$ & $1.9 \mathrm{~cm}$ & Máximo avance de la rueda derecha en un periodo \\
\hline
\end{tabular}

Tabla 7.3: Parámetros característicos del robot móvil

En primer lugar, se debe tener en cuenta que el término de variación de ángulo $\left(\frac{R[t]-L[t]}{B}\right)$ en el peor de los casos (con las ruedas girando en sentidos opuestos) nunca superará el valor

$$
\frac{R[t]-L[t]}{B} \leq \frac{R_{\text {máx }}-L_{\text {máx }}}{B}=0.2\left(=11^{\circ}\right)
$$

Para la secuencia de entradas supuestas en el ejemplo, el radio de curvatura es el indicado en la figura 7.11, donde se observa que el cociente nunca supera los $0.1 \mathrm{rad}\left(5.7^{\circ}\right) \mathrm{y}$, por tanto,se pueden hacer las aproximaciones

$$
\begin{aligned}
\sin \left(\frac{R[t]-L[t]}{B}\right) & \approx \frac{R[t]-L[t]}{B}, \\
\cos \left(\frac{R[t]-L[t]}{B}\right) & \approx 1 .
\end{aligned}
$$

Desarrollando los términos
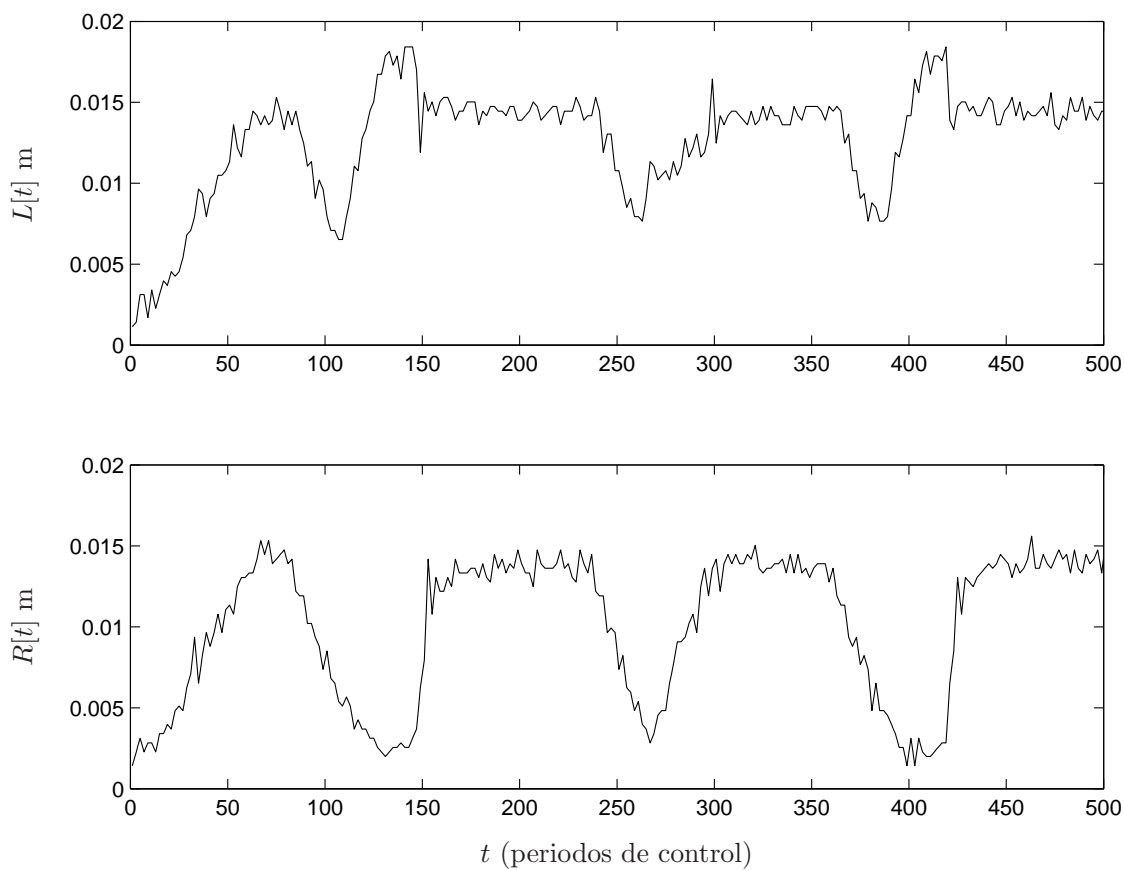

Figura 7.10: Secuencias de entrada medida en los encoders del robot móvil

$$
\sin \left(\theta[t]+\frac{R[t]-L[t]}{B}\right), \quad \cos \left(\theta[t]+\frac{R[t]-L[t]}{B}\right)
$$

en (7.7) y aplicando la aproximación anterior se llega a

$$
\left[\begin{array}{l}
x[t+1] \\
y[t+1] \\
\theta[t+1]
\end{array}\right]=\left[\begin{array}{l}
x[t] \\
y[t] \\
\theta[t]
\end{array}\right]+\left[\begin{array}{cc}
\frac{\cos \theta[t]}{2} & \frac{\cos \theta[t]}{2} \\
\frac{\sin \theta[t]}{2} & \frac{\sin \theta[t]}{2} \\
\frac{1}{B} & -\frac{1}{B}
\end{array}\right]\left[\begin{array}{c}
R[t] \\
L[t]
\end{array}\right] .
$$




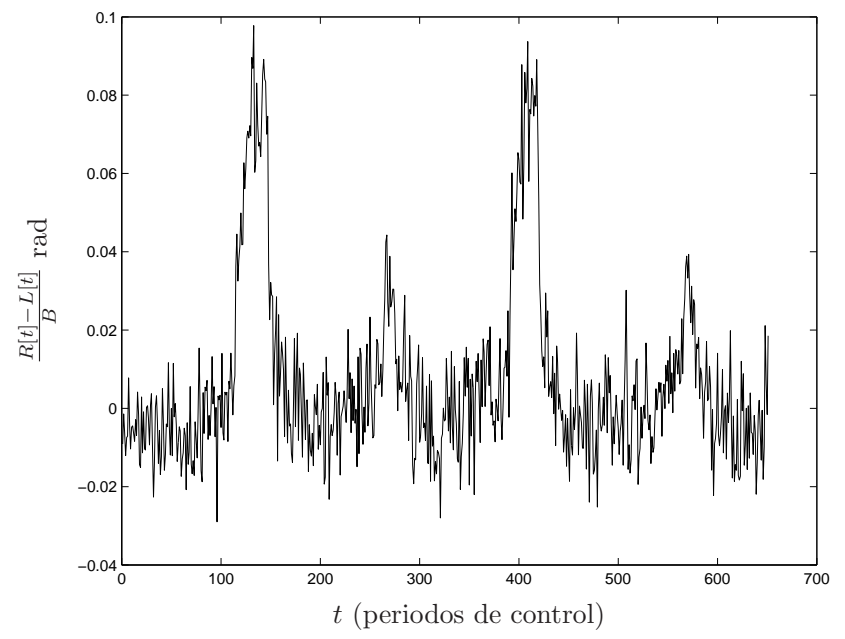

Figura 7.11: Variaciones del ángulo del vehículo a lo largo del tiempo

Teniendo ahora en cuenta la propagación del error en las señales $R[t]$ y $L[t]$ según (7.9):

$$
\begin{aligned}
L[t] & \rightarrow L[t]\left(1+\omega_{L}[t]\right), \\
R[t] & \rightarrow R[t]\left(1+\omega_{R}[t]\right),
\end{aligned}
$$

y añadiendo una perturbación del estado que modele el error del modelo por las aproximaciones realizadas se llega a una expresión completa de la forma

$$
\left[\begin{array}{l}
x[t+1] \\
y[t+1] \\
\theta[t+1]
\end{array}\right]=\underbrace{\left[\begin{array}{l}
x[t] \\
y[t] \\
\theta[t]
\end{array}\right]}_{\boldsymbol{A} \boldsymbol{x}[t]}+\underbrace{\left[\begin{array}{cc}
\frac{\cos \theta[t]}{2} \frac{\cos \theta[t]}{2} & \frac{\sin \theta[t]}{2} \\
\frac{1}{B} & -\frac{1}{B}
\end{array}\right]\left[\begin{array}{l}
R[t] \\
L[t]
\end{array}\right]}_{\boldsymbol{f}(\boldsymbol{x}[t], \boldsymbol{u}[t])}+\underbrace{\left[\begin{array}{ccccc}
\frac{R[t] \cos \theta[t]}{2} & \frac{L[t] \cos \theta[t]}{2} & 1 & 0 & 0 \\
\frac{R[t] \sin \theta[t]}{2} & \frac{L[t] \sin \theta[t]}{2} & 0 & 1 & 0 \\
\frac{-L[t]}{B} & 0 & 0 & 1
\end{array}\right]}_{\boldsymbol{B}_{v}} \underbrace{\left[\begin{array}{l}
\omega_{R}[t] \\
\omega_{L}[t] \\
\omega_{x}[t] \\
\omega_{y}[t] \\
\omega_{\theta}[t]
\end{array}\right]}_{\boldsymbol{v}[t]} .
$$

En este caso la matriz $\boldsymbol{A}$ es la matriz identidad (I). Ahora falta buscar la matriz $\boldsymbol{F}$ tal que

$$
\|\boldsymbol{f}(\boldsymbol{x}[t], \boldsymbol{u}[t])-\boldsymbol{f}(\hat{\boldsymbol{x}}[t], \boldsymbol{u}[t])\| \leq\|\boldsymbol{F}(\boldsymbol{x}[t]-\hat{\boldsymbol{x}}[t])\| .
$$

Es decir,

$$
\left\|\left[\begin{array}{c}
\frac{R[t]+L[t]}{2} \cos \theta[t] \\
\frac{R[t]+L[t]}{2} \sin \theta[t] \\
\frac{R[t]-L[t]}{B}
\end{array}\right]-\left[\begin{array}{c}
\frac{R[t]+L[t]}{2[t]+L[t]} \cos \hat{\theta}[t] \\
\frac{R[t]-L[t]}{B}
\end{array}\right]\right\| \leq\left\|\boldsymbol{F}\left[\begin{array}{l}
x[t]-\hat{x}[t] \\
y[t]-\hat{y}[t] \\
\theta[t]-\hat{\theta}[t]
\end{array}\right]\right\| .
$$

Es fácil demostrar que la matriz $\boldsymbol{F}$ tendrá la forma

$$
\boldsymbol{F}=\left[\begin{array}{ccc}
0 & 0 & f_{1} \\
0 & 0 & f_{2} \\
0 & 0 & 0
\end{array}\right]
$$

donde $f_{1}$ y $f_{2}$ deberán cumplir

$$
\begin{aligned}
& \left\|\frac{R[t]+L[t]}{2}(\cos \theta[t]-\cos \hat{\theta}[t])\right\| \leq\left\|f_{1}(\theta[t]-\hat{\theta}[t])\right\| \\
& \left\|\frac{R[t]+L[t]}{2}(\sin \theta[t]-\sin \hat{\theta}[t])\right\| \leq\left\|f_{2}(\theta[t]-\hat{\theta}[t])\right\| .
\end{aligned}
$$

Teniendo en cuenta las propiedades

$$
\begin{aligned}
\|\sin (a)-\sin (b)\|<\|a-b\|, & a, b \in[0,2 \pi], \\
\|\cos (a)-\cos (b)\|<\|a-b\|, & a, b \in[0,2 \pi],
\end{aligned}
$$


y teniendo en cuenta el límite de $R[t]$ y $L[t]$ en $1.9 \mathrm{~cm}$, se tiene que

$$
f_{1}=f_{2}=\frac{R_{\text {máx }}+L_{\text {máx }}}{2}=0.019,
$$

con lo que

$$
\boldsymbol{F}=\left[\begin{array}{ccc}
0 & 0 & 0.019 \\
0 & 0 & 0.019 \\
0 & 0 & 0
\end{array}\right]
$$

En este ejemplo, la matriz $\boldsymbol{B}_{v}$ es variante en el tiempo, con lo cual es necesario tomar un valor representativo de esta matriz. Para ello se puede tomar bien el valor medio de esta matriz, o bien el valor máximo que pueden tomar sus elementos para permanecer del lado de la seguridad. De esta forma, la matriz $\boldsymbol{B}_{v}$ queda

$$
\boldsymbol{B}_{v}=\left[\begin{array}{ccccc}
0.095 & 0.095 & 1 & 0 & 0 \\
0.095 & 0.095 & 0 & 1 & 0 \\
0.1 & -0.1 & 0 & 0 & 1
\end{array}\right]
$$

\subsubsection{Estimación de la posición}

A partir de las mediciones de los codificadores de las ruedas sensoras se obtienen los arcos $L[t]$ y $R[t]$ que son las entradas del modelo. Con estos valores se puede estimar la posición en $t+1$ del móvil corriendo el modelo (7.7) con la estimación $\hat{\boldsymbol{x}}[t]$ :

$$
\hat{\boldsymbol{x}}[t+1 \mid t]=\left[\begin{array}{c}
\hat{x}[t+1 \mid t] \\
\hat{y}[t+1 \mid t] \\
\hat{\theta}[t+1 \mid t]
\end{array}\right]=\left[\begin{array}{c}
\hat{x}[t] \\
\hat{y}[t] \\
\hat{\theta}[t]
\end{array}\right]+\left[\begin{array}{c}
r[t]\left[\sin \hat{\theta}[t]-\sin \left(\hat{\theta}[t]+\frac{R[t]-L[t]}{B}\right)\right] \\
r[t]\left[\cos \left(\hat{\theta}[t]+\frac{R[t]-L[t]}{B}\right)-\cos \hat{\theta}[t]\right] \\
\frac{R[t]-L[t]}{B}
\end{array}\right] .
$$

El problema de la estimación de la posición del móvil en bucle abierto es la gran deriva con el tiempo debida a factores aleatorios como imprecisiones en diámetros de ruedas, rugosidad del terreno, deslizamientos, etc. Esta deriva hace que si no se corrige la estimación de alguna forma, en poco tiempo el error cometido pueda llegar a ser enorme.

Para poder corregir la trayectoria estimada del vehículo se ha ubicado tres sensores de reflexión en la parte inferior del vehículo de manera que si el ángulo que lleva el vehículo es conocido (o se tiene una aproximación de él), cada vez que se activan de forma consecutiva un sensor lateral y el sensor central, se puede diferenciar qué tipo de línea -horizontal o vertical- ha atravesado el sensor central. En ese instante $\tau_{k}$ se considera que se ha tenido una medición de la posición $x\left(\tau_{k}\right)$ o $y\left(\tau_{k}\right)$ con mucha precisión, ya que estas mediciones se consideran múltiplo del tamaño de los azulejos, que se supone conocido de antemano. Como el paso del vehículo sobre las juntas de azulejos no se producirá en instantes de control (a periodo $T$ ), las mediciones son asíncronas, y, por ese motivo, es necesario utilizar una técnica de interpolación para obtener la medición equivalente al siguiente instante de muestreo. Esta interpolación llevará a un pequeño error en la medición que puede considerarse como un ruido de medida, tal y como se ha expuesto en $\S 4.7 .4$. Aplicando la técnica de interpolación de orden cero I3 (asignar la medición al instante inmediatamente anterior o al inmediatamente posterior, en función del instante exacto en el que se toma la medición), una cota del error de medida (tanto en la variable $x$ como en la $y$ ) viene dada por

$$
\begin{aligned}
\left\|w_{m_{x}}\right\|_{\infty} & =0.5\|\boldsymbol{c}(\boldsymbol{x}[t+1]-\boldsymbol{x}[t])\|_{\infty} \\
& =0.5\|\boldsymbol{c}(\boldsymbol{A} \boldsymbol{x}[t]+\boldsymbol{f}(\boldsymbol{x}[t], \boldsymbol{u}[t])-\boldsymbol{x}[t])\|_{\infty} \\
& =0.5\|\boldsymbol{c}(\boldsymbol{f}(\boldsymbol{x}[t], \boldsymbol{u}[t]))\|_{\infty} \\
& \leq 0.5\|L[t]+R[t]\|_{\infty} \leq 0.5\left(R_{\text {máx }}+L_{\text {máx }}\right)=0.019,
\end{aligned}
$$

es decir, que los errores de medida de $x$ e $y$ estarán comprendidos entre -0.019 y 0.019 m. El valor medio de este ruido de medida asociado a la interpolación es cero, y será mayor cuanto mayor sean los valores de las entradas, pudiéndose asumir una distribución normal de desviación típica aproximada

$$
\sigma_{m_{x}}=\sigma_{m_{y}}=\frac{0.019}{3}=0.0063 \mathrm{~m} .
$$

La estructura de 3 sensores que se observa en la figura 7.8 permite medir el ángulo con el que avanza el móvil cada vez que se cruza con una de las líneas del terreno (juntas de azulejos). El ángulo con el que avanza el vehículo se puede medir si se dan estas dos circunstancias: 
(i) El vehículo marcha aproximadamente en línea recta (con $L[t]$ y $R[t]$ de valor próximo),

(ii) El vehículo no se encuentra en una región próxima a dos aristas de azulejo (es decir, cerca de una esquina), ya que en ese caso es probable que la activación consecutiva de los sensores no se deba a la misma línea, sino a líneas de direcciones diferentes. En este caso no se medirá el ángulo pero se asumirá que se conocen tanto la posición $x$ como la posición $y$.

Bajo estas condiciones, si se mide el tiempo transcurrido desde que se activa uno de los detectores laterales hasta que se activa el detector central, se puede calcular de forma aproximada el ángulo de la trayectoria seguida por el móvil. Debido a las imprecisiones del montaje y a que las trayectorias no son siempre rectilíneas se asume que la medición de este ángulo tiene un error de medición asociado de media cero y desviación típica $3^{\circ}$.

Con todo lo expuesto, la ecuación que determina estas mediciones es

$$
\boldsymbol{y}_{k}=\left[\begin{array}{lll}
1 & 0 & 0 \\
0 & 0 & 1
\end{array}\right]\left[\begin{array}{l}
x\left[t_{k}\right] \\
y\left[t_{k}\right] \\
\theta\left[t_{k}\right]
\end{array}\right]
$$

cuando se atraviesa una línea horizontal,

$$
\boldsymbol{y}_{k}=\left[\begin{array}{lll}
0 & 1 & 0 \\
0 & 0 & 1
\end{array}\right]\left[\begin{array}{l}
x\left[t_{k}\right] \\
y\left[t_{k}\right] \\
\theta\left[t_{k}\right]
\end{array}\right]
$$

cuando se atraviesa una línea vertical, y

$$
\boldsymbol{y}_{k}=\left[\begin{array}{lll}
1 & 0 & 0 \\
0 & 1 & 0
\end{array}\right]\left[\begin{array}{l}
x\left[t_{k}\right] \\
y\left[t_{k}\right] \\
\theta\left[t_{k}\right]
\end{array}\right]
$$

si se atraviesa un cruce de juntas de azulejos, y éste es detectado correctamente.

Para saber cada cuántos periodos de control se tiene una muestra (es decir, para obtener el rango de $N, \mathcal{N})$, se ejecuta una simulación del proceso con las señales de entrada indicadas anteriormente y se obtiene la trayectoria de la figura 7.12, donde se han indicado dos detalles de la trayectoria. En ellos se puede observar el número de periodos de control que hay desde que el móvil atraviesa una junta de azulejos hasta que atraviesa la siguiente. A partir de la simulación se ha podido comprobar que, para las condiciones de funcionamiento utilizadas en la simulación, la $N$ puede variar entre 1 y 90 , es decir:

$$
\mathcal{N}=\{1,2, \ldots, 90\}
$$

La perturbación del estado $\boldsymbol{v}=\left[\omega_{x} \omega_{y} \omega_{\theta}\right]^{\top}$ se considera un ruido blanco de media cero y desviación típica

$$
\sigma_{x}=0.0014, \quad \sigma_{y}=0.0014, \quad \sigma_{\theta}=0.0014,
$$

que viene a interpretar los posibles errores de modelado debidos, por ejemplo, a las imperfecciones del terreno.

El funcionamiento del predictor es como sigue. En funcionamiento normal, el dispositivo estima la posición del móvil en bucle abierto utilizando la ecuación (7.13). Cuando hay una nueva medida (el móvil pasa sobre una junta de azulejos), la estimación inicial se corrige con la ecuación del predictor

$$
\hat{\boldsymbol{x}}[t]=\hat{\boldsymbol{x}}[t \mid t-1]+\boldsymbol{L}\left(s_{k}\right) \boldsymbol{\Delta}\left(s_{k}\right)\left(m_{k}-\boldsymbol{c} \hat{\boldsymbol{x}}[t \mid t-1]\right)
$$

donde $s_{k}$ es el parámetro que indica el tipo de muestreo que se ha dado, es decir, que indica tanto el valor de periodos que se había estado sin muestreo $\left(N_{k}\right)$, como los sensores que han estado accesibles en esa medición, dependiendo de qué tipo de cruce se haya producido. Como se tienen mediciones del ángulo, $n_{m}=3$, y $\boldsymbol{L}\left(s_{k}\right)$ es una matriz de orden $3 \times 3 . \boldsymbol{\Delta}\left(s_{k}\right)$ es la matriz de disponibilidad de orden $3 \times 3$ que contiene únicamente unos en aquellas posiciones de la diagonal correspondientes a los mediciones utilizadas:

- $\boldsymbol{\Delta}\left(s_{k}\right)=\left[\begin{array}{lll}1 & 0 & 0 \\ 0 & 0 & 0 \\ 0 & 0 & 1\end{array}\right]$ cuando se produce un cruce con una línea vertical, 

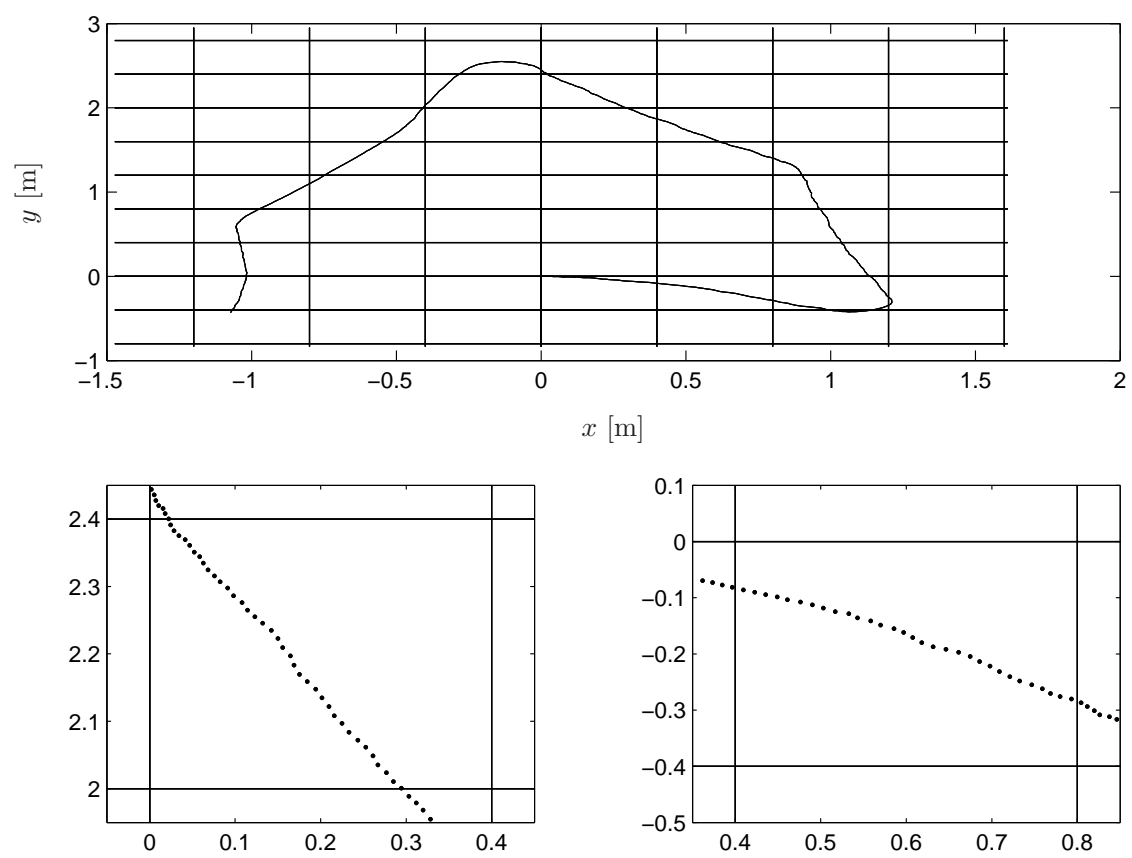

Figura 7.12: Trayectoria que sigue el robot

- $\boldsymbol{\Delta}\left(s_{k}\right)=\left[\begin{array}{lll}0 & 0 & 0 \\ 0 & 1 & 0 \\ 0 & 0 & 1\end{array}\right]$ cuando se produce un cruce con una línea horizontal, y

- $\boldsymbol{\Delta}\left(s_{k}\right)=\left[\begin{array}{lll}1 & 0 & 0 \\ 0 & 1 & 0 \\ 0 & 0 & 0\end{array}\right]$ cuando se produce un cruce con una esquina de un azulejo y se asume conocida la posición en $x$ y en $y$. En este caso se asume que no es posible obtener el ángulo $\theta$.

Con toda la información que se ha indicado se está en condiciones de aplicar el método de predicción expuesto en el teorema 5.6.2 (página 148). El número de matrices $\boldsymbol{L}\left(s_{k}\right)$ que es necesario calcular es muy elevado: 90 para las actualizaciones tras los cruces con líneas horizontales, 90 para las verticales, y 90 más para los cruces con las esquinas (se asumen conocidos $x$ e $y$ simultáneamente, pero se asume que el ángulo no es posible medirlo). Estas 270 matrices deberían almacenarse en la memoria del microcontrolador instalado en el robot. Para evitar esto, sólo se almacenan en el controlador del robot las matrices $\boldsymbol{L}$ para un conjunto más reducido de periodos intermuestreo

$$
\mathcal{N}^{\prime}=\{1,2,4,5,10,15,20,30,40,50,60,70,90\}
$$

y el resto de matrices necesarias se obtienen por interpolación en el propio vehículo cada vez que llega una nueva medición (véase \$4.8.3). En la figura 7.13 se muestran los valores que toman algunos de los elementos de la matriz $\boldsymbol{L}$ en función del número de periodos intermuestreo y de los sensores disponibles.

Procediendo de esta forma se ha realizado una simulación del predictor cuyo resultado se muestra en la figura 7.14, donde se observan los instantes donde el vehículo ha atravesado una junta y, por tanto, ha utilizado el valor de la medición. La predicción de bucle abierto muestra el gran error que se comete debido a la perturbación de entrada (las entradas reales del proceso no coinciden con las mediciones de los encoders) y a la perturbación del estado. En la figura 7.15 se detalla la evolución de cada uno de los estados y se compara con la predicción en bucle abierto y con la predicción propuesta. Los círculos indican que se ha tomado medición de esa variable. Si se desea implementar un predictor de menor coste computacional se puede calcular un predictor de ganancia constante. La resolución del problema de optimización asociado devuelve la matriz identidad como matriz de ganancias del predictor $(\boldsymbol{L}=\boldsymbol{I})$, es decir, que se trata de un predictor de sustitución. Si se realiza una simulación utilizando dicho predictor se obtiene el resultado de la figura 7.16

Si el error cometido en el cálculo del ángulo tras cada cruce con las juntas de azulejos se considera de desviación típica $\sigma_{m_{\theta}}=6^{\circ}$, se recalcula la matriz de ganancias (variante) y se obtiene el resultado que se muestra en la figura 7.17. La implementación de la matriz de ganancias en este caso también se ha 

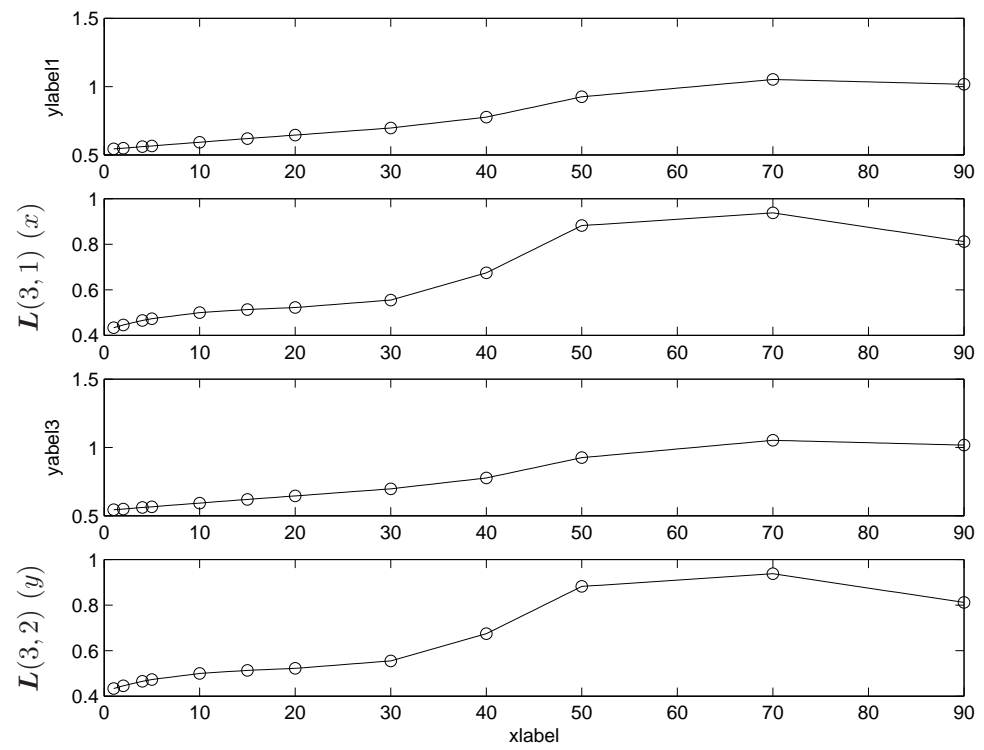

Figura 7.13: Diferentes valores de elementos de la matriz $\boldsymbol{L}$ en función del número de periodos intermuestreo. $\boldsymbol{L}(1,1)$ y $\boldsymbol{L}(3,1)$ son elementos utilizados cuando hay mediciones de $x$, mientras que $\boldsymbol{L}(2,2)$ y $\boldsymbol{L}(3,2)$ son elementos utilizados cuando hay mediciones de $y$

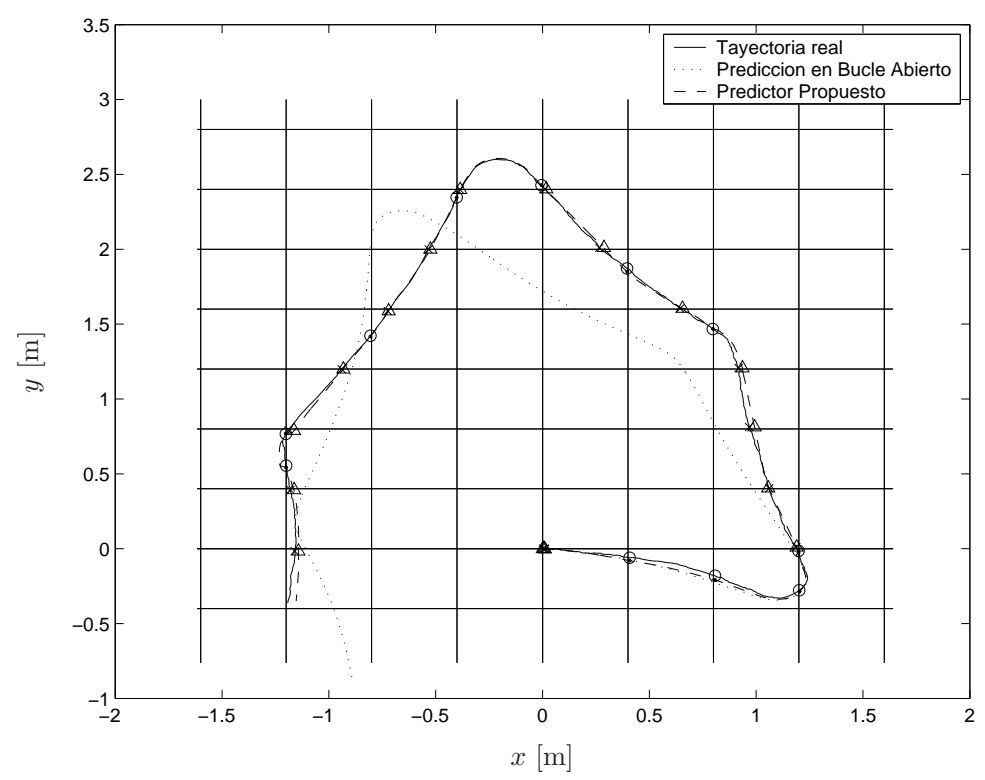

Figura 7.14: Trayectoria real, predicción en bucle abierto y predicción mediante la técnica propuesta

hecho mediante interpolación a partir de las obtenidas en el subconjunto $\mathcal{N}^{\prime}$ indicado anteriormente. Si se busca un predictor con ganancia constante, la matriz de ganancias que se obtiene es

$$
\boldsymbol{L}=\left[\begin{array}{ccc}
1.0345 & -0.0640 & 0.0024 \\
-0.0705 & 1.0376 & 0.0027 \\
0.0481 & 0.0486 & 0.8735
\end{array}\right]
$$

Si hace una simulación con este predictor se obtiene el resultado de la figura 7.18

Si no es posible medir con precisión el ángulo y sólo se dispone de la información relativa la posición en $x$ en $y$ del móvil tras cada paso sobre las juntas, es necesario redefinir las ecuaciones de medición $\mathrm{y}$, por tanto, la matriz $\boldsymbol{L}$, que ahora será de orden $3 \times 2$. Las nuevas ecuaciones de medición serán

$$
y[t]=\left[\begin{array}{lll}
1 & 0 & 0
\end{array}\right] \boldsymbol{x}[t]
$$



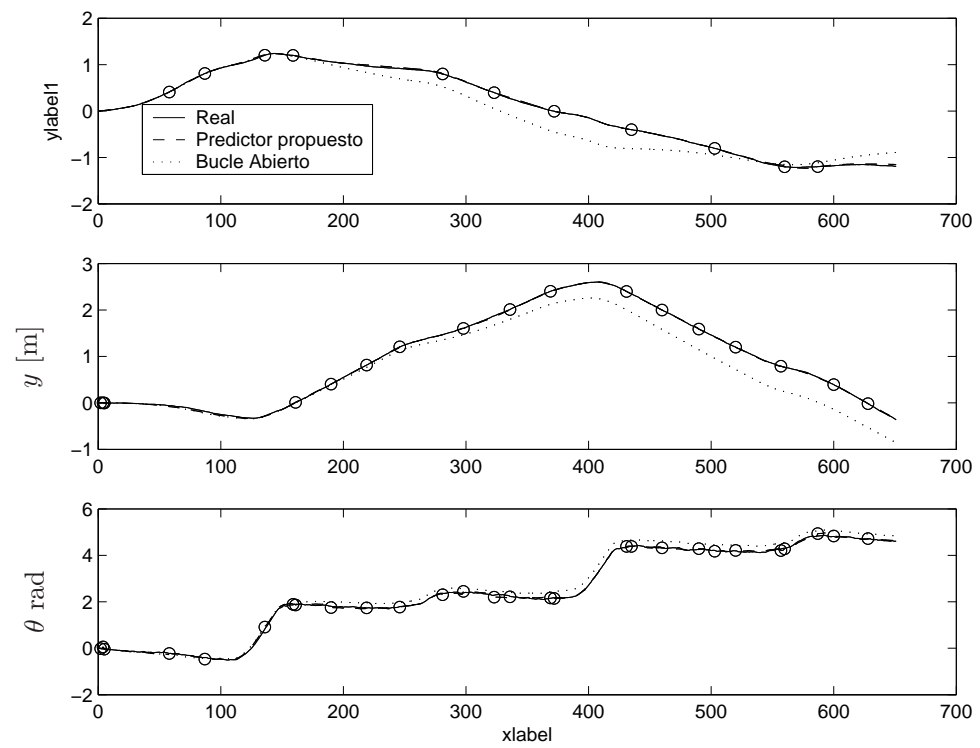

Figura 7.15: Trayectoria real, predicción en bucle abierto y predicción mediante la técnica propuesta

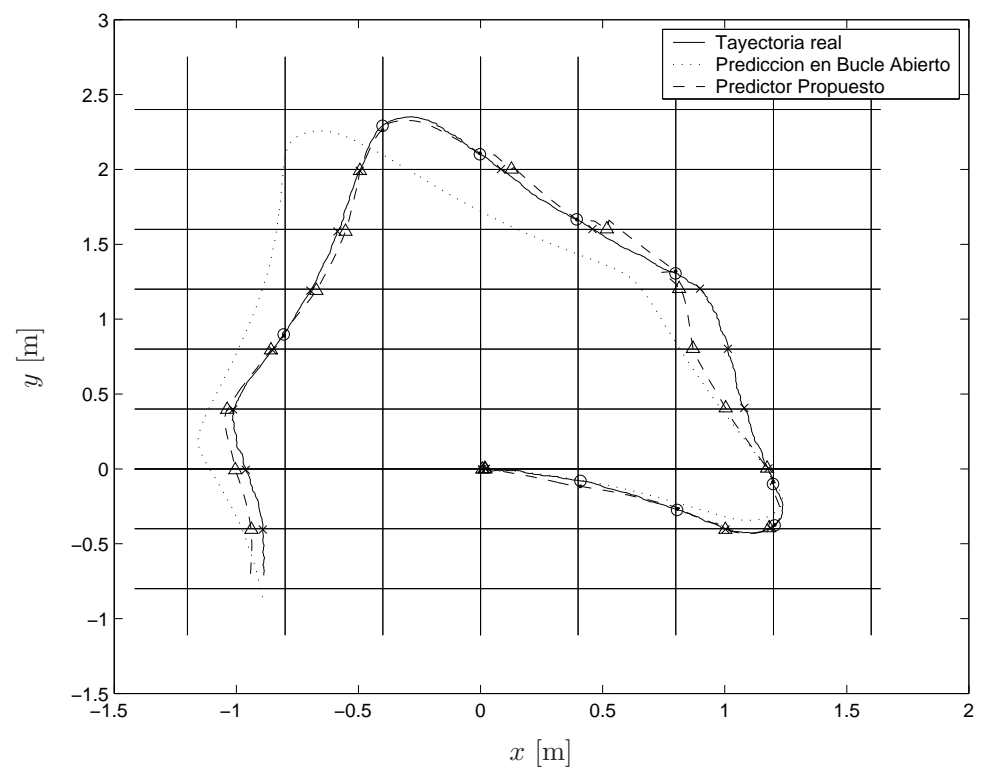

Figura 7.16: Trayectoria real, predicción en bucle abierto y predicción con ganancia constante (técnica de sustitución). $\sigma_{\theta}=3^{\circ}$

cuando hay un cruce con una arista vertical, más

$$
y[t]=\left[\begin{array}{lll}
0 & 1 & 0
\end{array}\right] \boldsymbol{x}[t]
$$

cuando hay un cruce con una arista horizontal. Procediendo como antes se ha llevado a cabo una simulación en la que se ha obtenido la trayectoria mostrada en la figura 7.19, donde se observa que el error cometido con el ángulo afecta principalmente a los tramos que siguen una trayectoria tal que sólo se tienen cruces con una de las direcciones principales. La simulación que se muestra corresponde a un predictor de ganancia constante con matriz de ganancias

$$
\boldsymbol{L}=\left[\begin{array}{cc}
1.003 & -0.007 \\
-0.007 & 1.003 \\
0.3976 & 0.3976
\end{array}\right]
$$

Las prestaciones obtenidas con un predictor con ganancia variable son muy similares, con lo que el predictor de ganancia constante es preferible por su menor coste computacional. 


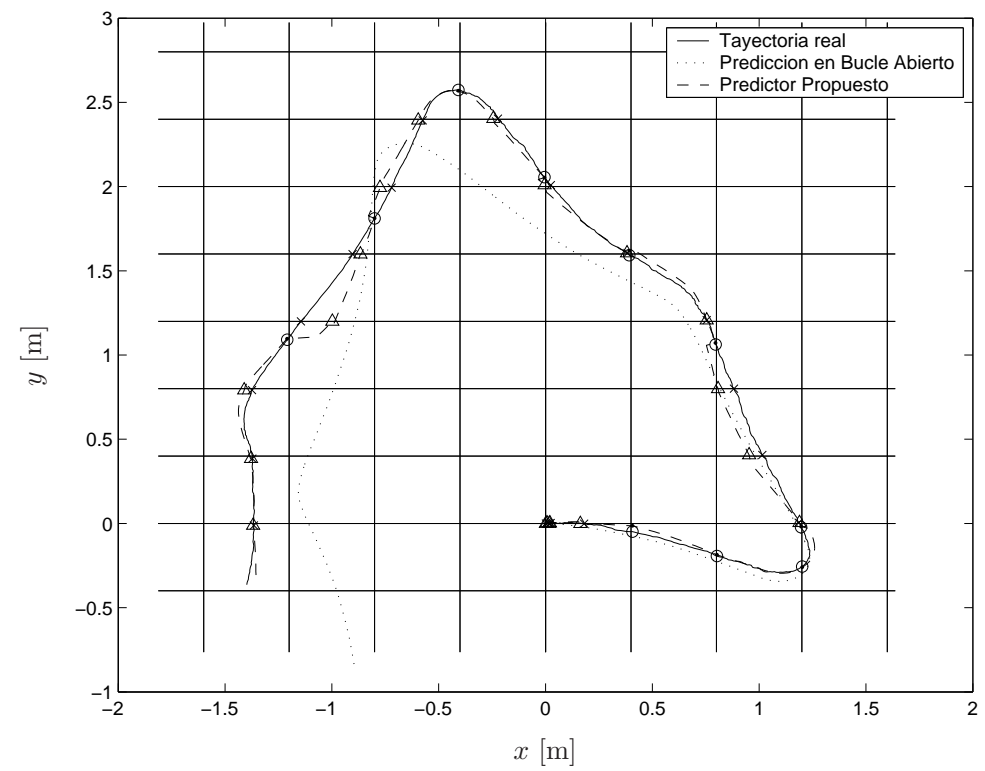

Figura 7.17: Trayectoria real, predicción en bucle abierto y predicción mediante la técnica propuesta. $\sigma_{\theta}=6^{\circ}$

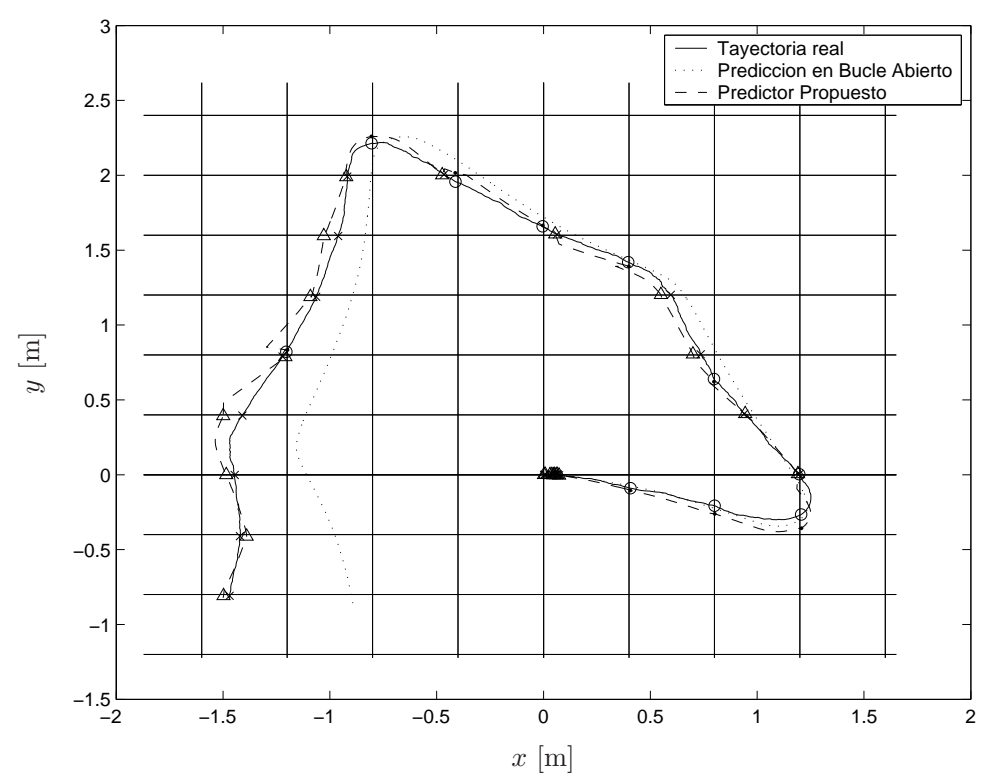

Figura 7.18: Trayectoria real, predicción en bucle abierto y predicción con ganancia constante. $\sigma_{\theta}=6^{\circ}$

\subsection{Estimación de corriente en un motor de inducción}

Supóngase un motor de inducción alimentado en su estator por un sistema trifásico de tensiones equilibradas definidas por

$$
\begin{aligned}
& u_{s A}(\tau)=U(\tau) \cos \left(\omega_{s} \tau\right) \\
& u_{s B}(\tau)=U(\tau) \cos \left(\omega_{s} \tau-120^{\circ}\right), \\
& u_{s C}(\tau)=U(\tau) \cos \left(\omega_{s} \tau-240^{\circ}\right)
\end{aligned}
$$




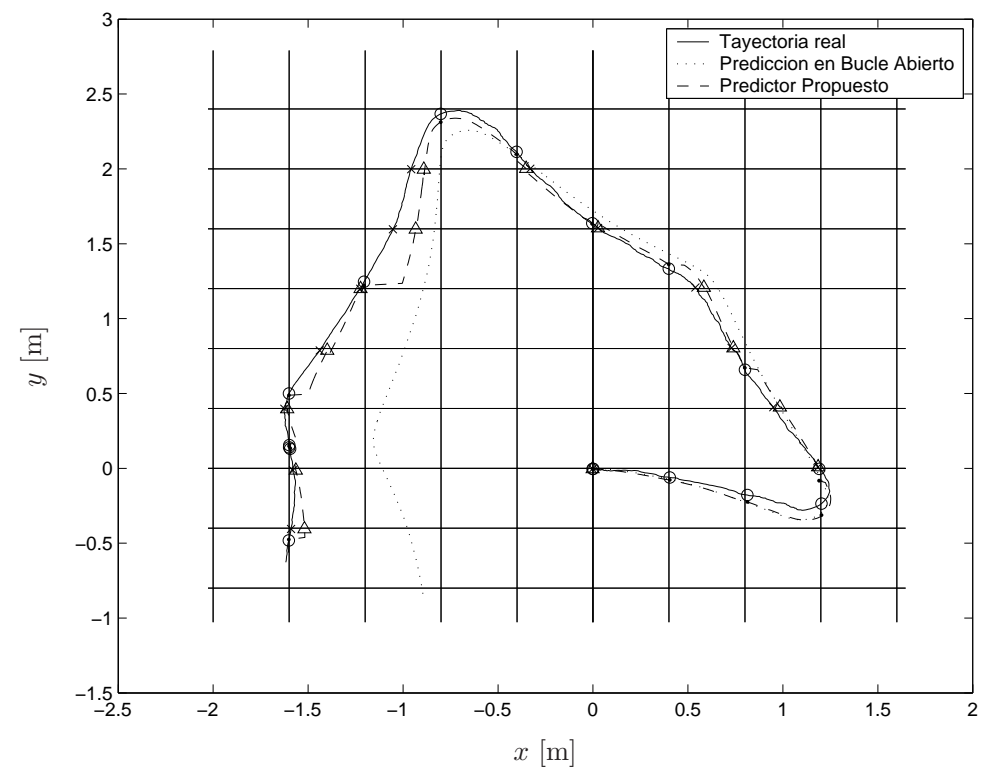

Figura 7.19: Trayectoria real, predicción en bucle abierto y predicción mediante la técnica propuesta. Medición únicamente de la posición $x$ e $y$.

siendo $U$ la tensión de pico y $\omega_{s}$ la frecuencia de sincronismo. Se definen el fasor espacial de tensiones como

$$
\begin{aligned}
\bar{u}_{s}(\tau) & =u_{s a}(\tau)+j u_{s b}(\tau) \\
u_{s a}(\tau) & =\frac{3}{2} U_{s A}(\tau), \\
u_{s b}(\tau) & =\frac{\sqrt{3}}{2}\left(U_{s B}(\tau)-U_{s C}(\tau)\right),
\end{aligned}
$$

siendo $u_{s a}(\tau)$ la parte real del fasor y $u_{s b}(\tau)$ la parte imaginaria. Nótese que el valor de la tensión de pico $U(\tau)$ se obtiene a partir de las componentes real e imaginaria del fasor como

$$
U(\tau)=\frac{2}{3} \sqrt{u_{s a}(\tau)^{2}+u_{s b}(\tau)^{2}}
$$

Las ecuaciones dinámicas que definen el funcionamiento del motor de inducción alimentado por una tensión $\bar{u}_{s}(\tau)$ son

$$
\begin{aligned}
\frac{\mathrm{d}}{\mathrm{d} t} i_{s a}(\tau) & =-\gamma i_{s a}(\tau)+\alpha \beta \varphi_{r a}(\tau)+\beta \omega(\tau) \varphi_{r b}(\tau)+\frac{1}{\sigma} u_{s a}(\tau) \\
\frac{\mathrm{d}}{\mathrm{d} t} i_{s b}(\tau) & =-\gamma i_{s b}(\tau)+\alpha \beta \varphi_{r b}(\tau)-\beta \omega(\tau) \varphi_{r a}(\tau)+\frac{1}{\sigma} u_{s b}(\tau) \\
\frac{\mathrm{d}}{\mathrm{d} t} \varphi_{r a}(\tau) & =-\alpha \varphi_{r a}(\tau)-\omega(\tau) \varphi_{r b}(\tau)+\alpha L_{m} i_{s a}(\tau) \\
\frac{\mathrm{d}}{\mathrm{d} t} \varphi_{r b}(\tau) & =-\alpha \varphi_{r b}(\tau)+\omega(\tau) \varphi_{r a}(\tau)+\alpha L_{m} i_{s b}(\tau) \\
\frac{\mathrm{d}}{\mathrm{d} t} \omega(\tau) & =\mu\left(\varphi_{r a}(\tau) i_{s b}(\tau)-\varphi_{r b}(\tau) i_{s a}(\tau)\right)-\frac{T_{L}(\tau)}{J}
\end{aligned}
$$

donde las variables de estado utilizadas son:

$$
\bar{i}_{s}(\tau)=i_{s a}(\tau)+j i_{s b}(\tau)
$$

el fasor de corrientes del estator,

$$
\bar{\varphi}_{r}(\tau)=\varphi_{r a}(\tau)+j \varphi_{r b}(\tau)
$$


el fasor de flujo magnético del rotor, y $\omega(\tau)$ la velocidad de giro del rotor. $T_{L}(\tau)$ es el par resistente ejercido sobre el eje del rotor. Las constantes $\alpha, \beta, \gamma$ y $\mu$ vienen dadas por

$$
\begin{aligned}
\sigma & =L_{s}\left(1-\frac{L_{m}^{2}}{L_{s} L_{r}}\right), \\
\beta & =\frac{L_{m}}{\sigma L_{r}} \\
\mu & =\frac{3}{2} \frac{L_{m}}{J L_{r}} \\
\alpha & =\frac{R_{r}}{L_{r}} \\
\gamma & =\frac{R_{s}}{\sigma}+\alpha L_{m} \beta,
\end{aligned}
$$

donde $L_{m}, L_{s}, L_{r}, R_{s}, R_{r}$ y $J$ son los parámetros del motor eléctrico cuyo significado y valor se detallan en la tabla 7.4 para el motor utilizado como ejemplo.

\begin{tabular}{lll} 
Resistencia del rotor & $R r$ & $4.3047 \Omega$ \\
Resistencia del estator & $R s$ & $9.65 \Omega$ \\
Inductancia mutua & $L m$ & $0.4475 \mathrm{H}$ \\
Inductancia del estator & $L s$ & $0.4718 \mathrm{H}$ \\
Inductancia del rotor & $L r$ & $0.4718 \mathrm{H}$ \\
Momento de inercia del rotor & $J$ & $0.0293 \mathrm{~kg} \mathrm{~m}^{2}$ \\
\hline
\end{tabular}

Tabla 7.4: Parámetros característicos del motor de inducción

Considérese el sistema de la figura 7.20, en el que se tiene un motor de inducción alimentado mediante un inversor PWM cuya tensión y frecuencia de alimentación viene marcada por un controlador digital. El motor de inducción está mecánicamente conectado a una reductora de ratio 1 : 10. La única medida que se dispone del proceso es la medida de velocidad proporcionada por un encoder de 50 pulsos/vuelta conectada al eje de salida.

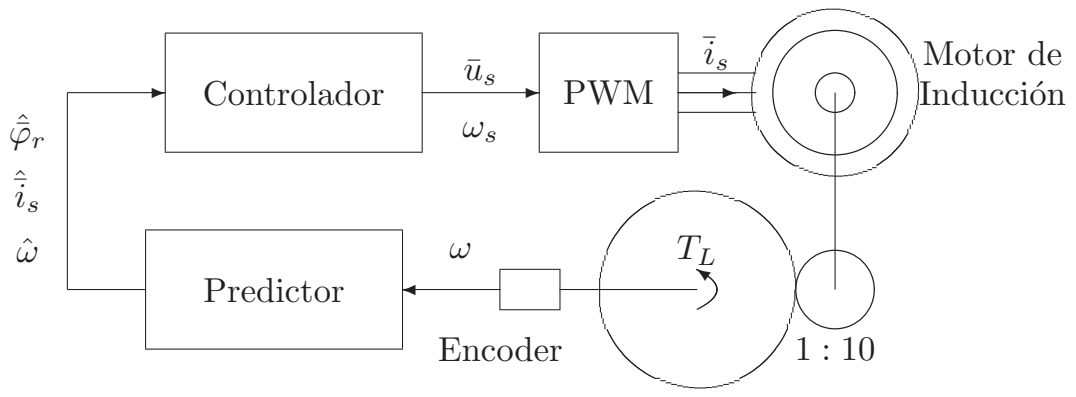

Figura 7.20: Estimación de variables en un motor de inducción con medición de la velocidad.

Supóngase que el sistema es alimentado a la frecuencia y tensión indicadas en la figura 7.21, donde también se muestra la carga no medible que afecta al eje de salida.

En la figura 7.22 se muestra la evolución de la velocidad del eje del rotor, la magnitud del fasor de corriente del estator dada por

$$
\left|\bar{i}_{s}(\tau)\right|=\sqrt{i_{s a}(\tau)^{2}+i_{s b}(\tau)^{2}}
$$

y la magnitud del flujo magnético en el rotor dada por

$$
\left|\varphi_{r}(\tau)\right|=\sqrt{\varphi_{r a}(\tau)^{2}+\varphi_{r b}(\tau)^{2}}
$$

Nótese la bajada de velocidad y aumento de corriente que se produce entre los instantes $\tau=2.5 \mathrm{~s}$ y $\tau=3.5$ s debido al aumento de la carga que se produce.

El objetivo de este ejemplo es estimar la corriente y el flujo magnético cada milisegundo $(T=0.001$ s) utilizando únicamente la medida de velocidad proporcionada por el encoder. 

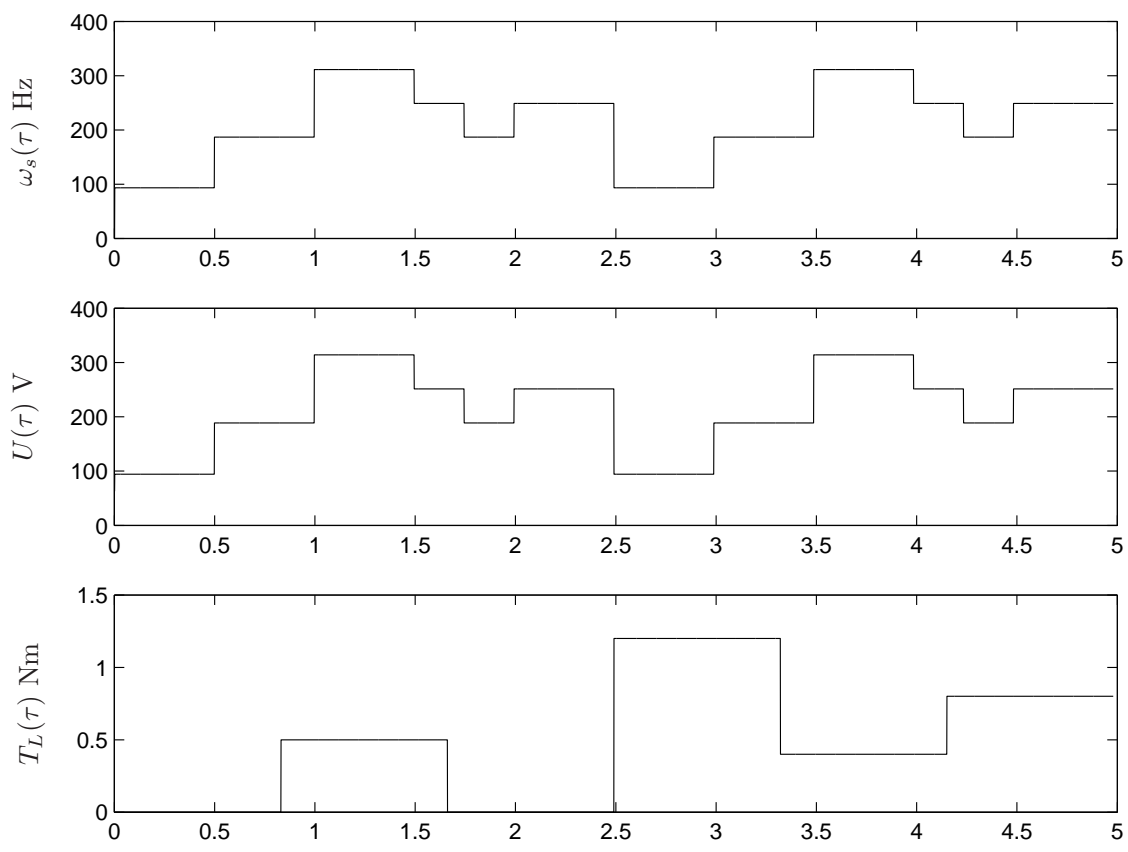

Figura 7.21: Señales de alimentación y carga del motor de inducción

En primer lugar, es necesario obtener un modelo discreto del motor de inducción. Si se utiliza la aproximación de la derivada, el sistema de ecuaciones diferenciales (7.21) queda

$$
\begin{aligned}
i_{s a}[t+1] & =i_{s a}[t]+T\left(-\gamma i_{s a}[t]+\alpha \beta \varphi_{r a}[t]+\beta \omega[t] \varphi_{r b}[t]+\frac{1}{\sigma} u_{s a}[t]\right) \\
i_{s b}[t+1] & =i_{s b}[t]+T\left(-\gamma i_{s b}[t]+\alpha \beta \varphi_{r b}[t]-\beta \omega[t] \varphi_{r a}[t]+\frac{1}{\sigma} u_{s b}[t]\right) \\
\varphi_{r a}[t+1] & =\varphi_{r a}[t]+T\left(-\alpha \varphi_{r a}[t]-\omega[t] \varphi_{r b}[t]+\alpha L_{m} i_{s a}[t]\right) \\
\varphi_{r b}[t+1] & =\varphi_{r b}[t]+T\left(-\alpha \varphi_{r b}[t]+\omega[t] \varphi_{r a}[t]+\alpha L_{m} i_{s b}[t]\right) \\
\omega[t+1] & =\omega[t]+T\left(\mu\left(\varphi_{r a}[t] i_{s b}[t]-\varphi_{r b}[t] i_{s a}[t]\right)-\frac{T_{L}[t]}{J}\right)
\end{aligned}
$$

La forma más simple de estimar las corrientes y el flujo magnético consiste en correr un modelo del proceso en bucle abierto asumiendo una perturbación $T_{L}$ nula. En la figura 7.23 se muestra una simulación de esta estimación en bucle abierto y se compara con la evolución real de la velocidad, la corriente y el flujo magnético. Se puede apreciar un gran aumento del error de estimación de corriente entre los instantes $\tau=2.5$ s y $\tau=3.5$ s debido al aumento de carga. Para mejorar la estimación de corriente se hace uso del encoder instalado en el proceso. La velocidad de giro del eje de salida (donde está ubicado el encoder) oscila entre 1 y $5 \mathrm{~Hz}$. Como en el encoder es de 50 pulsos por vuelta, se tendrá un flanco del encoder cada $20 \mathrm{~ms}$ cuando gire a $1 \mathrm{~Hz}$, y cada $4 \mathrm{~ms}$ cuando gire a $5 \mathrm{~Hz}$. Teniendo en cuenta el periodo de control de $T=1 \mathrm{~ms}$, esto significa que se tendrán pulsos de encoder (y, por lo tanto, medidas de velocidad) cada

$$
N \in \mathcal{N}=\{4,5,6, \ldots, 20\}
$$

instantes de control. Con esta información se va a tratar de construir un predictor capaz de estimar las corrientes y el flujo magnético en cada periodo de control. Como se trata de un sistema no lineal es 

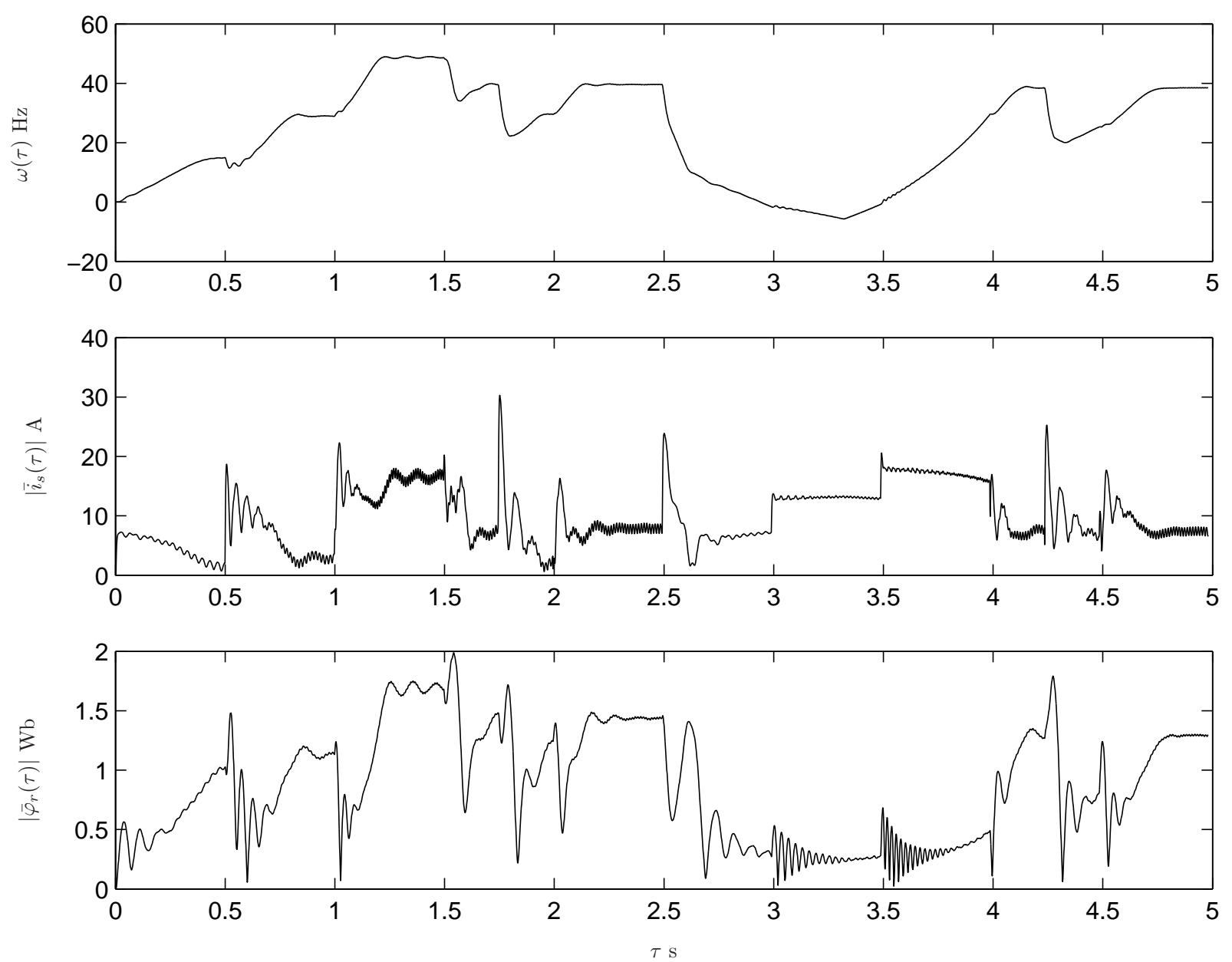

Figura 7.22: Evolución de la velocidad, corriente y flujo en el motor de inducción

necesario reescribir las ecuaciones en diferencias de la forma

$$
\begin{aligned}
& \underbrace{\left[\begin{array}{c}
i_{s a}[t+1] \\
i_{s b}[t+1] \\
\varphi_{r a}[t+1] \\
\varphi_{r b}[t+1] \\
\omega[t+1]
\end{array}\right]}_{\boldsymbol{x}[t+1]}=\underbrace{\left[\begin{array}{ccccc}
1-T \gamma & 0 & T \alpha \beta & 0 & 0 \\
0 & 1-T \gamma & 0 & T \alpha \beta & 0 \\
T \alpha L_{m} & 0 & 1-T \alpha & 0 & 0 \\
0 & T \alpha L_{m} & 0 & 1-T \alpha & 0 \\
0 & 0 & 0 & 0 & 1
\end{array}\right]\left[\begin{array}{c}
i_{s a}[t] \\
i_{s b}[t] \\
\varphi_{r a}[t] \\
\varphi_{r b}[t] \\
\omega[t]
\end{array}\right]}_{\boldsymbol{A} \boldsymbol{x}[t]}+ \\
& +\underbrace{\left[\begin{array}{c}
T \beta \omega[t] \varphi_{r b}[t]+\frac{u_{s a}}{\sigma} \\
-T \beta \omega[t] \varphi_{r a}[t]+\frac{u_{s b}}{\sigma} \\
-\omega[t] \varphi_{r b}[t] \\
\omega[t] \varphi_{r a}[t] \\
T \mu\left(\varphi_{r a}[t] i_{s b}[t]-\varphi_{r b}[t] i_{s a}[t]\right)
\end{array}\right]}_{\boldsymbol{f}(\boldsymbol{x}[t], \boldsymbol{u}[t])}+\underbrace{\left[\begin{array}{c}
0 \\
0 \\
0 \\
0 \\
\left.\frac{-T}{J}\right]
\end{array} T_{L}[t]\right.}_{\boldsymbol{B}_{v} \boldsymbol{w}_{x}[t]}
\end{aligned}
$$

siendo $\boldsymbol{x}[t]=\left[i_{s a}[t] i_{s b}[t] \varphi_{r a}[t] \varphi_{r b}[t] \omega[t]\right]^{\top}$ el vector de estados. La ecuación de mediciones viene dada por

$$
y[t]=\left[\begin{array}{lllll}
0 & 0 & 0 & 0 & 1
\end{array}\right] \boldsymbol{x}[t],
$$

mientras que la ecuación de salidas coincide con los estados:

$$
\boldsymbol{m}[t]=\boldsymbol{I} \boldsymbol{x}[t]
$$

Para poder aplicar la técnica expuesta en el capítulo $\$ 5$ es necesario obtener la matriz $\boldsymbol{F}$ tal que

$$
\|\boldsymbol{f}(\boldsymbol{x}[t], \boldsymbol{u}[t])-\boldsymbol{f}(\hat{\boldsymbol{x}}[t], \boldsymbol{u}[t])\| \leq\|\boldsymbol{F}(\boldsymbol{x}[t]-\hat{\boldsymbol{x}}[t])\| .
$$



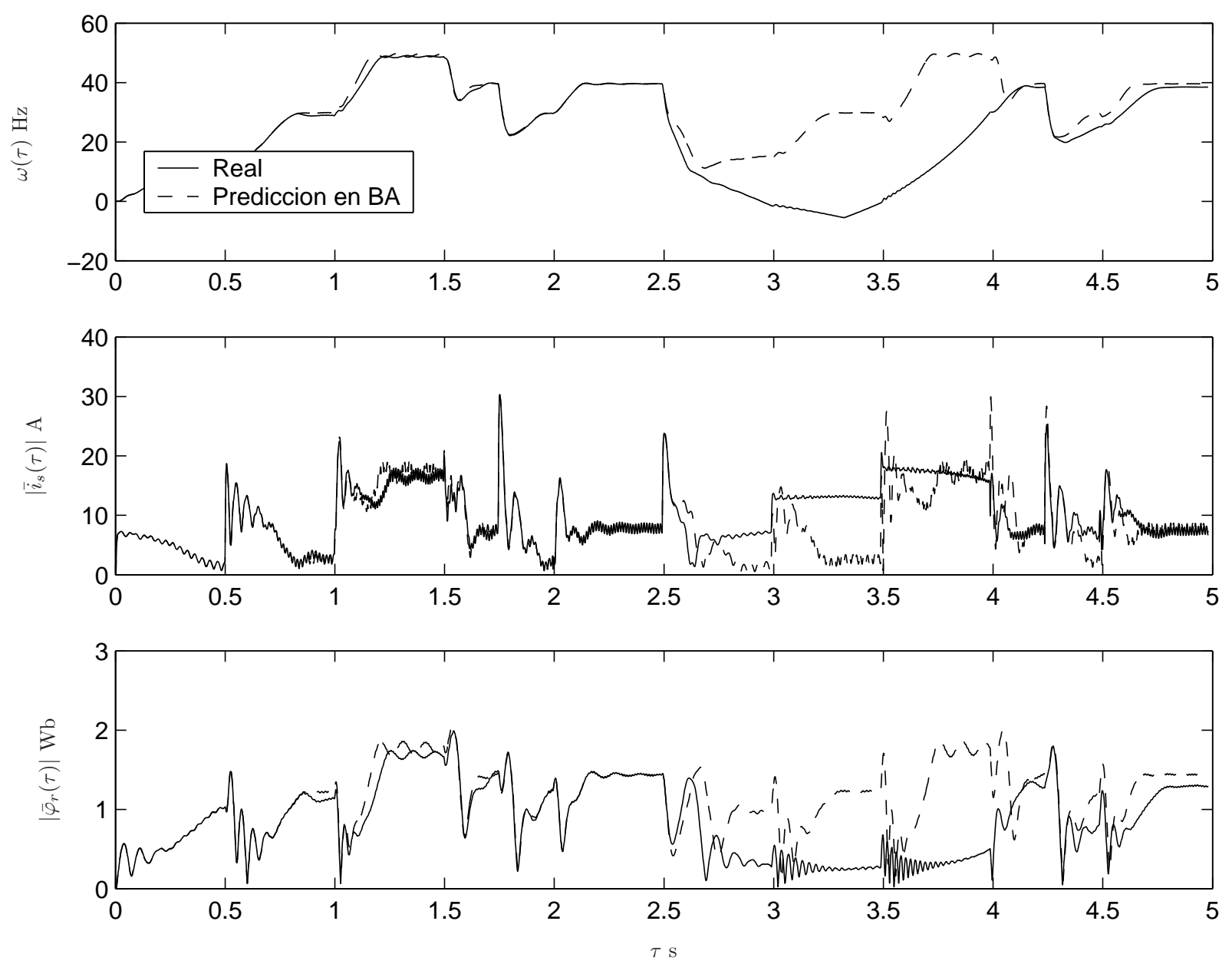

Figura 7.23: Predicción en bucle abierto de la velocidad, corriente y flujo en el motor de inducción

Observando la evolución de las salidas se observa que la velocidad tiene su máximo en $\omega_{\text {máx }}=50 \mathrm{~Hz}$, el flujo en $\varphi_{\text {máx }}=2 \mathrm{~Wb}$ y la corriente en $i_{\text {máx }}=20 \mathrm{~A}$. Con estos datos, la matriz $\boldsymbol{F}$ se puede escribir como

$$
\boldsymbol{F}=T \cdot\left[\begin{array}{ccccc}
0 & 0 & 0 & \beta \omega_{\text {máx }} & \beta \varphi_{\text {máx }} \\
0 & 0 & -\beta \omega_{\text {máx }} & 0 & -\beta \varphi_{\text {máx }} \\
0 & 0 & 0 & -\omega_{\text {máx }} & -\varphi_{\text {máx }} \\
0 & 0 & \omega_{\operatorname{máx}} & 0 & \varphi_{\text {máx }} \\
-\mu \varphi_{\operatorname{máx}} & \mu \varphi_{\operatorname{máx}} & \mu i_{\text {máx }} & -\mu i_{\text {máx }} & 0
\end{array}\right]
$$


Con esto, las matrices que definen el sistema son:

$$
\begin{aligned}
\boldsymbol{A} & =\left[\begin{array}{ccccc}
0.7144 & 0 & 0.1828 & 0 & 0 \\
0 & 0.7144 & 0 & 0.1828 & 0 \\
0.0041 & 0 & 0.9909 & 0 & 0 \\
0 & 0.0041 & 0 & 0.9909 & 0 \\
0 & 0 & 0 & 0 & 1.0000
\end{array}\right] \\
\boldsymbol{F} & =\left[\begin{array}{ccccc}
0 & 0 & 0 & 6.2933 & 0.0401 \\
0 & 0 & -6.2933 & 0 & -0.0401 \\
0 & 0 & 0 & -0.3142 & -0.0020 \\
0 & 0 & 0.3142 & 0 & 0.0020 \\
0.0971 & -0.0971 & 0.9712 & -0.9712 & 0
\end{array}\right] \\
\boldsymbol{B}_{v}= & {\left[\begin{array}{c}
0 \\
0 \\
0 \\
0 \\
0.0341
\end{array}\right] . }
\end{aligned}
$$

Mientras no se tiene medición, el predictor estima en bucle abierto el vector de estados utilizando la ecuación

$$
\begin{aligned}
\hat{i}_{s a}[t+1 \mid t] & =\hat{i}_{s a}[t]+T\left(-\gamma \hat{i}_{s a}[t]+\alpha \beta \hat{\varphi}_{r a}[t]+\beta \omega[t] \hat{\varphi}_{r b}[t]+\frac{1}{\sigma} u_{s a}[t]\right) \\
\hat{i}_{s b}[t+1 \mid t] & =\hat{i}_{s b}[t]+T\left(-\gamma \hat{i}_{s b}[t]+\alpha \beta \hat{\varphi}_{r b}[t]-\beta \omega[t] \hat{\varphi}_{r a}[t]+\frac{1}{\sigma} u_{s b}[t]\right) \\
\hat{\varphi}_{r a}[t+1 \mid t] & =\hat{\varphi}_{r a}[t]+T\left(-\alpha \hat{\varphi}_{r a}[t]-\omega[t] \hat{\varphi}_{r b}[t]+\alpha L_{m} \hat{i}_{s a}[t]\right) \\
\hat{\varphi}_{r b}[t+1 \mid t] & =\hat{\varphi}_{r b}[t]+T\left(-\alpha \hat{\varphi}_{r b}[t]+\omega[t] \hat{\varphi}_{r a}[t]+\alpha L_{m} \hat{i}_{s b}[t]\right) \\
\hat{\omega}[t+1 \mid t] & =\omega[t]+T \mu\left(\hat{\varphi}_{r a}[t] \hat{i}_{s b}[t]-\hat{\varphi}_{r b}[t] \hat{i}_{s a}[t]\right) .
\end{aligned}
$$

Cuando se tiene una medición, el vector de estados anterior se corrige mediante la ecuación

$$
\left[\begin{array}{c}
\hat{i}_{s a}[t+1] \\
\hat{i}_{s b}[t+1] \\
\hat{\varphi}_{r a}[t+1] \\
\hat{\varphi}_{r b}[t+1] \\
\hat{\omega}[t+1]
\end{array}\right]=\left[\begin{array}{c}
\hat{i}_{s a}[t+1 \mid t] \\
\hat{i}_{s b}[t+1 \mid t] \\
\hat{\varphi}_{r a}[t+1 \mid t] \\
\hat{\varphi}_{r b}[t+1 \mid t] \\
\hat{\omega}[t+1 \mid t]
\end{array}\right]+\boldsymbol{L}[t+1](\omega[t+1]-\hat{\omega}[t+1])
$$

donde $\boldsymbol{L}[t]$ es la matriz de corrección utilizada en función del número de periodos intermuestreo y es una matriz que varía dentro de un conjunto conocido a calcular.

Teniendo en cuenta el valor de pico de la perturbación $\|\boldsymbol{v}\|_{\infty}=\left\|T_{L}[t]\right\|_{\infty}=1.2 \mathrm{Nm}$, y asumiendo un ruido de medida de valor máximo $\left\|\boldsymbol{w}_{y}\right\|_{\infty}=1 \mathrm{rad} / \mathrm{s}$, se puede diseñar un predictor $\mathcal{H}_{\infty}$ siguiendo la técnica expuesta en el capítulo $\$ 5$ (véase teorema 5.6.2, página 148). Si se diseña el predictor con una ganancia constante, se obtiene el vector

$$
\boldsymbol{L}=\left[\begin{array}{lllll}
0.0553 & 0.0031 & -0.0091 & -0.0010 & 0.9988
\end{array}\right]^{\top}
$$

y se obtiene la simulación de la figura 7.24, donde el valor eficaz del error cometido en la estimación del módulo del fasor de corrientes es de $62 \mathrm{~mA}$, mientras que el valor de pico del error cometido es de 1.5 A. En la figura 7.25 se muestra un detalle de los instantes donde se aplica la carga de mayor valor, observándose el incremento instantáneo del error de estimación.

Las prestaciones obtenidas mediante la implementación del predictor con un vector de ganancias variante son similares a las del predictor de ganancia constante, por lo que es más conveniente utilizar el predictor de ganancia constante ya que tiene la ventaja de su simplicidad de implementación. 

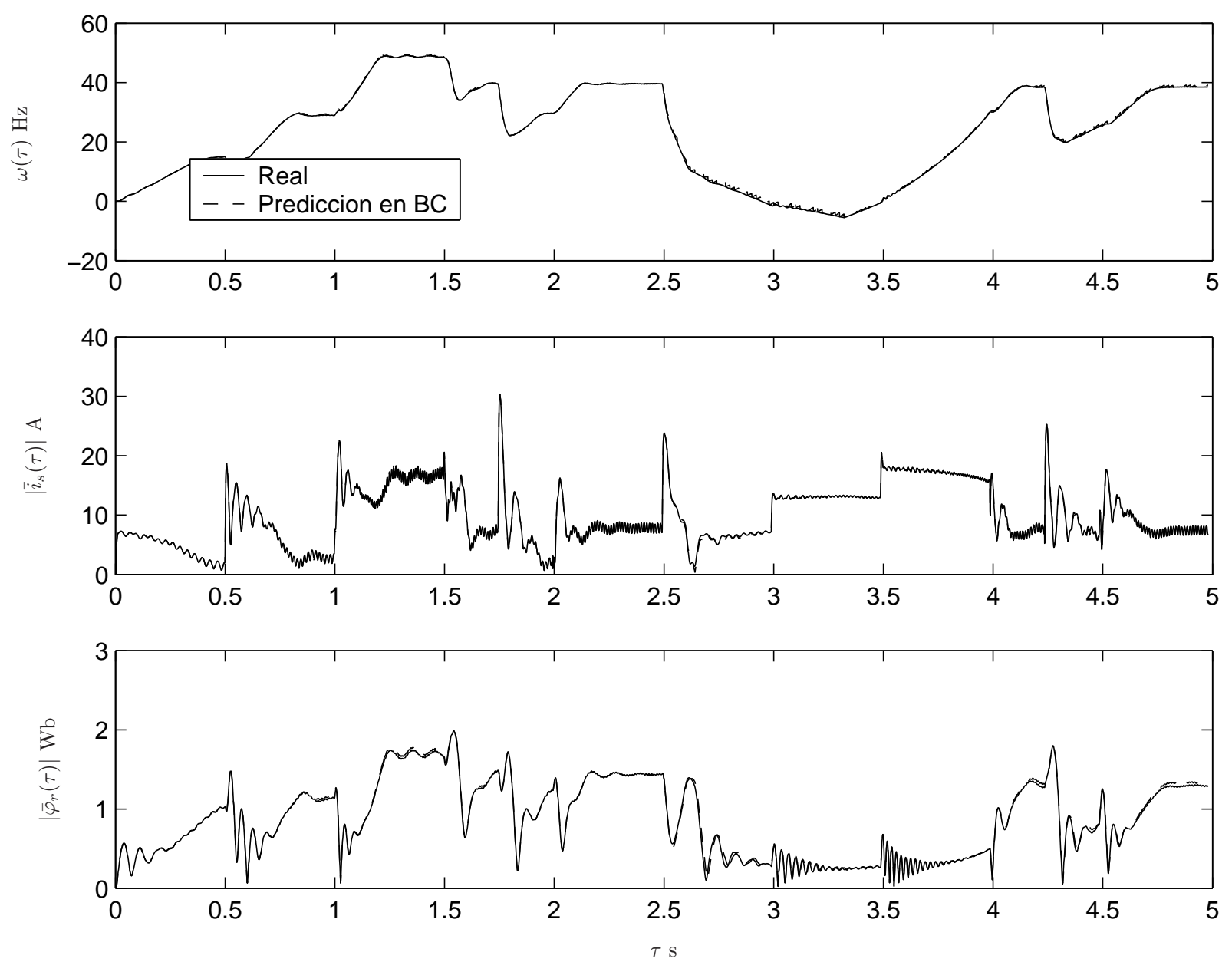

Figura 7.24: Predicción de la velocidad, corriente y flujo en el motor de inducción utilizando el predictor propuesto

\subsection{Estimación de sustancias en un biorreactor}

Las ecuaciones dinámicas que definen el funcionamiento de un biorreactor continuo son

$$
\begin{aligned}
& \dot{x}(\tau)=\mu(s(\tau)) x(\tau)-\frac{x(\tau) Q(\tau)}{V(\tau)} \\
& \dot{s}(\tau)=-\frac{1}{Y} \mu(s(\tau)) x(\tau)+\frac{\left(s_{F}(\tau)-s(\tau)\right) Q(\tau)}{V(\tau)} \\
& \mu(s)=\mu_{\text {máx }} \frac{s(\tau)}{k_{2} s(\tau)^{2}+s(\tau)+k_{1}}
\end{aligned}
$$

donde las diferentes variables y parámetros son las indicadas en la tabla 7.5, donde se han puesto los valores de los parámetros constantes Como se trata de un biorreactor continuo, el volumen permanecerá constante $(V(\tau)=V=4 \mathrm{l})$. La concentración del substrato de alimentación también se considera constante y de valor $s_{F}(\tau)=s_{F}=15 \mathrm{~g} / \mathrm{l}$. Si se discretizan las ecuaciones anteriores para un periodo $T$ utilizando la aproximación de la derivada, se tiene

$$
\begin{aligned}
x[t+1] & =x[t]+T \mu(s[t]) x[t]-T \frac{x[t] Q[t]}{V}, \\
s[t+1] & =s[t]-\frac{T}{Y} \mu(s[t]) x[t]+\frac{T\left(s_{F}-s[t]\right) Q[t]}{V}, \\
\mu(s[t]) & =\mu_{\text {máx }} \frac{s[t]}{k_{2} s[t]^{2}+s[t]+k_{1}} .
\end{aligned}
$$



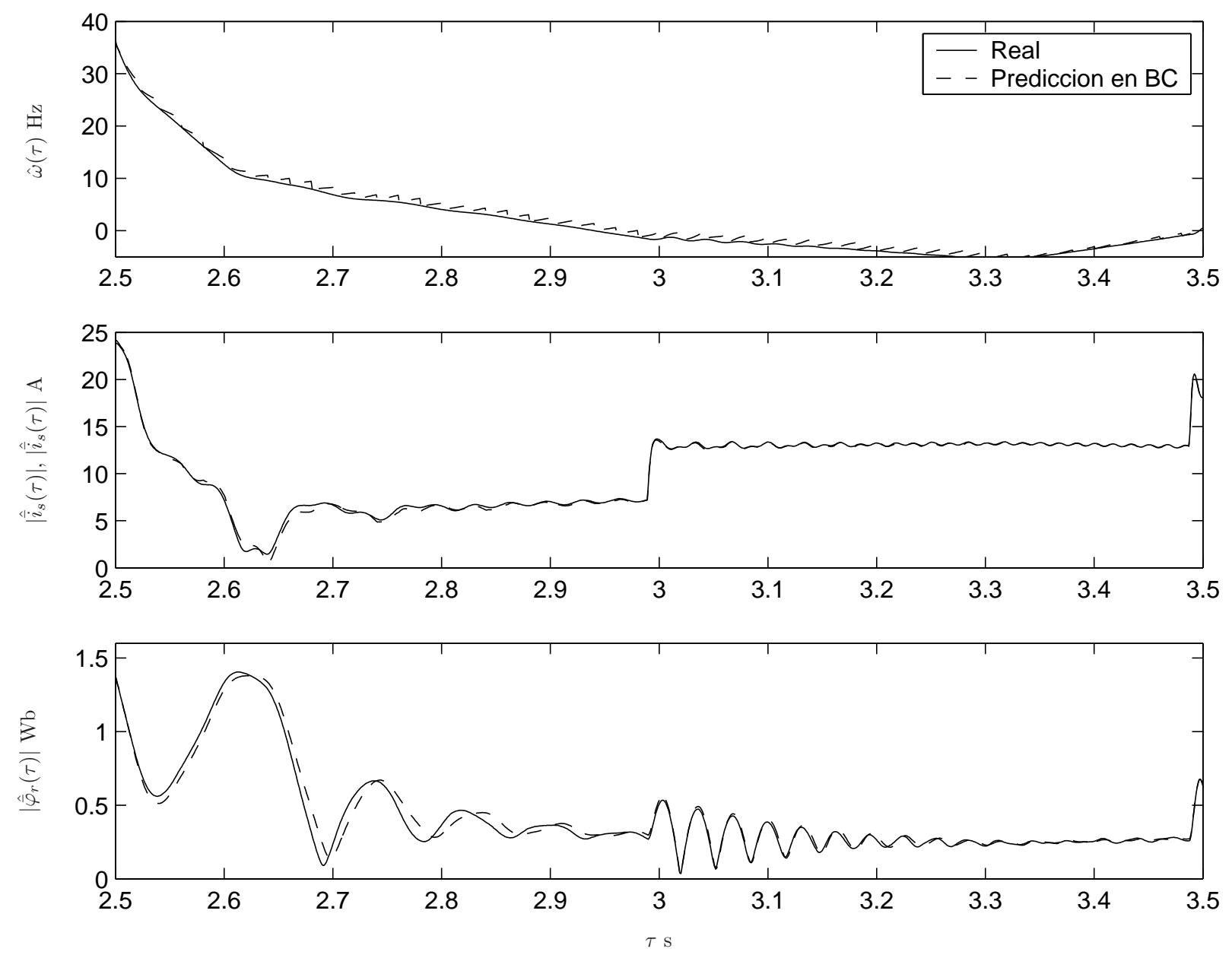

Figura 7.25: Predicción de la velocidad, corriente y flujo en el motor de inducción utilizando el predictor propuesto

Este sistema de ecuaciones se puede expresar como

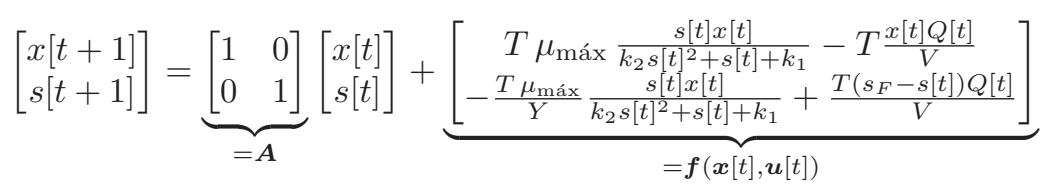

En este tipo de procesos, la disponibilidad de mediciones de salidas es muy escasa debido a la necesidad de realizar experimentos químicos para obtener los valores del substrato y de la biomasa. Estos experimentos requieren un tiempo de procesado de entre media hora y una hora, con lo que las mediciones que se obtienen son muy escasas y además vienen acompañadas de un retardo. Para poder realizar una estimación de la biomasa y el substrato en cada periodo de control se propone estudiar tres alternativas: predictor de bucle abierto, predictor de sustitución y predictor basado en la atenuación de perturbaciones. La estimación en bucle abierto se realiza mediante la ecuación

$$
\left[\begin{array}{l}
\hat{x}[t+1 \mid t] \\
\hat{s}[t+1 \mid t]
\end{array}\right]=\left[\begin{array}{ll}
1 & 0 \\
0 & 1
\end{array}\right]\left[\begin{array}{c}
\hat{x}[t] \\
\hat{s}[t]
\end{array}\right]+\left[\begin{array}{c}
T \hat{\mu}_{\text {máx }} \frac{\hat{s}[t] \hat{x}[t]}{\hat{k}_{2} \hat{s}[t]^{2}+\hat{s}[t]+\hat{k}_{1}}-T \frac{\hat{x}[t] Q[t]}{\hat{V}} \\
-\frac{T \hat{\mu}_{\text {máx }}}{\hat{Y}} \frac{\hat{s}[t] \hat{x}[t]}{\hat{k}_{2} \hat{s}[t]^{2}+\hat{s}[t]+\hat{k}_{1}}+\frac{T\left(\hat{s}_{F}-\hat{s}[t]\right) Q[t]}{\hat{V}}
\end{array}\right]
$$

donde $\hat{\mu}_{\text {máx }}, \hat{k}_{1}, \hat{k}_{2}, \hat{V}$ y $\hat{s}_{F}$ hacen referencia a los valores estimados que se tienen de los parámetros del modelo y cuyo valor es el mostrado en la tabla 7.6. La corrección de la estimación que se hace cada vez que está disponible una medición viene dada por la ecuación

$$
\left[\begin{array}{c}
\hat{x}[t+1] \\
\hat{s}[t+1]
\end{array}\right]=\left[\begin{array}{c}
\hat{x}[t+1 \mid t] \\
\hat{s}[t+1 \mid t]
\end{array}\right]+\boldsymbol{L}[t+1]\left(\left[\begin{array}{l}
m_{x}[t+1] \\
m_{s}[t+1]
\end{array}\right]-\left[\begin{array}{l}
\hat{x}[t+1 \mid t] \\
\hat{s}[t+1 \mid t]
\end{array}\right]\right)
$$




\begin{tabular}{llll}
\hline$x(\tau)$ & Concentración de biomasa & & $(\mathrm{g} / \mathrm{l})$ \\
$s(\tau)$ & Concentración de substratos & $(\mathrm{g} / \mathrm{l})$ \\
$Q(\tau)$ & Caudal de alimentación & & $(\mathrm{l} / \mathrm{h})$ \\
$V(\tau)$ & Volumen del tanque & 4 & $(\mathrm{l})$ \\
$s_{F}(\tau)$ & Concentración del substrato de alimentación & 15 & $(\mathrm{~g} / \mathrm{l})$ \\
$Y$ & Coeficiente de cosecha & 0.5 & \\
$\mu_{\text {máx }}$ & Máximo ratio de crecimiento & 1 & $(\mathrm{l} / \mathrm{h})$ \\
$k_{1}$ & Parámetro de saturación & 0.03 & $(\mathrm{l} / \mathrm{h})$ \\
$k_{2}$ & Parámetro de inhibición & 0.5 & $(\mathrm{l} / \mathrm{h})$ \\
$T$ & Periodo de control & 20 & $(\mathrm{~s})$ \\
\hline
\end{tabular}

Tabla 7.5: Parámetros de un biorreactor

\begin{tabular}{lll}
\hline$V(\tau)$ & 4.1 & $(\mathrm{l})$ \\
$s_{F}(\tau)$ & 15.1 & $(\mathrm{~g} / \mathrm{l})$ \\
$Y$ & 0.52 & \\
$\mu_{\text {máx }}$ & 1 & $(\mathrm{l} / \mathrm{h})$ \\
$k_{1}$ & 0.031 & $(1 / \mathrm{h})$ \\
$k_{2}$ & 0.52 & $(\mathrm{l} / \mathrm{h})$ \\
\hline
\end{tabular}

Tabla 7.6: Parámetros estimados del biorreactor

donde $m_{x}[t+1]$ y $m_{s}[t+1]$ hace referencia a las mediciones tomadas de la biomasa y el substrato, respectivamente, y que están afectadas de un ruido de medición:

$$
\begin{aligned}
& m_{x}[t]=x[t]+w_{x}[t], \\
& m_{s}[t]=s[t]+w_{s}[t] .
\end{aligned}
$$

Se asume que los ruidos de medición son señales aleatorias con media cero y desviación típica $\sigma_{x}=0.026$ $\mathrm{g} / \mathrm{l}, \mathrm{y} \sigma_{s}=0.025 \mathrm{~g} / \mathrm{l}$.

Tal y como se ha analizado en el capítulo 4, el efecto del error de modelado sobre el error de predicción es equivalente al efecto de una perturbación del estado. Para obtener esta perturbación equivalente se han realizado simulaciones con diferentes valores de $\hat{\mu}_{\text {máx }}, \hat{k}_{1}, \hat{k}_{2}, \hat{V}$ y $\hat{s}_{F}$ en el intervalo mostrado en la tabla 7.7, de manera que se ha obtenido una estimación del valor eficaz de la perturbación en cada estado

\begin{tabular}{lll}
\hline$\hat{V}(\tau)$ & $4.1 \pm 0.15$ & $(\mathrm{l})$ \\
$\hat{s}_{F}(\tau)$ & $15.1 \pm 0.15$ & $(\mathrm{~g} / \mathrm{l})$ \\
$\hat{Y}$ & $0.52 \pm 0.25$ & \\
$\hat{\mu}_{\text {máx }}$ & $1 \pm 0.05$ & $(\mathrm{l} / \mathrm{h})$ \\
$\hat{k}_{1}$ & $0.031 \pm 0.005$ & $(\mathrm{l} / \mathrm{h})$ \\
$\hat{k}_{2}$ & $0.52 \pm 0.04$ & $(\mathrm{l} / \mathrm{h})$ \\
\hline
\end{tabular}

Tabla 7.7: Intervalo de los parámetros estimados del biorreactor

de valor

$$
\begin{aligned}
\left\|v_{x}[t]\right\|_{R M S} & =\|x[t]-\hat{x}[t]\|_{R M S}=0.083 \mathrm{~g} / \mathrm{l} \\
\left\|v_{s}[t]\right\|_{R M S} & =\|s[t]-\hat{s}[t]\|_{R M S}=0.014 \mathrm{~g} / \mathrm{l} .
\end{aligned}
$$

Para poder diseñar un predictor de los propuestos en el capítulo 5 es necesario obtener la matriz $\boldsymbol{F}$ tal que

$$
\|\boldsymbol{f}(\boldsymbol{x}[t], \boldsymbol{u}[t])-\boldsymbol{f}(\hat{\boldsymbol{x}}[t], \boldsymbol{u}[t])\| \leq\|\boldsymbol{F} \cdot(\boldsymbol{x}[t]-\hat{\boldsymbol{x}}[t])\|
$$

En este ejemplo, esa búsqueda se hace de forma experimental, de manera que se propone una matriz $\boldsymbol{F}$ de la forma

$$
\boldsymbol{F}=\left[\begin{array}{ll}
a & b \\
c & d
\end{array}\right]
$$

y cada uno de los elementos se calcula de la siguiente forma: 
- Fijando $s[t]=\hat{s}[t]$, se realizan diversas simulaciones y se busca el máximo $a=\frac{f_{1}(\boldsymbol{x}[t], \boldsymbol{u}[t])-f_{1}(\hat{\boldsymbol{x}}[t], \boldsymbol{u}[t])}{x[t]-\hat{x}[t]}$

- Fijando $x[t]=\hat{x}[t]$, se realizan diversas simulaciones y se busca el máximo de $b=\frac{f_{1}(\boldsymbol{x}[t], \boldsymbol{u}[t])-f_{1}(\hat{\boldsymbol{x}}[t], \boldsymbol{u}[t])}{s[t]-\hat{s}[t]}$

- Fijando $s[t]=\hat{s}[t]$, se realizan diversas simulaciones y se busca el máximo $c=\frac{f_{2}(\boldsymbol{x}[t], \boldsymbol{u}[t])-f_{2}(\hat{\boldsymbol{x}}[t], \boldsymbol{u}[t])}{x[t]-\hat{x}[t]}$

- Fijando $x[t]=\hat{x}[t]$, se realizan diversas simulaciones y se busca el máximo de $d=\frac{f_{2}(\boldsymbol{x}[t], \boldsymbol{u}[t])-f_{2}(\hat{\boldsymbol{x}}[t], \boldsymbol{u}[t])}{s[t]-\hat{s}[t]}$

De esta forma se obtiene una matriz $\boldsymbol{F}$ dada por

$$
\boldsymbol{F}=\left[\begin{array}{ll}
0.0045 & 0.1961 \\
0.0089 & 0.3921
\end{array}\right]
$$

Para comprobar la validez de esta matriz obtenida experimentalmente se realiza una simulación en bucle abierto con dos condiciones iniciales diferentes $\boldsymbol{x}_{1}[0]$ y $\boldsymbol{x}_{2}[0]$, comprobándose que $\| \boldsymbol{f}\left(\boldsymbol{x}_{1}[t], \boldsymbol{u}[t]\right)-$ $\boldsymbol{f}\left(\hat{\boldsymbol{x}}_{2}[t], \boldsymbol{u}[t]\right)\|\leq\| \boldsymbol{F} \cdot\left(\boldsymbol{x}_{1}[t]-\hat{\boldsymbol{x}}_{2}[t]\right) \|$. La matriz $\boldsymbol{H}$ será nula en este caso, puesto que las mediciones corresponden directamente a estados del proceso, y no a funciones no lineales de éstos.

En base a todos estos datos indicados se ha diseñado un predictor para situaciones en las que se obtiene una medición cada 30 minutos $(N=90)$ obteniéndose una matriz de ganancias constante de valor

$$
L=\left[\begin{array}{cc}
1.0007 & -0.0095 \\
0.0601 & 0.1252
\end{array}\right],
$$

y una respuesta como la que se muestra en la figura 7.26, donde se muestran de forma conjunta la salida real del proceso, la predicción de bucle abierto, la predicción obtenida con un predictor de sustitución y la predicción obtenida con la técnica de predicción que se propone. Nótese que la predicción de bucle abierto arrastra un error de estimación considerable debido al error del modelo, mientras que la predicción obtenida con el método de sustitución, a pesar de mejorar los resultados de bucle abierto, provoca grandes errores de estimación en los instantes de medición debido al gran error de medida que acompaña a la medición de substrato. La predicción que se propone en este trabajo subsana estos dos problemas de error de modelado y ruido de medición obteniéndose una mejor estimación de las variables. En la figura 7.27 se muestra otra simulación del problema de estimación con un periodo de medición de 1 hora $(N=180)$. En este caso, la matriz de ganancias obtenida es

$$
L=\left[\begin{array}{cc}
1.0004 & -0.0071 \\
0.0490 & 0.1056
\end{array}\right]
$$

Finalmente, en la figura 7.28 se muestra una simulación en la que las mediciones se toman en un tiempo dado dentro del intervalo $[30,40]$ minutos. Para ello se ha diseñado un predictor que utiliza una matriz de ganancias diferente para cada número de periodos intermuestreo (30 matrices en total). De nuevo, la estimación de la biomasa y el substrato realizada con la predicción propuesta supera las prestaciones obtenidas con los predictores de bucle abierto y de sustitución. 

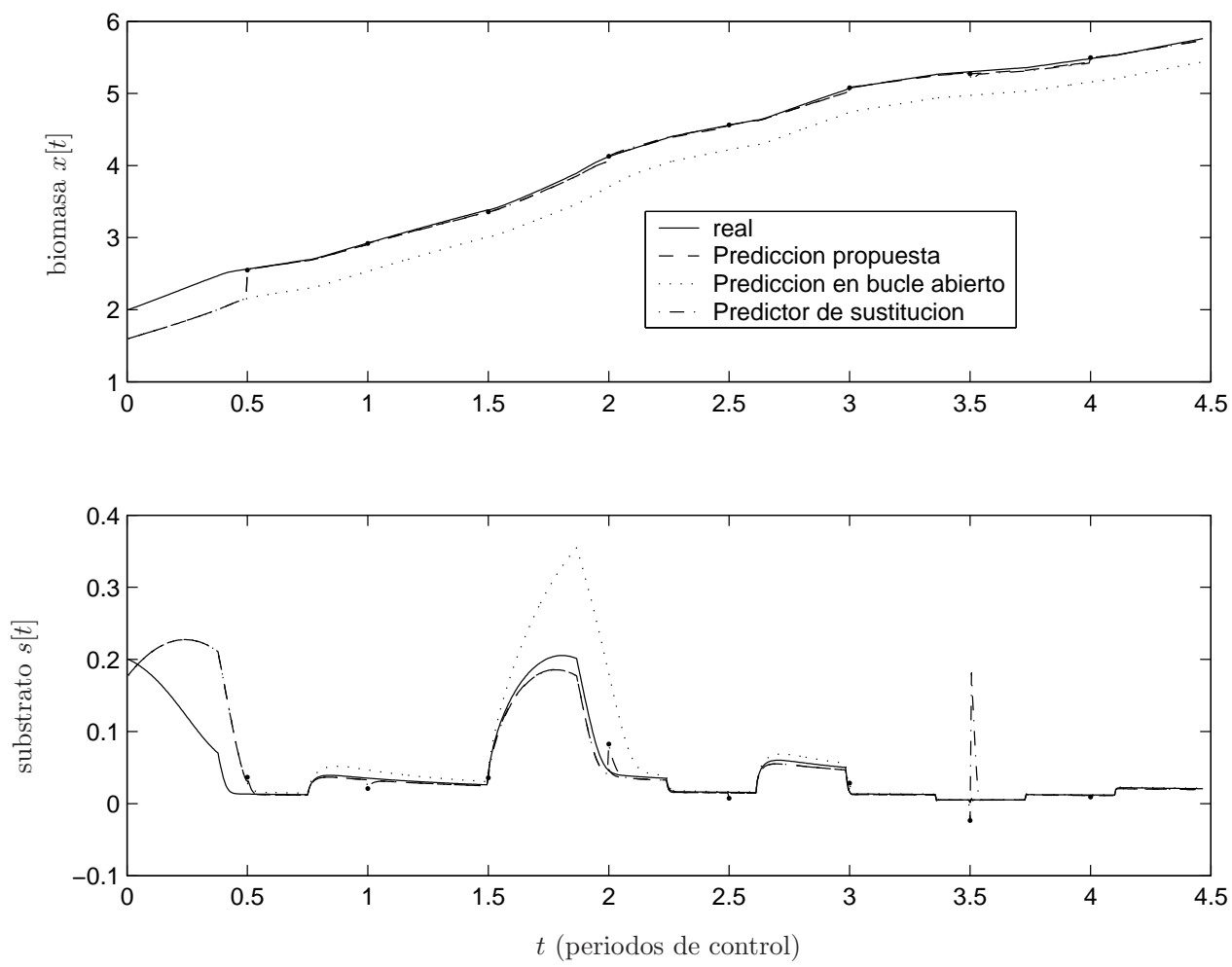

Figura 7.26: Salida, mediciones y diferentes predicciones para el biorreactor. Muestreo cada $30 \mathrm{~min}$.
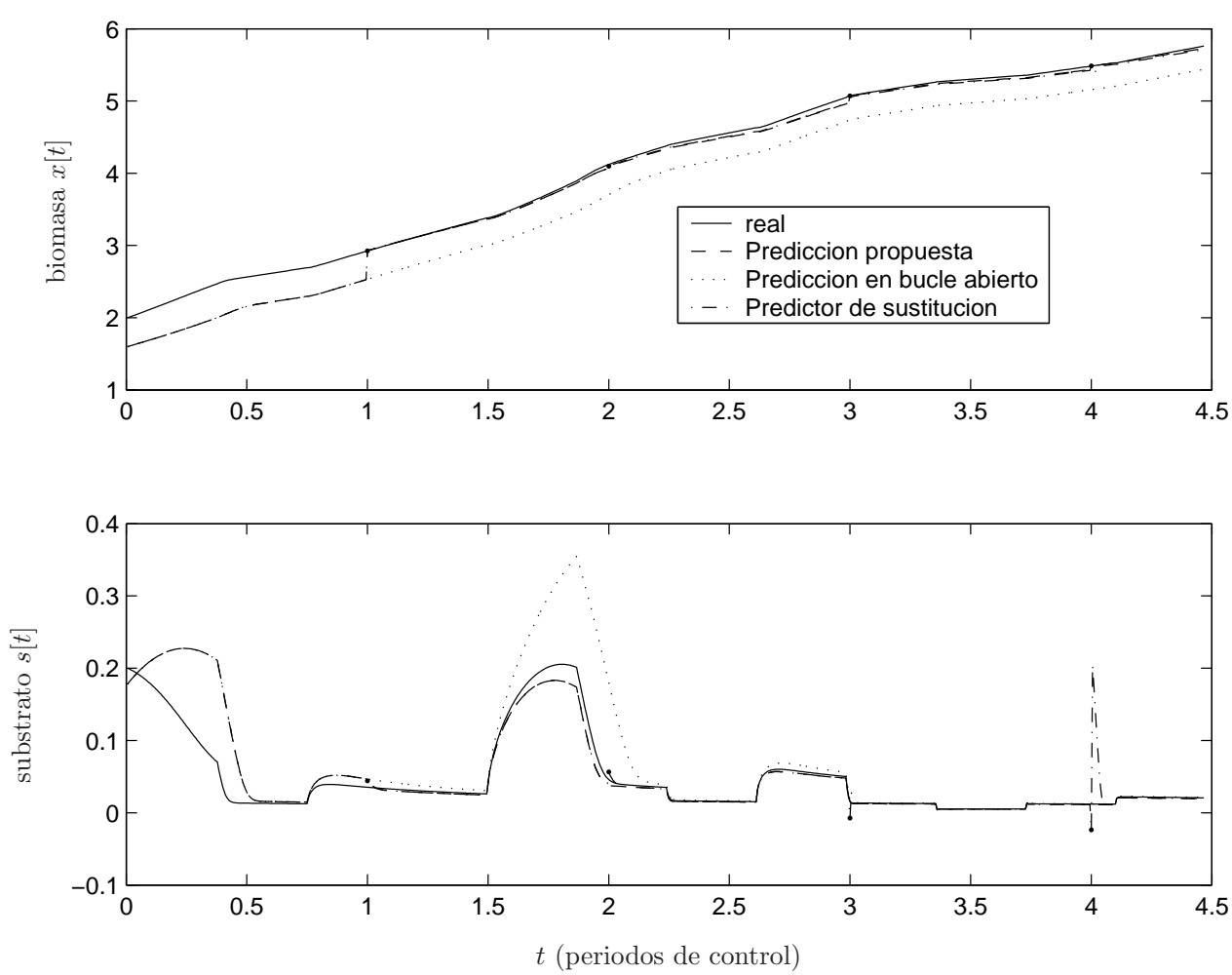

Figura 7.27: Salida, mediciones y diferentes predicciones para el biorreactor. Muestreo cada 1 hora 

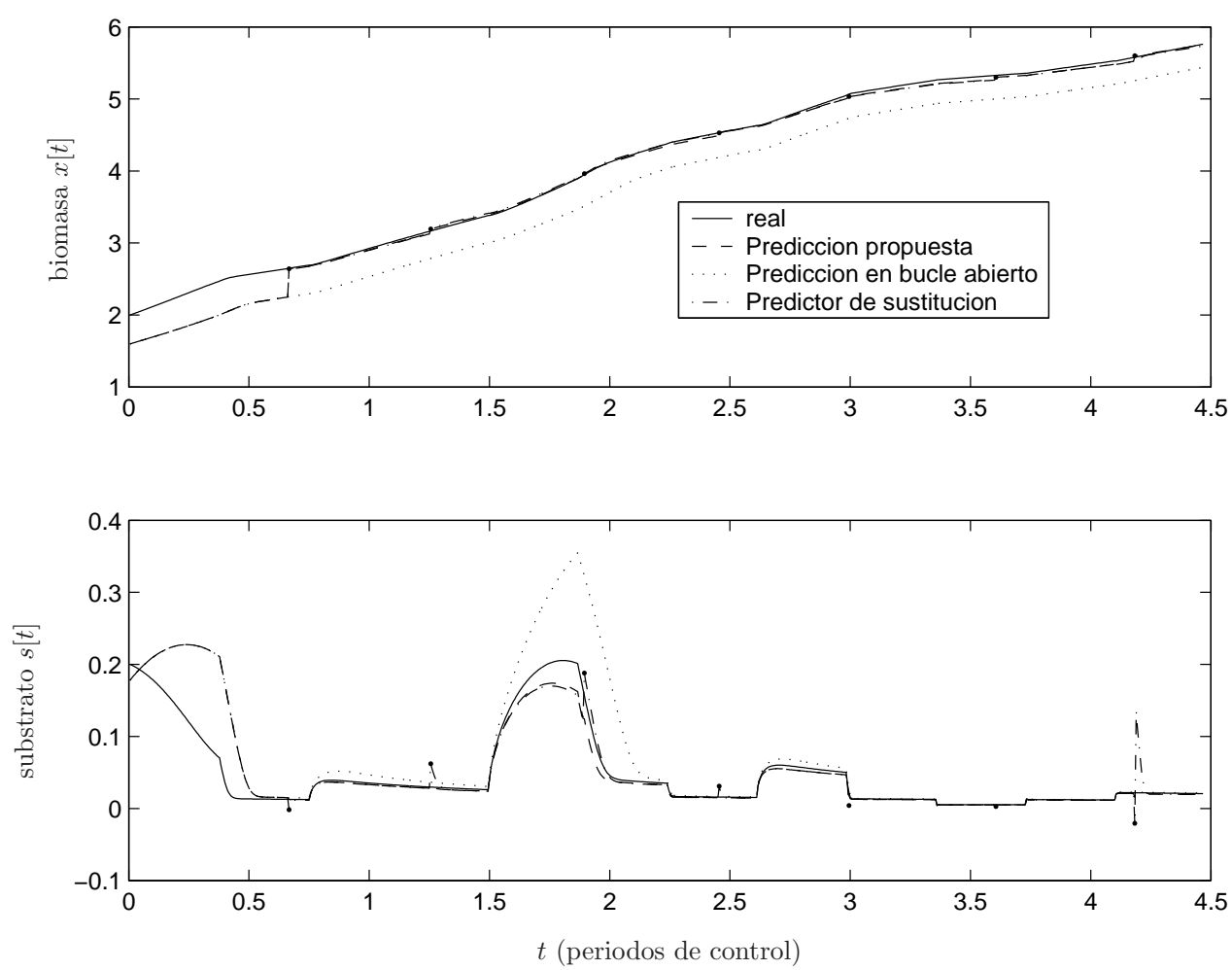

Figura 7.28: Salida, mediciones y diferentes predicciones para el biorreactor. Muestreo en el intervalo [30,40] minutos. 


\section{Conclusiones y trabajo futuro}

\subsection{Conclusiones}

En la presente tesis doctoral se ha analizado el problema de control de procesos en los que la escasez de medidas de la variable de salida y los retardos introducidos por el sistema de medición y transmisión de datos hace inviable la utilización de controladores convencionales. Se ha tratado una estructura de control inferencial en la que las acciones de control se actualizan a un periodo constante $T$, y en la que se utiliza un predictor para la predicción explícita de las salidas no disponibles a ese periodo de control. Se ha estudiado la predicción en un escenario general de muestreo escaso aleatorio o variante en el tiempo en el que las mediciones síncronas llegan con retardo respecto de la salida real. También se ha estudiado el caso de las mediciones asíncronas.

La primera parte del trabajo se ha dedicado al diseño de predictores, tanto en representación interna, como en representación entrada-salida.

La segunda parte del trabajo se ha dedicado al análisis de sistemas de control inferencial que hacen uso del predictor propuesto.

\subsubsection{Diseño de predictores}

Las contribuciones más importantes respecto al diseño de predictores se resumen a continuación.

- En el capítulo 3 se ha desarrollado un predictor (algoritmo 3.8) basado en la representación entradasalida que permite predecir directamente las salidas a periodo constante a partir de las mediciones escasas con retardo que se toman síncronamente con la actualización de la acción de control.

- En el capítulo 4 se ha desarrollado un predictor (algoritmo 4.12) basado en la representación en espacio de estados que permite predecir directamente las salidas y/o estados que se desean controlar a periodo constante a partir de mediciones de sensores que miden (de forma escasa y con retardos) algunas de las variables del proceso (estados, salidas o sus combinaciones) de forma síncrona con la actualización de la acción de control.

- En el capítulo 5 se ha desarrollado un predictor (algoritmo 4.12) para sistemas no lineales que cumplen con la condición de Lipschitz. El predictor propuesto estima las salidas y/o estados a controlar a partir de mediciones escasas de sensores que miden alguna variable del proceso.

- Se ha definido un parámetro variante en el tiempo $s_{k}=\left\{1, \ldots, n_{\mathcal{S}}\right\}$ que define el escenario de muestreo (combinación de periodos intermuestreo $N_{k}$, retardos en los sensores $d_{k}$ y sensores disponibles $\boldsymbol{\Delta}_{k}$ ) asociado a cada medición.

- El diseño de estos predictores se aborda calculando fuera de línea un conjunto finito de ganancias, habiendo una ganancia diferente para cada valor del parámetro $s_{k}$, a diferencia del filtro de Kalman, que calcula en línea una nueva ganancia con cada muestreo. De esta forma, cada vez que llega una medición se utiliza una de las ganancias almacenada según el valor que toma $s_{k}$. La implementación de este predictor tiene un coste computacional mucho menor que el filtro de Kalman con muestreo irregular. 
- Se ha demostrado que el error del predictor que utiliza esta ganancia tiene una dinámica variante en el tiempo definida por unas matrices $\mathcal{A}\left(s_{k}\right)$ y $\mathcal{B}\left(s_{k}\right)$ que dependen del parámetro $s_{k}$. Por este motivo, se han podido emplear técnicas de análisis y síntesis de sistemas lineales variantes en el tiempo de forma paramétrica para obtener la ganancia. Como el parámetro toma un conjunto finito de valores, son aplicables algunas técnicas basadas en desigualdades lineales matriciales (LMI).

- Las metodologías de diseño desarrolladas permiten reducir el coste computacional de implementación y de diseño dividiendo el conjunto $\mathcal{S}$ en un número de subconjuntos dando lugar a un número menor de ganancias a almacenar. De esta manera se pueden diseñar, por ejemplo, predictores que utilicen una ganancia diferente según el valor del retardo (sin atender a la disponibilidad de datos), o bien que utilicen una ganancia diferente según la disponibilidad de datos (sin atender al retardo). Finalmente, este procedimiento permite llevar a cabo el diseño del predictor más simple que consistiría en la utilización de una ganancia constante.

- El primer acercamiento al problema de diseño de las ganancias de los predictores se ha abordado asegurando la estabilidad nominal (en ausencia de perturbaciones) para un conjunto dado de escenarios de muestreo posibles $\mathcal{S}$.

- Posteriormente se ha tratado el diseño basado en la atenuación de perturbaciones y ruidos de medición. Dentro de este diseño se han propuesto diferentes estrategias que varían en función de la naturaleza de las perturbaciones:

- Cuando las perturbaciones son señales persistentes de valor eficaz conocido, el diseño $\mathcal{H}_{\infty}$ y el $\mathcal{H}_{2}$ premiten minimizar el valor eficaz del error de predicción cometido. El diseño $\mathcal{H}_{2}$ sólo es válido para señales de media cero con varianza conocida.

- Cuando las perturbaciones son señales de norma $\ell_{2}$ acotada (de energía finita), el diseño $\mathcal{H}_{2 g}$ permite acotar el valor máximo del error de predicción, mientras que el diseño $\mathcal{H}_{\infty}$ permite minimizar la norma $\ell_{2}$ del error de predicción.

- Cuando las perturbaciones son señales de las que sólo se conoce el valor máximo, el diseño $\ell_{1}$ permite minimizar el error de predicción máximo instantáneo, mientras que el diseño $\mathcal{H}_{\infty}$ permite minimizar el valor eficaz del error.

En todos los casos el método de diseño consiste en construir unas LMIs y utilizar herramientas estándar de minimización de funciones lineales sujetas a LMIs

- El diseño de predictores se ha analizado tanto desde el punto de vista determinista (todos los escenarios de muestreo son posibles con cada medición) como desde el punto de vista estocástico, donde los diferentes escenarios de muestreo tienen asignados una determinada probabilidad.

- Se ha demostrado que el efecto que tiene el error de modelado sobre el error de predicción es equivalente al de una perturbación y ruido de medida que dependen del error de modelado, de manera que las técnicas anteriores también son útiles para hacer el predictor robusto al error de modelado.

- Se ha analizado el efecto que tiene el retardo de la acción de control en la predicción y se han propuesto dos estrategias diferentes para atenuar su efecto:

- Si se trata de un retardo constante conocido, se ha obtenido un nuevo modelo de la planta que estima las salidas con el retardo de la entrada.

- Si se trata de un retardo variante en el tiempo y desconocido, se ha demostrado que el efecto del retardo sobre el error de predicción es equivalente al de una perturbación del estado. Se ha acotado el valor de esta perturbación equivalente, con lo que son aplicables todas las técnicas de diseño anteriores.

- Se ha estudiado la problemática de tener mediciones con retardo que vienen acompañadas de una etiqueta de tiempo incierta, que apunta a un instante de tiempo diferente de aquel en el que realmente se ha tomado la medida. Se ha demostrado que el efecto de esta incertidumbre sobre el error de predicción es equivalente al de un ruido de medida, de manera que las técnicas anteriores son útiles para hacer el predictor robusto a la incertidumbre de la etiqueta de tiempo. Uno de los ejemplos más típicos que se pueden encontrar es cuando las mediciones llegan con un retardo desconocido porque no vienen acompañadas de etiqueta de tiempo. 
- Se ha analizado la aplicación de los predictores a sistemas en los que las mediciones se toman de forma asíncrona con la actualización de la acción de control. Se han propuesto diferentes técnicas que se basan en la estimación de mediciones síncronas mediante una interpolación con la medición asíncrona, de manera que se puede utilizar el predictor propuesto. La diferencia entre la estimación de las mediciones en los instantes síncronos y el valor real tiene un efecto sobre el error de predicción equivalente al de un ruido de medida, con lo que se pueden utilizar las técnicas anteriores de atenuación de perturbaciones.

\subsubsection{Análisis de sistemas de control inferencial}

Las contribuciones más importantes respecto al análisis de sistemas de control inferencial se resumen a continuación.

- En el capítulo 6 se ha obtenido la ecuación que describe la dinámica de bucle cerrado de un sistema de control inferencial que utiliza un predictor basado en el modelo, tanto para el caso monovariable, como para el caso múltiples salidas, asumiendo un muestreo irregular con retardos.

- Se ha demostrado que se cumple el principio de separación de forma que es posible diseñar el predictor (con muestreo irregular y retardos) y el controlador (con muestreo convencional) por separado de forma que, si ambos son estables, el sistema de control inferencial también lo será.

- Se ha demostrado que el efecto del error de predicción sobre la salida controlada es el mismo que tiene un ruido de medida en un sistema de control con muestreo convencional. Partiendo de este hecho se ha propuesto una estrategia para diseñar el controlador convencional que minimiza el efecto de las perturbaciones sobre la salida a partir del conocimiento de la cota de la norma del error cometido con el predictor.

\subsection{Trabajo futuro}

En este trabajo han quedado pendientes de resolver algunos aspectos. Estos aspectos se proponen como líneas de investigación para futuros trabajos.

- En el trabajo se ha propuesto una técnica de diseño de predictores para sistemas multivariables que devuelve una solución siempre que el sistema sea estable o detectable para el escenario de muestreo dado. Sin embargo falta un estudio que permita decidir de antemano si es posible encontrar un predictor estable a partir de la definición de las características del muestreo. Otra formulación del problema consiste en buscar las condiciones de muestreo que permiten encontrar un predictor estable. Para este estudio resultarán útiles los resultados ya conocidos de observabilidad de sistemas conmutados.

- Una posible mejora de los predictores propuestos en este trabajo consiste en el desarrollo de predictores con acción de integral, de manera que sean capaces de atenuar el efecto que tienen las perturbaciones que varían lentamente con el tiempo. Este tipo de predictores también pueden ser útiles para atenuar el efecto del error de modelado y de elementos no lineales.

- Se han propuesto predictores para sistemas no lineales con medidas escasas. Sin embargo, no se ha propuesto ninguna solución a otros problemas que pueden presentarse durante la predicción en este tipo de sistemas como son: los retardos en las mediciones, el error de modelado en las funciones no lineales, las etiquetas de tiempo inciertas que pueden acompañar a cada medición o las mediciones asíncronas. Falta pues desarrollar predictores para sistemas no lineales que también aborden estas problemáticas.

- Se ha analizado la estabilidad del sistema de control inferencial con sistemas lineales. Sin embargo, sólo se ha hecho un inciso a la estabilidad local de sistemas de control inferencial con sistemas no lineales. Falta hacer un estudio de estabilidad con sistemas no lineales que hacen uso de predictores.

- No se ha estudiado el problema de la cuantificación de la transmisión de datos a través del canal de comunicación. Se debe estudiar cómo afecta el número de bits que se utiliza para definir los valores sobre el error de predicción, y cómo afecta al bucle global en general. 
- Una aplicación muy útil de los filtros es la detección de fallos. Queda pendiente la extensión de los algoritmos propuestos al caso de detección de fallos.

- Una de las aplicaciones de los predictores con muestreo aleatorio con retardo es la identificación de procesos a partir de un patrón de muestreo escaso. Falta un estudio de convergencia de algoritmos de identificación que utilizan los predictores propuestos en este trabajo.

- Faltaría también un estudio de estabilidad de un sistema de control adaptativo con muestreo escaso y retardos que hace uso del predictor propuesto y de algoritmos de identificación pseudolineales. 


\section{Fundamentos matemáticos}

\section{A.1 Introducción}

En este capítulo se muestran los fundamentos matemáticos que se han utilizado a lo largo del trabajo. Estos resultados están adaptados al caso discreto a partir de los resultados encontrados en la bibliografía para el caso continuo (véase $[9,18,59,63]$ ).

\section{A.2 Normas}

La utilidad de las normas reside en tener un único numero que da una medida global del tamaño de un vector, una matriz, una señal o un sistema. Para más detalles sobre normas de señales y sistemas continuos consúltese [63].

Definición A.2.1. Una norma de $\|e\|$ (que puede ser un vector, una matriz, una señal o un sistema) es un número real denotado por $\|e\|$ que cumple las siguientes propiedades:

1. No negativa: $\|e\|>0$.

2. Positiva: $\|e\|=0 \leftrightarrow e=0$.

3. Homogénea: $\|\alpha e\|=|\alpha| \cdot\|e\|, \forall \alpha \in \mathbb{C}$.

4. Desigualdad triangular: $\left\|e_{1}+e_{1}\right\| \leq\left\|e_{1}\right\|+\left\|e_{2}\right\|$.

Las normas consideradas en este trabajo pueden ser de cuatro objetos diferentes:

- $e$ es un vector constante,

- $e$ es una matriz constante,

- $e$ es una señal discreta dependiente del tiempo ( $e[t]$ ó $e_{k}$ ), donde para cada instante fijo $t, e$ es un escalar o vector constante,

- $e$ es un sistema dado por la función de transferencia discreta $G(z)$ o por la respuesta impulsional $g[t]$, donde para cada $z$ o $t$ fijo, $e$ es un escalar o matriz constante.

\section{A.2.1 Definiciones}

\section{Normas de vectores}

Se define la norma $p$ de un vector $\boldsymbol{v}=\left[v_{1}, v_{2}, \ldots, v_{n}\right]$ como

$$
\|\boldsymbol{v}\|_{p}=\left(\sum_{i=1}^{n}\left|v_{i}\right|^{p}\right)^{1 / p} .
$$

Las tres normas utilizadas en el trabajo son: 


$$
\begin{array}{ll}
\text { Norma } 1 \text { (norma suma) } & \|v\|_{1}=\sum_{i}\left|v_{i}\right| \\
\text { Norma } 2 \text { (norma euclídea) } & \|v\|_{2}=\sqrt{\sum_{i}\left|v_{i}\right|^{2}} \\
\text { Norma } \infty \text { (norma máx) } & \|v\|_{\infty}=\operatorname{máx}_{i}\left|v_{i}\right|
\end{array}
$$

\section{Normas de matrices}

Las normas de matrices cumplen, además de con las 4 propiedades de las normas de vectores, con la propiedad:

5. Multiplicativa: $\|\boldsymbol{A} B\| \leq\|\boldsymbol{A}\|\|\boldsymbol{B}\|$

Se define la norma $p$ de una matriz $\boldsymbol{A}=\left\{a_{i j}\right\}$ como

$$
\|\boldsymbol{A}\|_{p}=\left(\sum_{i} \sum_{j}\left|a_{i j}\right|^{p}\right)^{1 / p}
$$

Las tres normas utilizadas en el trabajo son

$$
\begin{array}{ll}
\text { Norma 1 (norma suma) } & \|\boldsymbol{A}\|_{1}=\sum_{i, j}\left|a_{i j}\right| \\
\text { Norma 2 (norma de Frobenius) } & \|\boldsymbol{A}\|_{2}=\sqrt{\sum_{i, j}\left|a_{i j}\right|^{2}}=\sqrt{\operatorname{tr}\left(\boldsymbol{A}^{\top} \boldsymbol{A}\right)} \\
\begin{array}{l}
\text { Norma } \infty \text { (norma de elemento } \\
\text { máximo) }
\end{array} & \|\boldsymbol{A}\|_{\infty}=\operatorname{máx}_{i, j}\left|a_{i j}\right|
\end{array}
$$

\section{Normas inducidas de matrices}

Las normas inducidas de matrices son importantes por su relación con la amplificación de señales en sistemas. Considérese la ecuación de un sistema $\boldsymbol{y}=\boldsymbol{A} \boldsymbol{u}$, donde $\boldsymbol{u}$ se considera el vector de entrada, $\boldsymbol{y}$ el vector de salida, y la amplificación o ganancia de la matriz $\boldsymbol{A}$ se define mediante el ratio $\|\boldsymbol{y}\| /\|\boldsymbol{u}\|$. Se define la norma $p$ inducida mediante

$$
\|\boldsymbol{A}\|_{i p}=\operatorname{máx}_{\boldsymbol{u} \neq 0} \frac{\|\boldsymbol{A} \boldsymbol{u}\|_{p}}{\|\boldsymbol{u}\|_{p}},
$$

e indica la máxima ganancia para todas las posibles direcciones de la entrada $\boldsymbol{u}$. Esta norma generaliza el concepto de ganancia de los sistemas monovariables. De la definición de la norma inducida se desprende la propiedad multiplicativa

6. Multiplicativa: $\|\boldsymbol{A} \boldsymbol{u}\|_{p} \leq\|\boldsymbol{A}\|_{i p}\|\boldsymbol{u}\|_{p}$.

Las tres normas utilizadas en este trabajo son:

$$
\begin{aligned}
& \text { Norma } 1 \text { inducida } \\
& \text { Norma } 2 \text { inducida } \\
& \text { Norma } \infty \text { inducida }
\end{aligned}
$$$$
\|\boldsymbol{A}\|_{i 1}=\operatorname{máx}_{j}\left(\sum_{i}\left|a_{i j}\right|\right)
$$$$
\|\boldsymbol{A}\|_{i 2}=\bar{\sigma}(\boldsymbol{A})=\operatorname{máx}_{i} \sqrt{\left|\lambda_{i}\left(\boldsymbol{A}^{\boldsymbol{\top}} \boldsymbol{A}\right)\right|}
$$$$
\|\boldsymbol{A}\|_{i \infty}=\operatorname{máx}_{i}\left(\sum_{j}\left|a_{i j}\right|\right)
$$

\section{Normas de señales}

Se considera ahora una señal temporal que puede ser un vector cuyas componentes varían su valor a lo largo del tiempo $\left(\boldsymbol{x}[t]=\left[x_{1}[t], \boldsymbol{x}_{2}[t], \ldots, x_{n}[t]\right]\right)$. El cálculo de normas de señales se realiza en dos pasos:

1. Obtener un valor para cada instante de tiempo usando una norma de vector.

2. Obtener el valor en el tiempo usando una norma temporal. 
Se define la norma $\ell_{p}$ de una señal vectorial discreta como

$$
\|\boldsymbol{x}[t]\|_{p}=\left(\sum_{t=0}^{\infty} \sum_{i}\left|x_{i}[t]\right|^{p}\right)^{1 / p} .
$$

Las tres normas utilizadas en este trabajo son:

$$
\begin{array}{ll}
\begin{array}{l}
\text { Norma } \ell_{1} \text { (valor absoluto } \\
\text { acumulado) }
\end{array} & \|\boldsymbol{x}[t]\|_{1}=\sum_{t=0}^{\infty} \sum_{i}\left|x_{i}[t]\right| \\
\begin{array}{l}
\text { Norma } \ell_{2} \text { (valor cuadráti- } \\
\text { co acumulado, energía) }
\end{array} & \|\boldsymbol{x}[t]\|_{2}=\sqrt{\sum_{t=0}^{\infty} \sum_{i}\left|x_{i}[t]\right|^{2}} \\
\text { Norma } \ell_{\infty} \text { (valor de pico) } & \|\boldsymbol{x}[t]\|_{\infty}=\operatorname{máx}_{t}\left(\operatorname{máx}_{i}\left|x_{i}[t]\right|\right) \\
\begin{array}{l}
\text { Norma RMS (valor eficaz } \\
\text { o potencia) }
\end{array} & \|\boldsymbol{x}[t]\|_{R M S}=\operatorname{lím}_{N \rightarrow \infty} \sqrt{\frac{1}{N} \sum_{t=0}^{N} \sum_{i}\left|x_{i}[t]\right|^{2}}
\end{array}
$$

\section{Normas de sistemas}

Sea un sistema discreto multivariable definido por su función de transferencia $\boldsymbol{G}(z)$ o por su respuesta impulsional $g[t]$. La norma de un sistema mide la ganancia que hay desde la señal de entrada dada hacia la salida cuando ambas se miden con una norma determinada. Las señales de entrada consideradas para medir esta ganancia son

- $u[t]$ señal impulsional acotada por $|u[0]|$,

- $u[t]$ señal acotada en energía por $\|u[t]\|_{2}$,

- $u[t]$ señal acotada en amplitud por $\|u[t]\|_{\infty}$,

- $u[t]$ señal acotada en valor eficaz por $\|u[t]\|_{\mathrm{RMS}}$,

- $u[t]$ ruido blanco de media cero y varianza $\sigma_{u}$,

mientras que las normas utilizadas para medir la salida son las normas $\ell_{2}, \ell_{\infty}$ y RMS. Las normas utilizadas en este trabajo son:

$$
\begin{array}{lll}
\text { Norma } \mathcal{H}_{\infty} & \text { Energía-energía } & \|G(z)\|_{\infty}=\operatorname{máx}_{u[t]} \frac{\|y[t]\|_{2}}{\|u[t]\|_{2}} \\
\text { Norma } \mathcal{H}_{2} & \text { Varianza-RMS } & \|G(z)\|_{2}=\frac{\|y[t]\|_{R M S}}{\sigma_{u}} \\
\text { Norma } \mathcal{H}_{2 g} & \text { Energía-pico } & \|G(z)\|_{2 g}=\operatorname{máx}_{u[t]} \frac{\|y[t]\|_{\infty}}{\|u[t]\|_{2}} \\
\text { Norma } \ell_{1} & \text { Pico-pico } & \|g[t]\|_{1}=\operatorname{máx}_{u[t]} \frac{\|y[t]\|_{\infty}}{\|u[t]\|_{\infty}}
\end{array}
$$

La norma $\mathcal{H}_{\infty}$ tiene otras formas de definirse, teniendo en cuenta que si $u[t]$ es un escalón, entonces $\|u[t]\|_{R M S}=\|u[t] \mid\|_{\infty} \mathrm{y}$, para una $u[t]$ cualquiera $\|u[t]\|_{R M S} \leq\|u[t]\|_{\infty}$ :

$$
\begin{aligned}
& \text { Norma } \mathcal{H}_{\infty} \\
& \text { RMS-RMS } \quad\|G(z)\|_{\infty}=\operatorname{máx}_{u[t]} \frac{\|y[t]\|_{R M S}}{\|u[t]\|_{R M S}} \\
& \text { Norma } \mathcal{H}_{\infty} \quad \text { pico-RMS } \quad\|G(z)\|_{\infty} \geq \operatorname{máx}_{u[t]} \frac{\|y[t]\|_{R M S}}{\|u[t]\|_{\infty}}
\end{aligned}
$$

En la tabla A.1 se resume cómo se relacionan las normas de las entradas y salidas a través de las normas de sistemas definidas. 


\begin{tabular}{|c|cccc}
\hline & $\|u[t]\|_{2}$ & $\|u[t]\|_{\infty}$ & $\|u[t]\|_{\mathrm{RMS}}$ & $\sigma_{u}$ \\
\hline$\|y[t]\|_{2}$ & $\|G(z)\|_{\infty}$ & $\infty$ & $\infty$ & $\infty$ \\
$\|y[t]\|_{\infty}$ & $\|G(z)\|_{2}$ & $\|g[t]\|_{1}$ & - & - \\
$\|y[t]\|_{\mathrm{RMS}}$ & 0 & $\|G(z)\|_{\infty}$ & $\|G(z)\|_{\infty}$ & $\|G(z)\|_{2}$ \\
\hline
\end{tabular}

Tabla A.1: Normas de sistemas

\section{A.3 Análisis de sistemas discretos vía LMI}

En este apartado se plantea la obtención de condiciones de estabilidad y las normas de sistemas discretos mediante la técnica de las desigualdades lineales matriciales (LMI). Esos planteamientos sirven como base de partida para los diferentes desarrollos de predictores realizados en los capítulos principales.

Tómese el sistema discreto multivariable definido mediante su representación interna

$$
\begin{aligned}
\boldsymbol{x}_{k+1} & =\boldsymbol{A} \boldsymbol{x}_{k}+\boldsymbol{B} \boldsymbol{u}_{k}, \\
\boldsymbol{y}_{k} & =\boldsymbol{C} \boldsymbol{x}_{k}+\boldsymbol{D} \boldsymbol{u}_{k} .
\end{aligned}
$$

La representación entrada-salida del mismo viene dada por

$$
\boldsymbol{y}(z)=G(z) \boldsymbol{u}(z)=\left(\boldsymbol{C}(z \boldsymbol{I}-\boldsymbol{A})^{-1} \boldsymbol{B}+\boldsymbol{D}\right) \boldsymbol{u}(z),
$$

mientras que la respuesta ante un impulso $\boldsymbol{u}_{0}$ es

$$
g_{k}= \begin{cases}\boldsymbol{D} \boldsymbol{u}_{0}, & k=0 \\ \boldsymbol{C} \boldsymbol{A}^{k-1} \boldsymbol{B} \boldsymbol{u}_{0}, & k>0\end{cases}
$$

\section{A.3.1 Estabilidad nominal}

Teorema A.3.1. El sistema (A.1) es cuadráticamente estable si existe una matriz $\boldsymbol{P}=\boldsymbol{P}^{\top} \succ \mathbf{0}$ (simétrica y definida positiva) tal que

$$
\boldsymbol{A}^{\top} \boldsymbol{P} \boldsymbol{A}-\boldsymbol{P} \prec \mathbf{0}
$$

Prueba A.3.1. Tómese la función de Lyapunov

$$
\mathcal{V}_{k}=\boldsymbol{x}_{k}^{\top} \boldsymbol{P} \boldsymbol{x}_{k}
$$

que será positiva siempre que se cumpla la condición $\boldsymbol{P} \succ \mathbf{0}$. El sistema será cuadráticamente estable si $\Delta \mathcal{V}_{k}=\mathcal{V}_{k+1}-\mathcal{V}_{k}<0$. La respuesta libre del sistema viene dada por

$$
\boldsymbol{x}_{k+1}=\boldsymbol{A} \boldsymbol{x}_{k}
$$

con lo que el incremento de la función de Lyapunov puede escribirse como

$$
\Delta \mathcal{V}_{k}=\boldsymbol{x}_{k+1}^{\top} \boldsymbol{P} \boldsymbol{x}_{k+1}-\boldsymbol{x}_{k} \boldsymbol{P} \boldsymbol{x}_{k}=\boldsymbol{x}_{k}^{\top} \boldsymbol{A}^{\top} \boldsymbol{P} \boldsymbol{A} \boldsymbol{x}_{k}-\boldsymbol{x}_{k}^{\top} \boldsymbol{P} \boldsymbol{x}_{k}=\boldsymbol{x}_{k}^{\top}\left(\boldsymbol{A}^{\top} \boldsymbol{P} \boldsymbol{A}-\boldsymbol{P}\right) \boldsymbol{x}_{k} .
$$

La condición (A.2) implica que el incremento $\Delta \mathcal{V}_{k}$ es negativo para todo $x_{k}$. En consecuencia, la función de Lyapunov decrecerá en cada instante y el sistema será cuadráticamente estable.

Antes de pasar a la obtención de las normas es necesario introducir el siguiente resultado, que es una herramienta básica en el tratamiento de desigualdades lineales matriciales.

Lema A.3.1 (Complementos de Schur). Si una matriz particionada

$$
M=\left[\begin{array}{ll}
A & B \\
C & D
\end{array}\right] \succ 0
$$

es definida positiva, entonces

$$
\boldsymbol{A}-\boldsymbol{B} \boldsymbol{D}^{-1} \boldsymbol{C} \succ \mathbf{0}
$$

$y$

$$
D \succ 0 .
$$


La matriz $\boldsymbol{A}-\boldsymbol{B} \boldsymbol{D}^{-1} \boldsymbol{C}$ es el complemento de Schur de $\boldsymbol{D}$ y satisface

$$
\boldsymbol{M}=\left[\begin{array}{cc}
\boldsymbol{I} & \boldsymbol{B} \boldsymbol{D}^{-1} \\
\mathbf{0} & \boldsymbol{I}
\end{array}\right]\left[\begin{array}{cc}
\boldsymbol{A}-\boldsymbol{B} \boldsymbol{D}^{-1} \boldsymbol{C} & \mathbf{0} \\
\mathbf{0} & \boldsymbol{D}
\end{array}\right]\left[\begin{array}{cc}
\boldsymbol{I} & \mathbf{0} \\
\boldsymbol{D}^{-1} \boldsymbol{C} & \boldsymbol{I}
\end{array}\right] .
$$

Si $\boldsymbol{M}$ es una matriz simétrica, entonces las condiciones $\boldsymbol{A}-\boldsymbol{B} \boldsymbol{D}^{-1} \boldsymbol{C} \succ 0$ y $\boldsymbol{D} \succ 0$ también implican que $\boldsymbol{M}$ es definida positiva.

De la misma forma, si $\boldsymbol{M}$ es definida positiva, entonces

$$
D-C A^{-1} B \succ 0
$$

$y$

$$
\boldsymbol{A} \succ \mathbf{0} .
$$

La matriz $\boldsymbol{D}-\boldsymbol{C A}^{-1} \boldsymbol{B}$ es el complemento de Schur de $\boldsymbol{A}$ y satisface

$$
M=\left[\begin{array}{cc}
\boldsymbol{I} & 0 \\
\boldsymbol{C A} \boldsymbol{A}^{-1} & \boldsymbol{I}
\end{array}\right]\left[\begin{array}{cc}
\boldsymbol{A} & \mathbf{0} \\
\mathbf{0} & \boldsymbol{D}-\boldsymbol{C A ^ { - 1 } B}
\end{array}\right]\left[\begin{array}{cc}
\boldsymbol{I} & \boldsymbol{A}^{-1} \\
0 & \boldsymbol{I}
\end{array}\right] .
$$

Si $\boldsymbol{M}$ es una matriz simétrica, entonces las condiciones $\boldsymbol{D}-\boldsymbol{C} \boldsymbol{A}^{-1} \boldsymbol{B} \succ 0$ y $\boldsymbol{D} \succ 0$ también implican que $M$ es definida positiva.

\section{A.3.2 Norma $\mathcal{H}_{\infty}$}

La norma $\mathcal{H}_{\infty}$ del sistema (A.1) viene definida por $\|G(z)\|_{\infty}=\operatorname{máx}_{u_{k}} \frac{\left\|y_{k}\right\|_{2}}{\left\|u_{k}\right\|_{2}}$. El siguiente teorema muestra cómo obtener el valor de esta norma mediante la utilización de LMI.

Teorema A.3.2. La norma $\mathcal{H}_{\infty}$ del sistema (A.1) viene dada por

$$
\|G(z)\|_{\infty}=\min _{\boldsymbol{P}} \gamma
$$

sujeto a

$$
\begin{aligned}
& \boldsymbol{P} \succ \mathbf{0}, \\
& {\left[\begin{array}{cc}
\boldsymbol{A}^{\top} \boldsymbol{P} \boldsymbol{A}-\boldsymbol{P}+\boldsymbol{C}^{\top} \boldsymbol{C} & \boldsymbol{A}^{\top} \boldsymbol{P} \boldsymbol{B}+\boldsymbol{C}^{\top} \boldsymbol{D} \\
\boldsymbol{B}^{\top} \boldsymbol{P} \boldsymbol{A}+\boldsymbol{D}^{\top} \boldsymbol{C} & \boldsymbol{B}^{\top} \boldsymbol{P} \boldsymbol{B}+\boldsymbol{D}^{\top} \boldsymbol{D}-\gamma^{2} \boldsymbol{I}
\end{array}\right] \prec \mathbf{0 .}}
\end{aligned}
$$

Prueba A.3.2. Premultiplicando la LMI (A.3b) por $\left[\boldsymbol{x}_{k}^{\top} \boldsymbol{u}_{k}^{\top}\right]$ y postmultiplicando por su transpuesta se tiene que

$$
\begin{gathered}
{\left[\begin{array}{ll}
\boldsymbol{x}_{k}^{\top} & \boldsymbol{u}_{k}^{\top}
\end{array}\right]\left[\begin{array}{cc}
\boldsymbol{A}^{\top} \boldsymbol{P} \boldsymbol{A}-\boldsymbol{P}+\boldsymbol{C}^{\top} \boldsymbol{C} & \boldsymbol{A}^{\top} \boldsymbol{P} \boldsymbol{B}+\boldsymbol{C}^{\boldsymbol{\top}} \boldsymbol{D} \\
\boldsymbol{B}^{\top} \boldsymbol{P} \boldsymbol{A}+\boldsymbol{D}^{\top} \boldsymbol{C} & \boldsymbol{B}^{\top} \boldsymbol{P} \boldsymbol{B}+\boldsymbol{D}^{\top} \boldsymbol{D}-\gamma^{2} \boldsymbol{I}
\end{array}\right]\left[\begin{array}{l}
\boldsymbol{x}_{k} \\
\boldsymbol{u}_{k}
\end{array}\right]=} \\
=\boldsymbol{x}_{k+1}^{\top} \boldsymbol{P} \boldsymbol{x}_{k+1}-\boldsymbol{x}_{k}^{\top} \boldsymbol{P} \boldsymbol{x}_{k}+\boldsymbol{y}_{k}^{\top} \boldsymbol{y}_{k}-\gamma^{2} \boldsymbol{u}_{k}^{\top} \boldsymbol{u}_{k}<0
\end{gathered}
$$

Asumiendo condiciones iniciales nulas $\boldsymbol{x}_{0}=0$ y sumando de $k=0$ a $k=N-1$ se llega a

$$
\boldsymbol{x}_{N}^{\top} \boldsymbol{P} \boldsymbol{x}_{N}+\sum_{k=0}^{N-1}\left(\boldsymbol{y}_{k}^{\top} \boldsymbol{y}_{k}-\gamma^{2} \boldsymbol{u}_{k}^{\top} \boldsymbol{u}_{k}\right)<0
$$

Como $\boldsymbol{P} \succ \mathbf{0}$ (A.3a), entonces $\boldsymbol{x}_{N}^{\top} \boldsymbol{P} \boldsymbol{x}_{N}>0, \mathrm{y}$

$$
\sum_{k=0}^{N-1}\left(\boldsymbol{y}_{k}^{\top} \boldsymbol{y}_{k}-\gamma^{2} \boldsymbol{u}_{k}^{\top} \boldsymbol{u}_{k}\right)<0 .
$$

Extendiendo el sumatorio hasta infinito se llega a $\left\|\boldsymbol{y}_{k}\right\|_{2}^{2}<\gamma^{2}\left\|\boldsymbol{u}_{k}\right\|_{2}^{2}$, con lo que

$$
\frac{\|\boldsymbol{y}\|_{2}}{\|\boldsymbol{u}\|_{2}}<\gamma
$$




\section{A.3.3 Norma $\mathcal{H}_{2}$}

La norma $\mathcal{H}_{2}$ del sistema (A.1) viene definida por $\|G(z)\|_{2}=\left(\frac{1}{2 \pi} \int_{0}^{2 \pi} \operatorname{tr}\left[G\left(e^{j \omega}\right)^{*} G\left(e^{j \omega}\right)\right] \mathrm{d} \omega\right)^{1 / 2}$. La norma $\mathcal{H}_{2}$ indica cual es la norma RMS de la salida ante un ruido blanco de media cero y una matriz de covarianzas diagonal $\left(\mathcal{E}\left\{\boldsymbol{u}_{k} \boldsymbol{u}_{k}^{\top}\right\}=\sigma \boldsymbol{I}\right)$.

El siguiente resultado es necesario para obtener la norma $\mathcal{H}_{2}$ y para el desarrollo de predictores cuyo diseño se basa en la atenuación $\mathcal{H}_{2}$ de las perturbaciones.

Teorema A.3.3 (Extraído de [61]). Sea $\boldsymbol{w}$ un vector estocástico con media $\boldsymbol{\mu}$ y matriz de covarianzas $\boldsymbol{W}$, y $\boldsymbol{P}$ una matriz cuadrada simétrica. Entonces

$$
\mathcal{E}\left\{\boldsymbol{w}^{\top} \boldsymbol{P} \boldsymbol{w}\right\}=\boldsymbol{\mu}^{\top} \boldsymbol{P} \boldsymbol{\mu}+\operatorname{tr}(\boldsymbol{P} \boldsymbol{W})
$$

Prueba A.3.3. Utilizando las propiedades del operador traza:

$$
\begin{aligned}
\mathcal{E}\left\{\boldsymbol{w}^{\top} \boldsymbol{P} \boldsymbol{w}\right\} & =\mathcal{E}\left\{\operatorname{tr}\left(\boldsymbol{P} \boldsymbol{w} \boldsymbol{w}^{\top}\right)\right\}=\operatorname{tr}\left(\boldsymbol{P} \mathcal{E}\left\{(\boldsymbol{w}-\boldsymbol{\mu})(\boldsymbol{w}-\boldsymbol{\mu})^{\top}+\boldsymbol{\mu} \boldsymbol{\mu}^{\top}\right\}\right) \\
& =\operatorname{tr}\left(\boldsymbol{P}\left(\boldsymbol{W}+\boldsymbol{\mu} \boldsymbol{\mu}^{\top}\right)\right)=\operatorname{tr}\left(\boldsymbol{\mu}^{\top} \boldsymbol{P} \boldsymbol{\mu}+\boldsymbol{P} \boldsymbol{W}\right) \\
& =\boldsymbol{\mu}^{\top} \boldsymbol{P} \boldsymbol{\mu}+\operatorname{tr}(\boldsymbol{P} \boldsymbol{W}) .
\end{aligned}
$$

El siguiente teorema muestra cómo obtener el valor de la norma $\mathcal{H}_{2}$ mediante la utilización de LMI para sistemas de una entrada y una salida.

Teorema A.3.4. La norma $\mathcal{H}_{2}$ del sistema (A.1) cuando $\boldsymbol{D}=\mathbf{0}$ viene dada por

$$
\|G(z)\|_{2}=\min _{\boldsymbol{P}, \boldsymbol{W}} \gamma
$$

sujeto a

$$
\begin{aligned}
& \boldsymbol{P} \succ \mathbf{0}, \\
& \boldsymbol{A}^{\top} \boldsymbol{P} \boldsymbol{A}-\boldsymbol{P}+\boldsymbol{C}^{\top} \boldsymbol{C} \prec \mathbf{0}, \\
& \boldsymbol{B}^{\top} \boldsymbol{P} \boldsymbol{B}+\boldsymbol{D}^{\top} \boldsymbol{D}-\boldsymbol{W} \prec \mathbf{0}, \\
& \operatorname{tr}(\boldsymbol{W})<\gamma .
\end{aligned}
$$

Prueba A.3.4. Tómese como función de Lyapunov $\mathcal{V}_{k}=\boldsymbol{x}_{k}^{\top} \boldsymbol{P} \boldsymbol{x}_{k}$. El valor esperado de la función de Lyapunov en un instante $k+1$ es

$\mathcal{E}\left\{\mathcal{V}_{k+1}\right\}=\mathcal{E}\left\{\boldsymbol{x}_{k+1}^{\top} \boldsymbol{P} \boldsymbol{x}_{k+1}\right\}=\mathcal{E}\left\{\left(\boldsymbol{A} \boldsymbol{x}_{k}+\boldsymbol{B} \boldsymbol{u}_{k}\right)^{\top} \boldsymbol{P}\left(\boldsymbol{A} \boldsymbol{x}_{k}+\boldsymbol{B} \boldsymbol{u}_{k}\right)\right\}=\mathcal{E}\left\{\boldsymbol{x}_{k}^{\top} \boldsymbol{A}^{\top} \boldsymbol{P} \boldsymbol{A} \boldsymbol{x}_{k}\right\}+\mathcal{E}\left\{\boldsymbol{u}_{k}^{\top} \boldsymbol{B}^{\top} \boldsymbol{P} \boldsymbol{B} \boldsymbol{u}_{k}\right\}$ donde se ha tenido en cuenta que

$$
\mathcal{E}\left\{\boldsymbol{x}_{k}^{\top} \boldsymbol{A}^{\top} \boldsymbol{P} \boldsymbol{B} u_{k}\right\}=\mathcal{E}\left\{\boldsymbol{x}_{k}^{\top} \boldsymbol{A}^{\top} \boldsymbol{P} \boldsymbol{B}\right\} \mathcal{E}\left\{u_{k}\right\}=0
$$

debido a la independencia entre $\boldsymbol{u}_{k}$ y $\boldsymbol{x}_{k}$ y que el valor esperado de $\boldsymbol{u}_{k}$ es el vector nulo. Utilizando los mismos argumentos, la esperanza de la norma euclídea del vector de salidas en un instante dado es

$$
\mathcal{E}\left\{\boldsymbol{y}_{k}^{\top} \boldsymbol{y}_{k}\right\}=\mathcal{E}\left\{\left(\boldsymbol{C} \boldsymbol{x}_{k}+\boldsymbol{D} \boldsymbol{u}_{k}\right)^{\top}\left(\boldsymbol{C} \boldsymbol{x}_{k}+\boldsymbol{D} \boldsymbol{u}_{k}\right)\right\}=\mathcal{E}\left\{\boldsymbol{x}_{k}^{\top} \boldsymbol{C}^{\boldsymbol{\top}} \boldsymbol{C} \boldsymbol{x}\right\}+\mathcal{E}\left\{\boldsymbol{u}_{k}^{\top} \boldsymbol{D}^{\boldsymbol{\top}} \boldsymbol{D} \boldsymbol{u}_{k}\right\}
$$

Premultiplicando la LMI (A.5b) por $\boldsymbol{x}_{k}^{\top}$ y postmultiplicando por su transpuesta se tiene que

$$
\boldsymbol{x}_{k}^{\top} \boldsymbol{A}^{\top} \boldsymbol{P} \boldsymbol{A} \boldsymbol{x}_{k}-\boldsymbol{x}_{k}^{\top} \boldsymbol{P} \boldsymbol{x}_{k}+\boldsymbol{x}_{k}^{\top} \boldsymbol{C}^{\top} \boldsymbol{C} \boldsymbol{x}_{k}<0
$$

Tomando esperanzas matemáticas y aplicando el resultado anterior se tiene que

$$
\mathcal{E}\left\{\mathcal{V}_{k+1}\right\}-\mathcal{E}\left\{\mathcal{V}_{k}\right\}+\mathcal{E}\left\{\boldsymbol{x}_{k}^{\top} \boldsymbol{C}^{\top} \boldsymbol{C} \boldsymbol{x}_{k}\right\}<\mathcal{E}\left\{\boldsymbol{u}_{k}^{\top} \boldsymbol{B}^{\top} \boldsymbol{P} \boldsymbol{B} \boldsymbol{u}_{k}\right\} .
$$


Por otra parte, premultiplicando la LMI (A.5c) por $\boldsymbol{u}_{k}^{\top}$ y postmultiplicando por su traspuesta se tiene que

$$
\boldsymbol{u}_{k}^{\top} \boldsymbol{B}^{\top} \boldsymbol{P} \boldsymbol{B} \boldsymbol{u}_{k}+\boldsymbol{u}_{k}^{\top} \boldsymbol{D}^{\top} \boldsymbol{D} \boldsymbol{u}_{k}<\boldsymbol{u}_{k}^{\top} \boldsymbol{W} \boldsymbol{u}_{k},
$$

con lo que la desigualdad (A.6) lleva a

$$
\mathcal{E}\left\{\mathcal{V}_{k+1}\right\}-\mathcal{E}\left\{\mathcal{V}_{k}\right\}+\mathcal{E}\left\{\boldsymbol{x}_{k}^{\top} \boldsymbol{C}^{\top} \boldsymbol{C} \boldsymbol{x}_{k}\right\}+\boldsymbol{u}_{k}^{\top} \boldsymbol{D}^{\top} \boldsymbol{D} \boldsymbol{u}_{k}<\mathcal{E}\left\{\boldsymbol{u}_{k}^{\top} \boldsymbol{W} \boldsymbol{u}_{k}\right\},
$$

que, aplicando el teorema A.3.3, se puede expresar como

$$
\mathcal{E}\left\{\mathcal{V}_{k+1}\right\}-\mathcal{E}\left\{\mathcal{V}_{k}\right\}+\mathcal{E}\left\{y_{k}^{\top} y_{k}\right\}<\operatorname{tr}\left(\boldsymbol{W} \sigma^{2} \boldsymbol{I}\right)=\sigma^{2} \operatorname{tr}(\boldsymbol{W}) .
$$

Utilizando la condición (A.5d), se tiene que

$$
\sigma^{2} \operatorname{tr}(\boldsymbol{W})<\gamma^{2} \sigma^{2}
$$

$\mathrm{y}$, por tanto

$$
\mathcal{E}\left\{\mathcal{V}_{k+1}\right\}-\mathcal{E}\left\{\mathcal{V}_{k}\right\}+\mathcal{E}\left\{y_{k}^{\top} y_{k}\right\}<\gamma_{2} \sigma^{2}
$$

Sumando esta expresión de $k=0$ a $k=N-1$, y asumiendo condiciones iniciales nulas, se tiene que

$$
\mathcal{E}\left\{\mathcal{V}_{N}\right\}+\sum_{k=0}^{N-1} \mathcal{E}\left\{y_{k}^{2}\right\}<N \gamma^{2} \sigma^{2}
$$

Como $\boldsymbol{P} \succ \mathbf{0}$, entonces $\mathcal{E}\left\{\mathcal{V}_{N}\right\} \succ \mathbf{0}$ y, tomando el límite cuando $N$ tiende a infinito

$$
\frac{\left\|y_{k}\right\|_{R M S}}{\sigma}<\gamma
$$

\section{A.3.4 Norma $\mathcal{H}_{2 g}$}

La norma $\mathcal{H}_{2}$ del sistema (A.1) viene definida por $\|G(z)\|_{2}=\operatorname{máx}_{u_{k}} \frac{\left\|y_{k}\right\|_{\infty}}{\left\|u_{k}\right\|_{2}}$. El siguiente teorema muestra cómo obtener el valor de esta norma mediante la utilización de LMI.

Teorema A.3.5. La norma $\mathcal{H}_{2}$ generalizada del sistema (A.1) cuando $\boldsymbol{D}=\mathbf{0}$ viene dada por

$$
\|G(z)\|_{2}=\min _{\boldsymbol{P}} \gamma
$$

sujeto a

$$
\begin{aligned}
& \boldsymbol{P} \succ \mathbf{0} \\
& {\left[\begin{array}{cc}
\boldsymbol{A}^{\top} \boldsymbol{P} \boldsymbol{A}-\boldsymbol{P} & \boldsymbol{A}^{\top} \boldsymbol{P B} \\
\boldsymbol{B}^{\top} \boldsymbol{P} \boldsymbol{A} & \boldsymbol{B}^{\top} \boldsymbol{P} \boldsymbol{B}-\gamma^{2} \boldsymbol{I}
\end{array}\right] \prec \mathbf{0},} \\
& \boldsymbol{P}-\boldsymbol{C}^{\top} \boldsymbol{C} \succ \mathbf{0} .
\end{aligned}
$$

Prueba A.3.5. Premultiplicando la LMI (A.7b) por $\left[\boldsymbol{x}_{k}^{\top} \boldsymbol{u}_{k}^{\top}\right]$ y postmultiplicando por su transpuesta se tiene que

$$
\begin{gathered}
{\left[\begin{array}{ll}
\boldsymbol{x}_{k}^{\top} & \boldsymbol{u}_{k}^{\top}
\end{array}\right]\left[\begin{array}{cc}
\boldsymbol{A}^{\top} \boldsymbol{P} \boldsymbol{A}-\boldsymbol{P} & \boldsymbol{A}^{\top} \boldsymbol{P} \boldsymbol{B} \\
\boldsymbol{B}^{\top} \boldsymbol{P} \boldsymbol{A} & \boldsymbol{B}^{\top} \boldsymbol{P} \boldsymbol{B}-\gamma^{2}
\end{array}\right]\left[\begin{array}{l}
\boldsymbol{x}_{k} \\
\boldsymbol{u}_{k}
\end{array}\right]=} \\
\boldsymbol{x}_{k+1}^{\top} \boldsymbol{P} \boldsymbol{x}_{k+1}-\boldsymbol{x}_{k}^{\top} \boldsymbol{P} \boldsymbol{x}_{k}-\gamma^{2} \boldsymbol{u}_{k}^{\top} \boldsymbol{u}_{k}<0 .
\end{gathered}
$$

Asumiendo condiciones iniciales nulas, $\boldsymbol{x}_{0}=0$, y sumando la expresión anterior de $k=0$ a $k=N-1$ se llega a

$$
\boldsymbol{x}_{N}^{\top} \boldsymbol{P} \boldsymbol{x}_{N}<\gamma^{2} \sum_{k=0}^{N-1}\left(\boldsymbol{u}_{k}^{\top} \boldsymbol{u}_{k}\right) .
$$

La LMI (A.7c) implica que

$$
\boldsymbol{x}_{N}^{\top} \boldsymbol{P} \boldsymbol{x}_{N}>\boldsymbol{x}_{N}^{\top} \boldsymbol{C}^{\top} \boldsymbol{C} \boldsymbol{x}_{N}=\boldsymbol{y}_{N}^{\top} \boldsymbol{y}_{N}
$$


y se llega a

$$
\boldsymbol{y}_{N}^{\top} \boldsymbol{y}_{N}<\gamma^{2} \sum_{k=0}^{N-1}\left(\boldsymbol{u}_{k}^{\top} \boldsymbol{u}_{k}\right)
$$

Tomando el instante $N$ como el instante en el que $\boldsymbol{y}_{N}^{\top} \boldsymbol{y}_{N}$ tiene su máximo valor, y considerando que

$$
\sum_{k=0}^{N-1}\left(\boldsymbol{u}_{k}^{\top} \boldsymbol{u}_{k}\right)<\sum_{k=0}^{\infty}\left(\boldsymbol{u}_{k}^{\top} \boldsymbol{u}_{k}\right), \quad \forall N
$$

entonces

$$
\frac{\left\|\boldsymbol{y}_{k}\right\|_{\infty}}{\left\|\boldsymbol{u}_{k}\right\|_{2}}<\gamma
$$

\section{A.3.5 Norma $\ell_{1}$}

La norma $\mathcal{H}_{2}$ del sistema (A.1) viene definida por $\left\|g_{k}\right\|_{1}=\operatorname{máx}_{u_{k}} \frac{\left\|y_{k}\right\|_{\infty}}{\left\|u_{k}\right\|_{\infty}}$. El siguiente teorema muestra cómo obtener el valor de esta norma mediante la utilización de LMI.

Teorema A.3.6. La norma $\ell_{1}$ generalizada del sistema (A.1) cuando $\boldsymbol{D}=\mathbf{0}$ viene dada por la minimización

$$
\left\|g_{k}\right\|_{1}=\operatorname{mín}_{\boldsymbol{P}, \lambda, \mu} \gamma
$$

sujeto a

$$
\begin{aligned}
& \boldsymbol{P} \succ \mathbf{0} \\
& \lambda \in[0,1] \\
& \mu>0 \\
& {\left[\begin{array}{ccc}
\boldsymbol{A}^{\top} \boldsymbol{P} \boldsymbol{A}-\boldsymbol{P}+\lambda \boldsymbol{P} & \boldsymbol{A}^{\top} \boldsymbol{P} \boldsymbol{B} \\
& \boldsymbol{B}^{\top} \boldsymbol{P} \boldsymbol{A} & \boldsymbol{B}^{\top} \boldsymbol{P} \boldsymbol{B}-\mu \boldsymbol{I}
\end{array}\right] \prec \mathbf{0}} \\
& {\left[\begin{array}{ccc}
\lambda \boldsymbol{P} & \mathbf{0} & \boldsymbol{C}^{\top} \\
\mathbf{0} & (\gamma-\mu) \boldsymbol{I} & \boldsymbol{D}^{\top} \\
\boldsymbol{C} & \boldsymbol{D} & \gamma \boldsymbol{I}
\end{array}\right] \succ \mathbf{0}}
\end{aligned}
$$

Prueba A.3.6. Premultiplicando la LMI (A.8d) por $\left[\boldsymbol{x}_{k}^{\top} \boldsymbol{u}_{k}^{\top}\right]$ y postmultiplicando por su transpuesta se tiene que

$$
\begin{aligned}
& {\left[\begin{array}{ll}
\boldsymbol{x}_{k}^{\top} & \boldsymbol{u}_{k}^{\top}
\end{array}\right]\left[\begin{array}{cc}
\boldsymbol{A}^{\top} \boldsymbol{P} \boldsymbol{A}-\boldsymbol{P}+\lambda \boldsymbol{P} & \boldsymbol{A}^{\top} \boldsymbol{P} \boldsymbol{B} \\
\boldsymbol{B}^{\top} \boldsymbol{P} \boldsymbol{A} & \boldsymbol{B}^{\top} \boldsymbol{P} \boldsymbol{B}-\mu
\end{array}\right]\left[\begin{array}{l}
\boldsymbol{x}_{k} \\
\boldsymbol{u}_{k}
\end{array}\right]=} \\
& =\boldsymbol{x}_{k+1}^{\top} \boldsymbol{P} \boldsymbol{x}_{k+1}-\boldsymbol{x}_{k}^{\top} \boldsymbol{P} \boldsymbol{x}_{k}+\lambda \boldsymbol{x}_{k}^{\top} \boldsymbol{P} \boldsymbol{x}_{k}-\mu \boldsymbol{u}_{k}^{\top} \boldsymbol{u}_{k}<0 .
\end{aligned}
$$

Definiendo $\mathcal{V}_{k}=\boldsymbol{x}_{k}^{\top} \boldsymbol{P} \boldsymbol{x}_{k}$ y $\boldsymbol{w}_{k}=\boldsymbol{u}_{k}^{\top} \boldsymbol{u}_{k}$ se tiene la inecuación en diferencias

$$
\mathcal{V}_{k+1}-(1-\lambda) \mathcal{V}_{k}<\mu \boldsymbol{w}_{k}
$$

El máximo de $\mathcal{V}_{k}$ se tiene cuando $\Delta \mathcal{V}_{k}=0$ (derivada discreta nula), es decir, cuando $\mathcal{V}_{k+1}=\mathcal{V}_{k}\left(\boldsymbol{x}_{k+1}=\right.$ $\boldsymbol{x}_{k}$ ), con lo que se puede escribir que

$$
\lambda \mathcal{V}_{k}<\mu \boldsymbol{w}_{k}<\mu\left\|\boldsymbol{w}_{k}\right\|_{\infty}
$$

es decir,

$$
\boldsymbol{x}_{k}^{\top} \boldsymbol{P} \boldsymbol{x}_{k}<\frac{\mu}{\lambda}\left\|\boldsymbol{u}_{k}\right\|_{\infty}^{2} .
$$

Aplicando complementos de Schur en la LMI (A.8e) se tiene que

$$
\left[\begin{array}{ll}
\boldsymbol{x}_{k}^{\top} & \boldsymbol{u}_{k}^{\top}
\end{array}\right]\left(\left[\begin{array}{cc}
\lambda \boldsymbol{P} & \mathbf{0} \\
\mathbf{0} & \gamma-\mu
\end{array}\right]-\frac{1}{\gamma}\left[\begin{array}{l}
\boldsymbol{C}^{\boldsymbol{\top}} \\
\boldsymbol{D}^{\top}
\end{array}\right]\left[\begin{array}{ll}
\boldsymbol{C} & \boldsymbol{D}
\end{array}\right]\right)\left[\begin{array}{l}
\boldsymbol{x}_{k} \\
\boldsymbol{u}_{k}
\end{array}\right]>0
$$




$$
\lambda \boldsymbol{x}_{k}^{\top} \boldsymbol{P} \boldsymbol{x}_{k}+(\gamma-\mu) \boldsymbol{u}_{k}^{\top} \boldsymbol{u}_{k}-\frac{1}{\gamma} \boldsymbol{y}_{k}^{\top} \boldsymbol{y}_{k}>0,
$$

de donde se tiene una cota para la norma de $\boldsymbol{y}_{k}$ como

$$
\boldsymbol{y}_{k}^{\top} \boldsymbol{y}_{k}<\gamma\left(\lambda \boldsymbol{x}_{k}^{\top} \boldsymbol{P} \boldsymbol{x}_{k}+(\gamma-\mu) \boldsymbol{u}_{k}^{\top} \boldsymbol{u}_{k}\right)
$$

Considerando (A.9) y teniendo en cuenta que $\boldsymbol{u}_{k}^{\top} \boldsymbol{u}_{k}<\left\|\boldsymbol{u}_{k}\right\|_{\infty}^{2}$, se tiene que

$$
\boldsymbol{y}_{k}^{\top} \boldsymbol{y}_{k}<\gamma\left(\mu\left\|\boldsymbol{u}_{k}\right\|_{\infty}^{2}+(\gamma-\mu)\left\|\boldsymbol{u}_{k}\right\|_{\infty}^{2}\right)<\gamma^{2}\left\|\boldsymbol{u}_{k}\right\|_{\infty}^{2}
$$

y por tanto

$$
\frac{\|\boldsymbol{y}\|_{\infty}}{\|\boldsymbol{u}\|_{\infty}}<\gamma
$$

\section{A.4 Estabilidad de sistemas variantes en el tiempo de forma paramétri- ca}

En esta sección se reproducen los resultados relativos a los sistemas variantes en el tiempo de forma paramétrica, que son la base de los diseños expuestos en los capítulos de desarrollo de predictores. Estos resultados se pueden encontrar demostrados en [14] y [15].

Considérese el sistema discreto dinámico

$$
\boldsymbol{x}_{k+1}=\mathcal{A}\left(\boldsymbol{\xi}_{k}\right) \boldsymbol{x}_{k}
$$

donde $\boldsymbol{x} \in \mathbb{R}^{n}$ es el vector de estado, $\boldsymbol{\xi} \in \Xi \subset \mathbb{R}^{p}$ es un parámetro variante en el tiempo desconocido pero acotado. Tómese la estructura de la matriz dinámica $\mathcal{A}$ de la forma:

$$
\begin{aligned}
& \mathcal{A}\left(\boldsymbol{\xi}_{k}\right)=\sum_{i=1}^{N} \xi_{i, k} \boldsymbol{A}_{i}, \\
& \xi_{i, k} \geq 0, \quad \sum_{i=1}^{N} \xi_{i, k}=1 .
\end{aligned}
$$

El siguiente teorema da un resultado estándar acerca del problema de análisis de estabilidad

Teorema A.4.1. La solución nula del sistema (A.10) es uniformemente asintóticamente estable si y sólo si existe una función de Lyapunov

$$
\mathcal{V}\left(\boldsymbol{x}_{k}, \boldsymbol{\xi}_{k}\right)=\boldsymbol{x}_{k}^{\top} \mathcal{P}\left(\boldsymbol{\xi}_{k}\right) \boldsymbol{x}_{k}
$$

tal que

$$
\alpha_{1}\left(\left\|\boldsymbol{x}_{k}\right\|\right) \leq \mathcal{V}\left(\boldsymbol{x}_{k}, \boldsymbol{\xi}_{k}\right) \leq \alpha_{2}\left(\left\|\boldsymbol{x}_{k}\right\|\right)
$$

y cuya diferencia a lo largo de la solución de (A.10) es definida negativa y decreciente

$$
\mathcal{L}=\mathcal{V}\left(\boldsymbol{x}_{k+1}, \boldsymbol{\xi}_{k+1}\right)-\mathcal{V}\left(\boldsymbol{x}_{k}, \boldsymbol{\xi}_{k}\right) \leq-\alpha_{0}\left(\left\|\boldsymbol{x}_{k}\right\|\right)
$$

para todo $\boldsymbol{x} \in \mathbb{R}^{n} y \xi \in \Xi y$ donde $\alpha_{0}(\cdot), \alpha_{1}(\cdot)$ y $\alpha_{2}(\cdot)$ son funciones $k_{\infty} 1$.

Prueba A.4.1. Véase [14].

Este resultado es bastante general y no puede aplicarse en esta forma ya que no hay una forma sistemática de construir la función de Lyapunov $\mathcal{V}\left(\boldsymbol{x}_{k}, \xi_{k}\right)$ como una función del parámetro incierto y variante con el tiempo $\xi_{k}$. Basado en la estructura de la incertidumbre, se busca una función de Lyapunov paramétrica (FLP) de la forma

$$
\mathcal{V}\left(\boldsymbol{x}_{k}, \xi_{k}\right)=\boldsymbol{x}_{k}^{\top} \mathcal{P}\left(\xi_{k}\right) \boldsymbol{x}_{k}^{\top}
$$

\footnotetext{
${ }^{1}$ Una función $\alpha:[0, \infty) \rightarrow[0, \infty)$ es $k_{\infty}$ si es continua, estrictamente creciente, nula en el origen y no acotada $(\alpha(s) \rightarrow \infty$ cuando $s \rightarrow \infty)$
} 
con

$$
\mathcal{P}\left(\xi_{k}\right)=\sum_{i=1}^{N} \xi_{i, k} \boldsymbol{P}_{i}
$$

donde $\boldsymbol{P}_{i}$ son matrices constantes simétricas y definidas positivas de la dimensión apropiada. Este tipo de FLP satisface la ecuación (A.12) con

$$
\alpha_{2}\left(\left\|\boldsymbol{x}_{k}\right\|\right)=\sum_{i=1}^{N} \lambda_{\text {máx }}\left(\boldsymbol{P}_{i}\right)\left\|\boldsymbol{x}_{k}\right\|^{2}
$$

y

$$
\alpha_{1}\left(\left\|\boldsymbol{x}_{k}\right\|\right)=\boldsymbol{\varepsilon}\left\|\boldsymbol{x}_{k}\right\|^{2}
$$

con $\varepsilon$ un escalar suficientemente pequeño. La diferencia de la FLP a lo largo de la solución de (A.10) viene dada por

$$
\mathcal{L}=\mathcal{V}\left(\boldsymbol{x}_{k+1}, \boldsymbol{\xi}_{k+1}\right)-\mathcal{V}\left(\boldsymbol{x}_{k}, \boldsymbol{\xi}_{k}\right)=\boldsymbol{x}_{k}^{\top}\left(\mathcal{A}^{\top} \mathcal{P}_{+} \mathcal{A}-\mathcal{P}\right) \boldsymbol{x}_{k}
$$

con $\mathcal{A}$ dada por (A.11) y

$$
\begin{gathered}
\mathcal{P}=\sum_{i=1}^{N} \xi_{i, k} \boldsymbol{P}_{i}, \\
\mathcal{P}_{+}=\sum_{i=1}^{N} \xi_{i, k+1} \boldsymbol{P}_{i}=\sum_{j=1}^{N} \xi_{j, k} \boldsymbol{P}_{j} .
\end{gathered}
$$

Para evitar confusión a la hora de usar la terminología usual de estabilidad cuadrática, se define la estabilidad policuadrática.

Definición A.4.1. El sistema (A.10) es policuadráticamente estable si existe una función cuadrática de Lyapunov dependiente de parámetro (A.14) cuya diferencia es negativa definida decreciente.

La estabilidad policuadrática utiliza funciones de Lyapunov paramétricas que son cuadráticas en el estado y dependen de forma politópica de un parámetro incierto para comprobar si el sistema (A.10) tiene estabilidad asintótica uniforme. El siguiente teorema establece una condición necesaria y suficiente para averiguar si el sistema (A.10) es policuadráticamente estable.

Teorema A.4.2. El sistema (A.10) es policuadráticamente estable si y sólo si existen unas matrices simétricas definidas positivas $\boldsymbol{S}_{i}, \boldsymbol{S}_{j}$ y matrices $\boldsymbol{G}_{i}$ de las dimensiones apropiadas tales que

$$
\left[\begin{array}{cc}
\boldsymbol{G}_{i}+\boldsymbol{G}_{i}^{\top}-\boldsymbol{S}_{i} & \boldsymbol{G}_{i}^{\top} \boldsymbol{A}_{i}^{\top} \\
\boldsymbol{A}_{i} \boldsymbol{G}_{i} & \boldsymbol{S}_{j}
\end{array}\right] \succ \mathbf{0}
$$

para todo $i=1, \ldots, N$ y $j=1, \ldots, N$. En este caso, la FLP variante en el tiempo viene dada por (A.11) con

$$
\mathcal{P}\left(\xi_{k}\right)=\sum_{i=1}^{N} \xi_{i, k} \boldsymbol{S}_{i}^{-1} .
$$

Prueba A.4.2. Véase [14].

\section{A.5 Observabilidad y detectabilidad}

En esta sección se presentan los resultados básicos que permiten desarrollar el capítulo 4 de diseño de predictores para sistemas multivariables. 


\section{A.5.1 Sistemas lineales invariantes en el tiempo}

Considérese el sistema multivariable definido por las ecuaciones

$$
\begin{aligned}
\boldsymbol{x}[t+1] & =\boldsymbol{A} \boldsymbol{x}[t]+\boldsymbol{B} \boldsymbol{u}[t], \\
\boldsymbol{y}[t] & =\boldsymbol{C} \boldsymbol{x}[t]+\boldsymbol{D} \boldsymbol{u}[t]
\end{aligned}
$$

donde $\boldsymbol{x} \in \mathbb{R}^{n}$ es el estado, $\boldsymbol{u} \in \mathbb{R}^{n_{u}}$ es el vector de entradas de control e $\boldsymbol{y} \in \mathbb{R}^{n_{y}}$ es el vector de salidas. Para poder estimar el estado del sistema a partir de las mediciones de la salida, se plantea un observador de la forma

$$
\begin{aligned}
\hat{\boldsymbol{x}}[t+1] & =\boldsymbol{A} \hat{\boldsymbol{x}}[t]+\boldsymbol{B} \boldsymbol{u}[t]+\boldsymbol{L}(\boldsymbol{y}[t]-\hat{\boldsymbol{y}}[t]), \\
\hat{\boldsymbol{y}}[t] & =\boldsymbol{C} \hat{\boldsymbol{x}}[t]+\boldsymbol{D} \boldsymbol{u}[t],
\end{aligned}
$$

donde $\boldsymbol{L}$ es la matriz de ganancias del observador. Definiendo el error de estimación del estado como $\widetilde{\boldsymbol{x}}[t]=\boldsymbol{x}[t]-\hat{\boldsymbol{x}}[t]$ la dinámica del error de estimación viene definida por la ecuación

$$
\widetilde{\boldsymbol{x}}[t+1]=(\boldsymbol{A}-\boldsymbol{L} \boldsymbol{C}) \widetilde{\boldsymbol{x}}[t] .
$$

El observador será estable si es posible tomar una matriz $\boldsymbol{L}$ tal que los valores propios de $(\boldsymbol{A}-\boldsymbol{L} \boldsymbol{C})$ sean de módulo menor que la unidad. En ese caso se dirá que el par $(\boldsymbol{A}, \boldsymbol{C})$ es detectable.

Lema A.5.1. Se dice que $\lambda$ es un valor propio no observable del par $(\boldsymbol{A}, \boldsymbol{C})$ si y sólo si

$$
\text { rango }\left[\begin{array}{c}
\boldsymbol{A}-\lambda \boldsymbol{I} \\
\boldsymbol{C}
\end{array}\right]<n
$$

Si $\lambda$ es un valor propio no observable del par $(\boldsymbol{A}, \boldsymbol{C})$, entonces $\lambda$ es un valor propio de la matriz $(\boldsymbol{A}-\boldsymbol{L} \boldsymbol{C})$ para cualquier matriz $\boldsymbol{L}$. Una condición necesaria y suficiente para que el sistema sea detectable es que todos los valores propios no observables sean estables.

Lema A.5.2. El par $(\boldsymbol{A}, \boldsymbol{C})$ es detectable si y sólo si

$$
\text { rango }\left[\begin{array}{c}
\boldsymbol{A}-\lambda \boldsymbol{I} \\
\boldsymbol{C}
\end{array}\right]=n, \quad \forall \lambda,|\lambda| \geq 1
$$

para todos los valores propios $\lambda$ de $\boldsymbol{A}$ de módulo igual o mayor que uno.

Si el sistema es no observable pero sí detectable, al observador del estado se le llama observador asintótico ya que la dinámica vendrá determinada por el modo no observable.

Ejemplo A.5.1. El sistema discreto dado por

$$
\begin{aligned}
\boldsymbol{x}[t+1] & =\left[\begin{array}{ccc}
0 & 0 & -0.6 \\
0 & 0.7 & 0.8 \\
0 & 0 & 0.5
\end{array}\right] \boldsymbol{x}[t]+\left[\begin{array}{l}
1 \\
2 \\
3
\end{array}\right] u[t] \\
\boldsymbol{y}[t] & =\left[\begin{array}{lll}
1 & 0 & 2
\end{array}\right] \boldsymbol{x}[t]
\end{aligned}
$$

es no observable porque la salida permanecerá en cero si sólo se inicializara en un valor no nulo el segundo estado $\left(x_{1}[0]=0, x_{2}[0] \neq 0, x_{3}[0]=0\right)$. El sistema, sin embargo, sí que es detectable porque el estado no observable $\left(x_{2}\right)$ es estable (polo en 0.7 )

Una condición necesaria para que el filtro de Kalman pueda dar como resultado un observador óptimo es que el par $(\boldsymbol{A}, \boldsymbol{C})$ sea detectable. Esta misma condición se aplica también al caso de los predictores diseñados en el capítulo 4. En los predictores cuya matriz $\boldsymbol{C}$ varía con el tiempo (debido a los diferentes sensores disponibles con cada medición), es posible aprovechar las mediciones escasas que se tienen aunque el sistema sea no detectable frente a dicho conjunto de salidas. La idea consiste en extraer un subsistema que sólo contenga aquellos estados detectables, es decir, que se tiene que buscar un conjunto $\mathcal{E}$ de $n^{\prime}<n$ estados tales que

$$
\operatorname{rango}\left[\begin{array}{c}
\boldsymbol{A}^{\prime}-\lambda \boldsymbol{I} \\
\boldsymbol{C}^{\prime}
\end{array}\right]=n^{\prime}, \quad \forall|\lambda| \geq 1
$$


para todos los valores propios $\lambda$ de $\boldsymbol{A}^{\prime}$ de módulo igual o mayor que uno, donde $\boldsymbol{A}^{\prime}$ es la matriz formada por las filas y columnas de $\boldsymbol{A}$ que se indican en el conjunto $\mathcal{E}$, y $\boldsymbol{C}^{\prime}$ es la matriz formada por las columnas de $\boldsymbol{C}$ que se indican en el conjunto $\mathcal{E}$.

Para visualizar los estados no detectables se puede utilizar una realización que descompone el sistema en sus partes observable y no observable como la siguiente

$$
\begin{aligned}
\boldsymbol{x}[t+1] & =\left[\begin{array}{cc}
\boldsymbol{A}_{o} & \mathbf{0} \\
\boldsymbol{A}_{12} & \boldsymbol{A}_{n o}
\end{array}\right] \boldsymbol{x}[t]+\boldsymbol{B}^{\prime} \boldsymbol{u}[t], \\
\boldsymbol{y}[t] & =\left[\begin{array}{ll}
\boldsymbol{C}_{o} & \mathbf{0}
\end{array}\right] \boldsymbol{x}[t]+\boldsymbol{D}^{\prime} \boldsymbol{u}[t] .
\end{aligned}
$$

donde $\boldsymbol{A}_{o}$ y $\boldsymbol{C}_{o}$ forman el subsistema observable, y $\boldsymbol{A}_{n o}$ es el subsistema no observable. Los estados no observables y no detectables son aquellos cuyo elemento correspondiente en la submatriz $\boldsymbol{A}_{n o}$ es de módulo mayor que uno.

Si el sistema es diagonalizable, de forma que se puede obtener una representación en espacio de estados de la forma

$$
\begin{aligned}
\boldsymbol{x}[t] & =\left[\begin{array}{cccc}
\lambda_{1} & & & 0 \\
& \lambda_{2} & & \\
& & \ddots & \\
0 & & & \lambda_{n}
\end{array}\right] \boldsymbol{x}[t]+\boldsymbol{B} \boldsymbol{u}[t] \\
\boldsymbol{y}[t] & =\left[\begin{array}{cccc}
c_{11} & c_{12} & \cdots & c_{1 n} \\
\vdots & & & \vdots \\
c_{n_{y} 1} & c_{n_{y} 2} & \cdots & c_{n_{y} n}
\end{array}\right] \boldsymbol{x}[t]
\end{aligned}
$$

se tiene que cada estado tiene asignado un único valor propio. Los estados observables serán aquellos que tengan elementos no nulos en la columna de $\boldsymbol{C}$ correspondiente. Los estados no observables detectables serán aquellos que tienen nula la columna correspondiente de $\boldsymbol{C}$, pero tienen un valor propio asociado estable $\left(\left|\lambda_{i}\right|<1\right)$

\section{A.5.2 Sistemas conmutados}

En esta sección se reproducen los resultados básicos relativos al análisis de estabilidad y de observabilidad de sistemas conmutados lineales ${ }^{2}$ que se pueden encontrar en trabajos como [8, 38].

Sea un sistema lineal conmutado de la forma

$$
\begin{aligned}
\boldsymbol{x}_{k+1} & =\boldsymbol{A}\left(s_{k}\right) \boldsymbol{x}_{k}+\boldsymbol{B}\left(s_{k}\right) \boldsymbol{u}_{k}, \quad k \geq 1 \\
\boldsymbol{y}_{k+1} & =\boldsymbol{C}\left(s_{k}\right) \boldsymbol{x}_{k}
\end{aligned}
$$

donde $s_{k}$ indica el modo de funcionamiento en cada instante y toma valores en el conjunto $\{1, \ldots, N\}$, de manera que las matrices paramétricas $\boldsymbol{A}\left(s_{k}\right), \boldsymbol{B}\left(s_{k}\right)$ y $\boldsymbol{C}\left(s_{k}\right)$ conmutan entre $N$ diferentes valores conocidos. Un sistema conmutado es un tipo de sistema variante en el tiempo en el que las matrices de definición del sistema se conocen de antemano, varían todas ellas en función de un único parámetro que tiene un valor diferente en cada periodo.

Lema A.5.3. El sistema conmutado anterior es globalmente asintóticamente estable si y sólo si existe un número entero finito $N$ tal que

$$
\left\|\boldsymbol{A}\left(s_{1}\right) \boldsymbol{A}\left(s_{2}\right) \cdots \boldsymbol{A}\left(s_{N}\right)\right\|_{p}<1
$$

para cualquier secuencia $\left\{s_{k}\right\}, k=1, \ldots, N$.

Dada una secuencia de salidas $\left\{\boldsymbol{y}_{k}\right\}$, una secuencia de entradas $\left\{\boldsymbol{u}_{k}\right\}$ y una secuencia de modos de funcionamiento $\left\{s_{k}\right\}$ para $k=1, \ldots, N$, es posible reconstruir el estado $\boldsymbol{x}_{1}$ si y sólo si la siguiente matriz de observabilidad es de rango $n$

$$
\mathcal{O}(s)=\left[\begin{array}{c}
\boldsymbol{C}\left(s_{1}\right) \\
\boldsymbol{C}\left(s_{2}\right) \boldsymbol{A}\left(s_{1}\right) \\
\vdots \\
\boldsymbol{C}\left(s_{N}\right) \boldsymbol{A}\left(s_{N-1}\right) \cdots \boldsymbol{A}\left(s_{1}\right)
\end{array}\right] .
$$

\footnotetext{
${ }^{2}$ Switched linear systems en la literatura inglesa
} 
Definición A.5.1 (Observabilidad a lo largo de una trayectoria). Se dice que el conjunto de pares $\{(\boldsymbol{A}(1), \boldsymbol{C}(1)), \ldots,(\boldsymbol{A}(N), \boldsymbol{C}(N)\}$ son observables a lo largo de una trayectoria si y sólo si existe un entero $N$ tal que todas las subtrayectorias de longitud $N$ son observables. A ese entero más pequeño se le llama índice de observabilidad de la trayectoria.

Si un conjunto de pares no es observable (es decir, para todo $N$ siempre existe una trayectoria de longitud $N$ no observable), se dice que no es observable a lo largo de una trayectoria.

Sistemas conmutados markovianos. Si las posibles secuencias de funcionamiento $\left\{s_{k}\right\}$ vienen determinadas por una cadena de Markov de probabilidades de transición

$$
\alpha_{i j}=\mathcal{P}\left\{s_{k}=j \mid s_{k-1}=i\right\},
$$

el sistema conmutado anterior será globalmente estable en valor medio si y sólo si existen unas matrices $\boldsymbol{P}(i) \succ \mathbf{0}(i=1, \ldots, N)$ tales que

$$
\boldsymbol{A}(i)^{\top}\left(\sum_{j=1}^{N} \alpha_{i j} \boldsymbol{P}(j)\right) \boldsymbol{A}(i)-\boldsymbol{P}(i) \prec \mathbf{0}, i=1, \ldots, N .
$$

En [16, 8] se establecen los procedimientos para detectar si un sistema de este tipo es observable o no, mientras que en [5] se establecen las bases para el diseño de observadores para sistemas conmutados mediante técnicas LMI. En [50] se establece la generalización del test de observabilidad PBH (PopovBelevitch-Hautus) para sistemas variantes en el tiempo (dentro de los cuales se puede incluir a los sistemas conmutados) mediante la utilización de matrices de dimensión infinita, mientras que en [27] se utilizan las matrices de dimensión infinita para generalizar el test PBH al estudio de la estabilización de estos sistemas.

\section{A.6 Diseño de predictores para sistemas no lineales}

En esta sección se presentan los resultados básicos que se han obtenido para poder desarrollar el capítulo 5 de diseño de predictores para sistemas no lineales partiendo del siguiente lema.

Lema A.6.1. [72] Para cualquier par de vectores $\boldsymbol{x}, \boldsymbol{y} \in \mathbb{R}^{n}$ y cualquier matriz $\boldsymbol{P} \in \mathbb{R}^{n \times n}$ definida positiva se tiene que

$$
2 \boldsymbol{x}^{\top} \boldsymbol{y} \leq \boldsymbol{x}^{\top} \boldsymbol{P} \boldsymbol{x}+\boldsymbol{y}^{\top} \boldsymbol{P}^{-1} \boldsymbol{y} .
$$

Tomando este lema como base de partida, se han obtenido las herramientas matemáticas que se detallan en los teoremas siguientes y que permiten realizar de forma sencilla el análisis de la estabilidad y de la atenuación de perturbaciones de sistemas no lineales. Estas herramientas son imprescindibles en el diseño de predictores para sistemas no lineales.

\section{A.6.1 Estabilidad nominal}

El siguiente teorema muestra una herramienta imprescindible en el análisis de estabilidad de sistemas no lineales.

Teorema A.6.1. Sean $\boldsymbol{x}$ un vector y $\boldsymbol{A}, \boldsymbol{B}, \boldsymbol{P}$ matrices de dimensiones apropiadas, tal que $\boldsymbol{P}$ es una matriz simétrica definida positiva. Sea $\boldsymbol{y}$ un vector que satisface

$$
\|\boldsymbol{y}\| \leq\|\boldsymbol{F} \boldsymbol{x}\|
$$

siendo $\boldsymbol{F}$ una matriz de dimensiones apropiadas. Entonces, para cualquier $\varepsilon>0$

$$
(\boldsymbol{A} \boldsymbol{x}+\boldsymbol{B} \boldsymbol{y})^{\top} \boldsymbol{P}(\boldsymbol{A} \boldsymbol{x}+\boldsymbol{B} \boldsymbol{y}) \leq \boldsymbol{x}^{\top} \boldsymbol{W} \boldsymbol{x}
$$

con

$$
\boldsymbol{W}=\boldsymbol{A}^{\top}\left(\boldsymbol{P}+\boldsymbol{P} \boldsymbol{B}\left(\varepsilon \boldsymbol{I}-\boldsymbol{B}^{\top} \boldsymbol{P} \boldsymbol{B}\right)^{-1} \boldsymbol{B}^{\top} \boldsymbol{P}\right) \boldsymbol{A}+\varepsilon \boldsymbol{F}^{\top} \boldsymbol{F}
$$


Prueba A.6.1. Desarrollando la expresión de la izquierda en (A.33) se tiene que

$$
(\boldsymbol{A x}+\boldsymbol{B} \boldsymbol{y})^{\top} \boldsymbol{P}(\boldsymbol{A} \boldsymbol{x}+\boldsymbol{B} \boldsymbol{y})=\boldsymbol{x}^{\top} \boldsymbol{A}^{\top} \boldsymbol{P} \boldsymbol{A} \boldsymbol{x}+2 \boldsymbol{x}^{\top} \boldsymbol{A}^{\top} \boldsymbol{P} \boldsymbol{B} \boldsymbol{y}+\boldsymbol{y}^{\top} \boldsymbol{B}^{\top} \boldsymbol{P} \boldsymbol{B} \boldsymbol{y} .
$$

Sumando y restando $\varepsilon \boldsymbol{y}^{\top} \boldsymbol{y}$ en la parte derecha de la igualdad se tiene que

$$
(\boldsymbol{A} \boldsymbol{x}+\boldsymbol{B} \boldsymbol{y})^{\top} \boldsymbol{P}(\boldsymbol{A} \boldsymbol{x}+\boldsymbol{B} \boldsymbol{y})=\boldsymbol{x}^{\top} \boldsymbol{A}^{\top} \boldsymbol{P} \boldsymbol{A} \boldsymbol{x}+2 \boldsymbol{x}^{\top} \boldsymbol{A}^{\top} \boldsymbol{P} \boldsymbol{B} \boldsymbol{y}-\boldsymbol{y}^{\top}\left(\varepsilon \boldsymbol{I}-\boldsymbol{B}^{\top} \boldsymbol{P} \boldsymbol{B}\right) \boldsymbol{y}+\varepsilon \boldsymbol{y}^{\top} \boldsymbol{y}
$$

Aplicando el lema A.6.1 se tiene que

$$
(\boldsymbol{A x}+\boldsymbol{B} \boldsymbol{y})^{\top} \boldsymbol{P}(\boldsymbol{A} \boldsymbol{x}+\boldsymbol{B} \boldsymbol{y}) \leq \boldsymbol{x}^{\top} \boldsymbol{A}^{\top} \boldsymbol{P} \boldsymbol{A} \boldsymbol{x}+\boldsymbol{x}^{\top} \boldsymbol{A}^{\top} \boldsymbol{P} \boldsymbol{B}\left(\varepsilon \boldsymbol{I}-\boldsymbol{B}^{\top} \boldsymbol{P} \boldsymbol{B}\right)^{-1} \boldsymbol{B}^{\top} \boldsymbol{P} \boldsymbol{A} \boldsymbol{x}+\varepsilon \boldsymbol{y}^{\top} \boldsymbol{y}
$$

Teniendo en cuenta (A.32) se llega a

$$
(\boldsymbol{A x}+\boldsymbol{B} \boldsymbol{y})^{\top} \boldsymbol{P}(\boldsymbol{A} \boldsymbol{x}+\boldsymbol{B} \boldsymbol{y}) \leq \boldsymbol{x}^{\top}\left(\boldsymbol{A}^{\top} \boldsymbol{P} \boldsymbol{A}+\boldsymbol{A}^{\top} \boldsymbol{P} \boldsymbol{B}\left(\varepsilon \boldsymbol{I}-\boldsymbol{B}^{\top} \boldsymbol{P} \boldsymbol{B}\right)^{-1} \boldsymbol{B}^{\top} \boldsymbol{P} \boldsymbol{A}+\varepsilon \boldsymbol{F}^{\top} \boldsymbol{F}\right) .
$$

El resultado anterior se puede utilizar para analizar la estabilidad de un sistema no lineal definido por la ecuación

$$
\boldsymbol{x}_{k+1}=\boldsymbol{A} \boldsymbol{x}_{k}+\boldsymbol{f}\left(\boldsymbol{x}_{k}\right)
$$

en el que la función $\boldsymbol{f}\left(\boldsymbol{x}_{k}\right)$ cumple las siguientes condiciones

$$
\begin{aligned}
& \boldsymbol{f}(\mathbf{0})=\mathbf{0}, \\
& \left\|\boldsymbol{f}\left(\boldsymbol{x}_{k}\right)\right\| \leq\left\|\boldsymbol{F} \boldsymbol{x}_{k}\right\| .
\end{aligned}
$$

Teorema A.6.2. El sistema A.35 con las condiciones (A.36) es estable si existe un escalar $\varepsilon>0$ y una matriz $\boldsymbol{P}=\boldsymbol{P}^{\top} \succ \mathbf{0}$ tal que

$$
\left[\begin{array}{cc}
\boldsymbol{A}^{\top} \boldsymbol{P A}-\boldsymbol{P}+\varepsilon \boldsymbol{F}^{\top} \boldsymbol{F} & \boldsymbol{A}^{\top} \boldsymbol{P} \\
\boldsymbol{P A} & \boldsymbol{P}-\varepsilon I
\end{array}\right] \prec \mathbf{0} .
$$

Prueba A.6.2. Tómese la función de Lyapunov

$$
\mathcal{V}_{k}=\boldsymbol{x}_{k}^{\top} \boldsymbol{P} \boldsymbol{x}_{k}
$$

Entonces, el sistema (A.35) será estable si $\mathcal{V}_{k+1}-\mathcal{V}_{k}<0$. El incremento de la función de Lyapunov se puede escribir como

$$
\Delta \mathcal{V}_{k}=\left(\boldsymbol{A} \boldsymbol{x}_{k}+\boldsymbol{f}\left(\boldsymbol{x}_{k}\right)\right)^{\top} \boldsymbol{P}\left(\boldsymbol{A} \boldsymbol{x}_{k}+\boldsymbol{f}\left(\boldsymbol{x}_{k}\right)\right)-\boldsymbol{x}_{k}^{\top} \boldsymbol{P} \boldsymbol{x}_{k} .
$$

Aplicando el teorema A.6.1 al primer sumando (con $\boldsymbol{y} \equiv \boldsymbol{f}\left(\boldsymbol{x}_{k}\right)$ y $\left.\boldsymbol{B} \equiv \boldsymbol{I}\right)$ se tiene que

$$
\Delta \mathcal{V}_{k}=\boldsymbol{x}_{k}^{\top}\left(\boldsymbol{A}^{\top} \boldsymbol{P} \boldsymbol{A}+\boldsymbol{A}^{\top} \boldsymbol{P}(\varepsilon \boldsymbol{I}-\boldsymbol{P})^{-1} \boldsymbol{P} \boldsymbol{A}+\boldsymbol{\varepsilon} \boldsymbol{F}^{\top} \boldsymbol{F}-\boldsymbol{P}\right) \boldsymbol{x}_{k} .
$$

La función de Lyapunov decrece si

$$
\boldsymbol{A}^{\top} \boldsymbol{P} \boldsymbol{A}+\boldsymbol{A}^{\top} \boldsymbol{P}(\varepsilon \boldsymbol{I}-\boldsymbol{P})^{-1} \boldsymbol{P} \boldsymbol{A}+\varepsilon \boldsymbol{F}^{\top} \boldsymbol{F}-\boldsymbol{P} \prec \mathbf{0} .
$$

Aplicando complementos de Schur, esta última condición se puede escribir como

$$
\left[\begin{array}{cc}
\boldsymbol{A}^{\top} \boldsymbol{P A}-\boldsymbol{P}+\varepsilon \boldsymbol{F}^{\top} \boldsymbol{F} & \boldsymbol{A}^{\top} \boldsymbol{P B} \\
\boldsymbol{B}^{\top} \boldsymbol{P} \boldsymbol{A} & \boldsymbol{P}-\varepsilon I
\end{array}\right]
$$




\section{A.6.2 Atenuación de perturbaciones}

El siguiente teorema muestra una herramienta imprescindible en el análisis de atenuación de perturbaciones de sistemas no lineales.

Teorema A.6.3. Sean $\boldsymbol{x}$ y $\boldsymbol{u}$ vectores y $\boldsymbol{B}, \boldsymbol{P}$ matrices de dimensiones apropiadas, tal que $\boldsymbol{P}$ es una matriz simétrica definida positiva. Entonces, para cualquier $\boldsymbol{\Gamma} \succ \mathbf{0}$

$$
(\boldsymbol{x}+\boldsymbol{B} \boldsymbol{u})^{\top} \boldsymbol{P}(\boldsymbol{x}+\boldsymbol{B} \boldsymbol{u})-\boldsymbol{u}^{\top} \boldsymbol{\Gamma}^{2} \boldsymbol{u} \leq \boldsymbol{x}^{\top} \boldsymbol{W} \boldsymbol{x}
$$

con

$$
\boldsymbol{W}=\boldsymbol{P}+\boldsymbol{P} \boldsymbol{B}\left(\boldsymbol{\Gamma}^{2}-\boldsymbol{B}^{\top} \boldsymbol{P} \boldsymbol{B}\right)^{-1} \boldsymbol{B}^{\top} \boldsymbol{P}
$$

Prueba A.6.3. Desarrollando la expresión de la izquierda en (A.38) se tiene que

$$
(\boldsymbol{x}+\boldsymbol{B} \boldsymbol{u})^{\top} \boldsymbol{P}(\boldsymbol{x}+\boldsymbol{B} \boldsymbol{u})-\boldsymbol{u}^{\top} \boldsymbol{\Gamma}^{2} \boldsymbol{u}=\boldsymbol{x}^{\top} \boldsymbol{P} \boldsymbol{x}+2 \boldsymbol{x}^{\top} \boldsymbol{P} \boldsymbol{B} \boldsymbol{u}-\boldsymbol{u}^{\top}\left(\boldsymbol{\Gamma}^{2}-\boldsymbol{B}^{\top} \boldsymbol{P} \boldsymbol{B}\right) \boldsymbol{u} .
$$

Aplicando el lema A.6.1 se tiene que

$$
(\boldsymbol{x}+\boldsymbol{B} \boldsymbol{u})^{\top} \boldsymbol{P}(\boldsymbol{x}+\boldsymbol{B} \boldsymbol{u})-\boldsymbol{u}^{\top} \boldsymbol{\Gamma}^{2} \boldsymbol{u} \leq \boldsymbol{x}^{\top}\left(\boldsymbol{P}+\boldsymbol{P} \boldsymbol{B}\left(\boldsymbol{\Gamma}^{2}-\boldsymbol{B}^{\top} \boldsymbol{P} \boldsymbol{B}\right)^{-1} \boldsymbol{B}^{\top} \boldsymbol{P}\right) \boldsymbol{x} .
$$

Los dos teoremas anteriores se pueden utilizar para analizar el efecto de las perturbaciones en un sistema no lineal definido por las ecuaciones

$$
\begin{aligned}
\boldsymbol{x}_{k+1} & =\boldsymbol{A} \boldsymbol{x}_{k}+\boldsymbol{f}\left(\boldsymbol{x}_{k}\right)+\boldsymbol{w} \\
\boldsymbol{y}_{k} & =\boldsymbol{g}\left(\boldsymbol{x}_{k}\right)
\end{aligned}
$$

donde $\boldsymbol{f}\left(\boldsymbol{x}_{k}\right)$ y $\boldsymbol{g}\left(\boldsymbol{x}_{k}\right)$ cumplen las siguientes condiciones

$$
\begin{aligned}
& \boldsymbol{f}(\mathbf{0})=\mathbf{0}, \quad\left\|\boldsymbol{f}\left(\boldsymbol{x}_{k}\right)\right\| \leq\left\|\boldsymbol{F} \boldsymbol{x}_{k}\right\|, \\
& \boldsymbol{g}(\mathbf{0})=\mathbf{0}, \quad\left\|\boldsymbol{g}\left(\boldsymbol{x}_{k}\right)\right\| \leq\left\|\boldsymbol{G} \boldsymbol{x}_{k}\right\| .
\end{aligned}
$$

A modo de ejemplo, se muestra cómo calcular la norma $\mathcal{H}_{\infty}$ con ayuda de los teoremas A.6.1 y A.6.3.

Teorema A.6.4. La norma $\mathcal{H}_{\infty}$ del sistema (A.40) $\left(\gamma^{*}\right)$ viene dada por

$$
\gamma^{*}=\min _{P} \gamma
$$

sujeto a

$$
\left[\begin{array}{ccc}
\boldsymbol{A}^{\top} \boldsymbol{P} \boldsymbol{A}-\boldsymbol{P}+\varepsilon \boldsymbol{F}^{\top} \boldsymbol{F}+\boldsymbol{G}^{\top} \boldsymbol{G} & \boldsymbol{A}^{\top} \boldsymbol{P} & \boldsymbol{A}^{\top} \boldsymbol{P} \\
\boldsymbol{P A} & \boldsymbol{P}-\varepsilon \boldsymbol{I} & \boldsymbol{P} \\
\boldsymbol{P A} & \boldsymbol{P} & \boldsymbol{P}-\gamma^{2} \boldsymbol{I}
\end{array}\right] \prec \mathbf{0}
$$

Prueba A.6.4. La estabilidad del sistema está probada puesto que la matriz XX implica XX (ya que $\left.\boldsymbol{G}^{\top} \boldsymbol{G} \succ \mathbf{0}\right)$. Ahora, para obtener la norma $\mathcal{H}_{\infty}$ asúmanse condiciones iniciales nulas y tómese el siguiente índice

$$
J=\sum_{k=0}^{\infty}\left(\boldsymbol{y}_{k}^{\top} \boldsymbol{y}_{k}-\gamma^{2} \boldsymbol{w}^{\top} \boldsymbol{w}\right)
$$

Bajo condiciones iniciales nulas se tiene que

$$
J \leq \sum_{k=0}^{\infty}\left(\boldsymbol{y}_{k}^{\top} \boldsymbol{y}_{k}-\gamma^{2} \boldsymbol{w}^{\top} \boldsymbol{w}\right)+\left.\mathcal{V}_{k}\right|_{k \rightarrow \infty}-\left.\mathcal{V}_{k}\right|_{k=0}=\sum_{k=0}^{\infty}\left(\boldsymbol{y}_{k}^{\top} \boldsymbol{y}_{k}-\gamma^{2} \boldsymbol{w}^{\top} \boldsymbol{w}+\Delta \mathcal{V}_{k}\right)
$$

Desarrollando las funciones de Lyapunov se tiene que

$$
J \leq \sum_{k=0}^{\infty}\left(\boldsymbol{y}_{k}^{\top} \boldsymbol{y}_{k}-\gamma^{2} \boldsymbol{w}^{\top} \boldsymbol{w}\left(\boldsymbol{A} \boldsymbol{x}_{k}+\boldsymbol{f}\left(x_{k}\right)+\boldsymbol{w}_{k}\right)^{\top} \boldsymbol{P}\left(\boldsymbol{A} \boldsymbol{x}_{k}+\boldsymbol{f}\left(x_{k}\right)+\boldsymbol{w}_{k}\right)-\boldsymbol{x}_{k}^{\top} \boldsymbol{P} \boldsymbol{x}_{k}\right)
$$


donde se puede aplicar el teorema A.6.3 para obtener

$$
J \leq \sum_{k=0}^{\infty}\left(\boldsymbol{y}_{k}^{\top} \boldsymbol{y}_{k}\left(\boldsymbol{A} \boldsymbol{x}_{k}+\boldsymbol{f}\left(x_{k}\right)\right)^{\top} \boldsymbol{P}_{\gamma}\left(\boldsymbol{A} \boldsymbol{x}_{k}+\boldsymbol{f}\left(x_{k}\right)\right)-\boldsymbol{x}_{k}^{\top} \boldsymbol{P} \boldsymbol{x}_{k}\right),
$$

con

$$
\boldsymbol{P}_{\gamma}=\left(\boldsymbol{P}+\boldsymbol{P}\left(\gamma^{2} \boldsymbol{I}-\boldsymbol{P}\right)^{-1} \boldsymbol{P}\right) .
$$

Aplicando el teorema A.6.1 al resultado anterior se tiene que

$$
J \leq \sum_{k=0}^{\infty}\left(\boldsymbol{y}_{k}^{\top} \boldsymbol{y}_{k}+\boldsymbol{x}_{k}^{\top} \boldsymbol{P}_{F} \boldsymbol{x}_{k}\right)
$$

con

$$
\boldsymbol{P}_{F}=\boldsymbol{A}^{\boldsymbol{\top}}\left(\boldsymbol{P}_{\gamma}+\boldsymbol{P}_{\gamma}\left(\varepsilon \boldsymbol{I}-\boldsymbol{P}_{\gamma}\right)^{-1} \boldsymbol{P}_{\gamma}\right) \boldsymbol{A}+\boldsymbol{\varepsilon} \boldsymbol{F}^{\boldsymbol{\top}} \boldsymbol{F}-\boldsymbol{P} .
$$

Teniendo en cuenta que $\boldsymbol{y}_{k}^{\top} \boldsymbol{y}_{k}<\boldsymbol{x}_{k}^{\top} \boldsymbol{G}^{\top} \boldsymbol{G} \boldsymbol{x}_{k}$ se llega a

$$
J \leq \sum_{k=0}^{\infty} \boldsymbol{x}_{k}^{\top}\left(\boldsymbol{P}_{F}+\boldsymbol{G}^{\top} \boldsymbol{G}\right) \boldsymbol{x}_{k}
$$

La condición $\left\|y_{k}\right\|_{2}<\gamma^{2}\left\|\boldsymbol{w}_{k}\right\|_{2}$ se cumplirá si $J<0$, condición que será cierta siempre que

$$
\boldsymbol{P}_{F}+\boldsymbol{G}^{\top} \boldsymbol{G} \prec \mathbf{0} .
$$

Sustituyendo $\boldsymbol{P}_{F}$ por su valor y aplicando complementos de Schur se tiene que

$$
\left[\begin{array}{cc}
\boldsymbol{A}^{\top} \boldsymbol{P}_{\gamma} \boldsymbol{A}+\varepsilon \boldsymbol{F}^{\top} \boldsymbol{F}-\boldsymbol{P}+\boldsymbol{G}^{\top} \boldsymbol{G} & \boldsymbol{A}^{\top} \boldsymbol{P}_{\gamma} \\
\boldsymbol{P}_{\gamma} \boldsymbol{A} & \boldsymbol{P}_{\gamma}-\varepsilon \boldsymbol{I}
\end{array}\right] \prec \mathbf{0}
$$

Sustituyendo $\boldsymbol{P}_{\gamma}$ por su valor y aplicando complementos de Schur se tiene finalmente (A.42). 
Apéndice B

\section{Resultados auxiliares en el diseño de predictores}

\section{B.1 Diseño estocástico de predictores monovariables}

\section{B.1.1 Demostración del teorema 3.5.2 (diseño $\mathcal{H}_{\infty}$ )}

Aplicando la desigualdad (3.79) en (3.80), y aplicando complementos de Schur la PLMI (3.80) implica

$$
\left[\begin{array}{cc}
\left(\begin{array}{c}
\sum_{j=1}^{n_{\mathcal{S}}} \alpha_{i j} \mathcal{A}(j)^{\top} \boldsymbol{P}(j) \mathcal{A}(j) \\
-\boldsymbol{P}(i)+\boldsymbol{c}^{\top} \boldsymbol{c}
\end{array}\right) & \sum_{j=1}^{n_{\mathcal{S}}} \alpha_{i j} \mathcal{A}(j)^{\top} \boldsymbol{P}(j) \mathcal{B}(j) \\
\sum_{j=1}^{n_{\mathcal{S}}} \alpha_{i j} \boldsymbol{B}(j)^{\top} \boldsymbol{P}(j) \mathcal{A}(j) & \sum_{j=1}^{n_{\mathcal{S}}} \alpha_{i j} \boldsymbol{B}(j)^{\top} \boldsymbol{P}(j) \mathcal{B}(j)-\boldsymbol{\Gamma}^{2}
\end{array}\right] \prec 0
$$

Multiplicando esta expresión por $\left[E_{k-1}^{\top} \mathbb{V}_{k}^{\top} w_{k}^{\top}\right]$ por la izquierda y por su traspuesta por la derecha se tiene que

$$
\begin{aligned}
& \sum_{j=1}^{n_{\mathcal{S}}} \alpha_{i j}\left(\boldsymbol{\mathcal { A }}(j) E_{k-1}+\boldsymbol{B}(j)\left[\begin{array}{c}
\mathbb{V}_{k} \\
w_{k}
\end{array}\right]\right)^{\top} \boldsymbol{P}(j)\left(\mathcal{A}(j) E_{k-1}+\mathcal{B}(j)\left[\begin{array}{c}
\mathbb{V}_{k} \\
w_{k}
\end{array}\right]\right) \\
& -E_{k-1}^{\top} \boldsymbol{P}(i) E_{k-1}+e_{k-1}^{\top} e_{k-1}-\frac{\gamma_{v}^{2}}{3 n} \mathbb{V}_{k}^{\top} \mathbb{V}_{k}-\gamma_{w}^{2} w_{k}^{\top} w_{k}<0
\end{aligned}
$$

Definiendo la función de Lyapunov como

$$
\mathcal{V}\left(E_{k}, s_{k}\right)=E_{k}^{\top} \boldsymbol{P}\left(s_{k}\right) E_{k}
$$

y teniendo en cuenta la siguiente esperanza matemática

$$
\mathcal{E}\left\{\mathcal{V}\left(E_{k}, s_{k}\right) \mid s_{k-1}=i\right\}=\sum_{j=1}^{n_{\mathcal{S}}} \alpha_{i j}(\star)^{\top} \boldsymbol{P}(j) \underbrace{\left(\mathcal{A}(j) E_{k-1}+\mathcal{B}(j)\left[\begin{array}{c}
\mathbb{V}_{k} \\
w_{k}
\end{array}\right]\right)}_{\star},
$$

la ecuación (B.1) puede expresarse como

$$
\begin{aligned}
\mathcal{E}\{ & \left.\mathcal{V}\left(E_{k}, s_{k}\right) \mid k-1\right\}-E_{k-1}^{\top} \boldsymbol{P}\left(s_{k-1}\right) E_{k-1} \\
& +e_{k-1}^{\top} e_{k-1}-\frac{\gamma_{v}^{2}}{3 n} \mathbb{V}_{k}^{\top} \mathbb{V}_{k}-\gamma_{w}^{2} w_{k}^{\top} w_{k}<0 .
\end{aligned}
$$

Tomando esperanzas matemáticas condicionadas al instante $k-2$ se tiene que

$$
\begin{aligned}
\mathcal{E}\{\mathcal{E} & \left.\left\{\mathcal{V}\left(E_{k}, s_{k}\right) \mid k-1\right\} \mid k-2\right\}-\mathcal{E}\left\{\mathcal{V}\left(E_{k-1}, s_{k-1}\right) \mid k-2\right\} \\
& +\mathcal{E}\left\{e_{k-1}^{\top} e_{k-1}-\frac{\gamma_{v}^{2}}{3 n} \mathbb{V}_{k}^{\top} \mathbb{V}_{k}-\gamma_{w}^{2} w_{k}^{\top} w_{k} \mid k-2\right\}<0
\end{aligned}
$$


Teniendo en cuenta que la dinámica del error de predicción sólo depende del instante anterior,

$$
\mathcal{E}\left\{\mathcal{E}\left\{\mathcal{V}\left(E_{k}, s_{k}\right) \mid k-1\right\} \mid k-2\right\}=\mathcal{E}\left\{\mathcal{V}\left(E_{k}, s_{k}\right) \mid k-1\right\}
$$

y teniendo en cuenta que $v_{k} \mathrm{y} w_{k}$ son señales deterministas, se tiene que

$$
\begin{aligned}
\mathcal{E} & \left\{\mathcal{V}\left(E_{k}, s_{k}\right) \mid k-1\right\}-\mathcal{E}\left\{\mathcal{V}\left(E_{k-1}, s_{k-1}\right) \mid k-2\right\} \\
& +\mathcal{E}\left\{e_{k-1}^{\top} e_{k-1} \mid k-2\right\}-\frac{\gamma_{v}^{2}}{3 n} \mathbb{V}_{k}^{\top} \mathbb{V}_{k}-\gamma_{w}^{2} w_{k}^{\top} w_{k}<0
\end{aligned}
$$

Asumiendo un error de predicción inicial nulo, sumando la expresión anterior de $k=1$ a $k=K$, se tiene que

$$
\mathcal{E}\left\{\mathcal{V}\left(E_{K}, s_{K}\right) \mid K-1\right\}+\sum_{k=1}^{K}\left(\mathcal{E}\left\{e_{k-1}^{\top} e_{k-1} \mid k-2\right\}-\frac{\gamma_{v}^{2}}{3 n} \mathbb{V}_{k}^{\top} \mathbb{V}_{k}^{\top} \mathbb{V}_{k}-\gamma_{w}^{2} w_{k}^{\top}\right) .
$$

Como $\mathcal{E}\left\{\mathcal{V}\left(E_{K}, s_{K}\right) \mid s_{K-1}=i\right\}>0$ se tiene que

$$
\mathcal{E}\left\{\sum_{k=1}^{K} e_{k-1}^{\top} e_{k-1} \mid k-2\right\}<\sum_{k=1}^{K}\left(\frac{\gamma_{v}^{2}}{3 n} \mathbb{V}_{k}^{\top} \mathbb{V}_{k}+\gamma_{w}^{2} w_{k}^{\top} w_{k}\right)
$$

que implica (3.81) cuando se hace tender $K$ a infinito.

\section{B.1.2 Demostración del teorema 3.5 .3 (diseño $\mathcal{H}_{2}$ )}

Siguiendo el mismo razonamiento que en el teorema anterior, la LMI (3.82a) implica la convergencia a cero en valor medio del predictor.

Tómese ahora la función de Lyapunov $\mathcal{V}_{k}=E_{k}^{\top} \boldsymbol{P}\left(s_{k}\right) E_{k}$. El valor esperado de la función de Lyapunov condicionado a un instante anterior es

$$
\begin{aligned}
\mathcal{E}\left\{\mathcal{V}_{k} \mid k-1\right\} & =\mathcal{E}\left\{E_{k}^{\top} \boldsymbol{P} E_{k}\right\}=\mathcal{E}\left\{\left(\mathcal{A}\left(s_{k}\right) E_{k-1}+\mathcal{B}\left(s_{k}\right)\left[\begin{array}{c}
\mathbb{V}_{k} \\
w_{k}
\end{array}\right]\right)^{\top} \boldsymbol{P}\left(\mathcal{A}\left(s_{k}\right) E_{k-1}+\mathcal{B}\left(s_{k}\right)\left[\begin{array}{c}
\mathbb{V}_{k} \\
w_{k}
\end{array}\right]\right) \mid k-1\right\} \\
& =\mathcal{E}\left\{E_{k-1}^{\top} \mathcal{A}\left(s_{k}\right)^{\top} \boldsymbol{P}\left(s_{k}\right) \mathcal{A}\left(s_{k}\right) E_{k-1} \mid k-1\right\}+\mathcal{E}\left\{\left[\begin{array}{c}
\mathbb{V}_{k} \\
w_{k}
\end{array}\right]^{\top} \boldsymbol{B}\left(s_{k}\right)^{\top} \boldsymbol{P}\left(s_{k}\right) \mathcal{B}\left(s_{k}\right)\left[\begin{array}{c}
\mathbb{V}_{k} \\
w_{k}
\end{array}\right] \mid k-1\right\}
\end{aligned}
$$

donde se ha tenido en cuenta que el producto cruzado

$$
\mathcal{E}\left\{E_{k-1}^{\top} \mathcal{A}\left(s_{k}\right)^{\top} \boldsymbol{P}\left(s_{k}\right) \mathcal{B}\left(s_{k}\right)\left[\begin{array}{c}
\mathbb{V}_{k} \\
w_{k}
\end{array}\right]\right\}=\mathcal{E}\left\{E_{k-1}^{\top} \mathcal{A}\left(s_{k}\right)^{\top} \boldsymbol{P}\left(s_{k}\right) \mathcal{B}\left(s_{k}\right)\right\} \mathcal{E}\left\{\left[\begin{array}{c}
\mathbb{V}_{k} \\
w_{k}
\end{array}\right] \mid k-1\right\}=0
$$

es nulo debido a la independencia de $E_{k-1}$ con $\mathbb{V}_{k}$ y $w_{k}$, y que el valor esperado de las perturbaciones es cero. Para un $s_{k-1}=i$ dado, las esperanzas matemáticas de (B.6) se pueden calcular con ayuda de las probabilidades de transmisión, quedando

$$
\begin{aligned}
\mathcal{E}\left\{\mathcal{V}_{k} \mid k-1\right\}= & E_{k-1}^{\top}\left(\sum_{j=1}^{n_{\mathcal{S}}} \alpha_{i j} \mathcal{A}(j)^{\top} \boldsymbol{P}(j) \mathcal{A}(j)\right) E_{k-1} \\
& +\mathcal{E}\left\{\left[\begin{array}{c}
\mathbb{V}_{k} \\
w_{k}
\end{array}\right]^{\top}\left(\sum_{j=1}^{n_{\mathcal{S}}} \alpha_{i j} \mathcal{B}(j)^{\top} \boldsymbol{P}(j) \mathcal{B}(j)\right)\left[\begin{array}{c}
\mathbb{V}_{k} \\
w_{k}
\end{array}\right]\right\}
\end{aligned}
$$

Utilizando la condición (3.79) en la LMI (3.82a) y aplicando los complementos de Schur se tiene que

$$
\sum_{j=1}^{n_{\mathcal{S}}} \alpha_{i j} \mathcal{A}(j)^{\top} \boldsymbol{P}(j) \mathcal{A}(j)-\boldsymbol{P}(i)+\boldsymbol{c}^{\top} \boldsymbol{c}
$$

Premultiplicando y postmultiplicando esta expresión por $E_{k-1}^{\top}$ y $E_{k-1}$ se tiene que

$$
E_{k-1}^{\boldsymbol{\top}}\left(\sum_{j=1}^{n_{\mathcal{S}}} \alpha_{i j} \mathcal{A}(j)^{\top} \boldsymbol{P}(j) \mathcal{A}(j)\right) E_{k-1}-E_{k-1}^{\top} \boldsymbol{P}(i) E_{k-1}+e_{k-1}^{\top} e_{k-1}<0 .
$$


Si se calcula la esperanza matemática de esta desigualdad con respecto a un instante anterior $k-2$ y se aplica el resultado (B.7) se tiene que

$$
\mathcal{E}\left\{\mathcal{V}_{k} \mid k-1\right\}-\mathcal{E}\left\{\mathcal{V}_{k-1} \mid k-2\right\}+\mathcal{E}\left\{e_{k-1}^{\top} e_{k-1} \mid k-2\right\}<\mathcal{E}\left\{\left[\begin{array}{c}
\mathbb{V}_{k} \\
w_{k}
\end{array}\right]^{\top}\left(\sum_{j=1}^{n_{\mathcal{S}}} \alpha_{i j} \boldsymbol{B}(j)^{\top} \boldsymbol{P}(j) \boldsymbol{B}(j)\right)\left[\begin{array}{c}
\mathbb{V}_{k} \\
w_{k}
\end{array}\right]\right\}
$$

Utilizando la condición (3.79) en la LMI (3.82b) y aplicando los complementos de Schur se tiene que

$$
\sum_{j=1}^{n_{\mathcal{S}}} \alpha_{i j} \mathcal{B}(j)^{\top} \boldsymbol{P}(j) \mathcal{B}(j)-\Gamma^{2} \prec \mathbf{0} .
$$

Introduciendo este resultado en (B.8) y aplicando el teorema A.3.3 se llega a

$$
\mathcal{E}\left\{\mathcal{V}_{k} \mid k-1\right\}-\mathcal{E}\left\{\mathcal{V}_{k-1} \mid k-2\right\}+\mathcal{E}\left\{e_{k-1}^{\top} e_{k-1} \mid k-2\right\}<\mathcal{E}\left\{\left[\begin{array}{c}
\mathbb{V}_{k} \\
w_{k}
\end{array}\right]^{\top} \Gamma^{2}\left[\begin{array}{c}
\mathbb{V}_{k} \\
w_{k}
\end{array}\right]\right\}=\frac{\gamma_{v}^{2}}{3 n} 3 n \sigma_{v}^{2}+\gamma_{w}^{2} \sigma_{w}^{2}
$$

Sumando esta expresión de $k=1$ a $K$ y asumiendo condiciones iniciales nulas se obtiene

$$
\mathcal{E}\left\{\mathcal{V}_{K} \mid K-1\right\}+\sum_{k=1}^{K} \mathcal{E}\left\{e_{k-1}^{\top} e_{k-1} \mid k-2\right\}<K\left(\gamma_{v}^{2} \sigma_{v}^{2}+\gamma_{w}^{2} \sigma_{w}^{2}\right)
$$

Como $\boldsymbol{P}\left(s_{K}\right) \succ \mathbf{0}$, entonces $\mathcal{E}\left\{\mathcal{V}_{K} \mid K-1\right\}>0$. Tomando el límite cuando $K$ tiende a infinito se llega finalmente a

$$
\lim _{K \rightarrow \infty} \frac{1}{K} \sum_{k=1}^{K} \mathcal{E}\left\{e_{k-1}^{\top} e_{k-1} \mid k-2\right\}=\mathcal{E}\left\{\left\|e_{k}\right\|_{R M S}^{2}\right\}<\gamma_{v}^{2} \sigma_{v}^{2}+\gamma_{w}^{2} \sigma_{w}^{2} .
$$

\section{B.1.3 Demostración del teorema 3.5.4 (diseño $\mathcal{H}_{2 g}$ )}

La estabilidad asintótica del predictor queda asegurada ya que si la PLMI (3.84a) se cumple también lo hará la PLMI (3.74). Utilizando la condición (3.79) en la LMI (3.84a) y aplicando los complementos de Schur, se tiene que

$$
\mathcal{E}\left\{E_{k}^{\top} \boldsymbol{P}\left(s_{k}\right) E_{k} \mid k-1\right\}-E_{k-1}^{\top} \boldsymbol{P}\left(s_{k-1}\right) E_{k-1}<\frac{\gamma_{v}^{2}}{3 n} \mathbb{V}_{k}^{\top} \mathbb{V}_{k}^{\top}+\gamma_{w}^{2} w_{k}^{\top} w_{k}^{\top}
$$

Tomando esperanzas matemáticas condicionadas a $s_{k-2}$ en la expresión anterior se tiene que

$$
\mathcal{E}\left\{E_{k}^{\top} \boldsymbol{P}\left(s_{k}\right) E_{k} \mid k-1\right\}-\mathcal{E}\left\{E_{k-1}^{\top} \boldsymbol{P}\left(s_{k-1}\right) E_{k-1} \mid k-2\right\}<\frac{\gamma_{v}^{2}}{3 n} \mathbb{V}_{k}^{\top} \mathbb{V}_{k}^{\top}+\gamma_{w}^{2} w_{k}^{\top} w_{k}^{\top}
$$

Considerando condiciones nulas y tomando sumatorio de 1 a $K$ en ambos lados de la desigualdad se llega a

$$
\mathcal{E}\left\{E_{K}^{\top} \boldsymbol{P}\left(s_{K}\right) E_{K} \mid K-1\right\}<\sum_{k=1}^{K}\left(\frac{\gamma_{v}^{2}}{3 n} \mathbb{V}_{k}^{\top} \mathbb{V}_{k}^{\top}+\gamma_{w}^{2} w_{k}^{\top} w_{k}\right) .
$$

Aplicando ahora la condición (3.79) y los complementos de Schur en (3.84b), para posteriormente multiplicar por la izquierda por $E_{k}^{\top}$ y por la derecha por $E_{k}$ se tiene que

$$
E_{K}^{\top} \boldsymbol{P}\left(s_{K}\right) E_{K}>E_{K}^{\top} \boldsymbol{c}^{\top} \boldsymbol{c} E_{K}=e_{K}^{\top} e_{K} .
$$

Tomando esperanzas matemáticas con respecto a $s_{K-1}$ se tiene que

$$
\mathcal{E}\left\{E_{K}^{\top} \boldsymbol{P}\left(s_{K}\right) E_{K} \mid K-1\right\}>E_{K}^{\top} \boldsymbol{c}^{\top} \boldsymbol{c} E_{K}=\mathcal{E}\left\{e_{K}^{\top} e_{K} \mid K-1\right\} .
$$

Con esto, la desigualdad (B.10) lleva a

$$
\mathcal{E}\left\{e_{K}^{\top} e_{K} \mid K-1\right\}<\sum_{k=1}^{K}\left(\frac{\gamma_{v}^{2}}{3 n} \mathbb{V}_{k}^{\top} \mathbb{V}_{k}+\gamma_{w}^{2} w_{k}^{\top} w_{k}\right) .
$$

Tomando $K$ como el instante en el que el error se hace máximo, y teniendo en cuenta que

$$
\sum_{k=1}^{K}\left(\frac{\gamma_{v}^{2}}{3 n} \mathbb{V}_{k}^{\top} \mathbb{V}_{k}+\gamma_{w}^{2} w_{k}^{\top} w_{k}\right)<\lim _{K \rightarrow \infty} \sum_{k=1}^{K}\left(\frac{\gamma_{v}^{2}}{3 n} \mathbb{V}_{k}^{\top} \mathbb{V}_{k}+\gamma_{w}^{2} w_{k}^{\top} w_{k}\right)
$$

para cualquier $K$, se llega a la cota expresada en (3.65). 


\section{B.1.4 Demostración del teorema 3.5.5 (diseño $\ell_{1}$ )}

La estabilidad asintótica del predictor queda asegurada ya que si las PLMIs (3.86a) y (3.86b) se cumplen también lo hará la PLMI (3.74), con $\mu=1-\lambda$. Utilizando la condición (3.79) en la LMI (3.86b) y aplicando los complementos de Schur, se tiene que

$$
\mathcal{E}\left\{E_{k}^{\top} \boldsymbol{P}\left(s_{k}\right) E_{k} \mid k-1\right\}-E_{k-1} \boldsymbol{P}\left(s_{k-1}\right) E_{k-1}+\lambda E_{k-1} \boldsymbol{P}\left(s_{k-1}\right) E_{k-1}-\frac{\varepsilon_{v}^{2}}{3 n} \mathbb{V}_{k}^{\top} \mathbb{V}_{k}-\varepsilon_{w}^{2} w_{k}^{\top} w_{k}<0
$$

Definiendo las nuevas variables

$$
\mathcal{V}_{k}=\mathcal{E}\left\{E_{k}^{\top} \boldsymbol{P}\left(s_{k}\right) E_{k} \mid k-1\right\}
$$

y

$$
\omega_{k}=\frac{\varepsilon_{v}^{2}}{3 n} \mathbb{V}_{k}^{\top} \mathbb{V}_{k}+\varepsilon_{w}^{2} w_{k}^{\top} w_{k},
$$

se puede ver que la expresión (B.13) equivale a una inecuación en diferencias de la forma

$$
\mathcal{V}_{k}-(1-\lambda) \mathcal{V}_{k-1}<\omega_{k}
$$

Tomando la entrada de mayor amplitud posible definida como

$$
\left\|\omega_{k}\right\|_{\infty}=\varepsilon_{v}^{2}\left\|v_{k}\right\|_{\infty}+\varepsilon_{w}^{2}\left\|w_{k}\right\|_{\infty}
$$

se puede obtener el máximo valor esperado de $\mathcal{V}_{k}$ como el instante en el que $\mathcal{V}_{k}=\mathcal{V}_{k-1}$ con lo que se llega a

$$
\lambda \mathcal{V}_{k}<\omega_{k} \leq\left\|\omega_{k}\right\|_{\infty}
$$

Deshaciendo los cambios de variable se tiene que

$$
\mathcal{E}\left\{E_{k}^{\top} \boldsymbol{P}\left(s_{k}\right) E_{k} \mid k-1\right\}<\frac{1}{\lambda}\left(\varepsilon_{v}^{2}\left\|v_{k}\right\|_{\infty}+\varepsilon_{w}^{2}\left\|w_{k}\right\|_{\infty}\right) .
$$

Por otra parte, multiplicando la expresión (3.68c) por $\left[E_{k}^{\top} \mathbb{V}_{k}^{\top} w_{k}^{\top}\right]$ por la izquierda y por su traspuesta por la derecha se tiene que

$$
e_{k}^{\top} e_{k}<\lambda E_{k}^{\top} \boldsymbol{P}\left(s_{k}\right) E_{k}+\frac{\gamma_{v}^{2}-\varepsilon_{v}^{2}}{3 n} \mathbb{V}_{k}^{\top} \mathbb{V}_{k}+\left(\gamma_{w}^{2}-\varepsilon_{w}^{2}\right) w_{k}^{\top} w_{k}
$$

que, si se toman esperanzas matemáticas con respecto al muestreo en $k-1$, implica

$$
\mathcal{E}\left\{e_{k}^{\top} e_{k} \mid k-1\right\}<\lambda \mathcal{E}\left\{E_{k}^{\top} \boldsymbol{P}\left(s_{k}\right) E_{k} \mid k-1\right\}+\frac{\gamma_{v}^{2}-\varepsilon_{v}^{2}}{3 n} \mathbb{V}_{k}^{\top} \mathbb{V}_{k}+\left(\gamma_{w}^{2}-\varepsilon_{w}^{2}\right) w_{k}^{\top} w_{k}
$$

Teniendo en cuenta (B.15) se llega a

$$
\mathcal{E}\left\{e_{k}^{\top} e_{k} \mid k-1\right\}<\varepsilon_{v}^{2}\left\|v_{k}\right\|_{\infty}+\varepsilon_{w}^{2}\left\|w_{k}\right\|_{\infty}+\frac{\gamma_{v}^{2}-\varepsilon_{v}^{2}}{3 n} \mathbb{V}_{k}^{\top} \mathbb{V}_{k}+\left(\gamma_{w}^{2}-\varepsilon_{w}^{2}\right) w_{k}^{\top} w_{k},
$$

con lo que, teniendo en cuenta que

se llega a

$$
\begin{gathered}
\mathbb{V}_{k}^{\top} \mathbb{V}_{k}<3 n\left\|v_{k}\right\|_{\infty}^{2} \\
w_{k}^{\top} w_{k}<\left\|w_{k}\right\|_{\infty}^{2}
\end{gathered}
$$

$$
\mathcal{E}\left\{e_{k}^{\top} e_{k} \mid k-1\right\}<\gamma_{v}^{2}\left\|v_{k}\right\|_{\infty}^{2}+\gamma_{w}^{2}\left\|w_{k}\right\|_{\infty}^{2}
$$

\section{B.2 Diseño determinista de predictores multivariables}

\section{B.2.1 Demostración del teorema 4.5.2 (diseño $\mathcal{H}_{\infty}$ )}

Como la LMI (4.64) implica obviamente que

$$
\begin{aligned}
& {\left[\begin{array}{cc}
\boldsymbol{Q}\left(s_{k}\right)+\boldsymbol{Q}\left(s_{k}\right)^{\top}-\boldsymbol{P}\left(s_{k}\right) & \left(\boldsymbol{Q}\left(s_{k}\right)-\boldsymbol{X}\left(s_{k}\right) \boldsymbol{\Delta}\left(s_{k}\right) \boldsymbol{C}_{d}\left(s_{k}\right)\right) \boldsymbol{A}^{N\left(s_{k}\right)} \\
\left(\left(\boldsymbol{Q}\left(s_{k}\right)-\boldsymbol{X}\left(s_{k}\right) \boldsymbol{\Delta}\left(s_{k}\right) \boldsymbol{C}_{d}\left(s_{k}\right)\right) \boldsymbol{A}^{N\left(s_{k}\right)}\right)^{\top} & \boldsymbol{P}\left(s_{k-1}\right)
\end{array}\right]} \\
& -\left[\begin{array}{cc}
\mathbf{0} & \mathbf{0} \\
\mathbf{0} & \boldsymbol{C}_{y}^{\top} \boldsymbol{C}_{y}
\end{array}\right] \succ 0,
\end{aligned}
$$


y teniendo en cuenta que

$$
\left[\begin{array}{cc}
\mathbf{0} & \mathbf{0} \\
\mathbf{0} & \boldsymbol{C}_{y}^{\top} \boldsymbol{C}_{y}
\end{array}\right] \succeq 0
$$

entonces se cumple la PLMI (4.51) (con $\mu=1)$ y el predictor converge asintóticamente a cero (teorema 4.5.1).

Aplicando la condición (4.54) en la PLMI (4.64) e introduciendo la ganancia $\boldsymbol{L}\left(s_{k}\right)=\boldsymbol{Q}\left(s_{k}\right)^{-1} \boldsymbol{X}\left(s_{k}\right)$, se obtiene la expresión

$$
\left[\begin{array}{ccc}
\boldsymbol{Q}\left(s_{k}\right) \boldsymbol{P}\left(s_{k}\right)^{-1} \boldsymbol{Q}\left(s_{k}\right)^{\top} & \boldsymbol{Q}\left(s_{k}\right) \mathcal{A}\left(s_{k}\right) & \boldsymbol{Q}\left(s_{k}\right) \boldsymbol{B}\left(s_{k}\right) \\
\mathcal{A}\left(s_{k}\right)^{\top} \boldsymbol{Q}\left(s_{k}\right)^{\top} & \boldsymbol{P}\left(s_{k-1}\right)-\boldsymbol{C}_{y}^{\top} \boldsymbol{C}_{y} & \mathbf{0} \\
\mathcal{B}\left(s_{k}\right)^{\top} \boldsymbol{Q}\left(s_{k}\right)^{\top} & \mathbf{0} & \boldsymbol{\Gamma}^{2}
\end{array}\right] \succ 0 .
$$

donde se ha tenido en cuenta que

$$
\begin{aligned}
& \boldsymbol{M}_{A}\left(s_{k}\right)=\boldsymbol{Q}\left(s_{k}\right) \mathcal{A}\left(s_{k}\right), \\
& \boldsymbol{M}_{B}\left(s_{k}\right)=\boldsymbol{Q}\left(s_{k}\right) \mathcal{B}\left(s_{k}\right) .
\end{aligned}
$$

Aplicando los complementos de Schur sobre la matriz en (B.18) es fácil comprobar que se cumple la LMI

$$
\left[\begin{array}{cc}
\mathcal{A}\left(s_{k}\right)^{\top} \boldsymbol{P}\left(s_{k}\right) \mathcal{A}\left(s_{k}\right)-\boldsymbol{P}\left(s_{k-1}\right)+\boldsymbol{C}_{y}^{\top} \boldsymbol{C}_{y} & \boldsymbol{\mathcal { A }}\left(s_{k}\right)^{\top} \boldsymbol{P}\left(s_{k}\right) \mathcal{B}\left(s_{k}\right) \\
\mathcal{B}\left(s_{k}\right)^{\top} \boldsymbol{P}\left(s_{k}\right) \mathcal{A}\left(s_{k}\right) & \boldsymbol{B}\left(s_{k}\right)^{\top} \boldsymbol{P}\left(s_{k}\right) \mathcal{B}\left(s_{k}\right)-\boldsymbol{\Gamma}^{2}
\end{array}\right] \prec \mathbf{0}
$$

Multiplicando esta desigualdad por $\left[\widetilde{\boldsymbol{x}}_{k-1}^{\top} \mathbb{V}_{k}^{\top} \boldsymbol{w}_{k}^{\top}\right]$ por la izquierda y por su traspuesta por la derecha se tiene que

$$
\widetilde{\boldsymbol{x}}_{k}^{\top} \boldsymbol{P}\left(s_{k}\right) \widetilde{\boldsymbol{x}}_{k}-\widetilde{\boldsymbol{x}}_{k-1}^{\top} \boldsymbol{P}\left(s_{k-1}\right) \widetilde{\boldsymbol{x}}_{k-1}+\boldsymbol{e}_{k-1}^{\top} \boldsymbol{e}_{k-1}-\mathbb{V}_{k}^{\top} \boldsymbol{\Gamma}_{v}^{\prime 2} \mathbb{V}_{k}-\boldsymbol{w}_{k}^{\top} \boldsymbol{\Gamma}_{w}^{2} \boldsymbol{w}_{k}<0
$$

donde se ha tenido en cuenta la dinámica del error de predicción (4.41). Asumiendo un error de predicción inicial nulo $\left(\widetilde{\boldsymbol{x}}_{0}=\mathbf{0}\right)$ y sumando de $k=1$ a $k=K$ se tiene que

$$
\widetilde{\boldsymbol{x}}_{K}^{\top} \boldsymbol{P}\left(s_{K}\right) E_{K}+\sum_{k=1}^{K}\left(\boldsymbol{e}_{k-1}^{\top} \boldsymbol{e}_{k-1}-\mathbb{V}_{k}^{\top} \boldsymbol{\Gamma}_{v}^{\prime 2} \mathbb{V}_{k}-\boldsymbol{\Gamma}_{w}^{2} \boldsymbol{w}_{k}^{\top} \boldsymbol{w}_{k}\right)<0
$$

Como $\boldsymbol{P}\left(s_{k}\right)>0$, entonces $\widetilde{\boldsymbol{x}}_{K}^{\top} \boldsymbol{P}\left(s_{K}\right) \widetilde{\boldsymbol{x}}_{K} \succ \mathbf{0}$, llegándose a

$$
\sum_{k=1}^{K}\left(\boldsymbol{e}_{k-1}^{\top} \boldsymbol{e}_{k-1}-\mathbb{V}_{k}^{\top} \boldsymbol{\Gamma}_{v}^{\prime 2} \mathbb{V}_{k}-\boldsymbol{\Gamma}_{w}^{2} \boldsymbol{w}_{k}^{\top} \boldsymbol{w}_{k}\right)<0 .
$$

Cuando $K$ tiende a infinito se obtiene la norma $\ell_{2}$ de las señales cumpliéndose que

$$
\left\|e_{k}\right\|_{2}^{2}<\left\|\boldsymbol{\Gamma}_{v} \boldsymbol{v}_{k}\right\|_{2}^{2}+\left\|\boldsymbol{\Gamma}_{w} \boldsymbol{w}_{k}\right\|_{2}^{2}
$$

donde se ha tenido en cuenta que la norma $\left\|\mathbb{V}_{k}\right\|_{2}$ es $\beta$ veces mayor que $\left\|\boldsymbol{w}_{x, k}\right\|_{2}$ porque el vector $\mathbb{V}_{k}$ contiene $\beta$ valores de la historia de la señal $\boldsymbol{v}[t]$ según (4.42).

\section{B.2.2 Demostración del teorema 4.5.3 (diseño $\mathcal{H}_{2}$ )}

La estabilidad del predictor queda probada aplicando los mismos argumentos que en el teorema anterior con la LMI (4.71a).

Tómese ahora la función de Lyapunov $\mathcal{V}_{k}=\widetilde{\boldsymbol{x}}_{k}^{\top} \boldsymbol{P}\left(s_{k}\right) \widetilde{\boldsymbol{x}}_{k}$. El valor esperado de la función de Lyapunov en un instante $k$ es

$$
\begin{aligned}
\mathcal{E}\left\{\mathcal{V}_{k}\right\} & =\mathcal{E}\left\{\widetilde{\boldsymbol{x}}_{k}^{\top} \boldsymbol{P}\left(s_{k}\right) \widetilde{\boldsymbol{x}}_{k}\right\}=\mathcal{E}\{\underbrace{\left(\mathcal{A}\left(s_{k}\right) \widetilde{\boldsymbol{x}}_{k-1}+\mathcal{B}\left(s_{k}\right)\left[\begin{array}{c}
\mathbb{V}_{k} \\
\boldsymbol{w}_{k}
\end{array}\right]\right)^{\top} \boldsymbol{P}\left(s_{k}\right)(\star)}_{\star}\} \\
& =\mathcal{E}\left\{\widetilde{\boldsymbol{x}}_{k-1}^{\top} \mathcal{A}\left(s_{k}\right)^{\top} \boldsymbol{P}\left(s_{k}\right) \mathcal{A}\left(s_{k}\right) \widetilde{\boldsymbol{x}}_{k-1}\right\}+\mathcal{E}\left\{\left[\begin{array}{c}
\mathbb{V}_{k} \\
\boldsymbol{w}_{k}
\end{array}\right]^{\top} \boldsymbol{B}\left(s_{k}\right)^{\top} \boldsymbol{P}\left(s_{k}\right) \boldsymbol{B}\left(s_{k}\right)\left[\begin{array}{c}
\mathbb{V}_{k} \\
\boldsymbol{w}_{k}
\end{array}\right]\right\}
\end{aligned}
$$


donde se ha tenido en cuenta que el producto cruzado

$$
\mathcal{E}\left\{\widetilde{\boldsymbol{x}}_{k-1}^{\top} \mathcal{A}\left(s_{k}\right)^{\top} \boldsymbol{P}\left(s_{k}\right) \boldsymbol{B}\left(s_{k}\right)\left[\begin{array}{c}
\mathbb{V}_{k} \\
\boldsymbol{w}_{k}
\end{array}\right]\right\}=\mathcal{E}\left\{\widetilde{\boldsymbol{x}}_{k-1}^{\top} \mathcal{A}\left(s_{k}\right)^{\top} \boldsymbol{P}\left(s_{k}\right) \mathcal{B}\left(s_{k}\right)\right\} \mathcal{E}\left\{\left[\begin{array}{c}
\mathbb{V}_{k} \\
\boldsymbol{w}_{k}
\end{array}\right]\right\}=0
$$

es nulo debido a la independencia de $\widetilde{\boldsymbol{x}}_{k-1}$ con $\mathbb{V}_{k}$ y $\boldsymbol{w}_{k}$, y que el valor esperado de las perturbaciones es cero.

Utilizando la condición (4.54) en la LMI (4.71a) y aplicando los complementos de Schur se tiene que

$$
\mathcal{A}\left(s_{k}\right)^{\top} \boldsymbol{P}\left(s_{k}\right) \mathcal{A}\left(s_{k}\right)-\boldsymbol{P}\left(s_{k-1}\right)+\boldsymbol{C}_{y}^{\top} \boldsymbol{C}_{y}
$$

Premultiplicando y postmultiplicando esta expresión por $\widetilde{\boldsymbol{x}}_{k-1}^{\top}$ y $\widetilde{\boldsymbol{x}}_{k-1}$ se tiene que

$$
\widetilde{\boldsymbol{x}}_{k-1} \mathcal{A}\left(s_{k}\right)^{\top} \boldsymbol{P}\left(s_{k}\right) \mathcal{A}\left(s_{k}\right) \widetilde{\boldsymbol{x}}_{k-1}-\widetilde{\boldsymbol{x}}_{k-1}^{\top} \boldsymbol{P}\left(s_{k}\right) \widetilde{\boldsymbol{x}}_{k-1}+\boldsymbol{e}_{k-1}^{\top} \boldsymbol{e}_{k-1}<0 .
$$

Si se calcula la esperanza matemática de esta desigualdad y se aplica el resultado (B.22) se tiene que

$$
\mathcal{E}\left\{\mathcal{V}_{k}\right\}-\mathcal{E}\left\{\mathcal{V}_{k-1}\right\}+\boldsymbol{e}_{k-1}^{\top} \boldsymbol{e}_{k-1}<\mathcal{E}\left\{\left[\begin{array}{c}
\mathbb{V}_{k} \\
\boldsymbol{w}_{k}
\end{array}\right]^{\top} \boldsymbol{B}\left(s_{k}\right)^{\top} \boldsymbol{P}\left(s_{k}\right) \boldsymbol{B}\left(s_{k}\right)\left[\begin{array}{c}
\mathbb{V}_{k} \\
\boldsymbol{w}_{k}
\end{array}\right]\right\}
$$

Utilizando la condición (4.54) en la LMI (4.71b) y aplicando los complementos de Schur se tiene que

$$
\mathcal{B}\left(s_{k}\right)^{\top} \boldsymbol{P}\left(s_{k}\right) \mathcal{B}\left(s_{k}\right)-\Gamma^{2} \prec \mathbf{0} .
$$

Introduciendo este resultado en (B.23) y aplicando el teorema A.3.3 se llega a

$$
\mathcal{E}\left\{\mathcal{V}_{k}\right\}-\mathcal{E}\left\{\mathcal{V}_{k-1}\right\}+\boldsymbol{e}_{k-1}^{\top} \boldsymbol{e}_{k-1}<\mathcal{E}\left\{\left[\begin{array}{c}
\mathbb{V}_{k} \\
\boldsymbol{w}_{k}
\end{array}\right]^{\top} \boldsymbol{\Gamma}^{2}\left[\begin{array}{c}
\mathbb{V}_{k} \\
\boldsymbol{w}_{k}
\end{array}\right]\right\}=\sum_{i=1}^{n} \frac{\gamma_{v_{i}}^{2}}{\beta} \beta \sigma_{v_{i}}^{2}+\sum_{i=1}^{n_{m}} \gamma_{w_{i}}^{2} \sigma_{w_{i}}^{2}
$$

Sumando esta expresión de $k=1$ a $K$ y asumiendo condiciones iniciales nulas se obtiene

$$
\mathcal{E}\left\{\boldsymbol{v}_{n_{v}}\right\}+\sum_{k=1}^{N} \boldsymbol{e}_{k-1}^{\boldsymbol{\top}} \boldsymbol{e}_{k-1}<N\left(\sum_{i=1}^{n_{v}} \gamma_{v_{i}}^{2} \sigma_{v_{i}}^{2}+\sum_{i=1}^{n_{m}} \gamma_{w_{i}}^{2} \sigma_{w_{i}}^{2}\right) .
$$

Como $\boldsymbol{P}\left(s_{N}\right) \succ \mathbf{0}$, entonces $\mathcal{E}\left\{\boldsymbol{v}_{n_{v}}\right\}>0$. Tomando el límite cuando $N$ tiende a infinito se llega finalmente a

$$
\lim _{N \rightarrow \infty} \frac{1}{N} \sum_{k=1}^{N} \boldsymbol{e}_{k-1}^{\top} \boldsymbol{e}_{k-1}=\left\|\boldsymbol{e}_{k}\right\|_{R M S}^{2}<\sum_{i=1}^{n_{v}} \gamma_{v_{i}}^{2} \sigma_{v_{i}}^{2}+\sum_{i=1}^{n_{m}} \gamma_{w_{i}}^{2} \sigma_{w_{i}}^{2}
$$

\section{B.2.3 Demostración del teorema 4.5 .4 (diseño $\mathcal{H}_{2 g}$ )}

La estabilidad asintótica del predictor queda asegurada ya que si la PLMI (4.73a) se cumple también lo hará la PLMI (4.51). Utilizando la condición (4.54) en la LMI (4.73a) y aplicando los complementos de Schur, se tiene que

$$
\widetilde{\boldsymbol{x}}_{k}^{\top} \boldsymbol{P}\left(s_{k}\right) \widetilde{\boldsymbol{x}}_{k}-\widetilde{\boldsymbol{x}}_{k-1}^{\top} \boldsymbol{P}\left(s_{k-1}\right) \widetilde{\boldsymbol{x}}_{k-1}<\left[\begin{array}{c}
\mathbb{V}_{k} \\
\boldsymbol{w}_{k}
\end{array}\right]^{\top} \boldsymbol{\Gamma}^{2}\left[\begin{array}{c}
\mathbb{V}_{k} \\
\boldsymbol{w}_{k}
\end{array}\right]
$$

donde

$$
\left[\begin{array}{c}
\mathbb{V}_{k} \\
\boldsymbol{w}_{k}
\end{array}\right]^{\top} \boldsymbol{\Gamma}^{2}\left[\begin{array}{c}
\mathbb{V}_{k} \\
\boldsymbol{w}_{k}
\end{array}\right]=\frac{1}{\beta} \sum_{i=1}^{\beta} \sum_{j=1}^{n_{v}} \gamma_{v_{j}}^{2} v_{j}\left[t_{k}-i\right]^{2}+\sum_{i=1}^{n_{m}} \gamma_{w_{i}} w_{i, k}^{2}
$$

Considerando condiciones nulas y tomando sumatorio en ambos lados de la desigualdad de 1 a $K$ se tiene que

$$
\widetilde{\boldsymbol{x}}_{K}^{\top} \boldsymbol{P}\left(s_{K}\right) \widetilde{\boldsymbol{x}}_{K}<\sum_{k=1}^{K}\left(\left[\begin{array}{c}
\mathbb{V}_{k} \\
\boldsymbol{w}_{k}
\end{array}\right]^{\top} \boldsymbol{\Gamma}^{2}\left[\begin{array}{c}
\mathbb{V}_{k} \\
\boldsymbol{w}_{k}
\end{array}\right]\right) .
$$

Si se multiplica la PLMI (4.73b) por la izquierda por $\widetilde{\boldsymbol{x}}_{k}^{\top}$ y se multiplica por la derecha por $\widetilde{\boldsymbol{x}}_{k}$ se tiene que

$$
\widetilde{\boldsymbol{x}}_{K}^{\top} \boldsymbol{P}\left(s_{K}\right) \widetilde{\boldsymbol{x}}_{K}>\widetilde{\boldsymbol{x}}_{K}^{\top} \boldsymbol{C}_{y}^{\top} \boldsymbol{C}_{y} \widetilde{\boldsymbol{x}}_{K}=\boldsymbol{e}_{K}^{\top} \boldsymbol{e}_{K} .
$$


Con esto, la desigualdad (B.25) lleva a

$$
\boldsymbol{e}_{K}^{\top} \boldsymbol{e}_{K}<\sum_{k=1}^{K}\left(\left[\begin{array}{c}
\mathbb{V}_{k} \\
\boldsymbol{w}_{k}
\end{array}\right]^{\top} \boldsymbol{\Gamma}^{2}\left[\begin{array}{c}
\mathbb{V}_{k} \\
\boldsymbol{w}_{k}
\end{array}\right]\right)
$$

Tomando $K$ como el instante en el que el error se hace máximo, y teniendo en cuenta que

$$
\sum_{k=1}^{K}\left(\left[\begin{array}{c}
\mathbb{V}_{k} \\
\boldsymbol{w}_{k}
\end{array}\right]^{\top} \boldsymbol{\Gamma}^{2}\left[\begin{array}{c}
\mathbb{V}_{k} \\
\boldsymbol{w}_{k}
\end{array}\right]\right)<\lim _{K \rightarrow \infty} \sum_{k=1}^{K}\left(\left[\begin{array}{c}
\mathbb{V}_{k} \\
\boldsymbol{w}_{k}
\end{array}\right]^{\top} \boldsymbol{\Gamma}^{2}\left[\begin{array}{c}
\mathbb{V}_{k} \\
\boldsymbol{w}_{k}
\end{array}\right]\right)
$$

para cualquier $K$, se llega a la cota expresada en (4.74).

\section{B.2.4 Demostración del teorema 4.5 .5 (diseño $\ell_{1}$ )}

La estabilidad asintótica del predictor queda asegurada ya que si (4.75a) y (4.75b) se cumplen, también lo hará la PLMI (4.51), con $\mu=1-\lambda$.

Utilizando la condición (4.54) en la LMI (4.75b) y aplicando los complementos de Schur, se tiene que

$$
\widetilde{\boldsymbol{x}}_{k}^{\top} \boldsymbol{P}\left(s_{k}\right) \widetilde{\boldsymbol{x}}_{k}-\widetilde{\boldsymbol{x}}_{k-1} \boldsymbol{P}\left(s_{k-1}\right) \widetilde{\boldsymbol{x}}_{k-1}+\lambda \widetilde{\boldsymbol{x}}_{k-1} \boldsymbol{P}\left(s_{k-1}\right) \widetilde{\boldsymbol{x}}_{k-1}-\left[\begin{array}{c}
\mathbb{V}_{k} \\
\boldsymbol{w}_{k}
\end{array}\right]^{\top} \mathbf{\Upsilon}^{2}\left[\begin{array}{c}
\mathbb{V}_{k} \\
\boldsymbol{w}_{k}
\end{array}\right]<0 .
$$

Definiendo las nuevas variables

$$
\mathcal{V}_{k}=\widetilde{\boldsymbol{x}}_{k}^{\top} \boldsymbol{P}\left(s_{k}\right) \widetilde{\boldsymbol{x}}_{k}
$$

$\mathrm{y}$

$$
\omega_{k}=\left[\begin{array}{c}
\mathbb{V}_{k} \\
\boldsymbol{w}_{k}
\end{array}\right]^{\top} \boldsymbol{\Upsilon}^{2}\left[\begin{array}{c}
\mathbb{V}_{k} \\
\boldsymbol{w}_{k}
\end{array}\right]
$$

se puede ver que la expresión (B.27) equivale a una inecuación en diferencias de la forma

$$
\mathcal{V}_{k}-(1-\lambda) \mathcal{V}_{k-1}<\omega_{k},
$$

correspondiente a un sistema de primer orden de entrada $\omega_{k}$ y salida $\mathcal{V}_{k}$. Tomando la entrada de mayor amplitud posible definida como

$$
\left\|\omega_{k}\right\|_{\infty}=\sum_{i=1}^{n_{v}} \varepsilon_{v_{i}}^{2}\left\|v_{i, k}\right\|_{\infty}^{2}+\sum_{i=1}^{n_{m}} \varepsilon_{w_{i}}^{2}\left\|w_{i, k}\right\|_{\infty}^{2}
$$

se puede obtener el máximo valor $\mathcal{V}_{k}$ como el instante en el que $\mathcal{V}_{k}=\mathcal{V}_{k-1}$ con lo que se llega a

$$
\lambda \mathcal{V}_{k}<\omega_{k} \leq\left\|\omega_{k}\right\|_{\infty}
$$

Deshaciendo los cambios de variable se tiene que

$$
\widetilde{\boldsymbol{x}}_{k}^{\top} \boldsymbol{P}\left(s_{k}\right) \widetilde{\boldsymbol{x}}_{k}<\frac{1}{\lambda}\left(\sum_{i=1}^{n_{v}} \varepsilon_{v_{i}}^{2}\left\|v_{i, k}\right\|_{\infty}^{2}+\sum_{i=1}^{n_{m}} \varepsilon_{w_{i}}^{2}\left\|w_{i, k}\right\|_{\infty}^{2}\right) .
$$

Multiplicando la expresión (4.75c) por $\left[\widetilde{\boldsymbol{x}}_{k}^{\top} \mathbb{V}_{k}^{\top} \boldsymbol{w}_{k}^{\top}\right]$ por la izquierda y por su traspuesta por la derecha se tiene que

$$
\boldsymbol{e}_{k}^{\top} \boldsymbol{e}_{k}<\lambda \widetilde{\boldsymbol{x}}_{k}^{\top} \boldsymbol{P}\left(s_{k}\right) \widetilde{\boldsymbol{x}}_{k}+\left[\begin{array}{c}
\mathbb{V}_{k} \\
\boldsymbol{w}_{k}
\end{array}\right]^{\top}\left(\boldsymbol{\Gamma}^{2}-\boldsymbol{\Upsilon}^{2}\right)\left[\begin{array}{c}
\mathbb{V}_{k} \\
\boldsymbol{w}_{k}
\end{array}\right] .
$$

Teniendo en cuenta $(\overline{B .29})$ se llega a

$$
\boldsymbol{e}_{k}^{\top} \boldsymbol{e}_{k}<\sum_{i=1}^{n_{v}} \varepsilon_{v_{i}}^{2}\left\|v_{i, k}\right\|_{\infty}^{2}+\sum_{i=1}^{n_{m}} \varepsilon_{w_{i}}^{2}\left\|w_{i, k}\right\|_{\infty}^{2}+\left[\begin{array}{c}
\mathbb{V}_{k} \\
\boldsymbol{w}_{k}
\end{array}\right]^{\top}\left(\boldsymbol{\Gamma}^{2}-\boldsymbol{\Upsilon}^{2}\right)\left[\begin{array}{c}
\mathbb{V}_{k} \\
\boldsymbol{w}_{k}
\end{array}\right]
$$

con lo que, teniendo en cuenta que

$$
\left[\begin{array}{c}
\mathbb{V}_{k} \\
\boldsymbol{w}_{k}
\end{array}\right]^{\top}\left(\boldsymbol{\Gamma}^{2}-\boldsymbol{\Upsilon}^{2}\right)\left[\begin{array}{c}
\mathbb{V}_{k} \\
\boldsymbol{w}_{k}
\end{array}\right]<\sum_{i=1}^{n_{v}}\left(\gamma_{v_{i}}^{2}-\varepsilon_{v_{i}}^{2}\right)\left\|v_{i, k}\right\|_{\infty}^{2}+\sum_{i=1}^{n_{m}}\left(\gamma_{w_{i}}^{2}-\varepsilon_{w_{i}}^{2}\right)\left\|w_{i, k}\right\|_{\infty}^{2}
$$

se llega a

$$
\boldsymbol{e}_{k}^{\top} \boldsymbol{e}_{k}<\sum_{i=1}^{n_{v}} \gamma_{v_{i}}^{2}\left\|v_{i, k}\right\|_{\infty}^{2}+\sum_{i=1}^{n_{m}} \gamma_{w_{i}}^{2}\left\|w_{i, k}\right\|_{\infty}^{2}
$$




\section{B.3 Diseño estocástico de predictores multivariables}

\section{B.3.1 Demostración del teorema 4.6.2 (diseño $\mathcal{H}_{\infty}$ )}

Aplicando la desigualdad (4.82) en (4.83), y aplicando complementos de Schur se llega a

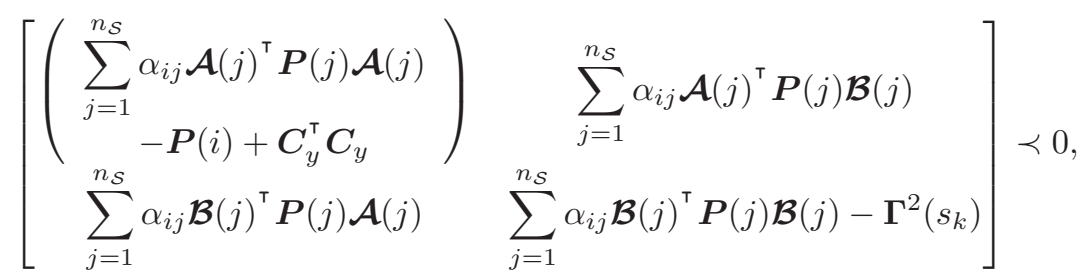

Multiplicando esta expresión por $\left[\widetilde{\boldsymbol{x}}_{k-1}^{\top} \mathbb{V}_{k}^{\top} \boldsymbol{w}_{k}^{\top}\right]$ por la izquierda y por su traspuesta por la derecha se tiene que

$$
\begin{gathered}
\sum_{j=1}^{n_{\mathcal{S}}} \alpha_{i j}\left(\boldsymbol{\mathcal { A }}(j) \widetilde{\boldsymbol{x}}_{k-1}+\mathcal{B}(j)\left[\begin{array}{r}
\mathbb{V}_{k} \\
\boldsymbol{w}_{k}
\end{array}\right]\right)^{\top} \boldsymbol{P}(j)\left(\boldsymbol{A}(j) \widetilde{\boldsymbol{x}}_{k-1}+\mathcal{B}(j)\left[\begin{array}{c}
\mathbb{V}_{k} \\
\boldsymbol{w}_{k}
\end{array}\right]\right) \\
-\widetilde{\boldsymbol{x}}_{k-1}^{\top} \boldsymbol{P}(i) \widetilde{\boldsymbol{x}}_{k-1}+\boldsymbol{e}_{k-1}^{\top} \boldsymbol{e}_{k-1}-\left[\begin{array}{r}
\mathbb{V}_{k} \\
\boldsymbol{w}_{k}
\end{array}\right]^{\top} \boldsymbol{\Gamma}^{2}\left[\begin{array}{r}
\mathbb{V}_{k} \\
\boldsymbol{w}_{k}
\end{array}\right]<0 .
\end{gathered}
$$

Definiendo la función de Lyapunov paramétrica como

$$
\mathcal{V}\left(\widetilde{\boldsymbol{x}}_{k}, s_{k}\right)=\widetilde{\boldsymbol{x}}_{k}^{\top} \boldsymbol{P}\left(s_{k}\right) \widetilde{\boldsymbol{x}}_{k},
$$

y teniendo en cuenta la siguiente esperanza matemática

$$
\mathcal{E}\left\{\mathcal{V}\left(\widetilde{\boldsymbol{x}}_{k}, s_{k}\right) \mid s_{k-1}=i\right\}=\sum_{j=1}^{n_{\mathcal{S}}} \alpha_{i j}(\star)^{\top} \boldsymbol{P}(j) \underbrace{\left(\mathcal{A}(j) \widetilde{\boldsymbol{x}}_{k-1}+\boldsymbol{\mathcal { B }}(j)\left[\begin{array}{c}
\mathbb{V}_{k} \\
\boldsymbol{w}_{k}
\end{array}\right]\right)}_{\star},
$$

la ecuación (B.31) puede expresarse como

$$
\begin{gathered}
\mathcal{E}\left\{\mathcal{V}\left(\widetilde{\boldsymbol{x}}_{k}, s_{k}\right) \mid k-1\right\}-\widetilde{\boldsymbol{x}}_{k-1}^{\top} \boldsymbol{P}\left(s_{k-1}\right) \widetilde{\boldsymbol{x}}_{k-1} \\
+\boldsymbol{e}_{k-1}^{\top} \boldsymbol{e}_{k-1}-\left[\begin{array}{r}
\mathbb{V}_{k} \\
\boldsymbol{w}_{k}
\end{array}\right]^{\top} \boldsymbol{\Gamma}^{2}\left[\begin{array}{c}
\mathbb{V}_{k} \\
\boldsymbol{w}_{k}
\end{array}\right]<0
\end{gathered}
$$

Tomando esperanzas matemáticas condicionadas al conocimiento del muestreo $s_{k-2}$ se tiene que

$$
\begin{aligned}
\mathcal{E}\left\{\mathcal{E}\left\{\mathcal{V}\left(\widetilde{\boldsymbol{x}}_{k}, s_{k}\right) \mid k-1\right\} \mid k-2\right\}-\mathcal{E}\left\{\mathcal{V}\left(\widetilde{\boldsymbol{x}}_{k-1}, s_{k-1}\right) \mid k-2\right\} \\
+\mathcal{E}\left\{\boldsymbol{e}_{k-1}^{\top} \boldsymbol{e}_{k-1}-\left[\begin{array}{r}
\mathbb{V}_{k} \\
\boldsymbol{w}_{k}
\end{array}\right]^{\top} \boldsymbol{\Gamma}^{2}\left[\begin{array}{r}
\mathbb{V}_{k} \\
\boldsymbol{w}_{k}
\end{array}\right] \mid k-2\right\}<0
\end{aligned}
$$

Teniendo en cuenta que la dinámica del error de predicción sólo depende del instante anterior,

$$
\mathcal{E}\left\{\mathcal{E}\left\{\mathcal{V}\left(\widetilde{\boldsymbol{x}}_{k}, s_{k}\right) \mid k-1\right\} \mid k-2\right\}=\mathcal{E}\left\{\mathcal{V}\left(\widetilde{\boldsymbol{x}}_{k}, s_{k}\right) \mid k-1\right\},
$$

y teniendo en cuenta que $\boldsymbol{w}_{x, k}$ y $\boldsymbol{w}_{k}$ son señales deterministas, se tiene que

$$
\begin{aligned}
\mathcal{E}\{ & \left.\mathcal{V}\left(\widetilde{\boldsymbol{x}}_{k}, s_{k}\right) \mid k-1\right\}-\mathcal{E}\left\{\mathcal{V}\left(\widetilde{\boldsymbol{x}}_{k-1}, s_{k-1}\right) \mid k-2\right\} \\
& +\mathcal{E}\left\{\boldsymbol{e}_{k-1}^{\top} \boldsymbol{e}_{k-1} \mid k-2\right\}-\left[\begin{array}{r}
\mathbb{V}_{k} \\
\boldsymbol{w}_{k}
\end{array}\right]^{\top} \boldsymbol{\Gamma}^{2}\left[\begin{array}{r}
\mathbb{V}_{k} \\
\boldsymbol{w}_{k}
\end{array}\right]<0 .
\end{aligned}
$$

Asumiendo un error de predicción inicial nulo, sumando la expresión anterior de $k=1$ a $k=K$, se tiene que

$$
\mathcal{E}\left\{\mathcal{V}\left(\widetilde{\boldsymbol{x}}_{K}, s_{K}\right) \mid K-1\right\}+\sum_{k=1}^{K}\left(\mathcal{E}\left\{\boldsymbol{e}_{k-1}^{\top} \boldsymbol{e}_{k-1} \mid k-2\right\}-\left[\begin{array}{c}
\mathbb{V}_{k} \\
\boldsymbol{w}_{k}
\end{array}\right]^{\top} \boldsymbol{\Gamma}^{2}\left[\begin{array}{c}
\mathbb{V}_{k} \\
\boldsymbol{w}_{k}
\end{array}\right]\right)
$$

Como $\mathcal{E}\left\{\mathcal{V}\left(\widetilde{\boldsymbol{x}}_{K}, s_{K}\right) \mid s_{K-1}=i\right\}>0$ se tiene que

$$
\mathcal{E}\left\{\sum_{k=1}^{K} \boldsymbol{e}_{k-1}^{\top} \boldsymbol{e}_{k-1} \mid k-2\right\}<\sum_{k=1}^{K}\left(\left[\begin{array}{c}
\mathbb{V}_{k} \\
\boldsymbol{w}_{k}
\end{array}\right]^{\top} \boldsymbol{\Gamma}^{2}\left[\begin{array}{c}
\mathbb{V}_{k} \\
\boldsymbol{w}_{k}
\end{array}\right]\right)
$$

que implica (4.84) cuando $K$ se hace tender a infinito. 


\section{B.3.2 Demostración del teorema 4.6 .3 (diseño $\mathcal{H}_{2}$ )}

Prueba B.3.1. Siguiendo el mismo razonamiento que en el teorema anterior, la LMI (4.85a) implica la convergencia a cero en valor medio del predictor.

Tómese ahora la función de Lyapunov $\mathcal{V}_{k}=\widetilde{\boldsymbol{x}}_{k}^{\top} \boldsymbol{P}\left(s_{k}\right) \widetilde{\boldsymbol{x}}_{k}$. El valor esperado de la función de Lyapunov condicionado a un instante anterior es

$$
\begin{aligned}
\mathcal{E}\left\{\mathcal{V}_{k} \mid k-1\right\}= & \mathcal{E}\left\{\widetilde{\boldsymbol{x}}_{k}^{\top} \boldsymbol{P}\left(s_{k}\right) \widetilde{\boldsymbol{x}}_{k} \mid k-1\right\}=\mathcal{E}\{\underbrace{\left(\mathcal{A}\left(s_{k}\right) \widetilde{\boldsymbol{x}}_{k-1}+\boldsymbol{\mathcal { B }}\left(s_{k}\right)\left[\begin{array}{c}
\mathbb{V}_{k} \\
\boldsymbol{w}_{k}
\end{array}\right]\right)^{\top}}_{\star} \boldsymbol{P}(\star) \mid k-1\} \\
= & \mathcal{E}\left\{\widetilde{\boldsymbol{x}}_{k-1}^{\top} \mathcal{A}\left(s_{k}\right)^{\top} \boldsymbol{P}\left(s_{k}\right) \mathcal{A}\left(s_{k}\right) \widetilde{\boldsymbol{x}}_{k-1} \mid k-1\right\} \\
& +\mathcal{E}\left\{\left[\begin{array}{c}
\mathbb{V}_{k} \\
\boldsymbol{w}_{k}
\end{array}\right]^{\top} \boldsymbol{B}\left(s_{k}\right)^{\top} \boldsymbol{P}\left(s_{k}\right) \mathcal{B}\left(s_{k}\right)\left[\begin{array}{c}
\mathbb{V}_{k} \\
\boldsymbol{w}_{k}
\end{array}\right] \mid k-1\right\}
\end{aligned}
$$

donde se ha tenido en cuenta que el producto cruzado

$$
\mathcal{E}\left\{\widetilde{\boldsymbol{x}}_{k-1}^{\top} \mathcal{A}\left(s_{k}\right)^{\top} \boldsymbol{P}\left(s_{k}\right) \mathcal{B}\left(s_{k}\right)\left[\begin{array}{c}
\mathbb{V}_{k} \\
\boldsymbol{w}_{k}
\end{array}\right]\right\}=\mathcal{E}\left\{\widetilde{\boldsymbol{x}}_{k-1}^{\top} \mathcal{A}\left(s_{k}\right)^{\top} \boldsymbol{P}\left(s_{k}\right) \mathcal{B}\left(s_{k}\right) \mid k-1\right\} \mathcal{E}\left\{\left[\begin{array}{c}
\mathbb{V}_{k} \\
\boldsymbol{w}_{k}
\end{array}\right]\right\}=0
$$

es nulo debido a la independencia de $\widetilde{\boldsymbol{x}}_{k-1}$ con $\mathbb{V}_{k}$ y $\boldsymbol{w}_{k}$, y que el valor esperado de las perturbaciones es cero. Para un $s_{k-1}=i$ dado, las esperanzas matemáticas de (B.22) se pueden calcular con ayuda de las probabilidades de transmisión, quedando

$$
\begin{aligned}
\mathcal{E}\left\{\mathcal{V}_{k} \mid k-1\right\}= & \widetilde{\boldsymbol{x}}_{k-1}^{\top}\left(\sum_{j=1}^{n_{\mathcal{S}}} \alpha_{i j} \mathcal{A}(j)^{\top} \boldsymbol{P}(j) \mathcal{A}(j)\right) \widetilde{\boldsymbol{x}}_{k-1} \\
& +\mathcal{E}\left\{\left[\begin{array}{c}
\mathbb{V}_{k} \\
\boldsymbol{w}_{k}
\end{array}\right]^{\top}\left(\sum_{j=1}^{n_{\mathcal{S}}} \alpha_{i j} \mathcal{B}(j)^{\top} \boldsymbol{P}(j) \mathcal{B}(j)\right)\left[\begin{array}{c}
\mathbb{V}_{k} \\
\boldsymbol{w}_{k}
\end{array}\right]\right\}
\end{aligned}
$$

Utilizando la condición (4.82) en la LMI (4.85a) y aplicando los complementos de Schur se tiene que

$$
\sum_{j=1}^{n_{\mathcal{S}}} \alpha_{i j} \mathcal{A}(j)^{\top} \boldsymbol{P}(j) \mathcal{A}(j)-\boldsymbol{P}(i)+\boldsymbol{C}_{y}^{\top} \boldsymbol{C}_{y}
$$

Premultiplicando y postmultiplicando esta expresión por $\widetilde{\boldsymbol{x}}_{k-1}^{\top}$ y $\widetilde{\boldsymbol{x}}_{k-1}$ se tiene que

$$
\widetilde{\boldsymbol{x}}_{k-1}^{\top}\left(\sum_{j=1}^{n_{\mathcal{S}}} \alpha_{i j} \mathcal{A}(j)^{\top} \boldsymbol{P}(j) \mathcal{A}(j)\right) \widetilde{\boldsymbol{x}}_{k-1}-\widetilde{\boldsymbol{x}}_{k-1}^{\top} \boldsymbol{P}(i) \widetilde{\boldsymbol{x}}_{k-1}+\boldsymbol{e}_{k-1}^{\top} \boldsymbol{e}_{k-1}<0 .
$$

Si se calcula la esperanza matemática de esta desigualdad con respecto a un instante anterior $k-2$ y se aplica el resultado (B.37) se tiene que

$$
\mathcal{E}\left\{\mathcal{V}_{k} \mid k-1\right\}-\mathcal{E}\left\{\mathcal{V}_{k-1} \mid k-2\right\}+\mathcal{E}\left\{\boldsymbol{e}_{k-1}^{\top} \boldsymbol{e}_{k-1} \mid k-2\right\}<\mathcal{E}\left\{\left[\begin{array}{c}
\mathbb{V}_{k} \\
\boldsymbol{w}_{k}
\end{array}\right]^{\top}\left(\sum_{j=1}^{n_{\mathcal{S}}} \alpha_{i j} \mathcal{B}(j)^{\top} \boldsymbol{P}(j) \boldsymbol{B}(j)\right)\left[\begin{array}{c}
\mathbb{V}_{k} \\
\boldsymbol{w}_{k}
\end{array}\right]\right\}
$$

Utilizando la condición (4.82) en la LMI (4.85b) y aplicando los complementos de Schur se tiene que

$$
\sum_{j=1}^{n_{\mathcal{S}}} \alpha_{i j} \mathcal{B}(j)^{\top} \boldsymbol{P}(j) \mathcal{B}(j)-\boldsymbol{\Gamma}^{2} \prec \mathbf{0} .
$$

Introduciendo este resultado en (B.38) y aplicando el teorema A.3.3 se llega a

$$
\begin{aligned}
\mathcal{E}\left\{\mathcal{V}_{k} \mid k-1\right\}-\mathcal{E}\left\{\mathcal{V}_{k-1} \mid k-2\right\}+\mathcal{E}\left\{\boldsymbol{e}_{k-1}^{\top} \boldsymbol{e}_{k-1} \mid k-2\right\} & <\mathcal{E}\left\{\left[\begin{array}{c}
\mathbb{V}_{k} \\
\boldsymbol{w}_{k}
\end{array}\right]^{\top} \boldsymbol{\Gamma}^{2}\left[\begin{array}{r}
\mathbb{V}_{k} \\
\boldsymbol{w}_{k}
\end{array}\right]\right\} \\
& =\sum_{i=1}^{n_{v}} \gamma_{v_{i}}^{2} \sigma_{v_{i}}^{2}+\sum_{i=1}^{n_{m}} \gamma_{w_{i}}^{2} \sigma_{w_{i}}^{2} .
\end{aligned}
$$


Sumando esta expresión de $k=1$ a $K$ y asumiendo condiciones iniciales nulas se obtiene

$$
\mathcal{E}\{\mathcal{V}-K \mid K-1\}+\sum_{k=1}^{K} \mathcal{E}\left\{\boldsymbol{e}_{k-1}^{\top} \boldsymbol{e}_{k-1} \mid k-2\right\}<K\left(\sum_{i=1}^{n_{v}} \gamma_{v_{i}}^{2} \sigma_{v_{i}}^{2}+\sum_{i=1}^{n_{m}} \gamma_{w_{i}}^{2} \sigma_{w_{i}}^{2}\right)
$$

Como $\boldsymbol{P}\left(s_{K}\right) \succ \mathbf{0}$, entonces $\mathcal{E}\left\{\mathcal{V}_{K} \mid K-1\right\}>0$. Tomando el límite cuando $K$ tiende a infinito se llega finalmente a

$$
\lim _{K \rightarrow \infty} \frac{1}{K} \sum_{k=1}^{K} \mathcal{E}\left\{\boldsymbol{e}_{k-1}^{\top} \boldsymbol{e}_{k-1} \mid k-2\right\}=\mathcal{E}\left\{\left\|\boldsymbol{e}_{k}\right\|_{R M S}^{2}\right\}<\sum_{i=1}^{n_{v}} \gamma_{v_{i}}^{2} \sigma_{v_{i}}^{2}+\sum_{i=1}^{n_{m}} \gamma_{w_{i}}^{2} \sigma_{w_{i}}^{2}
$$

\section{B.3.3 Demostración del teorema 4.6 .4 (diseño $\mathcal{H}_{2 g}$ )}

La estabilidad asintótica del predictor queda asegurada ya que si la PLMI (4.87a) se cumple también lo hará la PLMI (4.81). Utilizando la condición (4.82) en la LMI (4.73a) y aplicando los complementos de Schur, se tiene que

$$
\mathcal{E}\left\{\widetilde{\boldsymbol{x}}_{k}^{\top} \boldsymbol{P}\left(s_{k}\right) \widetilde{\boldsymbol{x}}_{k} \mid k-1\right\}-\widetilde{\boldsymbol{x}}_{k-1}^{\top} \boldsymbol{P}\left(s_{k-1}\right) \widetilde{\boldsymbol{x}}_{k-1}<\left[\begin{array}{c}
\mathbb{V}_{k} \\
\boldsymbol{w}_{k}
\end{array}\right]^{\top} \boldsymbol{\Gamma}^{2}\left[\begin{array}{c}
\mathbb{V}_{k} \\
\boldsymbol{w}_{k}
\end{array}\right]
$$

Tomando esperanzas matemáticas condicionadas a $s_{k-2}$ en la expresión anterior se tiene que

$$
\mathcal{E}\left\{\widetilde{\boldsymbol{x}}_{k}^{\top} \boldsymbol{P}\left(s_{k}\right) \widetilde{\boldsymbol{x}}_{k} \mid k-1\right\}-\mathcal{E}\left\{\widetilde{\boldsymbol{x}}_{k-1}^{\top} \boldsymbol{P}\left(s_{k-1}\right) \widetilde{\boldsymbol{x}}_{k-1} \mid k-2\right\}<\left[\begin{array}{c}
\mathbb{V}_{k} \\
\boldsymbol{w}_{k}
\end{array}\right]^{\top} \boldsymbol{\Gamma}^{2}\left[\begin{array}{c}
\mathbb{V}_{k} \\
\boldsymbol{w}_{k}
\end{array}\right] .
$$

Considerando condiciones nulas y tomando sumatorio de 1 a $K$ en ambos lados de la desigualdad se llega a

$$
\mathcal{E}\left\{\widetilde{\boldsymbol{x}}_{K}^{\top} \boldsymbol{P}\left(s_{K}\right) \widetilde{\boldsymbol{x}}_{K} \mid K-1\right\}<\sum_{k=1}^{K}\left(\left[\begin{array}{c}
\mathbb{V}_{k} \\
\boldsymbol{w}_{k}
\end{array}\right]^{\top} \boldsymbol{\Gamma}^{2}\left[\begin{array}{c}
\mathbb{V}_{k} \\
\boldsymbol{w}_{k}
\end{array}\right]\right) .
$$

Aplicando ahora la condición (4.82) y los complementos de Schur en (4.87b), para posteriormente multiplicar por la izquierda por $\widetilde{\boldsymbol{x}}_{k}^{\top}$ y por la derecha por $\widetilde{\boldsymbol{x}}_{k}$ se tiene que

$$
\widetilde{\boldsymbol{x}}_{K}^{\top} \boldsymbol{P}\left(s_{K}\right) \widetilde{\boldsymbol{x}}_{K}>\widetilde{\boldsymbol{x}}_{K}^{\top} \boldsymbol{C}_{y}^{\top} \boldsymbol{C}_{y} \widetilde{\boldsymbol{x}}_{K}=\boldsymbol{e}_{K}^{\top} \boldsymbol{e}_{K} .
$$

Tomando esperanzas matemáticas con respecto a $s_{K-1}$ se tiene que

$$
\mathcal{E}\left\{\widetilde{\boldsymbol{x}}_{K}^{\top} \boldsymbol{P}\left(s_{K}\right) \widetilde{\boldsymbol{x}}_{K} \mid K-1\right\}>\mathcal{E}\left\{\boldsymbol{e}_{K}^{\top} \boldsymbol{e}_{K} \mid K-1\right\}
$$

Con esto, la desigualdad (B.40) lleva a

$$
\mathcal{E}\left\{\boldsymbol{e}_{K}^{\top} \boldsymbol{e}_{K} \mid K-1\right\}<\sum_{k=1}^{K}\left(\left[\begin{array}{c}
\mathbb{V}_{k} \\
\boldsymbol{w}_{k}
\end{array}\right]^{\top} \boldsymbol{\Gamma}^{2}\left[\begin{array}{c}
\mathbb{V}_{k} \\
\boldsymbol{w}_{k}
\end{array}\right]\right)
$$

Tomando $K$ como el instante en el que el error se hace máximo, y teniendo en cuenta que

$$
\sum_{k=1}^{K}\left(\left[\begin{array}{r}
\mathbb{V}_{k} \\
\boldsymbol{w}_{k}
\end{array}\right]^{\top} \boldsymbol{\Gamma}^{2}\left[\begin{array}{r}
\mathbb{V}_{k} \\
\boldsymbol{w}_{k}
\end{array}\right]\right)<\lim _{K \rightarrow \infty} \sum_{k=1}^{K}\left(\left[\begin{array}{r}
\mathbb{V}_{k} \\
\boldsymbol{w}_{k}
\end{array}\right]^{\top} \boldsymbol{\Gamma}^{2}\left[\begin{array}{r}
\mathbb{V}_{k} \\
\boldsymbol{w}_{k}
\end{array}\right]\right)
$$

para cualquier $K$, se llega a la cota expresada en (4.88).

\section{B.3.4 Demostración del teorema 4.6.5 (diseño $\left.\ell_{1}\right)$}

La estabilidad asintótica del predictor queda asegurada ya que si las PLMIs (4.89a) y (4.89b) se cumplen también lo hará la PLMI (4.78), con $\mu=1-\lambda$. Utilizando la condición (4.82) en la LMI (4.89b) y aplicando los complementos de Schur, se tiene que

$$
\mathcal{E}\left\{\widetilde{\boldsymbol{x}}_{k}^{\top} \boldsymbol{P}\left(s_{k}\right) \widetilde{\boldsymbol{x}}_{k} \mid k-1\right\}-\widetilde{\boldsymbol{x}}_{k-1} \boldsymbol{P}\left(s_{k-1}\right) \widetilde{\boldsymbol{x}}_{k-1}+\lambda \widetilde{\boldsymbol{x}}_{k-1} \boldsymbol{P}\left(s_{k-1}\right) \widetilde{\boldsymbol{x}}_{k-1}-\left[\begin{array}{c}
\mathbb{V}_{k} \\
\boldsymbol{w}_{k}
\end{array}\right]^{\top} \boldsymbol{\Upsilon}^{2}\left[\begin{array}{c}
\mathbb{V}_{k} \\
\boldsymbol{w}_{k}
\end{array}\right]<0
$$


Definiendo las nuevas variables

$$
\mathcal{V}_{k}=\mathcal{E}\left\{\widetilde{\boldsymbol{x}}_{k}^{\top} \boldsymbol{P}\left(s_{k}\right) \widetilde{\boldsymbol{x}}_{k} \mid k-1\right\}
$$

y

$$
\omega_{k}=\left[\begin{array}{c}
\mathbb{V}_{k} \\
\boldsymbol{w}_{k}
\end{array}\right]^{\top} \boldsymbol{\Upsilon}^{2}\left[\begin{array}{c}
\mathbb{V}_{k} \\
\boldsymbol{w}_{k}
\end{array}\right]
$$

se puede ver que la expresión (B.43) equivale a una inecuación en diferencias de la forma

$$
\mathcal{V}_{k}-(1-\lambda) \mathcal{V}_{k-1}<\omega_{k}
$$

Tomando la entrada de mayor amplitud posible definida como

$$
\left\|\omega_{k}\right\|_{\infty}=\sum_{i=1}^{n_{v}} \varepsilon_{v_{i}}^{2}\left\|v_{i, k}\right\|_{\infty}^{2}+\sum_{i=1}^{n_{m}} \varepsilon_{w_{i}}^{2}\left\|w_{i, k}\right\|_{\infty}^{2}
$$

se puede obtener el máximo valor $\mathcal{V}_{k}$ como el instante en el que $\mathcal{V}_{k}=\mathcal{V}_{k-1}$ con lo que se llega a

$$
\lambda \mathcal{V}_{k}<\omega_{k} \leq\left\|\omega_{k}\right\|_{\infty}
$$

Deshaciendo los cambios de variable se tiene que

$$
\mathcal{E}\left\{\widetilde{\boldsymbol{x}}_{k}^{\boldsymbol{\top}} \boldsymbol{P}\left(s_{k}\right) \widetilde{\boldsymbol{x}}_{k} \mid k-1\right\}<\frac{1}{\lambda}\left(\sum_{i=1}^{n_{v}} \varepsilon_{v_{i}}^{2}\left\|v_{i, k}\right\|_{\infty}^{2}+\sum_{i=1}^{n_{m}} \varepsilon_{w_{i}}^{2}\left\|w_{i, k}\right\|_{\infty}^{2}\right) .
$$

Por otra parte, multiplicando la expresión (4.89c) por $\left[\widetilde{\boldsymbol{x}}_{k}^{\top} \mathbb{V}_{k}^{\top} \boldsymbol{w}_{k}^{\top}\right]$ por la izquierda y por su traspuesta por la derecha se tiene que

$$
\boldsymbol{e}_{k}^{\top} \boldsymbol{e}_{k}<\lambda \widetilde{\boldsymbol{x}}_{k}^{\top} \boldsymbol{P}\left(s_{k}\right) \widetilde{\boldsymbol{x}}_{k}+\left[\begin{array}{c}
\mathbb{V}_{k} \\
\boldsymbol{w}_{k}
\end{array}\right]^{\top}\left(\boldsymbol{\Gamma}^{2}-\boldsymbol{\Upsilon}^{2}\right)\left[\begin{array}{c}
\mathbb{V}_{k} \\
\boldsymbol{w}_{k}
\end{array}\right]
$$

que, si se toman esperanzas matemáticas con respecto al muestreo en $k-1$, implica

$$
\mathcal{E}\left\{\boldsymbol{e}_{k}^{\top} \boldsymbol{e}_{k} \mid k-1\right\}<\lambda \mathcal{E}\left\{\widetilde{\boldsymbol{x}}_{k}^{\top} \boldsymbol{P}\left(s_{k}\right) \widetilde{\boldsymbol{x}}_{k} \mid k-1\right\}+\left[\begin{array}{c}
\mathbb{V}_{k} \\
\boldsymbol{w}_{k}
\end{array}\right]^{\top}\left(\boldsymbol{\Gamma}^{2}-\boldsymbol{\Upsilon}^{2}\right)\left[\begin{array}{c}
\mathbb{V}_{k} \\
\boldsymbol{w}_{k}
\end{array}\right],
$$

Teniendo en cuenta (B.45) se llega a

$$
\mathcal{E}\left\{\boldsymbol{e}_{k}^{\top} \boldsymbol{e}_{k} \mid k-1\right\}<\sum_{i=1}^{n_{v}} \varepsilon_{v_{i}}^{2}\left\|v_{i, k}\right\|_{\infty}^{2}+\sum_{i=1}^{n_{m}} \varepsilon_{w_{i}}^{2}\left\|w_{i, k}\right\|_{\infty}^{2}+\left[\begin{array}{c}
\mathbb{V}_{k} \\
\boldsymbol{w}_{k}
\end{array}\right]^{\top}\left(\boldsymbol{\Gamma}^{2}-\boldsymbol{\Upsilon}^{2}\right)\left[\begin{array}{c}
\mathbb{V}_{k} \\
\boldsymbol{w}_{k}
\end{array}\right]
$$

con lo que, teniendo en cuenta que

$$
\left[\begin{array}{c}
\mathbb{V}_{k} \\
\boldsymbol{w}_{k}
\end{array}\right]^{\top}\left(\boldsymbol{\Gamma}^{2}-\boldsymbol{\Upsilon}^{2}\right)\left[\begin{array}{c}
\mathbb{V}_{k} \\
\boldsymbol{w}_{k}
\end{array}\right]<\sum_{i=1}^{n_{v}}\left(\gamma_{v_{i}}^{2}-\boldsymbol{\varepsilon}_{v_{i}}^{2}\right)\left\|v_{i, k}\right\|_{\infty}^{2}+\sum_{i=1}^{n_{m}}\left(\gamma_{w_{i}}^{2}-\varepsilon_{w_{i}}^{2}\right)\left\|w_{i, k}\right\|_{\infty}^{2}
$$

se llega a

$$
\mathcal{E}\left\{\boldsymbol{e}_{k}^{\top} \boldsymbol{e}_{k} \mid k-1\right\}<\sum_{i=1}^{n_{v}} \gamma_{v_{i}}^{2}\left\|v_{i, k}\right\|_{\infty}^{2}+\sum_{i=1}^{n_{m}} \gamma_{w_{i}}^{2}\left\|w_{i, k}\right\|_{\infty}^{2}
$$





\section{Notación}

La norma general seguida en la notación es que los escalares se escriben en letra matemática normal, los vectores en minúscula y negrita y las matrices en mayúscula y negrita. Para denotar señales continuas, discretas a periodo $T$ y discretas guiadas por eventos se han utilizado notaciones diferentes. Los conjuntos se nombran con letra caligráfica. Se utiliza letra caligráfica en negrita para denotar las matrices que definen la dinámica global de un sistema formado por diversos subsistemas.

\begin{tabular}{ll}
\hline & Señales y sistemas de tiempo continuo \\
\hline$G(s)$ & Sistema de tiempo continuo \\
$m(\tau)$ & Señal de medición continua \\
$u(\tau)$ & Señal de entrada continua \\
$\boldsymbol{u}(\tau)$ & Vector de entradas continuas \\
$v(\tau)$ & Perturbación de salida (representación entrada-salida) \\
$\boldsymbol{v}(\tau)$ & Perturbación del estado (representación interna) \\
$w(\tau)$ & Ruido de medida \\
$\boldsymbol{x}(\tau)$ & Vector de estado continuo \\
$y(\tau)$ & Señal de salida continua \\
$\boldsymbol{y}(\tau)$ & Vector de salidas continuas \\
$z(\tau)$ & Señal de salida controlada \\
\hline & Señales y sistemas de tiempo discreto a periodo $T$ \\
\hline$T$ & Periodo de actualización de la acción de control \\
$G(z)$ & Sistema de tiempo discreto a periodo $T$ \\
$u[t], y[t]$ & Señales de entrada y salida a periodo $T$ \\
$U[t], Y[t]$ & Vector con las últimas $n$ entradas o salidas a periodo $T$ \\
\hline & Señales y sistemas de tiempo discreto eventuales \\
\hline$d_{k}$ & Retardo asociado a la medición $k$-ésima \\
$e_{k},\left(\boldsymbol{e}_{k}\right)$ & Error de predicción \\
$\boldsymbol{\ell}_{k}$ & Vector de ganancias del predictor \\
$m_{k}$ & Medición $k$-ésima \\
$r_{k}$ & Referencia \\
$s_{k}$ & Parámetro de muestreo \\
$t_{k}$ & Instante de control en el que se produce la $k$-ésima medición \\
$u_{k},\left(\boldsymbol{u}_{k}\right)$ & Acción de control (MIMO) \\
$v_{k}$ & Perturbación \\
$\boldsymbol{x}_{k},\left(\boldsymbol{y}_{k}\right)$ & Estado de medida \\
$m_{k},\left(\boldsymbol{m}_{k}\right)$ & Señal(es) medida(s) del proceso \\
\hline & \\
\hline &
\end{tabular}




\begin{tabular}{ll}
\hline & Notación genérica \\
\hline $\boldsymbol{\ell}, \boldsymbol{h}$ & Vector fila o columna \\
$\boldsymbol{A}$ & Matriz \\
$\boldsymbol{A}^{\boldsymbol{\top}}$ & Transpuesta de $\boldsymbol{A}$ \\
$\boldsymbol{A}^{-1}$ & Inversa de $\boldsymbol{A}$ \\
$\boldsymbol{I}$ & Matriz Identidad \\
$\mathbf{0}$ & Matriz nula \\
$u(\tau)$ & Señal de tiempo continuo \\
$u[t]$ & Señal discreta a periodo de control $T$ \\
$m_{k}$ & Señal muestreada en el instante $t_{k}$ \\
\hline & Acrónimos \\
\hline LMI & Desigualdad lineal matricial (Linear Matrix Inequality) \\
SCI & Sistema de control inferencial \\
SISO & Sistema monovaraible (Single Input Single Output) \\
MIMO & Sistema multivariable (Multiple Input Multiple Output) \\
LTI & Sistema lineal e invariante en el tiempo \\
LTPV & Sistema lineal variante en el tiempo de forma paramétrica (linear \\
& time parameter varying) \\
\hline
\end{tabular}




\section{Bibliografía}

[1] P. Albertos. Block multirate input-output model for sampled-data control systems. IEEE Transactions on Automatic Control, 35:535-544, 1990.

[2] P. Albertos, I. Peñarrocha, and P. García. Virtual sensors under delayed scarce measurements. 7th IFAC Symposium on Cost Oriented Automation (SCOA-2004), 2004.

[3] P. Albertos, R. Sanchis, and I. Peñarrocha. Initializing parameter estimation algorithms under scarce measurements. 13th IFAC Symposium on System Identification (SYSID-2003), 2003.

[4] P. Albertos, R. Sanchis, and A. Sala. Output prediction under scarce data operation: Control applications. Automatica, 35:1671-1681, 1999.

[5] A. Alessandri and P.Coletta. Switching observers for continuous-time and discrete-time linear systems. Proceedings of the American Control Conference, 2001.

[6] M. Araki and T. Hagiwara. Pole assignment by multirate data output feedback. International Journal of Control, 44(6):1661-1673, 1986.

[7] Bamieh B., J.B. Pearson, B.A. Francis, and A. Tannenbaum. A lifting technique for linear periodic systems with applications to sampled-data control. Systems and Control Letters, 17:79-88, 1991.

[8] M. Babaali and M. Egerstedt. On the observability of piecewise linear systems. 43rd IEEE Conference on Decision and Control, pages 1879-1884, 2003.

[9] S. Boyd, L. Gaoui, E. Feron, and V. Balakrishnan. Linear Matrix Inequalities in Systems and Control Theory. SIAM. Philadelphia, 1994.

[10] J. Bu and M. Sznaier. A linear matrix inequality approach to synthesizing low-order suboptimal mixed $\ell_{1} / \mathcal{H}_{p}$ controllers. Automatica, 36:957-963, 2000.

[11] T. Chen and B. Francis. Optimal Sampled-Data Control Systems. Springer, 1995.

[12] K.S. Chong and L. Kleeman. Accurate odometry and error modelling for a mobile robot. IEEE International Conference on Robotics and Automation, pages 2783-2788, 1997.

[13] M-Y. Chow and Y. Tipsuwan. Network-based control systems: a tutorial. The 27th Annual Conference of the IEEE Industrial Electronics Society, pages 1593-160, 2001.

[14] J. Daafouz and J. Bernussou. Parameter dependent lyapunov functions for discrete time systems with time varying parametric uncertainties. Systems \& Control Letters, 43(5):355-359, 2001.

[15] M.C. de Oliveira, J. Bernossou, and J.C. Geromel. A new discrete-time robust stability condition. Systems \& Control letters, 37:261-265, 1999.

[16] J.B.R. do Val E.F. Costa. On the detectability and observability of discrete-time markov jump linear systems. Systems $\&$ Control Letters, 44:135-145, 2001.

[17] E. Fridman, U. Shaked, and V. Suplin. Input/output delay approach to robust sampled-data $\mathcal{H}_{\infty}$ control. Systems \& Control Letters, 54:271-282, 2005.

[18] L. El Ghaoui and S.-L. Niculescu, editors. Advances in Linear Matrix Inequality Methods in Control. Advances in Design and Control. SIAM, 2000. 
[19] J. Gonzalez and A. Ollero. Estimación de la posición de un robot móvil. Informática y Automática, 29(4):3-18, 1996.

[20] M.T. Guilandoust, A.J. Morris, and M.T. Tham. An adaptive estimation algorithm for inferential control. Industrial Engineering Chemistry: Process design and development, 27:1658-1664, 1988.

[21] B. Halder, B. Hassibi, and T. Kailath. Mixed $\mathcal{H}_{2} / \mathcal{H}_{\infty}$ estimation: Preliminary analytic characterization and a numerical solution. Proceedings of the 1996 IFAC World Congress, J:37-42, 1996.

[22] U. Halldorsson and M. Fikar H. Unbehauen. Multirate nonlinear predictive control. Proceedings of the American Control Conference, pages 4191-4196, 2002.

[23] B Hassibi and T. Kailath. On nonlinear filters for mixed $\mathcal{H}_{2} / \mathcal{H}_{\infty}$ estimation. Proceedings of the American Control Conference, 1997.

[24] F-H. Hsiao and S-T. Pan. Robust kalman filter synthesis for uncertain multiple time-delay stochastic systems. Journal of Dynamic Systems, Measurement, and Control, 1996.

[25] B. Hu and A.N. Michel. Robustness analysis of digital feedback control systems with time-varying sampling periods. Journal of the Franklin Institute, 337:117-130, 2000.

[26] B. Hu and A.N. Michel. Stability analysis of digital feedback control systems with time-varying sampling periods. Automatica, 36:897-905, 2000.

[27] V. Ionescu. A stabilizability criterion for time varying discrete system. Journal of Dynamic Systems, Measurement, and Control, 1996.

[28] D-S. Kim, Y.S. Lee, W.H. Kwon, and H.S. Park. Maximum allowable delay bounds of networked control systems. Control Engineering Practice, 11:1301-1313, 2003.

[29] J.H. Kim, S.J. Ahn, and S. Ahn. Guaranteed cost and $\mathcal{H}_{\infty}$ filtering for discrete-time poilytopic uncertain sytems with time delay. Journal of the Franklin Institute, 2005.

[30] G.M. Kranc. Input-output analysis of multirate feedback systems. IEE Trans. Auto. Control, 31(2):315-319, 1957.

[31] S. Lall and G. Dullerud. An LMI solution to the robust synthesis problem for multi-rate sampled-data systems. Automatica, 37:1909-1922, 2001.

[32] T. D. Larsen, N. A. Andersen, O. Ravn, and N. K. Poulsen. Incorporation of time delayed measurements in a discrete-time kalman filter. In Proceeding ofIEEE 37th Conference of Decision and Control, pages 3972-3977, December 1998.

[33] J.H. Lee and M. Morari. Robust inferential control of multi-rate sampled-data systems. Chemical engeneering science, 47:865-885, 1992.

[34] R.P. Leland. Poisson LQR design for asynchronous multirate controllers. IEEE transactions on signal processing, 40(1):115-117, 1995.

[35] H. Li and M. Fu. A linear matrix inequality approach to robust $\mathcal{H}_{\infty}$ filtering. IEEE transactions on signal processing, 45(9):2338-2350, 1997.

[36] Q. Li, S.L. Shah, and T. Chen. Analysis of dual-rate inferential control systems. Automatica, 38:1053-1059, 2002.

[37] W. Li, S.L. Shah, and D. Xiao. Kalman filters for non-uniformly sampled multirate systems. 16th IFAC world congress, 2005.

[38] H. Lin and P.J Antsaklis. Stability and stabilizability of switched linear systems: A short survey of recent results. IEEE International Symposium on Intelligent Control, 2005.

[39] H. Lin, G. Zhai, and P.J Antsaklis. Robust stability and disturbance attenuation analysis of a class of networked control systems. IEEE Conference on Decision and Control (CDC03), 2003.

[40] Q. Ling and M.D. Lemmon. Optimal dropout compensation in networked control systems. IEEE Conference on Decision and Control (CDC03), 2003. 
[41] R. Lozano, P. Castillo, P. García, and A. Dzul. Robust prediction-based control for unstable delay systems: Application to the yaw control of a mini-helicopter. Automatica, 40:603-612, 2004.

[42] X. Lu, H. Zhang, W. Wang, and K-L. Teo. Kalman filtering for multiple time-delay systems. Automatica, 41:1455-1461, 2005.

[43] A. S. Matveev and A. V. Savkin. Optimal design of networked control systems: computer control via asynchronous communication channels. International Journal of Control, 77(16):1426-1437, 2004.

[44] L. Mirkin and N. Raskin. Every stabilizing dead-time controller has an observer-predictor-based structure. Automatica, 39:1747-1754, 2003.

[45] L. Montestruque and P.J. Antsaklis. Networked control systems: A model-based approach. Proceedings of the 12thIEEE Mediterranean Conference on Control and Automation, 2004.

[46] D.Ñesic and A.R. Teel. Input-to-state stability of networked control systems. Automatica, 40:21212128, 2004.

[47] R.M. Palhares and P.L.D. Peres. Robust filtering with guaranteed energy-to-peak performance - an LMI approach. Automatica, 36:851-858, 2000.

[48] R.M. Palhares and P.L.D. Peres. Robust $\mathcal{H}_{\infty}$ filter design with pole constraints for discrete-time systems. Journal of the Franklin Institute, 337:713-723, 2000.

[49] I. Peñarrocha, A. Sala, R. Sanchis, and P. Albertos. Output prediction under random measurements. an LMI approach. 16th IFAC World Congress, 2005.

[50] M.A. Peters and P.A. Iglesias. A spectral test for observability and reachibility of time varying systems and the riccati difference equation. Proceedings of the 38th Conference on Decision \&3 Control, 1999.

[51] L. Qiu and T.Chen. Multirate sampled-data systems: all $\mathcal{H}_{\infty}$ suboptimal controllers and the minimum entropy controller. IEEE Transactions on Automatic Control, 44(3):537-550, 1999.

[52] J.P. Richard. Time-delay systems: an overview of some recent advances and open problems. Automatica, 39:1667-1694, 2003.

[53] V.S. Ritchey and G.F. Franklin. A stability criterion for asynchronous multirate linear systems. IEEE transactions on signal processing, 34:529-535, 1989.

[54] M.F. Sagfors and H.T. Toivonen. $\mathcal{H}_{\infty}$ and LQG control of asynchronous sampled-data systems. Automatica, 33:1663-1668, 1997.

[55] A. Sala. Computer control under time-varying sampling period: an LMI gridding approach. Automatica, 41, 2005.

[56] R. Sanchis. Control de Procesos Industriales con Medidas Escasas. Tesis Doctoral, Universitat Politècnica de València, 1999.

[57] R. Sanchis and P. Albertos. Recursive identification under scarce measurements. convergence analysis. Automatica, 38:535-544, 2002.

[58] R. Sanchis and P. Albertos. Virtual sensors: model based output prediction. First EurAsia Conference on Advances in Information and Communication Technology, 2002.

[59] C. Scherer, P. Gahinet, and M. Chilali. Multiobjective output-feedback control via LMI optimization. IEEE Transactions on automatic control, 42(7):896-911, 1997.

[60] M. Schinkel, W-H Chen, and A. Rantzer. Optimal control for systems with varying sampling rate. Proceedings of the American Control Conference, 2002.

[61] T. Söderström. Discrete-time stochastic systems. Estimation \& Control. Series in Systems and Control Engineering. Prentice Hall, 1994.

[62] J. Sheng, T. Chen, and S.L. Shah. Generalized predictive control for non-uniformly sampled systems. Journal of process control, pages 875-885, 2002. 
[63] S. Skogestad and I. Postlethwaite. Multivariable feedback control. Analysis and Design. John Wiley \& Sons, 1996.

[64] O.J.M. Smith. Closer control of loops with dead time. Chemical Engineering Progress, pages 217-219, 1959.

[65] W. Tan, H.J. Marquez, and T. Chen. Imc design of unstable processes with time delays. Journal of Process Control, 13:203-213, 2003.

[66] Y. Tipsuwan and M-Y. Chow. Control methodologies in networked control systems. Control Engineering Practice, 11:1099-1111, 2003.

[67] J. Tornero, J. Salt, and P. Albertos. LQ optimal control for multirate sampled data systems. 14th IFAC World Congress, 1999.

[68] P.G. Voulgaris. Control of asynchronous sampled-data systems. IEEE Transactions on Automatic Control, 39:1451-1455, 1994.

[69] G.C. Walsh, O. Beldiman, and L.G. Bushnell. Error encoding algorithms for networked control systems. Automatica, 38:261-267, 2002.

[70] H. Wang, H. Zhang, and G. Duan. State estimation schemes for descriptor systems with multi-time delayed measurements. 16th IFAC world congress, 2005.

[71] J. Wang, C. Wang, and H. Gao. Robust $\mathcal{H}_{\infty}$ filtering for LPV discrete-time state-delayed systems. Nature and Science, 2(2):36-45, 2004.

[72] Y. Wang, L. Xie, and C.E. de Souza. Robust control of a class of uncertain nonlinear systems. System Control Letters, 19:139-149, 1999.

[73] Z. Wang, B. Huang, and H. Unbehauen. Robust $\mathcal{H}_{\infty}$ observer design of linear state delayed systems with parametric uncertainty: the discrete-time case. Automatica, 35:1161-1167, 1999.

[74] L. Xie, L. Lu, D. Zhang, and H. Zhang. Improved robust $\mathcal{H}_{2}$ and $\mathcal{H}_{\infty}$ filtering for uncertain discretetime systems. Automatica, 40:873-880, 2004.

[75] S. Xu and T. Chen. Robust $\mathcal{H}_{\infty}$ filtering for uncertain impulsive stochastic systems under sampled measurements. Automatica, 39:509-516, 2003.

[76] S. Xu, J. Lu, S. Zhou, and C. Yang. Design of observers for a class of discrete-time uncertain nonlinear systems with time delay. Journal of the Franklin Institute, 341:295-308, 2004.

[77] J.K. Yook, D.M. Tilbury, and N.R. Soparkar. Trading computation for bandwidth: Reducing communication in distributed control systems using state estimators. IEEE Transactions on Control Systems Technology, 10(4):503-518, 2002.

[78] D. Yue, Q-L. Han, and J. Lam. Network-based robust $\mathcal{H}_{\infty}$ control of systems with uncertainty. Automatica, 41(6):999-1007, 2005.

[79] H. Zhang, M.V. Basin, and M. Skliar. Optimal state estimation with continuous, multirate and randomly sampled measurements. Proceedings of the American Control Conference, 2004.

[80] W. Zhang, M.S. Branicky, and S.M. Phillips. Stability of networked control systems. IEEE Control Systems Magazine, pages 84-99, 2001.

[81] P.V. Zhivoglyadov and R.H. Middleton. Networked control design for linear systems. Automatica, 39:743-750, 2003. 